Relations industrielles

Industrial Relations

\title{
Index analytique - Relations industrielles - Volume 36-45 (1981-1990)
}

Volume 45, numéro 4, 1990

URI : https://id.erudit.org/iderudit/050627ar

DOI : https://doi.org/10.7202/050627ar

Aller au sommaire du numéro

Éditeur(s)

Département des relations industrielles de l'Université Laval

ISSN

0034-379X (imprimé)

1703-8138 (numérique)

Découvrir la revue

Citer cet article

(1990). Index analytique - Relations industrielles - Volume 36-45 (1981-1990).

Relations industrielles / Industrial Relations, 45(4), 675-1093.

https://doi.org/10.7202/050627ar
Résumé de l'article

Index Analytique Relations Industrielles Volume 36 - Volume 45 (1981-1990)
Tous droits réservés (C Département des relations industrielles de l'Université Laval, 1990
Ce document est protégé par la loi sur le droit d'auteur. L'utilisation des services d'Érudit (y compris la reproduction) est assujettie à sa politique d'utilisation que vous pouvez consulter en ligne.

https://apropos.erudit.org/fr/usagers/politique-dutilisation/ 


\section{INDEX ANALYTIQUE CUMULATIVE INDEX \\ RELATIONS INDUSTRIELLES \\ INDUSTRIAL RELATIONS}

Volume 36 - Volume 45

(1981-1990)

Rapport des - Proceedings of

\section{CONGRÈS DES RELATIONS INDUSTRIELLES} (1981-1990)

SOMMAIRE - CONTENT

Avant-propos

Gérard Dion

Foreword

A- Index analytique des articles

Subject Index

B- Index méthodique des articles

Broad Subject Headings Index .................... 809

Liste des descripteurs génériques

List of Generic Keywords . . . . . . . . . . . . . . . . . . . . . . . 813

Sujets couverts par les descripteurs genériques

Description of Contents of Each Generic Keyword.......... 813

C- Index méthodique des ouvrages recensés

Broad Subject Headings Index of Book Reviews ............ 859

D- Index des auteurs des ouvrages recensés

Index of Authors of Books Reviewed.................. 897

E- Index des auteurs des articles et des recensions

Index of Authors of Articles and Book Reviews ................ 949

F- Table chronologique des références bibliographiques

Chronological Table of Bibliographical References .......... 979 
DIRECTEUR - EDITOR

DIRECTEUR-ADJOINT - ASSOCIATE EDITOR

SECRÉTAIRE - SECRETARY

RESPONSABLE DES RECENSIONS - BOOK REVIEW EDITOR

ATTACHÉE À LA RÉDACTION - MANAGING EDITOR

\author{
Gérard DION
}

Jean SEXTON

Michel AUDET

Jean BOIVIN

Claudine LECLERC

COMITÉ DE RÉDACTION - EDITORIAL BOARD: Jean Boivin, Gérard Bélanger, Jacques Bélanger, Laurent Bélanger, Jean Bernier, René Boulard, Esther Déom, Gilles Ferland, Gilles Laflamme, Alain Larocque, Carla Lipsig-Mummé, Jacques Mercier, Gregor Murray, Michel Pérusse, Lise Poulin-Simon, Claude Rondeau, Bernard Solasse, Alain Vinet: Université Laval; Gérard Hébert, Viateur Larouche: Université de Montréal; Roland Thériault: William M. Mercer Ltée ; Shirley B. Goldenberg: McGill University; Daniel Benedict: Concordia University; Solomon Barkin, University of Massachusetts; Dimitri Weiss: Université de Paris I; Thomas A. Kochan: Massachusetts Institute of Technology; Phil Beaumont, University of Glasgow; John Niland, University of New South Wales. COMITÉ DU DROIT DU TRAVAIL - LABOUR LAW COMMITTEE: Rodrigue Blouin, Pierre Verge, André C. Côté, Fernand Morin: Université Laval.

Publication du Département des relations industrielles de la Faculté des sciences sociales de l'Université Laval (Québec). Reconnue officiellement par l'Association canadienne des relations industrielles et subventionnée par le Conseil de recherches en sciences humaines du Canada et par le Fonds FCAR. Paraît en janvier, avril, juillet et octobre: ses articles n'engagent que la responsabilité des auteurs et sont répertoriés dans Index de périodiques canadiens, RADAR (Répertoire analytique d'articles des revues du Québec), Employment Relations Abstract, Index to Canadian Legal Periodical Literature, Canadian Business Periodical Index, Human Resources Abstracts, Current Contents, Social Sciences Citation Index (SSCI); CERCC (France).

Published quaterly by the Department of Industrial Relations, Faculty of Social Sciences, Laval University (Québec) with the assistance of a grant from the Social Sciences and Humanities Research Council and from the Fonds FCAR. It is of ficially recognized by the Canadian Industrial Relations Association. Issued each January, April, July and October. Opinions expressed are those of the authors alone and not necessarily opinions held by the editors. The articles appear in the Canadian Periodical Index, RADAR (Répertoire analytique d'articles des revues du Québec), the Employment Relations Abstract, the Index to Canadian Legal Periodical Literature, Canadian Business Periodical Index, Human Resources Abstracts, Social Sciences Citation Index (SSCI), CERC (France), Current Contents.

ARTICLES: ne sont acceptés pour publication que les textes originaux soumis en exclusivité; doivent être adressés en quatre (4) excmplaires au directeur de la revue. Normes de publication fournies sur demande. The Journal publishes original manuscripts only which are submitted in exclusivity. Should be sent in four (4) copies to the Editor. Style sheets on request. Département des relations industrielles, Université Laval, Québec, Qué. Canada, G1K 7P4. Tél. (418) 656-3358, Télécopieur - FAX (418) 656-3175.

\section{ADMINISTRATION ET ABONNEMENTS - ADMINISTRATION AND SUBSCRIPTION}

Les Presses de I'Université Laval, C.P. 2477, Québec, Canada, G1K 7P4 (418) 656-3809 Télécopieur - FAX (418) 656-2600

Abonnement annuel - Annual Subscription:

Institutions: Canada, 48 \$ CAN; Étranger -- Foreign, 48 \$ US

Individus - Individuals: Canada, $24 \$$ CAN; Etranger - Foreign, 24 \$ US

Numéro ordinaire - Regular issue: $10 \$$

Abréviation officielle de la revue. Official Abbreviation of the Journal: Relat. ind. ISSN 0034-379X Dépôt légal Bibliothèque nationale du Québec; quatrième trimestre 1990.

Courrier de la deuxième classe - Enr, no 1745. (C) PUL 1990, décembre 


\section{AVANT-PROPOS}

Le présent index analytique est le second complément à l'index cumulatif publié dans le numéro 4 du volume 25 de la revue Relations industrielles et des Congrès des relations industrielles de l'Université Laval. Cet index couvrait la période de 1945 à 1970 pour la revue et de 1946 à 1970 pour les congrès, c'est-à-dire depuis la formation de ces deux institutions.

Un premier complément à cet index venait s'ajouter dans le numéro 4 du volume 35, couvrant la période 1971 à 1980, tant pour la revue que pour les congrès. Dans le but d'être utile aux chercheurs, nous avons décidé de publier ce second complément qui couvre la période de 1981 à 1990 de la revue et des congrès.

Les rapports de congrès répertoriés constituent chacun un ouvrage portant un titre particulier:

La réduction de la durée du travail (1981);

Les régimes de retraite (1982);

La syndicalisation dans le secteur privé au Québec (1983);

Régimes de santé et sécurité et relations du travail (1984);

Le statut de salarié en milieu de travail (1985);

La mobilisation des ressources humaines: tendances et impact (1986);

Les lésions professionnelles (1987);

Les Chartes des droits et les relations industrielles (1988);

Acquisition ou fusion d'entreprises et emplois (1989);

Le défi de la gestion des emplois (1990).

La préparation de cet index analytique 1990 a été entièrement complétée par Jean-Eudes Desgagnés, qui avait été l'artisan principal des index analytiques publiés en 1970 et en 1980. La saisie et le traitement des données publiées dans ce numéro furent effectuées par Monique Guillemette et Marcelle Potvin du groupe Interaction du Collège de Jonquière. L'indexation des recensions parues dans Relations industrielles a été complétée par Claudine Leclerc, attachée à la rédaction de la revue. Finalement, la préparation de cet Index analytique 1990 a été rendue possible grâce à une subvention de Travail Canada.

$\grave{A}$ tous, nous offrons nos remerciements pour leur collaboration. 
FOREWORD

This cumulative index is the second supplement to the index published in Volume 25 Number 4 of Relations industrielles / Industrial Relations covering the contents of the Journal and of the annual Industrial Relations Conferences of Universite Laval. This first index covered the period 1945 to 1970 for the Journal and 1946 to 1970 for the Conferences, i.e. since the founding of these institutions.

A first supplement was added in Volume 35 number 4 for the period 1971 to 1980 for both the Journal and the Conferences. In order to assist interested researchers, it has been decided to publish this second supplement for the period 1981 to 1990 for both the Journal and the Conferences. The latter includes a series of volumes on specific themes:

La réduction de la durée du travail (1981);

Les régimes de retraite (1982);

La syndicalisation dans le secteur privé au Québec (1983);

Régimes de santé et sécurité et relations du travail (1984);

Le statut de salarié en milieu de travail (1985);

La mobilisation des ressources humaines: tendances et impact (1986);

Les lésions professionnelles (1987);

Les Chartes des droits et les relations industrielles (1988);

Acquisition ou fusion d'entreprises et emplois (1989);

Le défi de la gestion des emplois (1990).

The preparation of this 1990 Cumulative Index was entirely completed by Jean-Eudes Desgagnés, principal artisan of both the 1970 and 1980 Cumulative Indexes. Data treatment for this issue was made by Monique Guillemette and Marcelle Potvin of the Interaction Group of Collège de Jonquière. The indexation of book reviews was assured by Claudine Leclerc, the managing editor of the Journal. Finally, the preparation of this Cumulative Index 1990 was made possible by a grant from Labour Canada.

To all our contributors, a sincere message of thanks.

The Editor

Gérard Dion 


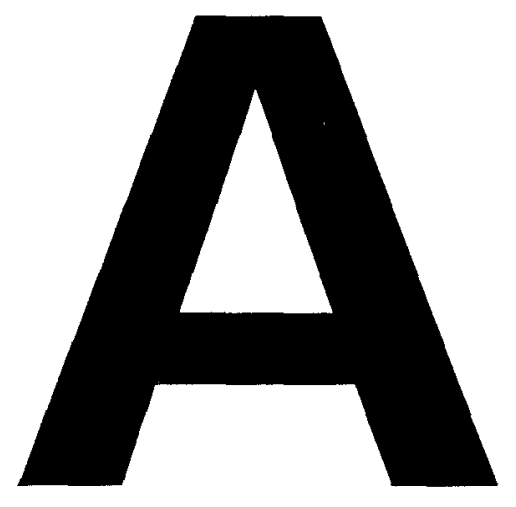

INDEX ANALYTIQUE DES ARTICLES

SUBJECT INDEX 
L'Index analytique signale les articles au moyen de descripteurs spécifiques, classés alphabétiquement et désignant des sujets précis. Pour chaque article, on retrouve l'auteur, le titre et la référence bibliographique, de même qu'un numéro d'accès permettant de renvoyer l'utilisateur à la section " $F$ " (Table chronologique des références bibliographiques) pour y obtenir le résumé de l'article.

\section{EXEMPLE:}

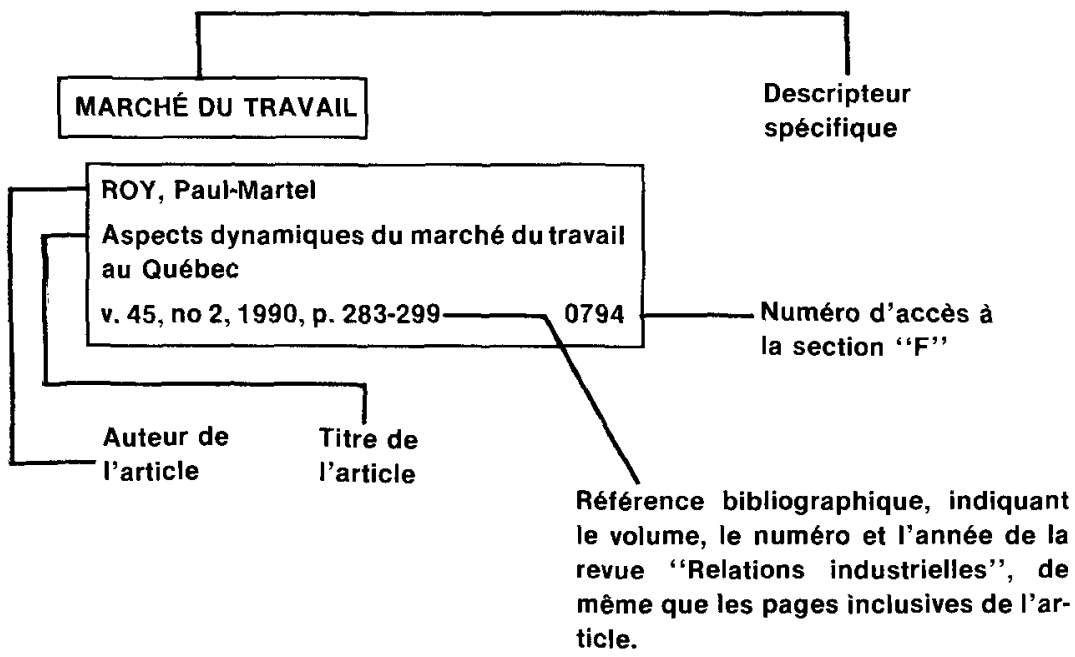

NOTE: Lorsqu'il s'agit d'un article paru dans un "Congrès des relations industrielles", la référence bibliographique indique pour chaque article, le numéro et l'année du Congrès de même que les pages inclusives de l'article. EXEMPLE: 45e Congrès, 1990, p. 7-14. 
Entries in the "Subject Index" consist of specific keywords classified in alphabetical order and assigned to the article to describe its subject contents. For each article one finds author, title and bibliographical reference, as well as an accession number which permits the user to refer to Section "F" (Chronological Table of Bibliographical Reference) in order to obtain the abstract of the article.

\section{EXAMPLE:}
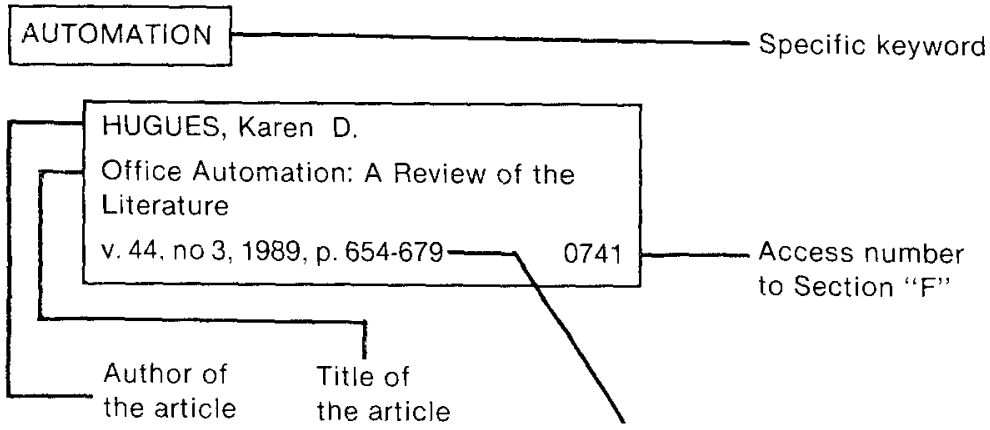

Bibliographical reference giving volume, number and year of "Industrial Relations" as well as the page reference.

NOTE: For articles published in "Congrès des relations industrielles", the bibliographical reference gives number and year of the Conference (Congrès) as well as page reference. EXAMPLE: 45e Congrès, 1990, p. 7-14. 



\section{A}

\section{ABATTOIRS (INDUSTRIE)}

FORREST, Anne

The Rise and Fall of National Bargaining in the Canadian Meat-Packing Industry.

v. 44 , no 2,1989 , p. $393-408$

0723

ABITIBI PRICE INC.

CASAVANT, Jean-Claude

La stratégie de gestion des ressources humaines chez Abitibi Price Inc.

41ième Congrès, 1986, p. 53-61 0908

BOUCHER, Lysette

Les limites des nouvelles approches en gestion des ressources humaines.

41ième Congrès, 1986, p. 77-84

0911

\section{ABSENTÉISME}

THÉRIAULT, Roland LESAGE, Pierre B. BOISVERT, Maurice

L'absentéisme: importance, nature et remèdes.

v. 36 , no 4,1981 , p. $775-802$

0073

DOLAN, Shimon L. ARSENAULT, André LIZOTTE, Jean-Paul ABENHAIM, Lucien L'absentéisme hospitalier au Québec: Aspects culturels et socio-démographiques.

v. 38 , no 1,1983, p. $45-57$

0179

LÉONARD, Christine

VAN AMÉRINGEN, Marie-Reine

DOLAN, Shimon L. ARSENAULT, André Absentéisme et assiduité au travail: Deux moyens d'adaptation au stress.

v. 42, no 4,1987 , p. $774-789 \quad 0609$

HACCOUN, Robert R. DUPONT, Serge Une analyse des comportements de travailleurs masculins et féminins selon deux formes d'absence au travail.

v. 43, no 1,1988 , p. $153-166$

0634

\section{ACCIDENTS DU TRAVAIL}

LAFLAMME, Lucie ARSENAULT, André Rémuneration, postes de travail et accidents: Une relation interactive.

v. 39 , no 3,1984 , p. $509-525$
ACCIDENTS DU TRAVAIL (suite)

BRODY, Bernard ROHAN, Paul

ROMPRÉ, Louise

Les accidents industriels au Canada: Le portrait d'une décennie

v. 40 , no 3,1985 , p. $545-566$

0409

SIMARD, Marcel BOUTEILLER, Dominique LÉVESQUE, Christian

Prévention des accidents du travail: Contexte législatif québécois et efficacité organisationnelle.

v. 40, no 4,1985 , p. $703-719$

0424

LAFLAMME, Lucie VINET, Alain

Accidents du travail et modernisation du processus de production: le cas de l'industrie forestière québécoise.

v. 43, no 3,1988 , p. $591-608$

0669

WEST, Leigh

The Exclusive Remedy Provision in Canadian Worker Compensation Law: The Need for Legislative Reform.

v. 43, no 4,1988 , p. $890-908 \quad 0689$

BRODY, Bernard LÉTOURNEAU, Yves POIRIER, André

Le coût des accidents du travail: État des connaissances.

v. 45 , no 1,1990 , p. $94-117 \quad 0776$

BEAUSOLEIL, Gilles

Les coûts et les benéfices du régime.

39ième Congrès, 1984, p. 207-242 0885

PÉRUSSE, Michel

Les lésions professionnelles - une problématique.

42ième Congrès, 1987, p. 1-8 0923

CHIASSON, Denis-Émile

Synopsis sur le nouveau régime.

42ième Congrès, 1987, p. 9-19 0924

LESAGE, Michel

Les lésions professionnelles: Point de vue d'un médecin.

42ième Congrès, 1987, p. 39-50 0926 
ACCIDENTS DU TRAVAIL (suite)

PRÉVOST, Charles

La sous-estimation des atteintes à la santé causées par les mauvaises conditions de travail.

42ième Congrès, 1987, p. 51-60 0927

FARQUHAR, Alec

Le régime des accidents du travail: Le cas de l'Ontario.

42ième Congrès, 1987, p. 115-130 0932

MORIN, Fernand

Le régime actuel d'indemnisation pour lésions professionnelles: Accessibilité et efficacité.

42ième Congrès, 1987, p. 131-155 0933

LEFÈBVRE, Marie-Claire

LEVASSEUR, Raymond

Commentaires - Le régime actuel d'indemnisation pour lésions professionnelles: Accessibilité et efficacité.

42ième Congrès, 1987, p. 155-176 0934

BERNIER, Lionel

Équité, indemnisation des victimes de lésions professionnelles et coûts à l'entreprise.

42ième Congrès, 1987, p. 177-211 0935

GINGRAS, Claude DUCHESNE, André Commentaires - Équité, indemnisation des victimes de lésions professionnelles et couts à l'entreprise.

42ième Congrès, 1987, p. 212-222 0936

SURET, Jean-Marc GENDRON, Michel BERNIER, Gilles

Le processus de gestion des risques, les lesions professionnelles et la CSST.

42ième Congrès, 1987, p. 223-241 0937

HARGUINDEGUY, Jean-Louis

BRODY, Bernard

Commentaires - Le processus de gestion des risques, les lésions professionnelles et la CSST.

42ième Congrès, 1987, p. 241-252 0938

\section{ACCORD DU LIBRE-ÉCHANGE}

ROY, Paul-Martel

Aspects dynamiques du marché du travail au Québec.

v. 45, no 2,1990 , p. $283-299$

0794

\section{ACCORDS ATYPIQUES}

VALLEEE, Guylaine

Les accords "atypiques" et le droit des rapports collectifs du travail.

v. 44 , no 3,1989 , p. $680-690 \quad 0742$

\section{ACCRÉDITATION SYNDICALE}

LAVERY, Daniel

Décisions rendues par le Conseil canadien des relations du travail.

v. 37, no 3,1982 , p. $684-696 \quad 0146$

LAVERY, Daniel

Décisions rendues par le Conseil canadien des relations du travail.

v. 37 , no 4,1982 , p. $944-959$

0167

LAVERY, Daniel

Décisions rendues par le Conseil canadien des relations du travail.

v. 38 , no 1,1983 , p. 159-173 0187

LAVERY, Daniel

Décisions rendues par le Conseil canadien des relations du travail.

v. 38 , no 3,1983 , p. $658-666$

0231

SOLOMON, Norman A.

The Negociation of First Agreement in Ontario.

v. 39 , no 1,1984 , p. 23-35

0269

SOLOMON, Norman A.

The Negociation of First Agreements under the Canada Labour Code.

v. 40 , no 3,1985, p. $458-472 \quad 0404$

LAMY, Francine

Décision rendue par le Conseil canadien des relations du travail

v. 40, no 4,1985, p. $880-885$

0434

SAINT-GERMAIN, Catherine

Décisions rendues par le Conseil canadien des relations du travail.

v. 41 , no 2,1986 , p. 397-411

0477

SAINT-GERMAIN, Catherine

Décisions rendues par le Conseil canadien des relations du travail v. 42 , no 2,1987, p. $415-427$

0569 


\section{ACCRÉDITATION SYNDICALE (sulte)}

SAINT-GERMAIN, Catherine

Décision rendue par le Conseil canadien des relations du travail.

v. 42 , no 3,1987, p. $628-635$

0589

TREMBLAY, Johane

Décisions rendues par le Conseil canadien des relations du travail.

v. 43, no 2,1988 , p. $447-452$

0655

MORIN, Fernand

D'un entrepreneur à un autre: l'accréditation ne suit pas

v. 44 , no 2,1989 , p. $315-336$

0719

CLARKE, Graham

Décision rendue par le Conseil canadien des relations du travail.

v. 45, no 3,1990, p. $616-624$

0820

\section{DESMARAIS, Jacques}

Les idées de réforme sur la syndicalisation au Québec depuis 1964.

38ième Congrès, 1983, p. 101-116 0864

LABERGE, Louis

Les préalables à une réorientation des relations du travail au Québec.

41ième Congrès, 1986, p. 173-175 0920

\section{ACQUISITIONS D'ENTREPRISES}

BÉLANGER, Laurent LIPSIG, Carla MORIN, Fernand PÉRUSSE, Michel Introduction - Acquisition ou fusion d'entreprises et emplois.

44ième Congrès, 1989, p. XV-XVI 0956

MORIN, Fernand

Acquisition ou fusion d'entreprises et emplois: La problematique sous-jacente.

44ième Congrès, 1989, p. 1-12 0957

BÉLANGER, Marc LE BRASSEUR, Lola L'ITALJEN, Paul MÉNARD, Marius Rappel de quelques expériences vécues. 44ième Congrès, 1989, p. 13-32 0958

CÓTÉ, André C. FONTAINE, Claude LESAGE, Paul

Aspects et implications juridiques des restructurations.

44ième Congrès, 1989, p. 33-84 0959
ACQUISITIONS D'ENTREPRISES (suite)

ANGERS, Georges GAGNON, Normand SIMARD, Jean

Le droit et le devoir à l'information.

44ième Congrès, 1989 , p. 85-100 0960

LAMARCHE, André GAUTHIER, Michel

BLAIS, Michel GAGNON, Denise

Les conditions de travail au lendemain d'une fusion ou d'une réorganisation.

44ième Congrès, 1989, p. 101-1270961

GAGNON, Astrid BLANCHETTE, Sylvain QUINTAL, Pierre

Fusion d'entreprises publiques.

44ième Congrès, 1989, p. 129-154 0962

GOSSELIN, Alain FRENETTE, Jean-Guy

DIONNE, Denis

Les acteurs d'une fusion ou d'une prise de pouvoir.

44ième Congrès, 1989, p. 155-173 0963

ROUSSEAU, Léontine

Annexe - Acquisitions, fusions, offres publiques d'achat: Notions de base et facteurs considérés.

44ième Congrès, 1989, p. 189-252 0965

ACTE DE L'AMÉRIQUE DU NORD BRITANNIQUE

JOSEPH, P.A.

Perfecting the Administrative Solution to Labour Disputes.

v. 38, no 4,1983, p. $863-868 \quad 0253$

ACTE dES MANUfACTURES DE QUÉBEC (1885)

CÔTÉ, André C.

L'Acte des manufactures de Québec, 1885: Un centenaire

v. 40, no 3,1985 , p. $623-628 \quad 0412$

\section{ACTION POLITIQUE SYNDICALE}

DION, Stéphane

Syndicats et politique au niveau municipal en France.

v. 39 , no 3.1984 , p. $466-485 \quad 0315$

MAC NEIL, Michael

Unions, Politics and Law in Canada.

v. 43 , no 4,1988, p. $847-868 \quad 0687$ 


\section{ACTION SOCIALE}

POOLE, Michael

Perceptions of Trade Union Members and the Social Action Perspective.

v. 36 , no 1,1981 , p. $35-62$

0002

\section{ACTION SYNDICALE}

BROSSARD, Michel

La strategie syndicale face aux groupes semi-autonomes de production: Hypothese pour l'analyse du cas Rushton.

v. 37, no 3,1982 , p. $670-683$

0145

BARKIN, Solomon

Troubled Worker Militancy: Challenges Confronting Western Industrial Relations Systems.

v. 38, no 4,1983, p. $713-729$

0245

\section{ACTION TRAVAIL DES FEMMES}

BOIVIN, Suzanne P.

Le Canadien National: Un cas riche d'enseignements.

43ième Congrès, 1988, p. 97-113 0947

\section{ADAMS, ROY J.}

MARTIN, RosS BLACK, Errol

Roy J. Adams' Proposal for a Training Levy Scheme.

v. 36 , no 2,1981 , p. $414-417 \quad 0033$

\section{ÂGE DES TRAVAILLEURS}

GUÉRIN, Gilles HÉBERT, Michel

Les obstacles rencontrés par des personnes de 45 à 64 ans à la recherche d'un emploi.

v. 45 , no 2,1990 , p. $235-267 \quad 0792$

LEBRASSEUR, Rolland

Retirement and Skill Issues in Northern Ontario Industries.

v. 45, no 2,1990 , p. $268-282 \quad 0793$

\section{AGENCE CANADIENNE D'EMPLOIS}

MAGUN, Sunder

The Placement Activity of the Canadian

Employment Agency.

v. 38 , no 1,1983, p. $72-94$

0181

\section{AGENTS DE COURTAGE IMMOBILIER}

DOLAN, Shimon L. TZINER, Aharon ROY, Denis

A Real Estate Agency's Level Analysis of the Climate-Performance Relationship.

v. 39 , no 1,1984, p. 167-176

0276

\section{AGENTS DE MAÎTRISE}

KNIGHT, Thomas R.

Correlates of Informal Grievance Resolution Among Fist-Line Supervisors

v. 41 , no 2,1986 , p. 281-298 0469

\section{ALBERTA}

AOUST, Claude d'

Les ententes individuelles et la convention collective.

v. 38 , no 1,1983 , p. 155-158 0186

FISHER, E.G. IVANKOVICH, I.F.

Alberta's Occupational Health and Safety Amendment Act, 1983.

v. 40 , no 1,1985, p. 115-139 0368

BRYCE, George K. MANGA, Pran

The Effectiveness of Health and Safety Committees.

v. 40, no 2,1985, p. $257-283 \quad 0385$

FISHER, E.G. KUSHNER, Stephen

Alberta's Construction Labour Relations During the Recent Downturn

v. 41 , no 4,1986 , p. $778-801$

0518

RESHEF, Yonatan

Negotiating Wage Settlements: A Structural Approach.

v. 44 , no 3,1989, p. $532-551 \quad 0735$

\section{ALBERTA GOVERNMENT TELEPHONE}

SAINT-GERMAIN, Catherine

Decision rendue par le Conseil canadien des relations du travail.

v. 42, no 3, 1987, p. 628-635 0589

\section{ALCOOLISME}

BEAUMONT, P.B.

The Diffusion of Human Resource Management Innovations.

v. 40, no 2,1985, p. $243-256 \quad 0384$

\section{ALIÉNATION}

BARTH, Richard T. BABA, Vishwanath V. Alienation Among Professionnal Engineers:

A Canadian - American Comparison.

v. 37 , no 1,1982, p. $126-140 \quad 0100$ 


\section{ALLEMAGNE DE L'OUEST}

MUCKENBERGER, Ulrich

The Regulation of Strike Law in Times of New Technologies and Deregulation: The Case of West Germany.

v. 45 , no 1,1990, p. $136-145 \quad 0778$

\section{AMÉRIQUE DU NORD}

JAIN, Hem C.

Disclosure of Corporate Information to Trade Unions in North America.

v. 36 , no 4,1981, p. $748-7740072$

CROWLEY, R.W. HUTH, E.

An International Comparison of Work Sharing Programs.

v. 38 , no 3,1983, p. $636-647 \quad 0230$

LIPSIG-MUMME, Carla

La crise du syndicalisme nord-américain: éléments d'interprétation.

v. 39, no 2,1984 , p. $275-284$

0294

JAIN, Hem C. GILES, Anthony

Workers' Participation in Western Europe: Implications for North America.

v. 40, no 4,1985, p. $747-7740426$

\section{AMÉRIQUE LATINE}

FALABELLA, Gonzalo

El sindicato en el ano 2000 temas, y desafios.

v. 44 , no 1,1989, p. 47-61

0701

\section{APPARICIO-VALDEZ, Luis}

La gestion empresarial en latinoamérica y su impacto en las relaciones laborales

v. 44 , no 1,1989 , p. 124-148 0704

\section{ZAPATA, Francisco}

La proteccion del empleo en las américas.

v. 44, no 1,1989, p. $177-194$

0707

LUCENA, Hector

Papel del Estado en las relaciones industriales en América Latina

v. 44 , no 1,1989, p. $249-282$

0710

\section{AMÉRIQUES}

SEXTON, Jean DION, Gérard

L'avenir des relations industrielles dans les Ameriques: Introduction.

v. 44 , no 1,1989, p. 1-2

0698

\section{AMIANTE}

SWIMMER, Gene LUCE, Sally R.

Asbestos Exposure and Attitudes Toward Occupational Health.

v. 40 , no 3,1985 , p. $529-544 \quad 0408$

\section{AMNISTIE}

AOUST, Claude d'

L'amnistie des fautes disciplinaires.

v. 43, no 4,1988 , p. $909-942$

0690

\section{ANALYSE LOGIT}

HOLMES, R.H. PINFIELD, L.T.

Logit Analysis of Employee Turnover in a Remote Community

v. 39, no 3,1984 , p. $553-569 \quad 0320$

\section{ANCIENNETÉ}

MORIN, Fernand

La survie de droits subjectifs à la convention collective.

v. 40 , no 4,1985 , p. $847-855$

0431

CHAYKOWSKI, Richard P.

SI_OTSVE, G.A.

Union Seniority Rules as a Determinant of Intra-Firm Job-Changes.

v. 41 , no 4,1986 , p. $720-737 \quad 0515$

\section{ANGLETERRE}

WARNER, Malcolm

Corporatism, Participation and Society

v. 38 , no 1,1983 , p. 27-44

0178

\section{ANTIGONISH}

GILSON, Clive H.J. SPENCER, lan S. GRANVILLE, S.

The Impact of a Strike on the Attitudes and Behavior of a Rural Community.

v. 44, no 4, 1989, p. 785-804 0753

\section{ANTISYNDICALISME}

BEAUMONT, P.B. TOWNLEY, B.

Non-Union American Plants in Britain:

Their Employment Practices.

v. 40, no 4,1985 , p. $810-825$

0429

BARKIN, Solomon

The Current Unilateralist Counterattack on Unionism and Collective Bargaining

v. 41 , no 1,1986 , p. $3-27$

0447 
ANTISYNDICALISME (suite)

BARBASH, Jack

The New Industrial Relations in the US:

Phase II.

v. 43, no 1,1988, p. $32-42$

0627

RESHEF, Yonatan

Changing Environments and Management IR Practices: Implications for U.S. Trade Unions.

v. 43, no 1,1988, p. $43-62$

0628

PERREAULT, Charles

L'entreprise peut-elle se passer d'un syndicat?

38ième Congrès, 1983, p. 173-182 0868

GAUTHIER, Fernand ROY, Paul-Martel

Commentaires - L'entreprise peut-elle se passer d'un syndicat?

38ième Congrès, 1983, p. 183-195 0869

\section{APPARTENANCE À L'ORGANISATION}

AMERNIC, J.H. ARANYA, N.

Organizational Commitment: Testing Two Theories.

v. 38 , no 2,1983 , p. $319-343 \quad 0206$

BABA, Vishwanath V. KNOOP, Robert Organizational Commitment and Independence Among Canadian Managers

v. 42, no 2,1987, p. $325-344$ 0564

\section{APPRENTISSAGE}

WEIERMAIR, Klaus

Apprenticeship Training in European Countries: The Lessons for Canada.

v. 37, no 3,1982, p. $557-574$

0139

\section{APPROCHE HISTORIQUE}

GILES, Anthony MURRAY, Grégor Towards an Historical Understanding of Industrial Relations Theory in Canada.

v. 43 , no 4 , 1988 , p. $780-811$

0684

\section{APPROCHE STRATÉGIQUE}

LAFLAMME, Gilles VALLÉE, Guylaine Changements technologiques et modes régulateurs des relations du travail. v. 42 , no 4,1987 , p. $702-715$

0605

\section{APPROCHE SYSTÉMIQUE}

LAROUCHE, Viateur DÉOM, Esther

L'approche systémique en relations indus-

trielles

v. 39 , no 1,1984, p. 114-145 0274

SHIROM, Arie

The Labor Relations System: A Proposed Conceptual Framework

v. 40, no 2,1985, p. $303-323 \quad 0387$

ARBITRAGE DES DIFFÉRENDS

ADAMS, Georges W.

The Ontario Experience with Interest arbitration: Problems in Detecting Policy

v. 36 , no 1,1981 , p. $225-250 \quad 0012$

BRUCE, Christopher J.

The Role of Information Concerning the Arbitrator's Preferences.

v. 36 , no 2,1981 , p. $386-402 \quad 0030$

CAIRE, Guy

Procédures de règlement pacifique des conflits collectifs en France.

v. 38, no 1, 1983, p. 3-27 0177

ANDIAPPAN, P. CATTANEO, R. Julian

MURPHY, John

Interest Arbitration in Ontario Hospitals:

Result of an Attitude Survey of Union and

Management Officials.

v. 39 , no 4,1984 , p. $680-694 \quad 0338$

HAMEED, Syed M.A. SEN, Joya

A Power Theory of Third Party Intervention in Labour Management Relations.

v. 42 , no 2,1987 , p. $243-255$

0559

SEXTON, Jean

L'arbitrage de première convention collective au Québec: 1978-1984

v. 42 , no 2,1987, p. $272-291$

0561

SUBBARAO, A.V.

Criteria in Arbitration of Wage Disputes: Theory and Practice in the Canadian Federal Public Service.

v. 43 , no 3,1988 , p. $547-570 \quad 0667$ 


\section{ARBITRAGE DES GRIEFS}

AOUST, Claude d'

Les réclamations à l'arbitrage de dommages résultant de la violation d'une clause de renonciation à la greve.

v. 36 , no 1,1981, p. 259-262

0014

AOUST, Claude d'

La juridiction de l'arbitre et de la Cour des petites créances devant la Cour suprême du Canada.

v. 36 , no 2,1981 , p. $418-424$

0034

MARTINEAU, LUC

Décisions rendues par le Conseil canadien des relations du travail.

v. 36 , no 3,1981 , p. $671-680$

0060

MOORE, William R.

Justice and the Grievance Procedure in the Federal Public Service.

v. 36 , no 4,1981 , p. $848-864$

0076

DESCHÊNES, Jean-Paul

L'évaluation des emplois et la convention collective.

v. 39 , no 2,1982 , p. $313-327$

0121

BEIGBEDER, Yves

Individual Grievance Procedures in United Nations Secretariats.

v. 37 , no 2,1982, p. 328-343

0122

AOUST, Claude d'

Les dispositifs d'une sentence arbitrale: quelques pièges à éviter.

v. 38, no 2,1983 , p. $415-417 \quad 0210$

AOUST, Claude d'

Effets d'annulation d'un avis de congédiement par un arbitre

v. 39, no 2,1984, p. $365-370$

0299

FISHER, E.G. SHERWOOD, L.M.

Fairness and Managerial Rights in Canadian Arbitral Jurisprudence

v. 39 , no 3,1984 , p. $538-552$

0319

GILSON, Clive H.J. GILLIS, L.P. Grievance Arbitration in Nova Scotia.

v. 42 , no 2,1987, p. $256-271$

0560
ARBITRAGE DES GRIEFS (sulte)

CARTER, Donald D.

Grievance Arbitration and the Charter: The Emergency Issues.

v. 44 , no 2,1989 , p. $337-353 \quad 0720$

MORIN, Fernand

Liberté d'expression et droit au travail:

l'arbitrage de la Cour supreme du Canada.

v. 44, no 4,1989, p. $921-932 \quad 0761$

ARBITRAGE DES PROPOSITIONS FINALES

BLACK, Errol SILVER, Jim

Contradictions and Limitations of Final Offer Selection: The Manibota Experience.

v. 45 , no 1,1990, p. $146-165$

0779

GRANT, Hugh $M$.

Contradictions and Limitations of Final Offer Selection: The Manitoba Experience.

A Comment.

v. 45 , no 1,1990 , p. $166-168 \quad 0780$

BLACK, Errol SILVER, Jim

FOS in Manitoba. A Rejoinder.

v. 45 , no 3,1990, p. $612-615$

0819

\section{ARBITRAGE OBLIGATOIRE}

FORREST, Anne

Bargaining Units and Bargaining Power

v. 41 , no 4,1986 , p. $840-850$

0523

ARMÉE

TZINER, Aharon DOLAN, Shimon L.

Identifying Female Officer Potential: An

Exploration in Predictors' Payoff

v. 40 , no 1,1985 , p. $87-98$

0366

\section{ASSIDUITÉ AU TRAVAIL}

LÉONARD, Christine

VAN AMÉRINGEN, Marie-Reine

DOLAN, Shimon L. ARSENAULT, André Absentéisme et assiduité au travail: Deux moyens d'adaptation au stress.

v. 42 , no 4,1987 , p. $774-789 \quad 0609$

ASSOCIATION CANADIENNE DES RELATIONS INDUSTRIELLES

HÉBERT, Gérard

Distinction 1982 de l'Association canadienne des relations industrielles: Gerard Dion.

v. 37, no 3, 1982, p. 475-476 0135 
ASSOCIATION CANADIENNE DES RELATIONS INDUSTRIELLES (suite)

HÉBERT, Gérard

L'état de la discipline en relations industrielles au Canada: Un brin d'histoire et une postface./The State of the Art in Industrial Relations: A Brief History and a Postcript.

v. 44 , no 4,1989 , p. $884-904$

0758

BARKIN, Solomon

The State of the Art in Industrial Relations:

A U.S. Commentary.

v. 44 , no 4,1989 , p. $905-914$

0759

WEISS, Dimitri

L'état de la discipline en relations industrielles au Canada: Un commentaire européen.

v. 44 , no 4,1989 , p. $914-920$

0760

\section{ASSOCIATIONS D'EMPLOYEURS}

THWAITES, James

Business "Organizes": The Early Years in Quebec.

v. 36 , no 2,1981 , p. $403-411$

0031

\section{ASSURANCE-CHÓMAGE}

GRUBEL, Herbert G. MAKI, Dennis R.

A Note on the Effects of Unemployment Insurance, Minimum Wage Legislation and Trade Union Growth on Reported Unemployment Rates in Canada, 1950-75

v. 36 , no 4,1981 , p. $922-927$

COUSINEAU, Jean-Miche!

Objectifs et modalites de l'assurance-chómage au Canada: 1940-1986

v. 41 , no 3,1986 , p. $451-468$

0486

\section{ATTITUDES}

RESHEF, Yonatan

A Typology of Shop Stewards: A Confirmatory Factor Analysis

v. 42 , no 1,1987 , p. $150-167$

0545

\section{ATTITUDES MILITANTES SYNDICALES}

MCSHANE, Steven L.

Sources of Attitudinal Union Militancy

v. 40, no 2,1985 , p. $284-302$

0386

AUROUX, J.

CAIRE, Guy

Les lois Auroux

v. 39 , no 2,1984, p. $235-258$
AUROUX, J. (suite)

DELAMOTTE, Yves

La loi et la négociation collective en France: Réflexion sur l'expérience 1981-1985.

v. 42 , no 1,1987 , p. $92-109$

\section{AUSTRALIE}

GURDON, Michael A.

Divergent Paths: Civil Service Employment Relations in Australia and Canada.

v. 42 , no 3,1987 , p. $566-575 \quad 0585$

\section{AUTOMATION}

HUGUES, Karen D.

Office Automation: A Review of the Litterature

v. 44 , no 3,1989, p. $654-679 \quad 0741$

VALASKAKIS, Kimon

Le temps libéré: à quels coûts?

36ième Congrès, 1981, p. 61-74 0836

POULIN-SIMON, Lise FORTIN, Bernard

Commentaires - Le temps libéré: à quels coûts?

36ième Congrès, 1981 , p. 75-86 0837

\section{AUTORITÉ}

RAINVILLE, Jean-Marie

La responsabilité fonctionnelle chez les cadres subalternes: Facteurs d'evolution des systèmes d'autorité.

v. 38 , no 4,1983, p. $831-846 \quad 0251$

\section{AVANTAGES SOCIAUX}

COUSINEAU, Jean-Michel

LACROIX, Robert

La détermination des avantages sociaux au Canada

v. 39 , no 1,1984, p. $3-22 \quad 0268$

GOBEILLE, Kenneth R.

Négocier la flexibilité: Le travail à temps partiel.

45ième Congrès, 1990, p. 48-58 0971

\section{AVOCATS}

OSBERG, Lars

A Note on the Incomes of Lawyers

v. 40 , no 4,1985 , p. $865-879$ 
AVOCATS (suite)

STAGER, David A.A.

Lawyers' Earnings in the Canadian Private and Public Sectors.

v. 43 , no 3,1988 , p. $571-590$

0668

\section{B}

\section{BANQUE ROYALE DU CANADA}

MARTINEAU, LUC

Décisions rendues par le Conseil canadien des relations du travail.

v. 36, no 1,1981 , p. 263-266

0015

MARTINEAU, LUC

Décisions rendues par le Conseil canadien des relations du travail.

v. 36, no 2,1981 , p. $424-429$

0035

\section{BANQUES}

PONAK, Allen MOORE, Larry F.

Canadian Bank Unionism: Perspectives and issues

v. 36, no 1,1981 , p. $3-34$

0001

LOWE, Graham S.

Causes of Unionization in Canadian Banks.

v. 36 , no 4,1981 , p. $865-893$

0077

\section{SAINT-GERMAIN, Catherine}

Décisions rendues par le Conseil canadien des relations du travail.

v. 41 , no 2,1986 , p. $397-411$

0477

TREMBLAY, Johane

Décisions rendues par le Conseil canadien des relations du travail.

v. 43, no 2,1988 , p. $447-452$

0655

GILSON, Clive H.J, SPENCER, lan S.

GRANVILLE, S.

The Impact of a Strike on the Attitudes and Behavior of a Rural Community.

v. 44 , no 4,1989, p. $785-804$

0753

\section{BEAUDRY, RENÉ}

RICHER, Jean-Charles

La Corporation professionnelle des conseillers en relations industrielles du Québec devant la Commission Beaudry.

v. 40, no 2,1985, p. $379-385$

0393

\section{BEHAVIORISME}

XISHAN, Yang MOORE, Larry F.

MARKGRAF, Herb

Behavioural Science Application in Vancouver Based Firms.

v. 38 , no 1,1983 , p. 120-141 0184

\section{BESOINS}

JAMAL, Muhammad BABA, Vishwanath V. MITCHELL, Vance F.

The Nature of Need Structure in Norrwork.

v. 37 , no 3,1982 , p. $618-633 \quad 0142$

\section{BONNE FOI}

BEMMELS, Brian FISHER, E.G.

NYLAND, Barbara

Canadian-American Jurisprudence on "Good Faith" Bargaining.

v. 41 , no 3,1986 , p. $596-621 \quad 0496$

SAINT-GERMAIN, Catherine

Décisions rendues par le Conseil canadien des relations du travail

v. 41, no 3,1986 , p. $622-637$

0497

\section{BOUVIER, ÉMILE}

HÉBERT, Gérard

In memoriam: Émile Bouvier, S.J. 19061985

v. 40 , no 2,1985 , p. 211-212 0381

LACOSTE, Paul

L'École des relations industrielles de l'Université de Montréal: Quarantième anniversaire.

v. 40 , no 2,1985 , p. $213-218 \quad 0382$

BRISEURS DE GRÈVE

CLARKE, Graham

Décisions rendues par le Conseil canadien des relations du travail.

v. 45, no 1, 1990, p. 187-197 0782

BUREAU INTERNATIONAL DU TRAVAIL BLANCHARD, Francis

Les relations professionnelles et les defis des années 80.

v. 37, no 1, 1982, p. 3-14 0094

\section{BUREAUTIQUE}

AUDET, Claude VINET, Alain

Bureautique et organisation du travail de secrétariat dans la fonction publique québécoise.

v. 43, no 4,1988 , p. $737-756 \quad 0682$ 
BUREAUX

HUGUES, Karen D.

Office Automation: A Review of the Litterature

v. 44 , no 3,1989, p. $654-679$

0741

\section{CADRES}

LE LOUARN, Jean-Yves

THÉRIAULT, Roland

TOULOUSE, Jean-Marie

Le travail des deux conjoints: Effets sur la progression de carrière du cadre.

v. 39 , no 1, 1984, p. 36-50

0270

GODIN, Jean LE LOUARN, Jean-Yves Les mentors ont-ils un effet sur la progression de carrière?

v. 41 , no 3,1986 , p. $505-518$

0489

BABA, Vishwanath V. KNOOP, Robert Organizational Commitment and Independence Among Canadian Managers

v. 42, no $2,1987, p, 325-344$

0564

GAGNON, Mona-Josée LEBEAU, Ernest JOHNSTON, Raymond

Commentaires - l'actualisation du mouvement syndical.

43ième Congrès, 1988, p. 222-236 0953

\section{CADRES SUBALTERNES}

RAINVILLE, Jean-Marie

La responsabilité fonctionnelle chez les cadres subalternes: Facteurs d'evolution des systèmes d'autorité.

v. 38 , no 4,1983 , p. $831-846$

0251

\section{CANADA}

PONAK, Allen MOORE, Larry F.

Canadian Bank Unionism: Perspectives and Issues

v. 36 , no 1,1981 , p. $3-34$

0001

DOWNIE, Bryan $M$.

Some Thoughts on Public Policy and Industrial Peace.

v. 36 , no 1,1981, p. $63-86$

0003

TUCK, Hugh

Canadian Railways and Unions in the Running Trades, 1865-1914

v. 36 , no 1,1981 , p. $106-131$

0005
CANADA (suite)

SWIDINSKI, Robert

The Effect of Bargaining Structure on Negotiated Wage Settlements.

v. 36, no 2,1981, p. $371-385 \quad 0029$

CHAISON, Gary N. ROSE, Joseph B.

The Structure and Growth of the Canadian National Unions.

v. 36, no 3,1981 , p. $530-551 \quad 0052$

LOWE, Graham S.

Causes of Unionization in Canadian Banks. v. 36 , no 4,1981 , p. $865-893 \quad 0077$

AOUST, Claude d' DELORME, François The Origin of the Freedom of Association and of the Right to Strike in Canada: An Historical Perspective.

v. 36, no 4,1981 , p. $894-921 \quad 0078$

GRUBEL, Herbert G. MAKI, Dennis R.

A Note on the Effects of Unemployment Insurance, Minimum Wage Legislation and Trade Union Growth on Reported Unemployment Rates in Canada, 1950-75

v. 36 , no 4,1981 , p. $922-927$

0079

MAFION, Gérald

La part des salaires dans le revenu national au Canada, 1910 à 1980.

v. 37, no 1,1982, p. $53-64 \quad 0097$

HASAN, Abrar GERA, Surendra

Reservation Wages in Canadian Labour Markets.

v. 37 , no 1,1982 , p. $65-92 \quad 0098$

BARTH, Richard T. BABA, Vishwanath V. Alienation Among Professionnal Engineers: A Canadian - American Comparison.

v. 37 , no 1,1982 , p. $126-140 \quad 0100$

FRANK, J.A. KELLY, Michael J.

MACNAUGHTON, Bruce D.

Legisiative Change and Strike Activity in Canada, 1926-1974

v. 37, no 2,1982, p. $267-283$

0119

JAIN, Harish C.

Race ans Sex Discrimination in Employment in Canada: Theories, Evidence and Policies.

v. 37, no 2,1982, p. $344-366 \quad 0123$ 


\section{CANADA (suite)}

MELTZ, Noah M.

Labour Market Information in Canada: The Current Situation and a Proposal.

v. 37, no 2,1982, p. 431.437

0127

MAGUN, Sunder

The Rise of Service Employment in the Canadian Economy.

v. 37 , no 3,1982 , p. $528-556$

0138

WEIERMAIR, Klaus

Apprenticeship Training in European Countries: The Lessons for Canada.

v. 37, no 3,1982 , p. $557-574$

0139

ADAMS, Roy J.

The Federal Government and Tripartism.

v. 37, no 3,1982, p. $606-617$

0141

CHAISON, Gary N. ANDIAPPAN, P.

Characteristics of Female Union Officer in Canada.

v. 37 , no 4,1982, p. $765-7790158$

AGARWAL, Naresh C.

Male-Female Pay Inequity and Public Policy in Canada and the U.S.

v. 37 , no 4,1982 , p. $780-804 \quad 0159$

MAKI, Dennis R.

Political Parties and Trade Union Growth in Canada.

v. 37 , no 4,1982 , p. $876-886 \quad 0163$

EASTMAN, Byron

Canadian Union Growth

v. 38 , no 1,1983, p. $58-71$

0180

SIMPSON, Wayne PETERS, Frank

The Economics of Mileage Restrictions for Railway Workers in Western Canada.

v. 38, no 1, 1983, p. 95-103 0182

MAKI, Dennis R.

Trade Unions and Productivity: Conventional Estimates.

v. 38 , no 2,1983 , p. $211-228 \quad 0201$

AMERNIC, J.H. ARANYA, N.

Organizational Commitment: Testing Two Theories.

v. 38 , no 2,1983, p. 319-343 0206

\section{CANADA (suite)}

JAIN, Harish C.

Micro-electronics Technology and Industrial Relations.

v. 38 , no 4,1983 , p. $869-879 \quad 0254$

COUSINEAU, Jean-Michel

LACROIX, Robert

La détermination des avantages sociaux au Canada

v. 39, no 1,1984, p. $3-220268$

WILLIAMS, C. Brian

The Impact of Labor Migration: The International Molders and Allied Workers Union in Canada, 1860-1885.

v. 39 , no 2,1984 , p. $335-364 \quad 0298$

DEVORETZ, D.J. REED, Clyde G.

Evidence from the Skilled-Unskilled Canadian Wage Index.

v. 39 , no 3,1984, p. $526-537 \quad 0318$

FISHER, E.G. SHERWOOD, L.M.

Fairness and Managerial Rights in Canadian Arbitral Jurisprudence

v. 39 , no 3,1984, p. $538-552$

0319

ADAMS, Roy J.

The Extent of Collective Bargaining in Canada

v. 39 , no 4,1984 , p. $655-667 \quad 0336$

JONES, J.C.H. LAUDADIO, L.

Organized Labour, Regional Political Bias and the Canadian Tariff Structure

v. 39, no 4,1984 , p. 695-709 0339

VERGE, Pierre

Evolution de la protection juridictionnelle de l'accès au syndicat.

v. 39 , no 4,1984, p. $710-743 \quad 0340$

ARTHURS, H.W.

Understanding "Understanding": Industrial Relations Research and Policy in Canada from 1969 to 1984...and Beyond. v. 39, no 4,1984, p. $753-761 \quad 0342$

MILNE, William J. ROSS, Thomas W. The Cyclical Variation of Wage Premiums in the Canadian Manufacturing Industries v. 39, no 4,1984 , p. $762-773 \quad 0343$ 
CANADA (sulte)

BENEDICT, Daniel

The 1984 GM Agreement in Canada: Significance and consequences.

v. 40 , no 1,1985 , p. $27-47$

0363

SOLOMON, Norman A.

The Negociation of First Agreements under the Canada Labour Code.

v. 40, no 3,1985, p. $458-472$

0404

BRODY, Bernard ROHAN, Paul

ROMPRÉ, Louise

Les accidents industriels au Canada: Le portrait d'une décennie

v. 40 , no 3,1985, p. $545-566$

0409

MAGUN, Sunder

The Effects of Technological Changes on the Labour Market in Canada

v. 40, no 4,1985 , p. $720-746$

0425

OSBERG, Lars

A Note on the Incomes of Lawyers

v. 40 , no 4,1985 , p. $865-879$

0433

BÉLANGER, Jacques MERCIER, Jacques Le plafonnement de la densité syndicale au Québec et au Canada.

v. 41 , no 1,1986 , p. $28-52$

0448

MAKI, Dennis R. MEREDITH, Lindsay N. The Effects of Unions on Profitability: Canadian Evidence

v. 41 , no 1,1986 , p. $54-68$

0449

KUMAR, Pradeep DOW, Bradley Econometric Analysis of Union Membership Growth in Canada, 1935-1981

v. 41 , no 2,1986 , p. $236-255$

0467

BEACH, Charles M. KALISKI, S.F. The Impact of Recession on the Distribution of Annual Unemployment.

v. 41 , no 2,1986 , p. 317-328

0471

DICKINSON, John A.

La législation et les travailleurs québécois, 1894-1914

v. 41, no 2,1986, p. $357-381$

0474

COUSINEAU, Jean-Michel

Objectifs et modalités de l'assurance-ch-

Omage au Canada: 1940-1986

v. 41 , no 3,1986 , p. $451-468$
CANADA (suite)

SOLOMON, Norman A. ANDIAPPAN, P.

SHAND, Dan

Canadian National Union Presidents: An

Empirical Study.

v. 41 , no 3,1986, p. $491-504 \quad 0488$

WILLIAMS, C. Brian

International Trade Unionism: The United Mine Workers in Eastern Canada, 1900-1920

v. 41 , no 3,1986 , p. $519-540 \quad 0490$

TANG, Roger Y.W. PONAK, Allen Employer Assessment of Strike Costs.

v. 41 , no 3,1986 , p. $552-571$

0492

EVANS, Martin G. ONDRACK, Daniel A. The Effect of Unionization on Wages: Some Canadian Evidence

v. 41 , no 3,1986 , p. $572-577$

0493

BEMMELS, Brian FISHER, E.G.

NYLAND, Barbara

Canadian-American Jurisprudence on "Good Faith" Bargaining.

v. 41, no 3, 1986, p. 596-621 0496

JAIN, Harish C. ANDIAPPAN, P.

Sexual Harassment in Employment in $\mathrm{Ca}$ nada: Issues and Policies.

v. 41 , no 4,1986 , p. $758-777$

0517

BETCHERMAN, Gordon

Labour Market Imbalances in Canada, 1966-1983

v. 41 , no 4,1986 , p. $802-816 \quad 0519$

ZEYTINOGLU, Isik Urla

The ILO Standards and Canadian Labour Légisiation.

v. 42, no 2,1987, p. $292-308 \quad 0562$

NG, Ignace

Determinants of Wildcat Strikes in Canada Manufacturing Industries.

v. 42 , no 2,1987, p. 386-397 0567

HÉBERT, Gérard

L'évolution du syndicalisme au Canada: Comment un mouvement devient institution.

v. 42 , no 3,1987, p. $500-519$

0581 


\section{CANADA (sulte)}

GURDON, Michael A.

Divergent Paths: Civil Service Employment Relations in Australia and Canada.

v. 42 , no 3,1987 , p. $566-575$

0585

DOOLEY, Martin D.

Within-Cohort Earnings Inequality Among Canadian Men: 1971-1982

v. 42 , no 3,1987 , p. $594-611$

0587

KUMAR, Pradeep

Recent Wage Deceleration in Canada: Short-run Response or Structural Change?

v. 42 , no 4,1987 , p. $687-701$

0604

JAIN, Harish C.

Recruitment of Racial Minorities in Canadian Police Forces.

v. 42, no 4,1987 , p. $790-805 \quad 0610$

MERCIER, Jacques

Effets du salaire minimum sur l'emploi: Résuitats des études économétriques canadiennes et québécoises.

v. 42, no 4,1987, p. $806-830$

0611

MCPHILLIPS, David C.

The Appropriate Bargaining Unit: The Need for Policy Consistency by Canadian Labour Boards.

v. 43, no 1,1988, p. $63-84$

0629

REID, Frank

Economic Aspects of Mandatory Retirement: The Canadian Experience.

v. 43 , no 1,1988 , p. 101-114

0631

CARTER, Donald D.

Canadian Labour Relations Under the Charter: Exploring the Implications.

v. 43 , no 2,1988, p. $305-321$

0648

KAMEL, Nawal ROY, Paul-Martel Temps supplémentaire et création d'emplois: Le cas du Canada, du Québec et de l'Ontario.

v. 43, no 2,1988 , p. $412-430 \quad 0653$

STAGER, David A.A.

Lawyers' Earnings in the Canadian Private and Public Sectors.

v. 43 , no 3,1988 , p. $571-590$

0668
CANADA (suite)

SEFTON MACDOWELL, Laurel

The Career of a Canadian Trade Union Leader: C.H. Millard 1937-1946

v. 43, no 3,1988, p. $609-632$

0670

FRICKE, John G.

Worker Participation in Canada: Some Lessons from the Past.

v. 43 , no 3,1988 , p. $633-658$

0671

KUMAR, Pradeep

Estimates of Unionism and Collective Bargaining in Canada.

v. 43 , no 4,1988 , p. $757-779 \quad 0683$

GILES, Anthony MURRAY, Grégor

Towards an Historical Understanding of Industrial Relations Theory in Canada.

v. 43 , no 4,1988 , p. $780-811 \quad 0684$

MAC NEIL, Michael

Unions, Politics and Law in Canada.

v. 43, no 4,1988 , p. $847-868$

0687

WEST, Leigh

The Exclusive Remedy Provision in Canadian Worker Compensation Law: The Need for Legislative Reform.

v. 43 , no 4,1988, p. $890-908 \quad 0689$

DION, Gérard HÉBERT, Gérard

L'avenir du syndicalisme au Canada.

v. 44 , no 1,1989 , p. $5-24$

0699

AUDET, Michel BÉLANGER, Laurent Nouveaux modes de gestion et relations industrielles au Canada.

v. 44, no 1,1989 , p. $62-96$

0702

MELTZ, Noah M.

Job Security in Canada

v. 44, no 1,1989, p. $149-161$

0705

SACK, Jeffrey LEE, Tanya

The Role of the State in Canadian Labour Relations.

v. 44 , no 1,1989 , p. $195-223 \quad 0708$

FORREST, Anne

The Rise and Fall of National Bargaining in the Canadian Meat-Packing Industry.

v. 44, no 2, 1989, p. 393-408 0723 


\section{CANADA (suite)}

JAIN, Harish C.

Racial Minorities and Affirmative Action/Employment Equity Législation in Canada.

v. 44 , no 3,1989 , p. $593-614$

0738

HUGUES, Karen D.

Office Automation: A Review of the Litterature

v. 44 , no 3,1989 , p. $654-679$

CÔTÉ, André C.

Nouvelles technologies et droit du travail au Canada.

v. 44, no 4,1989 , p. $751-768$

0751

LONG, Richard J.

Patterns of Workplace Innovation in Canada.

v. 44 , no 4,1989 , p. $805-826$

0754

ANDIAPPAN, P. CRESTOHL, Mark

SINGH, Jang $B$.

Racial Discrimination in Employment in Canada.

v. 44 , no 4,1989 , p. $827-849$

0755

HÉBERT, Gérard

L'état de la discipline en relations industrielles au Canada: Un brin d'histoire et une postface./The State of the Art in Industrial Relations: A Brief History and a Postcript.

v. 44, no 4,1989 , p. $884-904$

0758

BARKIN, Solomon

The State of the Art in Industrial Relations: A U.S. Commentary.

v. 44 , no 4,1989 , p. $905-914$

0759

WEISS, Dimitri

L'état de la discipline en relations industrielles au Canada: Un commentaire européen.

v. 44 , no 4,1989 , p. $914-920 \quad 0760$

MCWATTERS, Catherine $\mathrm{J}$.

BEACH, Charles $M$.

Factors Behind the Changes in Canada's

Family Income Distribution and the Share of the Middle Class.

v. 45 , no 1,1990, p. $118-135$

0777
CANADA (suite)

DOWNIE, Bryan M.

The Role of the State in Industrial Relations: A Comment.

v. 45 , no 1,1990 , p. $169-186 \quad 0781$

COUSINEAU, Jean-Michel

NAJEM, Elmustapha

L'effet du développement de la petite entreprise sur l'évolution du syndicalisme au Canada.

v. 45, no 3,1990 , p. $467-480 \quad 0812$

BÉGIN, Monique

Les politiques gouvernementales en matière de retraite.

37ième Conarès, 1982 , p. 171-180 0854

TRUDEAU, Guy J. OUELLET, Lionel La comparaison en matière de systèmes de santé et de sécurité du travail.

42ième Congrès, 1987, p. 101-1130931

GODBOUT, Clément RIOUX, Claude

Le libre-échange canado-américain et le marché du travail.

44ième Congrès, 1989, p. 175-188 0964

CUNNINGHAM, Barton MITCHELL, Lari Privatization in British Columbia: What the Experts Say Will Happen.

v. 45 , no 2,1990 , p. $382-403 \quad 0799$

\section{CANADAIR LIMITED}

PEACH, David A.

The Canadair-I.A.M. Productivity: Improvement Plan.

v. 37 , no 1,1982 , p. $177-197 \quad 0103$

\section{CANADIAN ADMIRAL}

BURKE, Ronald J.

Trainee Experiences in Industrial Retraining Programs: A Case Study

v. 39 , no 3.1984 , p. $570-577 \quad 0321$

BURKE, Ronald J.

Consequences of not Working Sixteen Months After a Plant Closing

v. 40 , no 1,1985 , p. $162-169 \quad 0370$

\section{CANADIEN NATIONAL}

BOIVIN, Suzanne P.

Le Canadien National: Un cas riche d'enseignements.

43ième Congrès, 1988, p. 97-113 0947 
CANADIEN NATIONAL (suite)

SIMARD, Monique TELLIER, Marie

Commentaires - Le Canadien National: Un cas riche d'enseignements.

43ième Congrès, 1988, p. 114-130 0948

\section{CANADIEN PACIFIQUE}

SIMPSON, Wayne PETERS, Frank

The Economics of Mileage Restrictions for Railway Workers in Western Canada.

v. 38, no 1,1983, p. $95-103$

0182

\section{CANADIENS-ANGLAIS}

RAINVILLE, Jean-Marie

Hierarchie ethnique dans la grande entreprise industrielle montréalaise.

v. 36, no 2,1981 , p. $336-360$

0027

\section{CANADIENS-FRANÇAIS}

RAINVILLE, Jean-Marie

Hierarchie ethnique dans la grande entreprise industrielle montréalaise.

v. 36 , no 2,1981 , p. $336-360$

0027

\section{CAPITAL HUMAIN}

COUSINEAU, Jean-Michel RABEAU, Yves Une méthodologie de comparaison des salaires pour les emplois spécifiques du secteur public.

v. 43 , no 1,1988, p. $85-100$

0630

\section{CAPITALISME}

WILLIAMS, C. Glyn

Joan Robinson on Unions, Distribution and Inflation.

v. 36 , no 3,1981 , p. $576-588 \quad 0054$

LIPSIG-MUMME, Carla

The Renaissance of Homeworking in Developped Economies.

v. 38 , no 3,1983 , p. $545-567$

0226

GALBRAITH, John Kenneth

Réflexions sur les problèmes de la retraite dans les années 80.

37ième Congrès, 1982, p. 193-213 0856

\section{CARRIÈRE PROFESSIONNELLE}

LE LOUARN, Jean-Yves

THÉRIAULT, Roland

TOULOUSE, Jean-Marie

Le travail des deux conjoints: Effets sur la progression de carrière du cadre.

v. 39 , no 1,1984, p. $36-50$

0270

\section{CARRIERE PROFESSIONNELLE (suite)}

FABI, Bruno

Privé ou public: choix et transfert du secteur organisationnel.

v. 39 , no 2,1984 , p. $313-334 \quad 0297$

GODIN, Jean LE LOUARN, Jean-Yves Les mentors ont-ils un effet sur la progression de carriere?

v. 41, no 3, 1986, p. 505-518 0489

BOURGEOIS, Robert-Paul WILS, Thierry Career Concepts, Personality and Values of Some Canadian Workers: An Exploratory Study

v. 42 , no 3,1987 , p. $528-543 \quad 0583$

\section{CASCADES INC.}

LEMAIRE, Alain

La communication directe chez Cascades Inc.

41ième Congrès, 1986, p. 63-68 0909

BOUCHER, Lysette

Les limites des nouvelles approches en gestion des ressources humaines.

41ième Congrès, 1986, p. 77-84 0911

\section{CATASTROPHE (THÉORIE)}

EASTMAN, Byron

A Catastrophe Theory on Union Behaviour.

v. 40, no 2,1985 , p. $340-350 \quad 0389$

\section{CENTRALE DES SYNDICATS DÉMOCRATI-} QUES

HÉTU, Jean-Paul

La nouvelle gestion des ressources humaines - mythe ou réalité?

41ième Congrès, 1986, p. 167-172 0919

\section{CENTRALE DES SYNDICATS NATIONAUX}

MARTINEAU, LUC

Décisions rendues par le Conseil canadien des relations du travail.

v. 36 , no 1,1981 , p. 263-266

0015

CENTRE DES REL. IND. (UNIV. MCGILL) GOLDENBERG, Shirley B.

HÉBERT, Gérard

Harry Douglas Woods, 1907-1983

v. 38 , no 4,1983 , p. 707-712

0244 
Relations Inoustrielles, vol. 45, no 4 (1990)

CENTRE DES REL. IND. (UNIV. QUEEN) KELLY, Laurence

Industrial Relations at Queen's: The First Fifty Years.

v. 42 , no 3,1987 , p. $475-499$

0580

\section{CENTRE HOSPITALIER DE VERDUN}

LEVINE, David

Négocier la flexibilite: Reconversion des heures de la liste de rappel en postes à temps complet.

45ième Congrès, 1990, p. 41-47 0970

CENTRE LOCAL DE SERVICES COMMUNAUTAIRES

BÉLANGER, Paul R. LÉVESQUE, Benoît Le mode de vie détermine-t-il l'éthique du travail? Etude de cas.

v. 42 , no 2,1987, p. $345-365$

0565

\section{CENTRES D'EMPLOIS DU CANADA}

MAGUN, Sunder

The Placement Activity of the Canadian Employment Agency.

v. 38 , no 1,1983 , p. $72-94$

0181

\section{CERCLES DE QUALITÉ}

BROSSARD, Michel

Les limites du modèle-type du fonctionnement des cercles de qualité

v. 44 , no 3,1989 , p. $552-568 \quad 0736$

\section{CERTIFICATS D'ACCRÉDITATION}

LAVERY, Daniel

Décisions rendues par le Conseil canadien des relations du travail.

v. 38 , no 3,1983 , p. $658-666$

0231

CHAMBERLAIN, $\mathbf{N}$.

MAKI, Dennis R. STRAND, Kenneth

The Determinants of Strike Activity: An Interindustry Analysis

v. 39 , no 1,1984 , p. $77-92$

0272

\section{CHANGEMENT D'EMPLOI}

DORNSTEIN, Miriam ZOREF, Uri

Motivation for Changing Jobs, Personal Background Characteristics and Perceived Opportunity

v. 41 , no 1,1986, p. $91-110$

0451
CHANGEMENT D'EMPLOI (suite)

CHAYKOWSKI, Richard P.

SLOTSVE, G.A.

Union Seniority Rules as a Determinant of Intra-Firm Job-Changes.

v. 41 , no 4,1986 , p. $720-737 \quad 0515$

CHANGEMENT ORGANISATIONNEL BROSSARD, Michel SIMARD, Marcel Problématique de la différenciation de la main-d'oeuvre et changement organisationnel.

v. 41 , no 2,1986 , p. $219-235 \quad 0466$

\section{CHANGEMENTS TECHNOLOGIQUES}

PEITCHINIS, Stephen G.

The Attitude of Trade Unions Towards Technological Changes.

v. 38, no 1, 1983, p. 104-119 0183

JAIN, Harish C.

Micro-electronics Technology and Industrial Relations.

v. 38, no 4,1983, p. $869-879 \quad 0254$

MAGUN, Sunder

The Effects of Technological Changer on the Labour Market in Canada

v. 40, no 4,1985 , p. $720-746$

0425

LAFLAMME, Gilles VALLÉE, Guylaine Changements technologiques et modes régulateurs des relations du travail.

v. 42 , no 4,1987 , p. $702-7150605$

CAMPBELL, Adrian WARNER, Malcolm Workplace Relations, Skills-Training and Technological Change at Plant-Level.

v. 43 , no 1,1988, p. 115-132 0632

CUNNINGHAM, Barton HULL, Dennis A Union Member's Perspective on Technological Change.

v. 43 , no 2,1988, p. $394-411 \quad 0652$

LAFLAMME, Lucie VINET, Alain

Accidents du travail et modernisation du processus de production: le cas de l'industrie forestière québécoise.

v. 43, no 3,1988 , p. 591-608 0669 
CHANGEMENTS TECHNOLOGIQUES (suite)

GAGNON, Yves-Chantal

LANDRY, Maurice

Les changements technologiques: Une stratégie d'étude exploratoire

v. 44 , no 2,1989 , p. $421-447$

0725

ZUREIK, Elia MOSCO, Vincent

LOCHHEAD, Clarence

Telephone Workers' Reaction to the New Technoiogy

v. 44 , no 3,1989 , p. $507-531$

0734

HUGUES, Karen D.

Office Automation: A Review of the Litterature

v. 44 , no 3,1989, p. $654-679$

0741

CÔTÉ, André C.

Nouvelles technologies et droit du travail au Canada.

v. 44 , no 4,1989 , p. $751-768$

0751

SMITH, Anthony E.

Innovation by Negotiation: Case Studies Among British White-Collar Unions.

v. 45 , no 1,1990 , p. $63-75$

0774

MUCKENBERGER, Ulich

The Regulation of Strike Law in Times of New Technologies and Deregulation: The Case of West Germany.

v. 45 , no 1,1990, p. $136-145$

0778

FRAPPIER-DESROCHERS, Monique La technologie: un substitut à la durée du travail?

36ième Congrès, 1981, p. 193-218 0843

\section{CHANTIER NAVAL DE LAUZON}

LISTON, Terrence

Négocier la flexibilité: La polyvalence des emplois.

45ième Congrès, 1990 , p. 65-69 0973

\section{CHARTE CANADIENNE DES DROITS ET LIBERTÉS}

ROBB, Roberta Edgecome

Occupational Segregation and Equal Pay for Work of Equal Value.

v. 39 , no 1, 1984, p. 146-166 0275
CHARTE CANADIENNE DES DROITS ET LIBERTÉS (suite)

LAMY, Francine

Décisions rendues par le Conseil canadien des relations du travail.

v. 40 , no 2,1985 , p. $386-398 \quad 0394$

CARTER, Donald D.

Canadian Labour Relations Under the Charter: Exploring the Implications.

v. 43 , no 2,1988 , p. $305-321 \quad 0648$

TREMBLAY, Johane

Décisions rendues par le Conseil canadien des relations du travail.

v. 43 , no 3,1988 , p. $686-694$

0673

WEST, Leigh

The Exclusive Remedy Provision in Canadian Worker Compensation Law: The Need for Legislative Reform.

v. 43, no 4,1988 , p. $890-908 \quad 0689$

DION, Gérard HÉBERT, Gérard

L'avenir du syndicalisme au Canada.

v. 44, no 1,1989 , p. $5-24$

0699

CARTER, Donald D.

Grievance Arbitration and the Charter: The Emergency Issues.

v. 44, no 2,1989, p. $337-353 \quad 0720$

MORIN, Fernand

Liberté d'expression et droit au travail: l'arbitrage de la Cour supreme du Canada.

v. 44 , no 4,1989 , p. 921-932 0761

PÉPIN, Marcel

L'actualisation du mouvement syndical.

43ième Congrès, 1988, p. 209-221 0952

GAGNON, Mona-Josée LEBEAU, Ernest JOHNSTON, Raymond

Commentaires - l'actualisation du mouvement syndical.

43ième Congrès, 1988, p. $222-2360953$

\section{CHARTES DES DROITS ET LIBERTÉS}

BLOUIN, Rodrigue FERLAND, Gilles

LAFLAMME, Gilles LAROCQUE, Alain

RONDEAU, Claude

Introduction - Les Chartes des droits et les relations industrielles.

43ième Congrès, 1988, p. XV

0941 


\section{CHARTES DES DROITS ET LIBERTÉS (suite)}

ROCHER, Guy

Les fondements de la société libérale, les relations industrielles et les Chartes.

43ième Congrès, 1988, p. 1-18 0942

ROJOT, Jacques

Droits collectifs et droits individuels: Les situations française, américaine et anglaise.

43ième Congrès, 1988, p. 19-49 0943

BERGERON, Jean-Louis

La gestion de l'embauche, de la promotion et du licenciement revue et corrigée par les Chartes.

43ième Congrès, 1988, p. 51-62 0944

DUCHARME, Claude

LECLERCQ, Dominique

WESTMORELAND-TRAORE, Juanita

Commentaires - La gestion de l'embau-

che, de la promotion et du licenciement revue et corrigée par les Chartes.

43ième Congrès, 1988, p. 63-82

0945

DUSSAULT, Ginette

À travail équivalent, salaire égal: Un droit difficile à appliquer?

43ième Congrès, 1988, p. 83-95 0946

\section{BARRÉ, Alain}

Le régime des rapports collectifs et les Chartes.

43ième Congrès, 1988, p. 131-156 0949

DESMARAIS, Jacques

Les moyens de pression: Les chartes en redéfinissent-elles les limites?

43ième Congrès, 1988, p. 157-195 0950

DULUDE, Gilles LOUMĖDE, Catherine

PARROT, Jean-Claude

Commentaires - Les moyens de pression: Les Chartes en redéfinissent-elles les limites?

43ième Congrès, 1988, p. 195-208 0951

GARANT, Patrice

Statut et pouvoirs des organismes du travail en regard des Chartes.

43ième Congrès, 1988, p. 237-254 0954

\section{CHARTES DES DROITS ET LIBERTÉS (suite)}

DUFOUR, Ghislain LABERGE, Louis

LAROSE, Gérald

Table ronde - Les Chartes impliquent-elles un réalignement des politiques syndicales et patronales?

43ième Congrès, 1988, p. 255-272 0955

\section{CHEMINEMENT DE CARRIÈRE}

BOURGEOIS, Robert-Paul WILS, Thierry Career Concepts, Personality and Values of Some Canadian Workers: An Exploratory Study

v. 42 , no 3,1987, p. $528-543 \quad 0583$

\section{CHEMINOTS}

TUCK, Hugh

Canadian Railways and Unions in the Running Trades, 1865-1914

v. 36 , no 1,1981 , p. 106-131 0005

\section{CHEMINS DE FER}

TUCK, Hugh

Canadian Railways and Unions in the Running Trades, 1865-1914

v. 36 , no 1,1981 , p. 106-131 0005

SIMPSON, Wayne PETERS, Frank

The Economics of Mileage Restrictions for Railway Workers in Western Canada.

v. 38, no 1, 1983, p. 95-103 0182

\section{CHÓMAGE}

GRUBEL, Herbert G. MAKI, Dennis R.

A Note on the Effects of Unemployment Insurance, Minimum Wage Legislation and Trade Union Growth on Reported Unemployment Rates in Canada, 1950-75

v. 36 , no 4,1981 , p. $922-927$

0079

HASAN, Abrar GERA, Surendra Reservation Wages in Canadian Labour Markets.

v. 37 , no 1,1982, p. $65-92$

0098

STURMTHAL, Adolf

Unemployment, Inflation and "Guest Workers": Comparative Study of Three European Countries.

v. 37 , no 4,1982 , p. $739-764$

0157 


\section{CHÓMAGE (sulte)}

MAGUN, Sunder

The Placement Activity of the Canadian

Employment Agency.

v. 38 , no 1,1983 , p. $72-94$

0181

JOLIVET, Thierry

La réduction de la durée du travail estelle créatrice d'emploi?

v. 38 , no 1,1983 , p. 142-154 0185

\section{DEMERS, Marie}

Chômage chez les jeunes: Conséquences psychologiques et sociales.

v. 38, no 4,1983, p. $785-814 \quad 0249$

\section{FORTIN, Pierre}

Le chómage des jeunes au Québec: Aggravation et concentration, 1966-1982

v. 39 , no 3,1984 , p. $419-448 \quad 0313$

MILNE, William J. ROSS, Thomas W. The Cyclical Variation of Wage Premiums in the Canadian Manufacturing Industries v. 39 , no 4,1984 , p. $762-773$

0343

\section{MERCIER, Jacques}

Les effets du salaire minimum sur l'emploi des jeunes au Québec.

v. 40, no 3,1985 , p. $431-457 \quad 0403$

BEACH, Charles M. KALISKI, S.F.

The Impact of Recession on the Distribution of Annual Unemployment.

v. 41 , no 2,1986, p. $317-328$

0471

\section{WEIERMAIR, Klaus}

Secular Changes in Youth Labour Markets and Youth Unemployment in Canada.

v. 41 , no 3,1986 , p. $469-490$

0487

\section{BETCHERMAN, Gordon}

Labour Market Imbalances in Canada, 1966-1983

v. 41 , no 4,1986 , p. $802-816 \quad 0519$

ROY, Paul-Martel

Licenciements collectifs, licenciements individuels et emploi au Québec: 1979-1984

v. 42, no 3,1987 , p. $577-593 \quad 0586$

\section{CHÓMAGE (suite)}

KUMAR, Pradeep

Recent Wage Deceleration in Canada: Short-run Response or Structural Change? v. 42, no 4,1987 , p. $687-701 \quad 0604$

\section{MERCIER, Jacques}

Effets du salaire minimum sur l'emploi: Résultats des études économétriques canadiennes et québécoises.

v. 42 , no 4,1987 , p. $806-830$

0611

\section{SODERSTROM, Lee}

Some Effects of Unemployment on the Health of Unemployed Quebec Workers.

v. 43 , no 2,1988, p. $341-377 \quad 0650$

\section{ZAPATA, Francisco}

La proteccion del empleo en las américas. v. 44, no 1, 1989, p. 177-194 0707

GRAYSON, J. Paul

The Political Consequences of Unemployment: An Application of the "Power Mode/" of Blue-Collar Radicalism

v. 44 , no 3,1989 , p. $635-653$

0740

ROY, Paul-Martel

Aspects dynamiques du marché du travail au Québec.

v. 45 , no 2,1990 , p. $283-299 \quad 0794$

AOUST, Fernand d' DÉOM, André

HARVEY, Pierre

Table ronde - La réduction du temps passé au travail: un moyen de lutte contre le chômage.

36ième Congrès, 1981, p. 219-246 0844

\section{CHÔMEURS}

BURKE, Ronald J.

Consequences of not Working Sixteen

Months After a Plant Closing

v. 40 , no 1,1985 , p. $162-169 \quad 0370$

\section{CIVILITÉ}

AOUST, Claude d' SAINT-JEAN, Sylvain TRUDEAU, Gilles

L'obligation de civilité du salarié

v. 41 , no 1,1986 , p. $157-180$

0455 


\section{CLASSE MOYENNE}

MCWATTERS, Catherine J.

BEACH, Charles M.

Factors Behind the Changes in Canada's Family Income Distribution and the Share of the Middle Class.

v. 45 , no 1,1990 , p. $118-135$

0777

\section{CLAUSES NON SALARIALES}

RONDEAU, Claude BADIN, François

Le contenu non salarial des conventions collectives dans les industries manufacturières au Quebec.

v. 41 , no 1,1986 , p. $69-90$

0450

\section{CLIMAT DE TRAVAIL}

DULUDE, Yves

Le conflit: la gestion au banc des accusés.

41ième Congrès, 1986, p. 111-133 0914

LAMARCHE, Pierre

Commentaires - Le conflit: la gestion au banc des accusés.

41ième Congrès, 1986, p. 133-136 0915

\section{LALANDE, Serge}

L'expérience de la compagnie Gaz Métropolitain.

41ième Congrès, 1986, p. 137-146 0916

LAMARCHE, André GAUTHIER, Michel BLAIS, Michel GAGNON, Denise Les conditions de travail au lendemain d'une fusion ou d'une réorganisation. 44ième Congrès, 1989, p. 101-127 0961

\section{CLIMAT ORGANISATIONNEL}

DOLAN, Shimon L. TZINER, Aharon ROY, Denis

A Real Estate Agency's Level Analysis of the Climate-Performance Relationship.

v. 39 , no 1,1984 , p. 167-176

0276

LORRAIN, Jean BRUNET, LUC

Climat organisationnel, satisfaction au travail et perception du syndicalisme

v. 39 , no 4,1984 , p. $668-679$

DASTMALCHIAN, Ali

ADAMSON, Raymond BLYTON, Paul Developing a Measure of Industrial Relations Climate

v. 41 , no 4,1986 , p. $851-859$

0524

\section{CODE CANADIEN DU TRAVAIL}

MARTINEAU, LUC

La "gestion" des conflits par le Conseil canadien des relations du travail.

v. 36 , no 3,1981 , p. $589-615$

0055

LAVERY, Daniel

Décisions rendues par le Conseil canadien des relations du travail.

v. 37 , no 4,1982 , p. $944-959$

0167

BEAULIEU, LUC

Décisions rendues par le Conseil canadien des relations du travail.

v. 38 , no 4,1983 , p. $880-903$

0255

BEAULIEU, LUC

Décisions rendues par le Conseil canadien des relations du travail.

v. 39 , no 1, 1984, p. 177-189

0277

BEAULIEU, LUC

Décisions rendues par le Conseil canadien des relations du travail

v. 39 , no 2,1984 , p. $371-385$

0300

BEAULIEU, LUC

Décisions rendues par le Conseil canadien des relations du travail.

v. 39, no 3,1984 , p. $605-618$

0323

POTHIER, Dianne

Décisions rendues par le Conseil canadien des relations du travail.

v. 39, no 4,1984, p. $774-782$

POTHIER, Dianne

Décisions rendues par le Conseil canadien des relations du travail.

v. 40 , no 1,1985, p. $170-174 \quad 0371$

SOLOMON, Norman A.

The Negociation of First Agreements under the Canada Labour Code.

v. 40, no 3,1985, p. $458-472 \quad 0404$

LAMY, Francine

Décision rendue par le Conseil canadien des relations du travail.

v. 40, no 3,1985 , p. $659-662$

0415

SAINT-GERMAIN, Catherine Décisions rendues par le Conseil cana'dien des relations du travail.

v. 41 , no 4,1986 , p. $860-868 \quad 0525$ 
CODE CANADIEN DU TRAVAIL (suite)

SAINT-GERMAIN, Catherine

Décision rendue par le Conseil canadien des relations du travail.

v. 42 , no 1,1987, p. 203-209

0549

TREMBLAY, Johane

Décisions rendues par le Conseil canadien des relations du travail.

v. 42 , no 4,1987 , p. $852-861$

0613

TREMBLAY, Johane

Décisions rendues par le Conseil canadien des relations du travail.

v. 43, no 3,1988 , p. $686-694$

0673

MARLEAU, Véronique L.

Décisions rendues par le Conseil canadien des relations du travail.

v. 43 , no 4,1988 , p. $953-962$

0692

MARLEAU, Véronique L.

Décisions rendues par le Conseil cana"dien des relations du travail.

v. 44, no 2,1989 , p. $448-462$

0726

MARLEAU, Véronique $L$.

Décisions rendues par le Conseil canadien des relations du travail.

v. 44, no 3,1989, p. $703-722$

0744

CLARKE, Graham

Décisions rendues par le Conseil canadien des relations du travail.

v. 44 , no 4,1989, p. $933-946$

0762

MARLEAU, Véronique $L$.

Décision rendue par le Conseil canadien des relations du travail.

v. 45 , no 2,1990, p. $414-423$

0801

CLARKE, Graham

Décision rendue par le Conseil canadien des relations du travail.

v. 45 , no 3,1990 , p. $616-624$

0820

\section{CODE DES PROFESSIONS DU QUÉBEC}

JOSEPH, P.A.

Perfecting the Administrative Solution to Labour Disputes.

v. 38 , no 4,1983 , p. $863-868$

0253
CODE DES PROFESSIONS DU QUÉBEC (suite)

BLOUIN, Rodrigue

Le titre réservé de CRI et la déontologie de la profession

v. 42, no 2,1987 , p. $309-3240563$

CODE DU TRAVAIL DE COLOMBIE BRITANNIQUE

JOSEPH, P.A.

Perfecting the Administrative Solution to Labour Disputes: The British Columbia Experiment.

v. 38 , no 2,1983 , p. $380-414 \quad 0209$

CODE DU TRAVAIL DU QUÉBEC

VERGE, Pierre

Syndicalisation de la grève.

v. 38 , no 3,1983 , p. $475-506$

0223

BLOUIN, Rodrigue

La réforme du Code du travail du Québec: une démarche fragmentaire.

v. 39, no 3.1984 , p. $578-604$

0322

MORIN, Fernand

Nouvelle présence de l'État dans les relations du travail.

v. 39 , no 4,1984 , p. $744-752$

0341

MORIN, Fernand

Les tenants et les aboutissants de la convention collective.

v. 40, no 2,1985, p. $371-378$

0392

PLANTE, Gilles

La permission d'appel au tribunal du travail

v. 41 , no 4,1986 , p. $817-834$

0521

MORIN, Fernand

La double personnalité d'un concierge!

v. 41 , no 4,1986 , p. $835-839$

0522

SEXTON, Jean

L'arbitrage de première convention collective au Québec: 1978-1984

v. 42 , no 2,1987, p. $272-291$

0561

MERCILLE, Pierre NADEAU, Bertin

OLIVIER, Madeleine

Table ronde - Organisation syndicale: difficultés et motifs de résistance.

38ième Congrès, 1983, p. 117-134 0865 
Relations Industrielles, vol. 45, no 4 (1990)

CODE DU TRAVAIL DU QUÉBEC (suite)

FRÉCHETTE, Raynald

La politique gouvernementale en matière de syndicalisation.

38ième Congrès, 1983, p. 197-206 0870

BONENFANT, Claire DUFOUR, Ghislain

HÉTU, Jean-Paul

Table ronde - Les réactions du milieu.

38ième Congrès, 1983, p. 207-223 0871

\section{CODE DU TRAVAIL FRANÇAIS}

CAIRE, GuY

Les lois Auroux

v. 39 , no 2,1984 , p. $235-258$

0292

\section{COLLECTIVITÉ LOCALE}

KRAHN, Harvey LOWE, Graham S.

Community Influences on Attitudes Towards Unions.

v. 39, no 1,1984, p. 93-113 0273

\section{COLOMBIE BRITANNIQUE}

MCPHILLIPS, David C.

Duty of Fair Representation: Recent Attitudes in British Columbia and Ontario.

v. 36 , no 4,1981 , p. $803-827$

0074

FISHER, E.G.

Strike Activity and Wildcat Strikes in British Columbia: 1945-1975.

v. 37, no 2, 1982, p. 284-312 0120

FISHER, E.G. PERCY, M.B.

The Impact of Unanticipated Output and Consumer Prices on Wildcat Strikes.

v. 38 , no 2,1983 , p. $254-276$

0203

JOSEPH, P.A.

Perfecting the Administrative Solution to Labour Disputes: The British Columbia Experiment.

v. 38, no 2,1983, p. $380-414$

0209

CUNNINGHAM, Barton HULL, Dennis A Union Member's Perspective on Technological Change.

v. 43 , no 2,1988, p. $394-411$

0652

DOWNIE, Bryan M.

The Role of the State in Industrial Relations: A Comment.

v. 45 , no 1,1990 , p. $169-186$

0781

\section{COLOMBIE BRITANNIQUE (suite)}

CUNNINGHAM, Barton MITCHELL, Lari

Privatization in British Columbia: What the

Experts Say Will Happen.

v. 45 , no 2,1990, p. $382-403 \quad 0799$

\section{COLS BLANCS}

SMITH, Anthony E.

Innovation by Negotiation: Case Studies Among British White-Collar Unions.

v. 45, no 1,1990, p. $63-75$

0774

\section{COLS BLEUS}

GRAYSON, J. Paul

The Political Consequences of Unemployment: An Application of the "Power Model" of Blue-Collar Radicalism

v. 44, no 3,1989 , p. $635-653 \quad 0740$

COMITÉ DES DÉLÉGUÉS DU PERSONNEL KAMINKA, Shlomit ROSEINSTEIN, Eliezer Shop Stewards, Workers, and Managers in Israëli Industry.

v. 38, no 3,1983, p. $598-617 \quad 0228$

\section{COMITÉS DE SANTÉ ET DE SÉCURITÉ}

BRYCE, George K. MANGA, Pran

The Effectiveness of Health and Safety Committees.

v. 40, no 2,1985, p. $257-283$

GEORGE, Kenneth

Les comités de santé et de sécurité du travail: Tables de concertation ou de négociation?

v. 40, no 3,1985, p. $512-528$

0407

\section{COMITÉS EXÉCUTIFS SYNDICAUX}

CHAISON, Gary N. ANDIAPPAN, P.

Characteristics of Female Union Officer in Canada.

v. 37 , no 4,1982 , p. $765-7790158$

\section{COMITÉS PARITAIRES}

LATULIPPE, Gérard P. O'FARRELL, Kevin Le comité paritaire: Anachronisme ou formule d'avenir.

v. 37 , no 3,1982, p. $634-655 \quad 0143$

\section{COMMISSION BEAUDRY}

RICHER, Jean-Charles

La Corporation professionnelle des con"seillers en relations industrielles du Quebec devant la Commission Beaudry. v. 40 , no 2,1985 , p. $379-3850393$ 


\section{COMMISSION DE LA SANTÉ ET SÉCURITÉ DU TRAVAIL \\ LESAGE, Michel \\ Les lésions professionnelles: Point de vue d'un médecin.}

42ième Congrès, 1987, p. $39-50 \quad 0926$

PRÉVOST, Charles

La sous-estimation des atteintes à la santé causées par les mauvaises conditions de travail.

42ième Congrès, 1987, p. 51-60 0927

MORIN, Fernand

Le régime actuel d'indemnisation pour lésions professionnelles: Accessibilité et efficacité.

42ième Congrès, 1987, p. 131-155 0933

SURET, Jean-Marc GENDRON, Michel BERNIER, Gilles

Le processus de gestion des risques, les lésions professionnelles et la CSST.

42ième Congrès, 1987, p. 223-241 0937

HARGUINDEGUY, Jean-Louis

BRODY, Bernard

Commentaires - Le processus de gestion des risques, les lésions professionnelles et la CSST.

42ième Congrès, 1987, p. 241-252 0938

TOBIN, Edmund LAROSE, Gérald

DUFOUR, Ghislain LABERGE, Louis

Table ronde - Financement de la santé et paritarisme.

42ième Congrès, 1987, p. 253-276 0939

JÉRÔME-FORGET, Monique

La politique québécoise en matière de lésions professionnelles à un point tournant.

42ième Congrès, 1987, p. 277-285 0940

\section{COMMISSION DES RELATIONS DU TRA-}

\section{VAIL DE L'ONTARIO}

PUPO, Norene DORIS DUFFY, Ann

The Ontario Labour Relations Board and the Part Time Workers.

v. 43 , no 3,1988 , p. $660-685$

0672
COMMISSION DES RELATIONS DU TRAVAIL DU QUÉBEC

BRUNET, Yves W. BEAULIEU, LUC

CANTIN, Isabelle

La nouvelle Commission québécoise des relations du travail (1988)

v. 43, no 2,1988, p. $231-304$

0647

\section{COMMISSIONS DES RELATIONS DU TRA-} VAIL

MCPHILLIPS, David C.

The Appropriate Bargaining Unit: The Need for Policy Consistency by Canadian Labour Boards.

v. 43 , no 1,1988, p. $63-84 \quad 0629$

\section{COMMON LAW}

MCSHANE, Steven L.

Reasonable Notice Criteria in Common Law Wrongful Dismissal Cases.

v. 38, no 3,1983, p. $618-6350229$

MCSHANE, Steven L. REDEKOP, Bruce Compensation Management and Canadian Wrongful Dismissal: Lessons from Litigation.

v. 45, no 2,1990, p. $357-381 \quad 0798$

\section{COMMUNAUTÉ ÉCONOMIQUE EURO-} PÉENNE

VANDAMME, François

L'information des travailleurs dans les grandes firmes: L'approche spécifique de la CEE.

v. 39 , no 1,1984, p. $51-76$

0271

COMMUNAUTÉ URBAINE DE MONTRÉAL MASSE, Gilles

Le temps passé au travail: un élément encore négociable?

36ième Congrès, 1981, p. 123-135 0839

\section{COMMUNAUTÉS RURALES}

GILSON, Clive H.J. SPENCER, lan S.

GRANVILIE, S.

The Impact of a Strike on the Attitudes and Behavior of a Rural Community.

v. 44 , no 4,1989 , p. $785-804 \quad 0753$ 
COMPARAISON DES SALAIRES

COUSINEAU, Jean-Michel RABEAU, Yves Une methodologie de comparaison des salaires pour les emplois spécifiques du secteur public.

v. 43 , no 1,1988 , p. $85-100$

0630

\section{COMPÉTENCE JURIDIQUE}

JOSEPH, P.A.

Perfecting the Administrative Solution to Labour Disputes.

v. 38 , no 4,1983 , p. $863-868$

\section{COMPORTEMENT SYNDICAL}

EASTMAN, Byron

A Catastrophe Theory on Union Behaviour. v. 40, no 2,1985 , p. $340-350 \quad 0389$

\section{COMPORTEMENTS}

RESHEF, Yonatan

A Typology of Shop Stewards: A Confirmatory Factor Analysis

v. 42 , no 1,1987 , p. $150-167 \quad 0545$

HACCOUN, Robert R. DUPONT, Serge Une analyse des comportements de travailleurs masculins et féminins selon deux formes d'absence au travail.

v. 43 , no 1,1988 , p. $153-166$

0634

\section{COMPORTEMENTS, SCIENCE DES}

XISHAN, Yang MOORE, Larry F.

MARKGRAF, Herb

Behavioural Science Application in Vancouver Based Firms.

v. 38 , no 1,1983 , p. 120-141 0184

\section{COMPTABLES AGRÉÉS}

AMERNIC, J.H. ARANYA, N.

Organizational Commitment: Testing Two Theories.

v. 38 , no 2,1983 , p. $319-343$

0206

\section{COMPTOIRS POSTAUX}

MARLEAU, Véronique L.

Décision rendue par le Conseil canadien des relations du travail.

v. 45 , no 2,1990, p. $414-423 \quad 0801$

\section{CONCERTATION}

GEORGE, Kenneth

Les comités de santé et de sécurité du travail: Tables de concertation ou de négociation?

v. 40 , no 3,1985 , p. $512-528$

0407

\section{CONCERTATION (suite)}

PLASSE, Micheline

Santé et sécurité du travail au Québec: Le défi de la concertation patronale - syndicale.

v. 42 , no 3,1987 , p. $544-565 \quad 0584$

OUELLET, Florian

Concertation et participation: mythe ou réalité?

39ième Congrès, 1984, p. 73-92 0877

DUMAS, Anne-Chantal SIMARD, Monique Commentaires - Concertation et participation: mythe ou realité.

39ième Congrès, 1984, p. 93-111 0878

\section{CONCIERGES}

MORIN, Fernand

La double personnalité d'un concierge!

v. 41, no 4,1986 , p. $835-8390522$

\section{CONCILIATION}

BEAUMONT, P.B.

Third Party Conciliation and Trade Union Recognition: Some British Evidence.

v. 37 , no 4,1982 , p. $827-842 \quad 0161$

HAMEED, Syed M.A. SEN, Joya

A Power Theory of Third Party Intervention in Labour Management Relations.

v. 42 , no 2,1987 , p. $243-255 \quad 0559$

ROSS, Claudette BROSSARD, Michel

La conciliation volontaire est-elle plus efficace que la conciliation obligatoire? Le cas du Québec.

v. 45 , no 1,1990 , p. $3-21$

0771

\section{CONCURRENCE SYNDICALE}

DELORME, François LAFRANCE, Victor La concurrence dans un contexte de pluralisme syndical: Quelques données sur le Québec.

v. 37 , no 3,1982, p. $575-605 \quad 0140$

MOUNT, Joan SIEGEL, Jacob P.

Resources and Results in Union Rivalry: A Case Study

v. 38 , no 4,1983 , p. $815-830 \quad 0250$ 


\section{CONDITIONS DE TRAVAIL}

BROSSARD, Michel

Revendications, stratégie syndicale et organisation du travail dans l'entreprise.

v. 36, no 3,1981 , p. $552-575$

0053

AOUST, Claude d'

Les ententes individuelles et la convention collective.

v. 38 , no 1,1983 , p. $155-158 \quad 0186$

MORIN, Fernand

Modification unilatérale des conditions de travail au terme d'une négociation collective!

v. 45 , no 3,1990 , p. $566-584 \quad 0817$

CARROTHERS, A.W.R.

Labour Law Through the Prism of Paccar v. 45 , no 3,1990 , p. 585-611 0818

CÔTÉ, André C.

Evolution des conditions de travail des salariés établies d'autorité.

40ième Congrès, 1985, p. 55-69 0891

PIUZE, Guy

Commentaires - Évolution des conditions de travail des salariés établies d'autorité.

40ième Congrès, 1985, p. 69-74 0892

\section{PRÉVOST, Charles}

La sous-estimation des atteintes à la santé causées par les mauvaises conditions de travail.

42ième Congrès, 1987, p. 51-60 0927

LAMARCHE, André GAUTHIER, Michel BLAIS, Michel GAGNON, Denise

Les conditions de travail au lendemain d'une fusion ou d'une réorganisation.

44ième Congrès, 1989, p. 101-127 0961

GAGNON, Astrid BLANCHETTE, Sylvain QUINTAL, Pierre

Fusion d'entreprises publiques.

44ième Congrès, 1989, p. 129-154 0962

MATHIEU, René

Négocier la flexibilité: La recherche d'équité par la négociation.

45ième Congrès, 1990, p. 82-92 0975

\section{CONFERENCE INTERNATIONALE DU} TRAVAIL

TAJGMAN, David

Political Resolutions in the International Labor Organizations: The experience since 1964.

v. 36 , no 3,1981 , p. $499-5290051$

\section{CONFLITS D'INTÉRÊT}

FASHOYIN, Tayo

State Regulation of Trade Disputes in Essential Services in Nigeria

v. 36, no 1, 1981, p. 207-222 0010

ADAMS, Georges W.

The Ontario Experience with Interest arbitration: Problems in Detecting Policy

v. 36 , no 1, 1981 , p. $225-250$

0012

\section{CAIRE, Guy}

Procédures de règiement pacifique des conflits collectifs en France.

v. 38 , no 1,1983 , p. $3-27$

0177

ANDIAPPAN, P. CATTANEO, R. Julian MURPHY, John

Interest Arbitration in Ontario Hospitals: Result of an Attitude Survey of Union and Management Officials.

v. 39, no 4,1984 , p. $680-694 \quad 0338$

\section{SUBBARAO, A.V.}

Impasse Choice in the Canadian Federal Service: An Innovation and an Intrigue

v. 40 , no 3,1985 , p. $567-590$

0410

KELLER, Berndt

Mediation as a Conflict-Solving Device in Collective Industrial Disputes.

v. 43 , no 2,1988 , p. $431-446$

0654

SUBBARAO, A.V.

Criteria in Arbitration of Wage Disputes: Theory and Practice in the Canadian Federal Public Service.

v. 43 , no 3,1988 , p. $547-570 \quad 0667$

\section{CONFLITS DU TRAVAIL}

MARTINEAU, LUC

La "gestion" des conflits par le Conseil canadien des relations du travail. v. 36 , no 3,1981 , p. $589-615$

0055 
Relations Industrielles, vol. 45, no 4 (1990)

CONFLITS DU TRAVAIL (suite)

FISHER, E.G.

Strike Activity and Wildcat Strikes in British Columbia: 1945-1975.

v. 37 , no 2,1982 , p. $284-312$

0120

JOSEPH, P.A.

Perfecting the Administrative Solution to Labour Disputes: The British Columbia Experiment.

v. 38, no 2,1983 , p. $380-414 \quad 0209$

\section{CONFLITS INTERPERSONNELS}

GARNIER, Bernard

La gestion des conflits interpersonne/s en milieu universitaire.

v. 38 , no 2,1983 , p. $277-296$

0204

\section{CONGÉDIEMENTS}

MARTINEAU, LUC

Décisions rendues par le Conseil canadien des relations du travail.

v. 36 , no 2,1981 , p. $424-429$

0035

MARTINEAU, LUC

Décisions rendues par le Conseil canadien des relations du travail.

v. 36 , no 3,1981 , p. $671-680$

0060

LAVERY, Daniel

Décisions rendues par le Conseil canadien des relations du travail.

v. 36 , no 4,1981 , p. $928-936$

0080

LAVERY, Daniel

Décisions rendues par le Conseil canadien des relations du travail.

v. 37 , no 2,1982 , p. $438-450$

MCSHANE, Steven L.

Reasonable Notice Criteria in Common Law Wrongful Dismissal Cases.

v. 38 , no 3,1983 , p. 618-635

0229

AOUST, Claude d'

Effets d'annulation d'un avis de congédiement par un arbitre

v. 39 , no 2,1984 , p. $365-370$

0299

MORIN, Fernand

Liberté d'expression et droit au travail: l'arbitrage de la Cour supréme du Canada. v. 44 , no 4 , 1989, p. 921-932

0761
CONGÉDIEMENTS (suite)

MCSHANE, Steven L. REDEKOP, Bruce

Compensation Management and Canadian

Wrongful Dismissal: Lessons from Litigation.

v. 45, no 2,1990, p. $357-381$

0798

\section{CONGRÈS DES RELATIONS INDUSTRIEL-} LES

HÉBERT, Gérard

Distinction 1982 de l'Association canadienne des relations industrielles: Gérard Dion.

v. 37 , no 3,1982 , p. $475-476 \quad 0135$

THWAITES, James LAJOIE, Mario

BOIS-BROCHU, Hélène

Supplément - Quarante ans au service des relations industrielles

40ième Congrès, 1985, p. 261-281 0904

\section{CONGRÈS DES RELATIONS INDUSTRIEL-}

\section{LES DES AMÉRIQUES}

SEXTON, Jean DION, Gérard

L'avenir des relations industrielles dans les Amériques: Introduction.

v. 44, no 1,1989, p. $1-2$

0698

\section{CONGRÈS DU TRAVAIL DU CANADA}

GILES, Anthony

The Canadian Labour Congress and Tripartism.

v. 37, no 1,1982 , p. $93-1250099$

\section{CONJOINTS}

LE LOUARN, Jean-Yves

THÉRIAULT, Roland

TOULOUSE, Jean-Marie

Le travail des deux conjoints: Effets sur la progression de carrière du cadre.

v. 39 , no 1,1984 , p. $36-50$

0270

\section{CONSEIL CANADIEN DES RELATIONS DU}

TRAVAIL

MARTINEAU, LUC

Décisions rendues par le Conseil canadien des relations du travail.

v. 36 , no 1,1981 , p. $263-266$

0015

MARTINEAU, LUC

Décisions rendues par le Conseil canadien des relations du travail.

v. 36 , no 2,1981 , p. $424-429$

0035 


\section{CONSEIL CANADIEN DES RELATIONS DU TRAVAIL (sulte) \\ MARTINEAU, LUC \\ La "gestion' des conflits par le Conseil canadien des relations du travail.}

v. 36 , no 3,1981 , p. $589-615$

0055

MARTINEAU, LUC

Décisions rendues par le Conseil canadien des relations du travail.

v. 36 , no 3,1981 , p. $671-680$

0060

LAVERY, Daniel

Décisions rendues par le Conseil canadien des relations du travail.

v. 36 , no 4,1981 , p. $928-936$

0080

LAVERY, Daniel

Décisions rendues par le Conseil canadien des relations du travail.

v. 37 , no 1,1982 , p. 226-234 0109

LAVERY, Daniel

Décisions rendues par le Conseil canadien des relations du travail.

v. 37 , no 2,1982 , p. $438-450$

0128

LAVERY, Daniel

Décisions rendues par le Conseil canadien des relations du travail.

v. 37 , no 3,1982 , p. $684-696 \quad 0146$

COULOMBE, Gilles

Le système de gestion des cas du Conseil canadien des relations du travail (CCRT).

v. 37, no 4,1982 , p. $928-943 \quad 0166$

LAVERY, Daniel

Décisions rendues par le Conseil canadien des relations du travail.

v. 37 , no 4,1982 , p. $944-959 \quad 0167$

LAVERY, Daniel

Décisions rendues par le Conseil canadien des relations du travail.

v. 38 , no 1,1983 , p. $159-173$

0187

LAVERY, Daniel

Décisions rendues par le Conseil canadien des relations du travail.

v. 38 , no 2,1983 , p. $418-432$

0211
CONSEIL CANADIEN DES RELATIONS DU

TRAVAIL (sulte)

LAVERY, Daniel

Décisions rendues par le Conseil canadien des relations $d u$ travail.

v. 38 , no 3,1983 , p. $658-666$

0231

BEAULIEU, LUC

Décisions rendues par le Conseil canadien des relations du travail.

v. 38, no 4,1983 , p. $880-903$

0255

BEAULIEU, LUC

Décisions rendues par le Conseil canadien des relations du travail.

v. 39 , no 1,1984 , p. 177-189

0277

BEAULIEU, LUC

Decisions rendues par le Conseil canadien des relations du travail

v. 39 , no 2,1984 , p. $371-385$

0300

BEAULIEU, LUC

Décisions rendues par le Conseil canadien des relations du travail.

v. 39 , no 3,1984 , p. $605-618 \quad 0323$

POTHIER, Dianne

Décisions rendues par le Conseil canadien des relations du travail.

v. 39 , no 4,1984 , p. $774-782$

0344

POTHIER, Dianne

Décisions rendues par le Conseil canadien des relations du travail.

v. 40 , no 1,1985 , p. $170-174$

0371

LAMY, Francine

Décisions rendues par le Conseil canadien des relations du travail.

v. 40 , no 2,1985 , p. $386-398 \quad 0394$

SOLOMON, Norman A.

The Negociation of First Agreements under the Canada Labour Code.

v. 40, no 3,1985, p. $458-472 \quad 0404$

LAMY, Francine

Décision rendue par le Conseil canadien des relations du travail.

v. 40 , no 3,1985, p. $659-662$

0415 


\section{CONSEIL CANADIEN DES RELATIONS DU} TRAVAIL (suite)

SAINT-GERMAIN, Catherine

Décisions rendues par le Conseil canadien des relations du travail.

v. 41 , no 2,1986, p. $397-411$

0477

SAINT-GERMAIN, Catherine Décisions rendues par le Conseil canadien des relations du travail v. 41 , no 3,1986 , p. $622-637$

SAINT-GERMAIN, Catherine Décisions rendues par le Conseil canadien des relations du travail. v. 41 , no 4,1986 , p. $860-868$

SAINT-GERMAIN, Catherine Décision rendue par le Conseil canadien des relations du travail.

v. 42, no 1,1987, p. $203-209$

0549

SAINT-GERMAIN, Catherine

Décisions rendues par le Conseil canadien des relations du travail

v. 42 , no 2,1987 , p. $415-427$

0569

SAINT-GERMAIN, Gatherine

Décision rendue par le Conseil canadien des relations du travail.

v. 42 , no 3,1987, p. $628-635$

0589

TREMBLAY, Johane

Décisions rendues par le Conseil canadien des relations du travail.

v. 42 , no 4,1987 , p. $852-861$

0613

TREMBLAY, Johane

Décisions rendues par le Conseil canadien des relations du travail.

v. 43 , no 1, †988, p. $188-195$

0637

TREMBLAY, Johane

Décisions rendues par le Conseil canadien des relations du travail.

v. 43 , no 2,1988 , p. $447-452$

0655

TREMBLAY, Johane

Décisions rendues par le Conseil canadien des relations du travail.

v. 43 , no 3,1988 , p. $686-694$
CONSEIL CANADIEN DES RELATIONS DU TRAVAIL (suite)

MARLEAU, Véronique $L$.

Décisions rendues par le Conseil canadien des relations du travail.

v. 43, no 4,1988 , p. $953-962$

0692

MARLEAU, Véronique L.

Décisions rendues par le Conseil canadien des relations du travail.

v. 44 , no 2,1989, p. $448-462$

0726

MARLEAU, Véronique L.

Décisions rendues par le Conseil canadien des relations du travail.

v. 44 , no 3, 1989, p. 703-722 0744

CLARKE, Graham

Décisions rendues par le Conseil canadien des relations du travail.

v. 44, no 4, 1989, p. 933-946 0762

CLARKE, Graham

Décisions rendues par le Conseil canadien des relations du travail.

v. 45, no 1, 1990, p. 187-197 0782

MARLEAU, Véronique $\mathrm{L}$.

Décision rendue par le Conseil canadien des relations du travail.

v. 45 , no 2,1990 , p. $414-423 \quad 0801$

CLARKE, Graham

Décision rendue par le Conseil canadien des relations du travail.

v. 45, no 3, 1990 , p. $616-624 \quad 0820$

CONSEIL DES RELATIONS DU TRAVAIL

VERGE, Pierre

La mission-type d'un Conseil des relations du travail moderne.

v. 36 , no 3,1981 , p. $663-670 \quad 0059$

\section{CONSEIL ECONOMIQUE DU CANADA}

BELLEMARE, Diane

Les emplois de l'avenir et les défis de la société.

45ième Congrès, 1990, p. 117-128 0978

LAVALLÉE, Diane CHARLAND, Gaston LEPAGE, Brigitte MASSÉ, Henri

MERCIER, Jean

Les emplois de lavenir et les defis de la société - Commentaires.

45ième Congrès, 1990், p. 128-148 0979 


\section{CONSEILLERS EN RELATIONS INDUS- TRIELLES}

BLOUIN, Rodrigue

Le titre réservé de CRI et la déontologie de la profession

v. 42, no 2,1987 , p. $309-324 \quad 0563$

\section{CONSEILS SCOLAIRES}

BEATTY, Carol GANDZ, Jeffrey

After the Strike: Changing the Teacher. Board Relationship.

v. 44, no 3,1989 , p. $569-592$

0737

\section{CONSTRUCTION (INDUSTRIE)}

MILLER, Roger

Les formes d'organisation dans l'industrie de la construction au Québec.

v. 37, no 1,1982 , p. 164-176

0102

ROSE, Joseph B. WETZEL, Kurt

Outcomes of Bargaining Structures in the Ontario and Saskatchewan Construction Industries.

v. 41 , no 2,1986 , p. $256-280$

0468

FISHER, E.G. KUSHNER, Stephen

Alberta's Construction Labour Relations During the Recent Downturn

v. 41 , no 4,1986 , p. $778-801$

0518

\section{CONTRAT DE TRAVAIL}

PLISZKIEWICZ, Marek

Le licenciement en droit polonais.

v. 36, no 1,1981, p. 251-259

0013

AOUST, Claude d'

Les ententes individuelles et la convention collective.

v. 38, no 1, 1983, p. 155-158 0186

\section{CONTRAT SOCIAL}

STURMTHAL, Adolf

Unemployment, Inflation and "Guest Wor-

kers": Comparative Study of Three European Countries.

v. 37, no 4,1982 , p. 739-764 0157

\section{CONTREMAITTRES}

MORIN, Fernand

La survie de droits subjectifs à la convention collective.

v. 40, no 4,1985 , p. $847-855$

0431

\section{CONTREMAÎTRES (suite)}

KNIGHT, Thomas R.

Correlates of Informal Grievance Resolution Among Fist-Line Supervisors

v. 41, no 2,1986, p. 281-298

0469

DULUDE, Yves

Le conflit: la gestion au banc des accusés.

41ième Congrès, 1986, p. 111-133 0914

\section{LAMARCHE, Pierre}

Commentaires - Le conflit: la gestion au banc des accusés.

41ième Congrès, 1986, p. 133-136 0915

\section{CONTRO̊LE}

CHARLES, Lawrence $M$.

HUMPHREYS, Edward $\mathrm{H}$.

Bargaining to Achieve Teacher Control in Ontario

v. 40, no 3,1985, p. $495-511 \quad 0406$

GILSON, Clive H.J.

Changes in the Nature of Grievance Issues Over the Last Ten Years: Labor Management Relations and the "Frontier of Control".

v. 40 , no 4,1985 , p. $856-8640432$

\section{BARKIN, Solomon}

The Current Unilateralist Counterattack on Unionism and Collective Bargaining

v. 41 , no 1,1986, p. $3-27$

0447

\section{CONTRÓLE DES PRIX ET DES SALAIRES}

GILES, Anthony

The Canadian Labour Congress and Tripartism.

v. 37, no 1,1982 , p. $93-125 \quad 0099$

\section{CONTRÓLE DES SALAIRES}

AULD, D.A.L. WILTON, D.A.

The Impact of Public Sector Wage Controls in Ontario

v. 42 , no 1,1987 , p. 132-149 0544

\section{CONTRÓLE JUDICIAIRE}

MORIN, Fernand

Le "raisonnable" déraisonnable! ou la rationalité du raisonnable

v. 40, no 3,1985, p. $646-658 \quad 0414$ 


\section{CONTRÓLE JUDICIAIRE (suite)}

AOUST, Claude d'

Le contrôle judiciaire des décisions arbitrales: Réflexions sur les moyens d'y échapper.

v. 41 , no 2,1986 , p. $348-356$

0473

MORIN, Fernand

Le rationnel et le raisonnable: Deux nécessités distinctes et conjugées en droit.

v. 41 , no 3,1986 , p. $578-582$

0494

\section{CONTRÓLE OUVRIER}

BÉLANGER, Jacques

Le contrôle ouvrier sur l'organisation du travail: Étude de cas en Grande-Bretagne. v. 41, no 4, 1986, p. 704-719 0514

\section{CONTRÓLE SYNDICAL}

LAFLAMME, Gilles VALLÉE, Guylaine Changements technologiques et modes régulateurs des relations du travail.

v. 42 , no 4,1987 , p. $702-7150605$

\section{CONVENTIONS COLLECTIVES}

PONAK, Allen MOORE, Larry F.

Canadian Bank Unionism: Perspectives and issues

v. 36 , no 1,1981 , p. 3-34

0001

\section{BRISSON, Chantal}

La sante au travail et la convention collective

v. 36 , no 1,1981, p. $152-178$

0007

MARTINEAU, LUC

Décisions rendues par le Conseil canadien des relations du travail.

v. 36 , no 1,1981 , p. $263-266$

0015

SWIDINSKI, Robert

The Effect of Bargaining Structure on Negotiated Wage Settlements.

v. 36, no 2,1981, p. $371-385$

0029

HÉBERT, Gérard

Les relations du travail au Québec: Bilan des années 1970

v. 36 , no 4,1981 , p. $715-747$

0071

MCPHILLIPS, David C.

Duty of Fair Representation: Recent Attitudes in British Columbia and Ontario.

v. 36 , no 4,1981 , p. $803-827$

\section{CONVENTIONS COLLECTIVES (suite)}

CHOUINARD, Jacques VIEL, André

L'efficacité des clauses d'indexation des salaires

v. 37, no 1, 1982, p. 207-214 0105

COUSINEAU, Jean-Michel

LACROIX, Robert

L'efficacité des clauses d'indexation des salaires: Réplique.

v. 36 , no 1,1982 , p. $214-2200106$

CHOUINARD, Jacques

ROBICHAUD, Jean-Marc

L'efficacité des clauses d'indexation des salaires.

v. 37 , no 1,1982 , p. $220-224 \quad 0107$

MICHAUD, Sharon ROY, Jean-Charles L'efficacité des clauses d'indexation des salaires: Réplique.

v. 37, no 1,1982, p. $224-2250108$

DESCHÊNES, Jean-Paul

L'évaluation des emplois et la convention collective.

v. 39 , no 2,1982 , p. $313-327$

0121

AOUST, Claude d'

Les ententes individuelles et la convention collective.

v. 38, no 1,1983 , p. 155-158 0186

VEILLEUX, Diane

Les clauses de promotion et de transfert chez les infirmières et les infirmiers.

v. 38, no 2, 1983, p. 229-253 0202

BERNIER, Jean

L'extension juridique des conventions collectives au Québec: Une approche comparative.

v. 38 , no 3,1983 , p. $532-544$

0225

BOULARD, René MARCHAND, Michel La loi sur la santé et la sécurité du travail et les conventions collectives.

v. 38, no 4, 1983, p. 847-862 0252

SOLOMON, Norman A.

The Negociation of First Agreement in Ontario.

v. 39 , no 1,1984, p. 23-35 0269 
CONVENTIONS COLLECTIVES (suite) PONAK, Allen THOMPSON, Mark

Faculty Collective Bargaining: The Voice of Experience

v. 39, no 3,1984, p. $449-465$

0314

ADAMS, Roy J.

The Extent of Collective Bargaining in Canada

v. 39 , no 4,1984 , p. $655-667$

0336

MORIN, Fernand

Les tenants et les aboutissants de la convention collective.

v. 40, no 2,1985 , p. $371-378$

0392

SOLOMON, Norman A.

The Negociation of First Agreements under the Canada Labour Code.

v. 40, no 3,1985, p. $458-472$

0404

MORIN, Fernand

La survie de droits subjectifs à la conven tion collective.

v. 40, no 4,1985 , p. $847-855$

0431

RONDEAU, Claude BADIN, François

Le contenu non salarial des conventions collectives dans les industries manufacturières au Québec.

v. 41 , no 1,1986, p. $69-90$

0450

SAINT-GERMAIN, Catherine

Décisions rendues par le Conseil canadien des relations du travail.

v. 41 , no 2,1986 , p. $397-411$

0477

FORREST, Anne

Bargaining Units and Bargaining Power

v. 41 , no 4,1986 , p. $840-850$

0523

SEXTON, Jean

L'arbitrage de première convention collective au Québec: 1978-1984

v. 42 , no 2,1987 , p. $272-291$

0561

MELTZ, Noah M.

Job Security in Canada

v. 44 , no 1,1989 , p. $149-161$

0705

MORIN, Fernand

D'un entrepreneur à un autre: l'accréditation ne suit pas

v. 44 , no 2,1989 , p. $315-336$

0719
CONVENTIONS COLLECTIVES (suite)

RESHEF, Yonatan

Negotiating Wage Settlements: A Structural Approach.

v. 44, no 3,1989, p. $532-551 \quad 0735$

VALLÉE, Guylaine

Les accords "atypiques" et le droit des rapports collectifs du travail.

v. 44 , no 3,1989 , p. $680-690 \quad 0742$

HÉBERT, Gérard

Le renouvellement du régime des décrets de convention collective.

v. 45 , no 2,1990 , p. $404-413 \quad 0800$

MORIN, Fernand

Modification unilatérale des conditions de travail au terme d'une négociation collective!

v. 45, no 3,1990 , p. $566-584 \quad 0817$

CARROTHERS, A.W.R

Labour Law Through the Prism of Paccar v. 45 , no 3,1990 , p. $585-611 \quad 0818$

MASSE, Gilles

Le temps passé au travail: un elément encore négociable?

36ième Congrès, 1981, p. 123-135 0839

DESCHÊNES, Jean-Paul

Commentaires - Le temps passé au travail: un élément encore négociable?

36ième Congrès, 1981, p. 136-1440840

BOULARD, René

L'impact de la Loi sur les conventions collectives.

39ième Congrès, 1984, p. 137-157 0880

LISTON, Terrence

Négocier la flexibilité: La polyvalence des emplois.

45ième Congrès, 1990, p. 65-69 0973

\section{CONVENTIONS INTERNATIONALES}

ROSSILION, Claude

L'OIT et l'élimination de la discrimination dans l'emploi

v. 40 , no 1,1985 , p. $03-266^{\circ} 0362$ 
CONVENTIONS INTERNATIONALES (suite) ZEYTINOGLU, Isik Urla

The ILO Standards and Canadian Labour Législation.

v. 42 , no 2,1987 , p. $292-308$

0562

\section{CONVICTIONS RELIGIEUSES}

SAINT-GERMAIN, Catherine

Decisions rendues par le Conseil canadien des relations du travail

v. 41 , no 3,1986, p. $622-637$

0497

\section{COOPÉRATION EMPLOYEURS-SALARIÉS} MROCZKOWSKI, Tomasz

Theory Z: Myths, Realities and Alternatives. v. 38 , no 2,1983 , p. $297-318$

0205

\section{CORPORATION DES CONSEILLERS EN} REL. IND.

RICHER, Jean-Charles

La Corporation professionnelle des conseillers en relations industrielles du Québec devant la Commission Beaudry.

v. 40, no 2,1985, p. $379-385$

0393

BLOUIN, Rodrigue

Le titre réservé de CRI et la déontologie de la profession

v. 42 , no 2,1987, p. $309-324$

0563

\section{CORPORATIONS PROFESSIONNELLES}

DUSSAULT, Gilles

The Future of Professional Monopolies.

v. 40 , no 2,1985 , p. $324-339$

0388

\section{CORPORATISME}

WARNER, Malcolm

Corporatism, Participation and Society

v. 38 , no 1,1983 , p. $27-44$

\section{COUPLES}

LE LOUARN, Jean-Yves

THÉRIAULT, Roland

TOULOUSE, Jean-Marie

Le travail des deux conjoints: Effets sur la progression de carrière du cadre.

v. 39 , no 1,1984 , p. $36-50$

0270

\section{COUR D'APPEL DU QUÉBEC}

MORIN, Fernand

La survie de droits subjectifs à la convention collective.

v. 40 , no 4,1985 , p. $847-855$

0431

\section{COUR DES PETITES CRÉANCES}

AOUST, Claude d'

La juridiction de l'arbitre et de la Cour des petites créances devant la Cour suprême du Canada.

v. 36, no 2, 1981, p. 418-424 0034

\section{COUR SUPRÊME DU CANADA}

AOUST, Claude d'

Les réclamations à l'arbitrage de dommages résultant de la violation d'une clause de renonciation à la grève.

v. 36, no 1,1981 , p. $259-262 \quad 0014$

AOUST, Claude d'

La juridiction de l'arbitre et de la Cour des petites créances devant la Cour supréme du Canada.

v. 36, no 2,1981 , p. $418-424$

0034

JOSEPH, P.A.

Perfecting the Administrative Solution to Labour Disputes.

v. 38 , no 4,1983 , p. $863-868 \quad 0253$

SUBBARAO, A.V.

Impasse Choice in the Canadian Federal Service: An Innovation and an Intrigue

v. 40 , no 3,1985 , p. $567-590 \quad 0410$

MORIN, Fernand

Le "raisonnable" déraisonnable! ou la rationalité du raisonnable

v. 40 , no 3,1985 , p. $646-658 \quad 0414$

MORIN, Fernand

Le rationnel et le raisonnable: Deux nécessités distinctes et conjugées en droit.

v. 41 , no 3,1986 , p. $578-582$

0494

TOUPIN, Sylvain

Le harcèlement sexuel en milieu de travail. v. 43 , no 3,1988 , p. $531-546 \quad 0666$

MORIN, Fernand

D'un entrepreneur à un autre: l'accréditation ne suit pas

v. 44, no 2,1989 , p. $315-336 \quad 0719$

MORIN, Fernand

Liberté d'expression et droit au travail: l'arbitrage de la Cour suprême du Canada. v. 44, no 4, 1989, p. 921-932 0761 


\section{COUR SUPRÊME DU CANADA (suite)}

MORIN, Fernand

Modification unilatéraje des conditions de travail au terme d'une négociation collective!

v. 45 , no 3,1990, p. $566-584$

0817

CARROTHERS, A.W.R.

Labour Law Through the Prism of Paccar v. 45, no 3,1990, p. $585-611$

0818

\section{PÉPIN, Marcel}

L'actualisation du mouvement syndical. 43ième Congrès, 1988, p. 209-221 0952

GAGNON, Mona-Joseee LEBEAU, Ernest JOHNSTON, Raymond

Commentaires - lactualisation du mouvement syndical.

43ième Congrès, 1988, p. 222-236 0953

\section{COURTAGE IMMOBILIER}

DOLAN, Shimon L. TZINER, Aharon ROY, Denis

A Real Estate Agency's Level Analysis of the Climate-Performance Relationship.

v. 39 , no 1, 1984, p. 167-176

\section{COÛTS D'UNE GRÈVE}

TANG, Roger Y.W. PONAK, Allen Employer Assessment of Strike Costs.

v. 41 , no 3,1986 , p. $552-571$

0492

\section{CRISE DU TRAVAIL}

BÉLANGER, Paul R. LÉVESQUE, Benoît Le mode de vie détermine-t-il l'éthique du travail? Etude de cas.

v. 42 , no 2,1987, p. $345-3650565$

\section{CROISSANCE SYNDICALE}

KUMAR, Pradeep DOW, Bradley Econometric Analysis of Union Membership Growth in Canada, 1935-1981

v. 41 , no 2,1986, p. $236-255$

0467

GILSON, Clive H.J. SPENCER, Ian S. Trade Union Growth: A Marketing Model. v. 42 , no 4,1987 , p. $756-773 \quad 0608$

\section{CUIVRE (INDUSTRIE)}

FISHER, E.G. PERCY, M.B.

The Impact of Unanticipated Output and Consumer Prices on Wildcat Strikes.

v. 38 , no 2,1983 , p. $254-276 \quad 0203$

\section{CULINAR INC.}

NÉRON, Roger

Le projet d'entreprise de Culinar Inc.

41ième Congrès, 1986, p. 69-76 0910

BOUCHER, Lysette

Les limites des nouvelles approches en gestion des ressources humaines.

41ième Congrès, 1986, p. 77-84 0911

\section{DANGER IMMINENT}

LAVERY, Daniel

Décisions rendues par le Conseil canadien des relations du travail.

v. 36 , no 4,1981 , p. $928-936 \quad 0080$

\section{DÉCISION, PRISE DE}

PECCEI, Ricardo WARNER, Malcolm

Industrial Relations, Strategic Importance, and Decision - Making.

v. 36 , no 1,1981, p. 132-151 0006

MEALIA, David W.

A Macro/Micro Décision Model for the Training and Development Specialist.

v. 37 , no 3,1982 , p. $656-669$

0144

\section{DÉCISIONS ARBITRALES}

AOUST, Claude d'

Le contrôle judiciaire des décisions arbitrales: Réflexions sur les moyens d'y échapper.

v. 41 , no 2,1986 , p. $348-356 \quad 0473$

\section{DÉCRETS DE CONVENTION COLLECTIVE} HÉBERT, Gérard

Le renouvellement du régime des décrets de convention collective.

v. 45 , no 2,1990 , p. $404-413 \quad 0800$

\section{DÉLAI-CONGÉ}

MCSHANE, Steven L.

Reasonable Notice Criteria in Common Law Wrongful Dismissal Cases.

v. 38, no 3, 1983, p. 618-635 0229

DÉLÉGUÉS DU PERSONNEL

KAMINKA, Shlomit ROSEINSTEIN, Eliezer Shop Stewards, Workers, and Managers in Israëli Industry.

v. 38 , no 3,1983 , p. $598-617 \quad 0228$ 
Relations lndustrielles, vol. 45, no 4 (1990)

DÉLÉGuÉs DU PERSONNEL (suite) RESHEF, Yonatan

A Typology of Shop Stewards: A Confirmatory Factor Analysis

v. 42 , no 1,1987 , p. $150-167$

0545

\section{DÉMOCRATIE INDUSTRIELLE}

WARNER, Malcolm

Corporatism, Participation and Society

v. 38 , no 1,1983 , p. $27-44$

RICHARDS, John MAUSER, Gary

HOLMES, Richard

What Do Workers Want? Attitudes Towards Collective Bargaining and Participation in Management.

v. 43, no 1,1988 , p. $133-152$

0633

\section{DÉMOTIONS}

CHAYKOWSKI, Richard P.

SLOTSVE, G.A.

Union Seniority Rules as a Determinant of Intra-Firm Job-Changes.

v. 41 , no 4,1986 , p. $720-737$

0515

\section{DENSITÉ SYNDICALE}

BÉLANGER, Jacques MERCIER, Jacques Le plafonnement de la densité syndicale au Québec et au Canada.

v. 41 , no 1,1986, p. $28-52$

0448

KUMAR, Pradeep

Estimates of Unionism and Collective Bargaining in Canada.

v. 43, no 4,1988 , p. $757-779$

\section{DÉONTOLOGIE}

BLOUIN, Rodrigue

Le titre réservé de $C R I$ et la déontologie de la profession

v. 42 , no 2,1987 , p. $309-324$

0563

\section{DÉPARTEMENT DES RELATIONS INDUS-}

TRIELLES (UNIV. LAVAL)

HÉBERT, Gérard

Distinction 1982 de l'Association canadienne des relations industrielles: Gérard Dion.

v. 37, no 3,1982 , p. $475-476 \quad 0135$

THWAITES, James LAJOIE, Mario BOIS-BROCHU, Hélène

Supplément - Quarante ans au service des relations industrielles

40ième Congrès, 1985, p. 261-281 0904

\section{DÉRÉGLEMENTATION}

MUCKENBERGER, Ulrich

The Regulation of Strike Law in Times of New Technologies and Deregulation: The Case of West Germany.

v. 45 , no 1,1990 , p. $136-145 \quad 0778$

\section{DÉVELOPPEMENT ET FORMATION}

MEALIA, David W.

A Macro/Micro Décision Model for the Training and Development Specialist.

v. 37 , no 3,1982 , p. $656-669$

0144

\section{DICTIONNAIRE CANADIEN DESRELATIONS}

DU TRAVAIL

HÉBERT, Gérard

Distinction 1982 de I'Association canadienne des relations industrielles: Gerard Dion.

v. 37 , no 3,1982 , p. $475-476 \quad 0135$

\section{DIFFÉRENCES SALARIALES}

AGARWAL, Naresh C.

Male-Female Pay Inequity and Public Policy in Canada and the U.S.

v. 37, no 4, 1982, p. 780-804 0159

DEVORETZ, D.J. REED, Clyde G.

Evidence from the Skilled-Unskilled Canadian Wage Index.

v. 39, no 3, 1984, p. 526-537

0318

\section{GUNDERSON, Morley}

Spline Functions Estimates of the Impact of Equal Pay Légis/ation: The Ontario Experience

v. 40 , no 4,1985, p. $775-792 \quad 0427$

OHTSU, Makoto VERMA, Anil

Intra-Organizational Bargaining: Wage Ditferentials Among Saskatchewan Schoolteachers.

v. 42 , no 3,1987 , p. $612-627 \quad 0588$

\section{DIFFÉRENCIATION OUVRIÈRE}

BROSSARD, Michel SIMARD, Marcel

Problématique de la différenciation de la main-d'oeuvre et changement organisationnel.

v. 41 , no 2,1986 , p. $219-235$

0466 


\section{DIFFERENDS}

ADAMS, Georges W.

The Ontario Experience with Interest arbitration: Problems in Detecting Policy

v. 36 , no 1,1981 , p. $225-250$

0012

BRUCE, Christopher J.

The Role of Information Concerning the Arbitrator's Preferences.

v. 36 , no 2,1981, p. 386-402 0030

CAIRE, Guy

Procédures de règlement pacifique des conflits collectifs en France.

v. 38 , no 1,1983 , p. $3-27$

JOSEPH, P.A.

Perfecting the Administrative Solution to Labour Disputes: The British Columbia Experiment.

v. 38, no 2,1983 , p. $380-414$

0209

ANDIAPPAN, P. CATTANEO, R. Julian MURPHY, John

Interest Arbitration in Ontario Hospitals: Result of an Attitude Survey of Union and Management Officials.

v. 39 , no 4,1984 , p. $680-694$

0338

SEXTON, Jean

L'arbitrage de première convention collective au Québec: 1978-1984

v. 42, no 2,1987 , p. $272-291$

0561

SUBBARAO, A.V.

Criteria in Arbitration of Wage Disputes: Theory and Practice in the Canadian Federal Public Service.

v. 43, no 3,1988, p. $547-570$

0667

DION, GÉRARD

HÉBERT, Gérard

Distinction 1982 de l'Association canadienne des relations industrielles: Gerard Dion.

v. 37, no 3,1982, p. $475-476 \quad 0135$

\section{DIRIGEANTS SYNDICAUX}

CHAISON, Gary N. ANDIAPPAN, P.

Characteristics of Female Union Officer in Canada.

v. 37 , no 4,1982 , p. $765-779 \quad 0158$
DIRIGEANTS SYNDICAUX (sulte)

SOLOMON, Norman A. ANDIAPPAN, P.

SHAND, Dan

Canadian National Union Presidents: An

Empirical Study.

v. 41 , no 3,1986 , p. $491-504$

0488

SEFTON MACDOWELL, Laurel

The Career of a Canadian Trade Union

Leader: C.H. Millard 1937-1946

v. 43, no 3,1988 , p. $609-632$

0670

\section{DISCRIMINATION}

JAIN, Harish C.

Race ans Sex Discrimination in Employment in Canada: Theories, Evidence and Policies.

v. 37, no 2,1982, p. $344-366 \quad 0123$

ROBB, Roberta Edgecome

Occupational Segregation and Equal Pay for Work of Equal Value.

v. 39 , no 1,1984 , p. $146-166$

0275

ROSSILION, Claude

L'OIT et l'élimination de la discrimination dans l'emploi

v. 40 , no 1,1985, p. $03-26$

0362

BURT, Sandra

Voluntary Affirmative Action. Does it Work?

v. 41, no 3, 1986, p. 541-551 0491

JAIN, Harish C. ANDIAPPAN, P.

Sexual Harassment in Employment in $\mathrm{Ca}$ nada: Issues and Policies.

v. 41, no 4,1986 , p. $758-777 \quad 0517$

ANDIAPPAN, P. CRESTOHL, Mark

SINGH, Jang B.

Racial Discrimination in Employment in Canada.

v. 44 , no 4,1989 , p. $827-8490755$

GUÉRIN, Gilles HÉBERT, Michel

Les obstacles rencontrés par des personnes de 45 à 64 ans à la recherche d'un emploi.

v. 45, no 2,1990, p. $235-267 \quad 0792$

SWIMMER, Gene

Gender Based Differences in Promotions of Clerical Workers.

v. 45, no 2,1990, p. $300-310 \quad 0795$ 


\section{DISCRIMINATION (sulte)}

DUCHARME, Claude

LECLERCQ, Dominique

WESTMORELAND-TRAORE, Juanita

Commentaires - La gestion de l'embau-

che, de la promotion et du licenciement

revue et corrigee par les Chartes.

43ième Congrès, 1988, p. 63-82

0945

DUSSAULT, Ginette

A travail equivalent, salaire égal: Un droit difficile à appliquer?

43ième Congrès, 1988, p. 83-95 0946

BOIVIN, Suzanne P.

Le Canadien National: Un cas riche d'enseignements.

43ième Congrès, 1988, p. 97-113 0947

SIMARD, Monique TELLIER, Marie

Commentaires - Le Canadien National: Un cas riche d'enseignements.

43ième Congrès, 1988, p. 114-130 0948

\section{DISPARITÉ SALARIALE}

DOOLEY, Martin D.

Within-Cohort Earnings Inequality Among Canadian Men: 1971-1982

v. 42, no 3,1987 , p. $594-611$

0587

\section{DIVULGATION DE L'INFORMATION}

JAIN, Hem C.

Disclosure of Corporate Information to Trade Unions in North America.

v. 36 , no 4,1981 , p. $748-774$

0072

\section{DOFASCO}

STOREY, Robert $\mathrm{H}$.

The Struggle to Organize Stelco and Dofasco

v. 42 , no 2,1987, p. $366-385$

0566

\section{DOMMAGES}

AOUST, Claude d'

Les réclamations à l'arbitrage de dommages résultant de la violation d'une clause de renonciation à la grève.

v. 36 , no 1,1981 , p. $259-262$

0014

\section{DOUBLE ALLÉGEANCE}

THACKER, James W. ROSEN, Hjalmar Dynamics of Employee Reactance to Company and Union Dual Allegiance Revisited and Expanded.

v. 41 , no 1,1986 , p. 128-144

0453

\section{DOUBLE EMPLOI}

JAMAL, Muhammad

CRAWFORD, Ronald $\mathrm{L}$.

Moonlighters: A Product of Deprivation or Aspiration?

v. 36 , no 2,1981 , p. $325-3350026$

\section{DOYENS D'UNIVERSITÉ}

GARNIER, Bernard

La gestion des conflits interpersonnels en milieu universitaire.

v. 38, no 2,1983 , p. $277-296 \quad 0204$

DROIT AU TRAVAIL

MORIN, Fernand

Libené d'expression et droit au travail: l'arbitrage de la Cour supreme du Canada.

v. 44 , no 4, 1989, p. 921-932 0761

\section{DROIT DE GRÈVE}

AOUST, Claude d' DELORME, François The Origin of the Freedom of Association and of the Right to Strike in Canada: An Historical Perspective.

v. 36 , no 4,1981 , p. $894-921$

0078

VERGE, Pierre

Syndicalisation de la grève.

v. 38 , no 3,1983 , p. $475-506$

0223

\section{MORIN, Fernand}

Rapports collectifs du travail dans les secteurs publics québécois ou le nouvel équilibre selon la loi du 19 juin 1985.

v. 40, no 3,1985, p. $629-645$

0413

\section{BARPÉ, Alain}

Le cadre juridique de la négociation collective dans le secteur public au Québec: une perspective internationale.

v. 42, no 4,1987 , p. $831-851$

0612

\section{DROIT DE REFUS}

MARLEAU, Véronique $L$.

Décisions rendues par le Conseil canadien des relations du travail.

v. 43 , no 4,1988 , p. $953-962 \quad 0692$

CLARKE, Graham

Décisions rendues par le Conseil canadien des relations du travail.

v. 44 , no 4,1989 , p. $933-9460762$ 


\section{DROIT DE REFUS (suite)}

DE KONINCK, Maria HEENAN, Roy

Droit de refus et retrait préventif: succès ou échec?

39ième Congrès, 1984, p. 159-175 0881

\section{DROITS DE LA DIRECTION}

LAVERY, Daniel

Decisions rendues par le Conseil canadien des relations du travail.

v. 38 , no 3,1983 , p. $658-666$

FISHER, E.G. SHERWOOD, L.M.

Fairness and Managerial Rights in Canadian Arbitral Jurisprudence

v. 39 , no 3,1984 , p. $538-552$

\section{BARKIN, Solomon}

The Flexibility Debate in Western Europe: The Current Drive to Restore Managements' Rights Over Personnel and Wages.

v. 42 , no 1,1987, p. $12-44$

0539

\section{DROITS DE SUCCESSION}

\section{LAVERY, Daniel}

Décisions rendues par le Conseil canadien des relations du travail.

v. 38 , no 1,1983 , p. 159-173

0187

\section{DROITS ET LIBERTÉS}

CARTER, Donald D.

Canadian Labour Relations Under the Charter: Exploring the Implications.

v. 43 , no 2,1988, p. $305-321$

0648

ANDIAPPAN, P. CRESTOHL, Mark SINGH, Jang B.

Racial Discrimination in Employment in Canada.

v. 44 , no 4,1989 , p. $827-849$

0755

BLOUIN, Rodrigue FERLAND, Gilles LAFLAMME, Gilles LAROCQUE, Alain RONDEAU, Claude

Introduction - Les Chartes des droits et les relations industrielles.

43ième Congrès, 1988, p. XV

0941

ROCHER, Guy

Les fondements de la societé liberale, les relations industrielles et les Chartes.

43ième Congrès, 1988, p. 1-18

0942

\section{DROITS ET LIBERTÉS (suite)}

ROJOT, Jacques

Droits collectifs et droits individue/s: Les situations française, américaine et anglaise.

43ième Congrès, 1988, p. $19-49 \quad 0943$

BERGERON, Jean-Louis

La gestion de l'embauche, de la promotion et du licenciement revue et corrigée par les Chartes.

43ième Congrès, 1988, p. 51-62 0944

DUCHARME, Claude

LECLERCQ, Dominique

WESTMORELAND-TRAORE, Juanita

Commentaires - La gestion de l'embauche, de la promotion et du licenciement revue et corrigée par les Chartes.

43ième Congrès, 1988, p. 63-82 0945

DUSSAULT, Ginette

À travail équivalent, salaire égal: Un droit difficile à appliquer?

43ième Congrès, 1988, p. 83-95 0946

BARRÉ, Alain

Le régime des rapports collectifs et les Chartes.

43ième Congrès, 1988, p. 131-156 0949

\section{DESMARAIS, Jacques}

Les moyens de pression: Les chartes en redéfinissent-elles les limites?

43ième Congrès, 1988, p. 157-195 0950

DULUDE, Gilles LOUMĖDE, Catherine

PARROT, Jean-Claude

Commentaires - Les moyens de pression:

Les Chartes en redéfinissent-elles les limites?

43ième Congrès, 1988, p. 195-208 0951

PÉPIN, Marcel

L'actualisation du mouvement syndical. 43ième Congrès, 1988, p. 209-221 0952

GAGNON, Mona-Josée LEBEAU, Ernest JOHNSTON, Raymond

Commentaires - l'actualisation du mouvement syndical.

43ième Congrès, 1988, p. $222-2360953$ 
DROITS ET LIBERTÉS (sulte)

DUFOUR, Ghislain LABERGE, Louis

LAROSE, Gérald

Table ronde - Les Chartes impliquent-elles un réalignement des politiques syndicales et patronales?

43ième Congrès, 1988, p. 255-272 0955

\section{DUNLOP, JOHN T.}

CHERMESH, Ran

Conflict in the Israeli Industrial Relations

System: A Critical Description.

v. 36 , no 3,1981 , p. $630-647$

0057

DIMMOCK, Stuart J. SETHI, Amarjit S.

The Role of ldeology and Power in Systems Theory: Some Fundamental Shortcomings.

v. 41 , no 4,1986 , p. $738-757$

0516

\section{DUPLESSIS, MAURICE}

COPP, Terry

The Rise of Industrial Unions in Montreal 1935-1945

v. 37 , no 4,1982 , p. $843-875$

0162

\section{DURÉE DE LA CONVENTION}

MORIN, Fernand

Les tenants et les aboutissants de la convention collective.

v. 40 , no 2,1985, p. $371-3780392$

\section{DURÉE DU TRAVAIL}

JOLIVET, Thierry

La réduction de la durée du travail estelle créatrice d'emploi?

v. 38 , no 1,1983 , p. $142-154$

0185

BOULARD, René DESCHÊNES, Jean-Paul LAROCQUE, Alain RONDEAU, Claude Introduction - La réduction de la durée du travail.

36ième Congrès, 1981, p. 13-14 0832

LAROCQUE, ALAIN

La réduction du temps de travail: un droit ou un privilege?

36ième Congrès, 1981, p. 15-29

0833

VALASKAKIS, Kimon

Le temps libéré: à quels coûts?

36ième Congrès, 1981, p. 61-74

\section{DURÉE DU TRAVAIL (suite)}

POULIN-SIMON, Lise FORTIN, Bernard

Commentaires - Le temps libéré: à quels couts?

36ième Congrès, 1981, p. 75-86 0837

FERLAND, Gilles HAMELIN, Jean-Marie PROULX, Pierre-Paul

Table ronde - Sommes-nous tous égaux face à la diminution du temps passé au travail?

36ième Congrès, 1981, p. 87-121 0838

DESCHÊNES, Jean-Paul

Commentaires - Le temps passé au travail: un élément encore négociable?

36ième Congrès, 1981, p. 136-1440840

BEAUSOLEIL, Gilles GAUTHIER, Hervé

La législation: consolidation ou innovation?

36ième Congrès, 1981, p. 145-172 0841

LOCKWELL, LUC M. LORTIE, Pierre

Commentaire - La législation: consolidation ou innovation?

36ième Congrès, 1981, p. 173-192 0842

FRAPPIER-DESROCHERS, Monique

La technologie: un substitut à la durée du travail?

36ième Congrès, 1981, p. 193-218 0843

AOUST, Fernand d' DẺOM, André

HARVEY, Pierre

Table ronde - La réduction du temps passé au travail: un moyen de lutte contre le chômage.

36ième Congrès, 1981, p. 219-246 0844

CROZIER, Michel

La diminution du temps de travail: un phénomène inéluctable?

36ième Congrès, 1981, p. 247-263 0845

\section{E}

ÉCOLE DES RELATIONS INDUSTRIELLES (UNIV. DE MONTRÉAL)

HÉBERT, Gérard

In memoriam: Émile Bouvier, S.J. 19061985 
ÉCOLE DES RELATIONS INDUSTRIELLES
(UNIV. DE MONTREAL) (sulte)
LACOSTE, Paul
L'Ecole des relations industrielles de l'Uni-
versité de Montréal: Quarantième anniver-
saire.
v. 40, no 2,1985, p. $213-218$
0382

\section{ÉCONOMIE CANADIENNE}

MARION, Gérald

La part des salaires dans le revenu national au Canada, 1910 à 1980.

v. 37 , no 1,1982 , p. $53-64$

0097

MAGUN, Sunder

The Rise of Service Employment in the Canadian Economy.

v. 37 , no 3,1982 , p. $528-556$

0138

MAKI, Dennis $R$.

Trade Unions and Productivity: Conventional Estimates.

v. 38, no 2,1983 , p. $211-228$

0201

MILNE, William J. ROSS, Thomas W. The Cyclical Variation of Wage Premiums in the Canadian Manufacturing Industries v. 39 , no 4,1984 , p. $762-773$

0343

BEACH, Charles M. KALISKI, S.F.

The Impact of Recession on the Distribution of Annual Unemployment.

v. 41 , no 2,1986 , p. $317-328$

0471

KUMAR, Pradeep

Recent Wage Deceleration in Canada: Short-run Response or Structural Change? v. 42 , no 4,1987 , p. $687-701$

0604

COUSINEAU, Jean-Michel

NAJEM, Elmustapha

L'effet du développement de la petite entreprise sur l'évolution du syndicalisme au Canada.

v. 45 , no 3,1990 , p. $467-480 \quad 0812$

\section{ÉCONOMIE MIXTE}

WILLIAMS, C. Glyn

Joan Robinson on Unions, Distribution and Inflation.

v. 36 , no 3,1981 , p. $576-588$

0054

\section{ÉCONOMIE QUÉBÉCOISE}

INGERMAN, Sidney

La syndicalisation dans le contexte économique québécois.

38ième Congrès, 1983, p. 37-69 0860

BONIN, Bernard

Commentaires - La syndicalisation dans le contexte économique québécois.

38ième Congrès, 1983, p. 70-76 0861

\section{ÉCONOMISTES}

BARKIN, Solomon

Institutional Economics and the American

Trade Union Movement.

v. 43 , no 3,1988 , p. $491-508 \quad 0664$

\section{EDMONTON}

KRAHN, Harvey LOWE, Graham S.

Community Influences on Attitudes Towards Unions.

v. 39 , no 1,1984 , p. $93-113 \quad 0273$

\section{EFFECTIFS SYNDICAUX}

EASTMAN, Byron

Canadian Union Growth

v. 38 , no 1,1983 , p. $58-71$

0180

\section{ADAMS, Roy J.}

The Extent of Collective Bargaining in Canada

v. 39, no 4,1984 , p. $655-667$

0336

\section{CAMERON, Samuel}

Historical Variations in the Impact of Union Density on Strike Frequency: Some U.K. Evidence

v. 40 , no 2,1985, p. $367-370 \quad 0391$

KUMAR, Pradeep DOW, Bradley

Econometric Analysis of Union Membership Growth in Canada, 1935-1981

v. 41 , no 2,1986 , p. $236-255 \quad 0467$

KUMAR, Pradeep

Estimates of Unionism and Collective Bargaining in Canada.

v. 43 , no 4,1988 , p. $757-779 \quad 0683$

COUSINEAU, Jean-Michel

NAJEM, Elmustapha

L'effet du développement de la petite entreprise sur l'évolution du syndicalisme au Canada.

v. 45 , no 3,1990, p. $467-480 \quad 0812$ 


\section{ÉGALITÉ SALARIALE}

AGARWAL, Naresh C.

Male-Female Pay Inequity and Public Policy in Canada and the U.S.

v. 37 , no 4,1982 , p. $780-804$

0159

ROBB, Roberta Edgecome

Occupational Segregation and Equal Pay for Work of Equal Value.

v. 39, no 1,1984 , p. 146-166 0275

GUNDERSON, Morley

Spline Functions Estimates of the Impact of Equal Pay Législation: The Ontario Experience

v. 40, no 4,1985 , p. $775-792$

0427

ELDRIDGE, J.E.T.

CHERMESH, Ran

Conflict in the Israeli Industrial Relations

System: A Critical Description.

v. 36 , no 3,1981 , p. $630-647$

0057

\section{ÉLECTRONIQUE (INDUSTRIE)}

PROULX, Pierre-Paul

Man̂triser la technologie: pourquoi, quelles technologies, comment?

v. 41, no 2,1986, p. $382-389$

0475

\section{EMBAUCHE}

BERGERON, Jean-Louis

La gestion de l'embauche, de la promotion et du licenciement revue et corrigée par les Chartes.

43ième Congrès, 1988, p. 51-62 0944

DUCHARME, Claude

LECLERCQ, Dominique

WESTMORELAND-TRAORE, Juanita

Commentaires - La gestion de l'embau-

che, de la promotion et du licenciement revue et corrigee par les Chartes.

43ième Congrès, 1988, p. 63-82 0945

\section{EMPLOI}

HASAN, Abrar GERA, Surendra

Reservation Wages in Canadian Labour Markets.

v. 37 , no 1,1982, p. $65-92$

0098

JAIN, Harish C.

Race ans Sex Discrimination in Employment in Canada: Theories, Evidence and Policies.

v. 37 , no 2,1982 , p. $344-366 \quad 0123$

\section{EMPLOI (suite)}

MAGUN, Sunder

The Rise of Service Employment in the Canadian Economy.

v. 37, no 3,1982 , p. $528-556 \quad 0138$

STURMTHAL, Adolf

Unemployment, Inflation and "Guest Workers": Comparative Study of Three European Countries.

v. 37 , no 4,1982 , p. $739-764 \quad 0157$

MAGUN, Sunder

The Placement Activity of the Canadian Employment Agency.

v. 38 , no 1,1983, p. $72-94$

0181

JOLIVET, Thierry

La réduction de la durée du travail estelle créatrice d'emploi?

v. 38, no 1, 1983, p. 142-154 0185

JAIN, Harish C.

Micro-electronics Technology and Industrial Relations.

v. 38 , no 4,1983 , p. $869-879 \quad 0254$

FORTIN, Pierre

Le chômage des jeunes au Québec: Aggravation et concentration, 1966-1982

v. 39 , no 3,1984 , p. $419-448 \quad 0313$

ROSSILION, Claude

L'OIT et l'élimination de la discrimination dans l'emploi

v. 40 , no 1,1985, p. $03-26$

0362

\section{SAUNDERS, George}

Employment and the Productivity Slowdown: 1958-1980

v. 40, no 2,1985, p. $219-242 \quad 0383$

MERCIER, Jacques

Les effets du salaire minimum sur l'emploi des jeunes au Québec.

v. 40, no 3,1985, p. $431-457 \quad 0403$

MAGUN, Sunder

The Effects of Technological Changes on the Labour Market in Canada

v. 40 , no 4,1985 , p. $720-746 \quad 0425$ 


\section{EMPLOI (sulte)}

BEAUMONT, P.B. TOWNLEY, B.

Non-Union American Plants in Britain:

Their Employment Practices.

v. 40, no 4,1985 , p. $810-825$

0429

DORNSTEIN, Miriam ZOREF, Uri

Motivation for Changing Jobs, Personal

Background Characteristics and Perceived

Opportunity

v. 41 , no 1,1986, p. $91-110$

0451

ROY, Paul-Martel

Licenciements collectifs, licenciements individuels et emploi au Québec: 1979-1984

v. 42 , no 3,1987, p. $577-593$

0586

MERCIER, Jacques

Effets du salaire minimum sur l'emploi: Résultats des études économétriques canadiennes et québécoises.

v. 42 , no 4,1987 , p. $806-830$

0611

REID, Frank

Economic Aspects of Mandatory Retirement: The Canadian Experience.

v. 43 , no 1,1988 , p. 101-114

0631

KAMEL, Nawal ROY, Paul-Martel

Temps supplémentaire et création d'emplois: Le cas du Canada, du Québec et de l'Ontario.

v. 43 , no 2,1988 , p. $412-430$

0653

MELTZ, Noah M.

Job Security in Canada

v. 44 , no 1,1989 , p. $149-161$

0705

LOVELL, Malcolm R. Jr.

Employment Security

v. 44 , no 1. 1989 , p. $162-176$

0706

\section{ZAPATA, Francisco}

La proteccion del empleo en las américas.

v. 44 , no 1,1989 , p. 177-194

0707

JAIN, Harish C.

Racial Minorities and Affirmative Action/Employment Equity Législation in Canada.

v. 44 , no 3,1989 , p. $593-614$

0738

\section{EMPLOI (sulte)}

GUÉRIN, Gilles HÉBERT, Michel

Les obstacles rencontrés par des personnes de 45 à 64 ans à la recherche d'un emploi.

v. 45, no 2,1990, p. $235-267$

0792

ROY, Paul-Martel

Aspects dynamiques du marché du travail au Québec.

v. 45 , no 2,1990 , p. $283-299$

0794

LANGLOIS, Simon

Le travail à temps partiel: Vers une polarisation de plus en plus nette.

v. 45 , no 3,1990 , p. $548-5650816$

BERNIER, Colette

Évolution du statut du salarié en raison de nouvelles formes d'emploi: L'exemple du travail à temps partiel au Québec.

40ième Congrès, 1985, p. 137-160 0896

DÉOM, Esther

Commentaires - Evolution du statut du salarié en raison des nouvelles formes d'emplois: L'exemple du travail à temps partiel au Québec.

40ième Congrès, 1985, p. 161-170 0897

BÉLANGER, Laurent LIPSIG, Carla MORIN, Fernand PÉRUSSE, Michel Introduction - Acquisition ou fusion d'entreprises et emplois.

44ième Congrès, 1989, p. XV-XVI 0956

MORIN, Fernand

Acquisition ou fusion d'entreprises et emplois: La problématique sous-jacente.

44ième Congrès, 1989, p. 1-12 0957

BÉLANGER, MarC LE BRASSEUR, Lola L'ITALIEN, Paul MÉNARD, Marius Rappel de quelques expériences vécues. 44ième Congrès, 1989, p. 13-32 0958

CÓTÉ, André C. FONTAINE, Claude LESAGE, Paul

Aspects et implications juridiques des restructurations.

44ième Congrès, 1989, p. 33-84 0959 


\section{EMPLOI (suite)}

ANGERS, Georges GAGNON, Normand

SIMARD, Jean

Le droit et le devoir a l'information.

44ième Congrès, 1989, p. 85-100 0960

GAGNON, Astrid BLANCHETTE, Sylvain QUINTAL, Pierre

Fusion d'entreprises publiques.

44ième Congrès, 1989, p. 129-154 0962

MERCIER, Jacques POULIN-SIMON, Lise Le défi de la gestion des emplois: La problématique.

45ième Congrès, 1990, p. 1-5

0966

BÉLAND, Claude

Gestion des emplois et sécurité économique des employés.

45ième Congrès, 1990, p. 7-14 0967

BLONDIN, Michel

Le defi de la gestion de l'emploi: Pourquoi le relever?

45ième Congrès, 1990, p. 15-23

0968

\section{LEVINE, David}

Négocier la flexibilite: Reconversion des heures de la liste de rappel en postes a temps complet.

45ième Congrès, 1990, p. 41-47

0970

GOBEILLE, Kenneth R.

Négocier la flexibilité: Le travail à temps partiel.

45ième Congrès, 1990 , p. 48-58

0971

GODIN, Michel

Négocier la flexibilité: La sous-traitance.

45ième Congrès, 1990, p. 59-64 0972

LISTON, Terrence

Négocier la flexibilité: La polyvalence des emplois.

45ième Congrès, 1990, p. 65-69

0973

BROUILLET, Normand

Négocier la flexibilité: Réaction syndicale aux stratégies patronales.

45ième Congrès, 1990, p. 70-81

0974

\section{EMPLOI (suite)}

MATHIEU, René

Négocier la flexibilite: La recherche d'equité par la négociation.

45ième Congrès, 1990, p. 82-92 0975

\section{BELLEMARE, Diane}

Les emplois de l'avenir et les défis de la société.

45ième Congrès, 1990, p. 117-128 0978

LAVALLÉE, Diane CHARLAND, Gaston

LEPAGE, Brigitte MASSÉ, Henri

MERCIER, Jean

Les emplois de l'avenir et les défis de la société - Commentaires.

45ième Congrès, 1990, p. 128-148 0979

\section{EMPLOIS ATYPIQUES}

\section{LE LOUARN, Jean-Yves}

Les emplois atypiques et l'efficacité de la gestion des ressources humaines.

45ième Congrès, 1990, p. 93-103 0976

BOUDREAULT, Pierre GARON, Jacques PAGÉ, Lorainne PAQUETTE, Pierre Les emplois atypiques et l'efficacité de la gestion des ressources humaines - Commentaires.

45ième Congrès, 1990, p. 103-116 0977

\section{EMPLOIS, ÉVALUATION DES}

DESCHÊNES, Jean-Paul

L'évaluation des emplois et la convention collective.

v. 39 , no 2,1982, p. 313-327 0121

\section{EMPLOYÉ}

LAVERY, Daniel

Décisions rendues par le Conseil canadien des relations du travail.

v. 36 , no 4,1981 , p. $928-9360080$

\section{LAVERY, Daniel}

Décisions rendues par le Conseil canadien des relations du travail.

v. 37, no 4, 1982, p. 944-959 0167

THACKER, James W. ROSEN, Hjalmar

Dynamics of Employee Reactance to Company and Union Dual Allegiance Revisited and Expanded.

v. 41 , no 1,1986 , p. $128-144$ 
EMPLOYÉ (suite)

SALES, Carol A. LEVANONI, Eliahu KNOOP, Robert

Employee Performance as a Function of Job Orientation and Job Design.

v. 44 , no 2,1989 , p. $409-420$

\section{EMPLOYÉ SUBSTITUT}

LAVERY, Daniel

Décisions rendues par le Conseil canadien des relations du travail.

v. 38 , no 2,1983 , p. $418-432$

\section{EMPLOYÉS DE BANQUE}

PONAK, Allen MOORE, Larry F.

Canadian Bank Unionism: Perspectives and Issues

v. 36 , no 1,1981 , p. $3-34$

0001

SAINT-GERMAIN, Catherine

Décisions rendues par le Conseil canadien des relations du travail.

v. 41 , no 2,1986, p. $397-411$

0477

TREMBLAY, Johane

Décisions rendues par le Conseil canadien des relations du travail.

v. 43, no 2,1988, p. $447-452$

0655

\section{EMPLOYÉS DE BUREAU}

VINET, Alain BÉGIN, Clermont

THÉRIAULT, Gilles

La santé des employés de bureau: Le cas de la fonction publique québécoise.

v. 37, no 4,1982 , p. $887-904$

HUGUES, Karen D.

Office Automation: A Review of the Litterature

v. 44 , no 3,1989, p. $654-679$

0741

SMITH, Anthony E.

Innovation by Negotiation: Case Studies Among British White-Collar Unions.

v. 45 , no 1,1990 , p. $63-75$

0774

SWIMMER, Gene

Gender Based Differences in Promotions of Clerical Workers.

v. 45 , no 2,1990 , p. $300-310$

0795

\section{EMPLOYÉS DE SOUTIEN}

MOUNT, Joan SIEGEL, Jacob P.

Resources and Results in Union Rivalry: $A$

Case Study

v. 38 , no 4,1983 , p. $815-830 \quad 0250$

\section{EMPLOYÉS DU TÉLÉPHONE}

ZUREIK, Elia MOSCO, Vincent

LOCHHEAD, Clarence

Telephone Workers' Reaction to the New Technology

v. 44 , no 3,1989 , p. $507-531 \quad 0734$

\section{EMPLOYÉS MUNICIPAUX}

DION, Stéphane

Syndicats et politique au niveau municipal en France.

v. 39 , no 3.1984 , p. $466-485 \quad 0315$

MCSHANE, Steven L.

Sources of Attitudinal Union Militancy

v. 40, no 2,1985 , p. $284-302 \quad 0386$

\section{EMPLOYEURS}

THWAITES, James

Business "Organizes": The Early Years in Quebec.

v. 36 , no 2 , 1981 , p. $403-411$

MORIN, Fernand

Nouvelle présence de l'État dans les relations du travail.

v. 39 , no 4,1984 , p. $744-752$

0341

BARKIN, Solomon

The Current Unilateralist Counterattack on Unionism and Collective Bargaining

v. 41 , no 1, 1986, p. 3-27

0447

THACKER, James W. ROSEN, Hjalmar Dynamics of Employee Reactance to Company and Union Dual Allegiance Revisited and Expanded.

v. 41 , no 1,1986 , p. $128-144 \quad 0453$

\section{BURT, Sandra}

Voluntary Affirmative Action. Does it Work? v. 41 , no 3,1986 , p. 541-551 0491

TANG, Roger Y.W. PONAK, Allen Employer Assessment of Strike Costs.

v. 41 , no 3,1986 , p. $552-571$

0492 


\section{EMPLOYEURS (suite)}

BARKIN, Solomon

The Flexibility Debate in Western Europe:

The Current Drive to Restore Manage-

ments' Rights Over Personnel and Wages.

v. 42 , no 1,1987 , p. $12-44$

0539

WEST, Leigh

The Exclusive Remedy Provision in Canadian Worker Compensation Law: The Need for Legislative Reform.

v. 43, no 4,1988 , p. $890-908 \quad 0689$

CLARKE, Graham

Decisions rendues par le Conseil canadien des relations du travail.

v. 45 , no 1,1990 , p. 187-197

0782

CHAYKOWSKI, Richard P.

Union and Firm Preferences for Bargaining Outcomes in the Private Sector.

v. 45 , no 2,1990, p. $326-356$

0797

\section{ENGAGEMENT POLITIQUE}

GRAYSON, J. Paul

The Political Consequences of Unemployment: An Application of the "Power Model" of Blue-Collar Radicalism

v. 44 , no 3,1989 , p. $635-653$

0740

\section{ENGAGEMENT SYNDICAL}

NG, Ignace

Determinants of Union Commitment Among University Faculty.

v. 44 , no 4,1989, p. 769-784

0752

\section{ENSEIGNANTS}

\section{DÉOM, Esther}

La négociation collective chez les fonctionnaires et les enseignants québécois: 1975-1976.

v. 37, no 1,1982 , p. 141-163

0101

TOUPIN, Louis LESSARD, Claude CORMIER, Roger A. VALOIS, Paul La satisfaction au travail chez les ensei'gnantes et enseignants au Québec.

v. 37, no 4,1982 , p. $805-826 \quad 0160$

HARRIS, Philip RAUSCH, Peter

RYAN, Samuel G.

Ineffectiveness in Teacher Bargaining: The Anatomy of a First Strike.

v. 37 , no 4,1982 , p. $905-926 \quad 0165$

\section{ENSEIGNANTS (suite)}

GALLAGHER, Daniel G. WETZEL, Kurt Local Employer and Union Perceptions of Two-Tier Bargaining

v. 39 , no 3,1984 , p. $486-508$

0316

CHARLES, Lawrence $M$.

HUMPHREYS, Edward $\mathrm{H}$.

Bargaining to Achieve Teacher Control in Ontario

v. 40 , no 3,1985 , p. $495-511 \quad 0406$

OHTSU, Makoto VERMA, Anil

Intra-Organizational Bargaining: Wage Differentials Among Saskatchewan Schoolteachers.

v. 42 , no 3,1987 , p. $612-627 \quad 0588$

BEATTY, Carol GANDZ, Jeffrey

After the Strike: Changing the TeacherBoard Relationship.

v. 44 , no 3,1989 , p. $569-592 \quad 0737$

\section{ENSEIGNEMENT UNIVERSITAIRE}

LE LOUARN, Jean-Yves

Les professeurs d'université et leur travail.
v. 37, no 2,1982 , p. $385-402$
0125

GILSON, Clive H.J.

Bargaining Exercises: Beyond Simulation, a Touch of Reality.

v. 41 , no 2,1986 , p. $390-396 \quad 0476$

\section{ENTENTES INDIVIDUELLES}

AOUST, Claude d'

Les ententes individuelles et la convention collective.

v. 38 , no 1,1983, p. $155-158 \quad 0186$

\section{ENTENTES SALARIALES}

LACROIX, Robert

A Microeconometric Analysis of the Effects of Strikes on Wages

v. 41 , no 1,1986 , p. 111-127 0452

RESHEF, Yonatan

Negotiating Wage Settlements: A Structural Approach.

v. 44, no 3, 1989 , p. $532-551 \quad 0735$ 


\section{ENTREPRENEURS}

MORIN, Fernand

La double personnalité d'un concierge!

v. 41, no 4,1986 , p. $835-839$

0522

\section{ENTREPRENEURS INDÉPENDANTS}

SAINT-GERMAIN, Catherine

Decisions rendues par le Conseil cana-

dien des relations du travail.

v. 41 , no 4,1986 , p. $860-868$

0525

TREMBLAY, Johane

Décisions rendues par le Conseil canadien des relations du travail.

v. 42 , no 4,1987 , p. $852-861$

0613

MARLEAU, Véronique L.

Décision rendue par le Conseil canadien des relations du travail.

v. 45, no 2,1990, p. $414-423$

0801

\section{ENTREPRISES}

WEISS, Dimitri

Politique, entreprise, syndicats

v. 36 , no 1,1981, p. $192-206$

0009

JAIN, Hem C.

Disclosure of Corporate Information to Trade Unions in North America.

v. 36, no 4,1981 , p. $748-774$

0072

THÉRIAULT, Roland LESAGE, Pierre B. BOISVERT, Maurice

L'absentéisme: importance, nature et remèdes.

v. 36 , no 4,1981 , p. $775-802$

0073

VANDAMME, François

L'information des travailleurs dans les grandes firmes: L'approche spécifique de la CEE.

v. 39 , no 1,1984 , p. $51-76$

0271

MILLER, Roger

La stratégie d'entreprise et la gestion des ressources humaines.

v. 40 , no 1.1985 , p. $68-86$

0365

TANG, Roger Y.W. PONAK, Allen Employer Assessment of Strike Costs.

v. 41 , no 3,1986, p. $552-571$

0492

\section{ENTREPRISES (sulte)}

LONG, Richard J. WARNER, Malcolm

Organizations, Participation and Recession: An Analysis of Recent Evidence.

v. 42 , no 1, 1987 , p. $65-91$

0541

FRICKE, John G.

Worker Participation in Canada: Some Lessons from the Past.

v. 43 , no 3,1988 , p. $633-658$

0671

ICHNIOWSKI, Casey DELANEY, John T. LEWIN, David

The New Resource Management in U.S. Workplaces: is it Really New and is it Only Nonunion?

v. 44 , no 1,1989 , p. $97-123$

0703

MORIN, Fernand

D'un entrepreneur à un autre: l'accréditation ne suit pas

v. 44 , no 2,1989 , p. $315-336$

0719

RONDEAU, Claude BÉLANGER, Jacques Le syndicalisme dans l'entreprise: tendances récentes et analyse.

38ième Congrès, 1983, p. 15-36 0859

PERREAULT, Charles

L'entreprise peut-elle se passer d'un syndicat?

38ième Congrès, 1983, p. 173-1820868

GAUTHIER, Fernand ROY, Paul-Martel Commentaires - L'entreprise peut-elle se passer d'un syndicat?

38ième Congrès, 1983, p. 183-195 0869

BOUCHARD, Robert CLÉMENT, Pierre R. GIASSON, Étienne GUILLEMETTE, Michel Table ronde - La santé et la sécurité dans l'entreprise.

39ième Congrès, 1984, p. 113-135 0879

BÉLANGER, Laurent

Nouvelles formes d'organisation du travail, nouveaux modes de gestion et leur incidence sur le statut du salarié.

40ième Congrès, 1985, p. 171-196 0898 
Relations Industrielles, vol. 45, no 4 (1990)

\section{ENTREPRISES (suite)}

CÔTÉ, Marcel

Commentaires - Nouvelles formes d'organisation du travail, nouveaux modes de gestion et leur incidence sur le statut du salarié.

40ième Congrès, 1985, p. 196-203 0899

BÉLANGER, Laurent LIPSIG, Carla MORIN, Fernand PÉRUSSE, Michel Introduction - Acquisition ou fusion d'entreprises et emplois.

44ième Congrès, 1989, p. XV-XVI 0956

MORIN, Fernand

Acquisition ou fusion d'entreprises et emplois: La problématique sous-jacente.

44ième Congrès, 1989, p. 1-12 0957

BÉLANGER, Marc LE BRASSEUR, Lola L'ITALIEN, Paul MÉNARD, Marius Rappel de quelques expériences vécues. 44ième Congrès, 1989, p. 13-32 0958

CÓTÉ, André C. FONTAINE, Claude LESAGE, Paul

Aspects et implications juridiques des restructurations.

44ième Congrès, 1989, p. 33-84 0959

ANGERS, Georges GAGNON, Normand SIMARD, Jean

Le droit et le devoir à l'information.

44ième Congrès, 1989, p. 85-100 0960

LAMARCHE, André GAUTHIER, Michel BLAIS, Michel GAGNON, Denise

Les conditions de travail au lendemain d'une fusion ou d'une réorganisation.

44ième Congrès, 1989, p. 101-127 0961

GOSSELIN, Alain FRENETTE, Jean-Guy DIONNE, Denis

Les acteurs d'une fusion ou d'une prise de pouvoir.

44ième Congrès, 1989, p. 155-173 0963

ROUSSEAU, Léontine

Annexe - Acquisitions, fusions, offres publiques d'achat: Notions de base et facteurs considérés.

44ième Congrès, 1989, p. 189-252 0965

\section{ENTREPPISES (suite)}

FRAPPIER-DESROCHERS, Monique

La pratique de gestion des ressources humaines dans les entreprises.

45ième Congrès, 1990, p. 25-39 0969

BROUILLET, Normand

Négocier la flexibilité: Réaction syndicale aux stratégies patronales.

45ième Congrès, 1990, p. 70-81

0974

\section{ENTREPRISES AMÉRICAINES}

BEAUMONT, P.B. TOWNLEY, B.

Non-Union American Plants in Britain:

Their Employment Practices.

v. 40, no 4,1985 , p. $810-825$

0429

\section{ENTREPRISES DE CONSTRUCTION}

MILLER, Roger

Les formes d'organisation dans l'industrie de la construction au Québec.

v. 37 , no 1,1982 , p. $164-176 \quad 0102$

\section{ENTREPRISES DU TROISIÈME TYPE}

LAROUCHE, Viateur

La mobilisation des ressources humaines

- orientations récentes.

41ième Congrès, 1986, p. 31-51 0907

SÉRIEYX, Hevé

L'entreprise du troisième type.

41ième Congrès, 1986, p. 85-97

0912

WILS, Thierry

Les travailleurs seront-ils du troisième type?

41ième Congrès, 1986, p. 99-109 0913

\section{ENTREPRISES INDUSTRIELLES}

RAINVILLE, Jean-Marie

Hierarchie ethnique dans la grande entreprise industrielle montrealaise.

v. 36 , no 2,1981 , p. $336-360 \quad 0027$

\section{ENTREPRISES PUBLOUES}

GAGNON, Astrid BLANCHETTE, Sylvain QUINTAL, Pierre

Fusion d'entreprises publiques.

44ième Congrès, 1989, p. 129-1540962

ÉQUITÉ

FISHER, E.G. SHERWOOD, L.M.

Fairness and Managerial Rights in Canadian Arbitral Jurisprudence

v. 39, no 3,1984, p. $538-552$ 
ÉQUITÉ (suite)

MATHIEU, René

Négocier la flexibilite: La recherche d'equité par la négociation.

45ième Congrès, 1990, p. 82-92 0975

\section{ÉQUITÉ DANS L'EMPLOI}

JAIN, Harish C.

Racial Minorities and Affirmative Action/Employment Equity Législation in Canada.

v. 44 , no 3,1989 , p. $593-614 \quad 0738$

\section{ÉQUITÉ SALARIALE}

DUSSAULT, Ginette

À travail équivalent, salaire égal: Un droit difficile à appliquer?

43ième Congrès, 1988, p. 83-95 0946

SIMARD, Monique TELLIER, Marie

Commentaires - Le Canadien National: Un cas riche d'enseignements.

43ième Congrès, 1988, p. 114-130 0948

\section{ÉTAT}

MORIN, Fernand

Nouvelle présence de l'État dans les relations du travail.

v. 39 , no 4,1984 , p. $744-752$

0341

LUCENA, Hector

Papel del Estado en las relaciones industriales en América Latina

v. 44, no 1, 1989, p. 249-282

0710

DOWNIE, Bryan M.

The Role of the State in Industrial Relations: A Comment.

v. 45 , no 1,1990 , p. $169-186$

0781

CÔTÉ, André C.

Evolution des conditions de travail des salariés établies d'autorité.

40ième Congrès, 1985, p. 55-69 0891

PIUZE, Guy

Commentaires - Evolution des conditions de travail des salariés établies d'autorité.

40ième Congrès, 1985, p. 69-74 0892

MORIN, Fernand

L'institutionnalisation des rapports collectifs du travail: Réalité d'aujourd'hui et de demain.

40ième Congrès, 1985, p. 75-115 0893
ÉTAT (sulte)

GAGNON, Robert P.

Commentaires - L'institutionnalisation des rapports collectifs du travail: Réalite d'aujourd'hui et de demain.

40ième Congrès, 1985, p. 116-119 0894

\section{ÉTATS-UNIS}

PETERSON, Richard B. TRACY, Lane

CABELLY, Alan

Problem Solving in Labor Negotiations: Retest of a Model

v. 36 , no 1,1981, p. $87-105 \quad 0004$

BARTH, Richard T. BABA, Vishwanath V. Alienation Among Professionnal Engineers: A Canadian - American Comparison.

v. 37, no 1, 1982, p. 126-140 0100

AGARWAL, Naresh C.

Male-Female Pay Inequity and Public Policy in Canada and the U.S.

v. 37 , no 4,1982 , p. $780-804 \quad 0159$

MROCZKOWSKI, Tomasz

Theory Z: Myths, Realities and Alternatives. v. 38, no 2,1983 , p. 297-318 0205

BEAUMONT, P.B. TOWNLEY, B.

Non-Union American Plants in Britain: Their Employment Practices.

v. 40, no 4,1985 , p. $810-8250429$

BEMMELS, Brian FISHER, E.G.

NYLAND, Barbara

Canadian-American Jurisprudence on "Good Faith" Bargaining.

v. 41 , no 3,1986 , p. 596-621 0496

RESHEF, Yonatan

A Typology of Shop Stewards: A Confirmatory Factor Analysis

v. 42 , no 1,1987 , p. $150-167 \quad 0545$

BARBASH, Jack

The New Industrial Relations in the US: Phase II.

v. 43 , no 1,1988 , p. $32-42 \quad 0627$

RESHEF, Yonatan

Changing Environments and Management IR Practices: Implications for U.S. Trade Unions.

v. 43 , no 1,1988 , p. $43-62 \quad 0628$ 


\section{ÉTATS-UNIS (suite)}

BARKIN, Solomon

Institutional Economics and the American

Trade Union Movement.

v. 43 , no 3,1988 , p. $491-508$

0664

FREEMAN, Richard $\mathrm{B}$.

What Does the Future Hold for U.S. Unionism?

v. 44 , no 1,1989 , p. $25-46 \quad 0700$

ICHNIOWSKI, Casey DELANEY, John T. LEWIN, David

The New Resource Management in U.S. Workplaces: Is it Really New and is it Only Nonunion?

v. 44 , no 1,1989 , p. $97-123$

0703

LOVELL, Maicolm R. Jr.

Employment Security

v. 44 , no 1. 1989 , p. $162-176$

0706

KOCHAN, Thomas A.

MCKERSIE, Robert B.

Future Directions for American Labor and Human Resources Policy.

v. 44 , no 1,1989 , p. 224-248 0709

BARKIN, Solomon

Human Resources Management Examines itself and its Limitations

v. 44, no 3,1989, p. 691-702

0743

DILTS, David A. KARIM, Ahmad

The Effect of Mediators' Qualities and Strategies on Mediation Outcomes

v. 45 , no 1,1990, p. $22-37$

0772

BRUCE, Peter $G$.

The Processing of Unfair Labor Practice Cases in the United States and Ontario.

v. 45 , no 3,1990 , p. $481-511$

0813

TRUDEAU, Guy J. OUELLET, Lionel La comparaison en matière de systèmes de santé et de sécurité du travail.

42ième Congrès, 1987, p. 101-113 0931

ROJOT, Jacques

Droits collectifs et droits individuels: Les situations française, americaine et anglaise.

43ième Congrès, 1988, p. 19-49 0943
ÉTATS-UNIS (suite)

GODBCUT, Clément RIOUX, Claude

Le libre-échange canado-américain et le marché du travail.

44ième Congrès, 1989, p. 175-188 0964

\section{ÉTHIQUE DU TRAVAIL}

BÉLANGER, Paul R. LÉVESQUE, Benoît Le mode de vie détermine-t-il l'éthique du travail? Etude de cas.

v. 42, no 2,1987, p. $345-365 \quad 0565$

\section{ÉTUDES EXPLORATOIRES}

GAGNON, Yves-Chantal

LANDRY, Maurice

Les changements technologiques: Une stratégie d'étude exploratoire

v. 44, no 2, 1989, p. 421-447 0725

EUROPE

WEIERMAIR, Klaus

Apprenticeship Training in European Countries: The Lessons for Canada.

v. 37, no 3, 1982, p. 557-574 0139

ADAMS, Roy J.

The Federal Government and Tripartism.

v. 37 , no 3,1982 , p. $606-617$

0141

STURMTHAL, Adolf

Unemployment, Inflation and "Guest Workers": Comparative Study of Three European Countries.

v. 37 , no 4,1982 , p. $739-764$

0157

BERNIER, Jean

L'extension juridique des conventions co/lectives au Québec: Une approche comparative.

v. 38 , no 3,1983 , p. $532-544 \quad 0225$

CROWLEY, R.W. HUTH, E.

An International Comparison of Work Sharing Programs.

v. 38 , no 3,1983 , p. $636-647 \quad 0230$

VANDAMME, François

L'information des travailleurs dans les grandes firmes: L'approche specifique de la CEE.

v. 39 , no 1,1984 , p. $51-76$

0271 


\section{EUROPE (suite)}

JAIN, Hem C. GILES, Anthony

Workers' Participation in Western Europe: Implications for North America.

v. 40, no 4,1985, p. $747-774$

0426

BARKIN, Solomon

The Flexibility Debate in Western Europe:

The Current Drive to Restore Managements' Rights Over Personnel and Wages.

v. 42 , no 1,1987, p. $12-44$

0539

\section{ÉVALUATION DES EMPLOIS}

DESCHÊNES, Jean-Paul

L'évaluation des emplois et la convention collective.

v. 39, no 2,1982, p. $313-327$

0121

ÉVALUATION DU POTENTIEL hUMAIN

DIONNE, Pierre OUELLET, Gilles

Repenser l'évaluation.

v. 38 , no 2,1983 , p. $344-368$

0207

TZINER, Aharon DOLAN, Shimon L. Identifying Female Officer Potential: An Exploration in Predictors Payoff

v. 40 , no 1,1985, p. $87-98$

0366

\section{EXTENSION DES CONVENTIONS COLLEC. TIVES}

BERNIER, Jean

L'extension juridique des conventions collectives au Québec: Une approche comparative.

v. 38 , no 3,1983 , p. $532-544 \quad 0225$

HÉBERT, Gérard

Le renouvellement du régime des décrets de convention collective.

v. 45 , no 2,1990, p. $404-413 \quad 0800$

\section{$\mathbf{F}$}

\section{FACTEURS DE PRODUCTION}

SAUNDERS, George

Employment and the Productivity Slowdown: 1958-1980

v. 40, no 2,1985 , p. $219-242$

0383

\section{FAILLITES}

SAINT-GERMAIN, Catherine

Décision rendue par le Conseil canadien des relations du travail.

v. 42 , no 1,1987, p. $203-209$

0549

\section{FAMILLE}

COOK, Alice $\mathrm{H}$.

Family and Work: Challenger to Labor, Management and Government

v. 42 , no 3,1987 , p. $520-5270582$

MCWATTERS, Catherine J.

BEACH, Charles $M$.

Factors Behind the Changes in Canada's Family Income Distribution and the Share of the Middle Class.

v. 45 , no 1,1990, p. $118-135$

0777

\section{FARDEAU DE LA PREUVE}

TREMBLAY, Johane

Décisions rendues par le Conseil canadien des relations du travail.

v. 43 , no 3,1988 , p. $686-694$

0673

\section{FÉDÉRATION DU TRAVAIL DE SASKAT-} CHEWAN

SASS, Robert

The Saskatchewan Trade Union Act. 1983:

The Public Battle.

v. 40 , no 3,1985, p. 591-622 0411

\section{FEMMES}

LOWE, Graham S.

Causes of Unionization in Canadian Banks. v. 36 , no 4,1981 , p. $865-893 \quad 0077$

WALSH, William D.

A Time Series Analysis of Female Labour Force Participation Rates Disaggregated by Marital Status.

v. 37, no 2,1982 , p. $367-384 \quad 0124$

CHAISON, Gary N. ANDIAPPAN, P.

Characteristics of Female Union Officer in Canada.

v. 37 , no 4,1982 , p. $765-7790158$

AGARWAL, Naresh C.

Male-Female Pay Inequity and Public Policy in Canada and the U.S.

v. 37 , no 4,1982 , p. $780-804 \quad 0159$

ROBB, Roberta Edgecome

Occupational Segregation and Equal Pay for Work of Equal Value.

v. 39 , no 1,1984, p. $146-166 \quad 0275$ 


\section{FEMMES (suite)}

TZINER, Aharon DOLAN, Shimon L. Identifying Female Officer Potential: An Exploration in Predictors' Payoff

v. 40, no 1,1985, p. $87-98$

0366

GRANT, Michel ROSE, Ruth

Encadrement du travail à domicile dans l'industrie du vêtement au Québec.

v. 40, no 3,1985 , p. $473-494$

0405

\section{GUNDERSON, Morley}

Spline Functions Estimates of the Impact of Equal Pay Législation: The Ontario Experience

v. 40, no 4,1985 , p. $775-792$

0427

\section{LAMSON, Cynthia}

On the Line: Women and Fish Plant Jobs in Atlantic Canada.

v. 41 , no 1,1986, p. $145-156$

0454

\section{BURT, Sandra}

Voluntary Affirmative Action. Does it Work? v. 41 , no 3,1986 , p. $541-551$

0491

JAIN, Harish C. ANDIAPPAN, P.

Sexual Harassment in Employment in Canada: Issues and Policies.

v. 41 , no 4,1986 , p. $758-777$

0517

HACCOUN, Robert R. DUPONT, Serge Une analyse des comportements de travailleurs masculins et féminins selon deux formes d'absence au travail.

v. 43, no 1,1988 , p. $153-166$

0634

SAVOIE, Dominique LAROUCHE, Viateur Le harcèlement sexuel au travail: Définition et mesure du phénomène.

v. 43 , no 3,1988 , p. $509-530$

0665

TOUPIN, Sylvain

Le harcelement sexuel en milieu de travail. v. 43, no 3,1988 , p. $531-546$ 0666

SAVOIE, Dominique LAROUCHE, Viateur Le harcèlement sexuel au travail: Résultats de deux études québécoises.

v. 45 , no 1,1990, p. $38-62$

0773
FEMMES (suite)

SWIMMER, Gene

Gender Based Differences in Promotions of Clerical Workers.

v. 45 , no 2,1990 , p. $300-310 \quad 0795$

DE KONINCK, Maria HEENAN, Roy

Droit de refus et retrait préventif: succès ou échec?

39ième Congrès, 1984, p. 159-175 0881

BOIVIN, Suzanne P.

Le Canadien National: Un cas riche d'enseignements.

43ième Congrès, 1988, p. 97-113 0947

SIMARD, Monique TELLIER, Marie

Commentaires - Le Canadien National: Un cas riche d'enseignements.

43ième Congrès, 1988, p. 114-130 0948

\section{FERMETURES D'USINES}

BURKE, Ronald J.

Trainee Experiences in Industrial Retraining Programs: A Case Study

v. 39 , no 3. 1984 , p. $570-577$

0321

BURKE, Ronald J.

Consequences of not Working Sixteen Months After a Plant Closing

v. 40 , no 1,1985 , p. $162-169 \quad 0370$

\section{GRAYSON, J. Paul}

The Political Consequences of Unemployment: An Application of the "Power Mode/" of Blue-Collar Radicalism

v. 44 , no 3,1989 , p. $635-653 \quad 0740$

\section{FINANCES PUBLIQUES}

COUSINEAU, Jean-Michel

LACROIX, Robert

La détermination des avantages sociaux au Canada

v. 39 , no 1,1984 , p. $3-22$

0268

\section{FONCTION PUBLIQUE}

GURDON, Michael A.

Divergent Paths: Civil Service Employment Relations in Australia and Canada.

v. 42 , no 3,1987 , p. $566-575$

0585 


\section{FONCTION PUBLIQUE DU CANADA}

MOORE, William $R$.

Justice and the Grievance Procedure in the Federal Public Service.

v. 36 , no 4,1981 , p. $848-864$

0076

SUBBARAO, A.V.

Impasse Choice in the Canadian Federal Service: An Innovation and an Intrigue

v. 40, no 3,1985, p. $567-590$

0410

FINKELMAN, Jacob

Public Sector Collective Bargaining

v. 41 , no 4,1986 , p. $691-703$

SUBBARAO, A.V.

Criteria in Arbitration of Wage Disputes: Theory and Practice in the Canadian Federal Public Service.

v. 43 , no 3,1988 , p. $547-570$

0667

DASTMALCHIAN, Ali NG, Ignace Industrial Relations Climate and Grievance Outcomes.

v. 45 , no 2,1990 , p. $311-325$

0796

WILS, Thierry BOURGEOIS, Robert-Paul LABELLE, Christiane

Planification des ressources humaines dans la fonction publique fédérale.

v. 45 , no 3,1990, p. $\mathbf{5 1 2 - 5 3 0}$

0814

\section{FONCTION PUBLIQUE DU QUÉBEC}

\section{DÉOM, Esther}

La négociation collective chez les fonctionnaires et les enseignants québécois: 1975-1976.

v. 37, no 1,1982, p. $141-163$

0101

BOLDUC, Roch BOUCHARD, Lucien

HÉBERT, Gérard

Le régime québécois de négociation des secteurs public et parapublic

v. 37, no 2,1982 , p. $403-430$

0126

VINET, Alain BÉGIN, Clermont

THÉRIAULT, Gilles

La santé des employés de bureau: Le cas de la fonction publique québécoise.

v. 37 , no 4,1982 , p. $887-904$

0164
FONCTION PUBLIQUE DU QUÉBEC (suite)

AUDET, Claude VINET, Alain

Bureautique et organisation du travail de secrétariat dans la fonction publique québécoise.

v. 43 , no 4,1988 , p. $737-756 \quad 0682$

\section{FONCTION PUBLIQUE PROVINCIALE}

CHRISTENSEN, Sandra

Collective Bargaining in Provincial Public Administration.

v. 36 , no 3,1981, p. $616-6290056$

\section{FONCTIONNAIRES}

VINET, Alain BÉGIN, Clermont

THÉRIAULT, Gilles

La santé des employés de bureau: Le cas de la fonction publique québécoise.

v. 37, no 4, 1982, p. 887-904 0164

FONDS DE SOLIDARITÉ DES TRAVAILLEURS QUÉBÉCOIS

GOSSELIN, Alain FRENETTE, Jean-Guy

DIONNE, Denis

Les acteurs d'une fusion ou d'une prise de pouvoir.

44ième Congrès, 1989, p. 155-173 0963

FORÊT (INDUSTRIE)

LAFLAMME, Lucie VINET, Alain

Accidents du travail et modernisation du processus de production: le cas de l'industrie forestière québécoise.

v. 43, no 3,1988, p. $591-608$

0669

\section{FORMATION ET DÉVELOPPEMENT}

MEALIA, David W.

A Macro/Micro Décision Model for the Training and Development Specialist.

v. 37, no 3, 1982, p. 656-669 0144

\section{FORMATION PROFESSIONNELLE}

MARTIN, RosS BLACK, Errol

Roy J. Adams' Proposal for a Training Levy Scheme.

v. 36 , no 2,1981, p. $414-417 \quad 0033$

WEIERMAIR, Klaus

Apprenticeship Training in European Coun"tries: The Lessons for Canada.

v. 37 , no 3,1982 , p. 557-574 0139 
FORMATION PROFESSIONNELLE (suite) LATULIPPE, Gérard P. O'FARRELL, Kevin Le comite paritaire: Anachronisme ou formule d'avenir.

v. 37, no 3,1982, p. $634-655$

0143

BURKE, Ronald J.

Trainee Experiences in Industrial Retraining Programs: A Case Study

v. 39 , no 3. 1984 , p. $570-577$

0321

CAMPBELL, Adrian WARNER, Malcolm Workplace Relations, Skills-Training and Technological Change at Plant-Level.

v. 43 , no 1,1988 , p. 115-132 0632

\section{FORMATION SUPÉRIEURE}

ROHLING, Thomas A.

Screening and Human Capital Theory: An Empirical Test

v. 41 , no 4,1986 , p. $817-826$

0520

\section{FRAIS JUDICIAIRES}

BEAULIEU, LUC

Décisions rendues par le Conseil canadien des relations du travail.

v. 39 , no 3,1984 , p. $605-618$

0323

\section{FRANÇAIS (LANGUE)}

AUCLAIR, Robert

Genève ignore Québec en matière de vocabulaire

v. 36 , no 1,1981 , p. $223-224$

0011

\section{FRANCE}

CAIRE, Guy

Procédures de règlement pacifique des conflits collectifs en France.

v. 38, no 1, 1983, p. 3-27

0177

CAIRE, Guy

Les lois Auroux

v. 39 , no 2,1984 , p. $235-258$

0292

DION, Stéphane

Syndicats et politique au niveau municipal en France.

v. 39 , no 3,1984 , p. $466-485 \quad 0315$

DELAMOTTE, Yves

La loi et la négociation collective en France: Réflexion sur l'expérience 1981-1985.

v. 42 , no 1,1987, p. $92-109$

0542
FRANCE (suite)

ROJOT, Jacques

Droits collectifs et droits individuels: Les situations française, américaine et anglaise.

43ième Congrès, 1988, p. $19-490943$

\section{FUSIONS D'ENTREPRISES}

CLARKE, Graham

Décision rendue par le Conseil canadien des relations du travail.

v. 45 , no 3,1990 , p. $616-624 \quad 0820$

BÉLANGER, Laurent LIPSIG, Carla MORIN, Fernand PÉRUSSE, Michel Introduction - Acquisition ou fusion d'entreprises et emplois.

44ième Congrès, 1989, p. XV-XVI 0956

MORIN, Fernand

Acquisition ou fusion d'entreprises et emplois: La problématique sous-jacente.

44ième Congrès, 1989, p. 1-12 0957

BÉLANGER, Marc LE BRASSEUR, Lola

L'ITALIEN, Paul MÉNARD, Marius

Rappel de quelques expériences vécues

44ième Congrès, 1989, p. 13-32 0958

CÔTÉ, André C. FONTAINE, Claude

LESAGE, Paul

Aspects et implications juridiques des restructurations.

44ième Congrès, 1989, p. 33-84 0959

ANGERS, Georges GAGNON, Normand SIMARD, Jean

Le droit et le devoir à linformation.

44ième Congrès, 1989, p. 85-100 0960

LAMARCHE, André GAUTHIER, Michel

BLAIS, Michel GAGNON, Denise

Les conditions de travail au lendemain d'une fusion ou d'une réorganisation.

44ième Congrès, 1989, p. 101-1270961

GAGNON, Astrid BLANCHETTE, Sylvain QUINTAL, Pierre

Fusion d'entreprises publiques.

44ième Congrès, 1989, p. 129-154 0962 
FUSIONS D'ENTREPRISES (sulte) GOSSELIN, Alain FRENETTE, Jean-Guy DIONNE, Denis

Les acteurs d'une fusion ou d'une prise de pouvoir.

44jème Congrès, 1989, p. 155-173 0963

ROUSSEAU, Léontine

Annexe - Acquisitions, fusions, offres publiques d'achat: Notions de base et facteurs considerés.

44ième Congrès, 1989, p. 189-252 0965

\section{FUSIONS SYNDICALES}

CHAISON, Gary N.

A Note on the Critical Dimensions of the Union Merger Process.

v. 37, no 1,1982 , p. $198-206 \quad 0104$

\section{G}

\section{GAZ MÉTROPOLITAIN}

LALANDE, Serge

L'expérience de la compagnie Gaz Métropolitain.

41ième Congrès, 1986, p. 137-146 0916

\section{GENDARMERIE ROYALE DU CANADA}

SAINT-GERMAIN, Catherine

Décisions rendues par le Conseil canadien des relations du travail.

v. 41 , no 4,1986 , p. $860-868$

\section{GENERAL MOTORS}

BENEDICT, Daniel

The 1984 GM Agreement in Canada: Significance and Consequences.

v. 40, no 1,1985, p. $27-47$

\section{GESTION}

MERCIER, Jean

L'informatique, ses "filtres" et ses effets.

v. 41 , no 2,1986, p. 299-316

\section{GESTION DE L'ENTREPRISE}

BÉLANGER, Laurent

Nouvelles formes d'organisation du travail, nouveaux modes de gestion et leur incidence sur le statut du salarié.

40ième Congrès, 1985, p. 171-196 0898

\section{GESTION DE L'ENTREPRISE (sulte)}

CÔTÉ, Marcel

Commentaires - Nouvelles formes d'organisation du travail, nourveaux modes de gestion et leur incidence sur le statut du salarié.

40ième Congrès, 1985, p. 196-203 0899

BHÉRER, Harold

Le salarié et la gestion générale de l'entreprise.

40ième Congrès, 1985, p. 205-222 0900

GODBOUT, Clément

Cornmentaires - Le salarié et la gestion générale de l'entreprise.

40ième Congrès, 1985, p. 222-228 0901

BÉLANGER, Jacques

Annexe - La participation des travailleurs aux décisions dans l'entreprise.

40ième Congrès, 1985, p. 245-259 0903

\section{GESTION DES CONFLITS}

MARTINEAU, LUC

La "gestion" des conflits par le Conseil canadien des relations du travail.

v. 36 , no 3,1981 , p. $589-615$

0055

\section{GARNIER, Bernard}

La gestion des conflits interpersonnels en milieu universitaire.

v. 38, no 2,1983 , p. 277-296 0204

\section{GESTION DES EMPLOIS}

MERCIER, Jacques POULIN-SIMON, Lise Le défi de la gestion des emplois: La problermatique.

45ième Congrès, 1990, p. $1-50966$

BÉLAND, Claude

Gestion des emplois et sécurité économique des employes.

45ième Congrès, 1990, p. 7-14 0967

BLONDIN, Michel

Le défi de la gestion de l'emploi: Pourquoi le relever?

45ième Congrès, 1990, p. 15-23 0968

\section{LEVINE, David}

Négocier la flexibilité: Reconversion des heures de la liste de rappel en postes à temps complet.

45ième Congrès, 1990, p. 41-47 0970 
GESTION DES EMPLOIS (suite)

GOBEILLE, Kenneth R.

Négocier la flexibilité: Le travail à temps partiel.

45ième Congrès, 1990, p. 48-58 0971

GODIN, Michel

Négocier la flexibilite: La sous-traitance.

45ième Congrès, 1990, p. 59-64 0972

LISTON, Terrence

Négocier la flexibilité: La polyvalence des emplois.

45ième Congrès, 1990 , p. $65-690973$

BROUILLET, Normand

Négocier la flexibilité: Réaction syndicale aux stratégies patronales.

45ième Congrès, 1990, p. 70-81

0974

MATHIEU, René

Négocier la flexibilité: La recherche d'equité par la négociation.

45ième Congrès, 1990, p. 82-92 0975

LE LOUARN, Jean-Yves

Les emplois atypiques et l'efficacité de la gestion des ressources humaines.

45ième Congrès, 1990, p. 93-103 0976

BOUDREAULT, Pierre GARON, Jacques PAGÉ, Lorainne PAQUETTE, Pierre Les emplois atypiques et l'efficacité de la gestion des ressources humaines - Commentaires.

45ième Congrès, 1990, p. 103-116 0977

BELLEMARE, Diane

Les emplois de l'avenir et les défis de la société.

45ième Congrès, 1990, p. 117-128 0978

LAVALLÉE, Diane CHARLAND, Gaston

LEPAGE, Brigitte MASSÉ, Henri

MERCIER, Jean

Les emplois de l'avenir et les défis de la société - Commentaires.

45ième Congrès, 1990, p. 128-148 0979

\section{GESTION DES ORGANISATIONS}

AUDET, Michel BÉLANGER, Laurent Nouveaux modes de gestion et relations industrielles au Canada.

v. 44 , no 1,1989, p. $62-96 \quad 0702$
GESTION DES ORGANISATIONS (suite)

APPARICIO-VALDEZ, Luis

La gestion empresarial en latinoamérica y

su impacto en las relaciones laborales

v. 44 , no 1,1989 , p. $124-148$

0704

\section{GESTION DES RESSOURCES HUMAINES}

PIGANIOL, Claude

Relations professionnelles et gestion des ressources humaines.

v. 39 , no 2,1984, p. $285-300$

0295

MILLER, Roger

La stratégie d'entreprise et la gestion des ressources humaines.

v. 40, no 1. 1985, p. $68-86$

0365

BEAUMONT, P.B.

The Diffusion of Human Resource Management Innovations.

v. 40, no 2,1985 , p. $243-256$

0384

CAMIRÉ, André

La productivité des intelligences.

v. 43, no 2,1988, p. $322-340$

0649

ICHNIOWSKI, Casey DELANEY, John T. LEWIN, David

The New Resource Management in U.S. Workplaces: Is it Really New and is it Only Nonunion?

v. 44 , no 1,1989, p. 97-123

0703

KOCHAN, Thomas A.

MCKERSIE, Robert $B$.

Future Directions for American Labor and Human Resources Policy.

v. 44 , no 1,1989 , p. $224-248$

0709

WILS, Thierry LABELLE, Christiane GUÉRIN, Gilles LE LOUARN, Jean-Yves La gestion stratégique des ressources humaines: Un reniement du rôle social de lentreprise?

v. 44 , no 2,1989 , p. $354-375 \quad 0721$

BARKIN, Solomon

Human Resources Management Examines itself and its Limitations

v. 44, no 3,1989 , p. $691-702 \quad 0743$ 


\section{GESTION DES RESSOURCES HUMAINES (suite)}

AUDET, Michel BÉLANGER, Laurent

BOIVIN, Jean DÉOM, Esther

MERCIER, Jacques

Introduction - La mobilisation des ressources humaines: tendances et impact.

41ième Congrès, 1986, p. 13-14 0905

BOIVIN, Jean

Émergence d'une réalité nouvelle en relations industrielles.

41ième Congrès, 1986, p. 17-30 0906

LAROUCHE, Viateur

La mobilisation des ressources humaines

- orientations récentes.

41ième Congrès, 1986, p. 31-51 0907

CASAVANT, Jean-Claude

La stratégie de gestion des ressources humaines chez Abitibi Price Inc.

41ième Congrès, 1986, p. 53-61 0908

LEMAIRE, Alain

La communication directe chez Cascades Inc.

41ième Congrès, 1986, p. 63-68 0909

BOUCHER, Lysette

Les limites des nouvelles approches en gestion des ressources humaines.

41ième Congrès, 1986, p. 77-84

0911

DOCQUIER, Gérard

Ressources humaines et défis du syndicalisme.

41ième Congrès, 1986, p. 149-156 0917

HÉTU, Jean-Paul

La nouvelle gestion des ressources humaines - mythe ou réalite?

41ième Congrès, 1986, p. 167-172 0919

FRAPPIER-DESROCHERS, Monique

La pratique de gestion des ressources humaines dans les entreprises.

45ième Congrès, 1990, p. 25-39

0969

LE LOUARN, Jean-Yves

Les emplois atypiques et l'efficacité de la gestion des ressources humaines.

45ième Congrès, 1990, p. 93-103 0976

\section{GESTION DES RESSOURCES HUMAINES (suite)}

BOUDREAULT, Pierre GARON, Jacques PAGÉ, Lorainne PAQUETTE, Pierre

Les emplois atypiques et l'efficacité de la gestion des ressources humaines - Commentaires.

45ième Congrès, 1990, p. 103-116 0977

\section{GESTION DES RISQUES}

SURET, Jean-Marc GENDRON, Michel BERNIER, Gilles

Le processus de gestion des risques, les lésions professionnelles et la CSST.

42ième Congrès, 1987, p. 223-241 0937

HARGUINDEGUY, Jean-Louis

BRODY, Bernard

Commentaires - Le processus de gestion des risques, les lésions professionnelles et la CSST.

42ième Congrès, 1987, p. 241-252 0938

\section{GESTION PARTICIPATIVE}

BROSSARD, Michel

Les limites du modèle-type du fonctionnement des cercles de qualité

v. 44 , no 3,1989 , p. $552-568$

0736

LONG, Richard J.

Patterns of Workplace Innovation in Canada.

v. 44 , no 4,1989 , p. $805-826 \quad 0754$

HÉTU, Jean-Paul

La nouvelle gestion des ressources humaines - mythe ou réalité?

41ième Congrès, 1986, p. 167-172 0919

\section{GOUVERNEMENT DU CANADA}

ADAMS, Roy J.

The Federal Government and Tripartism.

v. 37 , no 3,1982 , p. $606-617$

0141

\section{GRANDE-BRETAGNE}

POOLE, Michael

Perceptions of Trade Union Members and the Social Action Perspective.

v. 36, no 1,1981 , p. 35-62 0002

BEAUMONT, P.B.

Third Party Conciliation and Trade Union Recognition: Some British Evidence.

v. 37 , no 4,1982 , p. $827-842 \quad 0161$ 


\section{GRANDE-BRETAGNE (suite)}

WARNER, Malcolm

Corporatism, Participation and Society

v. 38 , no 1,1983 , p. $27-44$

0178

BEAUMONT, P.B.

Statutory Recognition Provisions in Britain, 1976-80

v. 38, no 4,1983, p. $744-766$

0247

BEAUMONT, P.B.

The Diffusion of Human Resource Management Innovations.

v. 40, no 2,1985 , p. $243-256$

0384

BEAUMONT, P.B. TOWNLEY, B.

Non-Union American Plants in Britain: Their Employment Practices.

v. 40, no 4,1985 , p. $810-825$

0429

BÉLANGER, Jacques

Le controle ouvrier sur l'organisation du travail: Étude de cas en Grande-Bretagne. v. 41, no 4, 1986, p. 704-719 0514

CAMPBELL, Adrian WARNER, Malcolm Workplace Relations, Skills-Training and Technological Change at Plant-Level.

v. 43 , no 1,1988 , p. 115-132

0632

BEAUMONT, P.B. HARRIS, R.I.D.

High Technology Industries and Non-Union

Establishments in Britain.

v. 43, no 4,1988, p. $819-846 \quad 0686$

SMITH, Anthony E.

Innovation by Negotiation: Case Studies Among British White-Collar Unions.

v. 45 , no 1,1990 , p. $63-75$

0774

ROJOT, Jacques

Droits collectifs et droits individue/s: Les situations française, americaine et anglaise.

43ième Congrès, 1988, p. 19-49

0943

\section{GRANDES ENTREPRISES}

RAINVILLE, Jean-Marie

Hiérarchie ethnique dans la grande entreprise industrielle montréalaise.

v. 36 , no 2,1981 , p. $336-360$

0027

\section{GRANDES ENTREPRISES (suite)}

VANDAMME, François

L'information des travailleurs dans les grandes firmes: L'approche spécifique de la CEE.

v. 39 , no 1,1984 , p. 51-76 0271

\section{GRÈCE}

KATASANEVAS, Theodoros

Trade Unions in Greece

v. 40 , no 1,1985 , p. $99-114$

0367

\section{GRËVES}

AOUST, Claude d'

Les réclamations à l'arbitrage de dommages résultant de la violation d'une clause de renonciation à la grève.

v. 36, no 1,1981, p. $259-262 \quad 0014$

MARTINEAU, LUC

Décisions rendues par le Conseil canadien des relations du travail.

v. 36 , no 2,1981 , p. $424-429$

0035

\section{LAVERY, Daniel}

Décisions rendues par le Conseil canadien des relations du travail.

v. 37 , no 1,1982 , p. $226-234$

0109

FRANK, J.A. KELLY, Michael J.

MACNAUGHTON, Bruce D.

Legis/ative Change and Strike Activity in Canada, 1926-1974

v. 37 , no 2,1982 , p. 267-283 0119

HARRIS, Philip RAUSCH, Peter

RYAN, Samuel G.

Ineffectiveness in Teacher Bargaining: The Anatomy of a First Strike.

v. 37 , no 4,1982 , p. $905-926$

0165

LAVERY, Daniel

Décisions rendues par le Conseil canadien des relations du travail.

v. 38 , no 2,1983, p. $418-432$

0211

VERGE, Pierre

Syndicalisation de la grève.

v. 38 , no 3,1983 , p. $475-506$

0223

COUSINEAU, Jean-Michel

LACROIX, Robert

Le climat des relations du travail à Montréal et à Toronto

v. 38 , no 4,1983 , p. $730-743 \quad 0246$ 


\section{GRÈVES (sulte)}

CAMERON, Samuel

An International Comparison of the Volatility of Strike Behavior

v. 38 , no 4,1983 , p. $767-784$

0248

MAKI, Dennis R. STRAND, Kenneth

The Determinants of Strike Activity: An Interindustry Analysis

v. 39 , no 1, 1984, p. 77-92

0272

BEAULIEU, LUC

Décisions rendues par le Conseil canadien des relations du travail.

v. 39 , no 1,1984 , p. 177-189

0277

BENEDICT, Daniel

The 1984 GM Agreement in Canada: Significance and Consequences.

v. 40 , no 1,1985 , p. $27-47$

0363

CAMERON, Samuel

Historical Variations in the Impact of Union Density on Strike Frequency: Some U.K. Evidence

v. 40, no 2,1985, p. $367-370$

0391

LAMY, Francine

Décision rendue par le Conseil canadien des relations du travail.

v. 40 , no 3,1985 , p. $659-662$

0415

LACROIX, Robert

A Microeconometric Analysis of the Effects of Strikes on Wages

v. 41 , no 1,1986 , p. 111-127

0452

RESHEF, Yonatan FRIED, Yatzhak

Strikes in Israell: The Histadrut in a Sectorial Perspective, 1965-1982

v. 41 , no 2,1986 , p. $329-347$

0472

TANG, Roger Y.W. PONAK, Allen Employer Assessment of Strike Costs.

v. 41 , no 3,1986 , p. $552-571$

0492

TREMBLAY, Johane

Décisions rendues par le Conseil canadien des relations du travail.

v. 43 , no 1,1988 , p. $188-195$

0637

LACROIX, Robert LESPÉRANCE, André Les nouvelles lois du travail et l'activité de grève.

v. 43, no 4,1988 , p. $812-828$

0685
GRÈVES (suite)

BEATTY, Carol GANDZ, Jeffrey

After the Strike: Changing the Teacher-

Board Relationship.

v. 44, no 3,1989 , p. $569-592$

0737

GILSON, Clive H.J. SPENCER, lan S. GRANVILLE, S.

The Impact of a Strike on the Attitudes and Behavior of a Rural Community.

v. 44, no 4,1989 , p. $785-804 \quad 0753$

MUCKENBERGER, Ulirich

The Regulation of Strike Law in Times of New Technologies and Deregulation: The Case of West Germany.

v. 45 , no 1,1990 , p. $136-145$

0778

CLARKE, Graham

Décisions rendues par le Conseil canadien des relations du travail.

v. 45 , no 1,1990 , p. 187-197

0782

DESMARAIS, Jacques

Les moyens de pression: Les chartes en redéfinissent-elles les limites?

43ième Congrès, 1988, p. 157-195 0950

DULUDE, Gilles LOUMÈDE, Catherine

PARROT, Jean-Claude

Commentaires - Les moyens de pression:

Les Chartes en redéfinissent-elles les limites?

43ième Congrès, 1988, p. 195-208 0951

\section{GRÈVES SAUVAGES}

FISHER, E.G.

Strike Activity and Wildcat Strikes in British Columbia: 1945-1975.

v. 37, no 2,1982 , p. 284-312 0120

FISHER, E.G. PERCY, M.B.

The Impact of Unanticipated Output and Consumer Prices on Wildcat Strikes.

v. 38 , no 2,1983 , p. 254-276 0203

NG, Ignace

Determinants of Wildcat Strikes in Canada Manufacturing Industries.

v. 42 , no 2,1987 , p. $386-397$

0567 


\section{GRIEFS}

AOUST, Claude d'

Les réclamations à l'arbitrage de dommages résultant de la violation d'une clause de renonciation à la grève.

v. 36, no 1,1981, p. $259-262$

0014

AOUST, Claude d'

La juridiction de l'arbitre et de la Cour des petites créances devant la Cour suprême du Canada.

v. 36 , no 2,1981, p. $418-424$

0034

MARTINEAU, LUC

Décisions rendues par le Conseil canadien des relations du travail.

v. 36 , no 3,1981, p. $671-680$

0060

MOORE, William $\mathbf{R}$.

Justice and the Grievance Procedure in the Federal Public Service.

v. 36 , no 4,1981 , p. $848-864$

0076

DESCHÊNES, Jean-Paul

L'évaluation des emplois et la convention collective.

v. 39, no 2,1982 , p. $313-327$

0121

BEIGBEDER, Yves

Individual Grievance Procedures in United Nations Secretariats.

v. 37, no 2,1982, p. $328-343$

0122

AOUST, Claude d'

Les dispositifs d'une sentence arbitrale: quelques pièges à éviter.

v. 38 , no 2,1983, p. $415-417$

0210

AOUST, Claude d'

Effets d'annulation d'un avis de congédiement par un arbitre

v. 39 , no 2,1984 , p. $365-370$

0299

GILSON, Clive H.J.

Changes in the Nature of Grievance Issues Over the Last Ten Years: Labor Management Relations and the "Frontier of Control".

v. 40 , no 4,1985, p. $856-864$

0432
GRIEFS (suite)

KNIGHT, Thomas R.

Correlates of Informal Grievance Resolution Among Fist-Line Supervisors

v. 41 , no 2,1986 , p. $281-298$

0469

GILSON, Clive H.J. GILLIS, L.P.

Grievance Arbitration in Nova Scotia.

v. 42 , no 2,1987, p. $256-271$

0560

KNIGHT, Thomas R.

The Role of The Duty of Fair Representation in Union Grievance Decisions.

v. 42, no 4,1987, p. $716-736$

0606

CARTER, Donald D.

Grievance Arbitration and the Charter: The Emergency issues.

v. 44 , no 2,1989 , p. $337-353 \quad 0720$

DASTMALCHIAN, Ali NG, Ignace

Industrial Relations Climate and Grievance Outcomes.

v. 45 , no 2,1990 , p. $311-325 \quad 0796$

\section{GROUPEMENTS PATRONAUX}

THWAITES, James

Business "Organizes": The Early Years in Quebec.

v. 36, no 2,1981 , p. $403-411$

0031

\section{GROUPES ETHNIQUES}

RAINVILLE, Jean-Marie

Hiérarchie ethnique dans la grande entreprise industrielle montréalaise.

v. 36 , no 2,1981 , p. $336-360 \quad 0027$

GROUPES SEMI-AUTONOMES DE PRODUCTION

BROSSARD, Michel

La stratégie syndicale face aux groupes semi-autonomes de production: Hypothèse pour l'analyse du cas Rushton.

v. 37 , no 3,1982 , p. $670-683$

0145

BROSSARD, Michel SIMARD, Marcel

Problématique de la différenciation de la main-d'oeuvre et changement organisationnel.

v. 41 , no 2,1986 , p. $219-235$

0466 


\section{H}

HARCÈLEMENT RACIAL

ANDIAPPAN, P. CRESTOHL, Mark

SINGH, Jang $B$.

Racial Discrimination in Employment in Canada.

v. 44 , no 4,1989 , p. $827-849$

0755

HARCËLEMENT SEXUEL

JAIN, Harish C. ANDIAPPAN, P.

Sexual Harassment in Employment in Canada: Issues and Policies.

v. 41 , no 4,1986 , p. $758-777$

0517

SAVOIE, Dominique LAROUCHE, Viateur Le harcèlement sexuel au travail: Définition et mesure du phénomène.

v. 43 , no 3,1988 , p. $509-530$

0665

TOUPIN, Sylvain

Le harcelement sexuel en milieu de travail. v. 43 , no 3,1988 , p. $531-546 \quad 0666$

SAVOIE, Dominique LAROUCHE, Viateur Le harcèlement sexuel au travail: Résultats de deux études québécoises.

v. 45 , no 1,1990 , p. 38-62

0773

HAUTE TECHNOLOGIE (INDUSTRIE)

BEAUMONT, P.B. HARRIS, R.I.D.

High Technology Industries and Non-Union

Establishments in Britain.

v. 43, no 4,1988 , p. $819-846$

0686

HEINRICH, H.W.

BRODY, Bernard LÉTOURNEAU, Yves POIRIER, André

Le cout des accidents du travail: État des connaissances.

v. 45 , no 1,1990 , p. $94-117$

0776

\section{HEURES DE TRAVAIL}

JOLIVET, Thierry

La réduction de la durée du travail estelle créatrice d'emploi?

v. 38 , no 1,1983, p. $142-154$

0185

\section{HISTADRUT}

RESHEF, Yonatan FRIED, Yatzhak Strikes in Israell: The Histadrut in a Sectorial Perspective, 1965-1982

v. 41 , no 2,1986 , p. $329-347$

0472

\section{HISTOIRE}

GILES, Anthony MURRAY, Grégor

Towards an Historical Understanding of Industrial Relations Theory in Canada.

v. 43 , no 4,1988, p. $780-811 \quad 0684$

\section{HISTOIRE ECONOMIQUE}

MARION, Gérald

La part des salaires dans le revenu national au Canada, 1910 à 1980.

v. 37 , no 1,1982 , p. $53-64$

0097

\section{HOMMES}

DOOLEY, Martin D.

Within-Cohort Earnings Inequality Among Canadian Men: 1971-1982

v. 42 , no 3,1987 , p. $594-611$

0587

HACCOUN, Robert R. DUPONT, Serge Une analyse des comportements de travailleurs masculins et féminins selon deux formes d'absence au travail.

v. 43 , no 1,1988 , p. $153-166$

0634

\section{HÔPITAUX}

DOLAN, Shimon L. ARSENAULT, André LIZOTTE, Jean-Paul ABENHAIM, Lucien L'abi:entéisme hospitalier au Québec: Aspects culture/s et socio-démographiques.

v. 38 , no 1,1983 , p. $45-57$

0179

RAINVILLE, Jean-Marie

La responsabilité fonctionnelle chez les cadres subalternes: Facteurs d'evolution des systèmes d'autorité.

v. 38 , no 4,1983 , p. $831-846$

0251

ANDIAPPAN, P. CATTANEO, R. Julian MURPHY, John

Interest Arbitration in Ontario Hospitals: Result of an Attitude Survey of Union and Management Officials.

v. 39 , no 4,1984 , p. $680-694$

0338

KRUGER, Arthur

Collective Bargaining in Ontario Public Hospitals

v. 40 , no 1,1985, p. $48-67$

0364 
HÓPITAUX (suite)

WETZEL, Kurt GALLAGHER, Daniel G. MAXEY, Charles T.

Impact of Nursing Unionism in the Hospital Industry: The Saskatchewan Experience.

v. 40 , no 4,1985 , p. $793-809$

0428

HACCOUN, Robert R. DUPONT, Serge Une analyse des comportements de travailleurs masculins et féminins selon deux formes d'absence au travail.

v. 43 , no 1,1988 , p. $153-166$

0634

LEVINE, David

Négocier la flexibilité: Reconversion des heures de la liste de rappel en postes à temps complet.

45ième Congrès, 1990, p. 41-47 0970

\section{HORAIRES DE TRAVAIL}

LAROUCHE, Viateur TRUDEL, Johanne La qualité de vie au travail et l'horaire variable.

v. 38 , no 3,1983 , p. $568-597$

0227

MASSE, Gilles

Le temps passé au travail: un elément encore négociable?

36ième Congrès, 1981, p. 123-135 0839

\section{I}

\section{IDÉOLOGIE}

DIMMOCK, Stuart J. SETHI, Amarjit S.

The Role of ldeology and Power in Systems Theory: Some Fundamental Shortcomings.

v. 41 , no 4,1986 , p. $738-757 \quad 0516$

\section{INDÉPENDANCE}

BABA, Vishwanath V. KNOOP, Robert

Organizational Commitment and Independence Among Canadian Managers

v. 42 , no 2,1987 , p. $325-344$

0564

\section{INDEXATION DES SALAIRES}

CHOUINARD, Jacques VIEL, André

L'efficacité des clauses d'indexation des salaires

v. 37 , no 1,1982 , p. 207-214 0105

COUSINEAU, Jean-Michel

LACROIX, Robert

L'efficacité des clauses d'indexation des salaires: Réplique.

v. 36 , no 1,1982 , p. $214-220 \quad 0106$

CHOUINARD, Jacques

ROBICHAUD, Jean-Marc

L'efficacité des clauses d'indexation des salaires.

v. 37 , no 1, 1982, p. 220-224 0107

MICHAUD, Sharon ROY, Jean-Charles

L'efficacité des clauses d'indexation des salaires: Réplique.

v. 37, no 1,1982 , p. 224-225 0108

\section{INDICE DES PRIX À LA CONSOMMATION}

FISHER, E.G. PERCY, M.B.

The Impact of Unanticipated Output and Consumer Prices on Wildcat Strikes.

v. 38, no 2,1983, p. $254-276 \quad 0203$

\section{INDUSTRIALISATION}

DICKINSON, John A.

La législation et les travailleurs québécois, 1894-1914

v. 41, no 2,1986, p. $357-381 \quad 0474$

INDUSTRIE DE LA CONSTRUCTION

MILLER, Roger

Les formes d'organisation dans l'industrie de la construction au Québec.

v. 37, no 1,1982 , p. 164-176 0102

ROSE, Joseph B. WETZEL, Kurt

Outcomes of Bargaining Structures in the Ontario and Saskatchewan Construction Industries.

v. 41 , no 2,1986 , p. $256-280 \quad 0468$

FISHER, E.G. KUSHNER, Stephen

Alberta's Construction Labour Relations During the Recent Downturn

v. 41 , no 4,1986 , p. $778-801 \quad 0518$ 
INDUSTRIE DE LA PÊCHE

GURDON, Michael A. WRIGHT, David J. Collective Bargaining in the Maritime Fishing Industry: Recent Developments

v. 39 , no 2,1984 , p. $259-274$

0293

LAMSON, Cynthia

On the Line: Women and Fish Plant Jobs in Atlantic Canada.

v. 41 , no 1,1986, p. $145-156$

0454

\section{INDUSTRIE DES ABATTOIRS}

FORREST, Anne

The Rise and Fall of National Bargaining in the Canadian Meat-Packing Industry.

v. 44 , no 2,1989 , p. $393-408$

0723

INDUSTRIE DES TÉLÉCOMMUNICATIONS

CUNNINGHAM, Barton HULL, Dennis

A Union Member's Perspective on Technological Change.

v. 43 , no 2,1988 , p. $394-411 \quad 0652$

\section{INDUSTRIE DES TEXTILES}

SIMARD, Marcel BOUTEILLER, Dominique LÉVESQUE, Christian

Prévention des accidents du travail: Contexte législatif québécois et efficacité organisationnelle.

v. 40 , no 4,1985 , p. $703-719$

0424

\section{INDUSTRIE DU CUIVRE}

FISHER, E.G. PERCY, M.B.

The Impact of Unanticipated Output and Consumer Prices on Wildcat Strikes.

v. 38, no 2,1983, p. $254-276$

0203

\section{INDUSTRIE DU MEUBLE}

LAFLAMME, Lucie ARSENAULT, André Rémunération, postes de travail et accidents: Une relation interactive.

v. 39, no 3,1984, p. $509-525$

0317

\section{INDUSTRIE DU VÊTEMENT}

GRANT, Michel ROSE, Ruth

Encadrement du travail à domicile dans l'industrie du vêtement au Québec.

v. 40, no 3,1985 , p. $473-494$

0405

VINET, Alain VÉZINA, Michel

BRISSON, Chantal

Des lésions professionnelles méconnues: le cas des opératrices de l'industrie du vêtement.

42ième Congrès, 1987, p. 21-38 0925

\section{INDUSTRIE FORESTIÈRE}

LAFLAMME, Lucie VINET, Alain

Accidents du travail et modernisation du processus de production: le cas de l'industrie forestière québécoise.

v. 43, no 3,1988 , p. 591-608

0669

\section{INDUSTRIE PÉTROCHIMIQUE}

EVANS, Martin G. ONDRACK, Daniel A. The Effect of Unionization on Wages: Some Canadian Evidence

v. 41 , no 3,1986 , p. $572-577$

0493

\section{INDUSTRIES}

MAKI, Dennis R. STRAND, Kenneth

The Determinants of Strike Activity: An Interindustry Analysis

v. 39 , no 1,1984, p. 77-92

0272

LEBRASSEUR, Rolland

Retirement and Skill Issues in Northern Ontario Industries.

v. 45 , no 2,1990, p. $268-282$

0793

\section{INDUSTRIES CANADIENNES}

MAGUN, Sunder

The Effects of Technological Changes on the Labour Market in Canada

v. 40, no 4,1985 , p. $720-746 \quad 0425$

\section{INDUSTRIES DE HAUTE TECHNOLOGIE}

BEAUMONT, P.B. HARRIS, R.I.D.

High Technology Industries and Non-Union Establishments in Britain.

v. 43 , no 4,1988 , p. $819-846$

0686

\section{INDUSTRIES ÉLECTRONIQUES}

PROULX, Pierre-Paul

Maitriser la technologie: pourquoi, quelles technologies, comment?

v. 41 , no 2,1986 , p. $382-389$

0475

\section{INDUSTRIES MANUFACTURIÈRES}

MILNE, William J. ROSS, Thomas W.

The Cyclical Variation of Wage Premiums in the Canadian Manufacturing Industries v. 39 , no 4,1984, p. $762-773$

0343

MAKI, Dennis R. MEREDITH, Lindsay N. The Effects of Unions on Profitability: Canadian Evidence

v. 41, no 1,1986 , p. $54-68$

0449 
INDUSTRIES MANUFACTURIÈRES (suite) RONDEAU, Claude BADIN, François Le contenu non salarial des conventions collectives dans les industries manufacturières au Québec.

v. 41 , no 1,1986 , p. $69-90$

0450

NG, Ignace

Determinants of Wildcat Strikes in Canada Manufacturing Industries.

v. 42, no 2,1987, p. $386-397$

0567

\section{INDUSTRIES MONTRÉALAISES}

COPP, Terry

The Rise of Industrial Unions in Montreal 1935-1945

v. 37, no 4,1982 , p. $843-87500162$

\section{INFIRMIERS ET INFIRMIÈRES}

VEILLEUX, Diane

Les clauses de promotion et de transfert chez les infirmières et les infirmiers.

v. 38 , no 2,1983 , p. $229-253 \quad 0202$

WETZEL, Kurt GALLAGHER, Daniel G. MAXEY, Charles T.

Impact of Nursing Unionism in the Hospital Industry: The Saskatchewan Experience.

v. 40, no 4,1985 , p. $793-809$

0428

\section{INFLATION}

WILLIAMS, C. Glyn

Joan Robinson on Unions, Distribution and Inflation.

v. 36 , no 3,1981 , p. $576-588$

0054

WILLIAMS, C. Glyn

The Role of Unions in Inflation: A Survey Article

v. 37 , no 3,1982 , p. $498-527$

\section{STURMTHAL, Adolf}

Unemployment, Inflation and "Guest Workers": Comparative Study of Three European Countries.

v. 37, no 4,1982, p. $739-764$

0157

MILNE, William J. ROSS, Thomas W. The Cyclical Variation of Wage Premiums in the Canadian Manufacturing Industries v. 39 , no 4,1984 , p. $762-773$

0343

\section{INFLATION (suite)}

KUMAR, Pradeep

Recent Wage Deceleration in Canada:

Short-run Response or Structural Change?

v. 42 , no 4,1987 , p. $687-701 \quad 0604$

\section{INFORMATION}

BRUCE, Christopher J.

The Role of Information Concerning the Arbitrator's Preferences.

v. 36, no 2, 1981, p. 386-402 0030

JAIN, Hem C.

Disclosure of Corporate Information to Trade Unions in North America.

v. 36, no 4,1981 , p. $748-774 \quad 0072$

MELTZ, Noah $M$.

Labour Market Information in Canada: The Current Situation and a Proposal.

v. 37 , no 2,1982 , p. $431-437$

0127

VANDAMME, François

L'information des travailleurs dans les grandes firmes: L'approche spécifique de la CEE.

v. 39 , no 1,1984 , p. $51-76$

0271

ANGERS, Georges GAGNON, Normand SIMARD, Jean

Le droit et le devoir à l'information.

44ième Congrès, 1989, p. 85-100 0960

INFORMATIQUE

MERCIER, Jean

L'informatique, ses "filtres" et ses effets.

v. 41 , no 2,1986 , p. $299-316 \quad 0470$

PROULX, Pierre-Paul

Maitriser la technologie: pourquoi, quelles technologies, comment?

v. 41 , no 2,1986, p. $382-389 \quad 0475$

TEMPLER, Andrew

SOLOMON, Norman A.

Unions and Technology: A Survey of Union Use of Information Technology.

v. 43 , no 2,1988 , p. $378-393 \quad 0651$

\section{INFORMATISATION}

CUNNINGHAM, Barton HULL, Dennis

A Union Member's Perspective on Technological Change.

v. 43, no 2,1988 , p. $394-411$

0652 


\section{INGENIEURS}

BARTH, Richard T. BABA, Vishwanath V. Alienation Among Professionnal Engineers:

A Canadian - American Comparison.

v. 37, no 1, 1982, p. 126-140 0100

INNOVATIONS EN MILIEU DE TRAVAIL

LONG, Richard J.

Patterns of Workplace Innovation in Canada.

v. 44, no 4,1989 , p. $805-826 \quad 0754$

\section{INSTITUTIONNALISME}

BARKIN, Solomon

Institutional Economics and the American Trade Union Movement.

v. 43 , no 3,1988 , p. $491-508 \quad 0664$

\section{INTERNATIONAL ASSOCIATION OF MACHI-} NISTS

PEACH, David A.

The Canadair-I.A.M. Productivity: Improvement Plan.

v. 37, no 1,1982, p. 177-197 0103

\section{INTERNATIONAL MOLDERS UNION}

WILLIAMS, C. Brian

The Impact of Labor Migration: The International Molders and Allied Workers Union in Canada, 1860-1885.

v. 39, no 2,1984, p. $335-364$

0298

\section{INTERVENTIONNISME}

MORIN, Fernand

Nouvelle présence de l'État dans les relations du travail.

v. 39, no 4,1984 , p. $744-752$

0341

\section{APPARICIO-VALDEZ, Luis}

La gestion empresarial en latinoamérica y su impacto en las relaciones laborales

v. 44, no 1,1989, p. $124-148$

0704

SACK, Jeffrey LEE, Tanya

The Role of the State in Canadian Labour Relations.

v. 44 , no 1,1989, p. $195-223$

0708

KOCHAN, Thomas A.

MCKERSIE, Robert $B$.

Future Directions for American Labor and Human Resources Policy.

v. 44, no 1, 1989, p. 224-248

0709

\section{INTERVENTIONNISME (suite)}

LUCENA, Hector

Papel del Estado en las relaciones industriales en América Latina

v. 44 , no 1,1989 , p. $249-282$

0710

WRIGHT, Michael D.

The Legislation of Labour Relations at Canada Post.

v. 44 , no 4,1989 , p. $866-883 \quad 0757$

DOWNIE, Bryan $M$.

The Role of the State in Industrial Relations: A Comment.

v. 45, no 1,1990, p. $169-186$

0781

DÉPATIE, Raymond

Commentaire - Les aspects économiques de la réforme des pensions.

37ième Congrès, 1982, p. 98-105 0851

BONENFANT, Claire GUÉRARD, Yves LAFONTAINE, Réal LAFOREST, Martial

Table ronde - La protection du revenu à la retraite: une responsabilité partagée?

37ième Congrès, 1982, p. 107-145 0852

HÉBERT, Hervé PERRON, Jacques

POULIN-SIMON, Lise

Table ronde - Qui doit administrer les régimes de retraite: lemployeur ou le syndicat?

37ième Congrès, 1982, p. 147-169 0853

CÔTÉ, André C.

Évolution des conditions de travail des salariés établies d'autorité.

40ième Congrès, 1985, p. 55-69 0891

PIUZE, Guy

Commentaires - Évolution des conditions de travail des salariés établies d'autorité. 40ième Congrès, 1985, p. 69-74 0892

MORIN, Fernand

L'institutionnalisation des rapports collectifs du travail: Réalité d'aujourd'hui et de demain.

40ième Congrès, 1985, p. 75-115 0893

GAGNON, Robert P.

Commentaires - L'institutionnalisation des rapports collectifs du travail: Réalité d'aujourd'hui et de demain.

40ième Congrès, 1985, p. 116-1190894 
IOWA

DILTS, David A. KARIM, Ahmad The Effect of Mediators' Qualities and Strategies on Mediation Outcomes v. 45 , no 1, 1990, p. $22-37$

0772

\section{ISRAËL}

CHERMESH, Ran

Conflict in the Israeli Industrial Relations System: A Critical Description.

v. 36 , no 3,1981 , p. $630-647$

0057

KAMINKA, Shlomit ROSEINSTEIN, Eliezer Shop Stewards, Workers, and Managers in Israeli Industry.

v. 38 , no 3,1983 , p. $598-617$

0228

RESHEF, Yonatan FRIED, Yatzhak

Strikes in Israell: The Histadrut in a Sectorial Perspective, 1965-1982

v. 41 , no 2,1986 , p. $329-347$

0472

\section{ITALIE}

WEISS, Dimitri

Du concept de "professionnalité" dans les relations industrielles italiennes.

v. 38 , no 2,1983 , p. $369-379$

0208

\section{$J$}

\section{JAPON}

MROCZKOWSKI, Tomasz

Theory Z: Myths, Realities and Alternatives.

v. 38 , no 2,1983 , p. 297-318

0205

\section{JEUNES}

DEMERS, Marie

Chómage chez les jeunes: Conséquences psychologiques et sociales.

v. 38 , no 4,1983 , p. $785-814$

0249

FORTIN, Pierre

Le chômage des jeunes au Québec: Aggravation et concentration, 1966-1982

v. 39, no 3,1984 , p. $419-448$

MERCIER, Jacques

Les effets du salaire minimum sur l'emploi des jeunes au Québec.

v. 40, no 3,1985, p. $431-457$

\section{JEUNES (sulte)}

WEIERMAIR, Klaus

Secular Changes in Youth Labour Markets and routh Unemployment in Canada.

v. 41 , no 3,1986 , p. $469-490 \quad 0487$

\section{JOURNALISTES}

DÉOM, Esther

La syndicalisation des journalistes dans quelques quotidiens québécois.

v. 42 , no 4,1987, p. $737-755 \quad 0607$

\section{JURISPRUDENCE}

BEMMELS, Brian FISHER, E.G.

NYLAND, Barbara

Canadian-American Jurisprudence on "Good Faith" Bargaining.

v. 41, no 3,1986 , p. $596-621 \quad 0496$

\section{JUSTICE}

MOORE, William R.

Justice and the Grievance Procedure in the Federal Public Service,

v. 36 , no 4,1981 , p. $848-864 \quad 0076$

\section{K}

KUHN, J.W.

MAKI, Dennis R. STRAND, Kenneth

The Determinants of Strike Activity: An Interindustry Analysis

v. 39 , no 1,1984, p. $77-92$

0272

$\mathbf{L}$

\section{LÉGISLATION DU TRAVAIL}

FRANK, J.A. KELLY, Michael J.

MACNAUGHTON, Bruce D.

Legislative Change and Strike Activity in Canada, 1926-1974

v. 37, no 2,1982 , p. 267-283 0119

DICKINSON, John A.

La législation et les travailleurs québécois, 1894-1914

v. 41 , no 2,1986, p. $357-381 \quad 0474$ 


\section{LÉGISLATION DU TRAVAIL (suite)}

ZEYTINOGLU, Isik Urla

The ILO Standards and Canadian Labour Législation.

v. 42 , no 2,1987, p. $292-308$

0562

LACROIX, Robert LESPÉRANCE, André Les nouvelles lois du travail et l'activité de grève.

v. 43 , no 4,1988 , p. $812-828$

0685

SACK, Jeffrey LEE, Tanya

The Role of the State in Canadian Labour Relations.

v. 44 , no 1,1989 , p. $195-223 \quad 0708$

WRIGHT, Michael D.

The Legislation of Labour Relations at Canada Post.

v. 44 , no 4,1989, p. $866-883$

0757

MUCKENBERGER, Ulrich

The Regulation of Strike Law in Times of New Technologies and Deregulation: The Case of West Germany.

v. 45 , no 1,1990, p. $136-145$

0778

DOWNIE, Bryan M.

The Role of the State in Industrial Relations: A Comment.

v. 45 , no 1,1990 , p. $169-186$

0781

BEAUSOLEIL, Gilles GAUTHIER, Hervé La législation: consolidation ou innovation?

36ième Congrès, 1981, p. 145-172 0841

GAGNON, Jean-Denis

Les notions de salarié en droit du travail. 40ième Congrès, 1985, p. 33-47 0889

DOUCET, René PARENT, Louise

Commentaires - Les notions de salarié en droit du travail.

40ième Congrès, 1985, p. 48-54 0890

MARCHAND, Jean

Les rapports collectifs du travail: Rétrospective et perspective.

40ième Congrès, 1985, p. 229-244 0902

GARANT, Patrice

Statut et pouvoirs des organismes du travail en regard des Chartes.

43ième Congrès, 1988, p. 237-254 0954

\section{LÉSIONS PROFESSIONNELLES}

PÉRUSSE, Michel

Les lésions professionnelles - une problématique.

42ième Congrès, 1987, p. 1-8 0923

CHIASSON, Denis-Émile

Synopsis sur le nouveau régime.

42ième Congrès, 1987, p. 9-19 0924

VINET, Alain VÉZINA, Michel

BRISSON, Chantal

Des lésions professionnelles méconnues: le cas des opératrices de l'industrie du vêtement.

42ième Congrès, 1987, p. 21-38 0925

LESAGE, Michel

Les lésions professionnelles: Point de vue d'un médecin.

42ième Congrès, 1987, p. 39-50 0926

PRÉVOST, Charles

La sous-estimation des atteintes à la santé causées par les mauvaises conditions de travail.

42ième Congrès, 1987 , p. 51-60 0927

\section{ARSENAULT, André}

La reconnaissance d'une maladie professionnelle est-elle négociable?

42ième Congrès, 1987, p. 61-72 0928

DUGUAY, Pierre BOUCHARD, Robert

GONTHIER, Jean-Marie

Commentaires - La reconnaissance d'une maladie professionnelle est-elle négociable?

42ième Congrès, 1987, p. 72-86 0929

LAFLAMME, Gilles LAROCQUE, Alain Lésions et maladies professionnelles: Un objet de négociation?

42ième Congrès, 1987 , p. 89-100 0930

\section{MORIN, Fernand}

Le régime actuel d'indemnisation pour lésions professionnelles: Accessibilité et efficacité.

42ième Congrès, 1987, p. 131-155 0933 


\section{LÉSIONS PROFESSIONNELLES (suite)}

LEFÈBVRE, Marie-Claire

LEVASSEUR, Raymond

Commentaires - Le régime actuel d'indemnisation pour lésions professionnelles: Accessibilité et efficacité.

42ième Congrès, 1987, p. 155-176 0934

BERNIER, Lionel

Équité, indemnisation des victimes de lesions professionnelles et coûts à l'entreprise.

42ième Congrès, 1987, p. 177-211 0935

GINGRAS, Claude DUCHESNE, André

Commentaires - Équité, indemnisation des victimes de lésions professionnelles et coûts à l'entreprise.

42ième Congrès, 1987, p. 212-222 0936

SURET, Jean-Marc GENDRON, Michel BERNIER, Gilles

Le processus de gestion des risques, les lésions professionnelles et la CSST.

42ième Congrès, 1987, p. 223-241 0937

HARGUINDEGUY, Jean-Louis

BRODY, Bernard

Commentaires - Le processus de gestion des risques, les lésions professionnelles et la CSST.

42ième Congrès, 1987, p. 241-252 0938

TOBIN, Edmund LAROSE, Gérald

DUFOUR, Ghislain LABERGE, Louis

Table ronde - Financement de la santé et paritarisme.

42ième Congrès, 1987, p. 253-276 0939

JÉRÔME-FORGET, Monique

La politique québécoise en matière de lésions professionnelles à un point tournant.

42ième Congrès, 1987, p. 277-285 0940

\section{LIBÉRALISME}

\section{ROCHER, Guy}

Les fondements de la société liberale, les relations industrielles et les Chartes.

43ième Congrès, 1988, p. 1-18

\section{LIBERTÉ D'ASSOCIATION}

AOUST, Claude d' DELORME, François

The Origin of the Freedom of Association and of the Right to Strike in Canada: An Historical Perspective.

v. 36 , no 4,1981 , p. $894-921$

0078

ZEYTINOGLU, Isik Urla

The ILO Standards and Canadian Labour Législation.

v. 42 , no 2,1987 , p. 292-308 0562

PÉPIN, Marcel

L'actualisation du mouvement syndical.

43ième Congrès, 1988, p. 209-221 0952

GAGNON, Mona-Josée LEBEAU, Ernest JOHNSTON, Raymond

Commentaires - l'actualisation du mouvement syndical.

43ième Congrès, 1988, p. 222-236 0953

\section{LIBERTÉ D'EXPRESSION}

TREMBLAY, Johane

Décisions rendues par le Conseil canadien des relations du travail.

v. 43, no 1,1988, p. $188-195 \quad 0637$

MORIN, Fernand

Liberté d'expression et droit au travail: l'arbitrage de la Cour suprême du Canada.

v. 44, no 4,1989 , p. 921-932 0761

\section{LIBRE-ÉCHANGE}

ROY, Paul-Martel

Aspects dynamiques du marché du travail au Québec.

v. 45, no 2,1990 , p. $283-299 \quad 0794$

GODBOUT, Clément RIOUX, Claude

Le libre-échange canado-américain et le marché du travail.

44ième Congrès, 1989, p. 175-188 0964

\section{LICENCIEMENTS}

PLISZKIEWICZ, Marek

Le licenciement en droit polonais.

v. 36, no 1, 1981, p. 251-259 0013

ROY, Paul-Martel

Licenciements collectifs, licenciements individuels et emploi au Québec: 19791984

v. 42 , no 3,1987, p. $577-593 \quad 0586$ 
LICENCIEMENTS (suite)

MORIN, Fernand

Un préavis de licenciements ou son équivalent.

v. 43, no 4,1988 , p. $943-952$

0691

BERGERON, Jean-Louis

La gestion de l'embauche, de la promotion et du licenciement revue et corrigée par les Chartes.

43ième Congrès, 1988, p. 51-62 0944

DUCHARME, Claude

LECLERCQ, Dominique

WESTMORELAND-TRAORE, Juanita

Commentaires - La gestion de l'embauche, de la promotion et du licenciement revue et corrigée par les Chartes.

43ième Congrès, 1988, p. 63-82

0945

\section{LIGNE DE PIQUETAGE}

MARLEAU, Véronique $L$.

Décisions rendues par le Conseil canadien des relations du travail.

v. 43, no 4,1988 , p. $953-962$

0692

CLARKE, Graham

Décisions rendues par le Conseil canadien des relations du travail.

v. 44 , no 4,1989 , p. $933-946$

0762

\section{LOCALITÉS ISOLÉES}

HOLMES, R.H. PINFIELD, L.T.

Logit Analysis of Employee Turnover in a Remote Community

v. 39, no 3,1984 , p. $553-569$

0320

\section{LOCK-OUT}

TANG, Roger Y.W. PONAK, Allen

Employer Assessment of Strike Costs.

v. 41 , no 3,1986 , p. $552-571$

\section{CLARKE, Graham}

Décisions rendues par le Conseil canadien des relations du travail.

v. 45 , no 1, 1990, p. 187-197 0782

\section{LOGICIELS}

PROULX, Pierre-Paul

Maîtriser la technologie: pourquoi, quelles technologies, comment?

v. 41 , no 2,1986 , p. $382-389$

0475

\section{LOGIT (MÉTHODE D'ANALYSE)}

HOLMES, R.H. PINFIELD, L.T.

Logit Analysis of Employee Turnover in a Remote Community

v. 39 , no 3,1984 , p. $553-569 \quad 0320$

\section{LOI CANADIENNE SUR LES DROITS DE LA}

PERSONNE

BOIVIN, Suzanne P.

Le Canadien National: Un cas riche d'enseignements.

43ième Congrès, 1988, p. 97-113 0947

SIMARD, Monique TELLIER, Marie

Commentaires - Le Canadien National: Un cas riche d'enseignements.

43ième Congrès, 1988, p. 114-130 0948

\section{LOI SUR LA SANTÉ ET LA SÉCURITÉ DU} TRAVAIL

BOULARD, René MARCHAND, Michel

La loi sur la santé et la sécurité du travail et les conventions collectives.

v. 38 , no 4,1983 , p. $847-8620252$

SIMARD, Marcel BOUTEILLER, Dominique LÉVESQUE, Christian

Prévention des accidents du travail: Contexte législatif québécois et efficacité organisationnelle.

v. 40 , no 4,1985 , p. $703-719 \quad 0424$

PLASSE, Micheline

Santé et sécurité du travail au Québec: Le défi de la concertation patronale - syndicale.

v. 42, no 3,1987 , p. $544-565 \quad 0584$

BLOUIN, Rodrigue BOULARD, René

DESCHÊNES, Jean-Paul

PÉRUSSE, Michel

Introduction - Régimes de santé et sécurité et relations du travail.

39ième Congrès, 1984, p. 15-16 0873

PÉRUSSE, Michel

Régimes de santé et sécurité et relations du travail.

39ième Congrès, 1984, p. 17-31 0874 


\section{LOI SUR LA SANTÉ ET LA SÉcuRITÉ DU TRAVAIL (suite)}

\section{SIMARD, Marcel}

Priorités en santé et sécurité du travail: secteur public et secteur privé.

39ième Congrès, 1984, p. 33-50 0875

LEDUC, Jean-Guy LEMELIN, Maurice VINET, Alain

Commentaires - Priorités en santé et sécurite du travail: secteur public et secteur privé.

39ième Congrès, 1984, p. 50-72 0876

BOUCHARD, Robert CLÉMENT, Pierre R. GIASSON, Étienne GUILLEMETTE, Michel Table ronde - La santé et la sécurité dans l'entreprise.

39ième Congrès, 1984, p. 113-135 0879

BOULARD, René

L'impact de la Loi sur les conventions collectives.

39ième Congrès, 1984, p. 137-157 0880

DE KONINCK, Maria HEENAN, Roy

Droit de refus et retrait préventif: succès ou échec?

39ième Congrès, 1984, p. 159-175 0881

BRUNET, Jacques

Santé et sécurité du travail: une affaire de professionnels?

39ième Congrès, 1984, p. 195-206 0884

DUFOUR, Ghislain FAVA, Frank

HÉTU, Jean-Paul LABERGE, Louis

LAROSE, Gérald SAUVÉ, Robert

Table ronde - Déceptions et espoirs.

39ième Congrès, 1984, p. 243-276 0886

\section{LOI SUR LE MAINTIEN DES SERVICES POSTAUX \\ WRIGHT, Michael D. \\ The Legislation of Labour Relations at Canada Post. \\ v. 44 , no 4,1989 , p. $866-883 \quad 0757$}

LOI SUR LES NORMES DU TRAVAIL DU QUÉBEC

LAPORTE, Pierre

Le caractère d'ordre public des dispositions de la Loi sur les normes du travail.

v. 42 , no 2,1987 , p. $398-414$

0568
LOI SUR LES NORMES DU TRAVAIL DU QUÉBEC (suite)

MORIN, Fernand

Un préavis de licenciements ou son équivalent.

v. 43 , no 4,1988 , p. $943-952 \quad 0691$

LOI SUR LES SYNDICATS DE SASKATCHEWAN

SASS, Robert

The Saskatchewan Trade Union Act. 1983:

The Public Battle.

v. 40 , no 3,1985 , p. $591-622 \quad 0411$

LOIS AUROUX

DELAMOTTE, Yves

La loi et la négociation collective en France: Réflexion sur l'expérience 1981-1985.

v. 42 , no 1,1987 , p. $92-109 \quad 0542$

\section{LOISIRS}

VALASKAKIS, Kimon

Le temps libére: à que/s coûts?

36ième Congrès, 1981, p. 61-74 0836

POULIN-SIMON, Lise FORTIN, Bernard

Commentaires - Le temps libéré: à quels coûts?

36ième Congrès, 1981 , p. $75-86 \quad 0837$

LOIS SPÉCIALES DE RETOUR AU TRAVAIL WRIGHT, Michael D.

The Legislation of Labour Relations at Canada Post.

v. 44, no 4,1989 , p. $866-883 \quad 0757$

\section{LOYAUTÉ}

THACKER, James W. ROSEN, Hjalmar

Dynamics of Employee Reactance to Company and Union Dual Allegiance Revisited and Expanded.

v. 41 , no 1,1986 , p. 128-144 0453

\section{$\mathbf{M}$}

\section{MACHINERIE}

LARRIVÉE, Liette AMBROISE, Gérald d' Difficultés de recrutement dans les PME québécoises: Quelques causes et solutions possibles.

v. 44, no 3,1989 , p. $487-506 \quad 0733$ 


\section{MAIN-D'OEUVRE}

WALSH, William D.

A Time Series Analysis of Female Labour Force Participation Rates Disaggregated by Marital Status.

v. 37 , no 2,1982, p. $367-384$

0124

GODBOUT, Clément RIOUX, Claude Le libre-échange canado-américain et le marché du travail.

44ième Congrès, 1989, p. 175-188 0964

\section{MAIN-D'OEUVRE QUALIFIÉE}

LARRIVÉE, Liette AMBROISE, Gérald d'

Difficultés de recrutement dans les PME québecoises: Quelques causes et solutions possibles.

v. 44, no 3,1989 , p. $487-506 \quad 0733$

\section{MAITTRISE EN ADMINISTRATION DES AF-} FAIRES

\section{FABI, Bruno}

Prive ou public: choix et transfert du secteur organisationnel.

v. 39 , no 2,1984, p. $313-334$

0297

MAITTRISE EN RELATIONS INDUSTRIELLES LECLERC, Claudine

Thèses de maitrise en relations industrielles - Université Laval et Université de Montréal: 1940-1980

v. 36, no 2,1981 , p. $435-448$

0036

\section{MALADIES PROFESSIONNELLES}

SWIMMER, Gene LUCE, Sally R.

Asbestos Exposure and Attitudes Toward Occupational Health.

v. 40, no 3,1985, p. $529-544$

0408

TURCOTTE, Fernand

La prévention: une utopie?

39ième Congrès, 1984, p. 177-183 0882

DROUIN, Claude PLAMONDON, Denise Commentaires - La prévention: une utopie?

39ième Congrès, 1984, p. 183-194 0883

BEAUSOLEIL, Gilles

Les couts et les bénéfices du régime.

39ième Congrès, 1984, p. 207-242 0885

\section{MALADIES PROFESSIONNELLES (suite)}

PÉRUSSE, Michel

Les lésions professionnelles - une problématique.

42ième Congrès, 1987, p. 1-8 0923

CHIASSON, Denis-Émile

Synopsis sur le nouveau régime.

42ième Congrès, 1987, p. 9-19

0924

VINET, Alain VÉZINA, Michel

BRISSON, Chantal

Des lésions professionnelles méconnues: le cas des opératrices de l'industrie du vêtement.

42ième Congrès, 1987, p. 21-38 0925

LESAGE, Michel

Les lésions professionnelles: Point de vue d'un médecin.

42ième Congrès, 1987, p. 39-50 0926

\section{PRÉVOST, Charles}

La sous-estimation des atteintes à la santé causées par les mauvaises conditions de travail.

42ième Congrès, 1987, p. 51-60 0927

\section{ARSENAULT, André}

La reconnaissance d'une maladie professionnelle est-elle négociable?

42ième Congrès, 1987, p. 61-72 0928

DUGUAY, Pierre BOUCHARD, Robert

GONTHIER, Jean-Marie

Commentaires - La reconnaissance d'une maladie professionnelle est-elle négociable?

42ième Congrès, 1987, p. $72-86 \quad 0929$

LAFLAMME, Gilles LAROCQUE, Alain

Lésions et maladies professionnelles: Un objet de négociation?

42ième Congrès, 1987 , p. 89-100 0930

FARQUHAR, Alec

Le régime des accidents du travail: Le cas de l'Ontario.

42ième Congrès, 1987, p. 115-130 0932

MORIN, Fernand

Le régime actuel d'indemnisation pour lésions professionnelles: Accessibilité et efficacité.

42ième Congrès, 1987, p. 131-155 0933 
MALADIES PROFESSIONNELLES (suite)

LEFĖBVRE, Marie-Claire

LEVASSEUR, Raymond

Commentaires - Le régime actuel d'indemnisation pour lésions professionnelles: Accessibilité et efficacité.

42ième Congrès, 1987, p. 155-176 0934

BERNIER, Lionel

Équité, indemnisation des victimes de lésions professionnelles et coûts à l'entreprise.

42ième Congrès, 1987, p. 177-211 0935

GINGRAS, Claude DUCHESNE, André Commentaires - Équité, indemnisation des victimes de lésions professionnelles et coûts à l'entreprise.

42ième Congrès, 1987, p. 212-222 0936

SURET, Jean-Marc GENDRON, Michel BERNIER, Gilles

Le processus de gestion des risques, les lésions professionnelles et la CSST.

42ième Congrès, 1987, p. 223-241 0937

HARGUINDEGUY, Jean-Louis

BRODY, Bernard

Commentaires - Le processus de gestion des risques, les lesions professionnelles et la CSST.

42ième Congrès, 1987, p. 241-252 0938

\section{MALADIES VIRALES}

JAIN, Hem C.

AIDS: Need for Policy in the Workplace.

v. 44 , no 4,1989 , p. $850-865$

0756

\section{MANITOBA}

BLACK, Errol

In Search of "Industrial Harmony": The Process of Labour Law Reform in Manitoba, 1984.

v. 40, no 1,1985 , p. $140-161$

0369

BLACK, Errol SILVER, Jim

Contradictions and Limitations of Final Offer Selection: The Manibota Experience. v. 45, no 1,1990 , p. $146-165$

0779

GRANT, Hugh M.

Contradictions and Limitations of Final Offer Selection: The Manitoba Experience.

A Comment.

v. 45 , no 1,1990 , p. $166-168$

0780
MANITOBA (suite)

BLACK, Errol SILVER, Jim

FOS in Manitoba. A Rejoinder.

v. 45 , no 3, 1990, p. $612-615$

0819

\section{MANUFACTURES}

CÓTÉ, André C.

L'Acte des manufactures de Québec, 1885: Un centenaire

v. 40 , no 3,1985, p. $623-628 \quad 0412$

\section{MARCHÉ DU TRAVAIL}

HASAN, Abrar GERA, Surendra

Reservation Wages in Canadian Labour Markets.

v. 37 , no 1,1982 , p. $65-92 \quad 0098$

JAIN, Harish C.

Race ans Sex Discrimination in Employment in Canada: Theories, Evidence and Policies.

v. 37 , no 2,1982, p. $344-366$

0123

WALSH, William D.

A Time Series Analysis of Female Labour Force Participation Rates Disaggregated by Marital Status.

v. 37, no 2,1982, p. $367-384$

0124

MELTZ, Noah $M$.

Labour Market Information in Canada: The Current Situation and a Proposal.

v. 37 , no 2,1982 , p. $431-437$

0127

MAGUN, Sunder

The Placement Activity of the Canadian Employment Agency.

v. 38 , no 1,1983 , p. $72-94$

0181

LIPSIG-MUMME, Carla

The Renaissance of Homeworking in Developped Economies.

v. 38 , no 3,1983 , p. $545-567 \quad 0226$

COUSINEAU, Jean-Michel

LACROIX, Robert

La determination des avantages sociaux au Canada

v. 39 , no 1,1984, p. $3-22$

0268

ROBB, Roberta Edgecome

Occupational Segregation and Equal Pay for Work of Equal Value.

v. 39, no 1, 1984, p. 146-166 0275 
MARCHÉ DU TRAVAIL (suite)

MAGUN, Sunder

The Effects of Technological Changes on the Labour Market in Canada

v. 40 , no 4,1985 , p. $720-746$

0425

WEIERMAIR, Klaus

Secular Changes in Youth Labour Markets and Youth Unempioyment in Canada.

v. 41 , no 3,1986 , p. $469-490$

0487

BETCHERMAN, Gordon

Labour Market Imbalances in Canada, 1966-1983

v. 41 , no 4,1986 , p. $802-816$

0519

BARKIN, Solomon

The Flexibility Debate in Western Europe: The Current Drive to Restore Managements' Rights Over Personnel and Wages.

v. 42 , no 1,1987 , p. $12-44$

0539

REID, Frank

Economic Aspects of Mandatory Retirement: The Canadian Experience.

v. 43 , no 1,1988, p. $101-114$

0631

ROY, Paul-Martel

Aspects dynamiques du marché du travail au Québec.

v. 45 , no 2,1990 , p. $283-299 \quad 0794$

LANGLOIS, Simon

Le travail à temps partiel: Vers une polarisation de plus en plus nette.

v. 45, no 3,1990, p. $548-565$

0816

GODBOUT, Clément RIOUX, Claude

Le libre-échange canado-américain et le marché du travail.

44ième Congrès, 1989, p. 175-188 0964

BELLEMARE, Diane

Les emplois de l'avenir et les défis de la societé.

45ième Congrès, 1990, p. 117-128 0978

LAVALLÉE, Diane CHARLAND, Gaston LEPAGE, Brigitte MASSÉ, Henri MERCIER, Jean

Les emplois de l'avenir et les défis de la societé - Commentaires.

45ième Congrès, 1990, p. 128-148 0979

\section{MARIAGE}

LE LOUARN, Jean-Yves

THÉRIAULT, Roland

TOULOUSE, Jean-Marie

Le travail des deux conjoints: Effets sur la progression de carrière du cadre.

v. 39 , no 1,1984 , p. $36-50$

0270

\section{MARITIMES (PROV.)}

LAMSON, Cynthia

On the Line: Women and Fish Plant Jobs in Atlantic Canada.

v. 41 , no 1,1986 , p. $145-156 \quad 0454$

\section{MARKETING}

GILSON, Clive H.J. SPENCER, lan S.

Trade Union Growth: A Marketing Model.

v. 42 , no 4,1987, p. $756-773 \quad 0608$

\section{MÉDECINE DU TRAVAIL}

TURCOTTE, Fernand

La prévention: une utopie?

39ième Congrès, 1984, p. 177-183 0882

DROUIN, Claude PLAMONDON, Denise

Commentaires - La prévention: une utopie?

39ième Congrès, 1984, p. 183-194 0883

BRUNET, Jacques

Santé et sécurité du travail: une affaire de professionnels?

39ième Congrès, 1984, p. 195-206 0884

\section{MÉDIATION}

HAMEED, Syed M.A. SEN, Joya

A Power Theory of Third Party Intervention

in Labour Management Relations.

v. 42 , no 2,1987 , p. $243-255 \quad 0559$

KELLER, Berndt

Mediation as a Conflict-Solving Device in Collective Industrial Disputes.

v. 43, no 2,1988 , p. $431-446 \quad 0654$

DILTS, David A. KARIM, Ahmad

The Effect of Mediators' Qualities and Strategies on Mediation Outcomes

v. 45 , no 1,1990, p. $22-37$

0772

\section{MÉDIATION PRÉVENTIVE}

DULUDE, Yves

Le conflit: la gestion au banc des accusés.

41ième Congrès, 1986, p. 111-133 0914 


\section{MÉdIATION PRÉVENTIVE (sulte)}

LAMARCHE, Pierre

Commentaires - Le conflit: la gestion au banc des accusés.

41ième Congrès, 1986, p. 133-136 0915

LALANDE, Serge

L'expérience de la compagnie Gaz Métropolitain.

41ième Congrès, 1986, p. 137-146 0916

\section{MENTORS}

GODIN, Jean LE LOUARN, Jean-Yves

Les mentors ont-ils un effet sur la progression de carrière?

v. 41 , no 3,1986 , p. $505-518 \quad 0489$

\section{MESURES DISCIPLINAIRES}

LAVERY, Daniel

Décisions rendues par le Conseil canadien des relations du travail.

v. 37 , no 1,1982 , p. 226-234

0109

LAMY, Francine

Décision rendue par le Conseil canadien des relations du travail.

v. 40 , no 3,1985 , p. $659-662$

0415

AOUST, Claude d'

L'amnistie des fautes disciplinaires.

v. 43 , no 4,1988 , p. $909-942 \quad 0690$

\section{MESURES TARIFAIRES}

JONES, J.C.H. LAUDADIO, L.

Organized Labour, Regional Political Bias and the Canadian Tariff Structure

v. 39 , no 4,1984 , p. $695-709$

0339

\section{MÉTIERS}

LEBRASSEUR, Rolland

Retirement and Skill Issues in Northern Ontario Industries.

v. 45 , no 2,1990, p. 268-282 0793

\section{MEUBLE (INDUSTRIE)}

LAFLAMME, LUcie ARSENAULT, André Rémuneration, postes de travail et accidents: Une relation interactive.

v. 39 , no 3,1984 , p. $509-525$

0317

\section{MICROÉLECTRONIQUE}

JAIN, Harish C.

Micro-electronics Technology and Industrial Relations.

v. 38 , no 4,1983 , p. $869-879$

0254
MICROÉLECTRONIQUE (suite)

CAMPBELL, Adrian WARNER, Malcolm

Workplace Relations, Skills-Training and Technological Change at Plant-Level.

v. 43 , no 1,1988 , p. 115-132 0632

MIL DAVIE INC.

LISTON, Terrence

Négocier la flexibilité: La polyvalence des emplois.

45ième Congrès, 1990, p. 65-69 0973

MILTTANTISME SYNDICAL

WILLIAMS, C. Glyn

The Role of Unions in Inflation: A Survey Article

v. 37 , no 3,1982 , p. $498-527 \quad 0137$

BARKIN, Solomon

Troubled Worker Militancy: Challenges Confronting Western Industrial Relations Systems.

v. 38 , no 4,1983 , p. $713-729 \quad 0245$

MCSHANE, Steven L.

Sources of Attitudinal Union Militancy

v. 40, no 2,1985, p. $284-302$

0386

MILLARD, C.H.

SEFTON MACDOWELL, Laurel

The Career of a Canadian Trade Union Leader: C.H. Millard 1937-1946

v. 43 , no 3,1988 , p. $609-632$

0670

\section{MINE RUSHTON}

BROSSARD, Michel

La stratégie syndicale face aux groupes semi-autonomes de production: Hypothese pour l'analyse du cas Rushton.

v. 37 , no 3,1982 , p. $670-683$

0145

\section{MINEURS UNIS D'AMÉRIQUE}

WILLIAMS, C. Brian

International Trade Unionism: The United Mine Workers in Eastern Canada, 19001920

v. 41 , no 3,1986 , p. $519-540 \quad 0490$

MINORITÉS VISIBLES

JAIN, Harish C.

Recruitment of Racial Minorities in Cana"dian Police Forces.

v. 42, no 4, 1987, p. 790-805 0610 


\section{MINORITÉS VISIBLES (suite)}

JAIN, Harish C.

Racial Minorities and Affirmative Action/Employment Equity Législation in Canada.

v. 44 , no 3,1989 , p. $593-614$

0738

\section{MISES À PIED}

MORIN, Fernand

Un préavis de licenciements ou son équivalent.

v. 43 , no 4,1988 , p. $943-952 \quad 0691$

\section{MODE DE VIE}

BÉLANGER, Paul R. LÉVESQUE, Benoît Le mode de vie détermine-t-il l'éthique du travail? Étude de cas.

v. 42, no 2,1987, p. $345-365 \quad 0565$

\section{MODÈLES D'INNOVATION}

LONG, Richard J.

Patterns of Workplace Innovation in Canada.

v. 44 , no 4,1989 , p. $805-826$

0754

\section{MONOPOLES PROFESSIONNELS}

DUSSAULT, Gilles

The Future of Professional Monopolies.

v. 40 , no 2,1985 , p. $324-339$

0388

\section{MONTRÉAL}

RAINVILLE, Jean-Marie

Hierarchie ethnique dans la grande entreprise industrielle montréalaise.

v. 36 , no 2,1981 , p. $336-360$

0027

COPP, Terry

The Rise of Industrial Unions in Montreal 1935-1945

v. 37 , no 4,1982, p. $843-875$

0162

\section{COUSINEAU, Jean-Michel}

\section{LACROIX, Robert}

Le climat des relations du travail à Mon"tréal et à Toronto

v. 38 , no 4,1983, p. $730-743$

0246

GODIN, Michel

Négocier la flexibilité: La sous-traitance.

45ième Congrès, 1990, p. 59-64 0972

\section{MOTIVATION AU TRAVAIL}

BOURGEOIS, Robert-Paul WILS, Thierry

Career Concepts, Personality and Values of Some Canadian Workers: An Exploratory Study

v. 42 , no 3,1987 , p. $528-543 \quad 0583$

SALES, Carol A. LEVANONI, Eliahu

KNOOP, Robert

Employee Performance as a Function of Job Orientation and Job Design.

v. 44 , no 2,1989, p. $409-420$

0724

\section{MOUVEMENT OUVRIER}

ROBAKC, Léo

Les travailleurs du Québec au XXième siècle.

v. 40, no 4,1985 , p. $826-846 \quad 0430$

\section{MOYENS DE PRESSION}

DESMARAIS, Jacques

Les moyens de pression: Les chartes en redefinissent-elles les limites?

43ième Congrès, 1988, p. 157-195 0950

DULUDE, Gilles LOUMÈDE, Catherine

PARROT, Jean-Claude

Commentaires - Les moyens de pression:

Les Chartes en redefinissent-elles les limites?

43ième Congrès, 1988, p. 195-208 0951

\section{MULTINATIONALES}

PECCEI, Ricardo WARNER, Malcolm Industrial Relations, Strategic Importance, and Decision - Making.

v. 36, no 1,1981, p. $132-151 \quad 0006$

FAJANA, Sola

The Systems Approach as Theory for Multinational Industrial Relations in Developing Countries.

v. 44 , no 3,1989 , p. $615-634 \quad 0739$

\section{MUNICIPALITÉS}

DION, Stéphane

Syndicats et politique au niveau municipal en France.

v. 39, no 3.1984 , p. $466-485 \quad 0315$

GODIN, Michel

Négocier la flexibilité: La sous-traitance. 45ième Congrès, 1990, p. 59-64 0972 


\section{MUTATION D'EMPLOI}

DORNSTEIN, Miriam ZOREF, Uri

Motivation for Changing Jobs, Personal

Background Characteristics and Perceived

Opportunity

v. 41 , no 1,1986 , p. $91-110$

0451

CHAYKOWSKI, Richard P.

SLOTSVE, G.A.

Union Seniority Rules as a Determinant of Intra-Firm Job-Changes.

v. 41 , no 4,1986 , p. $720-737$

\section{$\mathbf{N}$}

\section{NÉGOCIATION COLLECTIVE}

DOWNIE, Bryan $M$.

Some Thoughts on Public Policy and Industrial Peace.

v. 36 , no 1,1981 , p. $63-86$

0003

PETERSON, Richard B. TRACY, Lane

CABELLY, Alan

Problem Solving in Labor Negotiations:

Retest of a Model

v. 36 , no 1,1981 , p. $87-105$

0004

BARKIN, Solomon

Productivity Measures in Collective Bargaining.

v. 36 , no 2,1981 , p. $361-370 \quad 0028$

\section{SWIDINSKI, Robert}

The Effect of Bargaining Structure on Negotiated Wage Settlements.

v. 36 , no 2,1981 , p. $371-385$

0029

CHRISTENSEN, Sandra

Collective Bargaining in Provincial Public Administration.

v. 36 , no 3,1981 , p. $616-629$

0056

HÉBERT, Gérard

Les relations du travail au Québec: Bilan des années 1970

v. 36 , no 4,1981 , p. $715-747$

0071

MCPHILLIPS, David C.

Duty of Fair Representation: Recent Attitudes in British Columbia and Ontario.

v. 36 , no 4,1981 , p. $803-827$

0074

\section{NÉGOCIATION COLLECTIVE (suite)}

DÉOM, Esther

La négociation collective chez les fonctionnaires et les enseignants québécois: 1975-1976.

v. 37 , no 1,1982 , p. 141-163

0101

\section{LAVERY, Daniel}

Décisions rendues par le Conseil canadien des relations du travail.

v. 37 , no 1, 1982, p. 226-234

0109

BOLDUC, Roch BOUCHARD, Lucien

HÉBERT, Gérarơ

Le régime québécois de négociation des secteurs public et parapublic

v. 37, no 2,1982 , p. $403-430$

0126

\section{LAVERY, Daniel}

Décisions rendues par le Conseil canadien des relations du travail.

v. 37 , no 2,1982 , p. $438-450$

0128

LAVERY, Daniel

Décisions rendues par le Conseil canadien des relations du travail.

v. 37 , no 3, 1982, p. 684-696 0146

HARRIS, Philip RAUSCH, Peter

RYAN, Samuel G.

Ineffectiveness in Teacher Bargaining: The Anatomy of a First Strike.

v. 37 , no 4,1982 , p. $905-926 \quad 0165$

\section{BERNIER, Jean}

L'extension juridique des conventions collectives au Québec: Une approche comparative.

v. 38 , no 3,1983 , p. $532-544 \quad 0225$

SOLOMON, Norman A.

The Negociation of First Agreement in Ontario

v. 39, no 1,1984 , p. 23-35 0269

GURDON, Michael A. WRIGHT, David J. Collective Bargaining in the Maritime Fishing Industry: Recent Developments

v. 39 , no 2,1984 , p. $259-2740293$

BEAULIEU, LUC

Décisions rendues par le Conseil canadien des relations du travail

v. 39 , no 2,1984 , p. $371-385 \quad 0300$ 
NÉGOCIATION COLLECTIVE (suite)

PONAK, Allen THOMPSON, Mark

Faculty Collective Bargaining: The Voice of Experience

v. 39 , no 3,1984 , p. $449-465$

0314

GALLAGHER, Daniel G. WETZEL, Kurt Local Employer and Union Perceptions of Two-Tier Bargaining

v. 39 , no 3,1984 , p. $486-508 \quad 0316$

ADAMS, Roy J.

The Extent of Collective Bargaining in Canada

v. 39 , no 4,1984 , p. $655-667 \quad 0336$

KRUGER, Arthur

Collective Bargaining in Ontario Public Hospitals

v. 40 , no 1,1985 , p. $48-67$

0364

EASTMAN, Byron

A Catastrophe Theory on Union Behaviour. v. 40, no 2,1985 , p. $340-350 \quad 0389$

CARROTHERS, A.W.R.

A Way of Thinking about Collective Bargaining: Circumstance, Policy, Law and Actuality.

v. 40, no 2,1985, p. 351

0390

SOLOMON, Norman A.

The Negociation of First Agreements under the Canada Labour Code.

v. 40 , no 3,1985 , p. $458-472 \quad 0404$

CHARLES, Lawrence M.

HUMPHREYS, Edward $\mathrm{H}$.

Bargaining to Achieve Teacher Control in Ontario

v. 40 , no 3,1985 , p. $495-5110406$

MORIN, Fernand

Rapports collectifs du travail dans les secteurs publics québécois ou le nouvel équilibre selon la loi du 19 juin 1985.

v. 40 , no 3,1985 , p. $629-645$

0413

GILSON, Clive H.J.

Changes in the Nature of Grievance Issues Over the Last Ten Years: Labor Management Relations and the "Frontier of Control".

v. 40 , no 4,1985 , p. $856-864$

0432
NÉgOCIATION COLLECTIVE (suite)

BARKIN, Solomon

The Current Unilateralist Counterattack on Unionism and Collective Bargaining

v. 41 , no 1,1986, p. $3-27$

0447

ROSE, Joseph B. WETZEL, Kurt

Outcomes of Bargaining Structures in the Ontario and Saskatchewan Construction Industries.

v. 41 , no 2,1986, p. $256-280$

0468

GILSON, Clive H.J.

Bargaining Exercises: Beyond Simulation, a Touch of Reality.

v. 41, no 2,1986, p. $390-396 \quad 0476$

BEMMELS, Brian FISHER, E.G.

NYLAND, Barbara

Canadian-American Jurisprudence on "Go"od Faith" Bargaining.

v. 41 , no 3,1986 , p. $596-621 \quad 0496$

SAINT-GERMAIN, Catherine

Décisions rendues par le Conseil canadien des relations du travail

v. 41 , no 3,1986 , p. $622-637$

0497

FINKELMAN, Jacob

Public Sector Collective Bargaining

v. 41, no 4,1986, p. $691-703$

0513

DELAMOTTE, Yves

La loi et la négociation collective en France: Réflexion sur l'expérience 1981-1985.

v. 42 , no. 1,1987 , p. $92-109$

0542

HAMEED, Syed M.A. SEN, Joya

A Power Theory of Third Party Intervention in Labour Management Relations.

v. 42, no 2,1987, p. $243-255$

0559

BARRÉ, Alain

Le cadre juridique de la négociation collective dans le secteur public au Québec: une perspective internationale.

v. 42 , no 4,1987 , p. $831-851$

0612

RICHARDS, John MAUSER, Gary

HOLMES, Richard

What Do Workers Want? Attitudes Towards Collective Bargaining and Participation in Management.

v. 43, no 1,1988, p. $133-152 \quad 0633$ 


\section{NÉGOCIATION COLLECTIVE (suite)}

SACK, Jeffrey LEE, Tanya

The Role of the State in Canadian Labour Relations.

v. 44 , no 1,1989 , p. 195-223

0708

FORREST, Anne

The Rise and Fall of National Bargaining in the Canadian Meat-Packing Industry.

v. 44 , no 2,1989 , p. $393-408$

0723

RESHEF, Yonatan

Negotiating Wage Settements: A Structural Approach.

v. 44 , no 3,1989, p. $532-551$

0735

SMITH, Anthony E.

Innovation by Negotiation: Case Studies Among British White-Collar Unions.

v. 45, no 1,1990 , p. $63-75$

0774

CHAYKOWSKI, Richard P.

Union and Firm Preferences for Bargaining Outcomes in the Private Sector.

v. 45 , no 2,1990, p. $326-356$

0797

CUNNINGHAM, Barton MITCHELL, Lari Privatization in British Columbia: What the Experts Say Will Happen.

v. 45 , no 2,1990, p. $382-403$

0799

MORIN, Fernand

Modification unilaterale des conditions de travail au terme d'une négociation collectivel

v. 45 , no 3, 1990 , p. $566-584$

0817

CARROTHERS, A.W.R.

Labour Law Through the Prism of Paccar v. 45 , no 3,1990 , p. $585-611 \quad 0818$

\section{DOCQUIER, Gérard}

Ressources humaines et défis du syndicalisme.

41ième Congrès, 1986, p. 149-156 0917

KOCHAN, Thomas A.

L'avenir de la négociation collective.

41ième Congrès, 1986, p. 181-189 0922

LAFLAMME, Gilles LAROCQUE, Alain

Lésions et maladies professionnelles: Un objet de négociation?

42ième Congrès, 1987, p. 89-100 0930
NÉGOCIATION COLLECTIVE (suite)

ROJOT, Jacques

Droits collectifs et droits individuels: Les situations française, américaine et anglaise.

43ième Congrès, 1988, p. 19-49 0943

MATHIEU, René

Négocier la flexibilité: La recherche d'équité par la négociation.

45ième Congrès, 1990, p. 82-92 0975

NIGÉRIA

FASHOYIN, Tayo

State Regulation of Trade Disputes in Essential Services in Nigeria

v. 36, no 1,1981, p. 207-222 0010

NORMES DU TRAVAIL

ADAMS, Roy J.

Employment Standards in Ontario: An Industrial Relations Systems Analysis.

v. 42 , no 1,1987 , p. $46-64 \quad 0540$

LAPORTE, Pierre

Le caractère d'ordre public des dispositions de la Loi sur les normes du travail.

v. 42, no 2,1987 , p. 398-414 0568

MORIN, Fernand

Un préavis de licenciements ou son équivalent.

v. 43 , no 4,1988 , p. $943-952 \quad 0691$

NOUVEAU-BRUNSWICK

GURDON, Michael A. WRIGHT, David J. Collective Bargaining in the Maritime Fishing Industry: Recent Developments

v. 39, no 2, 1984, p. 259-274

0293

\section{NOUVEAU PARTI DÉMOCRATIQUE}

BLACK, Errol

In Search of "Industrial Harmony": The Process of Labour Law Reform in Manitoba, 1984.

v. 40, no 1,1985, p. $140-161 \quad 0369$

\section{NOUVELLE-ÉCOSSE}

GILSON, Clive H.J. GILLIS, L.P. Grievance Arbitration in Nova Scotia. v. 42 , no 2,1987 , p. 256-271 


\section{NOUVELLE-ÉCOSSE (suite)}

GILSON, Clive H.J. SPENCER, lan S.

GRANVILLE, S.

The Impact of a Strike on the Attitudes and Behavior of a Rural Community.

v. 44 , no 4,1989 , p. $785-804$

0753

\section{0}

\section{OCCIDENT}

BARKIN, Solomon

Troubled Worker Militancy: Challenges Confronting Western Industrial Relations Systems.

v. 38 , no 4,1983, p. $713-729$

0245

\section{OCCUPATIONS}

CHAYKOWSKI, Richard $P$.

SLOTSVE, G.A.

Union Seniority Rules as a Determinant of Intra-Firm Job-Changes.

v. 41 , no 4,1986, p. $720-737 \quad 0515$

\section{OFFICIERS}

TZINER, Aharon DOLAN, Shimon L. Identifying Female Officer Potential: An Exploration in Predictors Payoff

v. 40, no 1,1985, p. $87-98$

0366

\section{ONTARIO}

ADAMS, Georges W.

The Ontario Experience with Interest arbitration: Problems in Detecting Policy

v. 36, no 1, 1981, p. 225-250 0012

MCPHILLIPS, David C.

Duty of Fair Representation: Recent Attitudes in British Columbia and Ontario.

v. 36 , no 4,1981 , p. $803-827$

0074

SOLOMON, Norman A.

The Negociation of First Agreement in Ontario.

v. 39 , no 1,1984, p. $23-35$

0269

ANDIAPPAN, P. CATTANEO, R. Julian MURPHY, John

Interest Arbitration in Ontario Hospitals: Result of an Attitude Survey of Union and Management Officials.

v. 39 , no 4,1984, p. $680-694$
ONTARIO (sulte)

KRUGER, Arthur

Collective Bargaining in Ontario Public Hospitals

v. 40 , no 1,1985 , p. $48-67 \quad 0364$

BURKE, Ronald J.

Consequences of not Working Sixteen Months After a Plant Closing

v. 40 , no 1,1985 , p. $162-169 \quad 0370$

MCSHANE, Steven L.

Sources of Attitudinal Union Militancy

v. 40 , no 2,1985 , p. $284-302 \quad 0386$

CHARLES, Lawrence $M$.

HUMPHREYS, Edward $\mathrm{H}$.

Bargaining to Achieve Teacher Control in Ontario

v. 40 , no 3,1985 , p. $495-511 \quad 0406$

SWIMMER, Gene LUCE, Sally R.

Asbestos Exposure and Attitudes Toward Occupational Health.

v. 40 , no 3,1985 , p. $529-544 \quad 0408$

GUNDERSON, Morley

Spline Functions Estimates of the Impact of Equal Pay Legislation: The Ontario Experience

v. 40, no 4,1985, p. $775-792 \quad 0427$

ROSE, Joseph B. WETZEL, Kurt

Outcomes of Bargaining Structures in the Ontario and Saskatchewan Construction Industries.

v. 41, no 2,1986 , p. $256-280 \quad 0468$

FORREST, Anne

Bargaining Units and Bargaining Power

v. 41 , no 4,1986 , p. $840-850 \quad 0523$

ADAMS, Roy J.

Employment Standards in Ontario: An Industrial Relations Systems Analysis.

v. 42 , no 1,1987, p. $46-64$

0540

AULD, D.A.L. WILTON, D.A.

The Impact of Public Sector Wage Controls in Ontario

v. 42 , no 1,1987 , p. $132-149$

0544 
ONTARIO (suite)

KAMEL, Nawal ROY, Paul-Martel

Temps supplémentaire et création d'emplois: Le cas du Canada, du Québec et de l'Ontario.

v. 43 , no 2,1988 , p. $412-430$

0653

PUPO, Norene DORIS DUFFY, Ann

The Ontario Labour Relations Board and the Part Time Workers.

v. 43, no 3,1988 , p. $660-685$

0672

DOWNIE, Bryan M.

The Role of the State in Industrial Relations: A Comment.

v. 45 , no 1,1990, p. $169-186$

0781

LEBRASSEUR, Rolland

Retirement and Skill Issues in Northern Ontario Industries.

v. 45 , no 2,1990 , p. $268-282$

0793

BRUCE, Peter G.

The Processing of Unfair Labor Practice Cases in the United States and Ontario.

v. 45 , no 3,1990 , p. $481-511$

0813

WALTERS, Vivienne DENTON, Margaret Workers' Knowledge of their Legal Rights and Resistance to Hazardous Work.

v. 45 , no 3,1990, p. $531-547 \quad 0815$

FARQUHAR, Alec

Le régime des accidents du travail: Le cas de l'Ontario.

42ième Congrès, 1987, p. 115-130 0932

\section{ORDINATEURS}

PROULX, Pierre-Paul

Maîtriser la technologie: pourquoi, quelles technologies, comment?

v. 41 , no 2,1986 , p. $382-389$

0475

ZUREIK, Elia MOSCO, Vincent

LOCHHEAD, Clarence

Telephone Workers' Reaction to the New Technology

v. 44 , no 3,1989 , p. $507-531$

0734

\section{ORDRE PUBLIC}

LAPORTE, Pierre

Le caractère d'ordre public des disposi"tions de la Loi sur les normes du travail. v. 42 , no 2,1987, p. $398-414$
ORGANISATION DE COOP. ET DE DÉV. ÉCON.

BARKIN, Solomon

The Flexibility Debate in Western Europe:

The Current Drive to Restore Managements' Rights Over Personnel and Wages.

v. 42 , no 1,1987 , p. $12-44$

0539

\section{ORGANISATION DES NATIONS UNIES} BEIGBEDER, Yves

Individual Grievance Procedures in United Nations Secretariats.

v. 37, no 2, 1982, p. 328-343 0122

\section{ORGANISATION DU TRAVAIL}

BROSSARD, Michel

Revendications, stratégie syndicale et organisatior du travail dans l'entreprise.

v. 36 , no 3,1981 , p. $552-575$

0053

BROSSARD, Michel SIMARD, Marcel

Problématique de la différenciation de la main-d'oeuvre et changement organisationnel.

v. 41 , no 2,1986, p. $219-235$

0466

\section{BÉLANGER, Jacques}

Le controle ouvrier sur l'organisation du travail: Étude de cas en Grande-Bretagne. v. 41, no 4,1986 , p. $704-719 \quad 0514$

AUDET, Claude VINET, Alain

Bureautique et organisation du travail de secrétariat dans la fonction publique québécoise.

v. 43, no 4,1988 , p. $737-756$

0682

\section{MATON, BOD}

Socio-Technical Systems: Conceptual and Implementation Problems

v. 43 , no 4,1988 , p. $869-8890688$

CÔTÉ, André C.

Nouvelles technologies et droit du travail au Canada.

v. 44, no 4,1989 , p. $751-768$

0751

\section{BÉLANGER, Laurent}

Nouvelles formes d'organisation du travail, nouveaux modes de gestion et leur incidence sur le statut du salarié.

40ième Congrès, 1985, p. 171-196 0898 
ORGANISATION DU TRAVAIL (suite) CÔTÉ, Marcel

Commentaires - Nouvelles formes d'organisation du travail, nouveaux modes de gestion et leur incidence sur le statut du salarié.

40ième Congrès, 1985, p. 196-203 0899

\section{ORGANISATION INDUSTRIELLE}

MILLER, Roger

Les formes d'organisation dans l'industrie de la construction au Québec.

v. 37, no 1, 1982, p. 164-176 0102

\section{ORGANISATION INTERNATIONALE DU} TRAVAIL

TAJGMAN, David

Political Resolutions in the International Labor Organizations: The experience since 1964.

v. 36 , no 3,1981 , p. $499-5290051$

ROSSILION, Claude

L'OIT et l'élimination de la discrimination dans l'emploi

v. 40, no 1,1985, p. $03-26$

0362

BLANCHARD, Francis

L'OIT face au problème des relations professionnelles

v. 42 , no 1,1987 , p. 3-11

0538

ZEYTINOGLU, Isik Urla

The ILO Standards and Canadian Labour Législation.

v. 42, no 2,1987 , p. $292-308$

0562

BARRE, Alain

Le cadre juridique de la négociation collective dans le secteur public au Québec: une perspective internationale.

v. 42 , no 4,1987 , p. $831-851$

0612

\section{ORGANISATIONS}

DASTMALCHIAN, Ali

ADAMSON, Raymond BLYTON, Paul Developing a Measure of Industrial Relations Climate

v. 41 , no 4,1986 , p. $851-859$

0524

LONG, Richard J. WARNER, Malcolm Organizations, Participation and Recession: An Analysis of Recent Evidence. v. 42 , no 1,1987 , p. $65-91$

\section{ORGANISATIONS (suite)}

BABA, Vishwanath V.

KNOOP, Robert

Organizational Commitment and Independence Among Canadian Managers

v. 42 , no 2,1987 , p. $325-344 \quad 0564$

PEPERMANS, Raymond

Line, staff et functional

v. 43, no 1,1988, p. $183-187 \quad 0636$

STRATTON, Kay RESHEF, Yonatan

Private Sector Unions and Strategic Planning: A Research Agenda

v. 45 , no 1,1990 , p. $76-93$

0775

\section{ORGANISATIONS INTERNATIONALES}

BEIGBEDER, Yves

Individual Grievance Procedures in United Nations Secretariats.

v. 37, no 2, 1982, p. 328-343 0122

\section{ORGANISATIONS, THÉORIE DES}

GAGNON, Yves-Chantal

LANDRY, Maurice

Les changements technologiques: Une stratégie d'étude exploratoire

v. 44, no 2, 1989, p. 421-447 0725

\section{ORGANISMES DU TRAVAIL}

GARANT, Patrice

Statut et pouvoirs des organismes du travail en regard des Chartes.

43ième Congrès, 1988, p. 237-254 0954

\section{ORIENTATION DES TÂCHES}

SALES, Carol A. LEVANONI, Eliahu KNOOP, Robert

Employee Performance as a Function of Job Orientation and Job Design.

v. 44, no 2,1989 , p. $409-420$

0724

\section{OUEST CANADIEN}

SIMPSON, Wayne PETERS, Frank

The Economics of Mileage Restrictions for Railway Workers in Western Canada.

v. 38, no 1, 1983, p. 95-103 0182 


\section{$\mathbf{P}$}

\section{PACCAR OF CANADA LTD}

MORIN, Fernand

Modification unilatérale des conditions de travail au terme d'une négociation collective!

v. 45, no 3,1990, p. $566-584$

0817

CARROTHERS, A.W.R.

Labour Law Through the Prism of Paccar v. 45 , no 3,1990 , p. 585-611 0818

\section{PAIX INDUSTRIELLE}

DOWNIE, Bryan $M$.

Some Thoughts on Public Policy and industrial Peace.

v. 36 , no 1,1981, p. $63-86$

0003

\section{PAPETIERS}

WILLIAMS, C. Brian

International Unionism: The Papermakers

in Eastern Canada, 1930-1945

v. 42, no 1,1987, p. $110-131$

0543

\section{PARADIGMES}

YOUNG, Stanley

Industrial Relations: A Paradigmatic Analysis.

v. 37 , no 1,1982, p. $32-52$

0096

ADAMS, Roy J.

Competing Paradigms in Industrial Relations.

v. 38 , no 3,1983 , p. $508-531$

0224

AUDET, Michel LAROUCHE, Viateur Paradigmes, écoles de pensée et théories en relations industrielles.

v. 43 , no 1,1988 , p. $3-31$

0626

\section{PARITARISME}

LATULIPPE, Gérard P. O'FARRELL, Kevin Le comité paritaire: Anachronisme ou formule d'avenir.

v. 37, no 3,1982, p. $634-655 \quad 0143$

TOBIN, Edmund LAROSE, Gérald DUFOUR, Ghislain LABERGE, Louis Table ronde - Financement de la santé et paritarisme.

42ième Congrès, 1987, p. 253-276 0939

\section{PARTAGE DE L'EMPLOI}

CROWLEY, R.W. HUTH, E.

An International Comparison of Work Sharing Programs.

v. 38, no 3,1983, p. $636-647 \quad 0230$

\section{PARTICIPATION OUVRIÈRE}

WARNER, Malcolm

Corporatism, Participation and Society

v. 38, no 1, 1983, p. 27-44

BAR-HAIM, Aviad

The Desire for Workers' Participation: Conflicting Forces in the Workplace.

v. 39 , no 2,1984 , p. $301-312$

0296

JAIN, Hem C. GILES, Anthony

Workers' Participation in Western Europe: Implications for North America.

v. 40, no 4,1985, p. $747-774 \quad 0426$

LONG, Richard J. WARNER, Malcolm Organizations, Participation and Recession: An Analysis of Recent Evidence.

v. 42 , no 1,1987 , p. $65-91$

0541

RICHARDS, John MAUSER, Gary

HOLMES, Richard

What Do Workers Want? Attitudes Towards Collective Bargaining and Participation in Management.

v. 43 , no 1,1988 , p. $133-152 \quad 0633$

FRICKE, John G.

Worker Participation in Canada: Some Lessons from the Past.

v. 43, no 3,1988, p. $633-658$

0671

BROSSARD, Michel

Les limites du modèle-type du fonctionnement des cercles de qualité

v. 44 , no 3,1989 , p. 552-568 0736

LONG, Richard J.

Patterns of Workplace Innovation in Canada.

v. 44 , no 4,1989 , p. $805-826 \quad 0754$

OUELLET, Florian

Concertation et participation: mythe ou réalité?

39ième Congrès, 1984, p. 73-92 0877 


\section{PARTICIPATION OUVRIÈRE (suite)}

DUMAS, Anne-Chantal SIMARD, Monique Commentaires - Concertation et participation: mythe ou realité.

39ième Congrès, 1984 , p. 93-111 0878

BHÉRER, Harold

Le salarié et la gestion genérale de l'entreprise.

40ième Congrès, 1985, p. 205-222 0900

GODBOUT, Clément

Commentaires - Le salarié et la gestion générale de l'entreprise.

40ième Congrès, 1985, p. 222-228 0901

BÉLANGER, Jacques

Annexe - La participation des travailleurs aux décisions dans l'entreprise.

40ième Congrès, 1985, p. 245-259 0903

DOCQUIER, Gérard

Ressources humaines et défis du syndicalisme.

41ième Congrès, 1986, p. 149-156 0917

\section{PARTIS POLITIQUES}

MAKI, Dennis R.

Political Parties and Trade Union Growth in Canada.

v. 37 , no 4,1982 , p. $876-886 \quad 0163$

\section{PATERNALISME}

MROCZKOWSKI, Tomasz

Theory Z: Myths, Realities and Alternatives.

v. 38, no 2, 1983, p. 297-318 0205

\section{PATRONAT}

THWAITES, James

Business "Organizes": The Early Years in Quebec.

v. 36, no 2,1981 , p. $403-411$

0031

\section{BARBASH, Jack}

The New Industrial Relations in the US: Phase II.

v. 43, no 1,1988, p. $32-42$

0627

RESHEF, Yonatan

Changing Environments and Management IR Practices: Implications for U.S. Trade Unions.

v. 43 , no 1,1988 , p. $43-62$

0628

\section{PATRONAT (suite)}

DUFOUR, Ghislain LABERGE, Louis

LAROSE, Gérald

Table ronde - Les Chartes impliquent-elles un réalignement des politiques syndicales et patronales?

43ième Congrès, 1988, p. 255-272 0955

BROUILLET, Normand

Négocier la flexibilité: Réaction syndicale aux stratégies patronales.

45ième Congrès, 1990, p. 70-81 0974

\section{PÊCHE (INDUSTRIE)}

GURDON, Michael A. WRIGHT, David J. Collective Bargaining in the Maritime Fishing Industry: Recent Developments

v. 39 , no 2,1984 , p. $259-274$

0293

LAMSON, Cynthia

On the Line: Women and Fish Plant Jobs in Atlantic Canada.

v. 41 , no 1,1986, p. $145-156$

0454

\section{PERMISSION D'APPEL}

PLANTE, Gilles

La permission d'appel au tribunal du travail

v. 41 , no 4,1986 , p. $817-834$

0521

\section{PERSONNALITÉ}

BOURGEOIS, Robert-Paul WILS, Thierry Career Concepts, Personality and Values of Some Canadian Workers: An Exploratory Study

v. 42 , no 3,1987 , p. $528-543 \quad 0583$

\section{PERSONNEL ITINÉRANT}

TUCK, Hugh

Canadian Railways and Unions in the Running Trades, 1865-1914

v. 36 , no 1,1981, p. 106-131 0005

PERSONNEL SPÉCIALISÉ

LARRIVÉE, Liette AMBROISE, Gérald d' Difficultés de recrutement dans les PME québécoises: Quelques causes et solutions possibles.

v. 44, no 3,1989, p. $487-506 \quad 0733$ 


\section{PETITES ENTREPRISES}

COUSINEAU, Jean-Michel

NAJEM, Elmustapha

L'effet du développement de la petite entreprise sur l'évolution du syndicalisme au Canada.

v. 45, no 3,1990, p. $467-480$

0812

\section{PETITES ET MOYENNES ENTREPRISES}

LARRIVÉE, Liette AMBROISE, Gérald d' Difficultés de recrutement dans les PME québécoises: Quelques causes et solutions possibles.

v. 44 , no 3,1989 , p. $487-506 \quad 0733$

\section{PÉTROCHIMIE (INDUSTRIE)}

EVANS, Martin G. ONDRACK, Daniel A. The Effect of Unionization on Wages: Some Canadian Evidence

v. 41 , no 3,1986 , p. $572-577$

0493

\section{PHARMACIENS}

BOULARD, René

Le professionnalisme au Québec: Une étude empirique.

v. 36 , no 3,1981 , p. $648-662$

0058

\section{PIQUeTAGe}

DESMARAIS, Jacques

Les moyens de pression: Les chartes en redéfinissent-elles les limites?

43ième Congrès, 1988, p. 157-195 0950

DULUDE, Gilles LOUMĖDE, Catherine

PARROT, Jean-Claude

Commentaires - Les moyens de pression:

Les Chartes en redéfinissent-elles les limites?

43ième Congrès, 1988, p. 195-208 0951

\section{PLANIFICATION}

WILS, Thierry BOURGEOIS, Robert-Paul LABELLE, Christiane

Planification des ressources humaines dans la fonction publique fédérale.

v. 45, no 3,1990 , p. $512-530$

0814

\section{PLANIFICATION STRATÉGIQUE}

STRATTON, Kay RESHEF, Yonatan

Private Sector Unions and Strategic Planning: A Research Agenda

v. 45 , no 1,1990 , p. $76-93$

0775

PLURALISME SYNDICAL

DELORME, François LAFRANCE, Victor La concurrence dans un contexte de pluralisme syndical: Quelques données sur le Québec.

v. 37 , no 3, 1982, p. $575-605 \quad 0140$

\section{POLICIERS}

JAIN, Harish C.

Recruitment of Racial Minorities in Canadian Police Forces.

v. 42 , no 4,1987, p. $790-805 \quad 0610$

COUSINEAU, Jean-Michel RABEAU, Yves Une méthodologie de comparaison des salaires pour les emplois spécifiques du secteur public.

v. 43 , no 1,1988 , p. $85-100$

0630

MASSE, Gilles

Le temps passé au travail: un élément encore négociable?

36ième Congrès, 1981, p. 123-135 0839

POLITIQUE

WEISS, Dimitri

Politique, entreprise, syndicats

v. 36 , no 1,1981 , p. $192-206$

0009

GRAYSON, J. Paul

The Political Consequences of Unemployment: An Application of the "Power Mode/" of Blue-Collar Radicalism

v. 44 , no 3,1989 , p. $635-653 \quad 0740$

\section{POLITIQUE DE MAIN-D'OEUVRE}

CROWLEY, R.W. HUTH, E.

An International Comparison of Work Sharing Programs.

v. 38, no 3,1983 , p. $636-647 \quad 0230$

FORTIN, Pierre

Le chômage des jeunes au Québec: Aggravation et concentration, 1966-1982

v. 39 , no 3,1984 , p. $419-448$

0313 


\section{POLITIQUE DE MAIN-D'OEUVRE (suite)}

BARKIN, Solomon

The Flexibility Debate in Western Europe:

The Current Drive to Restore Managements' Rights Over Personnel and Wages.

v. 42 , no 1,1987 , p. $12-44$

0539

JAIN, Harish C.

Racial Minorities and Affirmative Action/Employment Equity Législation in Canada.

v. 44 , no 3,1989 , p. $593-614$

0738

\section{POLITIQUE ÉCONOMIQUE}

ADAMS, Roy J.

The Federal Government and Tripartism.

v. 37 , no 3,1982 , p. $606-617$

0141

COUSINEAU, Jean-Michel

LACROIX, Robert

La determination des avantages sociaux au Canada

v. 39 , no 1,1984 , p. $3-22$

0268

\section{POLITIQUE ET SYNDICAT}

MAC NEIL, Michael

Unions, Politics and Law in Canada.

v. 43, no 4,1988 , p. $847-868$

0687

\section{POLITIQUE GOUVERNEMENTALE}

DOWNIE, Bryan $\mathrm{M}$.

Some Thoughts on Public Policy and Industrial Peace.

v. 36 , no 1,1981 , p. $63-86$

0003

FRÉCHETTE, Raynald

La politique gouvernementale en matière de syndicalisation.

38ième Congrès, 1983, p. 197-206 0870

BONENFANT, Claire DUFOUR, Ghislain

HÉTU, Jean-Paul

Table ronde - Les réactions du milieu.

38ième Congrès, 1983, p. 207-223 0871

\section{POLITIQUE INTERNATIONALE}

TAJGMAN, David

Political Resolutions in the International Labor Organizations: The experience since 1964.

v. 36 , no 3,1981 , p. $499-529$

0051

\section{POLITIQUE SALARIALE}

FERLAND, Gilles

La politique de rémunération dans les secteurs public et parapublic au Québec.

v. 36 , no 3,1981 , p. $475-498 \quad 0050$

PROULX, Pierre-Paul

Rémuneration dans les secteurs public et parapublic au Québec: Eléments d'une nouvelle politique.

v. 37, no 3,1982 , p. $477-497 \quad 0136$

POLITIQUE TARIFAIRE

JONES, J.C.H. LAUDADIO, L.

Organized Labour, Regional Political Bias and the Canadian Tariff Structure

v. 39 , no 4,1984 , p. $695-709 \quad 0339$

\section{POLOGNE}

PLISZKIEWICZ, Marek

Le licenciement en droit polonais.

v. 36 , no 1,1981 , p. 251-259

0013

\section{POSTES CANADIENNES}

SAINT-GERMAIN, Catherine

Décisions rendues par le Conseil canadien des relations du travail.

v. 41 , no 4,1986 , p. $860-868$

0525

TREMBLAY, Johane

Décisions rendues par le Conseil canadien des relations du travail.

v. 42 , no 4,1987 , p. $852-861$

0613

MARLEAU, Véronique $L$.

Décisions rendues par le Conseil cana"dien des relations du travail.

v. 44 , no 3,1989 , p. $703-722$

0744

WRIGHT, Michael D.

The Legislation of Labour Relations at Canada Post.

v. 44 , no 4,1989 , p. $866-883 \quad 0757$

MARLEAU, Véronique L.

Décision rendue par le Conseil canadien des relations du travail.

v. 45 , no 2,1990, p. $414-423 \quad 0801$

POSTES DE TRAVAIL

LAFLAMME, Lucie ARSENAULT, André Rémunération, postes de travail et accidents: Une relation interactive.

v. 39 , no 3,1984 , p. $509-525 \quad 0317$ 


\section{POTENTIEL HUMAIN}

DIONNE, Pierre OUELLET, Gilles

Repenser l'évaluation.

v. 38, no 2,1983 , p. $344-368$

0207

TZINER, Aharon DOLAN, Shimon L. Identifying Female Officer Potential: An Exploration in Predictors Payoff

v. 40, no 1,1985 , p. $87-98$

0366

\section{POUVOIR}

HAMEED, Syed M.A.

A Critique of Industrial Relations Theory.

v. 37 , no 1,1982 , p. $15-31$

0095

DIMMOCK, Stuart J. SETHI, Amarjit S.

The Role of Ideology and Power in Systems Theory: Some Fundamental Shortcomings.

v. 41 , no 4,1986, p. $738-757$

0516

HAMEED, Syed M.A. SEN, Joya

A Power Theory of Third Party Intervention in Labour Management Relations.

v. 42 , no 2,1987, p. $243-255$

0559

GRAYSON, J. Paul

The Political Consequences of Unemployment: An Application of the "Power Model" of Blue-Collar Radicalism

v. 44 , no 3,1989 , p. $635-653$

0740

\section{POUVOIR DISCIPLINAIRE}

AOUST, Claude d'

L'amnistie des fautes disciplinaires.

v. 43 , no 4,1988 , p. $909-942$

0690

\section{PRATIQUES DÉLOYALES}

MARTINEAU, LUC

Décisions rendues par le Conseil canadien des relations du travail.

v. 36 , no 2,1981 , p. $424-429$

0035

LAVERY, Daniel

Décisions rendues par le Conseil canadien des relations du travail.

v. 36 , no 4,1981 , p. $928-936$

0080

\section{LAVERY, Daniel}

Décisions rendues par le Conseil canadien des relations du travail.

v. 37, no 3,1982 , p. $684-696$

\section{PRATIQUES DÉLOYALES (suite)}

LAVERY, Daniel

Décisions rendues par le Conseil canadien des relations du travail.

v. 38 , no 2,1983 , p. $418-432$

0211

POTHIER, Dianne

Décisions rendues par le Conseil canadien des relations du travail.

v. 40 , no 1,1985 , p. $170-174$

0371

LAMY, Francine

Décisions rendues par le Conseil canadien des relations du travail.

v. 40, no 2,1985, p. $386-398$

0394

LAMY, Francine

Décision rendue par le Conseil canadien des relations du travail.

v. 40 , no 3, 1985, p. 659-662 0415

SAINT-GERMAIN, Catherine

Décisions rendues par le Conseil canadien des relations du travail

v. 41 , no 3,1986 , p. $622-637$

0497

TREMBLAY, Johane

Décisions rendues par le Conseil canadien des relations du travail.

v. 43, no 3,1988 , p. $686-694$

0673

CLARKE, Graham

Décisions rendues par le Conseil canadien des relations du travail.

v. 45, no 1, 1990, p. 187-197 0782

BRUCE, Peter G.

The Processing of Unfair Labor Practice Cases in the United States and Ontario.

v. 45, no 3,1990, p. $481-511 \quad 0813$

\section{LEBEL, Hélène}

Commentaires - L'impact du régime de relations du travail sur la syndicalisation.

38ième Congrès, 1983, p. 91-100 0863

\section{PRATIQUES D'EMPLOI}

BEAUMONT, P.B. TOWNLEY, B.

Non-Union American Plants in Britain:

Their Employment Practices.

v. 40 , no 4,1985 , p. $810-825 \quad 0429$ 


\section{PRÉAVIS DE LICENCIEMENT}

MCSHANE, Steven L.

Reasonable Notice Criteria in Common Law Wrongful Dismissal Cases.

v. 38 , no 3,1983 , p. $618-635$

0229

MORIN, Fernand

Un préavis de licenciements ou son équivalent.

v. 43, no 4,1988 , p. $943-952$

0691

\section{PRÉRETRAITE}

SCHELL, Bernadette

LEBRASSEUR, Rolland RENAUD, Robert Predictors of Acceptance of Early Retirement Offers for Workers.

v. 44 , no 2,1989, p. $376-392 \quad 0722$

\section{PRÉSIDENTS DE SYNDICAT}

SOLOMON, Norman A. ANDIAPPAN, P. SHAND, Dan

Canadian National Union Presidents: An Empirical Study.

v. 41 , no 3,1986, p. $491-504$

0488

\section{PRESSE ÉCRITE}

\section{DÉOM, Esther}

La syndicalisation des journalistes dans quelques quotidiens québécois.

v. 42, no 4,1987 , p. $737-755$

0607

\section{PRÉVENTION}

SIMARD, Marcel BOUTEILLER, Dominique LÉVESQUE, Christian

Prévention des accidents du travail: Contexte législatif québécois et efficacité organisationnelle.

v. 40, no 4,1985, p. $703-719$

0424

TURCOTTE, Fernand

La prévention: une utopie?

39ième Congrès, 1984, p. 177-183 0882

DROUIN, Claude PLAMONDON, Denise Commentaires - La prévention: une utopie?

39ième Congrès, 1984, p. 183-194 0883

SURET, Jean-Marc GENDRON, Michel BERNIER, Gilles

Le processus de gestion des risques, les lésions professionnelles et la CSST.

42ième Congrès, 1987, p. 223-241 0937
PRÉVENTION (sulte)

HARGUINDEGUY, Jean-Louis

BRODY, Bernard

Commentaires - Le processus de gestion des risques, les lésions professionnelles et la CSST.

42ième Congrès, 1987, p. 241-252 0938

\section{PRISE DE DÉCISION}

PECCEI, Ricardo WARNER, Malcolm

Industrial Relations, Strategic Importance, and Decision - Making.

v. 36, no 1, 1981, p. 132-151 0006

MEALIA, David W.

A Macro/Micro Décision Model for the Training and Development Specialist.

v. 37, no 3, 1982, p. 656-669 0144

\section{PRIVATISATION}

CUNNINGHAM, Barton MITCHELL, Lari

Privatization in British Columbia: What the Experts Say Will Happen.

v. 45 , no 2,1990, p. $382-403 \quad 0799$

PRIX

GILES, Anthony

The Canadian Labour Congress and Tripartism.

v. 37 , no 1,1982 , p. $93-1250099$

\section{PRODUCTIVITÉ}

BARKIN, Solomon

Productivity Measures in Collective Bargaining.

v. 36 , no 2,1981 , p. $361-370 \quad 0028$

PEACH, David A.

The Canadair-I.A.M. Productivity: Improvement Plan.

v. 37 , no 1,1982 , p. $177-1970103$

MAGUN, Sunder

The Rise of Service Employment in the Canadian Economy.

v. 37 , no 3,1982 , p. $528-556 \quad 0138$

MAKI, Dennis R.

Trade Unions and Productivity: Conventional Estimates.

v. 38 , no 2,1983, p. $211-228 \quad 0201$ 
PRODUCTIVITÉ (sulte)

SAUNDERS, George

Employment and the Productivity Slowdown: 1958-1980

v. 40, no 2,1985 , p. $219-242$

CAMIRÉ, André

La productivité des intelligences.

v. 43 , no 2,1988 , p. $322-340$

0649

DESCHÊNES, Jean-Paul

Commentaires - Le temps passé au travail: un élément encore négociable?

$36 i e ̀ m e$ Congrès, 1981, p. 136-144 0840

FRANCOEUR, Jean

Le syndicalisme et le nouveau travail.

41ième Congrès, 1986, p. 157-166 0918

\section{PRODUIT NATIONAL BRUT}

STURMTHAL, Adolf

Unemployment, Inflation and "Guest Workers": Comparative Study of Three European Countries.

v. 37, no 4,1982 , p. $739-764$

0157

\section{PROFESSEURS D'UNIVERSITÉ}

LE LOUARN, Jean-Yves

Les professeurs d'université et leur travail.

v. 37, no 2,1982, p. $385-402$

0125

GARNIER, Bernard

La gestion des conflits interpersonnels en milieu universitaire.

v. 38 , no 2,1983 , p. $277-296$

0204

PONAK, Allen THOMPSON, Mark

Faculty Collective Bargaining: The Voice of Experience

v. 39, no 3,1984, p. $449-465$

0314

$N G$, Ignace

Determinants of Union Commitment Among University Faculty.

v. 44, no 4, 1989, p. 769-784 0752

\section{PROFESSIONNALISME}

BOULARD, René

Le professionnalisme au Québec: Une étude empirique.

v. 36, no 3,1981, p. $648-662$

0058
PROFESSIONNALISME (suite)

CHARLES, Lawrence $M$.

HUMPHREYS, Edward $\mathrm{H}$.

Bargaining to Achieve Teacher Control in

Ontario

v. 40 , no 3,1985 , p. $495-511 \quad 0406$

\section{PROFESSIONNALITÉ}

WEISS, Dimitri

Du concept de "professionnalite" dans les relations industrielles italiennes.

v. 38 , no 2,1983 , p. $369-379 \quad 0208$

\section{PROFESSIONNELS}

BARTH, Richard T. BABA, Vishwanath V. Alienation Among Professionnal Engineers:

A Canadian - American Comparison.

v. 37 , no 1,1982 , p. $126-140 \quad 0100$

MARLEAU, Véronique L.

Décisions rendues par le Conseil canadien des relations du travail.

v. 44 , no 2,1989 , p. $448-462$

0726

\section{PROFESSIONNELS DE LA SANTÉ}

BRUNET, Jacques

Santé et sécurité du travail: une affaire de professionnels?

39ième Congrès, 1984, p. 195-206 0884

\section{PROFESSIONS}

DUSSAULT, Gilles

The Future of Professional Monopolies.

v. 40 , no 2,1985 , p. $324-339$

0388

\section{MAGUN, Sunder}

The Effects of Technological Changes on the Labour Market in Canada

v. 40 , no 4,1985 , p. $720-746 \quad 0425$

BLOUIN, Rodrigue

Le titre réservé de CRI et la déontologie de la profession

v. 42, no 2,1987, p. $309-324 \quad 0563$

COUSINEAU, Jean-Michel RABEAU, Yves Une méthodologie de comparaison des salaires pour les emplois spécifiques du secteur public.

v. 43 , no 1,1988 , p. $85-100 \quad 0630$ 


\section{PROFESSIONS LIBÉRALES}

AMERNIC, J.H. ARANYA, N.

Organizational Commitment: Testing Two Theories.

v. 38 , no 2,1983 , p. 319-343

0206

\section{PROFITS}

MAK, Dennis R. MEREDITH, Lindsay N. The Effects of Unions on Profitability: $\mathrm{Ca}$ nadian Evidence

v. 41 , no 1,1986 , p. 54-68

0449

\section{PROGRAMMES DE MAIN-D'OEUVRE}

BURKE, Ronald J.

Trainee Experiences in Industrial Retraining Programs: A Case Study

v. 39 , no 3.1984 , p. $570-577$

0321

GODBOUT, Clément RIOUX, Claude Le libre-échange canado-américain et le marché du travail.

44ième Congrès, 1989, p. 175-188 0964

\section{PROGRÈS TECHNOLOGIQUE}

VALASKAKIS, Kimon

Le temps liberré: à que/s coûts?

36 ième Congrès, 1981, p. 61-74

0836

POULIN-SIMON, Lise FORTIN, Bernard Commentaires - Le temps libéré: à quels coûts?

36 ième Congrès, 1981 , p. $75-86 \quad 0837$

\section{PROGRESSION DE CARRIÈRE}

GODIN, Jean LE LOUARN, Jean-Yves

Les mentors ont-ils un effet sur la progression de carrière?

v. 41 , no 3,1986 , p. $505-518$

0489

\section{PROMOTION SOCIALE}

BURT, Sandra

Voluntary Affirmative Action. Does it Work?

v. 41 , no 3,1986 , p. 541-551

0491

\section{PROMOTIONS}

VEILLEUX, Diane

Les clauses de promotion et de transfert chez les infirmières et les infirmiers.

v. 38, no 2,1983 , p. $229-253 \quad 0202$

GODIN, Jean LE LOUARN, Jean-Yves Les mentors ont-ils un effet sur la progression de carrière?

v. 41, no 3,1986, p. $505-518$

0489
PROMOTIONS (suite)

CHAYKOWSKI, Richard P.

SLOTSVE, G.A.

Union Seniority Rules as a Determinant of Intra-Firm Job-Changes.

v. 41 , no 4,1986 , p. $720-737 \quad 0515$

SWIMMER, Gene

Gender Based Differences in Promotions of Clerical Workers.

v. 45 , no 2,1990, p. $300-310 \quad 0795$

BERGERON, Jean-Louis

La gestion de l'embauche, de la promotion et du licenciement revue et corrigee par les Chartes.

43ième Congrès, 1988, p. 51-62 0944

DUCHARME, Claude

LECLERCQ, Dominique

WESTMORELAND-TRAORE, Juanita

Commentaires - La gestion de l'embauche, de la promotion et du licenciement revue et corrigée par les Chartes.

43ième Congrès, 1988, p. 63-82 0945

\section{PROPOSITIONS FINALES}

BRUCE, Christopher J.

The Role of Information Cuncerning the Arbitrator's Preferences.

v. 36 , no 2,1981, p. $386-402 \quad 0030$

BLACK, Errol SILVER, Jim

Contradictions and Limitations of Final Offer Selection: The Manibota Experience. v. 45 , no 1,1990 , p. 146-165 0779

GRANT, Hugh $M$.

Contradictions and Limitations of Final Offer Selection: The Manitoba Experience.

A Comment.

v. 45, no 1,1990 , p. $166-168 \quad 0780$

BLACK, Errol SILVER, Jim FOS in Manitoba. A Rejoinder.

v. 45 , no 3,1990 , p. $612-615$

0819

PROTOCOLES DE RETOUR AU TRAVAIL

LAVERY, Daniel

Décisions rendues par le Conseil cana"dien des relations du travail.

v. 38, no 2,1983 , p. 418-432

0211 
PROVIGO DISTRIBUTION INC.

GOBEILLE, Kenneth $R$.

Negocier la flexibilité: Le travail à temps partiel.

45ième Congrès, 1990 , p. $48-58$

0971

\section{PROVINCES MARITIMES}

GURDON, Michael A. WRIGHT, David J. Collective Bargaining in the Maritime Fishing Industry: Recent Developments

v. 39 , no 2,1984 , p. $259-274$

0293

LAMSON, Cynthia

On the Line: Women and Fish Plant Jobs in Atlantic Canada.

v. 41 , no 1,1986 , p. $145-156$

0454

\section{Q}

\section{QUALITÉ DE VIE AU TRAVAIL}

LAROUCHE, Viateur TRUDEL, Johanne

La qualité de vie au travail et l'horaire variable.

v. 38 , no 3,1983 , p. $568-597$

0227

\section{QUÉBEC (PROV.)}

BRISSON, Chantal

La santé au travail et la convention collective

v. 36 , no 1,1981 , p. $152-178$

0007

THWAITES, James

Business "Organizes": The Early Years in

Quebec.

v. 36 , no 2,1981 , p. $403-411$

0031

FERLAND, Gilles

La politique de rémunération dans les secteurs public et parapublic au Québec.

v. 36, no 3, 1981, p. 475-498 0050

BOULARD, René

Le professionnalisme au Québec: Une étude empirique.

v. 36 , no 3,1981 , p. $648-662$

0058

HÉBERT, Gérard

Les relations du travail au Québec: Bilan des années 1970

v. 36 , no 4,1981 , p. $715-747$

0071
QUÉBEC (PROV.) (sulte)

DÉOM, Esther

La négociation collective chez les fonctionnaires et les enseignants québecois: 1975-1976.

v. 37 , no 1,1982 , p. 141-163 0101

BOLDUC, Roch BOUCHARD, Lucien

HÉBERT, Gérard

Le régime québécois de négociation des secteurs public et parapublic

v. 37 , no 2,1982 , p. $403-430 \quad 0126$

DELORME, François LAFRANCE, Victor La concurrence dans un contexte de pluralisme syndical: Quelques données sur le Québec.

v. 37 , no 3,1982 , p. $575-605 \quad 0140$

LATULIPPE, Gérard P. O'FARRELL, Kevin Le comité paritaire: Anachronisme ou formule d'avenir.

v. 37 , no 3,1982 , p. $634-655 \quad 0143$

TOUPIN, Louis LESSARD, Claude CORMIER, Roger A. VALOIS, Paul La satisfaction au travail chez les enseignantes et enseignants au Québec.

v. 37, no 4,1982 , p. $805-826 \quad 0160$

VINET, Alain BÉGIN, Clermont

THÉRIAULT, Gilles

La santé des employés de bureau: Le cas de la fonction publique québécoise.

v. 37 , no 4,1982 , p. $887-904 \quad 0164$

DOLAN, Shimon L. ARSENAULT, André LIZOTTE, Jean-Paul ABENHAIM, Lucien L'absentéisme hospitalier au Québec: Aspects culturels et socio-démographiques.

v. 38 , no 1,1983, p. $45-57 \quad 0179$

VEILLEUX, Diane

Les clauses de promotion et de transfert chez les infirmieres et les infirmiers.

v. 38 , no 2,1983 , p. $229-253 \quad 0202$

BERNIER, Jean

L'extension juridique des conventions collectives au Quebec: Une approche comparative.

v. 38, no 3,1983 , p. $532-544 \quad 0225$ 


\section{QUÉBEC (PROV.) (suite)}

FORTIN, Pierre

Le chómage des jeunes au Québec: Aggravation et concentration, 1966-1982

v. 39 , no 3,1984 , p. $419-448$

0313

BLOUIN, Rodrigue

La réforme du Code du travail du Québec: une démarche fragmentaire.

v. 39 , no 3.1984 , p. $578-604$

0322

VERGE, Pierre

Evolution de la protection juridictionnelle de l'accès au syndicat.

v. 39 , no 4,1984 , p. $710-743$

0340

MORIN, Fernand

Nouvelle présence de l'État dans les relations du travail.

v. 39 , no 4,1984 , p. $744-752$

0341

MERCIER, Jacques

Les effets du salaire minimum sur l'emploi des jeunes au Québec.

v. 40 , no 3,1985, p. $431-457$

0403

GRANT, Michel ROSE, Ruth

Encadrement du travail à domicile dans l'industrie du vêtement au Québec.

v. 40, no 3,1985, p. $473-494$

0405

\section{GEORGE, Kenneth}

Les comités de santé et de sécurité du travail: Tables de concertation ou de négociation?

v. 40, no 3,1985 , p. $512-528$

0407

CÔTÉ, André C.

L'Acte des manufactures de Québec, 1885: Un centenaire

v. 40, no 3,1985 , p. $623-628$

0412

\section{MORIN, Fernand}

Rapports collectifs du travail dans les secteurs publics québécois ou le nouvel équilibre selon la loi du 19 juin 1985.

v. 40 , no 3,1985 , p. $629-645$

0413

ROBAKC, Léo

Les travailleurs du Québec au XXième siècle.

v. 40 , no 4,1985 , p. $826-846$

0430
QUÉBEC (PROV.) (suite)

BÉLANGER, Jacques MERCIER, Jacques Le plafonnement de la densité syndicale au Québec et au Canada.

v. 41 , no 1,1986 , p. $28-52$

0448

RONDEAU, Claude BADIN, François Le contenu non salarial des conventions collectives dans les industries manufacturières au Québec.

v. 41 , no 1,1986, p. $69-90$

0450

DICKINSON, John A.

La législation et les travailleurs québécois, 1894-1914

v. 41 , no 2,1986 , p. $357-381$

0474

WILLIAMS, C. Brian

International Unionism: The Papermakers in Eastern Canada, 1930-1945

v. 42 , no 1,1987 , p. $110-131$

0543

SEXTON, Jean

L'arbitrage de première convention collective au Québec: 1978-1984

v. 42 , no 2,1987, p. $272-291$

0561

PLASSE, Micheline

Santé et sécurité du travail au Québec: Le défi de la concertation patronale - syndicale.

v. 42 , no 3,1987 , p. 544-565 0584

ROY, Paul-Martel

Licenciements collectifs, licenciements individuels et emploi au Québec: 1979-1984

v. 42 , no 3,1987 , p. $577-593 \quad 0586$

DÉOM, Esther

La syndicalisation des journalistes dans quelques quotidiens québecois.

v. 42, no 4,1987 , p. 737-755 0607

MERCIER, Jacques

Effets du salaire minimum sur l'emploi:

Résultats des études économétriques canadiennes et québécoises.

v. 42 , no 4,1987 , p. $806-830 \quad 0611$

BARRÉ, Alain

Le cadre juridique de la négociation collective dans le secteur public au Québec: une perspective internationale.

v. 42 , no 4,1987 , p. $831-851$

0612 
QUÉBEC (PROV.) (suite)

BRUNET, Yves W. BEAULIEU, LuC

CANTIN, Isabelle

La nouvelle Commission québécoise des relations du travail (1988)

v. 43, no 2,1988 , p. $231-304$

0647

SODERSTROM, Lee

Some Effects of Unemployment on the Health of Unemployed Quebec Workers.

v. 43 , no 2,1988, p. $341-377 \quad 0650$

KAMEL, Nawal ROY, Paul-Martel

Temps supplémentaire et création d'emplois: Le cas du Canada, du Québec et de l'Ontario.

v. 43 , no 2,1988 , p. $412-430 \quad 0653$

LARRIVÉE, Liette AMBROISE, Gérald d' Difficultes de recrutement dans les PME québécoises: Quelques causes et solutions possibles.

v. 44, no 3,1989 , p. $487-506$

0733

ROSS, Claudette BROSSARD, Michel La conciliation volontaire est-elle plus efficace que la conciliation abligatoire? Le cas du Québec.

v. 45 , no 1,1990 , p. $3-21$

0771

SAVOIE, Dominique LAROUCHE, Viateur Le harcèlement sexuel au travail: Résultats de deux études québécoises.

v. 45 , no 1,1990 , p. $38-62$

0773

ROY, Paul-Martel

Aspects dynamiques du marché du travail au Québec.

v. 45 , no 2,1990 , p. $283-299$

0794

MARCHAND, Jean

Les rapports collectifs du travail: Rétrospective et perspective.

40ième Congrès, 1985, p. 229-244 0902

\section{QUEEN'S UNIVERSITY}

KELLY, Laurence

Industrial Relations at Queen's: The First Fifty Years.

v. 42 , no 3,1987 , p. $475-499$

0580

\section{QUOTIDIENS}

DÉOM, Esther

La syndicalisation des journalistes dans quelques quotidiens québécois.

v. 42 , no 4,1987, p. 737-755 0607

\section{R}

\section{RACISME}

JAIN, Marish C.

Race ans Sex Discrimination in Employment in Canada: Theories, Evidence and Policies.

v. 37, no 2,1982 , p. $344-366 \quad 0123$

JAIN, Harish C.

Racial Minorities and Affirmative Action/Employment Equity Législation in Canada.
v. 44 , no 3,1989 , p. $593-614$
0738

ANDIAPPAN, P. CRESTOHL, Mark

SINGH, Jang $B$.

Racial Discrimination in Employment in Canada.

v. 44 , no 4,1989 , p. $827-8490755$

\section{RAISONNABLE}

MORIN, Fernand

Le "raisonnable" déraisonnable! ou la rationalité du raisonnable

v. 40, no 3,1985 , p. $646-658$

0414

AOUST, Claude d'

Le contróle judiciaire des décisions arbitrales: Réflexions sur les moyens d'y échapper.

v. 41, no 2,1986, p. $348-356$

0473

MORIN, Fernand

Le rationnel et le raisonnable: Deux nécessités distinctes et conjugées en droit.

v. 41 , no 3,1986 , p. $578-582 \quad 0494$

\section{RAPPORT WOODS}

GOLDENBERG, Shirley B.

HÉBERT, Gérard

Harry Douglas Woods, 1907-1983

v. 38 , no 4,1983 , p. $707-712$

0244

ARTHURS, H.W.

Understanding 'Understanding": Industrial Relations Research and Policy in Canada from 1969 to 1984 ... and Beyond.

v. 39 , no 4,1984 , p. $753-761$

0342 


\section{RAPPORTS COLLECTIFS}

BARRÉ, Alain

Le régime des rapports collectifs et les Chartes.

43ième Congrès, 1988, p. 131-156 0949

\section{RÉCESSION ÉCONOMIQUE}

BEACH, Charles M. KALISKI, S.F.

The Impact of Recession on the Distribution of Annual Unemployment.

v. 41 , no 2,1986 , p. $317-328$

0471

FISHER, E.G. KUSHNER, Stephen

Alberta's Construction Labour Relations During the Recent Downturn

v. 41 , no 4,1986, p. $778-801$

0518

LONG, Richard J. WARNER, Malcolm Organizations, Participation and Recession: An Analysis of Recent Evidence.

v. 42 , no 1,1987, p. $65-91$

0541

\section{RECHERCHE D'EMPLOI}

HASAN, Abrar GERA, Surendra

Reservation Wages in Canadian Labour Markets.

v. 37 , no 1,1982 , p. $65-92$

0098

\section{RECHERCHE EMPIRIQUE}

BOULARD, René

Le professionnalisme au Québec: Une étude empirique.

v. 36 , no 3,1981 , p. $648-6620058$

\section{RECHERCHE EN RELATIONS INDUSTRIEL-} LES

ARTHURS, H.W.

Understanding "Understanding": Industrial Relations Research and Policy in Canada from 1969 to 1984... and Beyond.

v. 39 , no 4,1984 , p. $753-761$

0342

\section{RECHERCHE PARADIGMATIQUE}

YOUNG, Stanley

Industrial Relations: A Paradigmatic Analysis.

v. 37 , no 1,1982 , p. $32-52$

0096

ADAMS, Roy J.

Competing Paradigms in Industrial Rela"tions.

v. 38 , no 3,1983 , p. $508-531 \quad 0224$

\section{RECHERCHE THÉORIQUE}

CHAISON, Gary N.

A Note on the Critical Dimensions of the Union Merger Process.

v. 37 , no 1,1982 , p. $198-206 \quad 0104$

DIONNE, Pierre OUELLET, Gilles

Repenser l'évaluation.

v. 38 , no 2,1983 , p. $344-368$

0207

\section{RECONNAISSANCE SYNDICALE}

BEAUMONT, P.B.

Third Party Conciliation and Trade Union Recognition: Some British Evidence.

v. 37, no 4, 1982, p. 827-842 0161

BEAUMONT, P.B.

Statutory Recognition Provisions in Britain, 1976-80

v. 38 , no 4,1983 , p. $744-766$

0247

SEXTON, Jean

L'arbitrage de première convention collective au Québec: 1978-1984

v. 42 , no 2,1987 , p. $272-291$

0561

\section{RECAUTEMENT DU PERSONNEL}

JAIN, Harish C.

Recruitment of Racial Minorities in Canadian Police Forces.

v. 42 , no 4,1987 , p. $790-805$

0610

LARRIVÉE, Liette AMBROISE, Gérald d' Difficultés de recrutement dans les PME québécoises: Quelques causes et solutions possibles.

v. 44 , no 3,1989 , p. $487-506$

0733

\section{RÉDUCTION DE LA DURÉE DU TRAVAIL} JOLIVET, Thierry

La réduction de la durée du travail estelle créatrice d'emploi?

v. 38 , no 1,1983 , p. $142-154 \quad 0185$

\section{RÉGIME DES RENTES DU QUÉBEC}

FAILLE, Jacques

Les principaux régimes de retraite et leur contenu.

37ième Congrès, 1982, p. 45-74 0849

\section{BÉLANGER, Gérard}

Les aspects économiques de la réforme des pensions.

37ième Congrès, 1982, p. 75-98. 0850 
RÉGIME DES RENTES DU QUÉBEC (suite) DÉPATIE, Raymond

Commentaire - Les aspects économiques de la réforme des pensions.

37ième Congrès, 1982, p. 98-105 0851

BONENFANT, Claire GUÉRARD, Yves LAFONTAINE, Réal LAFOREST, Martial Table ronde - La protection du revenu à la retraite: une responsabilité partagée? 37ième Congrès, 1982, p. 107-145 0852

HÉBERT, Hervé PERRON, Jacques POULIN-SIMON, Lise

Table ronde - Qui doit administrer les régimes de retraite: l'employeur ou le syndicat?

37ième Congrès, 1982, p. 147-169 0853

\section{RÉGIMES DE RETRAITE}

DESCHÊNES, Jean-Paul FERLAND, Gilles SAINT-LAURENT, Jacques

SEXTON, Jean

Introduction - Les régimes de retraite.

37ième Congrès, 1982, p. 13-16 0846

SOLASSE, Bernard

La retraite: votre problème.

37ième Congrès, 1982, p. 17-34 0847

BENOIT, Michel

Des solutions aux problèmes de la retraite. 37ième Congrès, 1982, p. 35-44 0848

FAlLLE, Jacques

Les principaux régimes de retraite et leur contenu.

37ième Congrès, 1982, p. 45-74 0849

BÉLANGER, Gérard

Les aspects économiques de la réforme des pensions.

37ième Congrès, 1982, p. 75-98 0850

DÉPATIE, Raymond

Commentaire - Les aspects économiques de la réforme des pensions.

37ième Congrès, 1982, p. 98-105 0851

BONENFANT, Claire GUÉRARD, Yves LAFONTAINE, Réal LAFOREST, Martial Table ronde - La protection du revenu à la retraite: une responsabilité partagée? 37ième Congrès, 1982, p. 107-145 0852
RÉGIMES DE RETRAITE (suite)

HÉBERT, Hervé PERRON, Jacques

POULIN-SIMON, Lise

Table ronde - Qui doit administrer les régimes de retraite: l'employeur ou le syndicat?

37ième Congrès, 1982, p. 147-169 0853

BÉGIN, Monique

Les politiques gouvernementales en matière de retraite.

37ième Congrès, 1982, p. 171-180 0854

PARIZEAU, Jacques

Les politiques gouvernementales en matière de retraite.

37ième Congrès, 1982, p. 181-192 0855

GALBRAITH, John Kenneth

Réflexions sur les problèmes de la retraite dans les années 80.

37ième Congrès, 1982, p. 193-213 0856

LAMONTAGNE, Maurice

Commentaire - Réflexions sur les problèmes de la retraite dans les annees 80 .

37ième Congrès, 1982, p. 213-218 0857

\section{RÉGIONS ISOLÉES}

HOLMES, R.H. PINFIELD, L.T.

Logit Analysis of Employee Turnover in a Remote Community

v. 39 , no 3,1984, p. $553-569$

0320

\section{RÈGLEMENT DES GRIEFS}

KNIGHT, Thomas R.

Correlates of Informal Grievance Resolution Among Fist-Line Supervisors

v. 41 , no 2,1986 , p. $281-298$

0469

\section{RELATIONS DU TRAVAIL}

PECCEI, Ricardo WARNER, Malcolm

Industrial Relations, Strategic Importance, and Decision - Making.

v. 36 , no 1,1981 , p. 132-151 0006

HÉBERT, Gérard

Les relations du travail au Québec: Bilan des années 1970

v. 36 , no 4,1981 , p. $715-747$

0071

LAFLAMME, Gilles VALLÉE, Guylaine Changements technologiques et modes régulateurs des relations du travail.

v. 42, no 4,1987, p. $702-715$

0605 


\section{RELATIONS DU TRAVAIL (suite)}

DASTMALCHIAN, Ali NG, Ignace

Industrial Relations Climate and Grievance Outcomes.

v. 45 , no 2,1990, p. $311-325$

0796

LAFLAMME, Gilles

L'impact du régime de relations du travail sur la syndicalisation.

38ième Congrès, 1983, p. 77-90 0862

\section{LEBEL, Hélène}

Commentaires - L'impact du régime de relations du travail sur la syndicalisation.

38ième Congrès, 1983, p. 91-100 0863

MORIN, Fernand

L'institutionnalisation des rapports collectifs du travail: Réalité d'aujourd'hui et de demain.

40ième Congrès, 1985, p. 75-115 0893

\section{GAGNON, Robert $P$.}

Commentaires - L'institutionnalisation des rapports collectifs du travail: Réalité d'aujourd'hui et de demain.

40ième Congrès, 1985, p. 116-119 0894

MARCHAND, Jean

Les rapports collectifs du travail: Rétrospective et perspective.

40ième Congrès, 1985, p. 229-244 0902

\section{RELATIONS INDUSTRIELLES}

WEISS, Dimitri

Qu'est-ce que les relations industrielles?

v. 36 , no 2,1981 , p. $412-413$

0032

LECLERC, Claudine

Thèses de maittrise en relations industrie/les - Université Laval et Université de Montreal: 1940-1980

v. 36 , no 2,1981 , p. $435-448 \quad 0036$

LAROUCHE, Viateur DÉOM, Esther L'approche systémique en relations industrielles

v. 39 , no 1,1984, p. $114-145 \quad 0274$

SHIROM, Arie

The Labor Relations Sysiem: A Proposed Conceptual Framework

v. 40 , no 2,1985, p. 303-323

0387

\section{RELATIONS INDUSTRIELLES (suite)}

GILSON, Clive H.J.

Changes in the Nature of Grievance Issues Over the Last Ten Years: Labor Management Relations and the "Frontier of Contror.

v. 40, no 4,1985, p. $856-864$

0432

DIMMOCK, Stuart J. SETHI, Amarjit S.

The Role of Ideology and Power in Systems Theory: Some Fundamental Shortcomings.

v. 41, no 4,1986 , p. $738-757 \quad 0516$

\section{DASTMALCHIAN, Ali}

ADAMSON, Raymond BLYTON, Paul

Developing a Measure of Industrial Relations Climate

v. 41 , no 4,1986 , p. $851-859$

0524

\section{BARBASH, Jack}

Like Nature, Industrial Relations Abhors a Vaccum: The Case of the Union-Free Strategy.

v. 42 , no 1,1987 , p. $168-179 \quad 0546$

BOIVIN, Jean

Les relations industrielles: Une pratique et une discipline

v. 42 , no 1,1987 , p. $179-196$

0547

CLARKE, Oliver

Industrial Relations Theory and Practice: $A$ Note

v. 42 , no 1,1987, p. $196-202 \quad 0548$

KELLY, Laurence

Industrial Relations at Queen's: The First Fifty Years.

v. 42 , no 3,1987 , p. $475-499 \quad 0580$

AUDET, Michel LAROUCHE, Viateur Paradigmes, écoles de pensée et théories en relations industrielles.

v. 43 , no 1,1988, p. $3-31$

0626

BARBASH, Jack

The New Industrial Relations in the US: Phase II.

v. 43 , no 1,1988 , p. $32-42$

0627 


\section{RELATIONS INDUSTRIELLES (suite) \\ RESHEF, Yonatan}

Changing Environments and Management IR Practices: Implications for U.S. Trade Unions.

v. 43, no 1,1988 , p. $43-62$

0628

GILES, Anthony MURRAY, Grégor Towards an Historical Understanding of Industrial Relations Theory in Canada.

v. 43, no 4,1988, p. $780-811$

SEXTON, Jean DION, Gérard

L'avenir des relations industrielles dans les Amériques: Introduction.

v. 44 , no 1,1989, p. $1-2$

0698

AUDET, Michel BÉLANGER, Laurent Nouveaux modes de gestion et relations industrielles au Canada.

v. 44 , no 1,1989 , p. $62-96$

0702

APPARICIO-VALDEZ, Luis

La gestion empresarial en latinoamérica y su impacto en las relaciones laborales

v. 44 , no 1,1989 , p. $124-148$

0704

\section{HÉBERT, Gérard}

L'état de la discipline en relations industrielles au Canada: Un brin d'histoire et une postface./The State of the Art in Industrial Relations: A Brief History and a Postcript.

v. 44, no 4,1989, p. $884-904$

0758

BARKIN, Solomon

The State of the Art in Industrial Relations: A U.S. Commentary.

v. 44 , no 4,1989 , p. $905-914$

0759

WEISS, Dimitri

L'état de la discipline en relations industrielles au Canada: Un commentaire européen.

v. 44 , no 4,1989 , p. $914-920$

0760

THWAITES, James LAJOIE, Mario

BOIS-BROCHU, Hélène

Supplément - Quarante ans au service des relations industrielles

40ième Congrès, 1985, p. 261-281 0904
RELATIONS INDUSTRIELLES (suite)

BOIVIN, Jean

Émergence d'une réalité nouvelle en relations industrielles.

41ième Congrès, 1986, p. 17-30 0906

BLOUIN, Rodrigue FERLAND, Gilles

LAFLAMME, Gilles LAROCQUE, Alain

RONDEAU, Claude

Introduction - Les Chartes des droits et les relations industrielles.

43ième Congrès, 1988, p. XV

0941

ROCHER, Guy

Les fondements de la societé liberale, les relations industrielles et les Chartes.

43ième Congrès, 1988, p. 1-18 0942

RELATIONS INDUSTRIELLES (REVUE)

HÉBERT, Gérard

Distinction 1982 de l'Association cana-

dienne des relations industrielles: Gérard

Dion.

v. 37 , no 3,1982 , p. $475-476 \quad 0135$

\section{RELATIONS INTERPERSONNELLES}

AOUST, Claude d' SAINT-JEAN, Sylvain TRUDEAU, Gilles

L'obligation de civilité du salarié

v. 41 , no 1,1986 , p. $157-180$

0455

\section{RELATIONS PROFESSIONNELLES}

CHERMESH, Ran

Conflict in the Israeli Industrial Relations

System: A Critical Description.

v. 36 , no 3,1981 , p. $630-647$

0057

BLANCHARD, Francis

Les relations professionnelles et les défis des années 80.

v. 37 , no 1,1982 , p. 3-14

0094

HAMEED, Syed M.A.

A Critique of Industrial Relations Theory.

v. 37 , no 1,1982 , p. $15-31$

0095

YOUNG, Stanley

Industrial Relations: A Paradigmatic Analysis.

v. 37 , no 1,1982, p. $32-52$

0096 
RELATIONS PROFESSIONNELLES (suite) ADAMS, Roy J.

Competing Paradigms in Industrial Relations.

v. 38 , no 3,1983, p. $508-531$

0224

\section{BARKIN, Solomon}

Troubled Worker Militancy: Challenges Confronting Western Industrial Relations Systems.

v. 38 , no 4,1983 , p. $713-729$

0245

JAIN, Harish C.

Micro-electronics Technology and Industrial Relations.

v. 38 , no 4,1983 , p. $869-879$

0254

KRAHN, Harvey LOWE, Graham S.

Community Influences on Attitudes Towards Unions.

v. 39 , no 1,1984 , p. 93-113

0273

PIGANIOL, Claude

Relations professionnelles et gestion des ressources humaines.

v. 39 , no 2,1984 , p. $285-300$

0295

ARTHURS, H.W.

Understanding "Understanding": Industrial Relations Research and Policy in Canada from 1969 to 1984...and Beyond.

v. 39 , no 4,1984 , p. $753-761$

0342

BLANCHARD, Francis

L'OIT face au problème des relations professionnelles

v. 42 , no 1,1987 , p. $3-11$

0538

ADAMS, Roy J.

Employment Standards in Ontario: An Industrial Relations Systems Analysis.

v. 42 , no 1,1987 , p. $46-64$

0540

CARTER, Donald D.

Canadian Labour Relations Under the Charter: Exploring the Implications.

v. 43 , no 2,1988 , p. $305-321$

0648
RELATIONS PROFESSIONNELLES (suite)

LUCENA, Hector

Papel del Estado en las relaciones industriales en América Latina

v. 44 , no 1,1989 , p. $249-282 \quad 0710$

FAJANA, Sola

The Systems Approach as Theory for Multinational Industrial Relations in Developing Countries.

v. 44 , no 3, 1989 , p. $615-634 \quad 0739$

\section{RÉMUNÉRATION}

FERLAND, Gilles

La politique de rémunération dans les secteurs public et parapublic au Québec. v. 36 , no 3,1981 , p. $475-498 \quad 0050$

PROULX, Pierre-Paul

Rémunération dans les secteurs public et parapublic au Québec: Éléments d'une nouvelle politique.

v. 37 , no 3,1982 , p. $477-497 \quad 0136$

SIMPSON, Wayne PETERS, Frank

The Economics of Mileage Restrictions for Railway Workers in Western Canada.

v. 38, no i, 1983, p. 95-103 0182

LAFLAMME, Lucie ARSENAULT, André Rémunération, postes de travail et accidents: Une relation interactive.

v. 39 , no 3,1984 , p. 509-525 0317

MCSHANE, Steven L. REDEKOP, Bruce Compensation Management and Canadian Wrongful Dismissal: Lessons from Litigation.

v. 45, no 2,1990, p. $357-381 \quad 0798$

\section{RENDEMENT}

FISHER, E.G. PERCY, M.B.

The Impact of Unanticipated Output and Consumer Prices on Wildcat Strikes.

v. 38 , no 2,1983 , p. 254-276 0203

RENDEMENT AU TRAVAIL

DOLAN, Shimon L. TZINER, Aharon ROY, Denis

A Real Estate Agency's Level Analysis of the Climate-Performance Relationship.

v. 39 , no 1,1984, p. 167-176 0276 
RENDEMENT AU THAVAIL (suite)

SALES, Carol A. LEVANONI, Eliahu KNOOP, Robert

Employee Performance as a Function of Job Orientation and Job Design.

v. 44 , no 2,1989 , p. $409-420$

0724

\section{RENONCIATION À LA GRÈVE}

AOUST, Claude d'

Les réclamations à l'arbitrage de dommages résultant de la violation d'une clause de renonciation à la greve.

v. 36 , no 1,1981 , p. $259-262$

0014

\section{RENVOI INJUSTIFIÉ}

MCSHANE, Steven L.

Reasonable Notice Criteria in Common Law Wrongful Dismissal Cases.

v. 38 , no 3,1983 , p. $618-635$

0229

\section{RÉPARTITION DES BIENS}

WILLIAMS, C. Glyn

Joan Robinson on Unions, Distribution and Inflation.

v. 36 , no 3,1981 , p. $576-588$

0054

\section{RÉPARTITION DES REVENUS}

MCWATTERS, Catherine J.

BEACH, Charles $M$.

Factors Behind the Changes in Canada's Family Income Distribution and the Share of the Middle Class.

v. 45 , no 1,1990 , p. $118-135$

0777

\section{RÉPARTITION DU TRAVAIL}

VINET, Bernard

Le problème de la retraite.

v. 36 , no 4,1981 , p. $828-847$

0075

\section{REPRÉSENTATION JUSTE}

MARTINEAU, LUC

Décisions rendues par le Conseil canadien des relations du travail.

v. 36, no 3,1981 , p. $671-680$

0060

MCPHILLIPS, David C.

Duty of Fair Representation: Recent Attitudes in British Columbia and Ontario.

v. 36, no 4,1981 , p. $803-827$

0074

\section{LAVERY, Daniel}

Décisions rendues par le Conseil canadien des relations du travail.

v. 37 , no 1,1982 , p. 226-234

0109
REPRÉSENTATION JUSTE (suite)

LAVERY, Daniel

Décisions rendues par le Conseil canadien des relations du travail.

v. 37 , no 2,1982 , p. $438-450$

BEAULIEU, LUC

Décisions rendues par le Conseil canadien des relations du travail

v. 39 , no 2,1984 , p. $371-385$

0300

SAINT-GERMAIN, Catherine

Décisions rendues par le Conseil canadien des relations du travail

v. 42 , no 2,1987 , p. $415-427$

KNIGHT, Thomas R.

The Role of The Duty of Fair Representa"tion in Union Grievance Decisions.

v. 42 , no 4,1987 , p. $716-736 \quad 0606$

\section{RÉPUBLIQUE FÉDÉRALE D'ALLEMAGNE}

MUCKENBERGER, Ulrich

The Regulation of Strike Law in Times of New Technologies and Deregulation: The Case of West Germany.

v. 45 , no 1,1990, p. $136-145$

0778

\section{REQUÊTES EN ACCRÉDITATION}

LAVERY, Daniel

Décisions rendues par le Conseil canadien des relations du travail.

v. 37, no 4,1982 , p. $944-959$

0167

\section{RESPONSABILITÉ CIVILE}

WEST, Leigh

The Exclusive Remedy Provision in Canadian Worker Compensation Law: The Need for Legislative Reform.

v. 43 , no 4,1988 , p. $890-908 \quad 0689$

\section{RESPONSABILITÉ FONCTIONNELLE}

RAINVILLE, Jean-Marie

La responsabilité fonctionnelle chez les cadres subalternes: Facteurs d'évolution des systèmes d'autorité.

v. 38, no 4,1983 , p. $831-846 \quad 0251$

\section{RESSOURCES HUMAINES}

ROHLING, Thomas A.

Screening and Human Capital Theory: An Empirical Test

v. 41 , no 4,1986 , p. $817-826 \quad 0520$ 


\section{RESSOURCES HUMAINES (sulte)}

CAMIRÉ, André

La productivité des intelligences.

v. 43, no 2,1988, p. $322-340$

0649

ICHNIOWSK, Casey DELANEY, John T. LEWIN, David

The New Resource Management in U.S. Workplaces: Is it Really New and is it Only Nonunion?

v. 44, no 1,1989 , p. $97-123$

0703

KOCHAN, Thomas A.

MCKERSIE, Robert B.

Future Directions for American Labor and Human Resources Policy.

v. 44 , no 1,1989 , p. $224-248$

0709

WILS, Thierry LABELLE, Christiane GUÉRIN, Gilles LE LOUARN, Jean-Yves La gestion stratégique des ressources humaines: Un reniement du róle social de l'entreprise?

v. 44 , no 2,1989, p. $354-375$

0721

BARKIN, Solomon

Human Resources Management Examines itself and its Limitations

v. 44 , no 3,1989 , p. $691-702$

0743

WILS, Thierry BOURGEOIS, Robert-Paul LABELLE, Christiane

Planification des ressources humaines dans la fonction publique fédérale.

v. 45 , no 3,1990 , p. $512-530$

0814

AUDET, Michel BÉLANGER, Laurent BOIVIN, Jean DÉOM, Esther

MERCIER, Jacques

Introduction - La mobilisation des ressources humaines: tendances et impact.

41ième Congrès, 1986, p. 13-14 0905

BOIVIN, Jean

Émergence d'une réalité nouvelle en relations industrielles.

41ième Congrès, 1986, p. 17-30 0906

LAROUCHE, Viateur

La mobilisation des ressources humaines

- orientations récentes.

41ième Congrès, 1986, p. 31-51 0907
RESSOURCES HUMAINES (suite)

CASAVANT, Jean-Claude

La stratégie de gestion des ressources humaines chez Abitibi Price Inc.

41ième Congrès, 1986, p. 53-61 0908

\section{LEMAIRE, Alain}

La communication directe chez Cascades Inc.

41ième Congrès, 1986, p. 63-68 0909

NÉRON, Roger

Le projet d'entreprise de Culinar Inc.

41ième Congrès, 1986, p. 69-76 0910

BOUCHER, Lysette

Les limites des nouvelles approches en gestion des ressources humaines.

41ième Congrès, 1986, p. 77-84 0911

\section{DOCQUIER, Gérard}

Ressources humaines et défis du syndicalisme.

41 ième Congrès, 1986, p. 149-156 0917

HÉTU, Jean-Paul

La nouvelle gestion des ressources humaines - mythe ou réalité?

41ième Congrès, 1986, p. 167-172 0919

GOSSELIN, Alain FRENETTE, Jean-Guy DIONNE, Denis

Les acteurs d'une fusion ou d'une prise de pouvoir.

44ième Congrès, 1989, p. 155-173 0963

FRAPPIER-DESROCHERS, Monique

La pratique de gestion des ressources humaines dans les entreprises.

45ième Congrès, 1990, p. 25-39 0969

LE LOUARN, Jean-Yves

Les emplois atypiques et l'efficacité de la gestion des ressources humaines.

45ième Congrès, 1990, p. 93-103 0976

BOUDREAULT, Pierre GARON, Jacques PAGÉ, Lorainne PAQUETTE, Pierre

Les emplois atypiques et l'efficacité de la gestion des ressources humaines - Commentaires.

45ième Congrès, 1990, p. 103-116 0977 
Relations lndustrielles, vol. 45, no 4 (1990)

\section{RETRAIT PRÉVENTIF}

DE KONINCK, Maria HEENAN, Roy

Droit de refus et retrait préventif: succès ou échec?

39ième Congrès, 1984, p. 159-175 0881

\section{RETRAITE}

VINET, Bernard

Le probleme de la retraite.

v. 36 , no 4,1981 , p. $828-847$

0075

REID, Frank

Economic Aspects of Mandatory Retirement: The Canadian Experience.

v. 43 , no 1,1988 , p. $101-114$

0631

\section{SCHELL, Bernadette}

LEBRASSEUR, Rolland RENAUD, Robert Predictors of Acceptance of Early Retirement Offers for Workers.

v. 44, no 2,1989 , p. $376-392$

0722

LEBRASSEUR, Rolland

Retirement and Skill Issues in Northern

Ontario Industries.

v. 45, no 2,1990, p. $268-282$

0793

DESCHÊNES, Jean-Paul FERLAND, Gilles

SAINT-LAURENT, Jacques

SEXTON, Jean

Introduction - Les régimes de retraite.

37ième Congrès, 1982, p. 13-16 0846

SOLASSE, Bernard

La retraite: votre problème.

37ième Congrès, 1982, p. 17-34 0847

BENOIT, Michel

Des solutions aux problèmes de la retraite. 37ième Congrès, 1982, p. 35-44 0848

FAILLE, Jacques

Les principaux régimes de retraite et leur contenu.

37ième Congrès, 1982, p. 45-74 0849

BÉLANGER, Gérard

Les aspects économiques de la réforme des pensions.

37ième Congrès, 1982, p. 75-98 0850
RETRAITE (suite)

DÉPATIE, Raymond

Commentaire - Les aspects économiques de la réforme des pensions.

37ième Congrès, 1982, p. 98-105 0851

BONENFANT, Claire GUÉRARD, Yves LAFONTAINE, Réal LAFOREST, Martial

Table ronde - La protection du revenu à la retraite: une responsabilité partagée?

37ième Congrès, 1982, p. 107-145 0852

HÉBERT, Hervé PERRON, Jacques POULIN-SIMON, Lise

Table ronde - Qui doit administrer les régimes de retraite: l'employeur ou le syndicat?

37ième Congrès, 1982, p. 147-169 0853

BÉGIN, Monique

Les politiques gouvernementales en matière de retraite.

37ième Congrès, 1982, p. 171-180 0854

PARIZEAU, Jacques

Les politiques gouvernementales en matière de retraite.

37ième Congrès, 1982, p. 181-192 0855

GALBRAITH, John Kenneth

Réflexions sur les problèmes de la retraite dans les années 80.

37ième Congrès, 1982, p. 193-213 0856

LAMONTAGNE, Maurice

Commentaire - Réflexions sur les problèmes de la retraite dans les années 80 .

37ième Congrès, 1982, p. 213-218 0857

\section{REVENDICATIONS SYNDICALES}

BROSSARD, Michel

Revendications, stratégie syndicale et organisation du travail dans l'entreprise.

v. 36, no 3,1981 , p. $552-575 \quad 0053$

\section{REVENU FAMILIAL}

MCWATTERS, Catherine $\mathrm{J}$.

BEACH, Charles M.

Factors Behind the Changer in Canada's Family Income Distribution and the Share of the Middle Class.

v. 45 , no 1, 1990, p. 118-135 0777 


\section{REVENU NATIONAL}

MARION, Gérald

La part des salaires dans le revenu national au Canada, 1910 à 1980.

v. 37 , no 1,1982 , p. $53-64$

0097

\section{REVENUS}

OSBERG, Lars

$A$ Note on the Incomes of Lawyers

v. 40 , no 4 , 1985 , p. $865-879$

0433

STAGER, David A.A.

Lawyers' Earnings in the Canadian Private and Public Sectors.

v. 43 , no 3,1988 , p. $571-590$

0668

\section{REVUE INTERNATIONALE DU TRAVAIL}

AUCLAIR, Robert

Genève ignore Québec en matière de vocabulaire

v. 36 , no 1,1981 , p. 223-224

0011

\section{RIVALITE SYNDICALE}

MOUNT, Joan SIEGEL, Jacob P.

Resources and Results in Union Rivalry: A Case Study

v. 38 , no 4,1983 , p. $815-830$

0250

\section{ROBINSON, JOAN}

WILLIAMS, C. Glyn

Joan Robinson on Unions, Distribution and Inflation.

v. 36 , no 3,1981 , p. $576-588 \quad 0054$

\section{ROULEMENT DE LA MAIN-D'OEUVRE}

HOLMES, R.H. PINFIELD, L.T.

Logit Analysis of Employee Turnover in a Remote Community

v. 39 , no 3,1984 , p. $553-569$

0320

DORNSTEIN, Miriam ZOREF, Uri

Motivation for Changing Jobs, Personal Background Characteristics and Perceived Opportunity

v. 41 , no 1,1986 , p. $91-110$

\section{ROYAUME-UNI}

CAMERON, Samuel

Historical Variations in the Impact of Union Density on Strike Frequency: Some U.K. Evidence

v. 40, no 2,1985, p. $367-370$

0391

\section{RUSHTON (MINE)}

BROSSARD, Michel

La stratégie syndicale face aux groupes semi-autonomes de production: Hypothèse pour l'analyse du cas Rushton.

v. 37, no 3, 1982, p. 670-683 0145

\section{$\mathbf{S}$}

\section{SALAIRE D'ACCEPTATION}

HASAN, Abrar GERA, Surendra

Reservation Wages in Canadian Labour Markets.

v. 37 , no 1,1982, p. $65-92$

0098

\section{SALAIRE MINIMUM}

GRUBEL, Herbert G. MAKI, Dennis R.

A Note on the Effects of Unemployment Insurance, Minimum Wage Legis/ation and Trade Union Growth on Reported Unemployment Rates in Canada, 1950-75

v. 36 , no 4,1981 , p. $922-927$

0079

MERCIER, Jacques

Les effets du salaire minimum sur l'emploi des jeunes au Québec.

v. 40 , no 3,1985 , p. $431-457 \quad 0403$

MERCIER, Jacques

Effets ou salaire minimum sur l'emploi: Résultats des études économétriques canadiennes et québécoises.

v. 42 , no 4,1987 , p. $806-830$

0611

\section{SALAIRES}

SWIDINSKI, Robert

The Effect of Bargaining Structure on Negotiated Wage Settlements.

v. 36 , no 2,1981 , p. $371-385 \quad 0029$

AOUST, Claude d'

La juridiction de l'arbitre et de la Cour des petites créances devant la Cour supréme du Canada.

v. 36, no 2,1981 , p. $418-424$

FERLAND, Gilles

La politique de rémunération dans les secteurs public et parapublic au Québec. v. 36 , no 3,1981, p. $475-498 \quad 0050$ 


\section{SALAIRES (sulte)}

CHRISTENSEN, Sandra

Collective Bargaining in Provincial Public Administration.

v. 36, no 3,1981 , p. $616-629$

0056

MARION, Gérald

La part des salaires dans le revenu national au Canada, 1910 à 1980.

v. 37 , no 1,1982 , p. $53-64$

0097

GILES, Anthony

The Canadian Labour Congress and Tripartism.

v. 37 , no 1,1982 , p. $93-125$

0099

CHOUINARD, Jacques VIEL, André

L'efficacité des clauses d'indexation des salaires

v. 37, no 1, 1982, p. 207-214 0105

COUSINEAU, Jean-Michel

LACROIX, Robert

L'efficacité des clauses d'indexation des salaires: Réplique.

v. 36, no 1,1982 , p. $214-220 \quad 0106$

CHOUINARD, Jacques

ROBICHAUD, Jean-Marc

L'efficacité des clauses d'indexation des salaires.

v. 37, no 1,1982 , p. $220-224 \quad 0107$

MICHAUD, Sharon ROY, Jean-Charles

L'efficacité des clauses d'indexation des salaires: Réplique.

v. 37 , no 1,1982 , p. 224-225 0108

PROULX, Pierre-Paul

Rémunération dans les secteurs public et parapublic au Québec: Éléments d'une nouvelle politique.

v. 37, no 3,1982, p. $477-497 \quad 0136$

AGARWAL, Naresh C.

Male-Female Pay Inequity and Public Policy in Canada and the U.S.

v. 37, no 4,1982 , p. $780-804 \quad 0159$

SIMPSON, Wayne PETERS, Frank The Economics of Mileage Restrictions for Railway Workers in Western Canada. v. 38, no 1, 1983, p. 95-103 0182

\section{SALAIRES (suite)}

ROBB, Roberta Edgecome

Occupational Segregation and Equal Pay for Work of Equal Value.

v. 39 , no 1,1984, p. $146-166$

0275

DEVORETZ, D.J. REED, Clyde G.

Evidence from the Skilled-Unskilled Canadian Wage Index.

v. 39 , no 3,1984, p. $526-537 \quad 0318$

MILNE, William J. ROSS, Thomas W.

The Cyclical Variation of Wage Premiums in the Canadian Manufacturing Industries

v. 39 , no 4,1984 , p. $762-773$

0343

GUNDERSON, Morley

Spline Functions Estimates of the Impact of Equal Pay Législation: The Ontario Experience

v. 40, no 4,1985 , p. $775-792$

0427

OSBERG, Lars

A Note on the Incomes of Lawyers

v. 40 , no 4,1985 , p. $865-879$

0433

LACROIX, Robert

A Microeconometric Analysis of the Effects of Strikes on Wages

v. 41 , no 1,1986 , p. 111-127

0452

EVANS, Martin G. ONDRACK, Daniel A. The Effect of Unionization on Wages: Some Canadian Evidence

v. 41 , no 3,1986 , p. $572-577$

0493

AULD, D.A.L. WILTON, D.A.

The Impact of Public Sector Wage Controls in Ontario

v. 42 , no 1,1987 , p. $132-149$

0544

DOOLEY, Martin D.

Within-Cohort Earnings Inequality Among Canadian Men: 1971-1982

v. 42 , no 3,1987 , p. $594-611$

0587

KUMAR, Pradeep

Recent Wage Deceleration in Canada: Short-run Response or Structural Change? v. 42 , no 4,1987 , p. $687-701 \quad 0604$ 


\section{SALAIRES (sulte)}

COUSINEAU, Jean-Michel RABEAU, Yves Une methodologie de comparaison des salaires pour les emplois spécifiques du secteur public.

v. 43 , no 1,1988 , p. $85-100$

0630

SUBBARAO, A.V.

Criteria in Arbitration of Wage Disputes: Theory and Practice in the Canadian Federal Public Service.

v. 43, no 3,1988, p. $547-570$

0667

STAGER, David A.A.

Lawyers' Earnings in the Canadian Private and Public Sectors.

v. 43 , no 3,1988 , p. $571-590$

0668

RESHEF, Yonatan

Negotiating Wage Settlements: A Structural Approach.

v. 44 , no 3,1989, p. $532-551$

0735

MCSHANE, Steven L. REDEKOP, Bruce Compensation Management and Canadian Wrongful Dismissal: Lessons from Litigation.

v. 45 , no 2,1990, p. $357-381 \quad 0798$

DUSSAULT, Ginette

À travail équivalent, salaire égal: Un droit difficile à appliquer?

43ième Congrès, 1988, p. 83-95 0946

SIMARD, Monique TELLIER, Marie

Commentaires - Le Canadien National: Un cas riche d'enseignements.

43ième Congrès, 1988, p. 114-130 0948

\section{SALARIÉ}

VERGE, Pierre

Syndicalisation de la grève.

v. 38 , no 3,1983 , p. $475-506$

0223

MORIN, Fernand

La survie de droits subjectifs à la conven"tion collective.

v. 40, no 4,1985 , p. $847-855$

0431

AOUST, Claude d' SAINT-JEAN, Sylvain TRUDEAU, Gilles

L'obligation de civilité du salarié

v. 41 , no 1,1986 , p. $157-180$

\section{SALARIÉ (suite)}

MORIN, Fernand

La double personnalité d'un concierge!

v. 41 , no 4,1986 , p. $835-839$

0522

BÉLANGER, Jacques BLOUIN, Rodrigue MORIN, Fernand SEXTON, Jean

Introduction - Le statut de salarié en milieu de travail.

40ième Congrès, 1985, p. 13-15 0887

BLOUIN, Rodrigue

Le statut de salarié en milieu de travail: la problématique.

40ième Congrès, 1985, p. 17-32 0888

GAGNON, Jean-Denis

Les notions de salarié en droit du travail. 40ième Congrès, 1985, p. 33-47 0889

DOUCET, René PARENT, Louise

Commentaires - Les notions de salarié en droit du travail.

40ième Congrès, 1985, p. 48-54 0890

CÔTÉ, André C.

Évolution des conditions de travail des salariés établies d'autorité.

40ième Congrès, 1985, p. 55-69 0891

PIUZE, Guy

Commentaires - Évolution des conditions de travail des salariés établies d'autorité. 40ième Congrès, 1985, p. 69-74 0892

DUCHARME, Claude SIMARD, Monique THIBAULT, Laurent

Table ronde - Le régime actuel de travail des salariés: où en sommes-nous?

40ième Congrès, 1985, p. 121-136 0895

BERNIER, Colette

Évolution du statut du salarié en raison de nouvelles formes d'emploi: L'exemple du travail à temps partiel au Québec.

40ième Congrès, 1985, p. 137-160 0896

DÉOM, Esther

Commentaires - Évolution du statut du salarié en raison des nouvelles formes d'emplois: L'exemple du travail à temps partiel au Québec.

40ième Congrès, 1985, p. 161-170 0897 


\section{SALARIÉ (sulte)}

BÉLANGER, Laurent

Nouvelles formes d'organisation du travail, nouveaux modes de gestion et leur incidence sur le statut du salarié.

40ième Congrès, 1985, p. 171-196 0898

CÔTÉ, Marcel

Commentaires - Nouvelles formes d'organisation du travail, nouveaux modes de gestion et leur incidence sur le statut du salarié.

40ième Congrès, 1985, p. 196-203 0899

BHÉRER, Harold

Le salarié et la gestion générale de l'entreprise.

40ième Congrès, 1985, p. 205-222 0900

GODBOUT, Clément

Commentaires - Le salarié et la gestion générale de l'entreprise.

40ième Congrès, 1985, p. 222-228 0901

BÉLANGER, Jacques

Annexe - La participation des travailleurs aux décisions dans l'entreprise.

40ième Congrès, 1985, p. 245-259 0903

\section{SANCTIONS DISCIPLINAIRES}

AOUST, Claude d'

L'amnistie des fautes disciplinaires.

v. 43 , no 4,1988 , p. $909-942 \quad 0690$

\section{SANTE}

SODERSTROM, Lee

Some Effects of Unemployment on the Health of Unemployed Quebec Workers.

v. 43 , no 2,1988 , p. $341-377$

0650

\section{SANTE AU TRAVAIL}

BRISSON, Chantal

La santé au travail et la convention collective

v. 36 , no 1,1981 , p. $152-178 \quad 0007$

LATULIPPE, Gérard P. O'FARRELL, Kevin Le comité paritaire: Anachronisme ou formule d'avenir.

v. 37, no 3,1982 , p. $634-655 \quad 0143$
SANTÉ AU TRAVAIL (suite)

VINET, Alain BÉGIN, Clermont

THÉRIAULT, Gilles

La santé des employés de bureau: Le cas de la fonction publique québécoise.

v. 37, no 4,1982, p. $887-904 \quad 0164$

BOULARD, René MARCHAND, Michel La loi sur la santé et la sécurité du travail et les conventions collectives.

v. 38 , no 4,1983 , p. $847-86200252$

FISHER, E.G. IVANKOVICH, I.F.

Alberta's Occupational Health and Safety Amendment Act, 1983.

v. 40 , no 1,1985 , p. $115-139 \quad 0368$

BRYCE, George K. MANGA, Pran

The Effectiveness of Health and Safety Committees.

v. 40 , no 2,1985, p. $257-283 \quad 0385$

GEORGE, Kenneth

Les comités de santé et de sécurité du travail: Tables de concertation ou de négociation?

v. 40, no 3,1985, p. $512-528 \quad 0407$

SWIMMER, Gene LUCE, Sally R.

Asbestos Exposure and Attitudes Toward Occupational Health.

v. 40, no 3,1985, p. $529-544 \quad 0408$

CÔTÉ, André C.

L'Acte des manufactures de Québec, 1885: Un centenaire

v. 40, no 3,1985 , p. $623-628 \quad 0412$

PLASSE, Micheline

Santé et sécurité du travail au Québec: Le defi de la concertation patronale - syndicale.

v. 42, no 3,1987, p. $544-565 \quad 0584$

JAIN, Hem C.

AIDS: Need for Policy in the Workplace.

v. 44, no 4,1989 , p. $850-865$

0756

WALTERS, Vivienne DENTON, Margaret Workers' Knowledge of their Legal Rights and Resistance to Hazardous Work.

v. 45 , no 3,1990, p. $531-547 \quad 0815$ 
SANTÉ AU TRAVAIL (sulte)

BLOUIN, Rodrigue BOULARD, René

DESCHÊNES, Jean-Paul

PÉRUSSE, Michel

Introduction - Régimes de santé et sécurité et relations du travail.

39ième Congrès, 1984, p. 15-16 0873

PÉRUSSE, Michel

Régimes de santé et sécurité et relations du travail.

39ième Congrès, 1984, p. 17-31

0874

SIMARD, Marcel

Priorités en santé et sécurité du travail: secteur public et secteur privé.

39ième Congrès, 1984, p. 33-50 0875

LEDUC, Jean-Guy LEMELIN, Maurice VINET, Alain

Commentaires - Priorités en santé et sécurité du travail: secteur public et secteur privé.

39ième Congrès, 1984 , p. $50-72 \quad 0876$

OUELLET, Florian

Concertation et participation: mythe ou réalité?

39ième Congrès, 1984, p. 73-92 0877

DUMAS, Anne-Chantal SIMARD, Monique Commentaires - Concertation et participation: mythe ou realité.

39ième Congrès, 1984, p. 93-111 0878

BOUCHARD, Robert CLÉMENT, Pierre R. GIASSON, Étienne GUILLEMETTE, Michel Table ronde - La santé et la sécurité dans l'entreprise.

39ième Congrès, 1984, p. 113-135 0879

BOULARD, René

L'impact de la Loi sur les conventions collectives.

39ième Congrès, 1984, p. 137-157 0880

DE KONINCK, Maria HEENAN, Roy

Droit de refus et retrait préventif: succès ou échec?

39ième Congrès, 1984, p. 159-175 0881

TURCOTTE, Fernand

La prévention: une utopie?

39ième Congrès, 1984, p. 177-183 0882
SANTÉ AU TRAVAIL (sulte)

DROUIN, Claude PLAMONDON, Denise

Commentaires - La prévention: une utopie?

39ième Congrès, 1984, p. 183-194 0883

BRUNET, Jacques

Santé et sécurité du travail: une affaire de professionne/s?

39ième Congrès, 1984, p. 195-206 0884

BEAUSOLEIL, Gilles

Les coûts et les bénéfices du régime.

39ième Congrès, 1984, p. 207-242 0885

DUFOUR, Ghislain FAVA, Frank

HÉTU, Jean-Paul LABERGE, Louis

LAROSE, Gérald SAUVÉ, Robert

Table ronde - Déceptions et espoirs.

39ième Congrès, 1984, p. 243-276 0886

TRUDEAU, Guy J. OUELLET, Lionel

La comparaison en matière de systèmes de santé et de sécurité du travail.

42ième Congrès, 1987, p. 101-113 0931

FARQUHAR, Alec

Le régime des accidents du travail: Le cas de l'Ontario.

42ième Congrès, 1987, p. 115-130 0932

TOBIN, Edmund LAROSE, Gérald

DUFOUR, Ghislain LABERGE, Louis

Table ronde - Financement de la santé et paritarisme.

42ième Congrès, 1987, p. 253-276 0939

JÉRÓME-FORGET, Monique

La politique québécoise en matière de lésions professionnelles à un point tournant.

42ième Congrès, 1987, p. 277-285 0940

\section{SASKATCHEWAN}

GALLAGHER, Daniel G. WETZEL, Kurt Local Employer and Union Perceptions of Two-Tier Bargaining

v. 39 , no 3, 1984, p. 486-508 0316

BRYCE, George K. MANGA, Pran

The Effectiveness of Health and Safety Committees.

v. 40, no 2,1985 , p. $257-283 \quad 0385$ 
SASKATCHEWAN (sulte)

SASS, Robert

The Saskatchewan Trade Union Act. 1983:

The Public Battle.

v. 40 , no 3,1985, p. 591-622

0411

WETZEL, Kurt GALLAGHER, Daniel G. MAXEY, Charles T.

Impact of Nursing Unionism in the Hospital Industry: The Saskatchewan Experience.

v. 40, no 4,1985, p. $793-809$

0428

ROSE, Joseph B. WETZEL, Kurt

Outcomes of Bargaining Structures in the

Ontario and Saskatchewan Construction Industries.

v. 41 , no 2,1986 , p. $256-280$

0468

OHTSU, Makoto VERMA, Anil Intra-Organizational Bargaining: Wage Differentials Among Saskatchewan Schoolteachers.

v. 42, no 3,1987 , p. $612-627$

0588

NG, Ignace

Determinants of Union Commitment Among University Faculty.

v. 44, no 4,1989 , p. $769-784$

0752

\section{SATISFACTION AU TRAVAIL}

WHITE, Terrence $\mathrm{H}$.

The Relative Importance of Work as a Factor in Life Satisfaction

v. 36 , no 1,1981 , p. $179-191$

0008

LE LOUARN, Jean-Yves

Les professeurs d'université et leur travail. v. 37, no 2,1982 , p. $385-402$

0125

TOUPIN, Louis LESSARD, Claude CORMIER, Roger A. VALOIS, Paul La satisfaction au travail chez les ensei'gnantes et enseignants au Québec.

v. 37 , no 4,1982 , p. $805-826$

0160

BAR-HAIM, Aviad

The Desire for Workers' Participation: Conflicting Forces in the Workplace.

v. 39, no 2,1984, p. $301-312$

0296

LORRAIN, Jean BRUNET, LUC

Climat organisationnel, satisfaction au travail et perception du syndicalisme

v. 39 , no 4,1984 , p. $668-679$

0337
SATISFACTION AU TRAVAIL (suite)

SALES, Carol A. LEVANONI, Eliahu

KNOOP, Robert

Employee Performance as a Function of Job Orientation and Job Design.

v. 44 , no 2,1989 , p. $409-420 \quad 0724$

\section{SATISFACTION DANS LA VIE}

WHITE, Terrence $H$.

The Relative Importance of Work as a

Factor in Life Satisfaction

v. 36 , no 1,1981 , p. $179-191 \quad 0008$

JAMAL, Muhammad

BABA, Vishwanath $V$.

MITCHELL, Vance F.

The Nature of Need Structure in Nonwork.

v. 37, no 3,1982 , p. $618-633 \quad 0142$

\section{SCIENCE DES COMPORTEMENTS}

XISHAN, Yang MOORE, Larry $F$.

MARKGRAF, Herb

Behavioural Science Application in Vancouver Based Firms.

v. 38 , no 1,1983 , p. 120-141 0184

\section{SECRÉTAIRES}

AUDET, Claude VINET, Alain

Bureautique et organisation du travail de secrétariat dans la fonction publique québécoise.

v. 43, no 4,1988, p. $737-756 \quad 0682$

\section{SECRÉTARIATS DES NATIONS UNIES}

BEIGBEDER, Yves

Individual Grievance Procedures in United

Nations Secretariats.

v. 37, no 2,1982 , p. $328-343 \quad 0122$

\section{SECTEUR DES SERVICES}

MAGUN, Sunder

The Rise of Service Employment in the Canadian Economy.

v. 37 , no 3,1982 , p. $528-556 \quad 0138$

\section{SECTEUR PRIVÉ}

CHRISTENSEN, Sandra

Collective Bargaining in Provincial Public Administration.

v. 36, no 3,1981 , p. $616-6290056$

FABI, Bruno

Privé ou public: choix et transfert du secteur organisationnel.

v. 39 , no 2,1984 , p. $313-334$

0297 
SECTEUR PRIVÉ (suite)

AULD, D.A.L. WILTON, D.A.

The Impact of Public Sector Wage Controls in Ontario

v. 42 , no 1,1987, p. $132-149 \quad 0544$

STRATTON, Kay RESHEF, Yonatan

Private Sector Unions and Strategic Planning: A Research Agenda

v. 45 , no 1,1990, p. $76-93$

0775

CHAYKOWSKI, Richard P.

Union and Firm Preferences for Bargaining

Outcomes in the Private Sector.

v. 45 , no 2,1990, p. $326-356$

0797

COUSINEAU, Jean-Michel

NAJEM, Elmustapha

L'effet du développement de la petite entreprise sur l'évolution du syndicalisme au Canada.

v. 45, no 3,1990 , p. $467-480 \quad 0812$

RONDEAU, Claude BÉLANGER, Jacques BOIVIN, Jean SEXTON, Jean

Introduction - La syndicalisation dans le secteur privé au Québec.

38ième Congrès, 1983, p. 13-14 0858

RONDEAU, Claude BÉLANGER, Jacques Le syndicalisme dans l'entreprise: tendances récentes et analyse.

38ième Congrès, 1983, p. 15-36 0859

INGERMAN, Sidney

La syndicalisation dans le contexte économique québécois.

38ième Congrès, 1983, p. 37-69 0860

\section{BONIN, Bernard}

Commentaires - La syndicalisation dans le contexte économique québécois.

38ième Congrès, 1983, p. 70-76

0861

LAFLAMME, Gilles

L'impact du régime de relations du travail sur la syndicalisation.

38ième Congrès, 1983, p. 77-90

0862

LEBEL, Hélène

Commentaires - L'impact du régime de relations du travail sur la syndicalisation.

38ième Congrès, 1983, p. 91-100 0863

\section{SECTEUR PRIVÉ (sulte)}

DESMARAIS, Jacques

Les idées de réforme sur la syndicalisation au Québec depuis 1964.

38ième Congrès, 1983, p. 101-116 0864

MERCILLE, Pierre NADEAU, Bertin

OLIVIER, Madeleine

Table ronde - Organisation syndicale: difficultés et motifs de résistance.

38ième Congrès, 1983, p. 117-1340865

SIMARD, Monique SLIGER, Raymond

Pourquoi et comment accroitre la syndicalisation dans le secteur privé?

38ième Congrès, 1983, p. 135-162 0866

DION, Gérard

Commentaires - Pourquoi et comment accroître la syndicalisation dans le secteur privé?

38ième Congrès, 1983, p. 162-171 0867

PERREAULT, Charles

L'entreprise peut-elle se passer d'un syndicat?

38ième Congrès, 1983 , p. 173-182 0868

GAUTHIER, Fernand ROY, Paul-Martel

Commentaires - L'entreprise peut-elle se passer d'un syndicat?

38ième Congrès, 1983, p. 183-195 0869

FRÉCHETTE, Raynald

La politique gouvernementale en matière de syndicalisation.

38ième Congrès, 1983, p. 197-206 0870

BONENFANT, Claire DUFOUR, Ghislain HÉTU, Jean-Paul

Table ronde - Les réactions du milieu.

38ième Congrès, 1983, p. 207-223 0871

SIMARD, Marcel

Priorités en santé et sécurité du travail: secteur public et secteur privé.

39ième Congrès, 1984, p. 33-50 0875

LEDUC, Jean-GuY LEMELIN, Maurice

VINET, Alain

Commentaires - Priorités en santé et sécurité du travail: secteur public et secteur privé.

39ième Congrès, 1984, p. 50-72 0876 
SECTEURS PUBLIC ET PARAPUBLIC ADAMS, Georges W.

The Ontario Experience with Interest arbitration: Problems in Detecting Policy

v. 36, no 1, 1981, p. 225-250 0012

FERLAND, Gilles

La politique de rémunération dans les secteurs public et parapublic au Quebec.

v. 36, no 3,1981 , p. $475-498$

0050

CHRISTENSEN, Sandra

Collective Bargaining in Provincial Public Administration.

v. 36, no 3,1981 , p. $616-629$

0056

DÉOM, Esther

La négociation collective chez les fonctionnaires et les enseignants québécois: 1975-1976.

v. 37 , no 1. 1982, p. 141-163 0101

BOLDUC, Roch BOUCHARD, Lucien

HÉBERT, Gérard

Le régime québécois de négociation des secteurs public et parapublic

v. 37, no 2,1982, p. $403-430$

0126

PROULX, Pierre-Paul

Rémunération dans les secteurs public et parapublic au Québec: Eléments d'une nouvelle politique.

v. 37 , no 3,1982, p. $477-497$

0136

FABI, Bruno

Prive ou public: choix et transfert du secteur organisationnel.

v. 39 , no 2,1984 , p. $313-334$

0297

KRUGER, Arthur

Collective Bargaining in Ontario Public Hospitals

v. 40 , no 1,1985 , p. $48-67$

0364

\section{MORIN, Fernand}

Rapports collectifs du travail dans les secteurs publics québécois ou le nouvel équilibre selon la loi du 19 juin 1985.

v. 40 , no 3,1985, p. $629-645$

0413

FINKELMAN, Jacob

Public Sector Collective Bargaining

v. 41 , no 4 , 1986, p. $691-703$

0513
SECTEURS PUBLIC ET PARAPUBLIC (suite) AULD, D.A.L. WILTON, D.A.

The Impact of Public Sector Wage Controls in Ontario

v. 42 , no 1,1987 , p. $132-149$

BARRÉ, Alain

Le cadre juridique de la négociation collective dans le secteur public au Québec: une perspective internationale.

v. 42 , no 4,1987 , p. $831-851$

0612

COUSINEAU, Jean-Michel RABEAU, Yves Une méthodologie de comparaison des salaires pour les emplois spécifiques du secteur public.

v. 43 , no 1,1988 , p. $85-100 \quad 0630$

DILTS, David A. KARIM, Ahmad

The Effect of Mediators' Qualities and Strategies on Mediation Outcomes

v. 45 , no 1,1990, p. $22-37 \quad 0772$

SIMARD, Marcel

Priorités en santé et sécurité du travail: secteur public et secteur privé.

39ième Congrès, 1984, p. 33-50 0875

LEDUC, Jean-Guy LEMELIN, Maurice

VINET, Alain

Commentaires - Priorités en santé et sécurité du travail: secteur public et secteur privé.

39ième Congrès, 1984 , p. 50-72 0876

\section{SÉCURITÉ AU TRAVAIL}

BRISSON, Chantal

La santé au travail et la convention collective

v. 36 , no 1,1981 , p. $152-178 \quad 0007$

LATULIPPE, Gérard P. O'FARRELL, Kevin Le comité paritaire: Anachronisme ou formule d'avenir.

v. 37 , no 3,1982 , p. $634-655 \quad 0143$

BOULARD, René MARCHAND, Michel La loi sur la santé et la sécurité du travail et les conventions collectives.

v. 38, no 4,1983 , p. $847-862 \quad 0252$ 
SÉCURITÉ AU TRAVAIL (suite)

LAFLAMME, Lucie ARSENAULT, André

Rémunération, postes de travail et accidents: Une relation interactive.

v. 39 , no 3,1984 , p. $509-525$

0317

FISHER, E.G. IVANKOVICH, I.F.

Alberta's Occupational Health and Safety Amendment Act, 1983.

v. 40 , no 1,1985, p. $115-139$

0368

BRYCE, George K. MANGA, Pran

The Effectiveness of Health and Safety Committees.

v. 40, no 2,1985, p. $257-283$

0385

\section{GEORGE, Kenneth}

Les comités de santé et de sécurité du travail: Tables de concertation ou de négociation?

v. 40 , no 3,1985, p. $512-528$

0407

BRODY, Bernard ROHAN, Paul

ROMPRÉ, Louise

Les accidents industriels au Canada: Le portrait d'une décennie

v. 40 , no 3,1985, p. 545.566

0409

CÓTÉ, André C.

L'Acte des manufactures de Québec, 1885: Un centenaire

v. 40, no 3,1985, p. $623-628$

0412

SIMARD, Marcel BOUTEILLER, Dominique LÉVESQUE, Christian

Prévention des accidents du travail: Contexte législatif québécois et efficacité organisationnelle.

v. 40 , no 4,1985 , p. $703-719 \quad 0424$

\section{PLASSE, Micheline}

Santé et sécurité du travail au Québec: Le defi de la concertation patronale - syndicale.

v. 42 , no 3,1987 , p. $544-565$

0584

LAFLAMME, Lucie VINET, Alain

Accidents du travail et modernisation du processus de production: le cas de lindustrie forestière québécoise.

v. 43 , no 3,1988 , p. 591-608

0669
SÉCURITÉ AU TRAVAIL (suite)

BRODY, Bernard LÉTOURNEAU, Yves

POIRIER, André

Le cout des accidents du travail: Etat des connaissances.

v. 45 , no 1, 1990 , p. $94-117$

0776

WALTERS, Vivienne DENTON, Margaret Workers' Knowledge of their Legal Rights and Resistance to Hazardous Work.

v. 45 , no 3,1990, p. $531-547 \quad 0815$

BLOUIN, Rodrigue BOULARD, René DESCHÊNES, Jean-Paul

PÉRUSSE, Michel

Introduction - Régimes de santé et sécurité et relations du travail.

39ième Congrès, 1984, p. 15-16 0873

PÉRUSSE, Michel

Régimes de santé et sécurité et relations du travail.

39ième Congrès, 1984, p. 17-31 0874

SIMARD, Marcel

Priorités en santé et sécurité du travail: secteur public et secteur privé.

39ième Congrès, 1984, p. 33-50 0875

LEDUC, Jean-Guy LEMELIN, Maurice

VINET, Alain

Commentaires - Priorités en santé et sécurite du travail: secteur public et secteur prive.

39ième Congrès, 1984, p. 50-72 0876

OUELLET, Florian

Concertation et participation: mythe ou réalité?

39ième Congrès, 1984, p. 73-92 0877

DUMAS, Anne-Chantal SIMARD, Monique Commentaires - Concertation et participation: mythe ou realité.

39ième Congrès, 1984, p. 93-111 0878

BOUCHARD, Robert CLÉMENT, Pierre R. GIASSON, Étienne GUILLEMETTE, Michel Table ronde - La santé et la sécurité dans l'entreprise.

39ième Congrès, 1984, p. 113-135 0879 
SÉCUPITÉ AU TRAVAIL (suite)

BOULARD, René

L'impact de la Loi sur les conventions collectives.

39ième Congrès, 1984, p. 137-157 0880

DE KONINCK, Maria HEENAN, Roy

Droit de refus et retrait préventif: succès ou echec?

39ième Congrès, 1984, p. 159-175 0881

BRUNET, Jacques

Santé et sécurité du travail: une affaire de professionnels?

39ième Congrès, 1984, p. 195-206 0884

\section{BEAUSOLEIL, Gilles}

Les couts et les bénéfices du régime.

39ième Congrès, 1984, p. 207-242 0885

DUFOUR, Ghislain FAVA, Frank HÉTU, Jean-Paul LABERGE, Louis LAROSE, Gérald SAUVÉ, Robert Table ronde - Déceptions et espoirs. 39ième Congrès, 1984, p. 243-276 0886

TRUDEAU, GuY J. OUELLET, Lionel La comparaison en matière de systèmes de santé et de sécurité du travail.

42ième Congrès, 1987, p. 101-113 0931

FARQUHAR, Alec

Le régime des accidents du travail: Le cas de l'Ontario.

42ième Congrès, 1987, p. 115-130 0932

TOBIN, Edmund LAROSE, Gérald

DUFOUR, Ghislain LABERGE, Louis

Table ronde - Financement de la santé et paritarisme.

42ième Congrès, 1987, p. 253-276 0939

JÉRÓME-FORGET, Monique

La politique québécoise en matière de lésions professionnelles à un point tournant.

42ième Congrès, 1987, p. 277-285 0940

\section{SÉCURITÉ D'EMPLOI}

MELTZ, Noah $M$.

Job Security in Canada

v. 44 , no 1,1989, p. $149-161$

\section{SÉCURITÉ D'EMPLOI (sulte)}

LOVELL, Maicolm R. Jr.

Employment Security

v. 44 , no 1. 1989 , p. $162-176$

0706

ZAPATA, Francisco

La proteccion del empleo en las américas.

v. 44 , no 1,1989 , p. 177-194

0707

BÉLANGER, Laurent LIPSIG, Carla MORIN, Fernand PÉRUSSE, Michel Introduction - Acquisition ou fusion d'entreprises et emplois.

44ième Congrès, 1989, p. XV-XVI 0956

MORIN, Fernand

Acquisition ou fusion d'entreprises et emplois: La problématique sous-jacente.

44ième Congrès, 1989, p. 1-12 0957

BÉLANGER, MarC LE BRASSEUR, Lola L'TTALIEN, Paul MÉNARD, Marius Rappel de quelques expériences vécues. 44ième Congrès, 1989, p. 13-32 0958

CÓTÉ, André C. FONTAINE, Claude LESAGE, Paul

Aspects et implications juridiques des restructurations.

44ième Congrès, 1989, p. 33-84 0959

ANGERS, Georges GAGNON, Normand SIMARD, Jean

Le droit et le devoir à l'information.

44ième Congrès, 1989, p. 85-100 0960

LAMARCHE, André GAUTHIER, Michel BLAIS, Michel GAGNON, Denise Les conditions de travail au lendemain d'une fusion ou d'une réorganisation.

44ième Congrès, 1989, p. 101-127 0961

GAGNON, Astrid BLANCHETTE, Sylvain QUINTAL, Pierre

Fusion d'entreprises publiques.

44ième Congrès, 1989, p. 129-154 0962

MERCIER, Jacques POULIN-SIMON, Lise Le defi de la gestion des emplois: La problématique.

45ième Congrès, 1990, p. 1-5 0966 


\section{SÉCURITE D'EMPLOI (suite)}

BÉLAND, Claude

Gestion des emplois et sécurité économique des employés.

45ième Congrès, 1990, p. 7-14 0967

\section{BLONDIN, Michel}

Le deffi de la gestion de l'emploi: Pourquoi le relever?

45ième Congrès, 1990, p. 15-23

0968

LEVINE, David

Négocier la flexibilité: Reconversion des heures de la liste de rappel en postes à temps complet.

45ième Congrès, 1990, p. 41-47 0970

MATHIEU, René

Négocier la flexibilité: La recherche d'équité par la négociation.

45ième Congrès, 1990, p. 82-92

LE LOUARN, Jean-Yves

Les emplois atypiques et l'efficacité de la gestion des ressources humaines.

45ième Congrès, 1990, p. 93-103 0976

BOUDREAULT, Pierre GARON, Jacques PAGÉ, Lorainne PAQUETTE, Pierre

Les emplois atypiques et l'efficacité de la gestion des ressources humaines - Commentaires.

45ième Congrès, 1990, p. 103-116 0977

\section{SÉCURITÉ SYNDICALE}

AUCLAIR, Robert

Geneve ignore Québec en matière de vocabulaire

v. 36 , no 1,1981 , p. $223-224$

0011

SAINT-GERMAIN, Catherine

Décisions rendues par le Conseil canadien des relations du travail

v. 41, no 3,1986, p. $622-637$

0497

\section{SÉLECTION DES OFFICIERS}

TZINER, Aharon DOLAN, Shimon $L$.

Identifying Female Officer Potential: An Exploration in Predictors Payoff

v. 40 , no 1,1985 , p. $87-98$

\section{SÉLECTION DU PERSONNEL}

JAIN, Harish C.

Recruitment of Racial Minorities in Canadian Police Forces.

v. 42 , no 4,1987 , p. $790-805 \quad 0610$

SINGH, Jang B. CROCKER, Olga

Operative and Espoused Personnel Selection Criteria of Managers.

v. 43 , no 1, p. 167-182

0635

\section{SENTENCES ARBITRALES}

AOUST, Claude d'

Les dispositifs d'une sentence arbitrale: quelques pièges à eviter.

v. 38, no 2,1983, p. $415-417 \quad 0210$

MORIN, Fernand

Le "raisonnable" déraisonnablel ou la rationalité du raisonnable

v. 40 , no 3,1985 , p. $646-658$

0414

MORIN, Fernand

Le rationnel et le raisonnable: Deux nécessités distinctes et conjugées en droit.

v. 41 , no 3,1986 , p. $\mathbf{5 7 8 - 5 8 2}$

0494

\section{SERVICE DE MÉDIATION PRÉVENTIVE}

DULUDE, Yves

Le conflit: la gestion au banc des accusés.

41ième Congrès, 1986, p. 111-1330914

LAMARCHE, Pierre

Commentaires - Le conflit: la gestion au banc des accusés.

41ième Congrès, 1986, p. 133-136 0915

\section{LALANDE, Serge}

L'expérience de la compagnie Gaz Métropolitain.

41ième Congrès, 1986, p. 137-146 0916

\section{SERVICE DE PLACEMENT}

MAGUN, Sunder

The Placement Activity of the Canadian Employment Agency.

v. 38 , no 1,1983 , p. $72-94 \quad 0181$ 
Relajions Industrielles, vol. 45, no 4 (1990)

\section{SERVICES}

MAGUN, Sunder

The Rise of Service Employment in the Canadian Economy.

v. 37 , no 3,1982 , p. $528-556$

0138

\section{SERVICES ESSENTIELS}

FASHOYIN, Tayo

State Regulation of Trade Disputes in Essential Services in Nigeria

v. 36 , no 1,1981 , p. 207-222

0010

SUBBARAO, A.V.

Impasse Choice in the Canadian Federal Service: An Innovation and an Intrigue

v. 40, no 3,1985, p. $567-590$

0410

\section{BARRÉ, Alain}

Le cadre juridique de la négociation co/lective dans le secteur public au Québec: une perspective internationale.

v. 42, no 4,1987, p. $831-851$

0612

\section{SERVICES LÉGAUX}

OSBERG, LarS

A Note on the Incomes of Lawyers

v. 40, no 4,1985, p. $865-879$

0433

STAGER, David A.A.

Lawyers' Earnings in the Canadian Private and Public Sectors.

v. 43, no 3,1988, p. $571-590 \quad 0668$

\section{SEXISME}

SWIMMER, Gene

Gender Based Differences in Promotions of Clerical Workers.

v. 45 , no 2,1990 , p. $300-310$

0795

\section{SIDA}

JAIN, Hem C.

AIDS: Need for Policy in the Workplace.

v. 44 , no 4,1989 , p. $850-865$

0756

\section{SIMULATION DE NÉGOCIATION}

GILSON, Clive H.J.

Bargaining Exercises: Beyond Simulation, a Touch of Reality.

v. 41 , no 2,1986 , p. $390-396$

0476

\section{SOCIALISATION}

AOUST, Claude d' SAINT-JEAN, Sylvain

TRUDEAU, Gilles

L'obligation de civilité du salarié

v. 41 , no 1,1986 , p. $157-180$

\section{SOCIÉTÉ CANADIENNE DES POSTES}

SAINT-GERMAIN, Catherine

Décisions rendues par le Conseil canadien des relations du travail.

v. 41, no 4,1986 , p. $860-868$

0525

TREMBLAY, Johane

Décisions rendues par le Conseil canadien des relations du travail.

v. 42 , no 4,1987 , p. $852-861$

0613

MARLEAU, Véronique L.

Décisions rendues par le Conseil canadien des relations du travail.

v. 44, no 3,1989, p. $703-722$

0744

WRIGHT, Michael D.

The Legislation of Labour Relations at Canada Post.

v. 44 , no 4,1989 , p. $866-883$

0757

MARLEAU, Véronique $L$.

Décision rendue par le Conseil canadien des relations du travail.

v. 45 , no 2,1990, p. $414-423 \quad 0801$

\section{SOCIÉTÉS D'ÉTAT}

SAINT-GERMAIN, Catherine

Décision rendue par le Conseil canadien des relations du travail.

v. 42, no 3, 1987, p. 628-635 0589

\section{SOCIÉTÉS MULTINATIONALES}

PECCEI, Ricardo WARNER, Malcolm

Industrial Relations, Strategic Importance, and Decision - Making.

v. 36, no 1,1981 , p. 132-151 0006

\section{SOLLICITATION SYNDICALE}

LAMY, Francine

Décisions rendues par le Conseil cana-

dien des relations du travail.

v. 40, no 2,1985, p. $386-398 \quad 0394$

\section{SOUS-TRAITANCE}

BEAULIEU, LUC

Décisions rendues par le Conseil canadien des relations du travail.

v. 39 , no 3,1984 , p. $605-618 \quad 0323$

GODIN, Michel

Négocier la flexibilité: La sous-traitance. 45ième Congrès, 1990, p. 59-64 0972 


\section{STATISTIQUE CANADA}

KUMAR, Pradeep

Estimates of Unionism and Collective Bargaining in Canada.

v. 43, no 4,1988, p. $757-779$

0683

\section{STATISTIQUES}

MELTZ, Noah M.

Labour Market Information in Canada: The Current Situation and a Proposal.

v. 37 , no 2,1982 , p. $431-437$

0127

\section{STATUT MATRIMONIAL}

WALSH, William D.

A Time Series Analysis of Female Labour Force Participation Rates Disaggregated by Marital Status.

v. 37 , no 2,1982, p. $367-384$

0124

\section{STELCO}

STOREY, Robert $\mathrm{H}$.

The Struggle to Organize Stelco and Dofasco

v. 42, no 2,1987, p. $366-385$

0566

\section{STRATÉGIES D'ENTREPRISE}

MILLER, Roger

La stratégie d'entreprise et la gestion des ressources humaines.

v. 40 , no 1.1985 , p. $68-86$

0365

WILS, Thierry LABELLE, Christiane GUÉRIN, Gilles LE LOUARN, Jean-Yves La gestion stratégique des ressources humaines: Un reniement du role social de l'entreprise?

v. 44 , no 2,1989, p. $354-375$

0721

\section{STRATÉGIES D'INVESTIGATION}

GAGNON, Yves-Chantal

LANDRY, Maurice

Les changements technologiques: Une stratégie d'étude exploratoire

v. 44 , no 2,1989 , p. $421-447$

0725

\section{STRATÉGIES PATRONALES}

THÉRIAULT, Roland LESAGE, Pierre B. BOISVERT, Maurice

L'absenteisme: importance, nature et remèdes.

v. 36, no 4,1981 , p. $775-802$
STRATEGIES PATRONALES (suite)

BROUILET, Normand

Négocier la flexibilité: Réaction syndicale aux stratégies patronales.

45ième Congrès, 1990, p. 70-81 0974

\section{STRATÉGIES SYNDICALES}

BROSSARD, Michel

Revendications, stratégie syndicale et organisation du travail dans l'entreprise.

v. 36 , no 3,1981 , p. $552-575 \quad 0053$

BROSSARD, Michel

La stratégie syndicale face aux groupes semi-autonomes de production: Hypothèse pour l'analyse du cas Rushton.

v. 37, no 3,1982 , p. $670-683 \quad 0145$

\section{STRESS}

LÉONARD, Christine

VAN AMÉRINGEN, Marie-Reine

DOLAN, Shimon L. ARSENAULT, André Absentéisme et assiduité au travail: Deux moyens d'adaptation au stress.

v. 42 , no 4,1987, p. $774-789 \quad .0609$

\section{STRUCTURE ORGANISATIONNELLE}

MILLER, Roger

Les formes d'organisation dans l'industrie de la construction au Québec.

v. 37, no 1, 1982, p. 164-176 0102

PEPERMANS, Raymond

Line, staff et functional

v. 43 , no 1,1988 , p. $183-187 \quad 0636$

\section{STRUCTURE SYNDICALE}

GILSON, Clive H.J. SPENCER, lan S.

Trade Union Growth: A Marketing Model.

v. 42 , no 4,1987 , p. $756-773 \quad 0608$

\section{STYLES DE GESTION}

CAMIRÉ, André

La productivité des intelligences.

v. 43 , no 2,1988, p. $322-340$

0649

\section{SUCCESSION, DROITS DE}

LAVERY, Daniel

Décisions rendues par le Conseil cana-

dien des relations du travail.

v. 38 , no 1, 1983, p. 159-173 0187 


\section{SUPERVISEURS}

KNIGHT, Thomas R.

Correlates of Informal Grievance Resolution Among Fist-Line Supenvisors

v. 41 , no 2,1986 , p. $281-298$

0469

\section{SYNDICALISATION}

PONAK, Allen MOORE, Larry F.

Canadian Bank Unionism: Perspectives and Issues

v. 36 , no 1,1981, p. 3-34

0001

LAMY, Francine

Décisions rendues par le Conseil canadien des relations du travail.

v. 40 , no 2,1985, p. $386-398$

0394

BÉLANGER, Jacques MERCIER, Jacques Le plafonnement de la densité syndicale au Québec et au Canada.

v. 41 , no 1,1986, p. $28-52$

0448

EVANS, Martin G. ONDRACK, Daniel A. The Effect of Unionization on Wages: Some Canadian Evidence

v. 41 , no 3,1986, p. $572-577$

0493

GEORGE, Kenneth

$L$ 'accès à la syndicalisation

v. 41 , no 3,1986 , p. $583-595$

0495

RONDEAU, Claude BÉLANGER, Jacques BOIVIN, Jean SEXTON, Jean

Introduction - La syndicalisation dans le secteur privé au Québec.

38ième Congrès, 1983, p. 13-14 0858

RONDEAU, Claude BÉLANGER, Jacques Le syndicalisme dans l'entreprise: tendances récentes et analyse.

38ième Congrès, 1983, p. 15-36 0859

INGERMAN, Sidney

La syndicalisation dans le contexte économique québécois.

38ième Congrès, 1983, p. 37-69 0860

BONIN, Bernard

Commentaires - La syndicalisation dans le contexte économique québécois.

38 ième Congrès, 1983, p. 70-76

\section{SYNDICALISATION (suite)}

LAFLAMME, Gilles

L'impact du régime de relations du travail sur la syndicalisation.

38ièmo Congrès, 1983, p. 77-90 0862

\section{LEBEL, Hélène}

Commentaires - L'impact du régime de relations du travail sur la syndicalisation.

38ième Congrès, 1983, p. 91-100 0863

DESMARAIS, Jacques

Les idées de réforme sur la syndicalisation au Québec depuis 1964.

38ième Congrès, 1983, p. 101-116 0864

MERCILLE, Pierre NADEAU, Bertin

OLIVIER, Madeleine

Table ronde - Organisation syndicale: difficultés et motifs de résistance.

38ième Congrès, 1983, p. 117-134 G865

SIMARD, Monique SLIGER, Raymond

Pourquoi et comment accroitre la syndicalisation dans le secteur privé?

38ième Congrès, 1983, p. 135-162 0866

DION, Gérard

Commentaires - Pourquoi et comment accroitre la syndicalisation dans le secteur privé?

38ième Congrès, 1983, p. 162-171 0867

\section{PERREAULT, Charles}

L'entreprise peut-elle se passer d'un syndicat?

38ième Congrès, 1983, p. 173-182 0868

GAUTHIER, Fernand ROY, Paul-Martel

Commentaires - L'entreprise peut-elle se passer d'un syndicat?

38ième Congrès, 1983, p. 183-195 0869

\section{FRÉCHETTE, Raynald}

La politique gouvernementale en matière de syndicalisation.

38ième Congrès, 1983, p. 197-206 0870

BONENFANT, Claire DUFOUR, Ghislain HÉTU, Jean-Paul

Table ronde - Les réactions du milieu.

38ième Congrès, 1983, p. 207-223 0871 


\section{SYNDICALISME}

BROSSARD, Michel

Revendications, stratégie syndicale et organisation du travail dans l'entreprise.

v. 36, no 3,1981 , p. $552-575$

0053

GRUBEL, Herbert G. MAKI, Dennis R. A Note on the Effects of Unemployment Insurance, Minimum Wage Legislation and Trade Union Growth on Reported Unemployment Rates in Canada, 1950-75

v. 36, no 4,1981 , p. $922-927$

0079

LORRAIN, Jean BRUNET, LuC

Climat organisationnel, satisfaction au travail et perception du syndicalisme

v. 39, no 4,1984 , p. $668-679$

0337

MCSHANE, Steven L.

Sources of Attitudinal Union Militancy

v. 40 , no 2,1985, p. $284-302$

0386

WETZEL, Kurt GALLAGHEA, Daniel G. MAXEY, Charles T.

Impact of Nursing Unionism in the Hospital Industry: The Saskatchewan Experience.

v. 40 , no 4,1985 , p. $793-809 \quad 0428$

\section{BARKIN, Solomon}

The Current Unilateralist Counterattack on Unionism and Collective Bargaining

v. 41, no 1,1986, p. 3-27

0447

BARBASH, Jack

Like Nature, Industrial Relations Abhors a Vaccum: The Case of the Union-Free Strategy.

v. 42 , no 1,1987 , p. $168-179 \quad 0546$

GILSON, Clive H.J. SPENCER, lan S. Trade Union Growth: A Marketing Model. v. 42, no 4,1987 , p. $756-773$

0608

FALABELLA, Gonzalo

El sindicato en el ano 2000 temas, y desafios.

v. 44, no 1,1989, p. $47-61$

0701

NG, Ignace

Determinants of Union Commitment Among University Faculty.

v. 44 , no 4,1989 , p. $769-784$

0752
SYNDICALISME (suite)

GILSON, Clive H.J. SPENCER, lan S.

GRANVILLE, S.

The Impact of a Strike on the Attitudes and Behavior of a Rural Community.

v. 44, no 4, 1989, p. 785-804

0753

CAIRE, Guy

Syndicalisme, nouvelles technologies et incertitudes économiques.

38ième Congrès, 1983, p. 225-265 0872

DOCQUIEA, Gérard

Ressources humaines et défis du syndicalisme.

41ième Congrès, 1986, p. 149-156 0917

FRANCOEUR, Jean

Le syndicalisme et le nouveau travail.

41 ième Congrès, 1986, p. 157-166 0918

\section{SYNDICALISME AMÉRICAIN}

BARBASH, Jack

The New Industrial Relations in the US: Phase II.

v. 43, no 1,1988, p. $32-42 \quad 0627$

RESHEF, Yonatan

Changing Environments and Management IR Practices: Implications for U.S. Trade Unions.

v. 43 , no 1,1988 , p. $43-62 \quad 0628$

BARKIN, Solomon

Institutional Economics and the American Trade Union Movement.

v. 43 , no 3,1988 , p. $491-508 \quad 0664$

FREEMAN, Richard B.

What Does the Future Hold for U.S. Unio"nism?

v. 44 , no 1,1989 , p. $25-46 \quad 0700$

\section{SYNDICALISME BRITANNIQUE}

POOLE, Michael

Perceptions of Trade Union Members and the Social Action Perspective.

v. 36, no 1, 1981, p. 35-62 0002

BEAUMONT, P.B.

Third Party Conciliation and Trade Union Recognition: Some British Evidence.

v. 37 , no 4,1982 , p. $827-842 \quad 0161$ 
Relations Industrielles, vol. 45 , no 4 (1990)

SYNDICALISME BRITANNIQUE (suite)

BEAUMONT, P.B.

Statutory Recognition Provisions in Britain, 1976-80

v. 38 , no 4,1983 , p. $\mathbf{7 4 4 - 7 6 6}$

0247

BEAUMONT, P.B. HARRIS, R.I.D.

High Technology Industries and Non-Union

Establishments in Britain.

v. 43 , no 4,1988 , p. $819-846$

0686

SMITH, Anthony $E$.

Innovation by Negotiation: Case Studies Among British White-Collar Unions.

v. 45 , no 1,1990 , p. $63-75$

0774

\section{SYNDICALISME CANADIEN}

TUCK, Hugh

Canadian Railways and Unions in the Running Trades, 1865-1914

v. 36 , no 1,1981 , p. 106-131

0005

CHAISON, Gary N. ROSE, Joseph B. The Structure and Growth of the Canadian National Unions.

v. 36 , no 3,1981 , p. $530-551$

0052

LOWE, Graham S.

Causes of Unionization in Canadian Banks. v. 36 , no 4,1981 , p. $865-893$

0077

AOUST, Claude d' DELORME, François The Origin of the Freedom of Association and of the Right to Strike in Canada: An Historical Perspective.

v. 36 , no 4,1981, p. $894-921 \quad 0078$

CHAISON, Gary N. ANDIAPPAN, P.

Characteristics of Female Union Officer in Canada.

v. 37 , no 4,1982 , p. $765-779$

0158

MAKI, Dennis R.

Political Parties and Trade Union Growth in Canada.

v. 37 , no 4,1982 , p. $876-886$

0163

EASTMAN, Byron

Canadian Union Growth

v. 38 , no 1,1983, p. $58-71$

0180

ADAMS, Roy $\mathrm{J}$.

The Extent of Collective Bargaining in Canada

v. 39, no 4,1984, p. $655-667$

0336
SYNDICALISME CANADIEN (suite)

JONES, J.C.H. LAUDADIO, L.

Organized Labour, Regional Political Bias and the Canadian Tariff Structure

v. 39 , no 4,1984 , p. $695-709 \quad 0339$

BENEDICT, Daniel

The 1984 GM Agreement in Canada: Significance and Consequences.

v. 40 , no 1,1985, p. $27-47$

0363

KUMAR, Pradeep DOW, Bradley

Econometric Analysis of Union Membership Growth in Canada, 1935-1981

v. 41, no 2,1986, p. $236-255$

0467

HÉBERT, Gérard

L'évolution du syndicalisme au Canada: Comment un mouvement devient institution.

v. 42 , no 3,1987 , p. $500-519 \quad 0581$

SEFTON MACDOWELL, Laurel

The Career of a Canadian Trade Union Leader: C.H. Millard 1937-1946

v. 43 , no 3,1988 , p. $609-632$

0670

KUMAR, Pradeep

Estimates of Unionism and Collective Bargaining in Canada.

v. 43, no 4,1988 , p. $757-7790683$

MAC NEIL, Michael

Unions, Politics and Law in Canada.

v. 43 , no 4,1988, p. $847-868 \quad 0687$

DION, Gérard HÉBERT, Gérard

L'avenir du syndicalisme au Canada.

v. 44 , no 1,1989 , p. $5-24$

0699

COUSINEAU, Jean-Michel

NAJEM, Elmustapha

L'effet du développement de la petite entreprise sur l'evolution du syndicalisme au Canada.

v. 45 , no 3,1990 , p. $467-480 \quad 0812$

PÉPIN, Marcel

L'actualisation du mouvement syndical.

43ième Congrès, 1988, p. 209-221 0952 
SYNDICALSME CANADIEN (suite)

GAGNON, Mona-Josée LEBEAU, Ernest JOHNSTON, Raymond

Commentaires - l'actualisation du mouvement syndical.

43ième Congrès, 1988, p. 222-236 0953

\section{SYNDICALISME FRANÇAIS}

DION, Stéphane

Syndicats et politique au niveau municipal en France.

v. 39 , no 3. 1984 , p. $466-485$

0315

\section{SYNDICALISME GREC}

KATASANEVAS, Theodoros

Trade Unions in Greece

v. 40, no 1,1985, p. $99-114$

0367

\section{SYNDICALISME INDUSTRIEL}

STOREY, Robert $\mathrm{H}$.

The Struggle to Organize Stelco and Dofasco

v. 42 , no 2,1987, p. $366-385$

0566

\section{SYNDICALISME INTERNATIONAL}

WILLIAMS, C. Brian

The Impact of Labor Migration: The International Molders and Allied Workers Union in Canada, 1860-1885.

v. 39 , no 2,1984, p. $335-364$

0298

\section{WILLIAMS, C. Brian}

International Trade Unionism: The United Mine Workers in Eastern Canada, 19001920

v. 41 , no 3,1986 , p. $519-540 \quad 0490$

WILLIAMS, C. Brian

International Unionism: The Papermakers

in Eastern Canada, 1930-1945

v. 42 , no 1, 1987, p. 110-131

0543

\section{SYNDICALISME NORD-AMÉRICAIN}

LIPSIG-MUMME, Carla

La crise du syndicalisme nord-américain: éléments d'interprétation.

v. 39, no 2,1984, p. $275-284$

\section{SYNDICALISME QUÉBÉCOIS}

\section{ROBAKC, Léo}

Les travailleurs du Québec au XXième siecle.

v. 40, no 4,1985, p. $826-846$

0430

\section{SYNDICALISME QUÉBÉCOIS}

DÉOM, Esther

La syndicalisation des journalistes dans quelques quotidiens québécois.

v. 42 , no 4,1987 , p. $737-755$

0607

MARCHAND, Jean

Les rapports collectifs du travail: Rétrospective et perspective.

40ième Congrès, 1985, p. 229-244 0902

LABERGE, Louis

Les préalables à une réorientation des relations du travail au Québec.

41ième Congrès, 1986, p. 173-175 0920

LAROSE, Gérald

L'adaptation du syndicalisme - un phénomène de continuité.

41ième Congrès, 1986, p. 177-179 0921

\section{SYNDICATS}

WEISS, Dimitri

Politique, entreprise, syndicats

v. 36 , no 1, 1981, p. $192-206$

0009

WILLIAMS, C. Glyn

Joan Robinson on Unions, Distribution and Inflation.

v. 36, no 3,1981 , p. $576-588 \quad 0054$

JAIN, Hem C.

Disclosure of Corporate Information to Trade Unions in North America.

v. 36 , no 4,1981 , p. $748-774 \quad 0072$

MCPHILLIPS, David C.

Duty of Fair Representation: Recent Attitudes in British Columbia and Ontario.

v. 36 , no 4,1981 , p. $803-827$

0074

LAVERY, Daniel

Décisions rendues par le Conseil canadien des relations du travail.

v. 37, no 1, 1982, p. 226-234 0109

LAVERY, Daniel

Décisions rendues par le Conseil canadien des relations du travail.

v. 37 , no 2,1982 , p. $438-450 \quad 0128$ 


\section{SYNDICATS (suite)}

WILLIAMS, C. Glyn

The Role of Unions in Inflation: A Survey Article

v. 37 , no 3,1982 , p. $498-527 \quad 0137$

DELORME, François LAFRANCE, Victor La concurrence dans un contexte de pluralisme syndical: Quelques données sur le Québec.

v. 37, no 3, 1982, p. 575-605 0140

BROSSARD, Michel

La strategie syndicale face aux groupes semi-autonomes de production: Hypothèse pour l'analyse du cas Rushton.

v. 37, no 3,1982 , p. $670-683$

0145

PEITCHINIS, Stephen G.

The Attitude of Trade Unions Towards Technological Changes.

v. 38, no 1, 1983, p. 104-119 0183

LAVERY, Daniel

Décisions rendues par le Conseil canadien des relations du travail.

v. 38 , no 1,1983 , p. $159-173$

MAKI, Dennis R.

Trade Unions and Productivity: Conventional Estimates.

v. 38 , no 2,1983 , p. $211-228$

0201

VERGE, Pierre

Syndicalisation de la grève.

v. 38 , no 3,1983 , p. $475-506$

BARKIN, Solomon

Troubled Worker Militancy: Challenges Confronting Western Industrial Relations Systems.

v. 38 , no 4,1983 , p. $713-729$

0245

MOUNT, Joan SIEGEL, Jacob $P$.

Resources and Results in Union Rivaly: $A$

Case Study

v. 38 , no 4,1983 , p. $815-830$

KRAHN, Harvey LOWE, Graham S. Community Influences on Attitudes Towards Unions.

v. 39 , no 1,1984, p. $93-113$

0273

\section{SYNDICATS (suite)}

BEAULIEU, LUC

Décisions rendues par le Conseil canadien des relations du travail

v. 39 , no 2,1984 , p. $\mathbf{3 7 1 - 3 8 5}$

0300

VERGE, Pierre

Évolution de la protection juridictionnelle de l'accès au syndicat.

v. 39 , no 4,1984 , p. $710-743$

0340

MORIN, Fernand

Nouvelle présence de l'État dans les relations du travail.

v. 39, no 4,1984, p. $744-752$

0341

EASTMAN, Byron

A Catastrophe Theory on Union Behaviour. v. 40 , no 2,1985 , p. $340-350 \quad 0389$

\section{SASS, Robert}

The Saskatchewan Trade Union Act. 1983.

The Public Battle.

v. 40 , no 3,1985, p. $591-622$

0411

LAMY, Francine

Décision rendue par le Conseil canadien des relations du travail

v. 40 , no 4,1985 , p. $880-885 \quad 0434$

MAKI, Dennis R. MEREDITH, Lindsay N. The Effects of Unions on Profitability: Canadian Evidence

v. 41 , no 1,1986 , p. $54-68$

0449

THACKER, James W. ROSEN, Hjalmar Dynamics of Employee Reactance to Company and Union Dual Allegiance Revisited and Expanded.

v. 41 , no i, 1986, p. 128-144 0453

SAINT-GERMAIN, Catherine

Décisions rendues par le Conseil canadien des relations du travail

v. 42 , no 2,1987 , p. $415-427$

0569

OHTSU, Makoto VERMA, Anil

Intra-Organizational Bargaining: Wage Differentials Among Saskatchewan Schoolteachers.

v. 42 , no 3,1987 , p. $612-627 \quad 0588$ 


\section{SYNDICATS (suite)}

KNIGHT, Thomas R.

The Role of The Duty of Fair Representation in Union Grievance Decisions.

v. 42 , no 4,1987 , p. $716-736$

0606

TREMBLAY, Johane

Décisions rendues par le Conseil canadien des relations du travail.

v. 43 , no 1,1988 , p. $188-195$

0637

TEMPLER, Andrew SOLOMON, Norman A.

Unions and Technology: A Survey of Union Use of Information Technology.

v. 43 , no 2,1988 , p. $378-393$

0651

CUNNINGHAM, Banton HULL, Dennis A Union Member's Perspective on Technological Change.

v. 43, no 2,1988, p. $394-411$

0652

MORIN, Fernand

D'un entrepreneur à un autre: l'accréditation ne suit pas

v. 44 , no 2,1989 , p. $315-336$

0719

CLARKE, Graham

Décisions rendues par le Conseil canadien des relations du travail.

v. 44, no 4,1989 , p. $933-946$

0762

STRATTON, Kay RESHEF, Yonatan Private Sector Unions and Strategic Planning: A Research Agenda

v. 45, no 1,1990, p. $76-93$

0775

CHAYKOWSKI, Richard P

Union and Firm Preferences for Bargaining Outcomes in the Private Sector.

v. 45, no 2,1990, p. $326-356$

0797

CUNNINGHAM, Barton MITCHELL, Lari Privatization in British Columbia: What the Experts Say Will Happen.

v. 45, no 2,1990 , p. $382-403$

0799

DULUDE, Yves

Le conflit: la gestion au banc des accu"sés.

41ième Congrès, 1986, p. 111-133 0914
SYNDICATS (suite)

LAMARCHE, Pierre

Commentaires - Le conflit: la gestion au banc des accusés.

41ième Congrès, 1986, p. 133-136 0915

HÉTU, Jean-Paul

La nouvelle gestion des ressources humaines - mythe ou réalite?

41 ième Congrès, 1986, p. 167-172 0919

DUFOUR, Ghislain LABERGE, Louis

LAROSE, Gérald

Table ronde - Les Chartes impliquent-elles un réalignement des politiques syndicales et patronales?

43ième Congrès, 1988, p. 255-272 0955

GOSSELIN, Alain FRENETTE, Jean-Guy DIONNE, Denis

Les acteurs d'une fusion ou d'une prise de pouvoir.

44ième Congrès, 1989, p. 155-173 0963

\section{SYNDICATS INDUSTRIELS}

COPP, Terry

The Rise of Industrial Unions in Montreal 1935-1945

v. 37 , no 4,1982 , p. $843-8750162$

\section{SYNDICATS NATIONAUX}

SOLOMON, Norman A. ANDIAPPAN, $P$. SHAND, Dan

Canadian National Union Presidents: An Empirical Study.

v. 41 , no 3,1986 , p. $491-504 \quad 0488$

\section{SYNDROMEIMMUNO-DÉFICITAIREACQUIS} JAIN, Hem C.

AIDS: Need for Policy in the Workplace.

v. 44 , no 4,1989 , p. $850-865$

0756

\section{SYSTÈME DE RELATIONS DU TRAVAIL} LAROUCHE, Viateur DÉOM, Esther L'approche systémique en relations industrielles

v. 39 , no 1,1984, p. $114-145$

0274

SHIROM, Arie

The Labor Relations System: A Proposed Conceptual Framework

v. 40 , no 2,1985, p. $303-323$

0387 
Relaijons Industrielles, vol. 45, no 4 (1990)

SYSTEME DE RELATIONS DU TRAVAIL (suite)

ADAMS, Roy J.

Employment Standards in Ontario: An industrial Relations Systems Analysis.

v. 42 , no 1,1987, p. $46-64$

0540

BARBASH, Jack

Like Nature, Industrial Relations Abhors a Vaccum: The Case of the Union-Free Strategy.

v. 42 , no 1,1987 , p. $168-179 \quad 0546$

\section{SYSTÈMES D'AUTORITÉ}

RAINVILLE, Jean-Marie

La responsabilité fonctionnelle chez les cadres subalternes: Facteurs d'evolution des systèmes d'autorité.

v. 38, no 4,1983 , p. $831-846$

0251

\section{SYSTÈMES SOCIO-TECHNIQUES}

MATON, BOb

Socio-Technical Systems: Conceptual and Implementation Problems

v. 43 , no 4,1988 , p. $869-8890688$

\section{SYSTÈMES, THÉORIE DES}

DIMMOCK, Stuart J. SETHI, Amarjit S.

The Role of Ideology and Power in Systems Theory: Some Fundamental Shortcomings.

v. 41 , no 4,1986 , p. $738-757$

0516

\section{FAJANA, Sola}

The Systems Approach as Theory for Muttinational Industrial Relations in Developing Countries.

v. 44 , no 3,1989 , p. $615-634$

0739

\section{$\mathbf{T}$}

\section{TÂCHES}

SALES, Carol A. LEVANONI, Eliahu KNOOP, Robert

Employee Performance as a Function of Job Orientation and Job Design.

v. 44, no 2,1989 , p. $409-420$

0724

\section{TAMISAGE, THÉORIE DU}

ROHLING, Thomas A.

Screening and Human Capital Theory: An Empirical Test

v. 41 , no 4,1986 , p. $817-826$

0520

\section{TARIFICATION}

JONES, J.C.H. LAUDADIO, L.

Organized Labour, Regional Political Bias and the Canadian Tariff Structure

v. 39 , no 4,1984 , p. $695-709 \quad 0339$

\section{TAYLORISME}

MATON, Bob

Socio-Technical Systems: Conceptual and Implementation Problems

v. 43 , no 4,1988 , p. $869-8890688$

\section{TECHNOLOGIE}

JAIN, Harish C.

Micro-electronics Technology and Industrial Relations.

v. 38 , no 4,1983 , p. $869-879 \quad 0254$

MERCIER, Jean

L'informatique, ses "filtres" et ses effets.

v. 41 , no 2,1986 , p. 299-316

0470

PROULX, Pierre-Paul

Maîtriser la technologie: pourquoi, quelles technologies, comment?

v. 41 , no 2,1986 , p. $382-389$

0475

TEMPLER, Andrew SOLOMON, Norman A.

Unions and Technology: A Survey of Union Use of Information Technology.

v. 43 , no 2,1988 , p. $378-393$

0651

ZUREIK, Elia MOSCO, Vincent

LOCHHEAD, Clarence

Telephone Workers' Reaction to the New Technology

v. 44 , no 3,1989 , p. $507-531 \quad 0734$

CÔTÉ, André C.

Nouvelles technologies et droit du travail au Canada.

v. 44, no 4,1989 , p. $751-768$

0751

FRAPPIER-DESROCHERS, Monique

La technologie: un substitut à la durée du travail?

36ième Congrès, 1981, p. 193-218 0843

CAIRE, Guy

Syndicalisme, nouvelles technologies et incertitudes économiques.

38ième Congrès, 1983, p. 225-265 0872 


\section{TÉLÉCOMMUNICATIONS}

ZUREIK, Elia MOSCO, Vincent

LOCHHEAD, Clarence

Telephone Workers' Reaction to the New

Technology

v. 44, no 3,1989 , p. $507-531$

0734

\section{TÉLÉCOMMUNICATIONS (INDUSTRIE)} CUNNINGHAM, Barton HULL, Dennis A Union Member's Perspective on Technological Change.

v. 43 , no 2,1988 , p. $394-411 \quad 0652$

\section{TÉLÉMATIQUE}

MERCIER, Jean

L'informatique, ses "filtres" et ses effets.

v. 41 , no 2,1986, p. 299-316

0470

\section{TÉLÉPHONIE}

ZUREIK, Elia MOSCO, Vincent

LOCHHEAD, Clarence

Telephone Workers' Reaction to the New

Technology

v. 44 , no 3,1989 , p. $507-531$

0734

\section{TEMPS DE TRAVAIL}

BOULARD, René DESCHÊNES, Jean-Paul LAROCQUE, Alain RONDEAU, Claude Introduction - La réduction de la durée du travail.

36ième Congrès, 1981, p. 13-14 0832

\section{LAROCQUE, ALAIN}

La réduction du temps de travail: un droit ou un privilège?

36ième Congrès, 1981, p. 15-29 0833

VALASKAKIS, Kimon

Le temps liberé: à quels couts?

36ième Congrès, 1981, p. 61-74

0836

POULIN-SIMON, Lise FORTIN, Bernard Commentaires - Le temps liberé: à quels coûts?

36ième Congrès, 1981, p. 75-86 0837

FERLAND, Gilles HAMELIN, Jean-Marie PROULX, Pierre-Paul

Table ronde - Sommes-nous tous égaux face à la diminution du temps passé au travail?

36ième Congrès, 1981 , p. $87-1210838$
TEMPS DE TRAVAIL (suite)

MASSE, Gilles

Le temps passé au travail: un élément encore négociable?

36ième Congrès, 1981, p. 123-135 0839

DESCHÊNES, Jean-Paul

Commentaires - Le temps passé au travail: un élément encore négociable?

36ième Congrès, 1981, p. 136-144 0840

BEAUSOLEIL, Gilles GAUTHIER, Hervé

La législation: consolidation ou innovation?

36ième Congrès, 1981 , p. 145-172 0841

LOCKWELL, Luc M. LORTIE, Pierre

Commentaire - La législation: consolidation ou innovation?

36ième Congrès, 1981, p. 173-192 0842

FRAPPIER-DESROCHERS, Monique

La technologie: un substitut à la durée du travail?

36ième Congrès, 1981, p. 193-218 0843

AOUST, Fernand d' DÉOM, André

HARVEY, Pierre

Table ronde - La réduction du temps passé au travail: un moyen de lutte contre le chómage.

36ième Congrès, 1981, p. 219-246 0844

CROZIER, Michel

La diminution du temps de travail: un phénomène inéluctable?

36ième Congrès, 1981, p. 247-263 0845

\section{TEMPS PARTIEL}

SARTIN, Pierrette

Le travail à temps partiel

v. 36 , no 2,1981, p. $307-324 \quad 0025$

PUPO, Norene DORIS DUFFY, Ann

The Ontario Labour Relations Board and the Part Time Workers.

v. 43, no 3,1988, p. $660-6850672$

LANGLOIS, Simon

Le travail à temps partiel: Vers une polarisation de plus en plus nette.

v. 45 , no 3,1990, p. $548-5650816$ 
TEMPS PARTIEL (sulte)

BERNIER, Colette

Evolution du statut du salarié en raison de nouvelles formes d'emploi: L'exemple du travail à temps partiel au Québec.

40ième Congrès, 1985, p. 137-160 0896

DÉOM, Esther

Commentaires - Evolution du statut du salarie en raison des nouvelles formes d'emplois: L'exemple du travail à temps partiel au Québec.

40ième Congrès, 1985, p. 161-170 0897

GOBEILLE, Kenneth R.

Négocier la flexibilité: Le travail à temps partiel.

45ième Congrès, 1990, p. 48-58 0971

\section{TEMPS SUPPLÉMENTAIRE}

KAMEL, Nawal ROY, Paul-Martel

Temps supplémentaire et création d'emplois: Le cas du Canada, du Québec et de l'Ontario.

v. 43, no 2,1988, p. $412-430$

0653

\section{TERMINOLOGIE}

WEISS, Dimitri

Politique, entreprise, syndicats

v. 36 , no 1,1981 , p. $192-206$

0009

AUCLAIR, Robert

Genève ignore Québec en matière de vocabulaire

v. 36 , no 1,1981 , p. $223-224$

0011

WEISS, Dimitri

Qu'est-ce que les relations industrielles?

v. 36 , no 2,1981 , p. $412-413$

0032

PEPERMANS, Raymond

Line, staff et functional

v. 43 , no 1,1988, p. $183-187$

0636

GAGNON, Jean-Denis

Les notions de salarié en droit du travail. 40ième Congrès, 1985, p. 33-47 0889

DOUCET, René PARENT, Louise

Commentaires - Les notions de salarié en droit du travail.

40ième Congrès, 1985, p. 48-54 0890
TEXTILES (INDUSTRIE)

SIMARD, Marcel BOUTEILLER, Dominique LÉVESQUE, Christian

Prévention des accidents du travail: Contexte législatif québécois et efficacité organisationnelle.

v. 40 , no 4,1985 , p. $703-719 \quad 0424$

\section{THÉORIE DE LA CATASTROPHE}

EASTMAN, Byron

A Catastrophe Theory on Union Behaviour.

v. 40, no 2,1985, p. $340-350 \quad 0389$

THÉORIE DE RECHERCHE

CHAISON, Gary N.

A Note on the Critical Dimensions of the Union Merger Process.

v. 37, no 1,1982 , p. 198-206 0104

\section{THÉORIE DES BESOINS}

JAMAL, Muhammad

BABA, Vishwanath $V$.

MITCHELL, Vance F.

The Nature of Need Structure in Nonwork.

v. 37, no 3, 1982, p. 618-633 0142

\section{THÉORIE DES ORGANISATIONS}

GAGNON Yves-Chantal

LANDRY, Maurice

Les changements technologiques: Une stratégie d'étude exploratoire

v. 44, no 2,1989, p. $421-447 \quad 0725$

THÉORIE DES RELATIONS INDUSTRIELLES HAMEED, Syed M.A.

A Critique of Industrial Relations Theory.

v. 37 , no 1,1982 , p. $15-31 \quad 0095$

AUDET, Michel LAROUCHE, Viateur Paradigmes, écoles de pensée et théories en relations industrielles.

v. 43 , no 1,1988 , p. 3-31 0626

GILES, Anthony MURRAY, Grégor

Towards an Historical Understanding of Industrial Relations Theory in Canada.

v. 43 , no 4,1988 , p. $780-811 \quad 0684$

HÉBERT, Gérard

L'état de la discipline en relations industrielles au Canada: Un brin d'histoire et une postface./The State of the Art in Industrial Relations: A Brief History and a Postcript.

v. 44 , no 4,1989 , p. $884-904 \quad 0758$ 
THÉORIE DES RELATIONS INDUSTRIELLES (suite)

BARKIN, Solomon

The State of the Art in Industrial Relations:

A U.S. Commentary.

v. 44 , no 4,1989 , p. $905-914$

0759

WEISS, Dimitri

L'état de la discipline en relations industrielles au Canada: Un commentaire européen.

v. 44 , no 4,1989 , p. $914-920$

0760

\section{THÉORIE DES SYSTĖMES}

LAROUCHE, Viateur DÉOM, Esther

L'approche systémique en relations industrielles

v. 39 , no 1,1984 , p. $114-145$

0274

SHIROM, Arie

The Labor Relations System: A Proposed Conceptual Framework

v. 40, no 2,1985, p. $303-323$

0387

DIMMOCK, Stuart J. SETHI, Amarjit S.

The Role of Ideology and Power in Systems Theory: Some Fundamental Shortcomings.

v. 41 , no 4,1986, p. $738-757 \quad 0516$

FAJANA, Sola

The Systems Approach as Theory for Multinational Industrial Relations in Developing Countries.

v. 44 , no 3,1989 , p. $615-634$

0739

\section{THÉORIE DU POUVOIR}

HAMEED, Syed M.A. SEN, Joya

A Power Theory of Third Party Intervention in Labour Management Relations.

v. 42 , no 2,1987, p. $243-255$

0559

\section{THÉORIE DU TAMISAGE}

ROHLING, Thomas A.

Screening and Human Capital Theory: An Empirical Test

v. 41 , no 4,1986 , p. $817-826 \quad 0520$

\section{THÉORIE Z}

\section{MROCZKOWSKI, Tomasz}

Theory Z: Myths, Realities and Alternatives.

v. 38 , no 2,1983 , p. $297-318$

0205
THĖSES DE MAîTRISE EN RELATIONS INDUSTRIELLES

LECLERC, Claudine

Thèses de maîtrise en relations industrielles - Université Laval et Université de Montréal: 1940-1980

v. 36, no 2, 1981, p. $435-448 \quad 0036$

\section{TIERS MONDE}

BLANCHARD, Francis

Les relations professionnelles et les défis des années 80.

v. 37 , no 1,1982 , p. 3-14 0094

\section{TORONTO}

COUSINEAU, Jean-Michel

LACROIX, Robert

Le climat des relations du travail à Montréal et à Toronto

v. 38 , no 4,1983 , p. $730-743 \quad 0246$

\section{TRAITEMENT DE TEXTE}

AUDET, Claude VINET, Alain

Bureautique et organisation du travail de secrétariat dans la fonction publique québécoise.

v. 43, no 4,1988 , p. $737-756 \quad 0682$

\section{TRANSFERTS}

VEILLEUX, Diane

Les clauses de promotion et de transfert chez les infirmières et les infirmiers.

v. 38 , no 2,1983 , p. 229-253 0202

TRAVAIL

WHITE, Terrence $\mathrm{H}$.

The Relative Importance of Work as a Factor in Life Satisfaction

v. 36 , no 1,1981 , p. $179-191 \quad 0008$

VINET, Bernard

Le problème de la retraite.

v. 36, no 4,1981 , p. $828-847$

0075

LE LOUARN, Jean-Yves

Les professeurs d'université et leur travail. v. 37 , no 2,1982 , p. 385-402 0125

JAMAL, Muhammad

BABA, Vishwanath $V$.

MITCHELL, Vance F.

The Nature of Need Structure in Nonwork.

v. 37 , no 3,1982, p. 618-633 0142 
TRAVAIL (suite)

LAROUCHE, Viateur TRUDEL, Johanne

La qualité de vie au travail et lhoraire variable.

v. 38 , no 3,1983 , p. $568-597$

0227

SAUNDERS, George

Employment and the Productivity Slowdown: 1958-1980

v. 40, no 2,1985 , p. $219-242$

0383

AOUST, Claude d' SAINT-JEAN, Sylvain TRUDEAU, Gilles

L'obligation de civilité du salarié

v. 41 , no 1,1986, p. $157-180$

0455

BROSSARD, Michel SIMARD, Marcel Problématique de la différenciation de la main-d'oeuvre et changementorganisationnel.

v. 41 , no 2,1986 , p. $219-2350466$

BÉLANGER, Jacques

Le controle ouvrier sur l'organisation du travail: Étude de cas en Grande-Bretagne. v. 41, no 4, 1986, p. 704-719 0514

COOK, Alice $\mathrm{H}$.

Family and Work: Challenger to Labor, Management and Government

v. 42, no 3,1987, p. $520-527$

0582

LÉONARD, Christine

VAN AMÉRINGEN, Marie-Reine

DOLAN, Shimon L. ARSENAULT, André

Absentéisme et assiduité au travail: Deux moyens d'adaptation au stress.

v. 42 , no 4,1987, p. $774-789$

0609

LONG, Richard J.

Patterns of Workplace Innovation in Canada.

v. 44 , no 4,1989 , p. $805-826$

0754

MONTMINY, Jean-Paul

La signification du travail en 1980: émergence de valeurs nouvelles?

36ième Congrès, 1981, p. 31-45 0834

DUFOUR, Ghislain RODRIGUE, Norbert Commentaires - La signification du travail en 1980: émergence de valeurs nouvelles?

36ième Congrès, 1981, p. 45-59 0835
TRAVAIL (sulte)

MASSE, Gilles

Le temps passé au travail: un élément encore négociable?

36ième Congrès, 1981, p. 123-135 0839

FRANCOEUR, Jean

Le syndicalisme et le nouveau travail.

41ième Congrès, 1986, p. 157-166 0918

\section{TRAVAIL A DOMICILE}

LIPSIG-MUMME, Carla

The Renaissance of Homeworking in Developped Economies.

v. 38 , no 3,1983 , p. $545-567 \quad 0226$

GRANT, Michel ROSE, Ruth

Encadrement du travail à domicile dans l'industrie du vêtement au Québec.

v. 40 , no 3,1985 , p. $473-494$

0405

\section{TRAVAIL À TEMPS PARTIEL}

SARTIN, Pierrette

Le travail à temps partiel

v. 36 , no 2,1981 , p. $307-324$

0025

PUPO, Norene DORIS DUFFY, Ann

The Ontario Labour Relations Board and the Part Time Workers.

v. 43 , no 3,1988 , p. $660-685$

0672

LANGLOIS, Simon

Le travail à temps partiel: Vers une polarisation de plus en plus nette.

v. 45 , no 3,1990 , p. $548-565 \quad 0816$

BERNIER, Colette

Évolution du statut du salarié en raison de nouvelles formes d'emploi: L'exemple du travail à temps partiel au Québec.

40ième Congrès, 1985, p. 137-160 0896

DÉOM, Esther

Commentaires - Évolution du statut du salarié en raison des nouvelles formes d'emplois: L'exemple du travail à temps partiel au Québec.

40ième Congrès, 1985, p. 161-170 0897

GOBEILLE, Kenneth R.

Négocier la flexibilité: Le travail à temps partiel.

45ième Congrès, 1990, p. 48-58 0971 


\section{TRAVAIL DANGEREUX}

WALTERS, Vivienne DENTON, Margaret Workers' Knowledge of their Legal Rights and Resistance to Hazardous Work.

v. 45 , no 3, 1990, p. 531-547 0815

TRAVAIL DE BUREAU

AUDET, Claude VINET, Alain

Bureautique et organisation du travail de secrétariat dans la fonction publique québécoise.

v. 43, no 4,1988 , p. $737-756$

0682

HUGUES, Karen D.

Office Automation: A Review of the Litterature

v. 44 , no 3,1989 , p. $654-679$

0741

TRAVAIL, DURÉE DU

JOLVET, Thierry

La réduction de la durée du travail estelle créatrice d'emploi?

v. 38 , no 1,1983 , p. $142-154 \quad 0185$

BOULARD, René DESCHÊNES, Jean-

-Paul

LAROCQUE, Alain RONDEAU, Claude Introduction - La réduction de la durée du travail.

36ième Congrès, 1981, p. 13-14 0832

LAROCQUE, ALAIN

La réduction du temps de travail: un droit ou un privilège?

36ième Congrès, 1981, p. 15-29 0833

VALASKAKIS, Kimon

Le temps libéré: à quels coûts?

36ième Congrès, 1981, p. 61-74

0836

POULIN-SIMON, Lise FORTIN, Bernard Commentaires - Le temps libéré: à quels coûts?

36ième Congrès, 1981 , p. 75-86 0837

FERLAND, Gilles HAMELIN, Jean-Marie PROULX, Pierre-Paul

Table ronde - Sommes-nous tous égaux face à la diminution du temps passé au travail?

36ième Congrès, 1981, p. 87-121 0838

DESCHÊNES, Jean-Paul

Commentaires - Le temps passé au travail: un élément encore négociable?

36ième Congrès, 1981, p. 136-144 0840
TRAVAIL, DURÉE DU (sulte)

BEAUSOLEIL, Gilles GAUTHIER, Hervé

La législation: consolidation ou innovation?

36ième Congrès, 1981, p. 145-172 0841

LOCKWELL, LUC M. LORTIE, Pierre

Commentaire - La législation: consolidation ou innovation?

36ième Congrès, 1981, p. 173-192 0842

FRAPPIER-DESROCHERS, Monique

La technologie: un substitut à la durée du travail?

36ième Congrès, 1981, p. 193-218 0843

AOUST, Fernand d' DÉOM, André

HARVEY, Pierre

Table ronde - La réduction du temps passé au travail: un moyen de lutte contre le chómage.

36ième Congrès, 1981, p. 219-246 0844

CROZIER, Michel

La diminution du temps de travail: un phénomène inéluctable?

36 ième Congrès, 1981, p. 247-263 0845

\section{TRAVAIL ÉQUIVALENT}

DUSSAULT, Ginette

A travail équivalent, salaire égal: Un droit difficile à appliquer?

43ième Congrès, 1988, p. 83-95 0946

\section{TRAVAIL PARTAGÉ}

CROWLEY, R.W. HUTH, E.

An International Comparison of Work Sharing Programs.

v. 38 , no 3,1983 , p. $636-647 \quad 0230$

\section{TRAVAILLEURS}

VANDAMME, François

L'information des travailleurs dans les grandes firmes: L'approche spécifique de la CEE.

v. 39 , no 1,1984 , p. $51-76$

0271

BAR-HAIM, Aviad

The Desire for Workers' Participation: Conflicting Forces in the Workplace.

v. 39 , no 2,1984 , p. $301-312$

0296 


\section{TRAVAILLEURS (sulte)}

SWIMMER, Gene LUCE, Sally $R$.

Asbestos Exposure and Attitudes Toward Occupational Health.

v. 40 , no 3,1985 , p. $529-544$

0408

DICKINSON, John A.

La législation et les travailleurs québécois, 1894-1914

v. 41 , no 2,1986 , p. $357-381$

0474

WALTERS, Vivienne DENTON, Margaret Workers' Knowledge of their Legal Rights and Resistance to Hazardous Work.

v. 45 , no 3, 1990 , p. $531-547 \quad 0815$

WILS, Thierry

Les travailleurs seront-ils du troisième type?

41ième Congrès, 1986, p. 99-109 0913

\section{TRAVAILLEURS ÂGÉS}

GUÉRIN, Gilles HÉBERT, Michel

Les obstacles rencontrés par des personnes de 45 à 64 ans à la recherche d'un emploi.

v. 45, no 2,1990, p. $235-267$

0792

LEBRASSEUR, Rolland

Retirement and Skill Issues in Northern Ontario Industries.

v. 45 , no 2,1990, p. $268-282$

0793

\section{TRAVAILLEURS FORESTIERS}

LAFLAMME, Lucie VINET, Alain

Accidents du travail et modernisation du processus de production: le cas de l'industrie forestière québécoise.

v. 43 , no 3,1988 , p. $591-608$

0669

\section{TRAVAILLEURS NON QUALIFIÉS}

DEVORETZ, D.J. REED, Clyde G.

Evidence from the Skilled-Unskilled Canadian Wage Index.

v. 39 , no 3,1984 , p. 526-537

0318

\section{TRAVAILIEURS QUALIFIÉS}

DEVORETZ, D.J. REED, Clyde G.

Evidence from the Skilled-Unskilled Canadian Wage Index.

v. 39, no 3,1984, p. $526-537$

0318
TRIBUNAL DES PROFESSIONS

JOSEPH, P.A.

Perfecting the Administrative Solution to Labour Disputes.

v. 38 , no 4,1983 , p. $863-868 \quad 0253$

TRIBUNAL DU TRAVAIL DU QUÉBEC

PLANTE, Gilles

La permission d'appel au tribunal du travail

v. 41, no 4,1986, p. $817-834 \quad 0521$

MORIN, Fernand

La double personnalité d'un concierge!

v. 41 , no 4,1986 , p. $835-8390522$

\section{TRIBUNAUX D'ARBITRAGE}

FISHER, E.G. SHERWOOD, L.M.

Fairness and Managerial Rights in Canadian Arbitral Jurisprudence

v. 39 , no 3,1984 , p. $538-552 \quad 0319$

MORIN, Fernand

Le "raisonnable" déraisonnable! ou la rationalité du raisonnable

v. 40 , no 3,1985 , p. $646-658 \quad 0414$

AOUST, Claude d'

Le contróle judiciaire des décisions arbitrales: Réflexions sur les moyens d'y échapper.

v. 41 , no 2,1986 , p. $348-356 \quad 0473$

MORIN, Fernand

Le rationnel et le raisonnable: Deux nécessités distinctes et conjugées en droit.

v. 41 , no 3,1986, p. $578-582$

0494

\section{TRIBUNAUX DU TRAVAIL}

GARANT, Patrice

Statut et pouvoirs des organismes du travail en regard des Chartes.

43ième Congrès, 1988, p. 237-254 0954

\section{TRIPARTISME}

GILES, Anthony

The Canadian Labour Congress and Tripartism.

v. 37, no 1,1982 , p. 93-125 0099

ADAMS, Roy J.

The Federal Government and Tripartism.

v. 37 , no 3,1982 , p. $606-617$

0141 


\section{U}

\section{UNITED PAPERMAKERS AND PAPERWOR- KERS}

WILLIAMS, C. Brian

International Unionism: The Papermakers in Eastern Canada, 1930-1945

v. 42 , no 1,1987, p. $110-131$

0543

\section{UNITÉS D'ACCRÉDITATION}

LAVERY, Daniel

Décisions rendues par le Conseil canadien des relations du travail.

v. 37, no 3,1982, p. $684-696$

LAVERY, Daniel

Décisions rendues par le Conseil canadien des relations du travail.

v. 38 , no 3,1983 , p. $658-666$

0231

\section{UNITÉS DE NÉGOCIATION}

SWIDINSKI, Robert

The Effect of Bargaining Structure on $\mathrm{Ne}$ gotiated Wage Settlements.

v. 36 , no 2,1981 , p. $371-385$

0029

FORREST, Anne

Bargaining Units and Bargaining Power

v. 41 , no 4,1986, p. $840-850$

0523

OHTSU, Makoto VERMA, Anil Intra-Organizational Bargaining: Wage Differentials Among Saskatchewan Schoolteachers.

v. 42, no 3,1987, p. $612-627$

0588

MCPHILLIPS, David C.

The Appropriate Bargaining Unit: The Need for Policy Consistency by Canadian Labour Boards.

v. 43 , no 1,1988, p. $63-84$

0629

MARLEAU, Véronique L.

Décisions rendues par le Conseil canadien des relations du travail.

v. 44 , no 2,1989 , p. $448-462$

0726

\section{CLARKE, Graham}

Décision rendue par le Conseil canadien des relations du travail.

v. 45 , no 3,1990, p. $616-624$

0820

\section{UNIVERSITÉ DE LA SASKATCHEWAN}

NG, Ignace

Determinants of Union Commitment Among University Faculty.

v. 44 , no 4,1989, p. $769-784 \quad 0752$

\section{UNIVERSITÉ DE MONTRÉAL}

LECLERC, Claudine

Thèses de maitrise en relations industrie/les - Université Laval et Université de Montréal: 1940-1980

v. 36, no 2,1981 , p. $435-448 \quad 0036$

LE LOUARN, Jean-Yves

Les professeurs d'université et leur travail. v. 37, no 2, 1982, p. 385-402 0125

HÉBERT, Gérard

In memoriam: Émile Bouvier, S.J. 19061985

v. 40 , no 2,1985 , p. $211-212 \quad 0381$

LACOSTE, Paul

L'École des relations industrielles de l'Université de Montréal: Quarantième anniversaire.

v. 40 , no 2,1985 , p. $213-2180382$

\section{UNIVERSITÉ LAURENTIENNE}

MOUNT, Joan SIEGEL, Jacob P.

Resources and Results in Union Rivalry: $A$ Case Study

v. 38 , no 4,1983 , p. $815-830 \quad 0250$

\section{UNIVERSITÉ LAVAL}

LECLERC, Claudine

Thèses de maitrise en relations industrielles - Université Laval et Université de Montréal: 1940-1980

v. 36, no 2,1981 , p. $435-448 \quad 0036$

HÉBERT, Gérard

Distinction 1982 de l'Association canadienne des relations industrielles: Gérard Dion.

v. 37 , no 3,1982 , p. $475-476 \quad 0135$

THWAITES, James LAJOIE, Mario

BOIS-BROCHU, Hélène

Supplément - Quarante ans au service des relations industrielles

40ième Congrès, 1985, p. 261-281 0904 
UNIVERSITÉ MCGILL

GOLDENBERG, Shirley B.

HÉBERT, Gérard

Harry Douglas Woods, 1907-1983

v. 38 , no 4,1983 , p. $707-712$

0244

\section{UNIVERSITÉ QUEEN}

KELLY, Laurence

Industrial Relations at Queen's: The First

Fifty Years.

v. 42 , no 3,1987, p. $475-499 \quad 0580$

\section{UNIVERSITÉS}

GARNIER, Bernard

La gestion des conflits interpersonnels en milieu universitaire.

v. 38, no 2,1983 , p. $277-296$

0204

PONAK, Allen THOMPSON, Mark

Faculty Collective Bargaining: The Voice of Experience

v. 39, no 3,1984, p. $449-465$

0314

\section{USINES DE FRUITS DE MER}

LAMSON, Cynthia

On the Line: Women and Fish Plant Jobs in Atlantic Canada.

v. 41 , no 1,1986 , p. $145-156$

0454

\section{V}

\section{VALEURS}

BOURGEOIS, Robert-Paul WILS, Thierry Career Concepts, Personality and Values of Some Canadian Workers: An Exploratory Study

v. 42 , no 3,1987, p. $528-543$

0583

\section{VALEURS DU TRAVAIL}

MONTMINY, Jean-Paul

La signification du travail en 1980: émergence de valeurs nouvelles?

36ième Congrès, 1981, p. 31-45 0834

DUFOUR, Ghislain RODRIGUE, Norbert Commentaires - La signification du travail en 1980: émergence de valeurs nouvelles?

36ième Congrès, 1981, p. 45-59 0835

\section{VANCOUVER}

XISHAN, Yang MOORE, Larry F.

MARKGRAF, Herb

Behavioural Science Application in Vancouver Based Firms.

v. 38 , no 1,1983 , p. $120-141$

RICHARDS, John MAUSER, Gary

HOLMES, Richard

What Do Workers Want? Attitudes Towards Collective Bargaining and Participation in Management.

v. 43, no 1,1988 , p. $133-152 \quad 0633$

\section{VENTE D'ENTREPRISE}

LAVERY, Daniel

Decisions rendues par le Conseil canadien des relations du travail.

v. 36 , no 4,1981 , p. $928-936 \quad 0080$

BEAULIEU, LUC

Décisions rendues par le Conseil canadien des relations du travail.

v. 38 , no 4,1983 , p. $880-903 \quad 0255$

POTHIER, Dianne

Décisions rendues par le Conseil canadien des relations du travail.

v. 39 , no 4,1984 , p. $774-782 \quad 0344$

SAINT-GERMAIN, Catherine

Décision rendue par le Conseil canadien des relations du travail.

v. 42 , no 1,1987 , p. 203-209 0549

TREMBLAY, Johane

Décisions rendues par le Conseil canadien des relations du travail.

v. 42 , no 4,1987 , p. $852-861 \quad 0613$

MARLEAU, Véronique L.

Décisions rendues par le Conseil canadien des relations du travail.

v. 44, no 3,1989, p. 703-722 0744

\section{VÊTEMENT (INDUSTRIE)}

GRANT, Michel ROSE, Ruth

Encadrement du travail à domicile dans l'industrie du vêtement au Québec.

v. 40, no 3,1985, p. $473-494 \quad 0405$ 
VÊTEMENT (INDUSTRIE) (sulte) VINET, Alain VÉZINA, Michel BRISSON, Chantal

Des lésions professionnelles méconnues: le cas des operatrices de lindustrie du vêtement.

42ième Congrès, 1987, p. 21-38

0925

VIE HORS-TRAVAIL

WHITE, Terrence $\mathrm{H}$.

The Relative Importance of Work as a Factor in Life Satisfaction

v. 36 , no 1,1981 , p. 179-191 0008

JAMAL, Muhammad BABA, Vishwanath V. MITCHELL, Vance F.

The Nature of Need Structure in Nonwork. v. 37, no 3, 1982, p. 618-633 0142

HACCOUN, Robert R. DUPONT, Serge Une analyse des comportements de travailleurs masculins et féminins selon deux formes d'absence au travail.

v. 43, no 1,1988 , p. $153-166$

\section{VOCABULAIRE}

WEISS, Dimitri

Politique, entreprise, syndicats

v. 36 , no 1,1981 , p. $192-206$

0009

AUCLAIR, Robert

Genève ignore Québec en matière de vocabulaire

v. 36, no 1,1981, p. $223-224$

0011

WEISS, Dimitri

Qu'est-ce que les relations industrielles? v. 36 , no 2,1981, p. $412-413$

0032

PEPERMANS, Raymond

Line, staff et functional

v. 43 , no 1,1988, p. $183-187$

0636

GAGNON, Jean-Denis

Les notions de salarié en droit du travail. 40ième Congrès, 1985, p. 33-47 0889

DOUCET, René PARENT, Louise

Commentaires - Les notions de salarié en droit du travail.

40ième Congrès, 1985, p. 48-54 0890

\section{VULGARISATION}

BEAUMONT, P.B.

The Diffusion of Human Resource Management Innovations.

v. 40, no 2,1985, p. $243-256 \quad 0384$

\section{W}

\section{WINNIPEG}

KRAHN, Harvey LOWE, Graham S.

Community Influences on Attitudes Towards Unions.

v. 39 , no 1,1984, p. $93-113$

0273

WOODS, H.D.

GOLDENBERG, Shirley $B$.

HÉBERT, Gérard

Harry Douglas Woods, 1907-1983

v. 38 , no 4,1983 , p. 707-712

0244

ARTHURS, H.W.

Understanding "Understanding": Industrial Relations Research and Policy in Canada from 1969 to 1984... and Beyond.

v. 39 , no 4,1984 , p. $753-761$

0342 


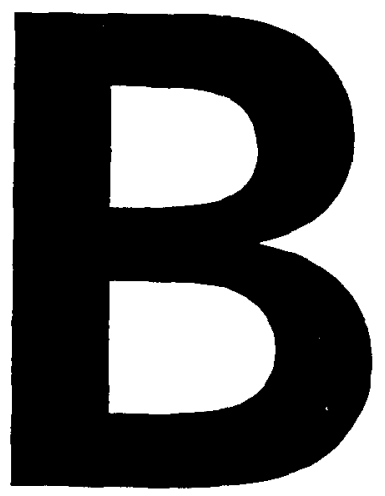

\section{INDEX MÉTHODIQUE DES ARTICLES BROAD SUBJECT HEADINGS INDEX}


L'Index méthodique groupe les articles analysés sous des descripteurs génériques correspondant aux divisions du domaine des relations industrielles. La liste de ces descripteurs apparaît au début de cette section.

\section{EXEMPLE:}

MAIN-D'OEUVRE - QUÉBEC $\quad \begin{aligned} & \text { Descripteur } \\ & \text { générique }\end{aligned}$

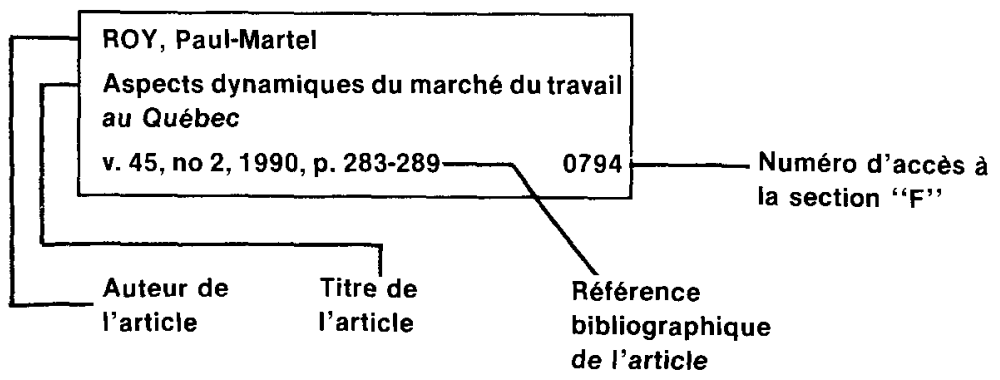


In this Index, articles are arranged under "Broad Subject Headings", the list of which is given at the beginning of this Section. The Broad Subject Headings correspond to main topics in Industrial Relations.

EXAMPLE:

MAIN-D'OEUVRE Broad subject Heading

\section{HUGUES, Karen D.}

Office Automation: A Review of the Literature

v. 44 , no 3,1989 , p. $654-679$ 0741

Author of

Title of the article

Bibliographical reference of the article 



\section{LISTE DES DESCRIPTEURS GÉNÉRIQUES}

LIST OF GENERIC KEYWORDS

DROIT DU TRAVAIL

ÉCONOMIQUE DU TRAVAIL

FEMMES ET TRAVAIL

GESTION DES ORGANISATIONS

GESTION DES RESSOURCES HUMAINES

MAIN-D'OEUVRE

MÉTHODOLOGIE

ORGANISATIONS PATRONALES

ORGANISATIONS SYNDICALES

RELATIONS DU TRAVAIL

SANTÉ ET SÉCURITÉ AU TRAVAIL

SÉCURITÉ SOCIALE

SOCIOLOGIE DU TRAVAIL

SYSTĖMES DES RELATIONS

INDUSTRIELLES

\author{
LABOUR LAW \\ LABOUR ECONOMICS \\ WOMEN AND WORK \\ MANAGEMENT OF ORGANIZATIONS \\ HUMAN RESOURCES MANAGEMENT \\ MANPOWER \\ METHODOLOGY \\ EMPLOYERS' ORGANIZATIONS \\ UNION ORGANIZATIONS \\ LABOUR RELATIONS \\ OCCUPATIONAL HEALTH AND SAFETY \\ SOCIAL SECURITY \\ SOCIOLOGY OF WORK \\ INDUSTRIAL RELATIONS SYSTEMS
}

\section{SUJETS COUVERTS PAR LES DESCRIPTEURS GÉNÉRIQUES}

DESCRIPTION OF CONTENTS OF EACH GENERIC KEYWORD

\begin{abstract}
DROIT DU TRAVAIL
Regroupe les articles présentant ou traitant de

- nomenclature, évolution, caractère et analyse critique des principales lois réglementant les relations du travail;

- tribunaux et organisations administratives chargés de l'application des lois;

- décisions, arrêts et sentences des cours de justice en matière de relations du travail.
\end{abstract}

\section{ÉCONOMIQUE DU TRAVAIL}

Regroupe des articles où les auteurs font appel, dans leur analyse, aux principes de l'économique du travail, la micro- et la macro-économique.

\section{LABOUR LAW}

Includes articles on

- nomenclature, evolution, characteristics, and critical analyses of the main labour law regulating labour relations;

- tribunals and administrative bodies emplementing the law;

- decisions and judgments rendered by the courts of justice and boards on industrial relations matters.

\section{LABOUR ECONOMICS}

Includes articles on labour economics, micro and macro-economics. 
Relations lndustrielles, vol. 45, no 4 (1990)

FEMMES ET TRAVAIL

Regroupe les articles traitant des femmes au travail en ce qui a trait à:

- l'emploi et le marché du travail;

- l'égalité;

- la discrimination, etc.

\section{GESTION DES ORGANISATIONS}

Regroupe les articies concernant tous les aspects de la gestion des organisations

- structure organisationnelle;

- prise de décision;

- culture organisationnelle;

- théorie de l'organisation, etc.

\section{GESTION DES RESSOURCES HUMAINES}

Regroupe des articles concernant

- le recrutement, la sélection et l'accueil;

- la formation et le développement;

- l'évaluation;

- la rémunération;

- la satisfaction et la motivation au travail, etc.

\section{MAIN-D'OEUVRE}

Regroupe les articles portant sur

- le marché du travail;

- le chômage et le nlein emploi;

- la composition de la main-d'ouvre;

- la mobilité et la migration des travailleurs, etc.

\section{MÉTHODOLOGIE}

Regroupe les articles présentant les résultats d'une enquête ou d'une recherche empirique en utilisant et décrivant

- une nouvelle méthode de recherche;

- un modèle déjà connu ou utilisé;

- une méthode particulière d'analyse statistique, etc.

\section{WOMEN AND WORK}

Includes articles on women at work and more specifically on

- employment and labour market;

- equality;

- discrimination, etc.

MANAGEMENT OF ORGANIZATIONS

Includes articles on all aspects of management of organizations

- organizational structure;

- decision making;

- organizational culture;

- theory of organization, etc.

\section{HUMAN RESOURCES MANAGEMENT}

Includes papers on

- recruitment, selection and socialization;

- training and development;

- performance appraisal;

- compensation;

- motivation and satisfaction at work, etc.

\section{MANPOWER}

Covers articles on

- labour market;

- unemployment and full employment;

- manpower composition;

- mobility and migration, etc.

\section{METHODOLOGY}

Includes articles presenting the results of a survey or empirical research using and describing

- a new research method;

- an already known model;

- a particular method of statistical analysis, etc. 


\section{ORGANISATIONS PATRONALES}

Regroupe les articles portant sur les différents aspects des groupements patronaux ou associations d'employeurs

- histoire, origine et développement;

- nature, structure, fonctions, etc.

\section{ORGANISATIONS SYNDICALES}

Regroupe les articles portant sur les differents aspects du syndicalisme ouvrier

- histoire, origine et développement;

- nature, structure, fonctions;

- action politique;

- démocratie syndicale, etc.

\section{RELATIONS DU TRAVAIL}

Regroupe les articles concernant les relations entre la direction des entreprises et les travailleurs syndiqués

- négociation collective;

- convention collective;

- conciliation et arbitrage;

- conflits du travail, etc.

\section{SANTÉ ET SÉCURITÉ AU TRAVAIL}

Regroupe les articles traitant de

- maladies professionnelles;

- prévention des accidents;

- hygiène dans l'entreprise;

- médecine industrielle, etc.

\section{SÉCURITÉ SOCIALE}

Regroupe les articles sur la protection de l'État contre les risques socio-économiques

- formes de sécurité sociale et législation;

- politique gouvernementale;

- financement de la sécurité sociale, etc.

\section{EMPLOYERS' ORGANISATIONS}

Includes articles on different aspects of employers' associations

- history, origin and development;

- nature, structure, functions, etc.

\section{UNION ORGANIZATIONS}

Includes articles on trade unionism

- origin and growth of the labour movement;

- trade unionism functions and structures;

- trade unions and political action;

- trade union democracy, etc.

\section{LABOUR RELATIONS}

Includes articles on union management relations

- collective bargaining;

- collective agreement;

- conciliation and arbitration;

- industrial conflict, etc.

\section{OCCUPATIONAL HEALTH AND SAFETY}

\section{Concerns}

- professional illness;

- accident prevention;

- health at the work place;

- industrial medecine, etc.

\section{SOCIAL SECURITY}

Includes articles on government protection against socio-economic risks

- social security;

- government policy;

- financing social security, etc 


\begin{abstract}
SOCIOLOGIE DU TRAVAIL
Regroupe les articles utilisant l'approche sociologique pour l'étude des problèmes reliés au monde du travail

- changements technologiques;

- rapports sociaux dans l'entreprise;

- organisation du travail, etc.
\end{abstract}

\section{SYSTĖME DES RELATIONS INDUSTRIELLES}

Regroupe les articles portant sur

- les systèmes de relations industrielles;

- l'analyse d'un système de relutions industrielles dans un pays en particulier;

- la théorie, la recherche, l'étude et l'enseignement des relations industrielles;

- la terminologie et le vocabulaire des relations industrielles.

\section{SOCIOLOGY OF WORK}

Presents papers using a sociological approach for the study of the world of work

- technological change;

- workplace relations;

- work organization, etc.

\section{INDUSTRIAL RELATIONS SYSTEMS}

Includes articles on

- industrial relations systems;

- analysis of a particular industrial relations system in a given country;

- theory, research, study and teaching of industrial relations;

- terminology and glossary of industrial relations. 


\section{DROIT DU TRAVAIL}

\section{DROIT DU TRAVAIL}

AOUST, Claude d'

Effets d'annulation d'un avis de congédiement par un arbitre

v. 39 , no 2,1984 , p. $365-370$

0299

AOUST, Claude d' SAINT-JEAN, Sylvain TRUDEAU, Gilles

L'obligation de civilité du salarié

v. 41 , no 1,1986 , p. $157-180$

AOUST, Claude d'

L'amnistie des fautes disciplinaires.

v. 43, no 4,1988 , p. $909-942$

0690

\section{DROIT DU TRAVAIL - ALBERTA}

AOUST, Claude d'

Les ententes individuelles et la convention collective

v. 38 , no 1,1983, p. $155-158$

0186

FISHER, E.G. IVANKOVICH, I.F.

Alberta's Occupational Health and Safety

Amendment Act, 1983.

v. 40, no 1,1985 , p. $115-139$

0368

\section{DROIT DU TRAVAIL - CANADA}

AOUST, Claude d'

Les réclamations à l'arbitrage de dommages résultant de la violation d'une clause de renonciation à la grève.

v. 36 , no 1,1981 , p. 259-262

0014

MARTINEAU, LUC

Décisions rendues par le Conseil canadien des relations du travail.

v. 36 , no 1,1981 , p. $263-266$

0015

AOUST, Claude d'

La juridiction de l'arbitre et de la Cour des petites créances devant la Cour suprême du Canada.

v. 36 , no 2,1981 , p. $418-424$

0034

MARTINEAU, LUC

Décisions rendues par le Conseil canadien des relations du travail.

v. 36 , no 2,1981 , p. $424-429$

0035

MARTINEAU, LUC

La "gestion" des conflits par le Conseil canadien des relations du travail.

v. 36 , no 3,1981 , p. $589-615$

\section{VERGE, Pierre}

La mission-type d'un Conseil des relations du travail moderne.

v. 36 , no 3,1981, p. $663-670$

0059

MARTINEAU, LUC

Décisions rendues par le Conseil canadien des relations du travail.

v. 36, no 3,1981, p. $671-680$

0060

MCPHILLIPS, David C.

Duty of Fair Representation: Recent Attitudes in British Columbia and Ontario.

v. 36 , no 4,1981 , p. $803-827$

0074

MOORE, William R.

Justice and the Grievance Procedure in the Federal Public Service.

v. 36 , no 4,1981 , p. $848-864$

0076

AOUST, Claude d' DELORME, François The Origin of the Freedom of Association and of the Right to Strike in Canada: An Historical Perspective.

v. 36 , no 4,1981 , p. $894-921$

0078

LAVERY, Daniel

Décisions rendues par le Conseil canadien des relations du travail.

v. 36 , no 4,1981 , p. $928-936 \quad 0080$

LAVERY, Daniel

Décisions rendues par le Conseil canadien des relations du travail.

v. 37, no 1,1982 , p. $226-234$

0109

FRANK, J.A. KELLY, Michael J.

MACNAUGHTON, Bruce D.

Legislative Change and Strike Activity in Canada, 1926-1974

v. 37 , no 2,1982 , p. $267-283$

LAVERY, Daniel

Décisions rendues par le Conseil canadien des relations du travail.

v. 37 , no 2,1982 , p. $438-450$

0128

LAVERY, Daniel

Décisions rendues par le Conseil canadien des relations du travail.

v. 37 , no 3,1982 , p. $684-696$

0146

LAVERY, Daniel

Décisions rendues par le Conseil canadien des relations du travail.

v. 37 , no 4,1982 , p. $944-959$

0167 
DROIT DU TRAVAIL - CANADA (suite)

LAVERY, Daniel

Décisions rendues par le Conseil canadien des relations du travail.

v. 38 , no 1,1983 , p. $159-173$

0187

LAVERY, Daniel

Décisions rendues par le Conseil canadien des relations du travail.

v. 38 , no 2,1983 , p. $418-432$

MCSHANE, Steven L.

Reasonable Notice Criteria in Common Law Wrongful Dismissal Cases.

v. 38 , no 3,1983, p. $618-635$

0229

LAVERY, Daniel

Décisions rendues par le Conseil canadien des relations du travail.

v. 38 , no 3,1983 , p. $658-666$

0231

JOSEPH, P.A.

Perfecting the Administrative Solution to Labour Disputes.

v. 38 , no 4,1983 , p. $863-868$

0253

BEAULIEU, LUC

Décisions rendues par le Conseil canadien des relations du travail.

v. 38 , no 4,1983 , p. $880-903$

0255

BEAULIEU, LUC

Décisions rendues par le Conseil canadien des relations du travail.

v. 39 , no 1,1984 , p. $177-189$

0277

BEAULIEU, LUC

Décisions rendues par le Conseil canadien des relations du travail

v. 39, no 2,1984, p. $371-385$

0300

FISHER, E.G. SHERWOOD, L.M.

Fairness and Managerial Rights in Canadian Arbitral Jurisprudence

v. 39, no 3,1984, p. $538-552$

0319

BEAULIEU, LUC

Décisions rendues par le Conseil canadien des relations du travail.

v. 39, no 3,1984 , p. $605-618$

0323

VERGE, Pierre

Évolution de la protection juridictionnelle de l'accès au syndicat.

v. 39, no 4,1984, p. $710-743$

0340
POTHIER, Dianne

Décisions rendues par le Conseil canadien des relations du travail.

v. 39 , no 4,1984 , p. $774-782$

0344

POTHIER, Dianne

Décisions rendues par le Conseil canadien des relations du travail.

v. 40, no 1,1985, p. $170-174$

0371

LAMY, Francine

Décisions rendues par le Conseil canadien des relations du travail.

v. 40, no 2,1985, p. $386-398$

0394

SOLOMON, Norman A.

The Negociation of First Agreements under the Canada Labour Code.

v. 40, no 3,1985 , p. $458-472 \quad 0404$

SUBBARAO, A.V.

Impasse Choice in the Canadian Federal Service: An Innovation and an Intrigue

v. 40, no 3,1985 , p. $567-590$

0410

MORIN, Fernand

Le "raisonnable" déraisonnable! ou la rationalité du raisonnable

v. 40 , no 3,1985 , p. $646-658$

0414

LAMY, Francine

Décision rendue par le Conseil canadien des relations du travail.

v. 40 , no 3,1985 , p. $659-662 \quad 0415$

LAMY, Francine

Décision rendue par le Conseil canadien des relations du travail

v. 40, no 4,1985 , p. $880-885$

0434

AOUST, Claude d'

Le contrôle judiciaire des décisions arbitrales: Réflexions sur les moyens d'y échapper.

v. 41 , no 2,1986 , p. $348-356$

0473

DICKINSON, John A.

La législation et les travailleurs québécois, 1894-1914

v. 41 , no 2,1986 , p. $357-381$

0474

SAINT-GERMAIN, Catherine

Décisions rendues par le Conseil canadien des relations du travail.

v. 41, no 2, 1986, p. 397-411 0477 
DROIT DU TRAVAIL - CANADA (suite)

MORIN, Fernand

Le rationnel et le raisonnable: Deux nécessités distinctes et conjugées en droit.

v. 41 , no 3,1986 , p. $578-582$

0494

BEMMELS, Brian FISHER, E.G.

NYLAND, Barbara

Canadian-American Jurisprudence on "Good Faith" Bargaining.

v. 41 , no 3,1986 , p. $596-621$

0496

SAINT-GERMAIN, Catherine

Décisions rendues par le Conseil canadien des relations du travail

v. 41 , no 3,1986 , p. $622-637$

0497

JAIN, Harish C. ANDIAPPAN, P.

Sexual Harassment in Employment in Canada: Issues and Policies.

v. 41 , no 4,1986 , p. $758-777$

0517

SAINT-GERMAIN, Catherine

Décisions rendues par le Conseil canadien des relations du travail.

v. 41 , no 4,1986 , p. $860-868$

0525

SAINT-GERMAIN, Catherine

Décision rendue par le Conseil canadien des relations du travail.

v. 42 , no 1,1987 , p. $203-209$

0549

ZEYTINOGLU, Isik Urla

The ILO Standards and Canadian Labour Législation.

v. 42 , no 2,1987, p. $292-308$

0562

SAINT-GERMAIN, Catherine

Décisions rendues par le Conseil canadien des relations du travail

v. 42 , no 2,1987 , p. $415-427$

0569

SAINT-GERMAIN, Catherine

Décision rendue par le Conseil canadien des relations du travail.

v. 42 , no 3,1987 , p. $628-635$

0589

TREMBLAY, Johane

Décisions rendues par le Conseil canadien des relations du travail.

v. 42 , no 4,1987 , p. $852-861$

0613

MCPHILLIPS, David C.

The Appropriate Bargaining Unit: The
Need for Policy Consistency by Canadian Labour Boards.

v. 43 , no 1,1988 , p. $63-84$

0629

TREMBLAY, Johane

Décisions rendues par le Conseil canadien des relations du travail.

v. 43 , no 1,1988 , p. 188-195 0637

TREMBLAY, Johane

Décisions rendues par le Conseil canadien des relations du travail.

v. 43, no 2,1988, p. $447-452$

0655

TOUPIN, Sylvain

Le harcèlement sexuel en milieu de travail. v. 43, no 3,1988 , p. $531-546 \quad 0666$

SUBBARAO, A.V.

Criteria in Arbitration of Wage Disputes: Theory and Practice in the Canadian Federal Public Service.

v. 43 , no 3,1988 , p. $547-570$

0667

TREMBLAY, Johane

Décisions rendues par le Conseil canadien des relations du travail.

v. 43 , no 3,1988 , p. $686-694$

0673

WEST, Leigh

The Exclusive Remedy Provision in Canadian Worker Compensation Law: The Need for Legislative Reform.

v. 43 , no 4,1988 , p. $890-908 \quad 0689$

MARLEAU, Véronique L.

Décisions rendues par le Conseil canadien des relations du travail.

v. 43 , no 4,1988 , p. $953-962$

0692

MORIN, Fernand

D'un entrepreneur à un autre: l'accréditation ne suit pas

v. 44 , no 2,1989 , p. $315-336$

0719

CARTER, Donald D.

Grievance Arbitration and the Charter: The Emergency Issues.

v. 44 , no 2,1989, p. $337-353$

0720

MARLEAU, Véronique $L$.

Décisions rendues par le Conseil canadien des relations du travail.

v. 44, no 2,1989 , p. $448-462$

0726 


\section{DROIT DU TRAVAIL - CANADA (suite)}

JAIN, Harish C.

Racial Minorities and Affirmative Action/Employment Equity Législation in Canada.

v. 44 , no 3,1989 , p. $593-614 \quad 0738$

MARLEAU, Véronique L.

Décisions rendues par le Conseil canadien des relations du travail.

v. 44 , no 3,1989 , p. $703-722$

0744

CÔTÉ, André C.

Nouvelles technologies et droit du travail au Canada.

v. 44, no 4,1989 , p. $751-768$

0751

ANDIAPPAN, P. CRESTOHL, Mark

SINGH, Jang $B$.

Racial Discrimination in Employment in Canada.

v. 44 , no 4,1989 , p. $827-849$

0755

WRIGHT, Michael D.

The Legislation of Labour Relations at Canada Post.

v. 44 , no 4,1989 , p. $866-883 \quad 0757$

MORIN, Fernand

Liberté d'expression et droit au travail: l'arbitrage de la Cour suprême du Canada.

v. 44 , no 4,1989 , p. $921-932$

0761

CLARKE, Graham

Décisions rendues par le Conseil canadien des relations du travail.

v. 44, no 4,1989 , p. $933-946$

0762

CLARKE, Graham

Décisions rendues par le Conseil canadien des relations du travail.

v. 45 , no 1 , 1990, p. 187-197

0782

MCSHANE, Steven L. REDEKOP, Bruce Compensation Management and Canadian Wrongful Dismissal: Lessons from Litigation.

v. 45 , no 2,1990 , p. $357-381 \quad 0798$

MARLEAU, Véronique L.

Décision rendue par le Conseil canadien des relations du travail.

v. 45, no 2,1990 , p. $414-423$

0801
MORIN, Fernand

Modification unilatérale des conditions de travail au terme d'une négociation collective!

v. 45 , no 3,1990 , p. $566-584$

0817

CARROTHERS, A.W.R.

Labour Law Through the Prism of Paccar v. 45 , no 3, 1990, p. $585-611 \quad 0818$ CLARKE, Graham

Décision rendue par le Conseil canadien des relations du travail.

v. 45 , no 3,1990 , p. $616-624 \quad 0820$

BOIVIN, Suzanne P.

Le Canadien National: Un cas riche d'enseignements.

43ième Congrès, 1988, p. 97-113 0947

SIMARD, Monique TELLIER, Marie

Commentaires - Le Canadien National:

Un cas riche d'enseignements.

43ième Congrès, 1988, p. 114-130 0948

DESMARAIS, Jacques

Les moyens de pression: Les chartes en redefinissent-elles les limites?

43ième Congrès, 1988, p. 157-195 0950

DULUDE, Gilles LOUMÈDE, Catherine

PARROT, Jean-Claude

Commentaires - Les moyens de pression: Les Chartes en redefinissent-elles les limites?

43ième Congrès, 1988, p. 195-208 0951

GARANT, Patrice

Statut et pouvoirs des organismes du travail en regard des Chartes.

43ième Congrès, 1988, p. 237-254 0954

CÔTÉ, André C. FONTAINE, Claude

LESAGE, Paul

Aspects et implications juridiques des restructurations.

44ième Congrès, 1989, p. 33-84 0959

\section{DROIT DU TRAVAIL -}

COLOMBIE BRITANNIQUE

JOSEPH, P.A.

Perfecting the Administrative Solution to Labour Disputes: The British Columbia Experiment.

v. 38 , no 2,1983 , p. $380-414 \quad 0209$ 
DROIT DU TRAVAIL - ÉTATS-UNIS

BEMMELS, Brian FISHER, E.G.

NYLAND, Barbara

Canadian-American Jurisprudence on "Good Faith' Bargaining.

v. 41 , no 3,1986 , p. $596-621$

0496

BRUCE, Peter G.

The Processing of Unfair Labor Practice Cases in the United States and Ontario.

v. 45, no 3,1990 , p. $481-511$

0813

DROIT DU TRAVAIL - FRANCE

CAIRE, GuY

Les lois Auroux

v. 39 , no 2,1984 , p. $235-258$

0292

\section{DELAMOTTE, Yves}

La loi et la négociation collective en France: Réflexion sur l'expérience 1981-1985.

v. 42 , no 1,1987 , p. $92-109$

0542

DROIT DU TRAVAIL - GRANDE-BRETAGNE BEAUMONT, P.B.

Statutory Recognition Provisions in Britain, 1976-80

v. 38 , no 4,1983 , p. $744-766$

0247

\section{DROIT DU TRAVAIL - MANITOBA}

BLACK, Errol

In Search of "Industrial Harmony": The Process of Labour Law Reform in Manitoba, 1984.

v. 40, no 1,1985 , p. $140-161$

0369

BLACK, Errol SILVER, Jim

Contradictions and Limitations of Final Offer Selection: The Manibota Experience.

v. 45 , no 1,1990, p. $146-165$

0779

GRANT, Hugh $M$.

Contradictions and Limitations of Final Offer Selection: The Manitoba Experience.

A Comment.

v. 45 , no 1,1990 , p. $166-168 \quad 0780$

BLACK, Errol SILVER, Jim FOS in Manitoba. A Rejoinder.

v. 45 , no 3,1990 , p. $612-615$

0819

\section{DROIT DU TRAVAIL - NIGÉRIA}

FASHOYIN, Tayo

State Regulation of Trade Disputes in Essential Services in Nigeria

v. 36 , no 1,1981 , p. 207-222

0010
DROIT DU TRAVAIL - ONTARIO

GUNDERSON, Morley

Spline Functions Estimates of the Impact of Equal Pay Législation: The Ontario Experience

v. 40, no 4,1985 , p. $775-792 \quad 0427$

FORREST, Anne

Bargaining Units and Bargaining Power

v. 41 , no 4,1986 , p. $840-850 \quad 0523$

PUPO, Norene DORIS DUFFY, Ann

The Ontario Labour Relations Board and the Part Time Workers.

v. 43 , no 3,1988 , p. $660-6850672$

BRUCE, Peter G.

The Processing of Unfair Labor Practice Cases in the United States and Ontario.

v. 45 , no 3,1990 , p. $481-511 \quad 0813$

DROIT DU TRAVAIL - POLOGNE

PLISZKIEWICZ, Marek

Le licenciement en droit polonais.

v. 36 , no 1,1981 , p. $251-259$

0013

DROIT DU TRAVAIL - QUÉBEC

VERGE, Pierre

Syndicalisation de la grève.

v. 38, no 3,1983 , p. $475-506$

0223

BERNIER, Jean

L'extension juridique des conventions collectives au Québec: Une approche comparative.

v. 38 , no 3,1983 , p. $532-544$

0225

BOULARD, René MARCHAND, Michel

La loi sur la santé et la sécurité du travail et les conventions collectives.

v. 38 , no 4,1983 , p. $847-862$

0252

JOSEPH, P.A.

Perfecting the Administrative Solution to Labour Disputes.

v. 38 , no 4,1983 , p. $863-868 \quad 0253$

BLOUIN, Rodrigue

La réforme du Code du travail du Québec: une démarche fragmentaire.

v. 39 , no 3. 1984 , p. $578-604$

0322

MORIN, Fernand

Nouvelle présence de l'État dans les relations du travail.

v. 39 , no 4,1984, p. $744-752$

0341 
DROIT DU TRAVAIL - QUÉBEC (suite) MORIN, Fernand

Les tenants et les aboutissants de la convention collective.

v. 40 , no 2,1985, p. $371-378$

0392

CÔTÉ, André C.

L'Acte des manufactures de Québec, 1885: Un centenaire

v. 40, no 3,1985, p. $623-628$

0412

MORIN, Fernand

Rapports collectifs du travail dans les secteurs publics québécois ou le nouvel équilibre selon la loi du 19 juin 1985.

v. 40, no 3,1985, p. $629-645$

0413

MORIN, Fernand

Rapports collectifs du travail dans les secteurs publics québécois ou le nouvel équilibre selon la loi du 19 juin 1985.

v. 40, no 3,1985, p. $629-645$

0413

SIMARD, Marcel

BOUTEILLER, Dominique

LÉVESQUE, Christian

Prévention des accidents du travail: Contexte législatif québécois et efficacité organisationnelle.

v. 40, no 4,1985, p. $703-719$

0424

MORIN, Fernand

La survie de droits subjectifs à la convention collective.

v. 40 , no 4,1985, p. $847-855$

0431

DICKINSON, John A.

La législation et les travailleurs québécois, 1894-1914

v. 41 , no 2,1986 , p. $357-381$

0474

PLANTE, Gilles

La permission d'appel au tribunal du travail

v. 41 , no 4,1986 , p. $817-834 \quad 0521$

MORIN, Fernand

La double personnalité d'un concierge!

v. 41, no 4,1986 , p. $835-839$

0522

SEXTON, Jean

L'arbitrage de première convention collective au Québec: 1978-1984

v. 42, no 2,1987, p. $272-291$

0561
BLOUIN, Rodrigue

Le titre réservé de $C R I$ et la déontologie de la profession

v. 42 , no 2,1987 , p. $309-324$

0563

\section{LAPORTE, Pierre}

Le caractere d'ordre public des dispositions de la Loi sur les normes du travail. v. 42 , no 2,1987 , p. $398-414$

0568

\section{BARRÉ, Alain}

Le cadre juridique de la négociation collective dans le secteur public au Québec: une perspective internationale.

v. 42 , no 4,1987 , p. $831-851$

0612

BRUNET, Yves W. BEAULIEU, LuC

CANTIN, Isabelle

La nouvelle Commission québécoise des relations du travail (1988)

v. 43 , no 2,1988 , p. 231-304

0647

MORIN, Fernand

Un préavis de licenciements ou son équivalent.

v. 43, no 4,1988 , p. $943-952$

0691

VALLÉE, Guylaine

Les accords "atypiques" et le droit des rapports coliectifs du travail.

v. 44, no 3,1989 , p. $680-690$

0742

ROSS, Claudette BROSSARD, Michel La conciliation volontaire est-elle plus efficace que la conciliation obligatoire? Le cas du Québec.

v. 45 , no 1,1990, p. $3-21$

0771

HÉBERT, Gérard

Le renouvellement du régime des décrets de convention collective.

v. 45, no 2,1990, p. $404-413$

0800

BEAUSOLEIL, Gilles GAUTHIER, Hervé La législation: consolidation ou innovation?

36ième Congrès, 1981, p. 145-172 0841

LOCKWELL, LUC M. LORTIE, Pierre

Commentaire - La législation: consolidation ou innovation?

36ième Congrès, 1981, p. 173-192 0842 
DROIT DU TRAVAIL - QUÉBEC (suite)

LEBEL, Hélène

Commentaires - L'impact du régime de relations du travail sur la syndicalisation. 38ième Congrès, 1983, p. 91-100 0863

DESMARAIS, Jacques

Les idées de réforme sur la syndicalisation au Québec depuis 1964.

38ième Congrès, 1983, p. 101-116 0864

MERCILLE, Pierre NADEAU, Bertin OLIVIER, Madeleine

Table ronde - Organisation syndicale: difficultés et motifs de résistance.

38ième Congrès, 1983, p. 117-134 0865

FRÉCHETTE, Raynald

La politique gouvernementale en matière de syndicalisation.

38ième Congrès, 1983, p. 197-206 0870

BONENFANT, Claire DUFOUR, Ghislain HÉTU, Jean-Paul

Table ronde - Les réactions du milieu.

38ième Congrès, 1983, p. 207-223 0871

SIMARD, Marcel

Priorités en santé et sécurité du travail: secteur public et secteur privé.

39ième Congrès, 1984, p. 33-50

0875

LEDUC, Jean-Guy LEMELIN, Maurice VINET, Alain

Commentaires - Priorités en santé et sécurité du travail: secteur public et secteur privé.

39ième Congrès, 1984 , p. $50-720876$

OUELLET, Florian

Concertation et participation: mythe ou réalité?

39ième Congrès, 1984, p. 73-92 0877

DE KONINCK, Maria HEENAN, Roy

Droit de refus et retrait préventif: succès ou échec?

39ième Congrès, 1984, p. 159-175 0881

BRUNET, Jacques

Santé et securité du travail: une affaire de professionnels?

39ième Congrès, 1984, p. 195-206 0884
BÉLANGER, Jacques BLOUIN, Rodrigue MORIN, Fernand SEXTON, Jean

Introduction - Le statut de salarié en milieu de travail.

40ième Congrès, 1985, p. 13-15 0887

BLOUIN, Rodrigue

Le statut de salarié en milieu de travail: la problématique.

40ième Congrès, 1985, p. 17-32 0888

GAGNON, Jean-Denis

Les notions de salarié en droit du travail.

40ième Congrès, 1985, p. 33-47 0889

DOUCET, René PARENT, Louise

Commentaires - Les notions de salarié en droit du travail.

40ième Congrès, 1985, p. 48-54 0890 CÔTÉ, André C.

Évolution des conditions de travail des salariés établies d'autorité.

40ième Congrès, 1985, p. 55-69 0891

PIUZE, Guy

Commentaires - Evolution des conditions de travail des salariés établies d'autorité. 40ième Congrès, 1985, p. 69-74 0892

\section{DROIT DU TRAVAIL - SASKATCHEWAN}

SASS, Robert

The Saskatchewan Trade Union Act. 1983: The Public Battle.

v. 40 , no 3,1985 , p. 591-622

0411

\section{ÉCONOMIQUE DU TRAVAIL}

\section{ÉCONOMIQUE DU TRAVAIL}

WILLIAMS, C. Glyn

Joan Robinson on Unions, Distribution and Inflation.

v. 36 , no 3,1981 , p. $576-588 \quad 0054$

WILLIAMS, C. Glyn

The Role of Unions in Inflation: A Survey Article

v. 37 , no 3,1982 , p. $498-527 \quad 0137$

JOLIVET, Thierry

La réduction de la durée du travail estelle créatrice d'emploi?

v. 38 , no 1,1983 , p. 142-154 0185 
ÉCONOMIQUE DU TRAVAIL (suite) MAKI, Dennis R. STRAND, Kenneth The Determinants of Strike Activity: An Interindustry Analysis

v. 39 , no 1,1984 , p. $77-92$

0272

CAMERON, Samuel

Historical Variations in the Impact of Union Density on Strike Frequency: Some U.K. Evidence

v. 40, no 2,1985, p. $367-370$

0391

LACROIX, Robert

A Microeconometric Analysis of the Effects of Strikes on Wages

v. 41 , no 1, 1986, p. 111-127

0452

CHAYKOWSKI, Richard P.

SLOTSVE, G.A.

Union Seniority Rules as a Determinant of Intra-Firm Job-Changes.

v. 41, no 4,1986 , p. $720-737$

0515

OHTSU, Makoto VERMA, Anil Intra-Organizational Bargaining: Wage Differentials Among Saskatchewan Schoolteachers.

v. 42 , no 3,1987 , p. $612-627 \quad 0588$

LACROIX, Robert LESPÉRANCE, André Les nouvelles lois du travail et l'activité de grève.

v. 43, no 4,1988 , p. $812-828 \quad 0685$

BRODY, Bernard LÉTOURNEAU, Yves POIRIER, André

Le coût des accidents du travail: État des connaissances.

v. 45 , no 1,1990 , p. $94-117$

0776

VALASKAKIS, Kimon

Le temps libéré: à quels coûts?

36ième Congrès, 1981, p. 61-74

0836

POULIN-SIMON, Lise FORTIN, Bernard Commentaires - Le temps liberé: à quels couts?

36ième Congrès, 1981 , p. $75-86 \quad 0837$

AOUST, Fernand d' DÉOM, André HARVEY, Pierre

Table ronde - La réduction du temps passé au travail: un moyen de lutte contre le chomage.

36ième Congrès, 1981, p. 219-246 0844
GALBRAITH, John Kenneth

Réflexions sur les problèmes de la retraite dans les années 80.

37ième Congrès, 1982, p. 193-213 0856

CAIRE, Guy

Syndicalisme, nouvelles technologies et incertitudes économiques.

38ième Congrès, 1983, p. 225-265 0872

ROUSSEAU, Léontine

Annexe - Acquisitions, fusions, offres publiques d'achat: Notions de base et racteurs considerés.

44ième Congrès, 1989, p. 189-252 0965

ÉCONOMIQUE DU TRAVAIL - CANADA

CHRISTENSEN, Sandra

Collective Bargaining in Provincial Public Administration.

v. 36 , no 3,1981 , p. $616-6290056$

GRUBEL, Herbert G. MAKI, Dennis R.

A Note on the Effects of Unemployment Insurance, Minimum Wage Legislation and Trade Union Growth on Reported Unemployment Rates in Canada, 1950-75

v. 36, no 4,1981 , p. $922-927$

0079

\section{MARION, Gérald}

La part des salaires dans le revenu national au Canada, 1910 à 1980.

v. 37 , no 1,1982 , p. $53-64$

0097

HASAN, Abrar GERA, Surendra

Reservation Wages in Canadian Labour Markets.

v. 37 , no 1, 1982, p. $65-92 \quad 0098$

GILES, Anthony

The Canadian Labour Congress and Tripartism.

v. 37 , no 1, 1982, p. 93-125 0099

MAGUN, Sunder

The Rise of Service Employment in the Canadian Economy.

v. 37 , no 3,1982 , p. $528-556 \quad 0138$

ADAMS, Roy J.

The Federal Government and Tripartism.

v. 37 , no 3,1982 , p. $606-617$

0141 


\section{ECONOMIQUE DU TRAVAIL - CANADA (suite)}

SIMPSON, Wayne PETERS, Frank

The Economics of Mileage Restrictions for Railway Workers in Western Canada.

v. 38, no 1, 1983, p. 95-103

0182

MAKI, Dennis R.

Trade Unions and Productivity: Conventional Estimates.

v. 38, no 2,1983, p. $211-228$

0201

FISHER, E.G. PERCY, M.B.

The Impact of Unanticipated Output and Consumer Prices on Wildcat Strikes.

v. 38 , no 2,1983 , p. 254-276 0203

COUSINEAU, Jean-Michel

LACROIX, Robert

La détermination des avantages sociaux au Canada

v. 39, no 1,1984, p. $3-22$

0268

ROBB, Roberta Edgecome

Occupational Segregation and Equal Pay for Work of Equal Value.

v. 39 , no 1,1984 , p. $146-166$

0275

DEVORETZ, D.J. REED, Clyde G.

Evidence from the Skilled-Unskilled Canadian Wage Index.

v. 39 , no 3,1984 , p. $526-537 \quad 0318$

JONES, J.C.H. LAUDADIO, L.

Organized Labour, Regional Political Bias and the Canadian Tariff Structure

v. 39 , no 4,1984 , p. $695-709$

0339

MILNE, William J. ROSS, Thomas W.

The Cyclical Variation of Wage Premiums in the Canadian Manufacturing Industries

v. 39 , no 4,1984 , p. $762-773$

0343

SAUNDERS, George

Employment and the Productivity Slowdown: 1958-1980

v. 40 , no 2,1985 , p. $219-242 \quad 0383$

BRODY, Bernard ROHAN, Paul

ROMPRÉ, Louise

Les accidents industriels au Canada: Le portrait d'une décennie

v. 40, no 3,1985 , p. $545-566$

0409
OSBERG, Lars

A Note on the Incomes of Lawyers

v. 40 , no 4,1985 , p. $865-879$

0433

BEACH, Charles M. KALISKI, S.F.

The Impact of Recession on the Distribution of Annual Unemployment.

v. 41 , no 2,1986 , p. 317-328

0471

TANG, Roger Y.W. PONAK, Allen

Employer Assessment of Strike Costs.

v. 41 , no 3,1986 , p. $552-571$

0492

EVANS, Martin G. ONDRACK, Daniel A. The Effect of Unionization on Wages: Some Canadian Evidence

v. 41 , no 3,1986 , p. $572-577 \quad 0493$

BETCHERMAN, Gordon

Labour Market Imbalances in Canada, 1966-1983

v. 41, no 4,1986, p. $802-816 \quad 0519$

DOOLEY, Martin D.

Within-Cohort Earnings Inequality Among Canadian Men: 1971-1982

v. 42 , no 3,1987 , p. $594-611$

0587

KUMAR, Pradeep

Recent Wage Deceleration in Canada: Short-run Response or Structural Change? v. 42 , no 4,1987 , p. $687-701 \quad 0604$

MERCIER, Jacques

Effets du salaire minimum sur l'emploi: Résultats des études économétriques canadiennes et québécoises.

v. 42, no 4,1987 , p. $806-830$

0611

REID, Frank

Economic Aspects of Mandatory Retirement: The Canadian Experience.

v. 43, no 1,1988, p. 101-114

0631

KAMEL, Nawal ROY, Paul-Martel

Temps supplémentaire et création d'enplois: Le cas du Canada, du Québec et de lOntario.

v. 43, no 2,1988, p. $412-430 \quad 0653$

STAGER, David A.A.

Lawyers' Earnings in the Canadian Private and Public Sectors.

v. 43 , no 3,1988 , p. $571-590 \quad 0668$ 


\section{ÉCONOMIQUE DU TRAVAIL - CANADA} (suite)

MCWATTERS, Catherine J.

BEACH, Charles M.

Factors Behind the Changes in Canada's Family income Distribution and the Share of the Middle Class.

v. 45, no 1, 1990, p. 118-135 0777

GODBOUT, Clément RIOUX, Claude Le libre-échange canado-américain et le marché du travail.

44ième Congrès, 1989, p. 175-188 0964

ÉCONOMIQUE DU TRAVAIL - EUROPE

STURMTHAL, Adolf

Unemployment, Inflation and "Guest Workers": Comparative Study of Three European Countries.

v. 37, no 4,1982 , p. $739-764 \quad 0157$

\section{ÉCONOMIQUE DU TRAVAIL - ONTARIO}

AULD, D.A.L. WILTON, D.A.

The Impact of Public Sector Wage Controls in Ontario

v. 42 , no 1,1987 , p. $132-149$

0544

\section{ÉCONOMIQUE DU TRAVALL - QUÉBEC}

ROY, Paul-Martel

Aspects dynamiques du marché du travail au Québec.

v. 45, no 2,1990 , p. $283-299$

0794

DÉPATIE, Raymond

Commentaire - Les aspects économiques de la réforme des pensions.

37ième Congrès, 1982, p. 98-105 0851

INGERMAN, Sidney

La syndicalisation dans le contexte économique québécois.

38ième Congrès, 1983 , p. 37-69 0860

BONIN, Bernard

Commentaires - La syndicalisation dans le contexte économique québécois.

38ième Congrès, 1983, p. 70-76

0861

BEAUSOLEIL, Gilles

Les coûts et les bénéfices du régime. 39ième Congrès, 1984, p. 207-242 0885

\section{FEMMES ET TRAVAIL}

\section{FEMMES ET TRAVAIL}

AGARWAL, Naresh C.

Male-Female Pay Inequity and Public Policy in Canada and the U.S.

v. 37, no 4, 1982, p. 780-804 0159

SAVOIE, Dominique LAROUCHE, Viateur Le harcèlement sexuel au travail: Définition et mesure du phénomène.

v. 43 , no 3,1988 , p. $509-530 \quad 0665$

SWIMMER, Gene

Gender Based Differences in Promotions of Clerical Workers.

v. 45 , no 2,1990 , p. $300-310 \quad 0795$

\section{FEMMES ET TRAVAIL - CANADA}

JAIN, Harish C.

Race ans Sex Discrimination in Employment in Canada: Theories, Evidence and Policies.

v. 37, no 2,1982, p. $344-366 \quad 0123$

WALSH, William D.

A Time Series Analysis of Female Labour Force Participation Rates Disaggregated by Marital Status.

v. 37, no 2,1982 , p. $367-384 \quad 0124$

CHAISON, Gary N. ANDIAPPAN, P. Characteristics of Female Union Officer in Canada.

v. 37 , no 4,1982 , p. $765-7790158$

ROBB, Roberta Edgecome

Occupational Segregation and Equal Pay for Work of Equal Value.

v. 39 , no 1,1984 , p. $146-166 \quad 0275$

LAMSON, Cynthia

On the Line: Women and Fish Plant Jobs in Atiantic Canada.

v. 41 , no 1,1986, p. $145-156$

0454

JAIN, Harish C. ANDIAPPAN, P.

Sexual Harassment in Employment in Canada: Issues and Policies.

v. 41 , no 4,1986 , p. $758-777$

0517

TOUPIN, Sylvain

Le harcèlement sexuel en milieu de travail.

v. 43, no 3,1988 , p. $531-546 \quad 0666$ 
FEMMES ET TRAVAIL - CANADA (suite)

DUCHARME, Claude

LECLERCQ, Dominique

WESTMORELAND-TRAORE, Juanita

Commentaires - La gestion de l'embauche, de la promotion et du licenciement revue et corrigée par les Chartes.

43ième Congrès, 1988, p. 63-82 0945

BOIVIN, Suzanne P.

Le Canadien National: Un cas riche d'enseignements.

43ième Congrès, 1988, p. 97-113 0947

SIMARD, Monique TELIER, Marie

Commentaires - Le Canadien National: Un cas riche d'enseignements.

43ième Congrès, 1988, p. 114-130 0948

\section{FEMMES ET TRAVAIL - ONTARIO}

GUNDERSON, Morley

Spline Functions Estimates of the Impact of Equal Pay Législation: The Ontario Experience

v. 40, no 4,1985 , p. $775-792 \quad 0427$

BURT, Sandra

Voluntary Affirmative Action. Does it Work?

v. 41 , no 3,1986 , p. 541-551

0491

FEMMES ET TRAVAIL - QUÉBEC

GRANT, Michel ROSE, Ruth

Encadrement du travail à domicile dans l'industrie du vêtement au Québec.

v. 40, no 3,1985 , p. $473-494$

0405

AUDET, Claude VINET, Alain

Bureautique et organisation du travail de secrétariat dans la fonction publique québécoise.

v. 43 , no 4,1988 , p. $737-7560682$

SAVOIE, Dominique LAROUCHE, Viateur Le harcèlement sexuel au travail: Résultats de deux études québécoises.

v. 45 , no 1,1990, p. $38-62$

0773

\section{GESTION DES ORGANISATIONS}

\section{GESTION DES ORGANISATIONS}

PECCEI, Ricardo WARNER, Malcolm

Industrial Relations, Strategic Importance, and Decision - Making.

v. 36 , no 1,1981 , p. 132-151 0006
BAR-HAlM, Aviad

The Desire for Workers' Participation: Conflicting Forces in the Workplace.

v. 39 , no 2,1984 , p. $301-312$

0296

\section{MILLER, Roger}

La stratégie d'entreprise et la gestion des ressources humaines.

v. 40, no 1.1985, p. $68-86$

0365

JAIN, Hem C. GILES, Anthony

Workers' Participation in Western Europe: Implications for North America.

v. 40 , no 4,1985 , p. $747-774 \quad 0426$

BROSSARD, Michel SIMARD, Marcel Problématique de la différenciation de la main-d'oeuvre et changement organisationnel.

v. 41 , no 2,1986 , p. $219-2350466$

MERCIER, Jean

L'informatique, ses "filtres" et ses effets.

v. 41 , no 2,1986 , p. $299-316 \quad 0470$

GODIN, Jean LE LOUARN, Jean-Yves

Les mentors ont-ils un effet sur la progression de carrière?

v. 41, no 3, 1986, p. 505-518 0489

LONG, Richard J. WARNER, Malcolm

Organizations, Participation and Recession: An Analysis of Recent Evidence.

v. 42 , no 1,1987 , p. $65-91$

0541

BABA, Vishwanath V. KNOOP, Robert Organizational Commitment and Independence Among Canadian Managers

v. 42 , no 2,1987 , p. $325-344 \quad 0564$

RICHARDS, John MAUSER, Gary

HOLMES, Richard

What Do Workers Want? Attitudes Towards Collective Bargaining and Participation in Management.

v. 43 , no 1,1988 , p. $133-152$

0633

PEPERMANS, Raymond

Line, staff et functional

v. 43 , no 1,1988 , p. $183-187$

0636

MATON, Bob

Socio-Technical Systems: Conceptual and Implementation Problems

v. 43 , no 4,1988 , p. $869-889 \quad 0688$ 


\section{GESTION DES ORGANISATIONS (suite)}

GAGNON, Yves-Chantal

LANDRY, Maurice

Les changements technologiques: Une stratégie d'étude exploratoire

v. 44 , no 2,1989 , p. $421-447$

0725

STRATTON, Kay RESHEF, Yonatan Private Sector Unions and Strategic Planning: A Research Agenda

v. 45 , no 1,1990 , p. $76-93$

0775

BÉLANGER, Laurent

Nouvelles formes d'organisation du travail, nouveaux modes de gestion et leur incidence sur le statut du salarié.

40ième Congrès, 1985, p. 171-196 0898

CÓTÉ, Marcel

Commentaires - Nouvelles formes d'organisation du travail, nouveaux modes de gestion et leur incidence sur le statut du salarié.

40ième Congrès, 1985, p. 196-203 0899

BÉLANGER, Jacques

Annexe - La participation des travailleurs aux décisions dans l'entreprise.

40ième Congrès, 1985, p. 245-259 0903

LAROUCHE, Viateur

La mobilisation des ressources humaines - orientations récentes.

41ième Congrès, 1986, p. 31-51 0907

SÉRIEYX, Hervé

L'entreprise du troisième type.

41ième Congrès, 1986, p. 85-97 0912

WILS, Thierry

Les travailleurs seront-ils du troisième type?

41ième Congrès, 1986, p. 99-109 0913

BÉLANGER, Laurent LIPSIG, Carla MORIN, Fernand PÉRUSSE, Michel Introduction - Acquisition ou fusion d'entreprises et emplois.

44ième Congrès, 1989, p. XV-XVI 0956

MORIN, Fernand

Acquisition ou fusion d'entreprises et emplois: La problématique sous-jacente.

44ième Congrès, 1989, p. 1-12 0957
ANGERS, Georges GAGNON, Normand SIMARD, Jean

Le droit et le devoir à linformation.

44ième Congrès, 1989, p. 85-100 0960

LAMARCHE, André GAUTHIER, Michel BLAIS, Michel GAGNON, Denise

Les conditions de travail au lendemain d'une fusion ou d'une réorganisation.

44ième Congrès, 1989, p. 101-127 0961

GOSSELIN, Alain FRENETTE, Jean-Guy DIONNE, Denis

Les acteurs d'une fusion ou d'une prise de pouvoir.

44ième Congrès, 1989, p. 155-173 0963

ROUSSEAU, Léontine

Annexe - Acquisitions, fusions, offres publiques d'achat: Notions de base et facteurs considérés.

44ième Congrès, 1989, p. 189-252 0965

\section{GESTION DES ORGANISATIONS -}

\section{AMÉRIQUE LATINE}

APPARICIO-VALDEZ, Luis

La gestion empresarial en latinoamérica $y$

su impacto en las relaciones laborales

v. 44 , no 1,1989 , p. $124-148$

0704

\section{GESTION DES ORGANISATIONS - CANADA} COULOMBE, Gilles

Le système de gestion des cas du Conseil canadien des relations du travail (CCRT).

v. 37 , no 4,1982 , p. $928-943 \quad 0166$

FRICKE, John G.

Worker Participation in Canada: Some Lessons from the Past.

v. 43 , no 3,1988 , p. $633-658$

0671

AUDET, Michel BÉLANGER, Laurent Nouveaux modes de gestion et relations industriel/es au Canada.

v. 44, no 1,1989, p. $62-96$

0702

LONG, Richard J.

Patterns of Workplace Innovation in Canada.

v. 44, no 4,1989 , p. $805-826 \quad 0754$ 
GESTION DES ORGANISATIONS - CANADA (suite)

CÓTÉ, André C. FONTAINE, Claude LESAGE, Paul

Aspects et implications juridiques des restructurations.

44ième Congrès, 1989, p. 33-84 0959

GESTION DES ORGANISATIONS - EUROPE

VANDAMME, François

L'information des travailleurs dans les grandes firmes: L'approche spécifique de la CEE.

v. 39 , no 1,1984, p. $51-76$

0271

\section{BARKIN, Solomon}

The Flexibility Debate in Western Europe: The Current Drive to Restore Managements' Rights Over Personnel and Wages.

v. 42, no 1,1987, p. $12-44 \quad 0539$

\section{GESTION DES ORGANISATIONS - GRANDE-BRETAGNE}

BEAUMONT, P.B. TOWNLEY, B.

Non-Union American Plants in Britain:

Their Employment Practices.

v. 40, no 4,1985 , p. $810-825$

\section{GESTION DES ORGANISATIONS - ONTARIO}

BURT, Sandra

Voluntary Affirmative Action. Does it Work?

v. 41, no 3,1986, p. $541-551$

0491

\section{GESTION DES ORGANISATIONS - QUÉBEC}

RAINVILLE, Jean-Marie

Hierarchie ethnique dans la grande entreprise industrielle montréalaise.

v. 36, no 2,1981 , p. $336-360$

0027

\section{MILLER, Roger}

Les formes d'organisation dans l'industrie de la construction au Québec.

v. 37 , no 1,1982 , p. $164-176$

0102

RAINVILLE, Jean-Marie

La responsabilite fonctionnelle chez les cadres subalternes: Facteurs d'évolution des systèmes d'autorité.

v. 38 , no 4,1983 , p. $831-846$

0251

FABI, Bruno

Privé ou public: choix et transfert du secteur organisationnel.

v. 39, no 2,1984, p. $313-334$

0297
BHÉRER, Harold

Le salarié et la gestion générale de l'entreprise.

40ième Congrès, 1985, p. 205-222 0900

GODBOUT, Clément

Commentaires - Le salarié et la gestion générale de l'entreprise.

40ième Congrès, 1985, p. 222-228 0901

BÉLANGER, MarC LE BRASSEUR, Lola L'ITALIEN, Paul MÉNARD, Marius

Rappel de quelques expériences vécues.

44ième Congrès, 1989, p. 13-32 0958

GAGNON, Astrid BLANCHETTE, Sylvain QUINTAL, Pierre

Fusion d'entreprises publiques.

44ième Congrès, 1989, p. 129-154 0962

\section{GESTION DES \\ RESSOURCES HUMAINES}

\section{GESTION DES RESSOURCES HUMAINES}

WHITE, Terrence $\mathrm{H}$.

The Relative Importance of Work as a Factor in Life Satisfaction

v. 36 , no 1,1981 , p. $179-191 \quad 0008$

JAMAL, Muhammad

CRAWFORD, Ronald L.

Moonlighters: A Product of Deprivation or Aspiration?

v. 36 , no 2,1981 , p. $325-335$

0026

MARTIN, RosS BLACK, Errol

Roy J. Adams' Proposal for a Training Ley Scheme.

v. 36 , no 2,1981 , p. $414-417 \quad 0033$

THÉFIAULT, Roland LESAGE, Pierre B. BOISVERT, Maurice

L'absenteisme: importance, nature et remèdes.

v. 36 , no 4,1981 , p. $775-802 \quad 0073$

BARTH, Fichard T. BABA, Vishwanath V. Alienation Among Professionnal Engineers:

A Canadian - American Comparison.

v. 37 , no 1,1982 , p. $126-140 \quad 0100$ 


\section{GESTION DES RESSOURCES HUMAINES (suite)}

JAMAL, Muhammad

BABA, Vishwanath V.

MITCHELL, Vance F.

The Nature of Need Structure in Nonwork.

v. 37, no 3, 1982, p. 818-633 0142

MEALIA, David W.

A Macro/Micro Décision Model for the Training and Development Specialist.

v. 37 , no 3,1982 , p. $656-669$

0144

XISHAN, Yang MOORE, Larry F.

MARKGRAF, Herb

Behavioural Science Application in Vancouver Based Firms.

v. 38 , no 1, 1983, p. $120-141$

0184

\section{GARNIER, Bernard}

La gestion des conflits interpersonnels en milieu universitaire.

v. 38, no 2,1983 , p. $277-296$

0204

AMERNIC, J.H. ARANYA, N.

Organizational Commitment: Testing Two Theories.

v. 38 , no 2,1983 , p. $319-343 \quad 0206$

DIONNE, Pierre OUELLET, Gilles

Repenser l'évaluation.

v. 38, no 2,1983, p. $344-368$

0207

LAROUCHE, Viateur TRUDEL, Johanne La qualité de vie au travail et l'horaire variable.

v. 38 , no 3,1983 , p. $568-597$

0227

DOLAN, Shimon L. TZINER, Aharon

ROY, Denis

A Real Estate Agency's Level Analysis of the Climate-Performance Relationship.

v. 39 , no 1,1984 , p. 167-176

0276

PIGANIOL, Claude

Relations professionnelles et gestion des ressources humaines.

v. 39 , no 2,1984 , p. $285-300$

0295

LORRAIN, Jean BRUNET, LUC

Climat organisationnel, satisfaction au travail et perception du syndicalisme

v. 39 , no 4,1984 , p. $668-679$
MILLER, Roger

La strategie d'entreprise et la gestion des ressources humaines.

v. 40 , no 1. 1985 , p. $68-86$

0365

TZINER, Aharon DOLAN, Shimon L. Identifying Female Officer Potential: An Exploration in Predictors' Payoff

v. 40, no 1,1985, p. $87-98$

0366

BEAUMONT, P.B.

The Diffusion of Human Resource Management Innovations.

v. 40, no 2,1985 , p. $243-256 \quad 0384$

DORNSTEIN, Míriam ZOREF, Uri

Motivation for Changing Jobs, Personal Background Characteristics and Perceived Opportunity

v. 41 , no 1,1986 , p. $91-110 \quad 0451$

THACKER, James W. ROSEN, Hjalmar Dynamics of Employee Reactance to Company and Union Dual Allegiance Revisited and Expanded.

v. 41 , no 1,1986, p. $128-144$

0453

GODIN, Jean LE LOUARN, Jean-Yves Les mentors ont-ils un effet sur la progression de carrière?

v. 41, no 3,1986 , p. $505-518 \quad 0489$

BABA, Vishwanath V. KNOOP, Robert Organizational Commitment and Independence Among Canadian Managers

v. 42 , no 2,1987 , p. $325-344$

0564

BOURGEOIS, Robert-Paul WILS, Thierry Career Concepts, Personality and Values of Some Canadian Workers: An Exploratory Study

v. 42 , no 3,1987, p. $528-543$

0583

\section{LÉONARD, Christine}

VAN AMÉRINGEN, Marie-Reine

DOLAN, Shimon L. ARSENAULT, André Absenterisme et assiduité au travail: Deux moyens d'adaptation au stress.

v. 42, no 4, 1987, p. 774-789 0609

HACCOUN, Robert R. DUPONT, Serge

Une analyse des comportements de travailleurs masculins et féminins selon deux formes d'absence au travail.

v. 43 , no 1,1988 , p. $153-166 \quad 0634$ 


\section{GESTION DES RESSOURCES HUMAINES} (suite)

SINGH, Jang B. CROCKER, Olga

Operative and Espoused Personnel Selection Criteria of Managers.

v. 43 , no 1, p. 167-182

0635

\section{CAMIRÉ, André}

La productivité des intelligences.

v. 43 , no 2,1988, p. $322-340$

0649

SAVOIE, Dominique LAROUCHE, Viateur Le harcelement sexuel au travail: Définition et mesure du phénomène.

v. 43 , no 3,1988 , p. $509-530$

0665

WILS, Thierry LABELLE, Christiane GUÉRIN, Gilles LE LOUARN, Jean-Yves La gestion stratégique des ressources humaines: un reniement du róle social de l'entreprise?

v. 44, no 2,1989, p. $354-375$

0721

SALES, Carol A. LEVANONI, Eliahu KNOOP, Robert

Employee Performance as a Function of Job Orientation and Job Design.

v. 44, no 2,1989, p. $409-420$

BROSSARD, Michel

Les limites du modele-type du fonctionnement des cercles de qualité

v. 44, no 3,1989 , p. $552-568$

JAIN, Hem C.

AIDS: Need for Policy in the Workplace.

v. 44, no 4,1989 , p. $850-865$

0756

BÉLANGER, Laurent

Nouvelles formes d'organisation du travail, nouveaux modes de gestion et leur incidence sur le statut du salarié.

40ième Congrès, 1985, p. 171-196 0898

CÓTÉ, Marcel

Commentaires - Nouvelles formes d'organisation du travail, nouveaux modes de gestion et leur incidence sur le statut du salarié.

40ième Congrès, 1985, p. 196-203 0899

AUDET, Michel BÉLANGER, Laurent BOIVIN, Jean DÉOM, Esther

MERCIER, Jacques

Introduction - La mobilisation des ressour- ces humaines: tendances et impact.

41ième Congrès, 1986, p. 13-14 0905

BOIVIN, Jean

Émergence d'une réalité nouvelle en relations industrielles.

41ième Congrès, 1986, p. 17-30 0906

LAROUCHE, Viateur

La mobilisation des ressources humaines - orientations récentes.

41ième Congrès, 1986, p. 31-51 0907

SÉRIEYX, Hervé

L'entreprise du troisième type.

41ième Congrès, 1986, p. 85-97 0912

WILS, Thierry

Les travailleurs seront-ils du troisième type?

41ième Congrès, 1986, p. 99-109 0913

DOCQUIER, Gérard

Ressources humaines et défis du syndicalisme.

41ième Congrès, 1986, p. 149-156 0917

BÉLANGER, Laurent LIPSIG, Carla

MORIN, Fernand PÉRUSSE, Michel Introduction - Acquisition ou fusion d'entreprises et emplois.

44ième Congrès, 1989, p. XV-XVI 0956

MORIN, Fernand

Acquisition ou fusion d'entreprises et emplois: La problématique sous-jacente.

44ième Congrès, 1989, p. 1-12 0957

ANGERS, Georges GAGNON, Normand SIMARD, Jean

Le droit et le devoir à l'information.

44ième Congrès, 1989, p. 85-100 0960

LAMARCHE, André GAUTHIER, Michel

BLAIS, Michel GAGNON, Denise

Les conditions de travail au lendemain d'une fusion ou d'une réorganisation.

44ième Congrès, 1989, p. 101-127 0961

GOSSELIN, Alain FRENETTE, Jean-Guy DIONNE, Denis

Les acteurs d'une fusion ou d'une prise de pouvoir.

44ième Congrès, 1989, p. 155-173 0963 


\section{GESTION DES RESSOURCES HUMAINES} (suite)

MERCIER, Jacques POULIN-SIMON, Lise Le defi de la gestion des emplois: La problématique.

45ième Congrès, 1990, p. 1-5 0966

BÉLAND, Claude

Gestion des emplois et sécurité économique des employés.

45ième Congrès, 1990, p. 7-14 0967

BLONDIN, Michel

Le défi de la gestion de l'emploi: Pourquoi le relever?

45ième Congrès, 1990, p. 15-23 0968

\section{GESTION DES RESSOURCES HUMAINES - CANADA}

JAIN, Harish C.

Recruitment of Racial Minorities in Canadian Police Forces.

v. 42 , no 4,1987 , p. $790-805$

0610

ZUREIK, Elia MOSCO, Vincent

LOCHHEAD, Clarence

Telephone Workers' Reaction to the New Technology

v. 44, no 3,1989 , p. $507-531$

0734

LONG, Richard $\downarrow$.

Patterns of Workplace Innovation in Canada.

v. 44 , no 4,1989 , p. $805-826$

0754

WILS, Thierry BOURGEOIS, Robert-Paul LABELLE, Christiane

Planification des ressources humaines dans la fonction publique fédérale.

v. 45 , no 3,1990, p. $512-530$

0814

BERGERON, Jean-Louis

La gestion de l'embauche, de la promotion et du licenciement revue et corrigée par les Chartes.

43ième Congrès, 1988, p. 51-62 0944

DUCHARME, Claude

LECLERCQ, Dominique

WESTMORELAND-TRAORE, Juanita

Commentaires - La gestion de l'embauche, de la promotion et du licenciement revue et corrigee par les Chartes.

43ième Congrès, 1988, p. 63-82

\section{GESTION DES RESSOURCES HUMAINES -} ÉTATS-UNIS

ICHNIOWSKI, Casey DELANEY, John T. LEWIN, David

The New Resource Management in U.S. Workplaces: Is it Really New and is it Only Nonunion?

v. 44 , no 1,1989 , p. $97-123 \quad 0703$

KOCHAN, Thomas A.

MCKERSIE, Robert B.

Future Directions for American Labor and Human Resources Policy.

v. 44, no 1, 1989, p. 224-248 0709

BARKIN, Solomon

Human Resources Management Examines itself and its Limitations

v. 44, no 3,1989 , p. $691-702 \quad 0743$

GESTION DES RESSOURCES HUMAINES EUROPE

WEIERMAIR, Klaus

Apprenticeship Training in European Countries: The Lessons for Canada.

v. 37, no 3,1982 , p. $557-574 \quad 0139$

\section{GESTION DES RESSOURCES HUMAINES -} QUÉBEC

LE LOUARN, Jean-Yves

Les professeurs d'université et leur travail. v. 37 , no 2,1982, p. $385-402$

0125

TOUPIN, Louis LESSARD, Claude CORMIER, Roger A. VALOIS, Paul La satisfaction au travail chez les enseignantes et enseignants au Québec.

v. 37 , no 4,1982 , p. $805-826 \quad 0160$

DOLAN, Shimon L. ARSENAULT, André LIZOTTE, Jean-Paul ABENHAIM, Lucien L'absentéisme hospitalier au Québec: Aspects culturels et socio-démographiques. v. 38 , no 1,1983 , p. $45-57$

0179

VEILLEUX, Diane

Les clauses de promotion et de transfert chez les infirmières et les infirmiers.

v. 38 , no 2,1983 , p. $229-253$

0202

LARRIVÉE, Liette AMBROISE, Gérald d' Difficultés de recrutement dans les PME québécoises: Quelques causes et solutions possibles.

v. 44 , no 3,1989 , p. $487-506 \quad 0733$ 


\section{GESTION DES RESSOURCES HUMAINES - QUÉBEC (sulte)}

SAVOIE, Dominique LAROUCHE, Viateur Le harcèlement sexuel au travail: Résultats de deux études québécoises.

v. 45 , no 1,1990 , p. $38-62$

0773

CASAVANT, Jean-Claude

La stratégie de gestion des ressources humaines chez Abitibi Price Inc.

41ième Congrès, 1986, p. 53-61 0908

LEMAIRE, Alain

La communication directe chez Cascades Inc.

41ième Congrès, 1986, p. 63-68 0909

NÉRON, Roger

Le projet d'entreprise de Culinar Inc.

41ième Congrès, 1986, p. 69-76 0910

BOUCHER, Lysette

Les limites des nouvelles approches en gestion des ressources humaines.

41ième Congrès, 1986, p. 77-84 0911

DULUDE, Yves

Le conflit: la gestion au banc des accusés.

41ième Congrès, 1986, p. 111-133 0914

LAMARCHE, Pierre

Commentaires - Le conflit: la gestion au banc des accusés.

41ième Congrès, 1986, p. 133-136 0915

LALANDE, Serge

L'experience de la compagnie Gaz Métropolitain.

41ième Congrès, 1986, p. 137-146 0916

HÉTU, Jean-Paul

La nouvelle gestion des ressources humaines - mythe ou réalité?

41ième Congrès, 1986, p. 167-172 0919

GAGNON, Astrid BLANCHETTE, Sylvain QUINTAL, Pierre

Fusion d'entreprises publiques.

44ième Congrès, 1989, p. 129-154 0962

FRAPPIER-DESROCHERS, Monique

La pratique de gestion des ressources humaines dans les entreprises.

45ième Congrès, 1990, p. 25-39
LEVINE, David

Négocier la flexibilite: Reconversion des heures de la liste de rappel en postes à temps complet.

45ièrne Congrès, 1990, p. $41.47 \quad 0970$

GOBEILLE, Kenneth R.

Négocier la flexibilite: Le travail à temps partiel.

45ième Congrès, 1990, p. 48-58 0971

BROUILLET, Normand

Négocier la flexibilité: Réaction syndicale aux stratégies patronales.

45ième Congrès, 1990, p. 70-81 0974

LE LOUARN, Jean-Yves

Les emplois atypiques et l'efficacité de la gestion des ressources humaines.

45ième Congrès, 1990, p. 93-103 0976

BOUDREAULT, Pierre GARON, Jacques PAGÉ, Lorainne PAQUETTE, Pierre

Les emplois atypiques et l'efficacité de la gestion des ressources humaines - Commentaires.

45ième Congrès, 1990, p. 103-116 0977

\section{MAIN-D'OEUVRE}

\section{MAIN-D'OEUVRE}

SARTIN, Pierrette

Le travail à temps partiel

v. 36 , no 2,1981, p. $307-324$

0025

LIPSIG-MUMME, Carla

The Renaissance of Homeworking in Developped Economies.

v. 38 , no 3,1983 , p. $545-567 \quad 0226$

CROWLEY, R.W. HUTH, E.

An International Comparison of Work Sharing Programs.

v. 38 , no 3,1983 , p. $636-647 \quad 0230$

\section{DEMERS, Marie}

Chômage chez les jeunes: Conséquences psychologiques et sociales.

v. 38 , no 4,1983 , p. $785-814 \quad 0249$

ROSSILION, Claude

L'OIT et l'élimination de la discrimination dans l'emploi

v. 40, no 1,1985, p. $03-26 \quad 0362$ 


\section{MAIN-D'OEUVRE (suite)}

DORNSTEIN, Miriam ZOREF, Uri

Motivation for Changing Jobs, Personal Background Characteristics and Perceived Opportunity

v. 41 , no 1,1986 , p. $91-110$

0451

GUÉRIN, Gilles HÉBERT, Michel

Les obstacles rencontrés par des personnes de 45 à 64 ans à la recherche d'un emploi.

v. 45, no 2,1990, p. $235-267$

0792

BOULARD, René DESCHÊNES, Jean-Paul LAROCQUE, Alain RONDEAU, Claude Introduction - La réduction de la durée du travail.

36ième Congrès, 1981, p. 13-14 0832

LAROCQUE, ALAIN

La réduction du temps de travail: un droit ou un privilège?

36ième Congrès, 1981, p. 15-29 0833

VALASKAKIS, Kimon

Le temps libéré: à quels coûts? 36ième Congrès, 1981, p. 61-74

0836

POULIN-SIMON, Lise FORTIN, Bernard Commentaires - Le temps libéré: à quels coûts?

36ième Congrès, 1981, p. 75-86 0837

FERLAND, Gilles HAMELIN, Jean-Marie PROULX, Pierre-Paul

Table ronde - Sommes-nous tous égaux face à la diminution du temps passé au travail?

36ième Congrès, 1981, p. 87-121 0838

MASSE, Gilles

Le temps passé au travail: un élément encore négociable?

36ième Congrès, 1981, p. 123-135 0839

DESCHÊNES, Jean-Paul

Commentaires - Le temps passé au travail: un élément encore négociable?

36ième Congrès, 1981, p. 136-144 0840

FRAPPIER-DESROCHERS, Monique

La technologie: un substitut à la durée du travail?

36ième Congrès, 1981, p. 193-218 0843
AOUST, Fernand d' DÉOM, André HARVEY, Pierre

Table ronde - La réduction du temps passé au travail: un moyen de lutte contre le chómage.

36ième Congrès, 1981, p. 219-246 0844

CROZIER, Michel

La diminution du temps de travail: un phénomène inéluctable?

36ième Congrès, 1981, p. 247-263 0845

DÉOM, Esther

Commentaires - Evolution du statut $d u$ salarié en raison des nouvelles formes d'emplois: L'exemple du travail à temps partiel au Québec.

40ième Congrès, 1985, p. 161-170 0897

GODBOUT, Clément RIOUX, Claude

Le libre-échange canado-américain et le marché du travail.

44ième Congrès, 1989, p. 175-188 0964

MERCIER, Jacques POULIN-SIMON, Lise Le défi de la gestion des emplois: La problématique.

45ième Congrès, 1990 , p. 1-5 0966

BÉLAND, Claude

Gestion des emplois et sécurité économique des employés.

45ième Congrès, 1990, p. 7-14 0967

BLONDIN, Michel

Le défi de la gestion de l'emploi: Pourquoi le relever?

45ième Congrès, 1990, p. 15-23 0968

BELLEMARE, Diane

Les emplois de l'avenir et les défis de la société.

45ième Congrès, 1990, p. 117.128 0978

\section{MAIN-D'OEUVRE - AMÉRIQUE LATINE} ZAPATA, Francisco

La proteccion del empleo en las américas. v. 44 , no 1, 1989, p. 177-194 0707 


\section{MAIN-D'OEUVRE - CANADA}

GRUBEL, Herbert G. MAKI, Dennis R.

A Note on the Effects of Unemployment Insurance, Minimum Wage Legislation and Trade Union Growth on Reported Unemployment Rates in Canada, 1950-75

v. 36 , no 4,1981 , p. $922-927$

0079

HASAN, Abrar GERA, Surendra

Reservation Wages in Canadian Labour Markets.

v. 37 , no 1,1982, p. $65-92$

0098

JAIN, Harish C.

Race ans Sex Discrimination in Employment in Canada: Theories, Evidence and Policies.

v. 37 , no 2,1982 , p. $344-366$

0123

MELTZ, Noah M.

Labour Market Information in Canada: The Current Situation and a Proposal.

v. 37 , no 2,1982, p. $431-437$

0127

MAGUN, Sunder

The Rise of Service Employment in the Canadian Economy.

v. 37 , no 3,1982 , p. $528-556$

0138

MAGUN, Sunder

The Placement Activity of the Canadian Employment Agency.

v. 38 , no 1,1983 , p. $72-94$

0181

JAIN, Harish C.

Micro-electronics Technology and Industrial Relations.

v. 38, no 4,1983 , p. $869-879$

0254

HOLMES, R.H. PINFIELD, L.T.

Logit Analysis of Employee Turnover in a Remote Community

v. 39 , no 3,1984, p. $553-569$

0320

BURKE, Ronald J.

Trainee Experiences in Industrial Retraining Programs: A Case Study

v. 39 , no 3.1984 , p. $570-577$

0321

MAGUN, Sunder

The Effects of Technological Changes on the Labour Market in Canada

v. 40, no 4,1985 , p. $720-746$

0425
LAMSON, Cynthia

On the Line: Women and Fish Plant Jobs in Atlantic Canada.

v. 41 , no 1,1986 , p. $145-156$

0454

BEACH, Charles M. KALISKI, S.F.

The Impact of Recession on the Distribution of Annual Unemployment.

v. 41 , no 2,1986 , p. $317-328$

0471

WEIERMAIR, Klaus

Secular Changes in Youth Labour Markets and Youth Unemployment in Canada.

v. 41 , no 3,1986 , p. $469-490$

0487

BETCHERMAN, Gordon

Labour Market Imbalances in Canada, 1966-1983

v. 41 , no 4,1986 , p. $802-816 \quad 0519$

DOOLEY, Martin D.

Within-Cohort Earnings Inequality Among Canadian Men: 1971-1982

v. 42 , no 3,1987 , p. $594-611$

0587

MERCIER, Jacques

Effets du salaire minimum sur l'emploi: Résultats des études économétriques canadiennes et québécoises.

v. 42 , no 4,1987 , p. $806-830 \quad 0611$

REID, Frank

Economic Aspects of Mandatory Retirement: The Canadian Experience.

v. 43, no 1,1988 , p. $101-114$

0631

KAMEL, Nawal ROY, Paul-Martel

Temps supplémentaire et création d'emplois: Le cas du Canada, du Québec et de l'Ontario.

v. 43 , no 2,1988 , p. $412-430 \quad 0653$

MELTZ, Noah M.

Job Security in Canada

v. 44 , no 1,1989 , p. 149-161 0705

JAIN, Harish C.

Racial Minorities and Affirmative Action/Employment Equity Législation in Canada.

v. 44 , no 3,1989 , p. $593-6140738$

GRAYSON, J. Paul

The Political Consequences of Unemployment: An Application of the "Power Model" of Blue-Collar Radicalism

v. 44 , no 3,1989 , p. $635-653 \quad 0740$ 
MAIN-D'OEUVRE - CANADA (suite)

HUGUES, Karen D.

Office Automation: A Review of the Litterature

v. 44 , no 3,1989 , p. $654-679$

0741

LAVALLÉE, Diane CHARLAND, Gaston LEPAGE, Brigitte MASSÉ, Henri MERCIER, Jean

Les emplois de l'avenir et les défis de la société - Commentaires.

45ième Congrès, 1990, p. 128-148 0979

MAIN-D'OEUVRE - ÉTATS-UNIS

LOVELL, Malcolm R. Jr.

Employment Security

v. 44 , no 1. 1989 , p. $162-176$

0706

MAIN-D'OEUVRE - EUROPE

BARKIN, Solomon

The Flexibility Debate in Western Europe: The Current Drive to Restore Managements' Rights Over Personnel and Wages.

v. 42 , no 1,1987, p. $12-44$

0539

MAIN-D'OEUVRE - GRANDE-BRETAGNE

CAMPBELL, Adrian WARNER, Malcolm

Workplace Relations, Skills-Training and Technological Change at Plant-Level.

v. 43, no 1,1988, p. 115-132

0632

\section{MAIN-D'OEUVRE - ONTARIO}

BURKE, Ronald J.

Consequences of not Working Sixteen

Months After a Plant Closing

v. 40, no 1,1985, p. $162-169$

0370

LEBRASSEUR, Rolland

Retirement and Skill Issues in Northern Ontario Industries.

v. 45, no 2,1990 , p. $268-282 \quad 0793$

\section{MAIN-D'OEUVRE - QUÉBEC}

FORTIN, Pierre

Le chómage des jeunes au Québec: Aggravation et concentration, 1966-1982

v. 39 , no 3,1984 , p. $419-448$

0313

MERCIER, Jacques

Les effets du salaire minimum sur l'emploi des jeunes au Québec.

v. 40 , no 3,1985 , p. $431-457$
GRANT, Michel ROSE, Ruth

Encadrement du travail à domicile dans l'industrie du vêtement au Québec.

v. 40, no 3,1985, p. $473-494$

0405

ROY, Paul-Martel

Licenciements collectifs, licenciements individuels et emploi au Québec: 19791984

v. 42 , no 3,1987 , p. $577-593 \quad 0586$

SODERSTROM, Lee

Some Effects of Unemployment on the Health of Unemployed Quebec Workers.

v. 43 , no 2,1988 , p. $341-377 \quad 0650$

LARRIVÉE, Liette AMBROISE, Gérald d' Difficultés de recrutement dans les PME québécoises: Quelques causes et solutions possibles.

v. 44 , no 3,1989 , p. $487-506$

0733

ROY, Paul-Martel

Aspects dynamiques du marché du travail au Québec.

v. 45 , no 2,1990, p. $283-299 \quad 0794$

LANGLOIS, Simon

Le travail à temps partiel: Vers une polarisation de plus en plus nette.

v. 45 . no 3,1990, p. $548-565 \quad 0816$

BEAUSOLEIL, Gilles GAUTHIER, Hervé La législation: consolidation ou innovation?

36ième Congrès, 1981, p. 145-172 0841

LOCKWELL, LUC M. LORTIE, Pierre

Commentaire - La législation: consolidation ou innovation?

36ième Congrès, 1981, p. 173-192 0842

BERNIER, Colette

Évolution du statut du salarié en raison de nouvelles formes d'emploi: L'exemple du travail à temps partiel au Québec.

40ième Congrès, 1985, p. 137-160 0896

FRAPPIER-DESROCHERS, Monique

La pratique de gestion des ressources humaines dans les entreprises.

45ième Congrès, 1990, p. 25-39 0969 


\section{MÉTHODOLOGIE}

\section{MÉTHODOLOGIE}

POOLE, Michael

Perceptions of Trade Union Members and the Social Action Perspective.

v. 36 , no 1,1981, p. 35-62

0002

PETERSON, Richard B. TRACY, Lane CABELLY, Alan

Problem Solving in Labor Negotiations: Retest of a Model

v. 36 , no 1,1981 , p. $87-105$

0004

MARTIN, Ross BLACK, Errol

Roy J. Adams' Proposal for a Training Levy Scheme.

v. 36 , no 2,1981 , p. $414-417$

0033

CHAISON, Gary N. ROSE, Joseph B. The Structure and Growth of the Canadian National Unions.

v. 36 , no 3,1981 , p. $530-551$

0052

BOULARD, René

Le professionnalisme au Québec: Une étude empirique.

v. 36, no 3,1981 , p. $648-662$

0058

THÉRIAULT, Roland LESAGE, Pierre B. BOISVERT, Maurice

L'absentéisme: importance, nature et remèdes.

v. 36, no 4,1981 , p. $775-802$

0073

YOUNG, Stanley

Industrial Relations: A Paradigmatic Analysis.

v. 37 , no 1,1982 , p. $32-52$

0096

BARTH, Richard T. BABA, Vishwanath V. Alienation Among Professionnal Engineers: A Canadian - American Comparison.

v. 37 , no 1,1982 , p. $126-140 \quad 0100$

DÉOM, Esther

La négociation collective chez les fonctionnaires et les enseignants québécois: 1975-1976.

v. 37 , no 1,1982 , p. 141-163 0101

CHAISON, Gary N.

A Note on the Critical Dimensions of the Union Merger Process.

v. 37, no 1,1982 , p. $198-206$

0104
WALSH, William D.

A Time Series Analysis of Female Labour Force Participation Rates Disaggregated by Marital Status.

v. 37 , no 2,1982, p. $367-384 \quad 0124$

WILLIAMS, C. Glyn

The Role of Unions in Inflation: A Survey Article

v. 37, no 3, 1982, p. $498-527 \quad 0137$

JAMAL, Muhammad

BABA, Vishwanath $V$.

MITCHELL, Vance F.

The Nature of Need Structure in Nonwork. v. 37, no 3, 1982, p. 618-633 0142

MEALIA, David W.

A Macro/Micro Décision Model for the Training and Development Specialist.

v. 37, no 3, 1982, p. 656-669 0144

MAKI, Dennis R.

Political Parties and Trade Union Growth in Canada.

v. 37 , no 4,1982 , p. $876-886 \quad 0163$

VINET, Alain BÉGIN, Clermont

THÉRIAULT, Gilles

La santé des employés de bureau: Le cas de la fonction publique québécoise.

v. 37, no 4,1982 , p. 887-904 0164

DOLAN, Shimon L. ARSENAULT, André LIZOTTE, Jean-Paul ABENHAIM, Lucien L'absentéisme hospitalier au Québec: Aspects culturels et socio-démographiques.

v. 38 , no 1,1983 , p. $45-57$

0179

EASTMAN, Byron

Canadian Union Growth

v. 38 , no 1,1983 , p. $58-71$

0180

MAKI, Dennis R.

Trade Unions and Productivity: Conventional Estimates.

v. 38, no 2,1983 , p. $211-228 \quad 0201$

AMERNIC, J.H. ARANYA, N.

Organizational Commitment: Testing Two Theories.

v. 38, no 2, 1983, p. 319-343 0206

DIONNE, Pierre OUELLET, Gilles

Repenser l'évaluation.

v. 38 , no 2,1983 , p. $344-368$

0207 


\section{MÉTHODOLOGIE (suite)}

ADAMS, Roy J.

Competing Paradigms in Industrial Relations.

v. 38 , no 3,1983 , p. $508-531$

0224

CAMERON, Samuel

An International Comparison of the Volatility of Strike Behavior

v. 38, no 4,1983 , p. 767-784

0248

COUSINEAU, Jean-Michel

\section{LACROIX, Robert}

La détermination des avantages sociaux au Canada

v. 39, no 1,1984, p. $3-22$

0268

MAKI, Dennis R. STRAND, Kenneth The Determinants of Strike Activity: An Interindustry Analysis

v. 39 , no 1,1984 , p. $77-92$

0272

LAROUCHE, Viateur DÉOM, Esther L'approche systémique en relations industrielles

v. 39 , no 1,1984 , p. $114-145 \quad 0274$

DEVORETZ, D.J. REED, Clyde G.

Evidence from the Skilled-Unskilled Canadian Wage Index.

v. 39 , no 3,1984 , p. $526-537 \quad 0318$

HOLMES, R.H. PINFIELD, L.T.

Logit Analysis of Employee Turnover in a Remote Community

v. 39 , no 3,1984, p. $553-569 \quad 0320$

MILNE, William J. ROSS, Thomas W.

The Cyclical Variation of Wage Premiums in the Canadian Manufacturing Industries

v. 39, no 4,1984 , p. $762-773$

0343

TZINER, Aharon DOLAN, Shimon $L$. Identifying Female Officer Potential: An Exploration in Predictors' Payoff

v. 40, no 1,1985 , p. $87-98$

SHIROM, Arie

The Labor Relations System: A Proposed Conceptual Framework

v. 40, no 2,1985 , p. $303-323 \quad 0387$

EASTMAN, Byron

A Catastrophe Theory on Union Behaviour.

v. 40, no 2,1985, p. $340-350 \quad 0389$
LACROIX, Robert

A Microeconometric Analysis of the Effects of Strikes on Wages

v. 41 , no 1,1986 , p. 111-127 0452

KUMAR, Pradeep DOW, Bradley

Econometric Analysis of Union Membership Growth in Canada, 1935-1981

v. 41 , no 2,1986 , p. $236-255$

0467

ROHLING, Thomas A.

Screening and Human Capital Theory: An Empirical Test

v. 41 , no 4,1986 , p. $817-826 \quad 0520$

DASTMALCHIAN, Ali

ADAMSON, Raymond BLYTON, Paul

Developing a Measure of Industrial Relations Climate

v. 41 , no 4,1986 , p. $851-859$

0524

RESHEF, Yonatan

A Typology of Shop Stewards: A Confirmatory Factor Analysis

v. 42 , no 1,1987 , p. $150-167$

0545

GILSON, Clive H.J. SPENCER, lan S. Trade Union Growth: A Marketing Model. v. 42 , no 4,1987 , p. $756-773 \quad 0608$

COUSINEAU, Jean-Michel RABEAU, Yves Une méthodologie de comparaison des salaires pour les emplois spécifiques du secteur public.

v. 43 , no 1,1988, p. $85-100$

0630

KUMAR, Pradeep

Estimates of Unionism and Collective Bargaining in Canada.

v. 43 , no 4,1988 , p. $757-779 \quad 0683$

LACROIX, Robert LESPÉRANCE, André Les nouvelles lois du travail et l'activité de grève.

v. 43 , no 4,1988 , p. $812-828 \quad 0685$

SCHELL, Bernadette

LEBRASSEUR, Rolland RENAUD, Robert Predictors of Acceptance of Early Retirement Offers for Workers.

v. 44 , no 2,1989 , p. $376-392 \quad 0722$ 


\section{MÉTHODOLOGIE (suite)}

GAGNON, Yves-Chantal

LANDRY, Maurice

Les changements technologiques: Une stratégie d'étude exploratoire

v. 44 , no 2,1989 , p. $421-447$

0725

VINET, Alain VÉZINA, Michel

BRISSON, Chantal

Des lésions professionnelles méconnues:

le cas des opératrices de l'industrie du vêtement.

42ième Congrès, 1987, p. 21-38

0925

\section{ORGANISATIONS PATRONALES}

\section{ORGANISATIONS PATRONALES - QUÉBEC}

THWAITES, James

Business "Organizes": The Early Years in Quebec.

v. 36 , no 2,1981 , p. $403-411$

0031

\section{ORGANISATIONS SYNDICALES}

\section{ORGANISATIONS SYNDICALES}

CHAISON, Gary N.

A Note on the Critical Dimensions of the Union Merger Process.

v. 37, no 1,1982 , p. 198-206 0104

BROSSARD, Michel

La stratégie syndicale face aux groupes semi-autonomes de production: Hypothèse pour l'analyse du cas Rushton.

v. 37 , no 3,1982 , p. $670-683$

0145

PEITCHINIS, Stephen G.

The Attitude of Trade Unions Towards Technological Changes.

v. 38, no 1, 1983, p. 104-119 0183

LIPSIG-MUMME, Carla

La crise du syndicalisme nord-américain: eléments d'interprétation.

v. 39 , no 2,1984, p. $275-284$

0294

GILSON, Clive H.J. SPENCER, Ian S. Trade Union Growth: A Marketing Model. v. 42 , no 4,1987 , p. 756-773

0608

STRATTON, Kay RESHEF, Yonatan Private Sector Unions and Strategic Planning: A Research Agenda v. 45 , no 1,1990, p. $76-93$
CAIRE, Guy

Syndicalisme, nouvelles technologies et incertitudes économiques.

38ième Congrès, 1983, p. 225-265 0872

FRANCOEUR, Jean

Le syndicalisme et le nouveau travail.

41ième Congrès, 1986, p. 157-166 0918

\section{ORGANISATIONS SYNDICALES -}

\section{AMÉRIQUE LATINE}

FALABELLA, Gonzalo

El sindicato en el ano 2000 temas, y desafios.

v. 44 , no 1,1989, p. $47-61 \quad 0701$

ORGANISATIONS SYNDICALES - CANADA PONAK, Allen MOORE, Larry $F$.

Canadian Bank Unionism: Perspectives and Issues

v. 36 , no 1,1981 , p. $3-34$

0001

TUCK, Hugh

Canadian Railways and Unions in the Running Trades, 1865-1914

v. 36 , no 1,1981 , p. $106-131 \quad 0005$

CHAISON, Gary N. ROSE, Joseph B.

The Structure and Growth of the Canadian National Unions.

v. 36, no 3,1981, p. $530-551$

LOWE, Graham S.

Causes of Unionization in Canadian Banks. v. 36 , no 4,1981 , p. $865-893$

AOUST, Claude d' DELORME, François The Origin of the Freedom of Association and of the Right to Strike in Canada: An Historical Perspective.

v. 36 , no 4,1981 , p. $894-921$

0078

GILES, Anthony

The Canadian Labour Congress and Tripartism.

v. 37 , no 1,1982 , p. $93-125$

0099

CHAISON, Gary N. ANDIAPPAN, P.

Characteristics of Female Union Officer in Canada.

v. 37 , no 4,1982 , p. $765-7790158$

MAKI, Dennis R.

Political Parties and Trade Union Growth in Canada.

v. 37 , no 4,1982 , p. $876-886 \quad .0163$ 
ORGANISATIONS SYNDICALES - CANADA (suite)

EASTMAN, Byron

Canadian Union Growth

v. 38 , no 1,1983, p. 58-71

0180

WILLIAMS, C. Brian

The Impact of Labor Migration: The International Molders and Allied Workers Union in Canada, 1860-1885.

v. 39 , no 2,1984 , p. $335-364$

0298

ADAMS, Roy J.

The Extent of Collective Bargaining in Canada

v. 39 , no 4,1984 , p. $655-667 \quad 0336$

JONES, J.C.H. LAUDADIO, L.

Organized Labour, Regional Political Bias and the Canadian Tariff Structure

v. 39 , no 4,1984 , p. $695-709$

0339

\section{VERGE, Pierre}

Evolution de la protection juridictionnelle de l'accès au syndicat.

v. 39, no 4,1984 , p. $710-743$

0340

BENEDICT, Daniel

The 1984 GM Agreement in Canada: Significance and Consequences.

v. 40 , no 1,1985 , p. $27-47$

0363

BÉLANGER, Jacques MERCIER, Jacques Le plafonnement de la densité syndicale au Québec et au Canada.

v. 41 , no 1,1986, p. $28-52$

0448

KUMAR, Pradeep DOW, Bradley

Econometric Analysis of Union Membership Growth in Canada, 1935-1981

v. 41 , no 2,1986 , p. $236-255$

0467

SOLOMON, Norman A. ANDIAPPAN, P. SHAND, Dan

Canadian National Union Presidents: An Empirical Study.

v. 41, no 3,1986, p. $491-504$

0488

WILLIAMS, C. Brian

International Trade Unionism: The United Mine Workers in Eastern Canada, $1900-1920$

v. 41 , no 3,1986, p. $519-540$

0490
STOREY, Robert $\mathrm{H}$.

The Struggle to Organize Stelco and Dofasco

v. 42, no 2,1987 , p. $366-385$

0566

HÉBERT, Gérard

L'évolution du syndicalisme au Canada: Comment un mouvement devient institution.

v. 42 , no 3,1987 , p. $500-519$

0581

\section{TEMPLER, Andrew}

\section{SOLOMON, Norman A.}

Unions and Technology: A Survey of Union Use of Information Technology.

v. 43, no 2,1988 , p. $378-393$

0651

SEFTON MACDOWELL, Laurel

The Career of a Canadian Trade Union Leader: C.H. Millard 1937-1946

v. 43, no 3,1988 , p. $609-632 \quad 0670$

\section{KUMAR, Pradeep}

Estimates of Unionism and Collective Bargaining in Canada.

v. 43 , no 4,1988 , p. $757-7790683$

MAC NEIL, Michael

Unions, Politics and Law in Canada.

v. 43 , no 4,1988 , p. $847-868$

0687

DION, Gérard HÉBERT, Gérard

L'avenir du syndicalisme au Canada.

v. 44 , no 1,1989 , p. 5-24

0699

COUSINEAU, Jean-Michel

NAJEM, Elmustapha

L'effet du développement de la petite entreprise sur l'évolution du syndicalisme au Canada.

v. 45, no 3,1990, p. $467-480$

0812

PERREAULT, Charles

L'entreprise peut-elle se passer d'un syndicat?

38ième Congrès, 1983, p. 173-182 0868

GAUTHIER, Fernand ROY, Paul-Martel Commentaires - L'entreprise peut-elle se passer d'un syndicat?

38ième Congrès, 1983, p. 183-195 0869

PÉPIN, Marcel

L'actualisation du mouvement syndical.

43ième Congrès, 1988, p. 209-221 0952 


ORGANISATIONS SYNDICALES - CANADA
(suite)
GAGNON, Mona-Josée LEBEAU, Ernest
JOHNSTON, Raymond
Commentaires - l'actualisation du mouve-
ment syndical.
43ième Congrè, 1988, p. $222-2360953$
ORGANISATIONS SYNDICALES -
ÉTATS-UNIS
RESHEF, Yonatan
A Typology of Shop Stewards: A Confirma-
tory Factor Analysis
v. 42, no 1, 1987, p. 150-167
BARKIN, Solomon
Institutional Economics and the American
Trade Union Movement.
v. 43, no 3,1988, p. $491-508$
FREEMAN, Richard B.
What Does the Future Hold for. U.S. Unio-
nism?
v. 44, no 1, 1989, p. $25-46$

ORGANISATIONS SYNDICALES - FRANCE DION, Stéphane

Syndicats et politique au niveau municipal en France.

v. 39 , no 3.1984 , p. $466-485$

0315

\section{ORGANISATIONS SYNDICALES - GRANDE-BRETAGNE}

POOLE, Michael

Perceptions of Trade Union Members and the Social Action Perspective.

v. 36 , no 1,1981 , p. $35-62$

0002

BEAUMONT, P.B.

Statutory Recognition Provisions in Britain, 1976-80

v. 38 , no 4,1983 , p. $744-766$

0247

BEAUMONT, P.B. HARRIS, R.I.D.

High Technology Industries and Non-Union Establishments in Britain.

v. 43, no 4,1988 , p. $819-846 \quad 0686$

\section{ORGANISATIONS SYNDICALES - GRÈCE}

KATASANEVAS, Theodoros

Trade Unions in Greece

v. 40 , no 1,1985 , p. $99-114$

0367
ORGANISATIONS SYNDICALES - ONTARIO MOUNT, Joan SIEGEL, Jacob P.

Resources and Results in Union Rivalry: $A$ Case Study

v. 38, no 4,1983, p. $815-830 \quad 0250$

MCSHANE, Steven L.

Sources of Attitudinal Union Militancy

v. 40 , no 2,1985 , p. $284-3020386$

ORGANISATIONS SYNDICALES - QUÉBEC DELORME, François LAFRANCE, Victor La concurrence dans un contexte de pluralisme syndical: Quelques données sur le Québec.

v. 37 , no 3,1982 , p. $575-605 \quad 0140$

COPP, Terry

The Rise of Industrial Unions in Montreal 1935-1945

v. 37, no 4, 1982, p. $843-8750162$

ROBAKC, Léo

Les travailleurs du Québec au XXième siècle.

v. 40 , no 4,1985 , p. $826-846 \quad 0430$

GEORGE, Kenneth

L'accès à la syndicalisation

v. 41, no 3, 1986, p. 583-595 0495

WILLIAMS, C. Brian

International Unionism: The Papermakers in Eastern Canada, 1930-1945

v. 42, no 1, 1987, p. 110131 0543

DÉOM, Esther

La syndicalisation des journalistes dans quelques quotidiens québécois.

v. 42 , no 4,1987 , p. $737-755 \quad 0607$

RONDEAU, Claude BÉLANGER, Jacques BOIVIN, Jean SEXTON, Jean

Introduction - La syndicalisation dans le secteur privé au Québec.

38ième Congrès, 1983, p. 13-14 0858

RONDEAU, Claude BÉLANGER, Jacques Le syndicalisme dans l'entreprise: tendances récentes et analyse.

38ième Congrès, 1983, p. 15-36 0859

INGERMAN, Sidney

La syndicalisation dans le contexte économique québécois.

38ième Congrès, 1983, p. 37-69 0860 
ORGANISATIONS SYNDICALES - QUÉBEC (suite)

BONIN, Bernard

Commentaires - La syndicalisation dans le contexte économique québécois.

38ième Congrès, 1983, p. 70-76

0861

LAFLAMME, Gilles

L'impact du régime de relations du travail sur la syndicalisation.

38ième Congrès, 1983, p. 77-90 0862

LEBEL, Hélène

Commentaires - L'impact du régime de relations du travail sur la syndicalisation.

38ième Congrès, 1983, p. 91-100 0863

DESMARAIS, Jacques

Les idées de réforme sur la syndicalisation au Québec depuis 1964.

38 ième Congrès, 1983, p. 101-116 0864

MERCILLE, Pierre NADEAU, Bertin

OLIVIER, Madeleine

Table ronde - Organisation syndicale: difficultés et motifs de résistance.

38ième Congrès, 1983, p. 117-134 0865

SIMARD, Monique SLIGER, Raymond

Pourquoi et comment accroître la syndicalisation dans le secteur privé?

38ième Congrès, 1983, p. 135-1620866

DION, Gérard

Commentaires - Pourquoi et comment accroitre la syndicalisation dans le secteur prive?

38ième Congrès, 1983, p. 162-171 0867

FRÉCHETTE, Raynald

La politique gouvernementale en matière de syndicalisation.

38ième Congrès, 1983, p. 197-206 0870

BONENFANT, Claire DUFOUR, Ghislain

HÉTU, Jean-Paul

Table ronde - Les réactions du milieu.

38ième Congrès, 1983, p. 207-223 0871

HÉTU, Jean-Paul

La nouvelle gestion des ressources humaines - mythe ou réalité?

41ième Congrès, 1986, p. 167-172 0919
LABERGE, Louis

Les préalables à une réorientation des relations du travail au Québec.

41ième Congrès, 1986, p. 173-175 0920

LAROSE, Gérald

L'adaptation du syndicalisme - un phénomène de continuité.

41ième Congrès, 1986, p. 177-179 0921

\section{ORGANISATIONS SYNDICALES -}

\section{SASKATCHEWAN}

SASS, Robert

The Saskatchewan Trade Union Act. 1983:

The Public Battle.

v. 40, no 3,1985, p. $591-622$

0411

WETZEL, Kurt GALLAGHER, Daniel G. MAXEY, Charles T.

Impact of Nursing Unionism in the Hospital Industry: The Saskatchewan Experience.

v. 40 , no 4,1985, p. $793-809 \quad 0428$

NG, Ignace

Determinants of Union Commitment Among University Faculty.

v. 44 , no 4,1989 , p. $769-784 \quad 0752$

\section{RELATIONS DU TRAVAIL}

\section{RELATIONS DU TRAVAIL}

PECCEI, Ricardo WARNER, Malcolm

Industrial Relations, Strategic Importance, and Decision - Making.

v. 36 , no 1,1981 , p. $132-151 \quad 0006$

\section{BARKIN, Solomon}

Productivity Measures in Collective Bargajning.

v. 36, no 2,1981, p. $361-370 \quad 0028$

BRUCE, Christopher J.

The Role of Information Concerning the Arbitrator's Preferences.

v. 36 , no 2,1981, p. $386-402 \quad 0030$

BROSSARD, Michel

Revendications, stratégie syndicale et organisation du travail dans l'entreprise.

v. 36 , no 3,1981, p. $552-575 \quad 0053$ 
RELATIONS DU TRAVAlL (suite)
JAIN, Hem C.
Disclosure of Corporate Information to
Trade Unions in North America.
v. 36, no 4, 1981, p. $748-774$
DESCHÊNES, Jean-Paul
L'évaluation des emplois et la convention
collective.

v. 39 , no 2,1982 , p. $313-327$

0121

BEIGBEDER, Yves

Individual Grievance Procedures in United Nations Secretariats.

v. 37, no 2,1982 , p. $328-343 \quad 0122$

HARRIS, Philip RAUSCH, Peter

RYAN, Samuel G.

Ineffectiveness in Teacher Bargaining: The Anatomy of a First Strike.

v. 37, no 4, 1982, p. 905-926 0165

CAMERON, Samuel

An International Comparison of the Volatility of Strike Behavior

v. 38 , no 4,1983, p. $767-784 \quad 0248$

PIGANIOL, Claude

Relations professionnelles et gestion des ressources humaines.

v. 39 , no 2,1984 , p. $285-300 \quad 0295$

EASTMAN, Byron

A Catastrophe Theory on Union Behaviour.

v. 40, no 2,1985 , p. $340-350$

0389

GILSON, Clive H.J.

Changes in the Nature of Grievance issues Over the Last Ten Years: Labor Management Relations and the "Frontier of Control".

v. 40 , no 4,1985, p. $856-864$

0432

KNIGHT, Thomas R.

Correlates of Informal Grievance Resolution Among Fist-Line Supervisors

v. 41 , no 2,1986 , p. $281-298$

0469

DASTMALCHIAN, Ali

ADAMSON, Raymond BLYTON, Paul

Developing a Measure of Industrial Relations Climate

v. 41, no 4,1986, p. $851-859 \quad 0524$
LAFLAMME, Gilles VALLÉE, Guylaine Changements technologiques et modes régulateurs des relations du travail. v. 42, no 4,1987 , p. $702-715 \quad 0605$

KELLER, Berndt

Mediation as a Conflict-Solving Device in Collective Industrial Disputes.

v. 43, no 2,1988 , p. $431-446 \quad 0654$

AOUST, Claude d'

L'amnistie des fautes disciplinaires.

v. 43 , no 4,1988 , p. $909-942$

0690

BEATTY, Carol GANDZ, Jeffrey

After the Strike: Changing the Teacher-Board Relationship.

v. 44 , no 3,1989 , p. $569-592$

0737

MASSE, Gilles

Le temps passé au travail: un élément encore négociable?

36ième Congrès, 1981, p. 123-135 0839

DESCHÊNES, Jean-Paul

Commentaires - Le temps passé au travail: un élément encore négociable?

36ième Congrès, 1981, p. 136-1440840

DOCQUIER, Gérard

Ressources humaines et defis du syndicalisme.

41ième Congrès, 1986, p. 149-156 0917

KOCHAN, Thomas A.

L'avenir de la négociation collective.

41ième Congrès, 1986, p. 181-189 0922

RELATIONS DU TRAVAIL - ALBERTA

FISHER, E.G. KUSHNER, Stephen

Alberta's Construction Labour Relations

During the Recent Downturn

v. 41 , no 4,1986 , p. $778-801 \quad 0518$

RESHEF, Yonatan

Negotiating Wage Settlements: A Structural Approach.

v. 44, no 3,1989, p. $532-551 \quad 0735$

RELATIONS DU TRAVAIL - AUSTRALIE

GURDON, Michael A.

Divergent Paths: Civil Service Employment Relations in Australia and Canada.

v. 42 , no 3,1987 , p. $566-575$

0585 


\section{RELATIONS DU TRAVAIL - CANADA} SWIDINSKI, Robert

The Effect of Bargaining Structure on Negotiated Wage Settlements.

v. 36, no 2,1981, p. $371-385$

0029

CHRISTENSEN, Sandra

Collective Bargaining in Provincial Public Administration.

v. 36 , no 3,1981 , p. $616-629$

0056

CHOUINARD, Jacques VIEL, André L'efficacité des clauses d'indexation des salaires

v. 37, no 1,1982 , p. 207-214 0105

COUSINEAU, Jean-Michel

LACROIX, Robert

L'efficacité des clauses d'indexation des salaires: Réplique.

v. 36 , no 1,1982 , p. $214-220 \quad 0106$

CHOUINARD, Jacques

ROBICHAUD, Jean-Marc

L'efficacité des clauses d'indexation des salaires.

v. 37 , no 1,1982 , p. $220-224$

0107

MICHAUD, Sharon ROY, Jean-Charles L'efficacité des clauses d'indexation des salaires: Réplique.

v. 37 , no 1,1982 , p. $224-2250108$

FRANK, J.A. KELLY, Michael J.

MACNAUGHTON, Bruce D.

Legislative Change and Strike Activity in Canada, 1926-1974

v. 37, no 2,1982 , p. $267-283$

0119

SIMPSON, Wayne PETERS, Frank

The Economics of Mileage Restrictions for Railway Workers in Western Canada.

v. 38 , no 1, 1983, p. 95-103

0182

FISHER, E.G. PERCY, M.B.

The Impact of Unanticipated Output and Consumer Prices on Wildcat Strikes.

v. 38 , no 2,1983 , p. $254-276$

0203

JAIN, Harish C.

Micro-electronics Technology and Industrial Relations.

v. 38 , no 4,1983 , p. $869-879$

0254
GURDON, Michael A. WRIGHT, David J. Collective Bargaining in the Maritime Fishing Industry: Recent Developments

v. 39 , no 2 , 1984 , p. $259-274$

0293

PONAK, Allen THOMPSON, Mark

Faculty Collective Bargaining: The Voice of Experience

v. 39 , no 3,1984 , p. $449-465 \quad 0314$

ADAMS, Roy J.

The Extent of Collective Bargaining in $\mathrm{Ca}$ nada

v. 39 , no 4,1984 , p. $655-667 \quad 0336$

BENEDICT, Daniel

The 1984 GM Agreement in Canada: Significance and Consequences.

v. 40, no 1,1985, p. $27-47$

0363

SOLOMON, Norman A.

The Negociation of First Agreements under the Canada Labour Code.

v. 40, no 3,1985, p. $458-472 \quad 0404$

ROSE, Joseph B. WETZEL, Kurt

Outcomes of Bargaining Structures in the Ontario and Saskatchewan Construction Industries.

v. 41 , no 2,1986 , p. $256-280 \quad 0468$

FINKELMAN, Jacob

Public Sector Collective Bargaining

v. 41 , no 4,1986 , p. $691-703 \quad 0513$

NG, ignace

Determinants of Wildcat Strikes in Canada Manufacturing Industries.

v. 42 , no 2,1987 , p. $386-397 \quad 0567$

GURDON, Michael A.

Divergent Paths: Civil Service Employment Relations in Australia and Canada.

v. 42 , no 3,1987 , p. $566-575 \quad 0585$

KNIGHT, Thomas R.

The Role of The Duty of Fair Representation in Union Grievance Decisions.

v. 42 , no 4,1987 , p. $716-736 \quad 0606$

SUBBARAO, A.V.

Criteria in Arbitration of Wage Disputes: Theory and Practice in the Canadian Federal Public Senvice.

v. 43, no 3,1988 , p. $547-570 \quad 0667$ 
RELATIONS DU TRAVAIL - CANADA (suite) MELTZ, Noah $M$.

Job Security in Canada

v. 44 , no 1,1989 , p. $149-161$

0705

\section{FORREST, Anne}

The Rise and Fall of National Bargaining in the Canadian Meat-Packing Industry.

v. 44 , no 2,1989 , p. $393-408$

0723

DASTMALCHIAN, Ali NG, Ignace Industrial Relations Climate and Grievance Outcomes.

v. 45 , no 2,1990, p. $311-325$

0796

CHAYKOWSKI, Richard P.

Union and Firm Preferences for Bargaining Outcomes in the Private Sector.

v. 45, no 2,1990, p. $326-356$

0797

PERREAULT, Charles

L'entreprise peut-elle se passer d'un syndicat?

38ième Congrès, 1983, p. 173-182 0868

GAUTHIER, Fernand ROY, Paul-Martel

Commentaires - L'entreprise peut-elle se passer d'un syndicat?

38ième Congrès, 1983, p. 183-195 0869

RELATIONS DU TRAVAIL .

COLOMBIE BRITANNIQUE

FISHER, E.G.

Strike Activity and Wildcat Strikes in British Columbia: 1945-1975.

v. 37 , no 2,1982 , p. $284-312 \quad 0120$

CUNNINGHAM, Barton MITCHELL, Lari

Privatization in British Columbia: What the Experts Say Will Happen.

v. 45, no 2,1990 , p. $382-403$

0799

RELATIONS DU TRAVAIL - ÉTATS-UNIS PETERSON, Richard B. TRACY, Lane CABELLY, Alan

Problem Solving in Labor Negotiations: Retest of a Model

v. 36 , no 1, 1981 , p. $87-105$

0004

DILTS, David A. KARIM, Ahmad

The Effect of Mediators' Qualities and Strategies on Mediation Outcomes

v. 45 , no 1,1990, p. $22-37$

0772
RELATIONS DU TRAVAIL - FRANCE

CAIRE, GuY

Procédures de règlement pacifique des conflits collectifs en France.

v. 38 , no 1,1983 , p. 3-27

0177

DELAMOTTE, Yves

La loi et la négociation collective en France: Réflexion sur l'expérience 1981-1985.

v. 42 , no 1,1987 , p. $92-109$

0542

\section{RELATIONS DU TRAVAIL.}

GRANDE-BRETAGNE

BEAUMONT, P.B.

Third Party Conciliation and Trade Union Recognition: Some British Evidence.

v. 37, no 4,1982, p. $827-842$

0161

CAMPBELL, Adrian WARNER, Malcolm Workplace Relations, Skills-Training and Technological Change at Plant-Level.

v. 43, no 1,1988 , p. $115-132$

0632

SMITH, Anthony E.

Innovation by Negotiation: Case Studies Among British White-Collar Unions.

v. 45 , no 1,1990 , p. $63-75$

0774

RELATIONS DU TRAVAIL - ISRAËL

RESHEF, Yonatan FRIED, Yatzhak

Strikes in Israël: The Histadrut in a Sectorial Perspective, 1965-1982

v. 41 , no 2,1986 , p. 329-347

0472

RELATIONS DU TRAVAIL - MANITOBA

BLACK, Errol

In Search of "Industrial Harmony": The Process of Labour Law Reform in Manitoba, 1984.

v. 40, no 1,1985, p. $140-161$

0369

BLACK, Errol SILVER, Jim

Contradictions and Limitations of Final Offer Selection: The Manibota Experience. v. 45 , no 1, 1990 , p. $146-165$

0779

GRANT, Hugh $M$.

Contradictions and Limitations of Final Offer Selection: The Manitoba Experience.

A Comment.

v. 45 , no 1,1990, p. $166-168 \quad 0780$

BLACK, Errol SILVER, Jim

FOS in Manitoba. A Rejoinder.

v. 45 , no 3,1990, p. $612-615$

0819 
RELATIONS DU TRAVAIL - NIGÉRIA

FASHOYIN, Tayo

State Regulation of Trade Disputes in Essential Services in Nigeria

v. 36 , no 1,1981 , p. $207-222$

0010

RELATIONS DU TRAVAIL NOUVELLE-ÉCOSSE

GILSON, Clive H.J. GILLIS, L.P.

Grievance Arbitration in Nova Scotia.

v. 42 , no 2,1987 , p. $256-271 \quad 0560$

\section{RELATIONS DU TRAVAIL. - ONTARIO}

ADAMS, Georges W.

The Ontario Experience with interest arbitration: Problems in Detecting Policy

v. 36 , no 1,1981 , p. $225-250$

0012

COUSINEAU, Jean-Michel

LACROIX, Robert

Le climat des relations du travail à Montréal et à Toronto

v. 38 , no 4,1983, p. $730-743$

0246

SOLOMON, Norman A.

The Negociation of First Agreement in Ontario.

v. 39 , no 1,1984, p. $23-35$

0269

ANDIAPPAN, P. CATTANEO, R. Julian MURPHY, John

Interest Arbitration in Ontario Hospitals: Result of an Attitude Survey of Union and Management Officials.

v. 39 , no 4,1984 , p. $680-694$

0338

KRUGER, Arthur

Collective Bargaining in Ontario Public Hospitals

v. 40, no 1,1985, p. $48-67 \quad 0364$

CHARLES, Lawrence $M$.

HUMPHREYS, Edward $\mathrm{H}$.

Bargaining to Achieve Teacher Control in Ontario

v. 40, no 3,1985 , p. $495-511 \quad 0406$

\section{RELATIONS DU TRAVAIL - QUÉBEC} FERLAND, Gilles

La politique de rémunération dans les secteurs public et parapublic au Québec. v. 36, no 3, 1981, p. $475-498 \quad 0050$
HÉBERT, Gérard

Les relations du travail au Québec: Bilan des années 1970

v. 36, no 4,1981 , p. $715-747$

0071

DÉOM, Esther

La négociation collective chez les fonctionnaires et les enseignants québécois: 1975-1976.

v. 37, no 1,1982 , p. 141-163 0101

PEACH, David A.

The Canadair-I.A.M. Productivity: Improvement Plan.

v. 37 , no 1,1982 , p. 177-197 0103

BOLDUC, Roch BOUCHARD, Lucien HÉBERT, Gérard

Le régime québécois de négociation des secteurs public et parapublic

v. 37, no 2,1982 , p. $403-430 \quad 0126$

\section{PROULX, Pierre-Paul}

Rémunération dans les secteurs public et parapublic au Québec: Éléments d'une nouvelle politique.

v. 37, no 3, 1982, p. $477-497 \quad 0136$

LATULIPPE, Gérard P. O'FARRELL, Kevin Le comité paritaire: Anachronisme ou formule d'avenir.

v. 37, no 3,1982 , p. $634-655 \quad 0143$

VEILLEUX, Diane

Les clauses de promotion et de transfert chez les infirmières et les infirmiers.

v. 38 , no 2,1983, p. $229-253 \quad 0202$

AOUST, Claude d'

Les dispositifs d'une sentence arbitrale: quelques pièges à éviter.

v. 38 , no 2,1983, p. $415-417 \quad 0210$

COUSINEAU, Jean-Michel

LACROIX, Robert

Le climat des relations du travail à Montréal et à Toronto

v. 38, no 4,1983 , p. $730-743 \quad 0246$

RICHER, Jean-Charles

La Corporation professionnelle des conseillers en relations industrielles du Québec devant la Commission Beaudry.

v. 40 , no 2,1985 , p. $379-385$

0393 
RELATIONS DU TRAVAIL - QUÉBEC (suite) MAKI, Dennis R. MEREDITH, Lindsay N. The Effects of Unions on Profitability: Canadian Evidence

v. 41 , no 1,1986 , p. $54-68$

0449

RONDEAU, Claude BADIN, François Le contenu non salarial des conventions collectives dans les industries manufacturières au Québec.

v. 41 , no 1,1986 , p. $69-90$

0450

SEXTON, Jean

L'arbitrage de première convention collective au Quebec: 1978-1984

v. 42, no 2,1987, p. $272-291$

0561

\section{BARRÉ, Alain}

Le cadre juridique de la négociation collective dans le secteur public au Québec: une perspective internationale.

v. 42 , no 4,1987 , p. $831-851$

0612

VALLÉE, Guylaine

Les accords "atypiques" et le droit des rapports collectifs du travail.

v. 44 , no 3,1989 , p. $680-690$

0742

ROSS, Claudette BROSSARD, Michel La conciliation volontaire est-elle plus efficace que la conciliation obligatoire? Le cas du Québec.

v. 45 , no 1,1990 , p. 3-21

0771

BLOUIN, Rodrigue BOULARD, René

DESCHÊNES, Jean-Paul

PÉRUSSE, Michel

Introduction - Régimes de santé et sécurité et relations du travail.

39ième Congrès, 1984, p. 15-16 0873

PÉRUSSE, Michel

Régimes de santé et sécurité et relations du travail.

39ième Congrès, 1984, p. 17-31 0874

DUMAS, Anne-Chantal SIMARD, Monique Commentaires - Concertation et participation: mythe ou réalité.

39ième Congrès, 1984, p. 93-111 0878

BOUCHARD, Robert CLÉMENT, Pierre R. GIASSON, Étienne GUILLEMETTE, Michel Table ronde - La santé et la sécurité dans l'entreprise.

39ième Congrès, 1984, p. 113-135 0879
BOULARD, René

L'impact de la Loi sur les conventions collectives.

39ième Congrès, 1984, p. 137-157 0880

BLOUIN, Rodrigue

Le statut de salarié en milieu de travail: la problématique.

40ième Congrès, 1985, p. 17-32 0888

DUCHARME, Claude SIMARD, Monique THIBAULT, Laurent

Table ronde - Le régime actuel de travail des salariés: où en sommes-nous?

40ième Congrès, 1985, p. 121-136 0895

CASAVANT, Jean-Claude

La stratégie de gestion des ressources humaines chez Abitibi Price Inc.

41ième Congrès, 1986, p. 53-61 0908

BOUCHER, Lysette

Les limites des nouvelles approches en gestion des ressources humaines.

41ième Congrès, 1986, p. 77-84 0911

DULUDE, Yves

Le conflit: la gestion au banc des accusés.

41ième Congrès, 1986, p. 111-133 0914

LAMARCHE, Pierre

Commentaires - Le conflit: la gestion au banc des accusés.

41ième Congrès, 1986, p. 133-136 0915

LALANDE, Serge

L'expérience de la compagnie Gaz Métropolitain.

41ième Congrès, 1986, p. 137-146 0916

LABERGE, Louis

Les préalables à une réorientation des relations du travail au Québec.

41 ième Congrès, 1986, p. 173-175 0920

LAFLAMME, Gilles LAROCQUE, Alain Lésions et maladies professionnelles: Un objet de négociation?

42ième Congrès, 1987, p. 89-100 0930

LEVINE, David

Négocier la flexibilité: Reconversion des heures de la liste de rappel en postes à temps complet.

45ième Congrès, 1990, p. 41-47 0970 
Relations lmdustrielles, vol. 45, no 4 (1990)

RELATIONS DU TRAVAIL - QUÉBEC (suite) GOBEILLE, Kenneth $R$.

Négocier la flexibilite: Le travail à temps partiel.

45ième Congrès, 1990, p. 48-58 0971

GODIN, Michel

Négocier la flexibilité: La sous-traitance.

45ième Congrès, 1990, p. 59-64 0972

LISTON, Terrence

Négocier la flexibilité: La polyvalence des emplois.

45ième Congrès, 1990, p. 65-69 0973

BROUILLET, Normand

Négocier la flexibilité: Réaction syndicale aux stratégies patronales.

45ième Congrès, 1990 , p. 70-81

0974

MATHIEU, René

Négocier la flexibilité: La recherche d'équité par la négociation.

45ième Congrès, 1990, p. 82-92

0975

\section{RELATIONS DU TRAVAIL - \\ SASKATCHEWAN}

GALLAGHER, Daniel G. WETZEL, Kurt Local Employer and Union Perceptions of Two-Tier Bargaining

v. 39, no 3,1984 , p. $486-508$

0316

\section{SANTÉ ET SÉCURITÉ AU TRAVAIL}

\section{SANTÉ ET SÉcuRITÉ AU TRAVAIL}

LAFLAMME, Lucie ARSENAULT, André Rémuneration, postes de travail et accidents: Une relation interactive.

v. 39 , no 3,1984 , p. $509-525$

JAIN, Hem C.

AIDS: Need for Policy in the Workplace.

v. 44 , no 4,1989 , p. $850-865$

0756

BRODY, Bernard LÉTOURNEAU, Yves POIRIER, André

Le cout des accidents du travail: État des connaissances.

v. 45 , no 1,1990 , p. $94-117$

0776

\section{SANTÉ ET SÉCURITÉ AU TRAVAIL -}

ALBERTA

FISHER, E.G. IVANKOVICH, I.F.

Alberta's Occupational Health and Safety Amendment Act, 1983.

v. 40 , no 1,1985 , p. $115-1390368$

\section{SANTÉ ET SÉCURITÉ AU TRAVAIL -}

\section{CANADA}

BRYCE, George K. MANGA, Pran

The Effectiveness of Health and Safety

Committees.

v. 40, no 2,1985, p. $257-283 \quad 0385$

BRODY, Bernard ROHAN, Paul

ROMPRE, Louise

Les accidents industriels au Canada: Le portrait d'une décennie

v. 40, no 3,1985 , p. $545-566 \quad 0409$

WEST, Leigh

The Exclusive Remedy Provision in Canadian Worker Compensation Law: The Need for Legislative Reform.

v. 43, no 4,1988 , p. $890-908 \quad 0689$

\section{SANTÉ ET SÉCURITÉ AU TRAVAIL -}

ONTARIO

SWIMMER, Gene LUCE, Sally R.

Asbestcs Exposure and Attitudes Toward Occupational Health.

v. 40 , no 3,1985 , p. $529-544 \quad 0408$

WALTERS, Vivienne DENTON, Margaret Workers' Knowledge of their Legal Rights and Resistance to Hazardous Work.

v. 45, no 3,1990, p. $531-547 \quad 0815$

FARQUHAR, Alec

Le régime des accidents du travail: Le cas de l'Ontario.

42ième Congrès, 1987, p. 115-130 0932

SANTÉ ET SÉCURITÉ AU TRAVAIL -

\section{QUÉBEC}

BRISSON, Chantal

La santé au travail et la convention collective

v. 36 , no 1,1981 , p. 152-178 0007

VINET, Alain BÉGIN, Clermont

THÉRIAULT, Gilles

La santé des employés de bureau: Le cas de la fonction publique québécoise.

v. 37 , no 4,1982 , p. $887-904 \quad 0164$ 


\section{SANTÉ ET SÉCURITÉ AU TRAVAIL - QUÉBEC (sulte)}

BOULARD, René MARCHAND, Michel La loi sur la santé et la sécurité du travail et les conventions collectives.

v. 38 , no 4,1983 , p. $847-862$

GEORGE, Kenneth

Les comités de santé et de sécurité du travail: Tables de concertation ou de négociation?

v. 40, no 3,1985 , p. $512-528$

0407

COTTÉ, André C.

L'Acte des manufactures de Québec, 1885: Un centenaire

v. 40 , no 3,1985 , p. $623-628$

0412

SIMARD, Marcel BOUTEILLER, Dominique LÉVESQUE, Christian

Prévention des accidents du travail: Contexte législatif québécois et efficacité organisationnelle.

v. 40 , no 4,1985, p. $703-719$

0424

\section{PLASSE, Micheline}

Santé et sécurité du travail au Québec: Le defi de la concertation patronale - syndicale.

v. 42 , no 3,1987 , p. $544-565$

0584

LAFLAMME, Lucie VINET, Alain

Accidents du travail et modernisation du processus de production: le cas de l'industrie forestière québécoise.

v. 43 , no 3,1988 , p. $591-608$

0669

BLOUIN, Rodrigue BOULARD, René DESCHÊNES, Jean-Paul

PÉRUSSE, Michel

Introduction - Régimes de santé et sécurité et relations du travail.

39ième Congrès, 1984, p. 15-16 0873

PÉRUSSE, Michel

Régimes de santé et sécurité et relations du travail.

39ième Congrès, 1984, p. 17-31

0874

SIMARD, Marcel

Priorités en santé et sécurité du travail: secteur public et secteur privé.

39ième Congrès, 1984, p. 33-50
LEDUC, Jean-Guy LEMELIN, Maurice VINET, Alain

Commentaires - Priorités en santé et sécurité du travail: secteur public et secteur privé.

39ième Congrès, 1984 , p. 50-72 0876

OUELLET, Florian

Concertation et participation: mythe ou réalité?

39ième Congrès, 1984, p. 73-92 0877

DUMAS, Anne-Chantal SIMARD, Monique Commentaires - Concertation et participation: mythe ou réalité.

39ième Congrès, 1984, p. 93-111 0878

BOUCHARD, Robert CLÉMENT, Pierre R. GIASSON, Étienne GUILLEMETTE, Michel Table ronde - La santé et la sécurité dans l'entreprise.

39ième Congrès, 1984, p. 113-135 0879

BOULARD, René

L'impact de la Loi sur les conventions collectives.

39ième Congrès, 1984, p. 137-157 0880

DE KONINCK, Maria HEENAN, Roy

Droit de refus et retrait préventif: succès ou echec?

39ième Congrès, 1984 , p. 159-175 0881

TURCOTTE, Fernand

La prévention: une utopie?

39ième Congrès, 1984, p. 177-183 0882

DROUIN, Claude PLAMONDON, Denise Commentaires - La prévention: une utopie?

39ième Congrès, 1984, p. 183-194 0883

BRUNET, Jacques

Santé et sécurité du travail: une affaire de professionnels?

39ième Congrès, 1984, p. 195-206 0884

BEAUSOLEIL, Gilles

Les coûts et les bénéfices du régime.

39ième Congrès, 1984, p. 207-242 0885

DUFOUR, Ghislain FAVA, Frank

HÉTU, Jean-Paul LABERGE, Louis

LAROSE, Gérald SAUVÉ, Robert

Table ronde - Déceptions et espoirs.

39ième Congrès, 1984, p. 243-276 0886 


\section{SANTÉ ET SÉCURITÉ AU TRAVAIL - QUÉBEC (suite) \\ PÉRUSSE, Michel}

Les lésions professionnelles - une problématique.

42ième Congrès, 1987, p. 1-8

0923

CHIASSON, Denis-Émile

Synopsis sur le nouveau régime.

42ième Congrès, 1987, p. 9-19

0924

VINET, Alain VÉZINA, Michel

BRISSON, Chantal

Des lésions professionnelles méconnues: le cas des opératrices de lindustrie du vêtement.

42ième Congrès, 1987, p. 21-38 0925

LESAGE, Michel

Les lésions professionnelles: Point de vue d'un médecin.

42ième Congrès, 1987, p. 39-50 0926

PRÉVOST, Charles

La sous-estimation des atteintes à la santé causées par les mauvaises conditions de travail.

42ième Congrès, 1987, p. 51-60

0927

ARSENAULT, André

La reconnaissance d'une maladie professionnelle est-elle négociable?

42ième Congrès, 1987, p. 61-72 0928

DUGUAY, Pierre BOUCHARD, Robert GONTHIER, Jean-Marie

Commentaires - La reconnaissance d'une maladie professionnelle est-elle négociable?

42ième Congrès, 1987, p. 72-86 0929

LAFLAMME, Gilles LAROCQUE, Alain

Lésions et maladies professionnelles: Un objet de négociation?

42ième Congrès, 1987, p. 89-100 0930

TRUDEAU, Guy J. OUELLET, Lionel

La comparaison en matière de systèmes de santé et de sécurité du travail.

42ième Congrès, 1987, p. 101-113 0931

\section{MORIN, Fernand}

Le régime actuel d'indemnisation pour lésions professionnelles: Accessibilité et efficacité.

42ième Congrès, 1987, p. 131-155 0933
LEFĖBVRE, Marie-Claire

LEVASSEUR, Raymond

Commentaires - Le régime actuel d'indemnisation pour lésions professionnelles: Accessibilité et efficacité.

42ième Congrès, 1987, p. 155-176 0934

BERNIER, Lionel

Equité, indemnisation des victimes de lésions professionnelles et coûts à l'entreprise.

42ième Congrès, 1987, p. 177-211 0935

GINGRAS, Claude DUCHESNE, André

Commentaires - Équité, indemnisation des victimes de lésions professionnelles et coûts à l'entreprise.

42ième Congrès, 1987, p. 212-222 0936

SURET, Jean-Marc GENDRON, Michel BERNIER, Gilles

Le processus de gestion des risques, les lésions professionnelles et la CSST.

42ième Congrès, 1987, p. 223-241 0937

HARGUINDEGUY, Jean-Louis

BRODY, Bernard

Commentaires - Le processus de gestion des risques, les lésions professionnelles et la CSST.

42ième Congrès, 1987, p. 241-252 0938

TOBIN, Edmund LAROSE, Gérald

DUFOUR, Ghislain LABERGE, Louis

Table ronde - Financement de la santé et paritarisme.

42ième Congrès, 1987, p. 253-276 0939

JÉRÔME-FORGET, Monique

La politique québécoise en matière de lésions professionnelles à un point tournant.

42ième Congrès, 1987, p. 277-285 0940

\section{SÉCURITÉ SOCIALE}

\section{SÉCURITE SOCIALE}

DESCHÊNES, Jean-Paul FERLAND, Gilles

SAINT-LAURENT, Jacques

SEXTON, Jean

Introduction - Les régimes de retraite.

37ième Congrès, 1982, p. 13-16 0846 
SÉCURITÉ SOCIALE (suite) BONENFANT, Claire GUÉRARD, Yves LAFONTAINE, Réal LAFOREST, Martial Table ronde - La protection du revenu à la retraite: une responsabilité partagée? 37ième Congrès, 1982, p. 107-145 0852

HÉBERT, HeNé PERRON, Jacques POULIN-SIMON, Lise

Table ronde - Qui doit administrer les régimes de retraite: l'employeur ou le syndicat?

37ième Congrès, 1982, p. 147-169 0853

GALBRAITH, John Kenneth

Réflexions sur les problèmes de la retraite dans les années 80.

37ième Congrès, 1982, p. 193-213 0856

LAMONTAGNE, Maurice

Commentaire - Réflexions sur les problèmes de la retraite dans les années 80 .

37ième Congrès, 1982, p. 213-218 0857

SÉCURITÉ SOCIALE - CANADA

COUSINEAU, Jean-Michel

Objectifs et modalités de l'assurance-chÓmage au Canada: 1940-1986

v. 41 , no 3,1986 , p. $451-468$

0486

BENOIT, Michel

Des solutions aux problèmes de la retraite. 37ième Congrès, 1982, p. 35-44 0848

BÉGIN, Monique

Les politiques gouvernementales en matière de retraite.

37ième Congrès, 1982, p. 171-180 0854

PARIZEAU, Jacques

Les politiques gouvernementales en matière de retraite.

37ième Congrès, 1982, p. 181-192 0855

\section{SÉCURITÉ SOCIALE - ONTARIO}

FARQUHAR, Alec

Le régime des accidents du travail: Le cas de l'Ontario.

42ième Congrès, 1987, p. 115-130 0932

\section{SÉCURITÉ SOCIALE - QUÉBEC}

SOLASSE, Bernard

La retraite: votre problème.

37ième Congrès, 1982, p. 17-34

0847
FAILLE, Jacques

Les principaux régimes de retraite et leur contenu.

37ième Congrès, 1982 , p. 45-74 0849

BÉLANGER, Gérard

Les aspects économiques de la réforme des pensions.

37ième Congrès, 1982, p. 75-98 0850

DÉPATIE, Raymond

Commentaire - Les aspects économiques de la réforme des pensions.

37ième Congrès, 1982, p. 98-105 0851

PÉRUSSE, Michel

Les lésions professionnelles - une problématique.

42ième Congrès, 1987, p. 1-8 0923

CHIASSON, Denis-Émile

Synopsis sur le nouveau régime.

42ième Congrès, 1987, p. 9-19

0924

LESAGE, Michel

Les lésions professionnelles: Point de vue d'un médecin.

42ième Congrès, 1987, p. 39-50 0926

TRUDEAU, GuY J. OUELLET, Lionel

La comparaison en matière de systèmes de santé et de sécurité du travail.

42ième Congrès, 1987, p. 101-113 0931

MORIN, Fernand

Le régime actuel d'indemnisation pour lésions professionnelles: Accessibilité et efficacité.

42ième Congrès, 1987, p. 131-155 0933

LEFÈBVRE, Marie-Claire

LEVASSEUR, Raymond

Commentaires - Le régime actuel d'indemnisation pour lésions professionnelles: Accessibilité et efficacité.

42ième Congrès, 1987, p. 155-176 0934

BERNIER, Lionel

Équite, indemnisation des victimes de lésionns professionnelles et coûts à l'entreprise. 


\section{SANTÉ ET SÉCURITÉ AU TRAVAIL - QUÉBEC (sulte)}

GINGRAS, Claude DUCHESNE, André

Commentaires - Équité, indemnisation des

victimes de lésions professionnelles et couts à l'entreprise.

42ième Congrès, 1987, p. 212-222 0936

SURET, Jean-Marc GENDRON, Michel BERNIER, Gilles

Le processus de gestion des risques, les lésions professionnelles et la CSST.

42ième Congrès, 1987, p. 223-241 0937

HARGUINDEGUY, Jean-Louis

BRODY, Bernard

Commentaires - Le processus de gestion des risques, les lésions professionnelles et la CSST.

42ième Congrès, 1987, p. 241-252 0938

TOBIN, Edmund LAROSE, Gérald

DUFOUR, Ghislain LABERGE, Louis

Table ronde - Financement de la santé et paritarisme.

42ième Congrès, 1987, p. 253-276 0939

JÉRÓME-FORGET, Monique

La politique québécoise en matière de lésions professionnelles à un point tournant.

42ième Congrès, 1987, p. 277-285 0940

\section{SOCIOLOGIE DU TRAVAIL}

\section{SOCIOLOGIE DU TRAVAIL}

WHITE, Terrence $\mathrm{H}$.

The Relative Importance of Work as a Factor in Life Satisfaction

v. 36 , no 1,1981 , p. 179-191

0008

TAJGMAN, David

Political Resolutions in the International Labor Organizations: The experience since 1964.

v. 36 , no 3,1981 , p. $499-529$

0051

VINET, Bernard

Le problème de la retraite.

v. 36 , no 4,1981 , p. $828-847$

0075

PEITCHINIS, Stephen G.

The Attitude of Trade Unions Towards Technological Changes.

v. 38 , no 1,1983 , p. 104-119

0183
DEMERS, Marie

Chómage chez les jeunes: Conséquences psychologiques et sociales.

v. 38 , no 4,1983 , p. $785-814$

0249

LE LOUARN, Jean-Yves

THÉRIAULT, Roland

TOULOUSE, Jean-Marie

Le travail des deux conjoints: Effets sur la progression de carrière du cadre.

v. 39 , no 1, 1984 , p. $36-50$

0270

MCSHANE, Steven L.

Sources of Attitudinal Union Militancy

v. 40, no 2,1985 , p. $284-302 \quad 0386$

DUSSAULT, Gilles

The Future of Professional Monopolies.

v. 40, no 2,1985, p. $324-339$

0388

AOUST, Claude d' SAINT-JEAN, Sylvain TRUDEAU, Gilles

L'obligation de civilité du salarié

v. 41 , no 1,1986 , p. $157-180$

0455

BROSSARD, Michel SIMARD, Marcel Problématique de la différenciation de la main-d'oeuvre et changement organisationnel.

v. 41 , no 2,1986 , p. 219-235 0466

MERCIER, Jean

L'informatique, ses "fiitres" et ses effets.

v. 41 , no 2,1986 , p. $299-316 \quad 0470$

PROULX, Pierre-Paul

Maîtriser la technologie: pourquoi, quelles technologies, comment?

v. 41, no 2,1986, p. $382-389$

0475

LONG, Richard J. WARNER, Malcolm Organizations, Participation and Recession: An Analysis of Recent Evidence.

v. 42 , no 1,1987 , p. $65-91$

0541

BÉLANGER, Paul R. LÉVESQUE, Benoît Le mode de vie determine-t-il l'éthique du travail? Étude de cas.

v. 42 , no 2,1987 , p. $345-365 \quad 0565$

COOK, Alice $\mathrm{H}$.

Family and Work: Challenger to Labor, Management and Government

v. 42 , no 3,1987 , p. $520-527$

0582 


\section{SOCIOLOGIE DU TRAVAIL (suite)}

RICHARDS, John MAUSER, Gary

HOLMES, Richard

What Do Workers Want? Attitudes Towards Collective Bargaining and Participation in Management.

v. 43 , no 1,1988 , p. $133-152$

0633

CUNNINGHAM, Barton HULL, Dennis A Union Member's Perspective on Technological Change.

v. 43 , no 2,1988 , p. $394-411$

0652

\section{MATON, Bob}

Socio-Technical Systems: Conceptual and Implementation Problems

v. 43, no 4,1988 , p. $869-8890688$

\section{SCHELL, Bernadette}

LEBRASSEUR, Rolland RENAUD, Robert Predictors of Acceptance of Early Retirement Offers for Workers.

v. 44, no 2,1989, p. 376-392

0722

GILSON, Clive H.J. SPENCER, lan S. GRANVILLE, S.

The Impact of a Strike on the Attitudes and Behavior of a Rural Community.

v. 44 , no 4,1989, p. $785-804$

0753

BOULARD, René DESCHÊNES, Jean-Paul LAROCQUE, Alain RONDEAU, Claude Introduction - La réduction de la durée du travail.

36ième Congrès, 1981, p. 13-14 0832

\section{LAROCQUE, ALAIN}

La réduction du temps de travail: un droit ou un privilège?

36ième Congrès, 1981, p. 15-29

0833

MONTMINY, Jean-Paul

La signification du travail en 1980: émergence de valeurs nouvelles?

36ième Congrès, 1981, p. 31-45 0834

DUFOUR, Ghislain RODRIGUE, Norbert Commentaires - La signification du travail en 1980: émergence de valeurs nouvelles?

36ième Congrès, 1981, p. 45-59 0835

FRANCOEUR, Jean

Le syndicalisme et le nouveau travail.

41ième Congrès, 1986, p. 157-166 0918
KRAHN, Harvey LOWE, Graham S.

Community Influences on Attitudes Towards Unions.

v. 39 , no 1,1984, p. $93-113$

0273

\section{GRAYSON, J. Paul}

The Political Consequences of Unemployment: An Application of the "Power Model" of Blue-Collar Radicalism

v. 44 , no 3,1989 , p. $635-653$

0740

HUGUES, Karen D.

Office Automation: A Review of the Litterature

v. 44 , no 3,1989 , p. $654-679$

0741

CÔTÉ, André C.

Nouvelles technologies et droit du travail au Canada.

v. 44, no 4,1989 , p. $751-768$

0751

ANDIAPPAN, P. CRESTOHL, Mark

SINGH, Jang $B$.

Racial Discrimination in Employment in Canada.

v. 44, no 4,1989 , p. $827-849 \quad 0755$

\section{SOCIOLOGIE DU TRAVAIL .}

GRANDE-BRETAGNE

BÉLANGER, Jacques

Le controle ouvrier sur l'organisation du travail: Etude de cas en Grande-Bretagne.

v. 41 , no 4,1986 , p. $704-719 \quad 0514$

SOCIOLOGIE DU TRAVAIL - QUÉBEC

SODERSTROM, Lee

Some Effects of Unemployment on the Health of Unemployed Quebec Workers.

v. 43, no 2,1988 , p. $341-377 \quad 0650$

AUDET, Claude VINET, Alain

Bureautique et organisation du travail de secrétariat dans la fonction publique québecoise.

v. 43, no 4,1988 , p. $737-756 \quad 0682$

\section{SYSTÈME DES RELATIONS INDUSTRIELLES}

\section{SYSTÈME DES RELATIONS}

\section{INDUSTRIELLES}

WEISS, Dimitri

Politique, entreprise, syndicats

v. 36 , no 1,1981, p. $192-206$ 


\section{SYSTÈME DES RELATIONS INDUSTRIELLES (suite)}

AUCLAIR, Robert

Genève ignore Québec en matière de vocabulaire

v. 36 , no 1,1981, p. $223-224$

0011

WEISS, Dimitri

Qu'est-ce que les relations industrielles?

v. 36, no 2, 1981, p. $412-413$

0032

BLANCHARD, Francis

Les relations professionnelles et les défis des années 80.

v. 37, no 1,1982 , p. 3-14

0094

HAMEED, Syed M.A.

A Critique of Industrial Relations Theory. v. 37 , no 1,1982, p. $15-31$

0095

YOUNG, Stanley

Industrial Relations: A Paradigmatic Analysis.

v. 37 , no 1,1982, p. $32-52$

0096

MROCZKOWSKI, Tomasz

Theory Z: Myths, Realities and Alternatives.

v. 38 , no 2,1983 , p. 297-318

0205

ADAMS, Roy J.

Competing Paradigms in Industrial Relations.

v. 38 , no 3,1983 , p. $508-531$

0224

BARKIN, Solomon

Troubled Worker Militancy: Challenges Confronting Western Industrial Relations Systems.

v. 38, no 4,1983, p. $713-729$

0245

LAROUCHE, Viateur DÉOM, Esther

L'approche systémique en relations industrielles

v. 39 , no 1,1984, p. $114-145$

0274

SHIROM, Arie

The Labor Relations System: A Proposed Conceptual Framework

-v. 40, no 2,1985, p. $303-323$

0387

CARROTHERS, A.W.R.

A Way of Thinking about Collective Bargaining: Circumstance, Policy, Law and ACtuality.

v. 40, no 2,1985, p. 351

0390
GILSON, Clive H.J.

Changes in the Nature of Grievance issues Over the Last Ten Years: Labor Management Relations and the "Frontier of Control.'.

v. 40, no 4,1985 , p. $856-864 \quad 0432$

\section{BARKIN, Solomon}

The Current Unilateralist Counterattack on Unionism and Collective Bargaining

v. 41 , no 1,1986 , p. $3-27$

0447

GILSON, Clive H.J.

Bargaining Exercises: Beyond Simulation, a Touch of Reality.

v. 41 , no 2,1986 , p. $390-396 \quad 0476$

DIMMOCK, Stuart J. SETHI, Amarjit S.

The Role of ldeology and Power in Systems Theory: Some Fundamental Shortcomings.

v. 41 , no 4,1986 , p. $738-757$

0516

ROHLING, Thomas A.

Screening and Human Capital Theory: An Empirical Test

v. 41 , no 4,1986 , p. $817-826 \quad 0520$

BLANCHARD, Francis

L'OIT face au problème des relations professionnelles

v. 42 , no 1,1987 , p. 3-11

0538

ADAMS, Roy J.

Employment Standards in Ontario: An industrial Relations Systems Analysis.

v. 42, no 1,1987 , p. $46-64$

0540

BARBASH, Jack

Like Nature, Industrial Relations Abhors a Vaccum: The Case of the Union-Free Strategy.

v. 42 , no 1,1987 , p. $168-179 \quad 0546$

BOIVIN, Jean

Les relations industrielles: Une pratique et une discipline

v. 42 , no 1,1987 , p. 179-196

0547

CLARKE, Oliver

Industrial Relations Theory and Practice: A Note

v. 42, no 1,1987, p. $196-202 \quad 0548$ 


\section{SYSTÈME DES RELATIONS INDUSTRIELLES (sulte)}

HAMEED, Syed M.A. SEN, Joya

A Power Theory of Third Party Intervention in Labour Management Relations.

v. 42 , no 2,1987 , p. $243-255$

0559

AUDET, Michel LAROUCHE, Viateur Paradigmes, écoles de pensée et théories en relations industrielles.

v. 43, no 1,1988, p. $3-31$

0626

SEXTON, Jean DION, Gérard

L'avenir des relations industrielles dans les Amériques: Introduction.

v. 44 , no 1,1989 , p. 1-2

0698

FAJANA, Sola

The Systems Approach as Theory for Multinational Industrial Relations in Developing Countries.

v. 44 , no 3,1989 , p. $615-634$

0739

BOIVIN, Jean

Émergence d'une réalité nouvelle en relations industrielles.

41ième Congrès, 1986, p. 17-30 0906

KOCHAN, Thomas A.

L'avenir de la négociation collective.

41ième Congrès, 1986, p. 181-189 0922

ROJOT, Jacques

Droits collectifs et droits individuels: Les situations française, américaine et anglaise.

43ième Congrès, 1988, p. 19-49 0943

\section{SYSTÈME DES RELATIONS}

INDUSTRIELLES - ALLEMAGNE

MUCKENBERGER, Ulrich

The Regulation of Strike Law in Times of New Technologies and Deregulation: The Case of West Germany.

v. 45 , no 1,1990 , p. $136-145$

0778

\section{SYSTÈME DES RELATIONS}

\section{INDUSTRIELLES - AMÉRIQUE LATINE}

LUCENA, Hector

Papel del Estado en las relaciones industriales en América Latina

v. 44 , no 1,1989 , p. $249-282$

0710

\section{SYSTĖME DES RELATIONS}

INDUSTRIELLES - ANGLETERRE

WARNER, Malcolm

Corporatism, Participation and Sociery

v. 38 , no 1,1983 , p. $27-44$

0178

\section{SYSTEMME DES RELATIONS}

INDUSTRIELLES - CANADA

DOWNIE, Bryan $M$.

Some Thoughts on Public Policy and Industrial Peace.

v. 36 , no 1,1981 , p. $63-86$

0003

ADAMS, Roy J.

The Federal Government and Tripartism.

v. 37 , no 3,1982 , p. $606-617$

0141

ARTHURS, H.W.

Understanding "Understanding": Industrial Relations Research and Policy in Canada from 1969 to 1984... and Beyond.

v. 39 , no 4,1984, p. $753-761$

0342

BÉLANGER, Jacques MERCIER, Jacques Le plafonnement de la densité syndicale au Québec et au Canada.

v. 41 , no 1,1986, p. $28-52$

0448

CARTER, Donald D.

Canadian Labour Relations Under the Charter: Exploring the Implications.

v. 43 , no 2,1988 , p. $305-321$

0648

FRICKE, John G.

Worker Participation in Canada: Some Lessons from the Past.

v. 43 , no 3,1988 , p. $633-658 \quad 0671$

GILES, Anthony MURRAY, Grégor Towards an Historical Understanding of Industrial Relations Theory in Canada.

v. 43 , no 4,1988 , p. $780-811 \quad 0684$

AUDET, Michel BÉLANGER, Laurent Nouveaux modes de gestion et relations industrielles au Canada.

v. 44 , no 1,1989 , p. $62-96$

0702

SACK, Jeffrey LEE, Tanya

The Role of the State in Canadian Labour Relations.

v. 44 , no 1,1989, p. $195-223$

0708 


\section{SYSTÈME DES RELATIONS} INDUSTRIELLES - CANADA (suite)

WRIGHT, Michael D.

The Legislation of Labour Relations at Canada Post.

v. 44 , no 4,1989, p. $866-883$

\section{HÉBERT, Gérard}

L'état de la discipline en relations industrielles au Canada: Un brin d'histoire et une postface./The State of the Art in Industrial Relations: A Brief History and a Postcript.

v. 44 , no 4,1989 , p. $884-904$

0758

\section{BARKIN, Solomon}

The State of the Art in Industrial Relations: A U.S. Commentary.

v. 44 , no 4,1989 , p. $905-914$

WEISS, Dimitri

L'état de la discipline en relations industrielles au Canada: Un commentaire européen.

v. 44 , no 4,1989 , p. $914-920$

0760

DOWNIE, Bryan M.

The Role of the State in Industrial Relations: A Comment.

v. 45 , no 1,1990 , p. $169-186$

0781

BLOUIN, Rodrigue FERLAND, Gilles LAFLAMME, Gilles LAROCQUE, Alain RONDEAU, Claude

Introduction - Les Chartes des droits et les relations industrielles.

43ième Congrès, 1988, p. XV

0941

ROCHER, Guy

Les fondements de la société libérale, les relations industrielles et les Chartes.

43ième Congrès, 1988, p. 1-18 0942

BERGERON, Jean-Louis

La gestion de l'embauche, de la promotion et du licenciement revue et corrigée par les Chartes.

43ième Congrès, 1988, p. 51-62 0944

\section{DUSSAULT, Ginette}

A travail équivalent, salaire égal: Un droit difficile à appliquer?

43ième Congrès, 1988, p. 83-95 0946
BARRÉ, Alain

Le régime des rapports collectifs et les Chartes.

43ième Congrès, 1988, p. 131-156 0949

DESMARAIS, Jacques

Les moyens de pression: Les chartes en redéfinissent-elles les limites?

43ième Congrès, 1988, p. 157-195 0950

DULUDE, Gilles LOUMĖDE, Catherine

PARROT, Jean-Claude

Commentaires - Les moyens de pression: Les Chartes en redéfinissent-elles les limites?

43ième Congrès, 1988, p. 195-208 0951

PÉPIN, Marcel

L'actualisation du mouvement syndical.

43ième Congrès, 1988, p. 209-221 0952

GAGNON, Mona-Josée LEBEAU, Ernest JOHNSTON, Raymond

Commentaires - l'actualisation du mouvement syndical.

43ième Congrès, 1988, p. 222-236 0953

\section{GARANT, Patrice}

Statut et pouvoirs des organismes du travail en regard des Chartes.

43ième Congrès, 1988, p. 237-254 0954

DUFOUR, Ghislain LABERGE, Louis

LAROSE, Gérald

Table ronde - Les Chartes impliquent-elles un réalignement des politiques syndicales et patronales?

43ième Congrès, 1988, p. 255-272 0955

\section{SYSTÈME DES RELATIONS}

INDUSTRIELLES - ÉTATS-UNIS

BARBASH, Jack

The New Industrial Relations in the US: Phase II.

v. 43 , no 1,1988 , p. $32-42$

0627

RESHEF, Yonatan

Changing Environments and Management 'IR Practices: Implications for U.S. Trade Unions.

v. 43 , no 1,1988, p. $43-62$

0628 
SYSTĖME DES RELATIONS

INDUSTRIELLES - ÉTATS-UNIS (suite)

KOCHAN, Thomas A.

MCKERSIE, Robert B.

Future Directions for American Labor and Human Resources Policy.

v. 44 , no 1,1989 , p. $224-248 \quad 0709$

\section{SYSTÈME DES RELATIONS}

INDUSTRIELLES - ISRAËL

CHERMESH, Ran

Conflict in the Israeli Industrial Relations System: A Critical Description.

v. 36, no 3,1981 , p. $630-647$

0057

KAMINKA, Shlomit ROSEINSTEIN, Eliezer Shop Stewards, Workers, and Managers in Israesli Industry.

v. 38 , no 3,1983 , p. $598-617 \quad 0228$

\section{SYSTĖME DES RELATIONS}

INDUSTRIELLES - ITALIE

WEISS, Dimitri

Du concept de "professionnalité" dans les relations industrielles italiennes.

v. 38 , no 2,1983 , p. $369-379 \quad 0208$

\section{SYSTÈME DES RELATIONS}

INDUSTRIELLES - ONTARIO

KELLY, Laurence

Industrial Relations at Queen's: The First Fifty Years.

v. 42 , no 3,1987 , p. $475-4990580$

\section{SYSTÈME DES RELATIONS}

INDUSTRIELLES - QUÉBEC

LECLERC, Claudine

Thèses de mantrise en relations industrie/les - Université Laval et Université de Montreal: 1940-1980

v. 36, no 2,1981 , p. $435-448 \quad 0036$

HÉBERT, Gérard

Distinction 1982 de l'Association canadienne des relations industrielles: Gérard Dion.

v. 37 , no 3,1982 , p. $475-476 \quad 0135$

GOLDENBERG, Shirley B.

HÉBERT, Gérard

Harry Douglas Woods, 1907-1983

v. 38, no 4,1983, p. $707-712$
HÉBERT, Gérard

In memoriam: Émile Bouvier, S.J. 1906-1985

v. 40 , no 2,1985, p. $211-212$

0381

\section{LACOSTE, Paul}

L'École des relations industrielles de I'Université de Montreal: Quarantième anniversaire.

v. 40, no 2,1985, p. $213-218 \quad 0382$

\section{LAFLAMME, Gilles}

L'impact du régime de relations du travail sur la syndicalisation.

38ième Congrès, 1983, p. 77-90 0862

MORIN, Fernand

L'institutionnalisation des rapports collectifs du travail: Réalité d'aujourd'hui et de demain.

40ième Congrès, 1985 , p. 75-115 0893

GAGNON, Robert P.

Commentaires - L'institutionnalisation des rapports collectifs du travail: Réalité d'aujourd'hui et de demain.

40ième Congrès, 1985, p. 116-119 0894

MARCHAND, Jean

Les rapports collectifs du travail: Rétrospective et perspective.

40ième Congrès, 1985, p. 229-244 0902

THWAITES, James LAJOIE, Mario BOIS-BROCHU, Hélène

Supplément - Quarante ans au service des relations industrielles

40ième Congrès, 1985, p. 261-281 0904 
$$
\text { . }
$$ 


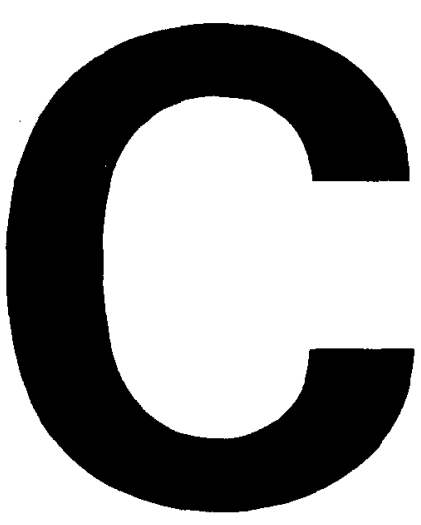

INDEX MÉTHODIQUE DES OUVRAGES

RECENSÉS

\author{
BROAD SUBJECT HEADINGS INDEX \\ OF BOOK REVIEWS
}


L'Index méthodique des ouvrages recensés groupe les ouvrages ayant fait l'objet d'un compte rendu sous les descripteurs génériques correspondant aux divisions du domaine des relations industrielles. La liste de ces descripteurs apparaît au début de la section " $B$ "' (page 809).

EXEMPLE:

FEMMES ET TRAVAIL

Descripteur

générique

DAVID, Hélène

Femmes et emploi: le défi de l'égalité.

Montréal, Les Presses de l'Université du

Québec, 1986, 477 pp., ISBN 2-7605-0393-3,

recensé par Carla Lipsig.

Auteur, titre, référence bibliographique de l'ouvrage recensé et auteur de la recension

v. 45 , no 1,1990 , p. $202-203$

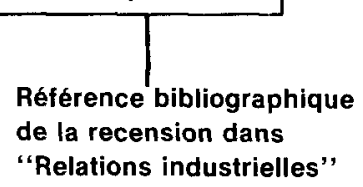


In this Index, Book Reviews are arranged under Broad Subject Headings, the list of which is given at the beginning of Section " $B$ " (page 809). The Broad Subject Headings correspond to main topics in Industrial Relations.

EXAMPLE: ORGANISATIONS SYNDICALES $ـ \begin{aligned} & \text { Broad Subject } \\ & \text { Heading }\end{aligned}$

WINDMULLER, J.P.

The International Trade Union Movement. Deventer, Kluwer, 1980, 174 pp., recensé par Gérard Dion.

v. 36 , no 2,1981 , p. $455-456$

Bibliographical reference

of book review in

"Industrial Relations"

Author, title, reference of book reviewed, and author of book reviewed 


\section{DROIT DU TRAVAIL}

\section{DROIT DU TRAVAIL}

VÉZINA, Claude

Les clauses d'ancienneté et l'arbitrage des griefs. Ottawa, Collection des travaux de la Faculté de droit de l'Université d'Ottawa, éditions de l'Université d'Ottawa, 1979, 125 pp., recensé par Rodrigue Blouin.

v. 36 , no 1,1981 , p. $288-289$

\section{B.I.T.}

Rapport de la Commission d'experts pour l'application des conventions et recommandations. Conférence internationale du travail, 66ième session, Genève, Rapport III, 1980, Partie 4A, 251 pp., Partie 4B, Travailleurs migrants, 190 pp., recensé par Alain Barré.

v. 36, no 1, 1981, p. 291-294

BÉRAUD, Jean-Marc

La suspension du contrat de travail. Paris, Editions Sirey, 1980, 294 pp., recensé par Rodrigue Blouin.

v. 36 , no 2,1981 , p. $451-452$

JAVILLIER, Jean-Claude

Le Droit du travail, 2ième éd., Paris, L.G.D.J., 1981, 700 pp., recensé par Dimitri Weiss.

v. 36, no 2,1981 , p. $452-453$

\section{B.I.T.}

Conciliation and Arbitration Procedures in Labour Disputes. A Comparative Study. Geneve, International Labour Office, 1980, 183 pp., recensé par Rodrigue Blouin.

v. 36 , no 2,1981 , p. $453-454$

\section{BÉRAUD, Jean-Marc}

La suspension du contrat de travail. Paris, Editions Sirey, 1980, 296 pp., recensé par Dimitri Weiss.

v. 36 , no 2,1981, p. $454-455$

BAR-NIV, Zvi H.

International Labour Law Reports, vol. 2, 1979, 376 pp., recensé par Dimitri Weiss. v. 36 , no 2,1981 , p. $454-455$

\section{B.I.T.}

Rapport de la Commission d'experts pour l'application des conventions et recom- mandations. Conférence internationale du travail, 67ième session, Genève, Rapport III (Partie 4A), BIT, 1981, 255 pp., recensé par Alain Barré.

v. 36 , no 4,1981 , p. $951-954$

B.I.T.

Age minimum. Etude d'ensemble de la commission d'experts pour l'application des conventions et recommandations, Conférence internationale du travail, 67ième session, Genève, Rapport III (Partie 4B), BIT, 1981, 238 pp., recensé par Alain Barré.

v. 36 , no 4,1981 , p. $951-954$

O.I.T.

International Labour Conventions and Recommandations. I.L.O., Geneva, 1982, 1167 pp., recensé par Alain Barré.

v. 38 , no 1,1983 , p. $189-190$

\section{AUBERT, Gabriel}

L'obligation de paix du travail - étude du droit suisse et comparé. Genève, Librairie de I'Université, Georg et Cie S.A., 1981, 306 pp., ISBN 2-8257-0078-9, recensé par Pierre Verge.

v. 38 , no 2,1983 , p. $442-444$

\section{BRITTON, Raymond L.}

The Arbitration Guide : A Case-Handing Manual Procedures and Practices in Dispute Resolutions. Englewood Cliffs, New Jersey, Prentice Hall, 1982, 298 pp., ISBN 0-13-043984-3, recensé par André C. Cóté. v. 38 , no 2,1983 , p. $446-448$

\section{BAR-NIV, Zvi H}

International Labour Law Reports, vol. 3. Al'phen aan den Rijn, Hollande, Sijthoff \& Noordhoff, 1980, 399 pp., ISBN 90-2860060-4, recensé par André C. Cóté. v. 38 , no 3,1983 , p. $690-691$

BLANPAIN, R. MILLARD, F. Comparative Labour Law and Industrial Relations. Deventer, Kluwer, Washington, the Bureau of National Affairs, 1982, 411 pp., ISBN 0-87179-392-2, recensé par Pierre Verge.

v. 39 , no 1,1984, p. $201-203$ 


\section{DROIT DU TRAVAIL (suite)}

LEARY, Virginia

International Labour Conventions and National Law. The Haque, Martinus Nijhoff Publishers, 1982, 191 pp., ISBN 90-247. 2551-8, recensé par Alain Barré.

v. 39 , no 1,1984 , p. $203-205$

BARBAGELATA, H.H.

Introduction aux institutions du travail en Amérique Latine. Louvain, Presses universitaires de Louvain, 1980, 303 pp., ISBN 90-6186-101-2, recensé par Pierre Verge. v. 39 , no 3,1984 , p. $632-633$

GOULD, W.B.

Japan's Reshaping of American Labor Law. Cambridge, Mass., The MIT Press, 1984, 193 pp., ISBN 0-262-07091-X, recensé par Pierre Verge.

v. 39 , no 3,1984, p. $642-643$

LAPERRIERE, René

Bibliographie du droit du travail canadien et québecois. Montréal, Les Éditions Yvon Blais Inc., 1984, 70 pp., ISBN 2-89073515-X, recensé par Rodrigue Blouin.

v. 39 , no 4,1984 , p. $812-813$

VERGE, Pierre

Le droit de grève, fondements et limites. Chicoutimi, Editions Yvon Blais, 1985, 229 pp., ISBN 2-89073-553-2, recensé par Fernand Morin.

v. 40, no 4,1985 , p. $907-910$

DESPAX, Michel PÉLISSIER, Jean

La gestion du personnel : aspects juridiques. Tome 1, 2ième éd., Paris, Éditions Cujas, 1984, 400 pp., ISBN 2-2548-40310 , recensé par Dimitri Weiss.

v. 41 , no 1,1986 , p. $190-191$

ZISKIND, .David

Concerning Human Aspirations - Essays in comparative Labor Law. Los Angeles, The Litlaw Foundation, 1985, 172 pp., ISBN O96-15761-1-1, recensé par Pierre Verge.

v. 41 , no 1,1986 , p. $196-197$

SCIARRA, Silvana

Contratto collettivo e contrattazione in azienda. Milano, Franco Angeli, 1985, 226 pp., recensé par Dimitri Weiss.

v. 41 , no 1,1986 , p. $198-200$
VON PRONDZYNSKI, F.

Freedom of Association and Industrial Relations. London, Mansell Publishing, 1987, 248 pp., ISBN 0-7201-1775-5, recensé par Pierre Verge.

v. 42 , no 3,1987 , p. $651-652$

RIVERO LAMAS, Juan

Limitacion de los poderes empresariales y democracia industrial, Leccion inaugural del curso academico 1986-1987. Zaragoza, Universidad de Zaragoza, 1986, 144 pp., Deposito Legal : Z.I. 631-86, recensé par Pierre Verge.

v. 42 , no 3,1987, p. $659-660$

\section{HÉBERT, Gérard TRUDEAU, Gilles}

Les normes minimales du travail au Canada et au Québec : Étude juridique et institutionnelle. Montréal, Éditions Yvon Blais, 1987, 192 pp., ISBN 2-89073-611-3, recensé par Pierre Laporte.

v. 42, no 4,1987, p. $876-877$

\section{ENGLAND, Geoffrey}

Part-time, Casual and Other Atypical Workers : A Legal View. Research and Current Issues Series No 48, Kingston, Industrial Relations Centre, Queen's University, 1987, 81 pp., ISBN 0-88886-154-0, recensé par Udo Mayer.

v. 42 , no 4,1987 , p. $880-881$

HEPPEL, Bob (Ed.)

The Making of Labour Law in Europe - A Comparative Study of Nine Countries up to 1945. London, Mansell Publishing Ltd, 1986, 401 pp., ISBN 0-7202-1697-X, recensé par Pierre Verge.

v. 42, no 4,1987 , p. $884-886$

\section{DELAMOTTE, Yves}

Le droit du travail en pratique. (3ième éd.), Paris, Les Éditions de l'organisation, 1987, 328 pp., ISBN 1-7081-0845-X, recensé par Gérard Dion.

v. 43 , no 2,1988 , p. 473 


\section{DROIT DU TRAVAIL (sulte)}

BEN-ISRAEL, Ruth

International Labour Standards : The Case of Freedom to Strike. Deventer, Kluwer, 1988, 133 pp., ISBN 90-6544-317-7, recensé par Pierre Verge.

v. 43, no 3,1988, p. $710-712$

B.I.T.

La liberté syndicale - Manuel d'education ouvrière. (2ième éd.), Genève, Bureau international du travail, $1988,153 \mathrm{pp}$, ISBN 92-2-205782-1, recensé par Pierre Verge.

v. 43, no 3,1988, p. $712-713$

\section{AUST, A.E.}

Le contrat d'emploi. Cowansville, Les Éditions Yvon Blais, 1988, 235 pp., ISBN 2-89073-675- $X$, recensé par Réjean Breton. v. 44 , no 2,1989 , p. $466-468$

DESPAX, Michel

Négociations, conventions et accords collectifs. (2ième éd.), tome sept de Droit du travail, publié sous la direction de G.H. Camerlynck, Paris, Dalloz, 1989, 586 pp., ISBN 2-247-00890-2, recensé par Pierre Verge.

v. 45, no 2,1990, p. $434-435$

\section{DROIT DU TRAVAIL - ALLEMAGNE}

KAHN-FREUND, $O$.

Labour Law and Politics in the Weimar Republic. Oxford, Basil Blackwell, 1981, ISBN 0-631-12825-5, recensé par Pierre Verge.

v. 37, no 2,1982, p. $454-455$

\section{KESSLER, Francis}

Le droit des conventions collectives de travail en République fédérale d'Allemagne. Francfort sur Mayenne, Peter Lang, 1988, 666 pp., ISBN 3-8204-1138-0, recensé par Pierre Verge.

v. 44, no 3,1989 , p. $728-729$

\section{DROIT DU TRAVAIL - BELGIQUE}

A l'enseigne du droit social belge. 3ième éd., dans Revue de l'Université de Bruxelles, 1978, Bruxelles, Éditions de l'Université de Bruxelles, 1982, 392 pp., recensé par Pierre Verge.

v. 38 , no 2,1983 , p. $445-446$
BLANPAIN, $R$.

Principes de droit du travail. BrugesBruxelles, La Charte, 1984, 313 pp., ISBN 90-6400-029-0, recensé par Pierre Verge.

v. 40, no 2,1985, p. $403-405$

\section{DROIT DU TRAVAIL - CANADA}

ARTHURS, H.W. CARTER, D.D.

GLASBEEK, H.J.

Labour Law and Industrial Relations in Canada. Deventer, Kluwer; Toronto; Butterworths, 1981, 291 pp., ISBN 90-3120139 (Kluwer), 0-409-81184-X (Butterworths), recensé par Pierre Verge.

v. 37 , no 1,1982 , p. $241-243$

TARNOPOLSKY, Walter Surma

Discrimination and the Law in Canada. Toronto, Richard De Boo Ltd, 1982, 595 pp., ISBN 0-88820-105-2, recensé par Rodrigue Blouin.

v. 38 , no 2,1983 , p. $441-442$

\section{DORSEY, J.}

Canada Labour Relations Board - Federal Law and Practice. Toronto, the Carswell Company 1983, 323 pp., ISBN 0-45935850-2, recensé par Pierre Verge.

v. 40, no 1,1985, p. $183-185$

\section{ARTHURS. H.W. CARTER, D.D.} GLASBEEK, H.J.

Labour Law and Industrial Relations in Canada. 2nd ed, Toronto, Butterworths, 1984, 316 pp., ISBN 0-409-81185-8, recensé par Pierre Verge.

v. 41 , no 1,1986 , p. $197-198$

ADAMS, G.W.

Canadian Labour Law - A Comprehensive Text. Aurora, Canada Law Book Inc., 1985, 983 pp., ISBN 0-888-04-030-X, recensé par Pierre Verge.

v. 41 , no 3,1986 , p. $659-661$

FOISY, C.H. LAVERY, D.E.

MARTINEAU, L.

Canada Labour Relations Board - Policies and Procedure. Toronto, Butterworths, 1986, 553 pp., ISBN 0-409-81975-1, recensé par Pierre Verge.

v. 41 , no 4,1986 , p. $875-877$ 
DROIT DU TRAVAIL - CANADA (suite) CARROTHERS, A.W.R. PALMER, E.E. RAYNER, W.B.

Collective Bargaining Law in Canada. 2nd Ed., Toronto, Butterworths, 1986, 785 pp., ISBN 0-409-81879-8, recensé par Pierre Verge.

v. 42 , no 1,1987, p. $219-221$

\section{BEATTY, D.M.}

Putting the Charter to Work Designing a Constitutional Labour Code. Kingston et Montreal, McGill-Queen's University Press, 1987, 251 pp., ISBN 0-7735-0601-2, recensé par Pierre Verge.

v. 42 , no 4,1987 , p. $877-880$

Labour Law. Cases, Material and Commentary. (4th ed.), compiled by The Labour Law Casebook Group, Industrial Relations Centre, Kingston, Queen's University, 1986, 593 pp., ISBN 0-88886-139-7, recensé par Fernand Morin.

v. 42, no 4,1987, p. $893-894$

Labour Law under the Charter. Kingston, Ontario, Queen's Law Journal and Industrial Relations Center. 1988, 332 pp., recensé par Gilles Trudeau.

v. 44 , no 4,1989 , p. $953-957$

ARTHURS, H.W. CARTER, D.D.

EUDGE, F. GLASBEEK, H.J.

Labour Law and Industrial Relations in Canada. 3rd ed., Toronto, Butterworths, 1988, 341 pp., ISBN 0-409-88891-5, ISBN 90-6544-389-4 (Kluwer), recensé par Pierre Verge.

v. 44, no 4,1989 , p. 959

\section{DROIT DU TRAVAIL - ÉTATS-UNIS \\ GOULD, W.B.}

A Primer on American Labor Law. Cambridge, Mass., The MIT Press, 1982, 242 pp., ISBN 0-262-070-87-1, recensé par Pierre Verge.

v. 38 , no 1,1983 , p. $180-181$

GOULD, W.B.

Strikes, Disputes Procedures and Arbitration - Essays on Labor Law. Westport, Greenwood Press, 1985, 313 pp., ISBN 0313-24468-5, recensé par Pierre Verge. v. 41, no 3,1986 , p. $650-651$

\section{DŔOIT DU TRAVAIL - FRANCE}

LYON-CAEN, Gérard PÉLISSIER, Jean

Les grands arrêts de Droit du travail, 2ième éd., Paris, Editions Sirey, 1980, 406 pp., recensé par Dimitri Weiss.

v. 36 , no 2,1981 , p. $454-455$

\section{DELAMOTTE, Yves}

Le droit du travail en pratique. Paris, Les Éditions d'organisation, 1983, 319 pp., ISBN 2-7081-0541-8, recensé par Gérard Dion.

v. 39, no 2,1984 , p. $402-403$

\section{VERDIER, Jean-Maurice}

Syndicats et droit syndical. 2ième éd., $v$. II, Le droit syndical dans l'entreprise, tome 5 du Traité de droit du travail, publié sous la direction de G.H. Camerlynck, Paris, Daloz, 1984, 475 pp., ISBN 2-247-005160 , recensé par Pierre Verge.

v. 40, no 1,1985, p. $178-180$

VERDIER, Jean-Maurice

Syndicats et droit syndical. 2ième éd., tome 5, vol. 1 du Traité de droit du travail publié sous la direction de G. $H$. Camerlynck, Paris, Dalloz, 1987, 671 pp., ISBN 2-247-00754-6, recensé par Pierre Verge.

v. 42, no 3,1987, p. $657-658$

DROIT DU TRAVAIL - GRANDE-BRETAGNE LEWIS, R. CLARK, J.

WEDDERBUFN, Lord of Charlton

Labour Law and Industrial Relations : Building on Kahn-Freund. Oxford, Clarendon Press, 1983, 250 pp., ISBN 0-19-825393-

1, recensé par Pierre Verge.

v. 38 , no 3,1983 , p. $677-678$

\section{DROIT DU TRAVAIL - QUÉBEC}

MALLETTE, NoëI

La gestion des relations du travail au Québec. Le cadre juridique et institutionnel. Scarborough, Collection administration, McGraw-Hill, 1980, 642 pp., recensé par Rodrigue Blouin.

v. 36, no 1, 1981, p. 275

MORIN, Fernand BLOUIN, Rodrigue Précis de l'arbitrage des griefs. Québec, Les Presses de I'Université Laval, 1980, 507 pp., recensé par Jean-Louis Dubé. v. 36 , no 2,1981 , p. $449-451$ 


\section{DROIT DU TRAVAIL - QUÉBEC (suite)}

NADEAU, Gaston

Le statut juridique du salarié-gréviste québécois. Québec, Les Presses de I'Université Laval, 1981, 186 pp., ISBN 2-76376938-1, recensé par Rodrigue Blouin. v. 37, no 4,1982 , p. $966-967$

\section{COPLAND LTEEE.}

Index et résumés de sentences arbitrales de griefs, vol. 1, 1970-1980. Montreal, Copland Ltée, 1982, ISBN 2-920391-002, recensé par Fernand Morin.

v. 37 , no 4,1982, p. $967-968$

\section{MORIN, Fernand}

Rapports collectifs du travail. Montreal, Éditions Thémis, 1982, 619 pp., ISBN 2920376-21-7, recensé par André C. Cóté. v. 38 , no 2,1983 , p. $437-440$

Code du travail F.M., lois connexes et règlements. Les Éditions FM à feliilles mobiles Enr., Farnham, 1981, pagination variée, recensé par Jean Sexton.

v. 38 , no 2,1983 , p. $440-441$

\section{AOUST, Claude d' LECLERC, $L$.}

TRUDEAU, Gilles

Les mesures disciplinaires : étude jurisprudentielle et doctrinale. Montréal, Élole de relations industrielles, Monographie no 13, 1982, 484 pp., ISBN 2-89067-012-0, ISSN 0708-9945, recensé par $R$. Blouin.

v. 38 , no 2,1983 , p. $444-445$

\section{CUTLER, Philip}

Code du travail du Québec. Montréal, Les Livres Toundra, 1983, 539 pp., ISBN 88776-135-6, recensé par Gilles Bachand. v. 38 , no 3,1983 , p. $676-677$

La médiation pré-arbitrale en matière de conflits de droits (griefs). Journée d'étude à l'École de relations industrielles, Université de Montreal, 1981, 91 pp., recensé par Rodrigue Blouin.

v. 39 , no 1,1984, p. $220-221$

\section{GAUTHIER-MONTPLAISIR, Francine} L'arbitrage des griefs et les infractions disciplinaires à caractère criminel. Cowansville, Les Éditions Yvon Blais Inc., 1983, 171 pp., ISBN 2-89073-170-7, recensé par André C. Cóté.

v. 39, no 4,1984, p. $795-797$
Index et résumés de sentences arbitrales de griefs. Tome II, Montréal, Coplanam, 1985, ISBN 2-920391-03-8, recensé par Rodrigue Blouin.

v. 41 , no 3,1986 , p. $658-659$

BRADET, Denis CLICHE, Bernard RACINE, Martin THIBAULT, France Droit de la santé et de la sécurité du travail - La loi et la jurisprudence commentée. Cowansville, Les Editions Yvon Blais, 1986, 300 pp., ISBN 2-89073-567-2, recensé par Gilles Trudeau.

v. 42 , no 1,1987, p. $215-216$

MORIN, Fernand BLOUIN, Rodrigue Arbitrage des griefs. Montréal, Les Éditions Yvon Blais, 1986, 554 pp., ISBN 289083-569-9, recensé par Gilles Plante. v. 42 , no 2,1987 , p. $432-434$

\section{LAPORTE, Pierre}

Code du travail du Québec (Législation, Jurisprudence et Doctrine). Avec la collaboration d'Hélène Ouimet, Montréal, Wilson \& Lafleur, 1987, 475 pp., ISBN 289127-058-4, recensé par Rodrigue Blouin. v. 42 , no 2,1987 , p. $436-437$

\section{DUBÉ, Jean-Louis DI LORIO, Nicola} Les normes du travail. Sherbrooke, Les Éditions Revue de Droit, Université de Sherbrooke, 1987, 442 pp., ISBN 2920003-08-9, recensé par Fernand Morin. v. 42 , no 3,1987 , p. $655-656$

\section{GAGNON, Robert P. LEBEL, Louis} VERGE, Pierre

Droit du travail. Québec, Presses de I'Université Laval, 1987, 933 pp., ISBN 27637-7123-8, recensé par Alain Barré. v. 43, no 4,1988 , p. $974-980$

\section{JOBIN, Carol}

Les relations du travail dans l'industrie de la construction. Montréal, Wilson \& Lafleur, 1989, 568 pp., ISBN 2-89127-085-1, recensé par Jean Sexton. v. 44 , no 2,1989 , p. 473

AUDET, Georges BONHOMME, Robert Le congédiement en droit québécois en matière de contrat individuel de travail. 2ième éd., Cowansville, Les Éditions Yvon Blais, 1988, 487 pp., ISBN 2-89073-678-4, recensé par Claude D'Aoust.

v. 44, no 3,1989, p. $732-733$ 
DROIT DU TRAVAIL - QUÉBEC (suite) RENAUD, Marc TRUDEAU, Gilles SAINTJJCQUES, Chantal DUBÉ, Louise Le droit de refus: une révolution tranquille - étude de la mise en oeuvre d'un nouveau droit. École des relations industrielles, Université de Montreal, Monographie, no 21, 1989, 329 p., ISBN 2-89067-0201, ISSN 0708-9945, recensé par Rodrigue Blouin.

v. 45, no 3,1990, p. $637-640$

BUREAU, Robert D. MACKAY, Pierre Le droit dans tous ses états. Montréal, Wilson \& Lafleur, 1987, 620 p., ISBN 289127-07602, recensé par Fernand Morin. v. 45 , no 3,1990 , p. $640-643$

\section{ÉCONOMIQUE DU TRAVAIL}

\section{ÉCONOMIQUE DU TRAVAIL}

EHRENBERG, Ronald G. (Ed.)

Aesearch in Labor Economics : An Annual Compilation of Research, Vol. 2, Greenwich, Connecticut, JAI Press Inc., 1978, recensé par Dennis $R$. Maki.

v. 36 , no 1,1981 , p. $289-291$

THUROW, Lester C.

The Zero-Sum Society. Distribution and the Possibilities for Economic Change. New York, Basic Books Inc., 1980, 230 pp., recensé par Alexander J. Matejko.

v. 36 , no 4 , 1981, p. 949-951

\section{$\mathrm{CROUCH}$, Colin}

Trade Unions : The Logic of Collective Action. Glasgow, Fontana Paperbacks, 1982, 251 pp., ISBN 0-00-635873X, recensé par Alexander J. Matejko.

v. 39 , no 1,1984 , p. $212-215$

BRADLEY, Keith GELB, Alan

Worker Capitalism. The New Industrial Relations. Cambridge, The MIT Press, 1983, 186 pp., ISBN 0-262-02191-0, recensé par Clive H.J. Gilson.

v. 40 , no 3,1985 , p. $678-680$

INGERMAN, Sidney $\mathrm{H}$.

Economics for Canadian Trade Unionists. 2nd Ed., Ottawa, Labour College of Canada, 1985, 208 pp., ISBN 0-969-0345-1-1, recensé par Peter Larson.

v. 40 , no 4,1985 , p. 906

\section{BRETON, Albert}

Le mariage, la population et le taux d'activité des femmes. Conseil économique du Canada, Ottawa, ministère des Approvisionnements et Services Canada, 1983, 36 pp., ISBN 0-660-91184-1, recensé par Jennifer Stoddart.

v. 41 , no 1,1986 , p. $203-204$

BELLEMARE, Diane POULIN-SIMON, Lise Le défi du plein emploi - un nouveau regard économique. Montréal, Éditions Saint-Martin, 1986, 530 pp., ISBN 2-89035131-9, recensé par Louis Ascah. v. 42 , no 2,1987 , p. $450-451$

TAHAR, Gabriel La réduction de la durée du travail. Paris, Collection Repères, Éditions La découverte, 1985, 127 pp., ISBN 2-7071-15525, recensé par Lise Chrétien.

v. 42 , no 3,1987, p. $646-648$

\section{ÉCONOMIQUE DU TRAVAIL - CANADA} CONSEIL ÉCONOMIQUE DU CANADA Pénuries et carences. Travailleurs qualifiés et emplois durant les années 80. Ottawa, ministère des Approvisionnements et Services Canada, 1982; 141 pp., ISBN 0-660-90874-3, recensé par Gérard Bélanver.

v. 37, no 3,1982, p. $704-709$

EASTMAN, Byron D.

Labour Market Theory and the Canadian Experience. Toronto, Harcourt Brace Jovanovich, 1987, 350 pp., ISBN 077-4730757, recensé par S.A. Alvi.

v. 42 , no 4,1987 , p. $894-895$

BROWN, Malcolm C.

Caring for Profit : Economic Dimensions of Canada's Health Industry. Vancouver, The Fraser Institute, 1987, 182 pp., ISBN O88975-106-04, recensé par Gérard Bélanger.

v. 44, no 1,1989, p. 291-292

\section{ÉCONOMIQUE DU TRAVAIL - JAPON}

MCMILLAN, Charles J.

The Japanese Industrial System. BerlinNew York, Walter de Gruyter, 1984, 356 pp., ISBN 3-11-008894-0, recensé par Alexander J. Matejko.

v. 39 , no 4,1984 , p. $799-801$ 
ÉCONOMIQUE DU TRAVAIL - QUÉBEC BOULET, Jac-André

La langue et le revenu du travail à Montréal. Ottawa, Ministère des Approvisionnements et Services Canada, Etude préparée par le Conseil économique du Canada, 1980, 135 pp., ISBN 0-660-90515-9, recensé par Jean-Marie Rainville.

v. 37, no 4,1982 , p. $971-973$

PAQUET, Gilles

La pensée économique au Québec français. Témoignages et perspectives. Montréal, Association canadiennefrançaise pour l'avancement des sciences, 1989, 364 pp., ISBN 2-89245-987-7, recensé par Gérard Dion.

v. 45 , no 2,1990, p. $441-442$

\section{FEMMES ET TRAVAIL}

\section{FEMMES ET TRAVAIL}

COOK, Alice $\mathrm{H}$

The Working Mother, A Survey of Problems and Programs in Nine Countries. 2nd ed., Ithaca, New York State School of Industrial and Labor Relations, Cornell University, 1978, 71 pp., recensé par Marie Lavigne

v. 36 , no 2,1981 , p. 458

\section{STEINBERG RATNER, Ronnie}

Equal Employment Policy for Women : Strategies for Implementation in the United States, Canada and Western Europe. Philadelphia, Temple University Press, 1980, 520 pp., recensé par Carole Swan.

v. 36 , no 3,1981 , p. $692-696$

\section{B.I.T.}

Normes et déclarations de principes intéressant particulièrement les travailleuses. Genève, BIT, 1981, 138 pp., recensé par Alain Barré.

v. 36 , no 3, 1981 , p. $696-697$

CATALYST, The Staff of

Upward Mobility. New-York, Holt, Rinehart and Winston, 1982, 292 pp., ISBN 0-03056163-9, recensé par Alexander J. Matejko.

v. 39 , no 2 , 1984 , p. $398-399$
PHILLIPS, Paul PHILLIPS, Erin

Women and Work : Inequality in the Labour Market. Toronto, James Lorimer \& Company, 1983, 200 pp., ISBN 0-8862608-8, recensé par Jennifer Stoddart.

v. 39, no 2,1984 , p. 400

ARMSTRONG, Pat ARMSTRONG, Hugh Une majorité laborieuse. Les femmes qui gagnent leur vie, mais à quel prix. Ottawa, Conseil consultatif canadien de la situation de la femme, 1983, 329 pp., ISBN 0-660-91026-8, recensé par Esther Déom.

v. 39 , no 4,1984 , p. $816-817$

\section{BRETON, Albert}

Le mariage, la population et le taux d'activité des femmes. Conseil économique du Canada, Ottawa, ministère des Approvisionnements et Services Canada, 1983, 36 pp., ISBN 0-660-91184-1, recensé par Jennifer Stoddart.

v. 41 , no 1,1986 , p. 203-204

AGGARWAL, Arjun P.

Sexual Harassment in the Workplace. Toronto, Butterworths, 1987, 230 pp., ISBN 0-409-80535-1, recensé par $P$. Andiappan. v. 43, no 2,1988 , p. $469-471$

KOZIARA, S MOSKOW, M.H.

TANNER, L.D.

Working Women : Past, Present, Future. Industrial Relations Research Association Series, Washington, The Bureau of National Affairs, 1987, 419 pp., ISBN 0-91344734-X, recensé par $P$. Andiappan.

v. 45 , no 2,1990 , p. $430-431$

\section{FEMMES ET TRAVAIL - CANADA}

WHITE, Julie

Les femmes et le syndicalisme. Ottawa, Conseil consultatif canadien de la situation de la femme, Centre d'édition du gouvernement du Canada, 1980, 147 pp., recensé par Marie Lavigne.

v. 36 , no 2,1981, p. $456-457$

\section{FEMMES ET TRAVAIL - ÉTATS-UNIS}

O'SULLIVAN, Judith GALLICK, Rosemary Workers and Allies : Female Participation in the American Trade Union Movement, 1824-1976. Washington, Smithsonian Institution Press, 1975, 96 pp., ISBN 0-87546138-1, recensé par Micheline Dumont. v. 43 , no 1,1988 , p. 205 
FEMMES ET TRAVAIL - QUÉBEC

VINET, Alain DUFRESNE, Francine

VÉZINA, Lucie

Condition féminine en milieu ouvrier. Qué-

bec, Institut québécois de recherche sur la culture, 1982, 221 pp., ISBN 2-89224018-2, recensé par Marie Françoise Marchis-Mouren.

v. 38 , no 3,1983 , p. $679-680$

DAVID, Hélène

Femmes et emploi : le défi de l'égalité. Montreal, Les Presses de I'Université du Québec, 1986, 477 pp., ISBN 2-76050393-3, recensé par Carla Lipsig.

v. 45 , no 1,1990 , p. $202-203$

Les principes de l'équité salariale et les approches dans le secteur public québécois. Montreal, Institut de recherche et d'information sur la rémunération, IRI-050, octobre 1989, 99 pp., ISBN 2-551-121930 , recensé par Esther Déom.

v. 45 , no 2,1990 , p. $438-439$

\section{GESTION DES ORGANISATIONS}

\section{GESTION DES ORGANISATIONS}

GUIOT, Jean M.

Organisations sociales et comportements. Montréal, Les éditions Agence d'Arc Inc., Paris, Les éditions Hommes et Techniques, 1980, 213 pp., recensé par Laurent Bélanger.

v. 36 , no 4,1981 , p. $946-948$

JACKSON, John H. MORGAN, Cyrill P. Organization Theory: A Macro Perspective for Management (2nd ed.). Englewood Cliffs, Prentice-Hall. Inc., 1982, 413 pp., ISBN 013-641415-X, recensé par Laurent Bélanger.

v. 37, no 2,1982 , p. $457-458$

CUMMINGS, Thomas G. MOLLOY, Edmond $S$.

Improving Productivity and the Quality of Work Life. New-York, Prager Publications, 1977, 305 pp., ISBN 0-03-022601-5, recensé par Alexander J. Matejko.

v. 37 , no 3,1982 , p. 715-717

LEFEBVRE, Gérard

Le coeur à l'ouvrage. Montréal, Les Édi- tions de l'Homme, 1982, 120 pp., ISBN 27619-0188-6, recensé par Dimitri Weiss. v. 38 , no 1,1983 , p. $187-188$

CO'_LERETTE, Pierre DELISLE, Gilles Le changement planifié. Montréal, Agence d'Arc, 1982, 213 pp., ISBN 0-89022045-1, recensé par Laurent Bélanger. v. 38 , no 1,1983 , p. $195-196$

LEMELIN, Maurice MCNEIL, Jeannine Productivité et qualité de vie au travail. Montréal, Les éditions Agence d'Arc Inc., 1982, ISBN 2-89022-044-3, recensé par Laurent Bélanger.

v. 38, no 2,1983, p. $452-453$

HAGE, Jerald

Theories of Organizations : Form, Process and Transformation. Newport, John Wiley \& Sons, 1980, 558 pp., ISBN 0471-338591 , recensé par $D$. Briolat, $P$. Gagnon, $H$. Sidibe et $Y$. Gasse.

v. 39 , no 1,1984 , p. 197-201

BRADLEY, Keith GELB, Alan Worker Capitalism. The New Industrial Relations. London, Heinemann, 1983, 186 pp., ISBN 0-435-82084-2, recensé par Alexander J. Matejko.

v. 39 , no 1,1984 , p. $208-209$

ONDRACK, Dan STUART, Timperley The Humanisation of Work. European Perspective. London, Armstrong Publishing, 1982, 239 pp., ISBN 0-9506540-86, recensé par Alexander J. Matejko.

v. 39 , no 2,1984 , p. $392-393$

Collection "Technologie et travail". Mon"tréal, Institut national de productivité, 1983, 8 textes de 43 pp. à 202 pp., recensé par Jean Nollet.

v. 39 , no 2,1984 , p. $400-402$

MLLLER, Danny FRIESEN, Peter H., Organizations: A Quantum View. Englewood Cliffs, Prentice-Hall, 1984, 320 pp., ISBN 0-13-641985-2, recensé par Michel Paquin.

v. 39 , no 3,1984 , p. $636-637$

BARANSON, Jack

Robots in Manufacturing: Key to International Competitiveness. Mt Airy, Maryland, Lomond Publications, 1983, 152 pp., ISBN 0-912338-39-3, recensé par Rolland Hurtubise.

v. 39 , no 4,1984 , p. $801-802$ 


\section{GESTION DES ORGANISATIONS (suite)} NIGHTINGALE, Donald V.

LONG, Richard J.

Gain and Equity Sharing. Quality of Working Life. Case Study Series, Ottawa, Minister of Supply and Services, Canada, 1984, 51 pp., ISBN 0-662-13138-X, recensé par Alexander J. Matejko.

v. 39, no 4,1984 , p. $813-814$

GOODSELL, Charles T.

The Case for Bureaucracy : A Public Administration Polemic. Chatham, Chatham House Publishers, 1983, 179 pp., ISBN O934540-17-9, recensé par Alexander $J$. Matejko.

v. 40 , no 1,1985, p. $188-190$

\section{HIRSCHHORN, Larry}

Beyond Mechanization: Work and Technology in a Postindustrial Age. Cambridge, MIT Press, 1984, 187 pp., ISBN 0-26208132-3, recensé par Daniel Benedict.

v. 40 , no 1,1985 , p. $192-194$

\section{CROCKER, Olga}

CHIU, Johnny Sik Leung

CHARNEY, Cyril

Quality Circles. A Guide to Participation and Productivity. Toronto, Methuen, 294 pp., ISBN 0-458-97360-2, recensé par Alexander J. Matejko.

v. 40, no 3,1985, p. $682-683$

MERCHANT, Kenneth A.

Control in Business Organization. Boston, Pitman, 1985, 161 pp., ISBN 0-273-019147 , recensé par Alexander J. Matejko.

v. 40 , no 3,1985, p. $685-686$

\section{BEAUDOIN, Pierre}

La gestion par projet : aspects stratégiques. Montréal, Agence d'Arc, 1984, 251 pp., ISBN 2-89-22-065-6, recensé par Paul Tessier.

v. 40, no 4,1985 , p. $898-899$

ALSTOM, Jon P.

The American Samurai. Blending American and Japanese Managerial Practices. Berlin-New York, Walter de Gruyter, 1986, 369 pp., ISBN 0-89925-063-7, recensé par Alexander J. Matejko.

v. 41 , no 3,1986, p. $662-664$
PETERS, TOM AUSTIN, Nancy

A Passion for Excellence. The Leadership Difference. New York, Random House, 1985, 437 pp., ISBN 0-394-54484, recensé par Alexander J. Matejko.

v. 41 , no 4,1986 , p. $881-882$

NAISBITT, John ABURDENE, Patricia Reinventing the Corporation. Transforming your Job and your Company for the New Information Society. New York, Warner Books, 1985, 308 pp., ISBN 0-446-512842, recensé par Alexander J. Matejko.

v. 41 , no 4,1986 , p. $881-882$

JACKSON, John H. MORGAN, Cyrill P. PROLILLO, J.G.P.

Organization Theory : A Macro Perspective for Managing. Englewood Cliffs, PrenticeHall, 1986, 387 pp., ISBN 0-13-641572-501 , recensé par James Thwaites.

v. 41 , no 4,1986 , p. $884-885$

\section{DESSLER, Gary}

Organization Theory : Integrating Structure and Behavior. Englewood Cliffs, PrenticeHall, 1986, 477 pp., ISBN 0-13-641-9038-01, recensé par James Thwaites.

v. 41 , no 4,1986 , p. $884-885$

DURAND, Jean-Pierre

DURAND-SEBAG, Joyce LOJKINE, Jean MAHIEU, Christian

L'enjeu informatique : former pour changer l'entreprise. Paris, Meridiens Klincksieck, 1986, 192 pp., ISBN 2-86563-1486 , recensé par Guylaine Vallée. v. 42 , no 3,1987 , p. $660-661$

PAQUIN, Michel

L'organisation du travail. Montreal, Agence d'Arc, 1986, 199 pp., ISBN 2-89022091-5, recensé par Alain Vinet. v. 42 , no 3,1987, p. $663-664$

GROOTINGS, Peter GUSTAVSEN, Bjorn HÉTHY, Lajos

New Forms of Work Organization and their Social and Economic Development. Budapest, Statistical Publishing House, 1986, 297 pp., ISBN 963-01-6862-6, recensé par Alexander J. Matejko.

v. 42 , no 3,1987 , p. $665-667$ 


\section{GESTION DES ORGANISATIONS (sulte)}

BOYER, LUC EQUILBEY, Noël

Le projet d'entreprise. Paris, Les Éditions d'Organisation, 1986, 135 pp., ISBN 27081-0749-6, recensé par Paul Beaulieu. v. 42 , no 4,1987 , p. $881-883$

RUBINSTEIN, Sidney P.

Participative Systems at Work : Creating Quality and Employment Security. New York, Human Sciences Press, 1987, 180 pp., ISBN 0-89885-338-9, recensé par Alexander J. Matejko.

v. 43, no 2,1988 , p. $471-472$

LEWCHUK, Wayne

American Technology and the British Vehicle Industry. Cambridge, Cambridge University Press, 1987, 304 pp., ISBN 0521 . 30269-2, recensé par Jacques Bélanger. v. 43, no 3,1988, p. $716-717$

FOOTE WHITE, William

KING WHITE, Kathleen

Making Mondragon : The Growth and Dynamics of the Worker Cooperative Complex. Ithaca, ILR Press, 1988, 317 pp., ISBN 0-87546-137-9, recensé par Hem C. Jain.

v. 44 , no 1,1989 , p. $289-291$

PETERS, TOM

Thriving on Chaos. Handbook for a Managerial Revolution. New York, Alfred A. Knopf, 1987, 561 pp., ISBN 0-394-56784-

6 , recensé par Alexander J. Matejko.

v. 44 , no 1,1989 , p. $292-293$

LAROCQUE, Alain BORDELEAU, Yvan

BOULARD, René FABI, Bruno

LAROUCHE, Viateur RONDEAU, Alain

Technologies nouvelles et aspects psycho-

logiques. Sillery, Presses de l'Université du Québec, 1987, 171 pp., ISBN 2-76050450-6, recensé par Jean Mercier.

v. 44 , no 2,1989 , p. $469-470$

GATTIKER, Urs E. LARWOOD, Laurie Managing Technological Development. Strategic and Human Resources Issues. Berlin, Walter de Gruyter, 1988, 232 pp., ISBN 3110110849, recensé par Alexander J. Matejko.

v. 44 , no 2,1989 , p. $470-472$
SNOW, Charles $\mathrm{C}$.

Strategy, Organization Design, and Human Resource Management. Greenwich, JAI Press, 1989, 368 pp., ISBN 0-89232-807$X$, recensé par Michel Audet.

v. 44, no 3,1989 , p. $734-736$

TURCOTTE, Pierre R.

La QVT une voie vers l'excellence. Montréal, Les Éditions Agence d'Arc, 1988, 276 pp., ISBN 289022-134-2 et ISBN 27624-0066-X, recensé par Roger Zaoré.

v. 44 , no 4,1989 , p. $962-963$

PASMORE, William A.

Designing Effective Organizations. The Sociotechnical Systems Perspective. Wiley Series on Organizational Assessment and Change, New York, John Wiley, 1988, 200 pp., ISBN 0-471-88785-4, recensé par Alexander J. Matejko.

v. 44 , no 4,1989 , p. $964-967$

BOONE, Louis E. KURTZ, David L. L'entreprise d'aujourd'hui. (2ième éd.), Montréal, Éditions études vivantes, 1989, 737 pp., ISBN 2-7607-0446-7, recensé par Laurent Bélanger.

v. 45 , no 2,1990 , p. 432

\section{GESTION DES ORGANISATIONS -}

\section{ALLEMAGNE}

LAWRENCE, Peter

Managers and Management in West Germany. London, Croom Helm, 1980, 202 pp., ISBN 0-85664-912-0, recensé par Michel Paquin.

v. 38 , no 2,1983 , p. $455-456$

\section{GESTION DES ORGANISATIONS - CANADA} MANSELL, Jacquie

Workplace Innovation in Canada. Reflections on the Past Prospects for the Future. A Study prepared for the Economic Council of Canada, Ottawa, Minister of Supply and Services Canada, 1987, 40 pp., ISBN 0-660-12554-4, recensé par Alexander J. Matejko.

v. 43 , no 1,1988 , p. 211 


\section{GESTION DES ORGANISATIONS - ÉTATS-UNIS}

PETERS, T.J. WATERMAN, R.H. Jr. In Search of Excellence : Lessons from America's Best-Run Companies. New York, Harper and Row, 1982, 350 pp., ISBN 0-06-015042-4, recensé par James Thwaites.

v. 39 , no 2,1984, p. $403-406$

KANTER, Rosabeth Moss

The Change Masters. Innovation and Entrepreneurship in the American Corporation. New York, Simon and Schuster, 1984, 432 pp., ISBN 0-671-42802-0, recensé par Alexander J. Matejko.

v. 40, no 2,1985, p. $411-412$

\section{GESTION DES ORGANISATIONS - JAPON}

THUROW, Lester C.

The Management Challenge. Japanese Views. Cambridge, The MIT Press, 1985, 237 pp., ISBN 0-262-20053-8, recensé par Helène Bois-Brochu.

v. 40, no 3,1985 , p. $683-685$

\section{GESTION DES RESSOURCES HUMAINES}

\section{GESTION DES RESSOURCES HUMAINES} COOPER, Cary L. MEMFORD, Enid The Quality of Working Life in Western and Eastern Europe. Westport, Greenwood Press, 1979, 348 pp, recensé par Alexander J. Matejko.

v. 36 , no 1,1981 , p. $285-288$

NAYLOR, James C. PRITCHARD, R.D. ILGEN, D.R.

A Theory of Behavior in Organizations. New York, Academic Press Inc., 1980, 299 pp., recensé par André Petit.

v. 36, no 3,1981, p. 703-704

PÉGANIOL, Claude

Techniques et politiques d'amélioration des conditions de travail. Paris, Entreprise moderne d'édition, 162 pp., recensé par Laurent Bélanger.

v. 36 , no 4,1981, p. $943-944$

BRIDDLE, Bruce J.

Role Theory : Expectations, ldentities and Behaviors. New York, Academic Press,
1979, 416 pp., recensé par André Petit. v. 36 , no 4,1981 , p. $944-945$

LEVY-LEBOYER, C.

Le psychologue et l'entreprise. Montreal, Masson et Presses de l'Université de Montréal, 1980, 169 pp., recensé par Laurent Belanger.

v. 36 , no 4,1981 , p. 948

HOUDE, Eugène

Emotivité et efficacité au travail. Montréal, Editions de l'Homme, 1982, 112 pp., ISBN 2-7619-0213-0, recensé par Bernard Angers.

v. 37 , no 2,1982 , p. $458-459$

GLUECK, William $F$.

Personnel : A Diganostic Approach, (3rd ed.). Business Publications Inc., 1982, 720 pp., ISBN 0-256-02429-4, recensé par Laurent Bélanger.

v. 37 , no 2,1982 , p. $459-460$

CUMMINGS, Thomas G.

MOLLOY, Edmond S.

Improving Productivity and the Quality of Work Life. New-York, Prager Publications, 1977, 305 pp., ISBN 0-03-022601-5, recensé par Alexander J. Matejko.

v. 37, rio 3, 1982, p. 715-717

LEFEBVRE, Gérard

Le coeur à l'ouvrage. Montréal, Les Éditions de l'Homme, 1982, 120 pp., ISBN 27619-0188-6, recensé par Dimitri Weiss.

v. 38 , no 1,1983 , p. $187-188$

KELLY, John E.

Scientific Management, Job Redesigi, and Work Performance. London, Academic Press, 1982, 257 pp., ISBN 012404020-9, recensé par Alain Vinet.

v. 38 , no 2,1983, p. $453-455$

FELDMAN, Daniel C. ARNOLD, Hugh J. Managing Individual and Group Behavior in Organizations. Toronto, McCrowill-Hill Series in Management, 1983, 613 pp., ISBN 0-8359-3006-4, recensé par Laurent Bélanger.

v. 38 , no 3,1983, p. $682-683$ 


\section{GESTION DES RESSOURCES HUMAINES (suite)}

CASCIO, Wayne E. AWARD, Elias M. Human Resources Management. An Information System Approach. Virginia, Reston Publishing Co., 1981, 594 pp., ISBN 0-07020386-5, recensé par Laurent Bélanger. v. 38, no 3,1983 , p. $683-684$

TURCOTTE, Pierre R.

Qualité de vie au travail : anti-stress et créativité. Montreal, Agences d'Arc, 1982, 184 pp., ISBN 2-89022-046-X, recensé par René Boulard.

v. 38 , no 3,1983 , p. $689-690$

DOLAN, Shimon ROY, Denis

La sélection des cadres : principes et problèmes contemporains. Monographie no 13, Montréal, École de Relations Industrielles, Université de Montréal, 1982, ISBN 2-89067-010-4, ISSN 0708-9945, recensé par Normand Pettersen.

v. 38 , no 4,1983 , p. $910-912$

THÉRIAULT, Roland

Gestion de la rémunération: Politiques et pratiques efficaces et équitables. Boucherville, Gaétan Morin Éditeur, 1983, 585 pp., ISBN 2-89105-109-2, recensé par Jacques Chouinard.

v. 38 , no 4,1983 , p. $913-915$

HALLORAN, Jack

Applied Human Relations, An Organizational Approach. (2nd ed), Englewood Cliffs, N.J., Prentice-Hall, 1983, 531 pp., ISBN O13-040808-5, recensé par Alain Rondeau. v. 38 , no 4,1983 , p. $920-922$

ARGYRIS, Chris

Reasoning, Learning and Action. San Francisco, Jossey-Bass, 1982, 499 pp., ISBN 0-87589-524-7, recensé par Naresh C. Agarwal.

v. 39 , no 1,1984 , p. $206-208$

BÉLANGER, Laurent PETIT, André

BERGERON, Jean-Louis

Gestion des ressources humaines : une approche globale et intégrée. Chicoutimi, Gaëtan Morin éditeur, 1983, 451 pp., ISBN 2-89105-072-X, recensé par Dimitri Weiss. v. 39 , no 2,1984 , p. $406-408$
RITA, Giuseppe De SILVESTRI, Umberto TEDESCHI, Michele WEISS, Dimitri ZUBOFF, Shoshana

II ruolo del fattore "uomo" nello scenario economico-produttivo degli anni "80:i quadri. Roma, STET, 1982, 84 pp., recensé par $P$. Joubert.

v. 39, no 3,1984, p. $637-638$

SLOANE, Arthur A.

Personnel, Managing Human Resources. Englewood Cliffs, Prentice Hall, 1983, 619 pp., ISBN 0-13-658278-8, recensé par Paul Tessier.

v. 39, no 4,1984 , p. $804-805$

DUBRIN, Andrew J.

Foundations of Organizational Behavior: An Applied Perspective. Englewood Cliffs, Prentice-Hall, 1984, 500 pp., ISBN 0-13329367- $X$, recensé par Laurent Bélanger. v. 39 , no 4,1984 , p. $805-806$

PIVETEAU, Jacques

L'entretien d'appréciation du personnel : pratiques en question. Toulouse, Éditions Erès, 1981, 207 pp., ISBN 2-86586-002-7, recensé par Roland Thériault.

v. 39 , no 4,1984 , p. $806-809$

NEILSEN, Eric $\mathrm{H}$.

Becoming an O.D. Practitioner. Englewood Cliffs, Prentice Hall, 1984, 280 pp., ISBN 0-13-071555-7, recensé par Laurent Bélanger.

v. 39 , no 4,1984 , p. $809-811$

\section{MINTZBERG, Henry}

Le manager au quotidien. Les dix róles du cadre. Montréal, Les Éditions Agence d'Arc, 1984, 220 pp., ISBN 2-7081-05582, recensé par Michel Paquin.

v. 39 , no 4,1984 , p. $811-812$

SERRUYS, Jacques W.

Phychologie du commandement. Bruxelles, Vander Éditeur, 1984, 230 pp., ISBN 2-8008-0000-3, recensé par Hélène BoisBrochu.

v. 40, no 2,1985, p. $414-415$ 


\section{GESTION DES RESSOURCES HUMAINES (suite)}

PINDER, Craig C.

Work Motivation. Theory, Issues and Applications. Glenview, Scott, Foresman and Corp., 1984, 365 pp., ISBN 0-673-157997 , recensé par Alexander J. Matejko.

v. 40, no 3,1985 , p. 680

\section{LANDY, Frank J.}

Psychology of Work Behavior. Homewood, The Dorsey Press, 1985, XIV-622-R4111 pp., ISBN 0-256-03046-4, recensé par Alexander J. Matejko.

v. 40 , no 4,1985 , p. $896-898$

DESPAX, Michel PÉLISSIER, Jean

La gestion du personnel : aspects juridiques. Tome 1, 2ième éd., Paris, Éditions Cujas, 1984, 400 pp., ISBN 2-2548-40310 , recensé par Dimitri Weiss.

v. 41 , no 1,1986 , p. $190-191$

WEISS, Dimitri

"La participation". Enciclopedia de Dirreccion y Administracion de la Empresa, Fasciculo 82, Barcelona, Ediciones Orbis, 1985, ISBN (fascicule) 84-7530-585-7; (oeuvre complète) 84-7530-583-0; (volume VI) 84-7634-230-6, recensé par $P$. Joubert.

v. 41 , no 2,1986 , p. $423-424$

BEAUMONT, P.B. PARTRIDGE, M.

Job Satisfaction in Public Administration. Londres, Royal Institute of Public Administration, 1983, 41 pp., ISBN 0-900628-324, recensé par Michel Paquin.

v. 41 , no 2,1986 , p. $426-427$

ROBBINS, Stephen $\mathbf{P}$.

Organizational Behavior. Concepts, Controversies and Applications. 3rd ed., Englewood Cliffs, Prentice-Hall, 1986, 554 pp., ISBN 0-13-641549-0-01, recensé par Hélène Bois.

v. 41 , no 4,1986 , p. $879-880$

PORTIS, Bernard FULLERTON, David F. INGRAM, Paul R.

Quality Circles in Canada - A Review of Case Histories on Employee Involvement Programs. London, School of Business Administration, University of Western Onta- rio, 1986, 77 pp., recensé par Jean-Louis Bergeron.

v. 41 , no 4,1986 , p. $885-886$

MILKOVICH, George T.

NEWMAN, Jerny M.

Compensation. Plano, Texas, Business Publications Inc., 1984, 549 pp., ISBN 0 256-02204-6, recensé par Gilles Ferland. v. 42 , no 1,1987 , p. $218-219$

KELLEY, Robert E.

The Gold Collar Worker. Reading Massachusetts, Addison-Wesley Publishing Co. 1985, 196 pp., ISBN 0-201-11739-8, recensé par Paul Beaulieu.

v. 42, no 3,1987, p. $664-665$

AGGARWAL, Arjun P.

Sexual Harassment in the Workplace. Toronto, Butterworths, 1987, 230 pp., ISBN 0-409-80535-1, recensé par P. Andiappan. v. 43, no 2,1988 , p. $469-471$

MEUNIER, Pierre-Marc

LAFLAMME, Marcel

Dynamisme humain dans l'excellence or ganisationnelle. Montréal, Agence d'Arc 1987, 431 pp., ISBN 2-89022-120-2, recensé par Roger Z. Vanié.

v. 43 , no 4,1988 , p. $972-973$

KLEINER, Morris M. BLOCK, Richard N. ROOMKIN, Myron

SALSBURG, Sydney W.

Human Resources and the Performance of the Firm. Madison, Industrial Relations Research Association Series, 1987, 343 pp., ISBN 0-913447-382, recensé par Bernard Portis.

v. 44 , no 1,1989 , p. 297

SNOW, Charles $\mathrm{C}$

Strategy, Organization Design, and Human Resource Management. Greenwich, JAl Press, 1989, 368 pp., ISBN 0-89232-807$X$, recensé par Michel Audet. v. 44 , no 3,1989 , p. $734-736$

FINCHAM, Robin RHODES, Peter S. The Individual, Work and Organization. Behavioural Studies in Business and Management Students. London, Weidenfeld and Nicolson, 1988, 336 pp., ISBN 0-29779139-7, recensé par Alexander J. Matejko.

v. 44 , no 4,1989 , p. 961-962 


\section{GESTION DES RESSOURCES HUMAINES (suite)}

TURCOTTE, Pierre R.

La QVT une voie vers l'excellence. Montréal, Les Éditions Agence d'Arc, 1988, 276 pp., ISBN 289022-134-2 et ISBN 27624-0066-X, recensé par Roger Zaoré.

v. 44, no 4,1989 , p. $962-963$

VECCHIO, Robert P.

Organizational Behavior. Chicago, Toronto, The Dryden Press, 1988, 576 pp., ISBN 0-000853-0, recensé par Bernard Portis.

v. 44 , no 4,1989 , p. $963-964$

ROTHWELL, W.J. KAZANAS, H.C.

Strategic Human Resources Planning and Management. Englewood Cliffs, PrenticeHall, 1988, 512 pp., ISBN 0-13-851643-X, recensé par Michel Audet.

v. 45, no 1,1990, p. $211-212$

BAMBER, Greg J. LANSBURY, Russell D. New Technology International Perspectives on Human Resources and Industrial Relations. Winchester, Unwin Hyman, 1989, 267 pp., ISBN 0-04-445123-7, recensé par Martin S. Serediak.

v. 45 , no 2,1990 , p. $435-438$

\section{BEATTY, Carol}

The Implementation of Technological Change. Kingston, Industrial Relations Centre, Queen's University, 1987, 137 p., ISBN 0-88886-172-9, recensé par Roger Zaoré.

v. 45, no 3,1990, p. $634-635$

\section{GESTION DES RESSOURCES HUMAINES - CANADA}

STONE, Thomas H. MELTZ, Noah M.

Personnel Management in Canada. Toronto Holt, Rinehart \& Winston, 1983, 411 pp., ISBN 0-03-921591-1, recensé par Alexander J. Matejko.

v. 39 , no 1,1984 , p. $205-206$

KAHN, Joan WESTLEY, William A.

The Working Conditions in Canadian Hospitals. Constraints and Opportunity. Ottawa, Minister of Supply and Services Canada, 1984, 112 pp., ISBN 0-662-52924-3, recensé par Alexander J. Matejko.

v. 39 , no 4,1984 , p. $815-816$
DOLAN, Shimon SCHULER, Randall S. Personnel and Human Resource Management in Canada. St-Paul, West Publishing, 1987, 620 pp., ISBN 0-314-32486-0, recensé par Michel Audet.

v. 42 , no 2,1987 , p. $440-443$

DOLAN, Shimon SCHULER, Randall S. Canadian Readings in Personnel and Human Resource Management. St-Paul, West Publishing Company, 1987, 467 pp., ISBN 0-314-32487-9, recensé par Michel Audet.

v. 43 , no 1,1988 , p. $203-205$

\section{GESTION DES RESSOURCES HUMAINES - ÉTATS-UNIS}

MCCARTHY, Maureen

ROSENBERG, Gail S.

Work Sharing: Case Studies. Kalamazoo, Michigan, W.E. Upjohn Institute for Employment Research, 1981, 277 pp., ISBN O911558-87-X, recensé par Alain Vinet.

v. 37 , no 4,1982 , p. 971

\section{GESTION DES RESSOURCES HUMAINES - GRANDE-BRETAGNE}

WHITE, Michael TREVOR, Malcolm

Under Japanese Management. The Experience of British Workers. London, Heinemann, 1983, 162 pp., ISBN 0-435-839357 , recensé par Alexander J. Matejko.

v. 39, no 2,1984 , p. $395-396$

\section{GESTION DES RESSOURCES HUMAINES -} INDES

SHARMA, Baldev R.

Not by Bread Alone : A Study of Organizational Climate and Employer-Employee Relations in India. New Delhi, Shri Ram Centre for Industrial Relations and Human Resources, 1987, 191 pp., resencé par Basu Sharma.

v. 43 , no 1,1988 , p. $208-209$

\section{GESTION DES RESSOURCES HUMAINES - JAPON}

THUROW, Lester C.

The Management Challenge. Japanese Views. Cambridge, The MIT Press, 1985, 237 pp., ISBN 0-262-20053-8, recensé par Hélène Bois-Brochu.

v. 40, no 3,1985, p. $683-685$ 
GESTION DES RESSOURCES HUMAINES QUEBEC

BOISVERT, Maurice

La qualité de la vie au travail. Regard sur l'expérience québécoise. Le Groupe d'étude de travail des HEC, Montréal, Éditions Agence d'Arc Inc., 1981, 461 pp., recensé par Lucie Vézina.

v. 37, no 3,1982 , p. $713-715$

Les principes de l'équité salariale et les approches dans le secteur public québécois. Montreal, Institut de recherche et d'information sur la rémunération, IRI-050, octobre 1989, 99 pp., ISBN 2-551-121930 , recensé par Esther Déom.

v. 45 , no 2,1990, p. $438-439$

\section{MAIN-D'OEUVRE}

\section{MAIN-D'OEUVRE}

B.I.T.

Rapport de la Commission d'experts pour l'application des conventions et recommandations. Conférence internationale du travail, 66ieme session, Geneve, Rapport III, 1980, Partie 4A, 251 pp., Partie 4B, Travailleurs migrants, $190 \mathrm{pp}$., recensé par Alain Barré.

v. 36 , no 1,1981 , p. 291-294

\section{STEINBERG RATNER, Ronnie}

Equal Employment Policy for Women : Strategies for Implementation in the United States, Canada and Western Europe. Philadelphia, Temple University Press, 1980, 520 pp., recensé par Carole Swan.

v. 36 , no 3,1981 , p. $692-696$

B.I.T.

Rapport de la Commission d'experts pour l'application des conventions et recommandations. Conférence internationale du travail, 67ième session, Genève, Rapport III (Partie 4A), BIT, 1981, 255 pp., recensé par Alain Barré.

v. 36 , no 4,1981 , p. $951-954$

B.I.T.

Age minimum. Étude d'ensemble de la commission d'experts pour l'application des conventions et recommandations, Conférence internationale du travail, 67ième session, Genève, Rapport III (Par- tie 4B), BIT, 1981, 238 pp., recensé par Alain Barré.

v. 36 , no 4 , 1981, p. 951-954

\section{DELPLANQUE, Bernard}

Le partage de l'emploi, vers une société sans chomage. Paris, Editions Entente, 1980, 141 pp., recensé par Paul Beaulieu. v. 37 , no 1,1982 , p. 248

\section{CEREQ}

L'évolution des systèmes de travail dans l'économie moderne. Paris, Éditions du CNRS, 1981, 287 pp., ISBN 2-222-02935$X$, recensé par Gérard Dion.

v. 37, no 2,1982, p. 460

MELTZ, Noah M. REID, Frank SWATZ, Gérald S.

Sharing the Work : An Analysis of the Issues in Worksharing and Jobsharing. Toronto, University of Toronto Press, 1981, 90 pp., recensé par Paul Blyton.

v. 37 , no 3,1982 , p. $717-718$

MARIC, D.

La durée du travail dans les pays en voie de développement. Genève, Bureau international du Travail, 1981, 138 pp., ISBN 92-2-202732-9, recensé par Alain Larocque.

v. 37 , no 3,1982, p. $718-719$

MCGAUGHEY, William Jr.

A Shorter Workweek in the 1980's. White Bear Lake, Minnesota, Thestterose Publications, 1981, 308 pp., ISBN 0-9605630$0-8$, recensé par Alain Larocque.

v. 37, no 3,1982, p. $718-719$

Le travail : quête de sens, quête d'emploi. Cahiers de recherche éthique, no 10 , Montreal, Fides, 1984, 238 pp., ISBN 27621-1222-2, recensé par Gilles Dussault. v. 40 , no 2,1985 , p. $407-408$

BRADLEY, Keith GELB, Alan Worker Capitalism. The New Industrial Relations. Cambridge, The MIT Press, 1983, 186 pp., ISBN 0-262-02191-0, recensé par Clive H.J. Gilson.

v. 40 , no 3,1985 , p. $678-680$ 


\section{MAIN-D'OEUVRE (sulte)} GRAIS, Bernard

Les mises à pied et le travail à temps réduit dans quelques pays de l'OCDE. Paris, Institut national de la statistique et des études économiques et Conclusions du groupe de travail de l'OCDE sur les statistiques de l'emploi et du chómage, 1983, 154 pp., ISBN 92-64-22391-6, recensé par Lise Chrétien.

v. 40 , no 4,1985, p. $904-905$

Entreprises d'intêrét collectif et création d'emplois. Initiatives locales de création d'emplois, OCDE, Paris, 1984, 77 pp., ISBN 92-64-22569-2, recensé par Louis Gosselin.

v. 41 , no 1,1986, p. $189-190$

\section{PACAULT, Emile}

La fin du chómage par le partage du travail. Édition établie avec introduction, notes et index par Maurice Lebel. Montréal, Éditions Paulines, 1985, 118 pp., ISBN 2-89039-046-2, recensé par Dimitri Weiss.

v. 41 , no 1,1986 , p. $193-196$

\section{B.I.T.}

Technological Change : The Tripartite Response, 1982-85. Genève, Bureau international du Travail, 1985, 355 pp., ISBN 92-2-105162-5, recensé par Rolland Hurtubise.

v. 41 , no 2,1986 , p. $433-435$

OFFE, Claus

Contradictions of the Welfare State. Edited and introduced by John Keane, Cambridge, MIT Press, 1984, 310 pp., ISBN 0262-65014-2, recensé par Anthony Giles. v. 41 , no 3,1986, p. $664-667$

SCHMIECHEN, James A.

Sweated Industries and Sweated Labor: The London Clothing Trades, 1860-1914. Urbana and Chicago, University of Illinois Press, 1984, 209 pp., ISBN 0-252-010248, recensé par James Thwaites.

v. 41 , no 4,1986, p. $877-878$

PONIATOWSKI, Michel

Les Technologies Nouvelles. Paris, Plon, 1986, 334 pp., ISBN 2-259-0143-9, recensé par Maurice Lebel.

v. 42 , no 2,1987 , p. $445-448$
TAHAR, Gabriel

La réduction de la durée du travail. Paris, Collection Reperes, Éditions La découverte, 1985, 127 pp., ISBN 2-7071-1552-

5 , recensé par Lise Chrétien.

v. 42 , no 3,1987 , p. $646-648$

\section{KELLEY, Robert E.}

The Gold Collar Worker. Reading Massachusetts, Addison-Wesley Publishing Co., 1985, 196 pp., ISBN 0-201-11739-8, recensé par Paul Beaulieu.

v. 42 , no 3,1987 , p. $664-665$

\section{ENGLAND, Geoffrey}

Part-time, Casual and Other Atypical Workers : A Legal View. Research and Current Issues Series No 48, Kingston, Industrial Relations Centre, Queen's University, 1987, 81 pp., ISBN 0-88886-154-0, recensé par Udo Mayer.

v. 42 , no 4,1987 , p. $880-881$

ROBERTSON, David 'NAREHAM, Jeff Technological Change in the Auto industry. Willowdale, CAW Technology Project, 56 pp., ISBN 0-969-2932-08, recensé par Bernard Portis.

v. 43 , no 1,1988 , p. $206-207$

La flexibilité des marchés du travail : essai bibliographique/Labour Market Flexibility : A Bibliography Essay. Série bibliographique no 12, Genève, Institut international d'études sociales, 1987, 101 pp., ISBN 929014-418-1, recensé par Michel Audet. v. 43 , no 2,1988 , p. $461-462$

WAY, Harold E. WEISS, Carla M. Plant Closings : A Selected Bibliography of Materials Published Through 1985. Ithaca, NYSSILR, Cornell University, 1988, 206 pp., recense par Jean Sexton.

v. 43, no 2,1988, p. 472

\section{SARFATI, Hedva KOBRIN, Catherine} La flexibilité du marché de l'emploi : un enjeu économique et social. Genève, Bureau international du travail, 1987, 384 pp., ISBN 92-2-205675-2, recensé par Lise Poulin-Simon.

v. 43, no 3,1988, p. $707-710$ 
MAIN-D'OEUVRE (suite)

GERHART, Paul F.

Saving Plants and Jobs : Union Management Negotiations in the Context of Threatened Plant Closing. Kalamazoo, W.E. Upjohn Institute for Employment Research, 1987, 109 pp., ISBN 0-88099-047-3, recensé par Norman A. Solomon.

v. 44 , no 1,1989 , p. $293-294$

PLASSARD, Jean-Michel

Discrimination sur le marché du travail et information imparfaite. Editions du CNRS, France, 1987, 386 pp., ISBN 2-222-04077-

9, recensé par Esther Déom.

v. 45 , no 1,1990 , p. $203-204$

COOK, Robert F.

Worker Dislocation: Case Studies of Causes and Cures. Kalamazoo, Michigan, W.E. Upjohn Institute for Employment Research, 1987, 219 pp., ISBN 0-88099-052$X$, recensé par Maurice J. Mazerolle.

v. 45 , no 2,1990 , p. $432-434$

\section{MAIN-D'OEUVRE - ANGLETERRE}

ASHTON, D.N. MAGUIRE, J.J.

Young Adult in the Labour Market. Research Paper no 55, Leicester, University of Leicester, 1986, 163 pp., recensé par Lise Poulin Simon.

v. 42 , no 4,1987, p. $887-889$

\section{MAIN-D'OEUVRE - AUSTRALIE}

WILKES, John

The Future of Work. Sydney, G. Allen and Unwin, Australia Institute of Political Science, 1981, 164 pp., ISBN 0-86861-283-9, recensé par Alexander J. Matejko.

v. 38 , no 3, 1983, p. 689

\section{MAIN-D'OEUVRE - CANADA}

DENTON, F.T. ROBB, A.L. SPENCER, B.G.

Unemployment and Labour Force Behaviour of Young People : Evidence from Canada and Ontario. Ontario Economic Council Research Series no 20, Toronto, University of Toronto Press, 1980, 215 pp., ISSN 0708-3688, ISBN 0-8020-3379-2, recensé par Jacques Mercier. v. 37 , no 1,1982 , p. $249-251$

CONSEIL ÉCONOMIQUE DU CANADA Pénuries et carences. Travailleurs quali- fiés et emplois durant les années 80. Ottawa, ministère des Approvisionnements et Services Canada, 1982, 141 pp., ISBN 0-660-90874-3, recensé par Gérard Bélanger.

v. 37, no 3,1982, p. $704-709$

\section{SEN, Joya}

The Unemployment of Youth : The Importance of Education for their Adjustment in the Canadian Labour Market. Toronto, Ontario Institute for Studies in Education, 1982, 125 pp., ISBN 0-7744-5053-3, recensé par Morley Gunderson. v. 38 , no 4,1983 , p. $925-927$

\section{STRUTHERS, James}

"No Fault of their Own". Unemployment and the Canadian Welfare State 19141940. The State and Economic Life Series, Mel Watkins and Leo Panitch eds, Toronto, University of Toronto Press, 1983, 238 pp., ISBN 0-8020-7, recensé par F.J.K. Griezic.

v. 39 , no 3,1984 , p. $638-641$

\section{EASTMAN, Byron D.}

Labour Market Theory and the Canadian Experience. Toronto, Harcourt Brace Jovanovich, 1987, 350 pp., ISBN 077-4730757, recensé par S.A. Alvi.

v. 42 , no 4,1987 , p. $894-895$

\section{SMUCKER, Joseph}

VAN DEN BERG, Axel

Capitalism vs Socialism? Canadian and Swedish Labour Market Policies Compared. Working Papers in Social Behaviour, no 88-8, Montréal, Mc Gill University, 1988, 75 pp., recensé par Alexander J. Matejko. v. 44 , no 1,1989 , p. $294-295$

LAFLAMME, Gilles MURRAY, Gregor BÉLANGER, Jacques FERLAND, Gilles Flexibilité and Labour Markets in Canada and United States. (Research Series 94), Geneva, International Institute for Labour Studies, 1989, 317 p., ISBN 92-9014-4629 , recensé par Brad Pragnell. v. 45 , no 3,1990 , p. $643-647$ 
MAIN-D'OEUVRE - ÉTATS-UNIS

MCCARTHY, Maureen

ROSENBERG, Gail S.

Work Sharing: Case Studies. Kalamazoo,

Michigan, W.E. Upjohn Institute for Employment Research, 1981, 277 pp., ISBN 0-911558-87-X, recensé par Alain Vinet.

v. 37 , no 4,1982 , p. 971

LAFLAMME, Gilles MURRAY, Gregor BÉLANGER, Jacques FERLAND, Gilles Flexibilité and Labour Markets in Canada and United States. (Research Series 94), Geneva, International Institute for Larour Studies, 1989, 317 p., ISBN 92-9014-4629, recensé par Brad Pragnell.

v. 45 , no 3,1990 , p. $643-647$

\section{MAIN-D'OEUVRE - EUROPE}

\section{ADAM, Jan}

Employment Policies in the Soviet Union and Eastern Europe. 2nd ed., London, The MacMillan Press, 1987, 224 pp., ISBN 0-333-41775-5, recensé par Chris Jecchinis.

v. 44, no 3,1989, p. $729-730$

\section{MAIN-D'OEUVRE - GRANDE-BRETAGNE} TAYLOR, Robert

Workers and the New Depression. London, Macmillan Press, 1981, 212 pp., ISBN 0-333-33411-6, recensé par Alexander J. Matejko.

v. 39, no 3,1984 , p. 636

\section{MAIN-D'OEUVRE - QUÉBEC \\ M.T.M.O.}

Problèmes et perspectives d'emploi dans le secteur hôtelier du Québec. Québec, Centre de recherche et de statistiques sur le marché du travail, MTMO, 1980, 262 pp., recensé par André Roy.

v. 36 , no 2,1981 , p. $460-461$

DELORME, François PARENT, Réjean Les licenciements collectifs au Québec : un bilan partiel du dispositif public en vigueur. Monographie 12, Montreal, Ecole de relations industrielles, Université de Montreal, 1982, 106 pp., ISBN 2-89067011-2 et ISSN 0708-9945, recensé par Jean Sexton.
LECLERC, Claudine SEXTON, Jean La sécurité d'emploi dans l'industrie de la construction au Québec, un réve impossible? Québec, Presses de l'Université Laval, 1983, 275 pp., ISBN 2-7637-7028. 2, recensé par Noël Mallette.

v. 39 , no 3,1984 , p. $643-645$

\section{MAIN-D'OEUVRE - SUÈDE}

SMUCKER, Joseph

VAN DEN BERG, Axel

Capitalism vs Socialism? Canadian and Swedish Labour Market Policies Compared. Working Papers in Social Behaviour, no 88-8, Montreal, McGill University, 1988, 75 pp., recensé par Alexander J. Matejko. v. 44 , no 1,1989 , p. 294-295

\section{MÉTHODOLOGIE}

\section{MÉTHODOLOGIE}

EHRENBERG, Ronald G. (Ed.)

Research in Labor Economics : An Annual Compilation of Research, Vol. 2, Greenwich, Connecticut, JAl Press Inc., 1978, recensé par Dennis $R$. Maki.

v. 36 , no 1,1981 , p. $289-291$

\section{ORGANISATIONS PATRONALES}

\section{ORGANISATIONS PATRONALES}

WINDMULLER, John P.

GLADSTONE, Alan

Employers' Associations and Industrial Relations : A Comparative Study. Oxford, Clarendon Press, 1986, 370 pp., ISBN 019-827260-X, recensé par James Thwaites. v. 44 , no 1,1989 , p. $295-297$

\section{ORGANISATIONS PATRONALES - CANADA} TRAVES, TOM

Essays in Canadian Business History. Toronto, McClelland and Steward, 1984, 212 pp., ISBN 0-7710-8570-2, recensé par James Thwaites.

v. 39 , no 4,1984 , p. $802-803$ 


\section{ORGANISATIONS SYNDICALES}

\section{ORGANISATIONS SYNDICALES}

WINDMULLER, John P.

The International Trade Union Movement. Deventer, Kluwer, 1980, 174 pp., recensé par Gérard Dion.

v. 36 , no 2,1981 , p. $455-456$

\section{MARSH, Arthur}

Trade Union Handbook, 2nd ed. Farnborough, England, Gower, 1980, 396 pp., recensé par Gérard Dion.

v. 36 , no 4,1981 , p. $948-949$

\section{CLARK, Paul $F$.}

The Mines Fight for Democracy. Arnold Miller and Reform of the United Mine Workers. New York State School of Industrial and Labour Relations, Cornell University, 1981, 190 pp., recensé par Paul Phillips.

v. 39 , no 1, 1984, p. $210-211$

\section{CROUCH, Colin}

Trade Unions : The Logic of Collective Action. Glasgow, Fontana Paperbacks, 1982, 251 pp., ISBN 0-00-635873X, recensé par Alexander J. Matejko.

v. 39 , no 1, 1984, p. 212-215

\section{REES, Albert}

The Economics of Trade Unions. Chicago and London, The University of Chicago Press, 1977, 200 pp., ISBN 0-226-707024, recensé par Alexander J. Matejko.

v. 39 , no 1,1984, p. $212-215$

ADDISON, John T. BURTON, John Trade Unions and Society. Vancouver, The Fraser Institute, 1984, 189 pp., ISBN 0-88975-056-4, recensé par Peter E. Larson.

v. 40 , no 1,1985, p. $180-181$

FREEMAN, Richard B.

MEDOFF, James L.

What do Unions do? New York, Basic Books Inc., 1984, 293 pp., ISBN 0-46509133-4, recensé par Alexander J. Matejko.

v. 40, no 1,1985 , p. $185-186$

LEAB, Daniel J.

The Labor History Reader. Champaign, University of Illinois Press, 1985, 470 pp.,
ISBN 0-252-01197-X, recense par Foster J.K. Griezic.

v. 41 , no 1,1986 , p. $202-203$

ROBERTS, B.C. JACOBI, Otto JESSOP, Bob KASTENDIEK, Hans REGINI, Marino

Industrial Relations in Europe. The Imperatives of Change. Beckenham, England, 1986, 279 pp., ISBN 0-7099-4212-5, and Economic Crisis. Trade Unions and the State. Beckenham, England, 1986, 295 pp., ISBN 0-7099-1447-4, recensés par Roy J. Adams.

v. 42 , no 2,1987 , p. $448-450$

LIPSET, Seymour Martin

Unions in Transition. Entering the Second Century. San Francisco, ICS Press, 1986, 506 pp., ISBN 0-917616-74-X, recensé par Carla Lipsig.

v. 42, no 2,1987 , p. $452-454$

CHAISON, Gary N.

When Union Merge. Lexington, Lexington Books, 1986, 186 pp., ISBN 0-669-110817 , recensé par $P$. Andiappan.

v. 42 , no 3,1987 , p. $649-651$

VON PRONDZYNSKI, F.

Freedom of Association and Industrial Relations. London, Mansell Publishing, 1987, 248 pp., ISBN 0-7201-1775-5, recensé par Pierre Verge.

v. $42, n c$ 3, 1987, p. 651-652

LARSON, Simeon NISSEN, Bruce

Theories of the Labor Movement. Detroit, Wayne State University Press, 1987, 395 pp., ISBN 0-8143-1815-0, recensé par Michel Audet.

v. 43 , no 1,1988 , p. $199-200$

B.I.T.

La liberté syndicale - Manuel d'éducation ouvrière. (2ième éd.), Genève, Bureau international du travail, $1988,153 \mathrm{pp}$., ISBN 92-2-205782-1, recensé par Pierre Verge.

v. 43, no 3,1988, p. $712-713$

SPYROPOULOS, G.

Trade Unions Today and Tomorrow. 2 vol, Masestricht, Presses universitaires européennes, 1985 et 1987, 266 et 212 pp., ISBN 90-70776-17-0 et ISBN 9070776-18-9, recensé par Pierre Verge. v. 44 , no 1,1989 , p. $287-289$ 


\section{ORGANISATIONS SYNDICALES (suite)} HECKSCHER, Charles C.

The New Unionism : Employee Involvement in the Changing Corporation. New York, Basic Books, 1988, 320 pp., ISBN O465-05098-0, recensé par Michel Grant. v. 45 , no 1,1990, p. $205-208$

Trade Unions of the World 1989-1990. (2ième éd.), Longman Group UK Limited, The High, Harlow, 1989, 480 pp., ISBN O582-03908-8, Distributed exclusively in United States and Canada by St James Press, ISBN 0-55862-014-1, recensé par Gérard Dion.

v. 45, no 2,1990, p. $429-430$

\section{ORGANISATIONS SYNDICALES - AUSTRALIE}

DONN, C.D.

The Australian Council of Trade Unions. History and Economic Policy. New York, University Press of America, 1983, 366 pp., ISBN 0-8191-2728-9, recensé par Gilles Dussault.

v. 39 , no 3,1984 , p. $630-631$

FORD, B. PLOWMAN, D.

Australian Unions : An Industrial Relations Perspectives. Melbourne, MacMillan Company of Australia, 1983, 576 pp., ISBN O333-33913-4, recensé par Gilles Dussault. v. 40 , no 1,1985 , p. $186-188$

\section{ORGANISATIONS SYNDICALES - CANADA} WHITE, Julie

Les femmes et le syndicalisme. Ottawa, Conseil consultatif canadien de la situation de la femme, Centre d'édition du gouvernement du Canada, 1980, 147 pp., recensé par Marie Lavigne.

v. 36 , no 2,1981 , p. $456-457$

\section{SALUTIN, Rick}

Kent Rowley, The Organizer, A Canadian Union Life. Toronto, James Lorimer, 1980, 163 pp., recense par F.J.K. Griezic.

v. 36 , no 3,1981 , p. $704-705$

MORTON, Desmond COOP, Terry Working People, An Illustrated History of Canadian Labour. Ottawa, Deneau \& Greenberg, 1980, 349 pp., recensé par F.J.K. Griezic.

v. 37, no 1,1982 , p. $243-245$
VAISEY, Douglas G.

The Labour Companion : A Bibliography of Canadian Labour History, Based on Materials Printed from 1950 to 1975. Halifax, Committee on Canadian Labour History, 1980, 128 pp., recensé par Mario Lajoie. v. 37 , no 1,1982 , p. $251-252$

FORSEY, Eugène

Trade Unions in Canada : 1812-1902. Toronto, University of Toronto Press, 1982, 600 pp., ISBN 0-8020-6388-8, recensé par James Thwaites.

v. 38 , no 1,1983 , p. $179-180$

ORGANISATIONS SYNDICALES - ESPAGNE SAGARDOY BENGOECHEA, J.A.

BLANCO, David Leon

El poder sindical en Espana. Barcelona, Editorial Planeta/Instituto de Estudios Economicos, 1982, 210 pp., ISBN 84-3207318-0, recensé par $P$. Joubert.

v. 38 , no 1,1983 , p. 192-194

\section{ORGANISATIONS SYNDICALES - \\ ÉTATS-UNIS}

DANIEL, Cletus E.

The ACLU and the Wagner ACt: An inquiry into the Depression. Era Crisis of American Liberalism. Cornell Studies in Ind. and Labor Rel. Number 20, N. Y. State School of Ind. and Labor Rel., Cornell Univ., Ithaca, N. Y., 1980, 142 pp., recensé par Hugh Tuck.

v. 36 , no 4,1981 , p. $954-956$

NEUFELD, Maurice F. LEAB, Daniel J. SWANSON, Dorothy

American Working Class History : A Representative Biography. New York - London, Bowker, 1983, 356 pp., ISBN 0-8352-17523, recensé par James Thwaites. v. 39 , no 2,1984 , p. 406

O'SULLIVAN, Judith GALLICK, Rosemary Workers and Allies : Female Participation in the American Trade Union Movement, 1824-1976. Washington, Smithsonian Institution Press, 1975, 96 pp., ISBN 0-87546138-1, recensé par Micheline Dumont. v. 43 , no 1,1988, p. 205 
ORGANISATIONS SYNDICALES - FRANCE MOURIAUX, René

Les syndicats dans la société française. Paris, Presses de la Fondation nationale des Sciences politiques, 1983, 272 pp., ISBN 2-7246-0480-6, recensé par Dimitri Weiss.

v. 38 , no 4,1983 , p. $922-924$

VERDIER, Jean-Maurice

Syndicats et droit syndical. 2ième éd., tome 5, vol. 1 du Traité de droit du travail publié sous la direction de G.-H. Camerlynck, Paris, Dalloz, 1987, 671 pp., ISBN 2-247-00754-6, recensé par Pierre Verge.

v. 42, no 3,1987, p. $657-658$

\section{ORGANISATIONS SYNDICALES -} GRANDE-BRETAGNE

MARTIN, ROSS M.

TUC : The Growth of a Pressure Group 1868-1976. Oxford, Oxford University Press, 1980, 394 pp., recensé par Jacques Bélanger.

v. 36 , no 4,1981 , p. $956-958$

MARSH, Arthur RYAN, Victoria Historical Directory of Trade Unions, vol. 1 : Non-Manual Unions. Farnborough, England, Gower, 1980, 256 pp., ISBN 0-56602160-9, recensé par Gérard Dion.

v. 37 , no 1,1982, p. $245-246$

NICHLOSON, Nigel URSELL, Gill BLYYTON, Paul

The Dynamics of White Collar Unionism : A Study of Local Union Participation. London, Academic Press, 1981, 268 pp., ISBN 0-12-518020-9, recensé par Jacques Bélanger.

v. 39 , no 1,1984, p. $218-220$

\section{ORGANISATIONS SYNDICALES - ITALIE} PIRZIO AMMASSARI, Gloria

MATTIOLI, Francesco

I sindicalisti. Bar, De Donato, 1982, 158 pp., CL 07-0501-2, recensé par Dimitri Weiss.

v. 38 , no 4,1983 , p. $922-924$
RICCI, Maurizio

La struttura organizzativa del movimento sindicale, Dalle origini al 1949. Milano, Franco Angeli, 1986, 250 pp., recensé par Dimitri Weiss.

v. 42, no 1, 1987, p. $223-224$

ORGANISATIONS SYNDICALES - QUÉBEC VIGEANT-GALLEY, Paulette

Les enseignants et le pouvoir : histoire de I'Alliance des professeurs de Montréal, les luttes syndicales et le développement social (1952-1958). Québec, CEQ-APM, décembre 1981, 128 pp., recensé par James Thwaites.

v. 37 , no 3,1982 , p. $712-713$

DEMERS, François

Chroniques impertinentes du 3ième (sic) Front commun Syndical, 1979-1980. Montréal, Nouvelle optique, 1982, $170 \mathrm{pp}$., ISBN 2-89017-035-7, recensé par Gérard Hébert.

v. 37 , no 4,1982 , p. $962-963$

\section{RELATIONS DU TRAVAIL}

\section{RELATIONS DU TRAVAIL}

NORTHRUP, Herbert $R$.

ROWAN, Richard L.

Multinational Collective Bargaining Attempts. Multinational Industrial Relations Series no 6, Industrial Research Unit, Philadelphia, The Wharton School, University of Pennsylvania, 1979, 580 pp., recensé par Jean Boivin.

v. 36 , no 1,1981 , p. $280-282$

VÉZINA, Claude

Les clauses d'ancienneté et l'arbitrage des griefs. Ottawa, Collection des travaux de la Faculté de droit de l'Université d'Ottawa, éditions de l'Université d'Ottawa, 1979, 125 pp., recensé par Rodrigue Blouin.

v. 36 , no 1,1981, p. $288-289$ 


\section{RELATIONS DU TRAVAIL (suite)}

B.I.T.

Conciliation and Arbitration Procedures in Labour Disputes. A Comparative Study. Genève, International Labour Office, 1980, 183 pp., recensé par Rodrigue Blouin.

v. 36 , no 2,1981 , p. $453-454$

FOSSUM, John A.

Labor Relations : Development, Structure, Process (Revised Edition). Dallas, Business Publications, 1982, 521 pp., ISBN 0-256-02688-2, recensé par Kurt Wetzel.

v. 37 , no 4,1982 , p. $968-969$

The Bargaining Process and Mediation. Toronto, Education Relations Commission, 1983, recense par E.G. Fisher.

v. 38 , no 4,1983 , p. $915-916$

KOLB, Deborah M.

The Mediators. Cambridge, Mass., The MIT Press, 1983, 230 pp., ISBN 0-26211088-1, recensé par Jean Sexton.

v. 39 , no 2,1984, p. $391-392$

ANTONIDES, Harry

Renewal in the Workplace: A Critical Look at Collective Bargaining. London, Ontario, Christian Labour Association of Canada, 1982, 82 pp., ISBN 0-919663-00-1, recensé par Alton W.J. Craig.

v. 39 , no 2,1984, p. $393-394$

FREEMAN, Richard B.

MEDOFF, James $L$.

What do Unions do? New York, Basic Books Inc., 1984, 29.3 pp., ISBN 0-46509133-4, recense par Alexander J. Matejko.

v. 40, no 1,1985, p. $185-186$

HANAMI, T. BLANPAIN, R.

Industrial Conflict Resolution in Market Economies. Deventer, Kluwer, 1984, 321 pp., ISBN 90-6544-1808, recensé par Pierre Verge.

v. 40, no 3,1985, p. $681-682$

PLANTE, Gilles

Le conflit du travail : strategie et tactique. Collection Relations du travail, Québec, Presses de l'Université Laval, 1984, 166 pp., ISBN 2-7637-7041-X, recensé par Marcel Simard.

v. 40, no 4,1985 , p. $891-892$

\section{DUPONT, Christophe}

La négociation. Conduite, thérie, application. Paris, Dalloz, 1982, 276 pp., ISBN 2-247-00315- $X$, recensé par Paul Beaulieu. v. 40 , no 4,1985, p. $892-893$

LEWICKI, Roy J. LITTERER, Joseph A. Negotiation. Homewood, Richard D. Irwin, 1985, 368 pp., ISBN 0-256-02633-5, recensé par Jean Sexton.

v. 40 , no 4,1985 , p. $894-895$

LEWICKI, Roy J. LITTERER, Joseph A. Negotiation : Readings, Exercices and Cases. Homewood, Richard D. Inwin, 1985, 633 pp., ISBN 0-256-02634-3, recensé par Gilles Ferland.

v. 40 , no 4,1985 , p. 895

RICHARDSON, Reed C.

Collective Bargaining by Objectives : A Positive Approach. 2nd ed., New Jersey, Prentice-Hall, 1985, 325 pp., ISBN 0-13140476-8-01, recensé par Esther Déom. v. 41 , no 1,1986 , p. 191-192

\section{SCIARRA, Silvana}

Contratto collettivo e contrattazione in azienda. Milano, Franco Angeli, 1985, 226 pp., recensé par Dimitri Weiss.

v. 41 , no 1,1986 , p. $198-200$

PLANTE, Gilles

Le conflit du travail : stratégie et tactique. Collection Relations du travail, Québec, Presses de I'Université Laval, 1984, 166 pp., ISBN 2-7637-7041-X, recensé par Yvan Simonis.

v. 41 , no 1,1986, p. $200-202$

B.I.T.

Collective Bargaining : A Response to the Recession in Industrialised Market Economy Countries. Genève, Bureau international du Travail, 1984, 275 pp., ISBN 92 20103628-6, recensé par Paul Beaulieu.

v. 41 , no 3,1986, p. $651-652$

WHEELER, Hoyt N.

Industrial Conflict. An Integrative Theory. Columbia, University of South Carolina Press, 1985, 293 pp., ISBN 0-87249-4594, recensé par Esther Déom.

v. 41, no 3,1986, p. $652-654$ 


\section{RELATIONS DU TRAVAIL (suite)} PEACH, David A. KUECHLE, David The Practise of Industrial Relations. 2no Ed., Scarborouogh, McGraw Ryerson, 1985, 407 pp., ISBN 0-07-548909-0, recensé par Norman A. Solomon.

v. 42 , no 1,1987 , p. $222-223$

WALL, J.A. Jr.

Negotiation : Theory and Practice. Glenview, Scott, Foresman and Company, 1985, 182 pp., ISBN 0-673-15865-9, recensé par Paul Beaulieu.

v. 42 , no 2,1987, p. 435

GEORGE, Kenneth

Entre syndicats et patrons, fragile alliance. Montréal, Les Editions Agence d'Arc, 1987, 137 pp., ISBN 2-89022-104-0, recensé par André Roy.

v. 43 , no 1,1988, p. 210

MORRIS, Gillian S.

Strikes in Essential Services. London and New York, Manselle Publishing Company, 1986, 221 pp., ISBN 0-7201-1791-7, recensé par Anthony Giles.

v. 43, no 2,1988 , p. $462-466$

\section{BEN-ISRAEL, Ruth}

International Labour Standards : The Case of Freedom to Strike. Deventer, Kluwer, 1988, 133 pp., ISBN 90-6544-317-7, recensé par Pierre Verge.

v. 43, no 3,1988, p. $710-712$

TIZIANO, Treu et al.

Public Service Labour Relations : Recent Trends and Future Prospects. Genève, Bureau international du travail, 1987, 287 pp., ISBN 92-2-106049-7, recensé par Maurice Lemelin.

v. 43 , no 4,1988 , p. 974

AARON, Benjamin NAJITA, Joyce M.

STERN, James $L$.

Public Sector Bargaining. 2nd ed., Industrial Relations Research Association Series, Washington, Bureau of National Affairs, 1988, 334 pp., ISBN 0-87179-566-3, ISBN 0-913447-37-4, recensé par Jean Boivin.

v. 44 , no 3,1989 , p. $730-732$
MCKELVEY, Jean T.

Cleared for Takeoff : Airline Labour Relations since Deregulation. Ithaca, ILR Press, Cornell University, 1988, 298 pp., ISBN 0-87546-110-7, recensé par Jean Boivin.

v. 44, no 3,1989, p. $733-734$

WOLTERS, Roger HOLLEY, William $\mathrm{H}$. Labour Relations : An Experiential and Case Approach. New York, the Dryden Press, 1988, 321 pp., ISBN 0-03-0693047, recensé par Mark Thompson.

v. 44 , no 4,1989 , p. $957-958$

STAUDOHAR, Paul D.

The Sports Industry and Collective Bargaining. Ithaca, ILR Press, Cornell University, 1986, 195 pp., ISBN 0-87546-118-2, recensé par Jean Boivin.

v. 45 , no 1,1990 , p. 212

WINDMULLER, John P.

Collective Bargaining in Industrialised Market Economies: A Reappraisal. Geneva, International Labour Office, 1987, 333 p., ISBN 92-2-105606-6, recensé par Anthony Giles.

v. 45 , no 3,1990, p. 630

HARRIS, Rosemary

Power and Powerlessness in Industry: An Analysis of the Industrial Relations of Production. London, U.K., Tavistock Publ., 1987, pp. VIII + 245, ISBN 0-422-609-X, recensé par Alexander J. Matejko.

v. 45, no 3,1990, p. 634

SIRARD, Ronald GAZAILLE, Alain Comprendre et appliquer une convention collective. Montréal, Wilson et Lafleur, 1989, 165 p., ISBN 2-89127-151-3, recensé par Hélène Gascon.

v. 45 , no 3,1990 , p. $635-637$

\section{RELATIONS DU TRAVAIL - AUSTRALIE}

Compulsory Arbitration - The Australian Way? Industrial Relations Society of Australia, Papers presented at the 1986 National Convention, Adelaide, September 1986, 192 pp., recensé par Pierre Verge. v. 42 , no 4,1987, p. $886-887$ 


\section{RELATIONS DU TRAVAIL - CANADA} CHRISTENSEN, Sandra

Unions and the Public Interest : Collective Bargaining in the Government Sector. Vancouver, The Fraser Institute, 1980, 95 pp., recensé par Jean Boivin.

v. 36 , no 1,1981 , p. $275-276$

\section{CHRISTENSEN, Sandra}

Unions and the Public Interest : Collective Bargaining in the Government Sector. Vancouver, The Fraser Institute, 1980, 95 pp., recensé par William Cunningham.

v. 36, no 2,1981 , p. $461-462$

FINKELMAN, Jacob

GOLDENBERG, Shirley B.

Collection Bargaining in the Public Service : the Federal Experience in Canada. Montreal, Institute for Research on Public Policy/L'Institut de recherches politiques, 1983, 825 pp., ISBN 0-920380-95-6 (vol. 1), ISBN 0-920380-97-2 (vol. 2), recensé par Maurice Lemelin.

v. 39, no 3,1984, p. $633-634$

\section{SEFTON MACDOWELL, Laurel}

"Remember Kirkland Lake" : The History and Effects of the Kirkland Lake Gold Miners' Strike 1941-42. Toronto, University of Toronto Press, 1983, 292 pp., ISBN O8020-5585-0, recensé par Hugh Tuck.

v. 39 , no 4,1984 , p. $803-804$

THOMPSON, Mark SWIMMER, Gene Conflict or Compromise : The Future of Public Sector Industrial Relations. Montréal, Institut de recherches politiques, 1984, 476 pp., ISBN 0-88645-001-2, recensé par Jean Boivin.

v. 40 , no 3,1985, p. $676-678$

\section{BEATTY, D.M.}

Putting the Charter to Work Designing a Constitutional Labour Code. Kingston et Montréal, McGill-Queen's University Press, 1987, 251 pp., ISBN 0-7735-0601-2, recensé par Pierre Verge.

v. 42 , no 4,1987 , p. $877-880$

RIDDELL, W. Craig

Labour-Management Cooperation in Canada. Toronto, University of Toronto Press, 1986, 205 pp., ISBN 0-8020-7257-7, recensé par Alexander J. Matejko.

v. 43, no 3,1988 , p. $714-715$
RELATIONS DU TRAVAIL - ÉTATS-UNIS SOMERS, Gérald C.

Collective Bargaining : Contemporary American Experience. Madison, WI., Industrial Relations Research Association, 1980, 588 pp., recense par Gerard Dion.

v. 36, no 4,1981 , p. 954

SIEGEL, Irving $H$. WEINBERT, Edgar

Labor-Management Cooperation. The American Experience. Kalamazoo, Michigan, W.E. Upjohn Institute for Employment Research, 1982, 316 pp., ISBN 091155899-3, recensé par Jean Sexton.

v. 37 , no 4,1982 , p. 973

\section{GROSS, James A.}

The Reshaping of the National Labor Relations Board : National Labor Policy in Transition 1937-1947. Albany, State University of New York Press, 1981, 381 pp., ISBN 0-87395-270-7, recensé par Robert H. Babcock.

v. 38 , no 1,1983 , p. $188-189$

\section{LESTER, Richard A.}

Labor Arbitration in State and Local Government. Princeton, Princeton University, 1984, 210 pp., ISSN 0079-5305, recensé par André Roy.

v. 40 , no 1,1985, p. $181-183$

KATZ, Harry C.

Shifting Gears : Changing Labor Relations in the U.S. Automobile Industry. London, MIT Press, 1985, 230 pp., ISBN 0-26211098-9, recensé par Michel Turner.

v. 40 , no 4,1985 , p. $900-901$

KATZ, Harry C.

Shifting Gears : Changing Labor Relations in the U.S. Automobile Industry. Cambridge, MIT Press, 1985, 230 pp., ISBN O262-11098-9, recensé par Solomon Barkin. v. 41 , no 3,1986 , p. $654-658$

HERMAN, E. Edward KUHN, Alfred SEEBER, Ronald

Collective Bargaining and Labor Relations. (2nd ed.), Englewood Cliffs, Prentice Hall, 1987, 621 pp., ISBN 0-13-140576-6, recensé par Norman A. Solomon.

v. 43 , no 1,1988 , p. $207-208$ 


\section{RELATIONS DU TRAVAIL - ÉTATS-UNIS (suite)}

LIPSKI, David B. DONN, Clifford B. Collective Bargaining in American Industry. Lexington, Lexington Books, 1987, 351 pp., ISBN 0-669-12595, recensé par Joseph B. Rose.

v. 43, no 4,1988 , p. $980-981$

KOCHAN, Thomas C. KATZ, Harry C. Collective Bargaining and Industrial Relations. Irwin Series in Management and the Behavioural Sciences, Homewood, 1988, 496 pp., ISBN 0-256-03025-1, recensé par Jean Boivin.

v. 45 , no 1, 1990, p. 210

\section{RELATIONS DU TRAVAIL - EUROPE} ANDERSEN, Svein $S$.

British and Norwegian Offshore Industrial Relations : Pluralism and Neo-Corporatism as Contexts of Strategic Adaptation. Aldershot, U.K., and Brookfield, Vermont, USA, Averbury/Gover, 1988, 213 pp., ISBN 0-566-055317, recensé par Alexander $J$. Matejko.

v. 45 , no 2,1990 , p. 440

\section{RELATIONS DU TRAVAIL - GRANDE-BRETAGNE}

FRIEDMAN, Henry MEREDEEN, Sander The Dynamics of Industrial Conflict, Lessons from Ford. London, Croom Helm, 1980, 386 pp., recensé par Hervé Gauthier.

v. 37 , no 1,1982 , p. $246-248$

\section{STOREY, John}

The Challenge to Management Control. Brookfie/d, Vermont, Renouf USA, 1981, 192 pp., ISBN 0-09-145941-9, recensé par Jacques Belanger.

v. 37, no 2,1982, p. $455-457$

DANIEL, W.W. MILLWARD, Neil

Workplace Industrial Relations in Britain. The DEIPSI/SSRC Survey, London, Heinemann Educational Books, 1983, 338 pp., ISBN 0-435-83191-7, recensé par Alexander J. Matejko.

v. 39 , no 1,1984 , p. 217-218

CRAIG, C. RUBERY, Jill

TARLING, Roger WILKINSON, Frank Abolition and After the Paper Box Wages
Council. Research Paper no 12, London, Department of Employment, June 1980, $98 \mathrm{pp}$., recensé par David Bright.

v. 39 , no 2,1984 , p. $396-398$

DAVIES, Annette

Industrial Relations and New Technology. UWIST /Croom Helm, London, Sydney, Dover, 1986, ISBN 0-7099-0882-2, recensé par Guy Fréchet.

v. 43, no 2,1988, p. $468-469$

\section{RELATIONS DU TRAVAIL - JAPON}

CHALMERS, Norma $\mathrm{J}$.

Industrial Relations in Japan. The Peripheral Workforce. New York, Routledge, Chapman \& Hall, 1989, 283 p., ISBN 0415-00008-4, recensé par Hem C. Jain.

v. 45 , no 3,1990 , p. $631-632$

\section{RELATIONS DU TRAVAIL -}

NOUVEAU-BRUNSWICK

WOODS, H.D.

Relations industrielles dans l'industrie de la construction du sud du Nouveau-Brunswick. Fredericton, N.B., Ministère du travail et de la main-d'oeuvre, mars 1981, 617 pp., recensé par Jean Sexton.

v. 37 , no 1,1982 , p. $240-241$

\section{RELATIONS DU TRAVAIL -}

\section{NOUVELLE-ÉCOSSE}

GILSON, C.H.J.

Strikes in Nova Scotia, 1970-1985. Hantsport, Lancelot Press, 1986, 191 pp., ISBN 0-88999-314-9, recensé par David Frank. v. 43 , no 1,1988 , p. $200-203$

\section{RELATIONS DU TRAVAIL -}

\section{NOUVELLE-ZÉLANDE}

HOLT, James

Compulsory Arbitration in New Zealand The First Forty Years. Auckland, Auckland University Press, 1986, 247 pp., ISBN 1 86940-0062, recensé par Donald D. Carter.

v. 44, no 2,1989, p. $468-469$

\section{RELATIONS DU TRAVAIL - QUÉBEC}

\section{MALLETTE, Noël}

La gestion des relations du travail au Québec. Le cadre juridique et institutionnel. Scarborough, Collection administration, McGraw-Hill, 1980, 642 pp., recensé par Rodrigue Blouin.

v. 36 , no 1,1981 , p. 275 
RELATIONS DU TRAVAIL - QUÉBEC (suite) BOIVIN, Jean GUILBAULT, Jacques Les relations patronales-syndicales au Québec. Chicoutimi, Gaẽtan Morin Éditeur, 1982, 309 pp., ISBN 2-89105-087-8, recensé par Dimitri Weiss.

v. 37 , no 4,1982 , p. $969-970$

GAGNON, Chantal HÉBERT, Gérard Les grèves illégales dans les hópitaux du Québec, 1977-1978. Montréal, École de relations industrielles, Université de Montreal, monographie no 14, 1982, 76 pp., ISBN 0708-9945, recense par Gilles Dussault.

v. 39 , no 1,1984, p. $209-210$

La médiation pré-arbitrale en matière de conflits de droits (griefs). Journée d'étude à l'École de relations industrielles, Université de Montréal, 1981, 91 pp., recensé par Rodrigue Blouin.

v. 39 , no 1,1984, p. 220-221

\section{LEMELIN, Maurice}

Les négociations collectives dans les secteurs public et parapublic. Expérience québécoise et regard sur l'extérieur. Montréal, Éditions Agence d'Arc, 1984, 381 pp., ISBN 2-89022-067-2, recensé par Jacques Bélanger.

v. 40 , no 3,1985 , p. $675-676$

TRUDEAU, Pierre Elliott

La grève de l'amiante. Montreal, Éditions du Jour, 1970, 430 pp., recensé par Jacques Gagnon.

v. 41 , no 3,1986, p. $669-670$

MEHLING, Jean

Analyse socio-économoique d'une grève. Montréal, HEC et Beauchemin, 1963, 218 pp., recensé par Jacques Gagnon.

v. 41 , no 3,1986 , p. $670-671$

PRATT, Michel

La grève à la United Aircraft. Montréal, Les Presses de l'Université du Québec, 1980, 115 pp., recensé par Jacques Gagnon.

v. 41 , no 3,1986 , p. $671-672$

MARTIN, Roger G.

Annuaire du travail 1986-1987. Montréal, Productions INFORT, 1986, recensé par
Gérard Dion.

v. 41 , no 4,1986 , p. $886-887$

JOBIN, Carol

Les relations du travail dans l'industrie de la construction. Montreal, Wilson \& Lafleur, 1989, 568 pp., ISBN 2-89127-085-1, recensé par Jean Sexton.

v. 44, no 2,1989, p. 473

BOIVIN, Jean GUILBAULT, Jacques Les relations patronales-syndicales. Boucherville (Québec), 2ième éd., Gaétan Morin éditeur, 1989, 301 p. ISBN 2-89105322-2, recensé par Jacques-André Lequin. v. 45, no 3,1990 , p. $627-629$

\section{SANTÉ ET SÉCURITÉ AU TRAVAIL}

\section{SANTÉ ET SÉCURITÉ AU TRAVAIL}

BOULARD, René PÉRUSSE, Lyne

Les moyens organisationnels de prévention des maladies industrielles et des accidents du travail : essai de typologie et bibliographie annotee. Québec, Collection instruments de travail, Departement des relations industrielles, Universite Laval, 1981, 183 pp., recensé par Bernard Boucher.

v. 36 , no 3,1981 , p. $697-699$

DOLAN, Shimon ARSENEAULT, André Stress, santé et rendement au travail, monographie no 5. Montréal, Ecole des relations industrielles de l'Université de Montréal, 1980, 186 pp., recensé par René Boulard.

v. 36 , no 3,1981 , p. $701-702$

STELLMAN, Jeanne M. DAUM, Susan M. Perdre sa vie à la gagner; manuel pour la santé des travailleurs. Montréal, Édition Parti pris, 1979, 493 pp., recensé par René Boulard et Michel Pérusse.

v. 36, no 3,1981, p. $702-703$

\section{LEWIS, Gary A.}

News from Somewhere : Connecting Health and Freedom at the Workplace. New York, Greenwood Press, 1986, 213 pp., ISBN 0-313-24869-9, recensé par Robert Sass.

v. 42 , no 3,1987 , p. $653-655$ 
SANTÉ ET SÉCURITE AU TRAVAIL (suite) DAWSON, Sandra WILLMAN, Paul BAMFORD, Martin CUNTON, Alain Safety at Work : The Limits of Self-Regulation. Cambridge, Cambridge University Press, 1988, 310 pp., ISBN 0-521-354978, recensé par Eric Tucker.

v. 44, no 4,1989 , p. $959-961$

\section{SANTÉ ET SÉCURITÉ AU TRAVAIL . CANADA}

WONG, Jim

Employment Injuries and Occupational IIInesses 1972-1981. Ottawa Minister of Supply and Services Canada, 1984, 134 pp., ISBN 0-662-53002-0, recensé par Alexander J. Matejko.

v. 39 , no 4,1984 , p. $814-815$

\section{SANTÉ ET SÉCURITÉ AU TRAVAIL -}

\section{ÉTATS-UNIS}

GERSUNY, Carl

Work Hazards and Industrial Conflict. Hanover, University Press of New England, 1981, 162 pp., Lib of Congress Catalog Card no 80-51506, recensé par René Boulard.

v. 38 , no 3,1983 , p. 686

\section{SANTÉ ET SÉCURITÉ AU TRAVAIL . QUÉBEC}

BRADET, Denis CLICHE, Bernard

RACINE, Martin THIBAULT, France

Droit de la santé et de la sécurité du travail - La loi et la jurisprudence commentée. Cowansville, Les Éditions Yvon Blais, 1986, 300 pp., ISBN 2-89073-567-2, recensé par Gilles Trudeau.

v. 42 , no 1,1987, p. $215-216$

PLASSE, Micheline

Santé et sécurité du travail. Montréal, Agence d'Arc inc., 1988, 155 p., ISBN 289022-138-5, recensé par Alain Vinet.

v. 45, no 3,1990, p. $632-633$

\section{SÉCURITÉ SOCIALE}

\section{SÉCURITÉ SOCIALE}

Sécurité sociale, chómage et retraite anticipée. Association internationale de la sécurité sociale, Études et recherches no 22, Geneve, 1985, 191 pp., ISBN 92843-2021-6, recensé par Lise Chrétien. v. 40 , no 4,1985 , p. $902-903$
GUILLEMARD, Anne-Marie

Le déclin du social, formation et crise des politiques de la vieillesse. Paris, Presses universitaires de France, 1986, 396 pp., ISBN 2-130-39565-1, recensé par Hé/ène David.

v. 42 , no 3,1987, p. $642-646$

\section{SÉCURITÉ SOCIALE - AUTRICHE}

TOMANDL, T. FUERBOECK, $\mathrm{K}$.

Social Partnership - The Austrian System of Industrial Relations and Social Insurance. Ithaca, ILR Press, 1986, $165 \mathrm{pp}$., ISBN 0-87546-116-6, recensé par Pierre Verge.

v. 42 , no 3,1987 , p. $662-663$

\section{SÉCURITÉ SOCIALE - BELGIQUE}

A l'enseigne du droit social belge. 3ième éd., dans Revue de l'Université de Bruxelles, 1978, Bruxelles, Editions de l'Université de Bruxelles, 1982, 392 pp., recensé par Pierre Verge.

v. 38 , no 2,1983 , p. $445-446$

\section{SÉCURITÉ SOCIALE - CANADA}

STRUTHERS, James

"No Fault of their Own". Unemployment and the Canadian Welfare State 19141940. The State and Economic Life Series, Mel Watkins and Leo Panitch eds, Toronto, University of Toronto Press, 1983, 238 pp., ISBN 0-8020-7, recensé par F.J.K. Griezic.

v. 39 , no 3,1984 , p. $638-641$

\section{SÉCURITÉ SOCIALE - GRANDE-BRETAGNE} SHRAGGE, Eric

Pensions Policy in Britain : A Socialist Analysis. London, Routledge \& Kegan Paul, 1984, 194 pp., ISBN 0-7100-9842-1, recensé par Anthony Giles.

v. 41 , no 2,1986 , p. $428-430$

\section{SOCIOLOGIE DU TRAVAIL}

\section{SOCIOLOGIE DU TRAVAIL}

BURAWOY, Michael

Manufacturing Consent : Changes in the Labor Process under Monopoly Capitalism. Chicago, III., The University of Chicago Press, 1979, $267 \mathrm{pp}$., recensé par Craig A. Zabala.

v. 36 , no 1,1981, p. $282-285$ 
Relations Industrielles, vol. 45, no 4 (1990)

SOCIOLOGIE DU TRAVAIL (suite)

COTE, Marcel TÉGA, Vasile

La démocratie industrielle / The Industrial

Democracy. Montréal, Les Editions Agen-

ce d'Arc, 1981, 518 pp., recensé par Jacques Bélanger

v. 36 , no 3,1981 , p. $689-690$

JAIN, Hem C.

Worker Participation : Success and Problems. New York, Praeger Publisher, 1980, 358 pp., recensé par Jacques Bélanger.

v. 36 , no 3,1981 , p. $690-692$

BRIDDLE, Bruce J.

Role Theory : Expectations, Identities and Behaviors. New York, Academic Press, 1979, 416 pp., recensé par André Petit.

v. 36, no 4,1981 , p. $944-945$

GRAWITZ, Madeleine

Lexique des sciences sociales. Paris, Dalloze, 1981, 376 pp., recensé par Dimitri Weiss.

v. 36 , no 4,1981 , p. $945-946$

GUIOT, Jean M.

Organisations sociales et comportements. Montréal, Les éditions Agence d'Arc Inc., Paris, Les éditions Hommes et Techniques, 1980, 213 pp., recensé par Laurent Bélanger.

v. 36 , no 4 , 1981 , p. $946-948$

\section{L.R.S.C.}

Labour Studies Research Bulletin. Labour Studies Resource Centre, Department of Political Science, Carleton University, 1981, 32 pp., recensé par James Thwaites.

v. 36 , no 4,1981 , p. 958

\section{BAUM, Gregory}

The Priority of Labour. A Commentary on Laborem Exercens, Encyclical Letter of Pope John Paul II. New York/Ramsey, Paulist Press, 1982, 152 pp., ISBN 0-8091-2479-3, recensé par Alexander J. Matejko.

v. 37 , no 4,1982 , p. $964-966$

WEISS, Dimitri

Politica, partidos y sindicatos en la empresa. Madrid, Instituto de Estudios laborales y de la Seguridad social, 1982, $136 \mathrm{pp.}$ ISBN 84-5007823-7, recensé par $P$. Joubert.

v. 38 , no 1,1983 , p. $192-194$

CROZIER, Michel FRIEDBERG, Erhard Actors and Systems. The Politics of Collective Action. Chicago, The University of Chicago Press, 1980, 333 pp., recensé par Alexander J. Matejko.

v. 38 , no 2,1983 , p. $448-452$

REYNAUD, Jean-Daniel

Sociologie des conflits du travail. Paris, Presses universitaires de France, 1982, 127 pp., recense par Alexander J. Matejko.

v. 38 , no 3,1983 , p. $681-682$

\section{LINDENFELD, Frank}

ROTHSCHILD-WHITT, Joice

Workplace Democracy and Social Change. Boston, Porter Sargent Publication, 447 pp., ISBN 0-87558-102-1, recensé par Alexander J. Matejko.

v. 38 , no 3,1983 , p. $687-688$

BOLTANSKI, LUC

Les cadres : la formation d'un groupe social. Paris, Editions Minuit, Collection 'Le sens commun' dirigée par Pierre Bourdieu, 1982, 523 pp., ISBN 2-70780617-7, recensé par Dimitri Weiss.

v. 38 , no 4 , 1983 , p. $929-934$

MAUAICE, Marc SELLIER, François

SILVESTRE, Jean-Jacques

Politique d'education et organisation industrielle en France et en Allemagne : essai d'analyse sociétale. Paris, PUF, collection "Sociologies" dirigée par Raymond Boudon et François Bourricaud, 1982, 382 pp., ISBN 2-13-037472-7, recensé par Dimitri Weiss.

v. 38 , no 4,1983 , p. $929-934$

ANTONIDES, Harry

Renewal in the Workplace : A Critical Look at Collective Bargaining. London, Ontario, Christian Labour Association of Canada, 1982, 82 pp., ISBN 0-919663-00-1, recensé par Alton W.J. Craig.

v. 39 , no 2,1984 , p. $393-394$ 
SOCIOLOGIE DU TRAVAIL (suite)

THOMPSON, Paul

The Nature of Work. An Introduction to Debates on the labour Process. London, The Macmillan Press, 1983, 305 pp., ISBN 0-333-33027-7, recensé par Alexander J. Matejko.

v. 39, no 3,1984, p. $641-642$

Le travail : quête de sens, quête d'emploi. Cahiers de recherche éthique, no 10, Montreal, Fides, 1984, 238 pp., ISBN 27621-1222-2, recensé par Gilles Dussault. v. 40, no 2,1985, p. $407-408$

\section{WEISS, Dimitri}

"La participation". Enciclopedia de Dirreccion y Administracion de la Empresa, Fasciculo 82, Barcelona, Ediciones Orbis, 1985, ISBN (fascicule) 84-7530-585-7; (oeuvre complète) 84-7530-583-0; (volume VI) 84-7634-230-6, recensé par $P$. Joubert.

v. 41 , no 2,1986 , p. $423-424$

THOMPSON, Kenneth

Work, Employment and Unemployment. Perspectives on Work and Society. Milton Keynes, Open University Press, 1984, 280 pp., ISBN 03-3510-5947, recensé par Alexander J. Matejko.

v. 41 , no 2,1986 , p. $425-426$

MARS, Gerald

Cheats at Work. An Anthropology of Workplace Crime. London, Unwin Paperbacks, 1983, 242 pp., ISBN 0-04-301166-7, recensé par Alexander J. Matejko.

v. 41 , no 2,1986 , p. $431-432$

WELLS, Don

Soft Sell : "Quality of Working Life" Programs and the Productivity Race. Canadian Centre for Policy Alternatives, 150 pp., ISBN 088627-040-5, recensé par Robert Sass.

v. 41 , no 2,1986, p. $432-433$

LUFT, Joseph

Group Process. An Introduction to Group Dynamics. 3rd ed., Alto, Cal., Mayfield Publishing Company, 1984, 237 pp., ISBN 0-87484-542-4, recensé par Alexander $J$. Matejko.

v. 41 , no 3,1986, p. 667
BERNSTEIN, Paul

Workplace Democratization. Its Internal Dynamics. New Brunswick, Transaction Books, 1983, 133 pp., ISBN 0-87855-7113 , recense par Alexander J. Matejko.

v. 41 , no 3,1986, p. $667-668$

\section{STODDART, Linda}

Conditions of Work and Quality of Working Life, a Directory of Institutions. Geneva, International Labour Office, 1986, 306 pp., ISBN 92-2-105328-8, recensé par Gérard Dion.

v. 41, no 4,1986 , p. $878-879$

MONLÉON, Jacques de

Marx et Aristote. Perspectives sur l'homme. Paris, FAC éditions, 1984, ISBN 0317-0179, recensé par Maurice Lebel. v. 41 , no 4,1986 , p. $883-884$

\section{GUITTON, Jean}

Le travail intellectuel. Paris, Aubier-Montaigne, 1951 et 1986, 189 pp., ISBN 2707 3409-2, recensé par Maurice Lebel.

v. 42 , no 1,1987, p. $225-227$

\section{GUILIEMARD, Anne-Marie}

Le déclin du social, formation et crise des politiques de la vieillesse. Paris, Presses universitaires de France, 1986, 396 pp., ISBN 2-130-39565-1, recensé par Hélène David.

v. 42 , no 3,1987 , p. $642-646$

GROOTINGS, Peter GUSTAVSEN, Bjorn HÉTHY, Lajos

New Forms of Work Organization and their Social and Economic Development. Budapest, Statistical Publishing House, 1986, 297 pp., ISBN 963-01-6862-6, recensé par Alexander J. Matejko.

v. 42 , no 3,1987, p. $665-667$

RINEHART, James W.

The Tyranny of Work Alienation and the Labour Process. (2nd éd.), Toronto, Harcourt Brace Jovanovich, 1987, 226 pp., ISBN 0-7747-3067-6, recensé par Alexander J. Matejko.

v. 43, no 2,1988, p. $466-468$ 
SOCIOLOGIE DU TRAVAIL (suite)

LEWCHUK, Wayne

American Technology and the British Vehicle Industry. Cambridge, Cambridge University Press, 1987, 304 pp., ISBN 0521 30269-2, recensé par Jacques Bélanger.

v. 43, no 3,1988 , p. $716-717$

WALKER, Michael A.

Freedom, Democracy and Economic We/fare. Vancouver, the Fraser Institute, 1988, 369 pp., ISBN 0-88975-116-1, recensé par Morley Gunderson.

v. 44 , no 2,1989 , p. $465-466$

CROZIER, Michel

L'entreprise à l'écoute. Paris, Inter Éditions, 1990, 218 p., recensé par Dimitri Weiss.

v. 45 , no 3,1990 , p. $647-649$

\section{SOCIOLOGIE DU TRAVAIL - ALLEMAGNE}

SCHANZ, Günter

Mitarbeiter beteiligung. Grundlagen - Befunde - Modelle. Munich, Verlag Franz Vahlen, 1985, 198 pp., ISBN 3-8006-11309, recensé par Alexander J. Matejko.

v. 42, no 1,1987, p. 224-225

\section{SOCIOLOGIE DU TRAVAIL - AUSTRALIE}

Industrial Democracy and Employee Participation. Digest of Case Studies. vol. 1, Department of Employment and Industrial Relations, Sydney, Australia, 1985, 207 pp., ISBN 0814-9739, recensé par Bernard Portis.

v. 42 , no 1,1987, p. 221-222

\section{SOCIOLOGIE DU TRAVAIL - CANADA}

MORTON, Desmond COOP, Terry

Working People, An Illustrated History of Canadian Labour. Ottawa, Deneau \& Greenberg, 1980, 349 pp., recensé par F.J.K. Griezic.

v. 37 , no 1,1982 , p. $243-245$

NIGHTINGALE, Donald V.

Workplace Democracy : An Inquiry into Employee Participation in Canadian Work Organizations. Toronto, University of Toronto Press, 1983, 313 pp., ISBN 0-80206471-1, recensé par Alexander J. Matejko. v. 38 , no 4,1983 , p. $927-929$
CHEN, Mervin Y.T. REGAN, Thomas G. Work in the Changing Canadian Society. Toronto, Butterworths, 1985, 289 pp., ISBN 0-409-82335- $X$, recensé par Alexander $J$. Matejko.

v. 40, no 2,1985, p. $405-407$

\section{PALMER, Bryon}

Working-Class Experience: The Rise and Reconstitution of Canadian Labour, 18001980. Toronto, Butterworths, 1983, 320 pp., recensé par Jerry $P$. White.

v. 40 , no 2,1985 , p. $408-409$

HAMOWY, Ronald

Canadian Medicine : A Study in Restricted Entry. Vancouver, The Fraser Institute, 1984, 394 pp., ISBN 0-88975-062-9, recensé par Gilles Dusseault.

v. 40 , no 2,1985 , p. $412-414$

HERON, Craig STOREY, Robert

On the Job : Confronting the Labour Process in Canada. Kingston and Montreal, McGill-Queen's University Press, 1986, 360 pp., ISBN 0-7735-0598-9, recensé par Robert H. Zieger.

v. 42 , no 1,1987 , p. $216-218$

SOCIOLOGIE DU TRAVAIL - ÉTATS-UNIS

GERSUNY, Carl

Work Hazards and Industrial Conflict. Hanover, University Press of New England, 1981, 162 pp., Lib of Congress Catalog Card no 80-51506, recensé par René Boulard.

v. 38 , no 3,1983, p. 686

GORDON, David EDWARDS, Richard $\mathrm{REICH}$, Michael

Segmented Work Divided Workers : The Historical Transformation of Labour in the United States. Cambridge, Cambridge University Press, 1982, 288 pp., recensé par Jerry $P$. White.

v. 40 , no 2,1985, p. $410-411$

\section{SOCIOLOGIE DU TRAVAIL -}

GRANDE-BRETAGNE

EDWARDS, P.K. SCULLION, Hugh

The Social Organization of Industrial Conflict : Control and Resistance in the Workplace. Oxford, Basil Blackwell, 1982, 314 pp., ISBN 0-631-13127-2, recensé par Jacques Bélanger.

v. 38 , no 1,1983, p. 181-184 


\section{SOCIOLOGIE DU TRAVAIL .} GRANDE-BRETAGNE (suite)

EDWARDS, P.K SCULLION, Hugh

The Social Organization of Industrial Conflict. Control and Resistance in the Workplace. Oxford, Basil Blackwell, 1982, 314 pp., ISBN 0-631-13127-2, recensé par Alexander J. Matejko.

v. 38, no 3,1983, p. $685-686$

\section{SOCIOLOGIE DU TRAVAIL - INDES}

SAHAI, Jugendra

Urban Complex of an Industrial City. Allahbad, India, Chugh Publishing Company, 1980, recensé par Hem C. Jain.

v. 38 , no 1,1983 , p. 194-195

\section{SOCIOLOGIE DU TRAVAIL - ITALIE}

GIANNINI, Mirella

Giustizia e Mezzogiorno. II caso dello statuto dei lavoratori. Venezia, Marsilio Editori, 1979, 162 pp., recensé par Danielle Picard.

v. 36, no 3,1981 , p. $699-701$

\section{SOCIOLOGIE DU TRAVAIL - ONTARIO}

PALMER, Bryan D.

A Culture in Conflict : Skilled Workers and Industrial Capitalism in Hamilton. Ontario, 1860-1914, Montreal, McGill-Queen's University Press, 1979, 331 pp., recensé par Hugh Tuck.

v. 36 , no 1,1981 , p. $277-280$

\section{SOCIOLOGIE DU TRAVAIL - POLOGNE KOZDROJ, Alicja}

Grupa pracownicza jako przedmiot i podmiot motywowania. The Polish Academy of Sciences, Institute of Management, Wroclaw Ossolineum, 1988, 307 pp., ISBN 8304-02806-9, recensé par Alexander J. Matejko.

v. 45 , no 1,1990 , p. $213-214$

\section{SOCIOLOGIE DU TRAVAIL - QUÉBEC}

COUSINEAU, Jacques

L'Élise d'ici et le social 1940-1960, 1. La Commission sacerdotale d'Études sociales, Montreal, Les Éditions Bellarmin,
1982, 287 pp., ISBN 2-89007-462-5, recensé par Gérard Dion.

v. 37 , no 3,1982 , p. $709-712$

VINET, Alain DUFRESNE, Francine VÉZINA, Lucie

Condition féminine en milieu ouvrier Québec, Institut québécois de recherche sur la culture, 1982, 221 pp., ISBN 289224-018-2, recensé par Marie Françoise Marchis-Mouren.

v. 38 , no 3,1983 , p. $679-680$

\section{SYSTÈME DES RELATIONS INDUSTRIELLES}

\section{SYSTĖME DES RELATIONS \\ INDUSTRIELLES}

GIUGNI, Gino MENGONI, Luigi

MORTILLAARO, Felice WEISS, Dimitri

Possono migliorare le relazioni industriali in Italia? Torino, Gruppo Dirigenti Fiat, 1981, 140 pp., recensé par $P$. Joubert. v. 38 , no 1,1983 , p. $190-192$

\section{WEISS, Dimitri}

Les relations du travail. Employeurs, personnel, syndicats, État. 5ième éd., Paris, Dunod, 1983, 448 pp., ISBN 2-04-0155457, ISSN 0335-3184, recensé par Gérard Dion.

v. 38 , no 4,1983 , p. $912-913$

ROMAGNOLI, Umberto WEISS, Dimitri Pluralità : Italia ed Europa nei conflitti industriali. Roma, ASAP, 1983, 288 pp., recensé par $P$. Joubert.

v. 38 , no 4,1983 , p. $916-920$

\section{BARKIN, Sol}

Worker Militancy and its Consequences, 2nd Ed. The Changing Climate of Western Industrial Relations, New York, Praeger Publisher, 1983, 400 pp., ISBN 0-03061793-6, recensé par Daniel Benedict. v. 39, no 3,1984, p. $626-630$

WEISS, Dimitri CHIROUZE, Yves Le consommérisme. Paris, Editions Sirey, collection Administration des entreprises, 1984, 387 pp., ISBN 2-248-00080-4, recensé par $P$. Joubert. v. 39 , no 3,1984 , p. $634-635$ 


\section{SYSTEME DES RELATIONS INDUSTRIELLES (sulte) \\ BARBASH, Jack}

The Elements of Industrial Relations. Madison, Wisc., The University of Wisconsin Press, 1984, 153 pp., ISBN 0-299-096106 , recensé par Fernand Morin.

v. 39 , no 4,1984 , p. $793-795$

CAMPBELL, Duncan C. AOWAN, Richard L.

Multinational Enterprises and the OECD Industrial Relations. Philadelphia, The Wharton School Industrial Research Unit, University of Pennsylvania, 1983, 280 pp., ISBN 0-89546-039-4 et ISSN 0149-0818, recensé par François Vandamme.

v. 39, no 4,1984, p. $797-799$

WILCZYNSKI, Jozef

Comparative Industrial Relations. Ideologies, Institutions, Practices and Problems under Social Systems with Special Reference to Socialist Planned Economies. London, The Macmillan Press, 256 pp., ISBN 0-333-33430-2, recensé par Alexander J. Matejko.

v. 40 , no 1,1985 , p. $190-192$

BEAN, $\boldsymbol{R}$.

Comparative Industrial Relations : An Introduction to Cross National Perspectives. London, Crom Helm, 1985, 261 pp., ISBN 0-7099-3251-9 recensé par Mark Thompson.

v. 40, no 4,1985, p. $903-904$

ROBERTS, B.C.

Industrial Relations in Europe. The Imperatives of Change. Dover, New Hampshire, Crown Holm, 1985, 277 pp., recensé par Solomon Barkin.

v. 41 , no 2,1986 , p. $417-422$

DION, Gérard

Dictionnaire canadien des relations du travail. 2ième éd., Québec, Les Presses de I'Université Laval, 1986, 993 pp., ISBN 2-7637-6975-6, recensé par Alton W.J. Craig.

v. 41 , no 3,1986, p. $644-646$

DION, Gérard

Dictionnaire canadien des relations du travail. 2ième éd., Québec, Les Presses de I'Université Laval, 1986, 993 pp., ISBN 2-7637-6975-6, recensé par Gérard Hébert.

v. 41 , no 3,1986, p. $646-650$

JURIS, H, THOMPSON, Mark

DANIELS, $W$.

Industrial Relations in a Decade of Economic Change. IRRA Series, Annual Research Volume, Madison, Wisconsin, IRRA, 1985, 407 pp., ISBN 0-913447-30-7, recensé par Hem C. Jain.

v. 42 , no 2,1987 , p. $443-445$

ROBERTS, B.C. JACOBI, OtTO

JESSOP, Bob KASTENDIEK, Hans

REGINI, Marino

Industrial Relations in Europe. The Imperatives of Change. Beckenham, England, 1986, 279 pp., ISBN 0-7099-4212-5, and Economic Crisis. Trade Unions and the State. Beckenham, England, 1986, 295 pp., ISBN 0-7099-1447-4, recensés par Roy J. Adams.

v. 42, no 2,1987, p. $448-450$

JACKSON, Michael P.

Industrial Relations - A textbook. 3rd ed, London, Croom Helm, 1985, 294 pp., ISBN 0-7099-1474-1, recense par Anthomy Giles.

v. 42 , no 3,1987, p. $667-669$

KERR, Clark STAUDOHAR, Paul D. Industrial Relations in a New Age. San Francisco, Jossey-Bass Inc., 1986, 419 pp., ISBN 0-55542-013-3, recensé par Norman A. Solomon.

v. 42 , no 4,1987 , p. $883-884$

MAINWARING, John

The International Labour Organisation : A Canadian View. Ottawa, Canadian Government Publishing Centre, 1986, 206 pp., recensé par Garfield Clack.

v. 42 , no 4,1987 , p. $889-890$

MAURICE, Marc SELLIER, François SILVESTRE, Jean-Jacques

The Social Foundations of Industrial Power : A Comparison of France and Germany. Traduit par Arthur Goldhammer, Cambridge, MIT Press, 1986, 292 pp., ISBN O. 262-13213-3, recensé par Anthony Giles. v. 43, no 3,1988 , p. $703-707$ 


\section{SYSTĖME DES RELATIONS INDUSTRIELLES (suite)}

BAMBER, Greg J. LANSBURY, Russell D. International and Comparative Industrial Relations. London, Allen \& Unwin, 1987, 289 pp., ISBN 0-04-331117-4, recensé par Anthony Giles.

v. 43, no 3,1988, p. $703-707$

New Departures in Industrial Relations : Developments in the U.S., the U.K. and Canada. Occasional Paper published by British - North American Committee, Toronto, C.D. Howe Institute, April 1988, 74 pp., ISBN 0-89068-092-2, recensé par Jack Barbash.

v. 43, no 4,1988 , p. $981-982$

HYMAN, Richard STREECK, Wolfgang New Technology and Industrial Relations. Oxford, Basil Blackwell, 1988, 309 pp., ISBN 0-631-15982-7, recensé par Jacques Bélanger.

v. 45 , no 1,1990, p. $208-209$

BAMBER, Greg J. LANSBURY, Russell D. New Technology International Perspectives on Human Resources and Industrial Relations. Winchester, Unwin Hyman, 1989, 267 pp., ISBN 0-04-445123-7, recensé par Martin S. Serediak.

v. 45, no 2,1990 , p. $435-438$

\section{SYSTÈME DES RELATIONS}

\section{INDUSTRIELLES - AUTRICHE}

TOMANDL, T. FUERBOECK, $\mathrm{K}$

Social Partnership - The Austrian System of Industrial Relations and Social Insurance. Ithaca, ILR Press, 1986, 165 pp., ISBN 0-87546-116-6, recensé par Pierre Verge.

v. 42, no 3,1987, p. $662-663$

\section{SYSTÈME DES RELATIONS \\ INDUSTRIELLES - CANADA \\ CRAVEN, Paul}

"An Impartial Umpire" : Industrial Relations and the Canadian State 1900-1911. Toronto, University of Toronto Press, 1980, 386 pp., ISBN 0-8020-5505-2, recensé par James Thwaites.

v. 37 , no 1,1982 , p. $252-253$

CRAIG, Alton W.J.

The System of Industrial Relations in Ca- nada. (2nd ed.), Englewood Cliffs, Prentice-Hall, 1986, $510 \mathrm{pp}$., ISBN 0-13881194-6, recensé par Norman A. Solomon.

v. 41 , no 4,1986 , p. $874-875$

KRAHN, Harvey J. LOWE, Graham S. Work, Industry and Canadian Society. Scarborough, Nelson Canada, 1988, 310 pp., ISBN 0-17-603414-5, recensé par Alexander J. Matejko.

v. 43 , no 3,1988 , p. $718-719$

ANDERSON, John C.

GUNDERSON, Morley PONAK, Allen

Union-Management Relations in Canada.

(2nd ed.), Don Mills, Ont., Addison-Wesley Publishers, 1989, 498 pp., ISBN 0-20118621-7, recensé par Gene Swimmer.

v. 45 , no 2,1990 , p. $428-429$

\section{SYSTÈME DES RELATIONS}

\section{INDUSTRIELLES - ÉTATS-UNIS}

STIEBER, Jack MCKERSIE, Robert B. QUINN MILLS, D.

U.S. Industrial Relations 1950-1980 : A Critical Assessment. Madison, Industrial Relations Research Association, 1981, 361 pp., recensé par Mahmood A. Zaidi. v. 38 , no 1,1983 , p. $184-187$

KATZ, Harry C.

Shifting Gears : Changing Labor Relations in the U.S. Automobile Industry. London, MIT Press, 1985, 230 pp., ISBN 0-26211098-9, recensé par Michel Turner.

v. 40 , no 4,1985 , p. $900-901$

\section{SYSTÈME DES RELATIONS}

INDUSTRIELLES - GRANDE-BRETAGNE

POOLE, Michael BROWN, William

RUBERY, Jill SISSON, Keith

TARLING, Roger WILKINSON, Frank Industrial Relations in the Future. Trends and Possibilities in Britain over the Next Decade. Agincourt, Methuen Publications, 1984, 148 pp., ISBN 0-7102-0145-1, recensé par Roy J. Adams.

v. 42 , no 2,1987, p. $437-439$ 
SYSTÈME DES RELATIONS

INDUSTRIELLES - GRANDE-BRETAGNE (suite)

MILLWARD, Neil STEVENS, Mark

British Workplace Industrial Relations 1980-1984 : The DE/ESRC/PSI/ACAS Surveys. Aldershot, Gower, 1986, 341 pp., ISBN 0-566-05396-9, recensé par Jacques Bélanger.

v. 42 , no 4,1987 , p. $891-893$

\section{SYSTÈME DES RELATIONS}

INDUSTRIELLES - INDES

VENKATCHALAM, V. SINGH, R.K.

The Political, Economic and Labor Climate in India. Philadelphia, The Wharton School Industrial Research Unit, University of Pennsylvania, 1982, 147 pp., ISBN 0-89546030-0, ISSN 149-0818, recensé par Hem C. Jain.

v. 39, no 1,1984 , p. $215-217$

\section{SYSTÈME DES RELATIONS INDUSTRIELLES - ITALIE BUSANA, Osvaldo SCHÜTT, Maria Teresa UNNIA, Mario L'impresa nel sistema socio-sindicale : dieci anni di contrattazione. Turin, ISVOR - FIAT, 1980, recensé par Dimitri Weiss. v. 36 , no 2,1981 , p. 459}

\section{SYSTÈME DES RELATIONS INDUSTRIELLES - JAPON \\ CONNAGHAN, Charles J.}

L'expérience japonaise. Relations industrielles au Japon moderne. Ottawa, Travail Canada, 1982, 143 pp., ISBN 0-662-91608-

5 , recensé par Raynald Bourassa.

v. 37 , no 3,1982 , p. 720

\section{SYSTÈME DES RELATIONS}

\section{INDUSTRIELLES - PHILIPPINES}

INFANTE, Jaime T.

The Political, Economic and Labor Climate in the Philippines. Industrial Research Unit, The Wharton School, University of Pennsylvania, 1980, 147 pp., recensé par Elias T. Ramos.

v. 37 , no 3,1982 , p. $720-722$ 


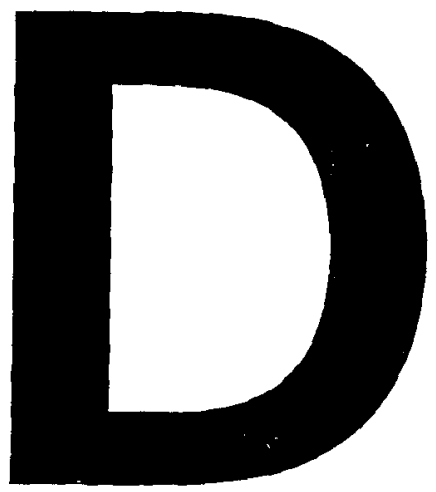

INDEX DES AUTEURS DES OUVRAGES RECENSÉS INDEX OF AUTHORS OF BOOKS REVIEWED 
Liste alphabétique des auteurs dont les ouvrages ont fait l'objet d'un compte rendu dans "Relations industrielles".

EXEMPLE:

DAVID, Hélène Auteur

DAVID, Hélène

Femmes et emploi: le défi de l'égalité. Montréal, Les Presses de l'université du Québec, 1986, 477 pp., ISBN 2-7605-0393-3, recensé par Carla Lipsig. Auteur, titre, référence de l'ouvrage recensé et auteur de la recension

v. 45, no 1,1990, p. $202-203$

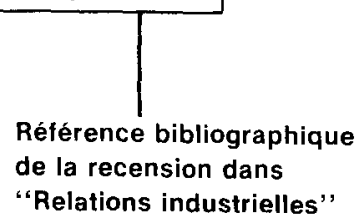


Alphabetical list of the authors whose books have been reviewed in "Industrial Relations".

EXAMPLE:

WINDMULLER, J.P. Author

WINDMULLER, J.P.
The International Trade Union Movement
Deventer, Kluwer, 1980, 174 pp., recensé
par Gérard Dion.

v. 36 , no 2,1981, p. $455-456$
Author, titie, reference of book reviewed, and author of book review

Bibliographical reference

of book review in

"Industrial Relations" 



\section{A}

AARON, Benjamin

AARON, Benjamin NAJITA, Joyce $M$.

STERN, James $L$.

Public Sector Bargaining. 2nd ed., Industrial Relations Research Association Series, Washington, Bureau of National Affairs, 1988, 334 pp., ISBN 0-87179-566-3, ISBN 0-913447-37-4, recensé par Jean Boivin. v. 44 , no 3,1989 , p. $730-732$

\section{ABURDENE, Patricia}

NAISBITT, John ABURDENE, Patricia Reinventing the Corporation. Transforming your Job and your Company for the New Information Society. New York, Warner Books, 1985, 308 pp., ISBN 0-446-512842, recensé par Alexander J. Matejko. v. 41 , no 4,1986 , p. $881-882$

ADAM, Jan

ADAM, Jan

Employment Policies in the Soviet Union and Eastern Europe. 2nd ed., London, The MacMillan Press, 1987, 224 pp., ISBN 0-333-41775-5, recensé par Chris Jecchinis.

v. 44, no 3,1989, p. $729-730$

ADAMS, G.W.

ADAMS, G.W.

Canadian Labour Law - A Comprehensive Text. Aurora, Canada Law Book Inc., 1985, 983 pp., ISBN 0-888-04-030-X, recensé par Pierre Verge.

v. 41 , no 3,1986 , p. $659-661$

ADDISON, John T.

ADDISON, John T. BURTON, John

Trade Unions and Society. Vancouver, The Fraser Institute, 1984, 189 pp., ISBN 0-88975-056-4, recensé par Peter E. Larson.

v. 40 , no 1,1985 , p. $180-181$

AGGARWAL, Arjun P.

AGGARWAL, Arjun P.

Sexual Harassment in the Workplace. Toronto, Butterworths, 1987, 230 pp., ISBN 0-409-80535-1, recensé par $P$. Andiappan. v. 43 , no 2,1988 , p. $469-471$
ALSTOM, Jon $P$.

ALSTOM, Jon P.

The American Samurai. Blending American and Japanese Managerial Practices. Berlin-New York, Walter de Gruyter, 1986, 369 pp., ISBN 0-89925-063-7, recensé par Alexander $J$. Matejko.

v. 41 , no 3,1986 , p. $662-664$

\section{ANDERSEN, Svein S.}

ANDERSEN, Svein S.

British and Norwegian Offshore Industrial Relations : Pluralism and Neo-Corporatism as Contexts of Strategic Adaptation. Aldershot, U.K., and Brookfield, Vermont, USA, Averbury/Gover, 1988, 213 pp., ISBN 0-566-055317, recensé par Alexander $J$. Matejko.

v. 45, no 2,1990 , p. 440

ANDERSON, John C.

ANDERSON, John $C$.

GUNDERSON, Morley PONAK, Allen

Union-Management Relations in Canada. (2nd ed.), Don Mills, Ont., Addison-Wesiey Publishers, 1989, 498 pp., ISBN 0-20118621-7, recensé par Gene Swimmer.

v. 45 , no 2,1990 , p. $428-429$

\section{ANTONIDES, Harry}

ANTONIDES, Harry

Renewal in the Workplace : A Critical Look at Collective Bargaining. London, Ontario, Christian Labour Association of Canada, 1982, 82 pp., ISBN 0-919663-00-1, recensé par Alton W.J. Craig.

v. 39 , no 2,1984 , p. $393-394$

\section{AOUST, Claude d'}

AOUST, Claude d' LECLERC, L.

TRUDEAU, Gilles

Les mesures disciplinaires : étude jurisprudentielle et doctrinale. Montréal, École de relations industrielles, Monographie no 13, 1982, 484 pp., ISBN 2-89067-012-0, ISSN 0708-9945, recensé par R. Blouin. v. 38 , no 2,1983 , p. $444-445$

\section{ARGYRIS, Chris}

ARGYRIS, Chris

Reasoning, Learning and Action. San Francisco, Jossey-Bass, 1982, 499 pp., ISBN 0-87589-524-7, recensé par Naresh C. Agarwal.

v. 39 , no 1,1984 , p. $206-208$ 


\section{ARMSTRONG, Hugh}

ARMSTRONG, Pat ARMSTRONG, Hugh Une majorité laborieuse. Les femmes qui gagnent leur vie, mais à quel prix. Ottawa, Conseil consultatif canadien de la situation de la femme, 1983, 329 pp., ISBN 0-660-91026-8, recensé par Esther Déom.

v. 39 , no 4,1984 , p. $816-817$

\section{ARMSTRONG, Pat}

ARMSTRONG, Pat ARMSTRONG, Hugh Une majorité laborieuse. Les femmes qui gagnent leur vie, mais à quel prix. Ottawa, Conseil consultatif canadien de la situation de la femme, 1983, 329 pp., ISBN 0-660-91026-8, recensé par Esther Déom.

v. 39 , no 4,1984 , p. $816-817$

ARNOLD, Hugh J.

FELDMAN, Daniel C. ARNOLD, Hugh J. Managing Individual and Group Behavior in Organizations. Toronto, McCrowill-Hill Series in Management, 1983, 613 pp., ISBN 0-8359-3006-4, recensé par Laurent Bêlanger.

v. 38, no 3,1983, p. $682-683$

\section{ARSENEAULT, André}

DOLAN, Shimon L. ARSENEAULT, André Stress, santé et rendement au travail, monographie no 5. Montréal, École des relations industrielles de l'Université de Montréal, 1980, 186 pp., recensé par René Boulard.

v. 36 , no 3,1981 , p. $701-702$

\section{ARTHURS, H.W.}

ARTHURS, H.W. CARTER, D.D.

EUDGE, F. GLASBEEK, H.J.

Labour Law and Industrial Relations in Canada. 3rd ed., Toronto, Butterworths, 1988,341 pp., ISBN 0-409-88891-5, ISBN 90-6544-389-4 (Kluwer), recensé par Pierre Verge.

v. 44 , no 4,1989 , p. 959

ARTHURS, H.W. CARTER, D.D.

GLASBEEK, H.J.

Labour Law and Industrial Relations in Canada. Deventer, Kluwer; Toronto; Butterworths, 1981, 291 pp., ISBN 90-3120139 (Kluwer), 0-409-81184-X (Butterworths), recensé par Pierre Verge.

v. 37 , no 1,1982 , p. 241-243
ARTHURS, H.W. CARTER, D.D.

GLASBEEK, H.J.

Labour Law and Industrial Relations in Canada. 2nd ed, Toronto, Butterworths, 1984, 316 pp., ISBN 0-409-81185-8, recensé par Pierre Verge.

v. 41 , no 1,1986 , p. 197-198

\section{ASHTON, D.N.}

ASHTON, D.N. MAGUIRE, J.J.

Young Adult in the Labour Market. Research Paper no 55, Leicester, University of Leicester, 1986, 163 pp., recensé par Lise Poulin Simon.

v. 42 , no 4,1987, p. $887-889$

\section{AUBERT, Gabriel}

AUBERT, Gabriel

L'obligation de paix du travail - étude du droit suisse et comparé. Genève, Librairie de I'Université, Georg et Cie S.A., 1981, 306 pp., ISBN 2-8257-0078-9, recensé par Pierre Verge.

v. 38, no 2,1983, p. $442-444$

\section{AUDET, Georges}

AUDET, Georges BONHOMME, Robert Le congédiement en droit québécois en matière de contrat individuel de travail. 2ième éd., Cowansville, Les Éditions Yvon Blais, 1988, 487 pp., ISBN 2-89073-6784 , recensé par Claude D'Aoust.

v. 44 , no 3,1989, p. $732-733$

\section{AUST, A.E.}

AUST, A.E.

Le contrat d'emploi. Cowansville, Les Éditions Yvon Blais, 1988, 235 pp., ISBN 2-89073-675- $X$, recensé par Réjean Breton. v. 44 , no 2,1989 , p. $466-468$

\section{AUSTIN, Nancy}

PETERS, Tom AUSTIN, Nancy

A Passion for Excellence. The Leadership Difference. New York, Random House, 1985, 437 pp., ISBN 0-394-54484, recensé par Alexander J. Matejko.

v. 41 , no 4,1986 , p. $881-882$

AWARD, Elias $\boldsymbol{M}$.

CASCIO, Wayne E. AWARD, Elias M.

Human Resources Management. An Information System Approach. Virginia, Reston Publishing Co., 1981, 594 pp., ISBN 0-07020386-5, recensé par Laurent Bélanger. v. 38 , no 3,1983 , p. $683-684$ 


\section{B}

\section{BAMBER, Greg J.}

BAMBER, Greg J. LANSBURY, Russell D. International and Comparative Industrial Relations. London, Allen \& Unwin, 1987, 289 pp., ISBN 0-04-331117-4, recensé par Anthony Giles.

v. 43, no 3,1988 , p. $703-707$

BAMBER, Greg J. LANSBURY, Russell D. New Technology International Perspectives on Human Resources and Industrial Relations. Winchester, Unwin Hyman, 1989, 267 pp., ISBN 0-04-445123-7, recensé par Martin S. Serediak.

v. 45 , no 2,1990 , p. $435-438$

\section{BAMFORD, Martin}

DAWSON, Sandra WILLMAN, Paul

BAMFORD, Martin CLINTON, Alain

Safety at Work: The Limits of Self-Regulation. Cambridge, Cambridge University Press, 1988, 310 pp., ISBN 0-521-354978, recensé par Eric Tucker.

v. 44 , no 4,1989 , p. $959-961$

\section{BAR-NIV, ZvI H.}

BAR-NIV, Zvi H.

International Labour Law Reports, vol. 2, 1979, 376 pp., recensé par Dimitri Weiss. v. 36 , no 2,1981 , p. $454-455$

BAR-NIV, Zvi $\mathrm{H}$.

International Labour Law Reports, vol. 3. Alphen aan den Rijn, Hollande, Sijthoff \& Noordhoff, 1980, 399 pp., ISBN 90-2860060-4, recensé par André C. Côté.

v. 38 , no 3,1983 , p. $690-691$

\section{BARANSON, Jack}

BARANSON, Jack

Robots in Manufacturing : Key to International Competitiveness. Mt Airy, Maryland, Lomond Publications, 1983, 152 pp., ISBN 0-912338-39-3, recensé par Rolland Hurtubise.

v. 39 , no 4,1984 , p. $801-802$

BARBAGELATA, H.H.

BARBAGELATA, H.H.

Introduction aux institutions du travail en Amérique Latine. Louvain, Presses universitaires de Louvain, 1980, 303 pp., ISBN 90-6186-101-2, recensé par Pierre Verge. v. 39 , no 3,1984 , p. $632-633$
BARBASH, Jack

BARBASH, Jack

The Elements of Industrial Relations. Madison, Wisc., The University of Wisconsin Press, 1984, 153 pp., ISBN 0-299-096106 , recensé par Fernand Morin.

v. 39 , no 4,1984 , p. $793-795$

\section{BARKIN, Sol}

BARKIN, Sol

Worker Militancy and its Consequences, $2 n d E d$. The Changing Climate of Western Industrial Relations, New York, Praeger Publisher, 1983, 400 pp., ISBN 0-03061793-6, recensé par Daniel Benedict.

v. 39 , no 3,1984 , p. $626-630$

\section{BAUM, Gregory}

BAUM, Gregory

The Priority of Labour. A Commentary on Laborem Exercens, Encyclical Letter of Pope John Paul II. New York/Ramsey, Paulist Press, 1982, 152 pp., ISBN 0-80912479-3, recensé par Alexander J. Matejko. v. 37 , no 4,1982 , p. $964-966$

BEAN, $R$.

BEAN, R.

Comparative Industrial Relations : An Introduction to Cross National Perspectives. London, Crom Helm, 1985, 261 pp., ISBN 0-7099-3251-9 recensé par Mark Thompson.

v. 40, no 4,1985 , p. $903-904$

\section{BEATTY, Carol}

BEATTY, Carol

The Implementation of Technological Change. Kingston, Industrial Relations Centre, Queen's University, 1987, 137 p., ISBN 0-88886-172-9, recense par Roger Zaoré.

v. 45, no 3,1990 , p. $634-635$

\section{BEATTY, D.M.}

\section{BEATTY, D.M.}

Putting the Charter to Work Designing a Constitutional Labour Code. Kingston et Montréal, McGill-Queen's University Press, 1987, 251 pp., ISBN 0-7735-0601-2, recensé par Pierre Verge.

v. 42 , no 4,1987, p. $877-880$ 


\section{BEAUDOIN, Pierre} BEAUDOIN, Pierre

La gestion par projet : aspects stratégiques. Montréal, Agence d'Arc, 1984, 251 pp., ISBN 2-89-22-065-6, recensé par Paul Tessier.

v. 40, no 4,1985, p. $898-899$

\section{BEAUMONT, P.B.}

BEAUMONT, P.B. PARTRIDGE, M.

Job Satisfaction in Public Administration. Londres, Royal Institute of Public Administration, 1983, 41 pp., ISBN 0-900628-324 , recensé par Michel Paquin.

v. 41 , no 2,1986 , p. $426-427$

\section{BÉLANGER, Jacques}

LAFLAMME, Gilles MURRAY, Gregor

BÉLANGER, Jacques FERLAND, Gilles Flexibilité and Labour Markets in Canada and United States. (Research Series 94), Geneva, International Institute for Labour Studies, 1989, 317 p., ISBN 92-9014-462-

9, recensé par Brad Pragnell.

v. 45, no 3,1990, p. $643-647$

\section{BÉLANGER, Laurent}

BÉLANGER, Laurent PETIT, André

BERGERON, Jean-Louis

Gestion des ressources humaines : une approche giobale et intégrée. Chicoutimi, Gaëtan Morin éditeur, 1983, 451 pp., ISBN 2-89105-072- $X$, recensé par Dimitri Weiss.

v. 39 , no 2,1984 , p. $406-408$

\section{BELLEMARE, Diane}

BELLEMARE, Diane POULIN-SIMON, Lise Le défi du plein emploi - un nouveau regard économique. Montréal, Éditions Saint-Martin, 1986, 530 pp., ISBN 2-89035131-9, recensé par Louis Ascah.

v. 42 , no 2,1987 , p. $450-451$

\section{BEN-ISRAEL, Ruth}

BEN-ISRAEL, Ruth

International Labour Standards: The Case of Freedom to Strike. Deventer, Kluwer, 1988, 133 pp., ISBN 90-6544-317-7, recensé par Pierre Verge.

v. 43, no 3,1988, p. $710-712$

\section{BÉRAUD, Jean-Marc}

BÉRAUD, Jean-Marc

La suspension du contrat de travail. Paris, Editions Sirey, 1980, 294 pp., recensé par Rodrigue Blouin.

v. 36, no 2,1981 , p. $451-452$

BÉRAUD, Jean-Marc

La suspension du contrat de travail. Paris, Editions Sirey, 1980, 296 pp., recensé par Dimitri Weiss.

v. 36 , no 2,1981 , p. $454-455$

\section{BERGERON, Jean-Louis}

BÉLANGER, Laurent PETIT, André

BERGERON, Jean-Louis

Gestion des ressources humaines : une approche globale et intégrée. Chicoutimi, Gaëtan Morin éditeur, 1983, 451 pp., ISBN 2-89105-072- $X$, recensé par Dimitri Weiss. v. 39 , no 2,1984 , p. $406-408$

\section{BERNSTEIN, Paul}

BERNSTEIN, Paul

Workplace Democratization. Its Internal Dynamics. New Brunswick, Transaction Books, 1983, 133 pp., ISBN 0-87855-7113 , recensé par Alexander J. Matejko.

v. 41, no 3,1986 , p. $667-668$

B.I.T.

B.I.T.

Rapport de la Commission d'experts pour l'application des conventions et recommandations. Conference internationale $d u$ travail, 66ième session, Genève, Rapport III, 1980, Partie 4A, 251 pp., Partie 4B, Travailleurs migrants, $190 \mathrm{pp}$., recensé par Alain Barré.

v. 36 , no 1,1981 , p. $291-294$

B.I.T.

Conciliation and Arbitration Procedures in Labour Disputes. A Comparative Study. Geneve, International Labour Office, 1980, 183 pp., recensé par Rodrigue Blouin.

v. 36 , no 2,1981 , p. $453-454$

B.I.T.

Normes et déclarations de principes inté'ressant particulierement les travailleuses. Genève, BIT, 1981, 138 pp., recensé par Alain Barré.

v. 36 , no 3,1981 , p. $696-697$

B.I.T.

Rapport de la Commission d'experts pour l'application des conventions et recommandations. Conférence internationale du travail, 67ieme session, Genève, Rapport III (Partie 4A), BIT, 1981, 255 pp., recensé par Alain Barré.

v. 36, no 4,1981 , p. $951-954$ 


\section{B.I.T. (suhie)}

B.I.T.

Age minimum. Etude d'ensemble de la commission d'experts pour l'application des conventions et recommandations, Conférence internationale du travail, 67ième session, Genève, Rapport III (Partie 4B), BIT, 1981, 238 pp., recensé par Alain Barré.

v. 36 , no 4,1981 , p. $951-954$

B.I.T.

Technological Change : The Tripartite Response, 1982-85. Genève, Bureau international du Travail, 1985, $355 \mathrm{pp}$., ISBN 92-2-105162-5, recensé par Rolland Hurtubise.

v. 41 , no 2,1986 , p. $433-435$

B.I.T.

Collective Bargaining : A Response to the Recession in Industrialised Market Economy Countries. Geneve, Bureau international du Travail, 1984, 275 pp., ISBN $92-$ 20103628-6, recensé par Paul Beaulieu.

v. 41 , no 3,1986, p. $651-652$

B.I.T.

La liberté syndicale - Manuel d'éducation ouvrière. (2ième éd.), Genève, Bureau international du travail, 1988, $153 \mathrm{pp}$., ISBN 92-2-205782-1, recensé par Pierre Verge.

v. 43, no 3,1988, p. $712-713$

\section{BLANCO, David Leon}

SAGARDOY BENGOECHEA, J.A.

BLANCO, David Leon

El poder sindical en Espana. Barcelona, Editorial Planeta/Instituto de Estudios Economicos, 1982, 210 pp., ISBN 84-3207318-0, recensé par $P$. Joubert.

v. 38 , no 1,1983 , p. $192-194$

\section{BLANPAIN, $R$.}

BLANPAIN, R.

Principes de droit du travail. BrugesBruxelles, La Charte, 1984, 313 pp., ISBN 90-6400-029-0, recensé par Pierre Verge. v. 40 , no 2,1985 , p. $403-405$

BLANPAIN, R. MILLARD, F.

Comparative Labour Law and Industrial Relations. Deventer, Kluwer, Washington, the Bureau of National Affairs, 1982, 411 pp., ISBN 0-87179-392-2, recensé par Pierre Verge.

v. 39 , no 1,1984 , p. $201-203$

HANAMI, T. BLANPAIN, R.

Industrial Conflict Resolution in Market Economies. Deventer, Kluwer, 1984, 321 pp., ISBN 90-6544-1808, recensé par Pierre Verge.

v. 40, no 3,1985 , p. $681-682$

\section{BLOCK, Richard N.}

KLEINER, Morris M. BLOCK, Richard N. ROOMKIN, Myron

SALSBURG, Sydney W.

Human Resources and the Performance of the Firm. Madison, Industrial Relations Research Association Series, 1987, 343 pp., ISBN 0-913447-382, recensé par Bernard Portis.

v. 44 , no 1,1989 , p. 297

\section{BLOUIN, Rodrigue}

MORIN, Fernand BLOUIN, Rodrigue Précis de l'arbitrage des griefs. Québec, Les Presses de I'Université Laval, 1980, 507 pp., recensé par Jean-Louis Dubé. v. 36, no 2,1981 , p. $449-451$

MORIN, Fernand BLOUIN, Rodrigue Arbitrage des griefs. Montréal, Les Editions Yvon Blais, 1986, 554 pp., ISBN 289083-569-9, recensé par Gilles Plante. v. 42 , no 2,1987, p. $432-434$

\section{BLYTON, Paul}

NICHLOSON, Nigel URSELL, Gill

BLYTON, Paul

The Dynamics of White Collar Unionism : A Study of Local Union Participation. London, Academic Press, 1981, 268 pp., ISBN 0-12-518020-9, recensé par Jacques Belanger.

v. 39 , no 1,1984 , p. $218-220$

\section{BOISVERT, Maurice}

BOISVERT, Maurice

La qualité de la vie au travail. Regard sur l'expérience québécoise. Le Groupe d'étude de travail des HEC, Montréal, Éditions Agence d'Arc Inc., 1981, 461 pp., recensé par Lucie Vézina.

v. 37, no 3,1982, p. $713-715$ 
BOIVIN, Jean

BOIVIN, Jean GUILBAULT, Jacques

Les relations patronales-syndicales au

Québec. Chicoutimi, Gaëtan Morin Éditeur, 1982, 309 pp., ISBN 2-89105-087-8, recensé par Dimitri Weiss.

v. 37 , no 4,1982 , p. $969-970$

BOIVIN, Jean GUILBAULT, Jacques

Les relations patronales-syndicales. Boucherville (Québec), 2ième éd., Gaétan Morin editeur, 1989, 301 p. ISBN 2-89105322-2, recensé par Jacques-André Lequin. v. 45 , no 3,1990 , p. 627-629

\section{BOLTANSKI, LUC}

BOLTANSKI, LUC

Les cadres : la formation d'un groupe social. Paris, Éditions Minuit, Collection "Le sens commun" dirigée par Pierre Bourdieu, 1982, 523 pp., ISBN 2-7078-0617-7, recensé par Dimitri Weiss.

v. 38 , no 4,1983 , p. $929-934$

\section{BONHOMME, Robert}

AUDET, Georges BONHOMME, Robert Le congédiement en droit québécois en matière de contrat individuel de travail. 2ième éd., Cowansville, Les Éditions Yvon Blais, 1988, 487 pp., ISBN 2-89073-6784 , recensé par Claude D'Aoust.

v. 44 , no 3,1989, p. $732-733$

BOONE, Louis E.

BOONE, Louis E. KURTZ, David L. L'entreprise d'aujourd'hui. (2ième éd.), Montréal, Éditions études vivantes, 1989, 737 pp., ISBN 2-7607-0446-7, recensé par Laurent Bélanger.

v. 45 , no 2,1990 , p. 432

\section{BORDELEAU, Yvan}

LAROCQUE, Alain BORDELEAU, Yvan BOULARD, René FABI, Bruno

LAROUCHE, Viateur RONDEAU, Alain

Technologies nouvelles et aspects psychologiques. Sillery, Presses de l'Université du Québec, 1987, 171 pp., ISBN 27605-0450-6, recensé par Jean Mercier. v. 44 , no 2,1989 , p. $469-470$

\section{BOULARD, René}

BOULARD, René PÉRUSSE, Lyne Les moyens organisationnels de prévention des maladies industrielles et des ac- cidents du travail : essai de typologie et bibliographie annotée. Québec, Collection instruments de travail, Departement des relations industrielles, Université Laval, 1981, 183 pp., recensé par Bernard Boucher.

v. 36 , no 3,1981 , p. $697-699$

LAROCQUE, Alain BORDELEAU, Yvan BOULARD, René FABI, Bruno

LAROUCHE, Viateur RONDEAU, Alain

Technologies nouvelles et aspects psychologiques. Sillery, Presses de l'Université du Québec, 1987, 171 pp., ISBN 27605-0450-6, recensé par Jean Mercier.

v. 44 , no 2,1989 , p. $469-470$

\section{BOULET, Jac-André}

BOULET, Jac-André

La langue et le revenu du travail à Montréal. Ottawa, Ministère des Approvisionnements et Services Canada, Étude préparée par le Conseil économique du Canada, 1980, 135 pp., ISBN 0-660-90515-9, recensé par Jean-Marie Rainville.

v. 37, no 4,1982, p. $971-973$

BOYER, LUC

BOYER, Luc EQUILBEY, Noël

Le projet d'entreprise. Paris, Les Éditions d'Organisation, 1986, $135 \mathrm{pp}$., ISBN 27081-0749-6, recensé par Paul Beaulieu.

v. 42 , no 4,1987 , p. $881-883$

\section{BRADET, Denis}

BRADET, Denis CLICHE, Bernard

RACINE, Martin THIBAULT, France

Droit de la santé et de la sécurité du travail - La loi et la jurisprudence commentée. Cowansville, Les Éditions Yvon Blais, 1986, 300 pp., ISBN 2-89073-567-2, recensé par Gilles Trudeau.

v. 42 , no 1,1987, p. $215-216$

\section{BRADLEY, Keith}

BRADLEY, Keith GELB, Alan

Worker Capitalism. The New Industrial Relations. London, Heinemann, 1983, 186 pp., ISBN 0-435-82084-2, recensé par Alexander J. Matejko.

v. 39 , no 1,1984 , p. $208-209$

BRADLEY, Keith GELB, Alan Worker Capitalism. The New Industrial Relations. Cambridge, The MIT Press, 1983, 186 pp., ISBN 0-262-02191-0, recensé par Clive H.J. Gilson.

v. 40 , no 3,1985 , p. $678-680$ 


\section{BRETON, Albert}

BRETON, Albert

Le mariage, la population et le taux d'activité des femmes. Conseil économique du Canada, Ottawa, ministère des Approvisionnements et Services Canada, 1983, 36 pp., ISBN 0-660-91184-1, recensé par Jennifer Stoddart.

v. 41 , no 1,1986 , p. 203-204

\section{BRIDDLE, Bruce J.}

BRIDDLE, Bruce J.

Role Theory : Expectations, Identities and Behaviors. New York, Academic Press, 1979, 416 pp., recensé par André Petit. v. 36 , no 4 , 1981, p. $944-945$

\section{BRITTON, Raymond L.}

BRITTON, Raymond L.

The Arbitration Guide : A Case-Handing Manual Procedures and Practices in Dispute Resolutions. Englewood Cliffs, New Jersey, Prentice Hall, 1982, 298 pp., ISBN 0-13-043984-3, recensé par André C. Cóté. v. 38, no 2,1983 , p. $446-448$

BROWN, Malcolm C.

BROWN, Malcolm C.

Caring for Profit : Economic Dimensions of Canada's Health Industry. Vancouver, The Fraser Institute, 1987, 182 pp., ISBN 0-88975-106-04, recense par Gérard Belanger.

v. 44 , no 1,1989 , p. $291-292$

\section{BROWN, William}

POOLE, Michael BROWN, William

RUBERY, Jill SISSON, Keith

TARLING, Roger WILKINSON, Frank Industrial Relations in the Future. Trends and Possibilities in Britain over the Next Decade. Agincourt, Methuen Publications, 1984, 148 pp., ISBN 0-7102-0145-1, recensé par Roy J. Adams.

v. 42 , no 2,1987 , p. $437-439$

\section{BURAWOY, Michael}

BURAWOY, Michael

Manufacturing Consent: Changes in the Labor Process under Monopoly Capitalism. Chicago, III., The University of Chicago Press, 1979, 267 pp., recensé par Craig A. Zabala.

v. 36, no 1,1981 , p. $282-285$

\section{BUREAU, Robert D.}

BUREAU, Robert D. MACKAY, Pierre

Le droit dans tous ses états. Montreal, Wilson \& Lafleur, 1987, 620 p., ISBN 289127-07602, recensé par Fernand Morin. v. 45 , no 3,1990 , p. $640-643$

\section{BURTON, John}

ADDISON, John T. BURTON, John Trade Unions and Society. Vancouver, The Fraser Institute, 1984, 189 pp., ISBN 0-88975-056-4, recensé par Peter E. Larson.

v. 40 , no 1,1985, p. $180-181$

\section{BUSANA, Osvaldo}

BUSANA, Osvaldo

SCHUTT, Maria Teresa UNNIA, Mario

L'impresa nel sistema socio-sindicale. dieci anni di contrattazione. Turin, ISVOR - FIAT, 1980, recensé par Dimitri Weiss.

v. 36, no 2,1981 , p. 459

\section{C}

\section{CAMPBELL, Duncan $C$.}

CAMPBELL, Duncan C

ROWAN, Richard L.

Multinational Enterprises and the OECD Industrial Relations. Philadelphia, The Wharton School Industrial Research Unit, University of Pennsylvania, 1983, 280 pp., ISBN 0-89546-039-4 et ISSN 0149-0818, recensé par François Vandamme.

v. 39 , no 4,1984 , p. $797-799$

CARROTHERS, A.W.R.

CARROTHEFIS, A.W.R. PALMER, E.E.

RAYNER, W.B.

Collective Bargaining Law in Canada. 2nd Ed., Toronto, Butterworths, 1986, 785 pp., ISBN 0-409-81879-8, recensé par Pierre Verge.

v. 42 , no 1,1987 , p. $219-221$

\section{CARTER, D.D.}

ARTHURS, H.W. CARTER, D.D.

EUDGE, F. GLASBEEK, H.J.

Labour Law and Industrial Relations in Canada. 3rd ed. Toronto, Butterworths, 1988, 341 pp., ISBN 0-409-88891-5, ISBN 90-6544-389-4 (Kluwer), recensé par Pierre Verge.

v. 44 , no 4,1989 , p. 959

ARTHURS, H.W. CARTER, D.D.

GLASBEEK, H.J.

Labour Law and Industrial Relations in Canada. Deventer, Kluwer; Toronto; Butterworths, 1981, 291 pp., ISBN 90-3120139 (Kluwer), 0-409-81184-X (Butterworths), recensé par Pierre Verge.

v. 37, no 1,1982 , p. $241-243$ 
CARTER, D.D. (suite)

ARTHURS, H.W. CARTER, D.D.

GLASBEEK, H.J.

Labour Law and Industrial Relations in Canada. 2nd ed, Toronto, Butterworths, 1984, 316 pp., ISBN 0-409-81185-8, recensé par Pierre Verge.

v. 41 , no 1,1986, p. $197-198$

CASCIO, Wayne E.

CASCIO, Wayne E. AWARD, Elias M. Human Resources Management. An Information System Approach. Virginia, Reston Publishing Co., 1981, 594 pp., ISBN 0-07020386-5, recensé par Laurent Bélanger. v. 38 , no 3,1983 , p. $683-684$

\section{CATALYST, The Staff of}

CATALYST, The Staff of

Upward Mobility. New-York, Holt, Rinehart and Winston, 1982, 292 pp., ISBN 0-03056163-9, recensé par Alexander J. Matejko.

v. 39 , no 2,1984 , p. $398-399$

\section{CEREQ}

CEREQ

L'évolution des systèmes de travail dans l'économie moderne. Paris, Éditions du CNRS, 1981, 287 pp., ISBN 2-222-02935$X$, recensé par Gérard Dion.

v. 37 , no 2,1982 , p. 460

\section{CHAISON, Gary N.}

CHAISON, Gary $N$.

When Union Merge. Lexington, Lexington Books, 1986, 186 pp., ISBN 0-669-110817 , recensé par $P$. Andiappan.

v. 42 , no 3,1987 , p. $649-651$

\section{CHALMERS, Norma $\mathrm{J}$.}

CHALMERS, Norma J.

Industrial Relations in Japan. The Peripheral Workforce. New York, Routledge, Chapman \& Hall, 1989, 283 p., ISBN O415-00008-4, recensé par Hem C. Jain.

v. 45, no 3,1990, p. $631-632$

\section{CHARNEY, Cyril}

CROCKER, OIga

CHIU, Johnny Sik Leung CHARNEY, Cyril Quality Circles. A Guide to Participation and Productivity. Toronto, Methuen, 294 pp., ISBN 0-458-97360-2, recensé par Alexander J. Matejko.

v. 40, no 3,1985, p. $682-683$

\section{CHEN, Mervin Y.T.}

CHEN, Menvin Y.T. REGAN, Thomas G. Work in the Changing Canadian Society. Toronto, Butterworths, 1985, 289 pp., ISBN 0-409-82335- $X$, recensé par Alexander $J$. Matejko.

v. 40 , no 2,1985 , p. $405-407$

\section{CHIROUZE, Yves}

WEISS, Dimitri CHIROUZE, Yves

Le consommérisme. Paris, Editions Sirey, collection Administration des entreprises, 1984, 387 pp., ISBN 2-248-00080-4, recensé par P. Joubert.

v. 39 , no 3,1984 , p. $634-635$

\section{CHIU, Johnny Sik Leung}

\section{CROCKER, Olga}

CHIU, Johnny Sik Leung CHARNEY, Cyri! Quality Circles. A Guide to Participation and Productivity. Toronto, Methuen, 294 pp., ISBN 0-458-97360-2, recensé par - Alexander J. Matejko.

v. 40 , no 3,1985 , p. $682-683$

\section{CHRISTENSEN, Sandra}

CHRISTENSEN, Sandra

Unions and the Public Interest : Collective Bargaining in the Government Sector. Vancouver, The Fraser Institute, 1980, 95 pp., recensé par Jean Boivin.

v. 36 , no 1,1981 , p. $275-276$

\section{CHRISTENSEN, Sandra}

Unions and the Public Interest: Collective Bargaining in the Government Sector. Vancouver, The Fraser Institute, 1980, 95 pp., recensé par William Cunningham. v. 36 , no 2,1981 , p. $461-462$

\section{CLARK, J.}

LEWIS, R. CLARK, J.

WEDDERBURN, Lord of Charlton

Labour Law and Industrial Relations : Building on Kahn-Freund. Oxford, Clarendon Press, 1983, 250 pp., ISBN 0-19-8253931 , recensé par Pierre Verge.

v. 38 , no 3,1983 , p. $677-678$

CLARK, Paul F.

CLARK, Paul F.

The Mines Fight for Democracy. Arnold Miller and Reform of the United Mine Workers. New York State School of Industrial and Labour Relations, Cornell University, 1981, 190 pp., recensé par Paul Phillips. v. 39 , no 1,1984 , p. 210-211 


\section{CLICHE, Bernard}

BRADET, Denis CLICHE, Bernard

RACINE, Martin THIBAULT, France

Droit de la santé et de la sécurité du travail - La loi et la jurisprudence commentée. Cowansville, Les Éditions Yvon Blais, 1986, 300 pp., ISBN 2-89073-567-2, recensé par Gilles Trudeau.

v. 42 , no 1,1987 , p. $215-216$

\section{CLINTON, Alain}

DAWSON, Sandra WILLMAN, Paul

BAMFORD, Martin CLINTON, Alain

Safety at Work: The Limits of Self-Regula-

tion. Cambridge, Cambridge University

Press, 1988, 310 pp., ISBN 0-521-35497-

8, recensé par Eric Tucker.

v. 44 , no 4,1989 , p. $959-961$

\section{COLLERETTE, Plerre}

COLLERETTE, Pierre DELISLE, Gilles Le changement planifié. Montréal, Agence d'Arc, 1982, 213 pp., ISBN 0-89022-0451, recensé par Laurent Bélanger.

v. 38, no 1, 1983, p. 195-196

CONNAGHAN, Charles J.

CONNAGHAN, Charles J.

L'experience japonaise. Relations industrielles au Japon moderne. Ottawa, Travail Canada, 1982, 143 pp., ISBN 0-662-916085 , recensé par Raynald Bourassa.

v. 37 , no 3,1982 , p. 720

\section{CONSEIL ÉCONOMIQUE DU CANADA} CONSEIL ÉCONOMIQUE DU CANADA

Pénuries et carences. Travailleurs qualifiés et emplois durant les années 80. Ottawa, ministère des Approvisionnements et Services Canada, 1982, 141 pp., ISBN 0-660-90874-3, recensé par Gérard Bélanger.

v. 37, no 3,1982 , p. $704-709$

\section{COOK, Alice $\mathrm{H}$.}

COOK, Alice $\mathrm{H}$.

The Working Mother, A Survey of Problems and Programs in Nine Countries. 2nd ed., Ithaca, New York State School of Industrial and Labor Relations, Cornell University, 1978, 71 pp., recensé par Marie Lavigne v. 36, no 2,1981, p. 458

\section{COOK, Robert F.}

COOK, Robert $F$.

Worker Dislocation : Case Studies of Causes and Cures. Kalamazoo, Michigan, W.E. Upjohn Institute for Employment Research, 1987, 219 pp., ISBN 0-88099-052$X$, recensé par Maurice J. Mazerolle.

v. 45 , no 2,1990, p. $432-434$

\section{COOP, Terry}

MORTON, Desmond COOP, Terry

Working People, An Illustrated History of Canadian Labour. Ottawa, Deneau \& Greenberg, 1980, 349 pp., recensé par F.J.K. Griezic.

v. 37, no 1,1982 , p. $243-245$

\section{COOPER, Cary L.}

COOPER, Cary L. MEMFORD, Enid

The Quality of Working Life in Western and Eastern Europe. Westport, Greenwood Press, 1979, 348 pp., recensé par Alexander J. Matejko.

v. 36 , no 1,1981 , p. $285-288$

\section{COPLAND LTÉE.}

COPLAND LTÉE.

Index et résumés de sentences arbitrales de griefs, vol. 1, 1970-1980. Montréal, Copland Ltée, 1982, ISBN 2-920391-002, recensé par Fernand Morin.

v. 37 , no 4,1982 , p. $967-968$

\section{CÔTÉ, Marcel}

CÔTÉ, Marcel TÉGA, Vasile

La démocratie industrielle / The Industrial Democracy. Montreal, Les Editions Agence d'Arc, 1981, 518 pp., recensé par Jacques Belanger.

v. 36 , no 3,1981 , p. $689-690$

\section{COUSINEAU, Jacques}

COUSINEAU, Jacques

L'Église d'ici et le social 1940-1960, 1. La Commission sacerdotale d'Études sociales, Montréal, Les Éditions Bellarmin, 1982, 287 pp., ISBN 2-89007-462-5, recensé par Gérard Dion.

v. 37, no 3,1982 , p. $709-712$

\section{CRAIG, Alton W.J.}

CRAIG, Alton W.J.

The System of Industrial Relations in Canada. (2nd ed.), Englewood Cliffs, PrenticeHall, 1986, 510 pp., ISBN 0-13-881194-6, recensé par Norman A. Solomon.

v. 41 , no 4,1986 , p. $874-875$ 
CRAIG, C.

CRAIG, C. RUBERY, Jill

TARLING, Roger WILKINSON, Frank Abolition and After the Paper Box Wages Council. Research Paper no 12, London, Department of Employment, June 1980, 98 pp., recensé par David Bright.

v. 39, no 2,1984 , p. $396-398$

CRAVEN, Paul

CRAVEN, Paul

"An Impartial Umpire" : Industrial Relations and the Canadian State 1900-1911. Toronto, University of Toronto Press, 1980 , 386 pp., ISBN 0-8020-5505-2, recensé par James Thwaites.

v. 37 , no 1,1982 , p. $252-253$

CROCKER, Olga

CROCKER, Olga

CHIU, Johnny Sik Leung CHARNEY, Cyril Quality Circles. A Guide to Participation and Productivity. Toronto, Methuen, 294 pp., ISBN 0-458-97360-2, recensé par Alexander J. Matejko.

v. 40, no 3,1985 , p. $682-683$

\section{CROUCH, Colin}

$\mathrm{CROUCH}$, Colin

Trade Unions : The Logic of Collective Action. Glasgow, Fontana Paperbacks, 1982, 251 pp., ISBN 0-00-635873X, recensé par Alexander J. Matejko.

v. 39, no 1, 1984, p. 212-215

\section{CROZIER, Michel}

CROZIER, Michel

L'entreprise à l'écoute. Paris, Inter Éditions, 1990, 218 p., recensé par Dimitri Weiss.

v. 45 , no 3, 1990 , p. $647-649$

CROZIER, Michel FRIEDBERG, Erhard Actors and Systems. The Politics of Collective Action. Chicago, The University of Chicago Press, 1980, 333 pp., recensé par Alexander J. Matejko.

v. 38 , no 2,1983 , p. $448-452$

CUMMINGS, Thomas G.

CUMMINGS, Thomas G.

MOLLOY, Edmond S.

Improving Productivity and the Quality of Work Life. New-York, Prager Publications, 1977, 305 pp., ISBN 0-03-022601-5, recensé par Alexander J. Matejko.

v. 37, no 3, 1982, p. 715-717

\section{CUTLER, Phillip}

CUTLER, Philip

Code du travail du Québec. Montréal, Les Livres Toundra, 1983, 539 pp., ISBN 88776-135-6, recensé par Gilles Bachand. v. 38 , no 3,1983, p. $676-677$

\section{D}

DANIEL, Cletus E.

DANIEL, Cletus E.

The ACLU and the Wagner Act : An inquiry into the Depression. Era Crisis of American Liberalism. Cornell Studies in Ind. and Labor Rel. Number 20, N. Y. State School of Ind. and Labor Rel., Cornell Univ., Ithaca, N. Y., 1980, 142 pp., recensé par Hugh Tuck.

v. 36 , no 4,1981 , p. $954-956$

DANIEL, W.W.

DANIEL, W.W. MILLWARD, Neil

Workplace Industrial Relations in Britain. The DE/PSI/SSRC Survey, London, Heinemann Educational Books, 1983, 338 pp., ISBN 0-435-83191-7, recensé par Alexander J. Matejko.

v. 39, no 1,1984, p. $217-218$

DANIELS, $\mathbf{W}$.

JURIS, $H$. THOMPSON, Mark

DANIELS, $W$.

Industrial Relations in a Decade of Economic Change. IRRA Series, Annual Research Volume, Madison, Wisconsin, IRRA, 1985, 407 pp., ISBN 0-913447-30-7, recensé par Hem C. Jain.

v. 42 , no 2,1987, p. $443-445$

\section{DAUM, Susan $M$.}

STELLMAN, Jeanne M. DAUM, Susan M. Perdre sa vie à la gagner; manuel pour la santé des travailleurs. Montréal, Édition Parti pris, 1979, 493 pp., recensé par René Boulard et Michel Pérusse.

v. 36 , no 3,1981 , p. $702-703$

\section{DAVID, Hélène}

DAVID, Hélène

Femmes et emploi : le défi de l'égalité. Montréal, Les Presses de l'Université du Québec, 1986, 477 pp., ISBN 2-76050393-3, recensé par Carla Lipsig.

v. 45 , no 1,1990, p. $202-203$ 


\section{DAVIES, Annette}

DAVIES, Annette

Industrial Relations and New Technology.

UWIST /Croom Helm, London, Sydney,

Dover, 1986, ISBN 0-7099-0882-2, recensé par Guy Fréchet.

v. 43, no 2,1988 , p. $468-469$

\section{DAWSON, Sandra}

DAWSON, Sandra WILLMAN, Paul

BAMFORD, Martin CLINTON, Alain

Safety at Work: The Limits of Self-Regula-

tion. Cambridge, Cambridge University

Press, 1988, 310 pp., ISBN 0-521-35497-

8 , recensé par Eric Tucker.

v. 44 , no 4,1989 , p. $959-961$

\section{DELAMOTTE, Yves}

DELAMOTTE, Yves

Le droit du travail en pratique. Paris, Les

Éditions d'organisation, 1983, 319 pp., ISBN 2-7081-0541-8, recensé par Gérard Dion.

v. 39 , no 2,1984 , p. $402-403$

\section{DELAMOTTE, Yves}

Le droit du travail en pratique. (3ième éd.), Paris, Les Éditions de l'organisation, 1987, 328 pp., ISBN 1-7081-0845-X, recensé par Gérard Dion.

v. 43, no 2,1988, p. 473

\section{DELISLE, Gilles}

COLLERETTE, Pierre DELISLE, Gilles

Le changement planifié. Montréal, Agence d'Arc, 1982, 213 pp., ISBN 0-89022-045-

1, recensé par Laurent Bélanger.

v. 38 , no 1,1983 , p. $195-196$

\section{DELORME, François}

DELORME, François PARENT, Réjean Les licenciements collectifs au Québec : un bilan partiel du dispositif public en vigueur. Monographie 12, Montréal, Ecole de relations industrielles, Université de Montréal, 1982, 106 pp., ISBN 2-89067011-2 et ISSN 0708-9945, recensé par Jean Sexton.

v. 38 , no 1,1983 , p. 178

\section{DELPLANQUE, Bernard}

\section{DELPLANQUE, Bernard}

Le partage de l'emploi, vers une société sans chomage. Paris, Editions Entente, 1980, 141 pp., recensé par Paul Beaulieu. v. 37 , no 1,1982, p. 248

\section{DEMERS, François}

DEMERS, François

Chroniques impertinentes du 3ième (sic)

Front commun Syndical, 1979-1980. Mon-

tréal, Nouvelle optique, 1982, 170 pp., ISBN 2-89017-035-7, recensé par Gerard Hébert.

v. 37 , no 4,1982 , p. $962-963$

DENTON, F.T.

DENTON, F.T. ROBB, A.L.

SPENCER, B.G.

Unemployment and Labour Force Behaviour of Young People : Evidence from Canada and Ontario. Ontario Economic Council Research Series no 20, Toronto, University of Toronto Press, 1980, 215 pp., ISSN 0708-3688, ISBN 0-8020-3379-2, recensé par Jacques Mercier. v. 37 , no 1,1982 , p. $249-251$

\section{DESPAX, Michel}

DESPAX, Michel

Négociations, conventions et accords collectifs. (2ième éd.), tome sept de Droit du travail, publié sous la direction de G.H. Camerlynck, Paris, Dalloz, 1989, 586 pp., ISBN 2-247-00890-2, recensé par Pierre Verge.

v. 45 , no 2,1990 , p. $434-435$

DESPAX, Michei PÉLISSIER, Jean La gestion du personnel : aspects juridiques. Tome 1, 2ième éd., Paris, Éditions Cujas, 1984, 400 pp., ISBN 2-2548-40310 , recensé par Dimitri Weiss.

v. 41 , no 1,1986 , p. 190-191

\section{DESSLER, Gary}

DESSLER, Gary

Organization Theory: Integrating Structure and Behavior. Englewood Cliffs, PrenticeHall, 1986, 477 pp., ISBN 0-13-641-9038-01, recensé par James Thwaites.

v. 41 , no 4,1986 , p. $884-885$

\section{LORIO, Nicola}

DUBÉ, Jean-Louis DI LORIO, Nicola Les normes du travail. Sherbrooke, Les Éditions Revue de Droit, Université de Sherbrooke, 1987, 442 pp., ISBN 2920003-08-9, recensé par Fernand Morin. v. 42 , no 3,1987, p. $655-656$ 


\section{DION, Gérard}

DION, Gérard

Dictionnaire canadien des relations $d u$ travail. 2ième ed., Québec, Les Presses de IUniversité Laval, 1986, 993 pp., ISBN 2-7637-6975-6, recensé par Alton W.J. Craig.

v. 41 , no 3,1986 , p. $644-646$

\section{DION, Gérard}

Dictionnaire canadien des relations du travail. 2ième éd., Québec, Les Presses de l'Université Laval, 1986, 993 pp., ISBN 2-7637-6975-6, recensé par Gérard Hébert.

v. 41 , no 3,1986 , p. $646-650$

\section{DOLAN, Shimon L.}

DOLAN, Shimon L. ARSENEAULT, André Stress, santé et rendement au travail, monographie no 5. Montreal, École des relations industrielles de l'Université de Montréal, 1980, 186 pp., recensé par René Boulard.

v. 36 , no 3,1981 , p. $701-702$

DOLAN, Shimon L. ROY, Denis

La sélection des cadres : principes et problèmes contemporains. Monographie no 13, Montréal, École de Relations Industrielles, Université de Montréal, 1982, ISBN 2-89067-010-4, ISSN 0708-9945, recensé par Normand Pettersen.

v. 38 , no 4,1983 , p. $910-912$

DOLAN, Shimon L. SCHULER, Randall S. Personnel and Human Resource Management in Canada. St-Paul, West Publishing, 1987, 620 pp., ISBN 0-314-32486-0, recensé par Michel Audet.

v. 42, no 2,1987 , p. $440-443$

DOLAN, Shimon L. SCHULER, Randall S. Canadian Readings in Personnel and Human Resource Management. St-Paul, West Publishing Company, 1987, 467 pp., ISBN 0-314-32487-9, recensé par Michel Audet.

v. 43 , no 1,1988 , p. $203-205$

DONN, C.D.

DONN, C.D.

The Australian Council of Trade Unions. History and Economic Policy. New York, University Press of America, 1983, 366 pp., ISBN 0-8191-2728-9, recensé par Gilles Dussault.

v. 39 , no 3,1984 , p. $630-631$

\section{DONN, Clifford B.}

LIPSKI, David B. DONN, Clifford B.

Collective Bargaining in American Industry. Lexington, Lexington Books, 1987, 351 pp., ISBN 0-669-12595, recensé par Joseph B. Rose.

v. 43 , no 4,1988 , p. $980-981$

\section{DORSEY, J.}

DORSEY, J.

Canada Labour Relations Board - Federal Law and Practice. Toronto, the Carswell Company 1983, 323 pp., ISBN 0-45935850-2, recensé par Pierre Verge.

v. 40, no 1,1985 , p. $183-185$

\section{DUBE, Jean-Louis}

DUBÉ, Jean-Louis DI LORIO, Nicola

Les normes du travail. Sherbrooke, Les Éditions Revue de Droit, Université de Sherbrooke, 1987, 442 pp., ISBN 2920003-08-9, recensé par Fernand Morin. v. 42, no 3,1987 , p. $655-656$

\section{DUBE, Louise}

RENAUD, Marc TRUDEAU, Gilles

SAINT-JACQUES, Chantal DUBE, Louise Le droit de refus: une révolution tranquille - étude de la mise en oeurre d'un nouveau droit. École des relations industrielles, Université de Montréal, Monographie, no 21, 1989, 329 p., ISBN 2-89067-0201, ISSN 0708-9945, recensé par Rodrigue Blouin.

v. 45 , no 3,1990 , p. $637-640$

DUBRIN, Andrew J.

DUBRIN, Andrew J.

Foundations of Organizational Behavior : An Applied Perspective. Englewood Cliffs, Prentice-Hall, 1984, 500 pp., ISBN 0-13329367-X, recensé par Laurent Bélanger. v. 39 , no 4,1984 , p. $805-806$

\section{DUFRESNE, Francine}

VINET, Alain DUFRESNE, Francine VÉZINA, Lucie

Condition féminine en milieu ouvrier. Québec, Institut québécois de recherche sur la culture, 1982, 221 pp., ISBN 289224-018-2, recensé par Marie Françoise Marchis-Mouren.

v. 38 , no 3,1983 , p. $679-680$ 
DUPONT, Christophe

DUPONT, Christophe

La négociation. Conduite, theorie, application. Paris, Dalloz, 1982, 276 pp., ISBN 2-247-00315-X, recensé par Paul Beaulieu. v. 40 , no 4,1985 , p. $892-893$

DURAND, Jean-Pierre

DURAND, Jean-Pierre

DURAND-SEBAG, Joyce LOJKINE, Jean MAHIEU, Christian

L'enjeu informatique : former pour changer l'entreprise. Paris, Méridiens Klincksieck, 1986, 192 pp., ISBN 2-86563-148-6, recensé par Guylaine Vallée.

v. 42 , no 3,1987 , p. $660-661$

\section{DURAND-SEBAG, Joyce}

DURAND, Jean-Pierre

DURAND-SEBAG, Joyce LOJKINE, Jean MAHIEU, Christian

L'enjeu informatique : former pour changer l'entreprise. Paris, Méridiens Klincksieck, 1986, 192 pp., ISBN 2-86563-148-6, recensé par Guylaine Vallée.

v. 42 , no 3,1987 , p. $660-661$

\section{E}

\section{EASTMAN, Byron D.}

EASTMAN, Byron D.

Labour Market Theory and the Canadian Experience. Toronto, Harcourt Brace Jovanovich, 1987, 350 pp., ISBN 077-4730757, recensé par S.A. Alvi.

v. 42 , no 4,1987 , p. $894-895$

\section{EDWARDS, P.K.}

EDWARDS, P.K. SCULLION, Hugh The Social Organization of Industrial Conflict : Control and Resistance in the Workplace. Oxford, Basil Blackwell, 1982, 314 pp., ISBN 0-631-13127-2, recensé par Jacques Bélanger.

v. 38, no 1,1983, p. 181-184

EDWARDS, P.K. SCULLION, Hugh The Social Organization of Industrial Conflict. Control and Resistance in the Workplace. Oxford, Basil Blackwell, 1982, 314 pp., ISBN 0-631-13127-2, recensé par Alexander J. Matejko.

v. 38, no 3,1983, p. $685-686$
EDWARDS, Richard

GORDON, David EDWARDS, Richard

REICH, Michael

Segmented Work Divided Workers : The Historical Transformation of Labour in the United States. Cambridge, Cambridge University Press, 1982, 288 pp., recensé par Jerry $P$. White.

v. 40, no 2,1985, p. $410-411$

\section{EHRENBERG, Ronaid G. (Ed.)}

EHRENBERG, Ronald G. (Ed.)

Research in Labor Economics : An Annual Compilation of Research, Vol. 2, Greenwich, Connecticut, JAl Press Inc., 1978, recensé par Dennis $R$. Maki.

v. 36 , no 1,1981 , p. 289-291

\section{ENGLAND, Geoffrey}

ENGLAND, Geoffrey

Part-time, Casual and Other Atypical Workers : A Legal View. Research and Current Issues Series No 48, Kingston, Industrial Relations Centre, Queen's University, 1987, 81 pp., ISBN 0-88886-154-0, recensé par Udo Mayer.

v. 42 , no 4,1987 , p. $880-881$

\section{EQUILBEY, NoèI}

BOYER, LUC EQUILBEY, Noël

Le projet d'entreprise. Paris, Les Éditions d'Organisation, 1986, 135 pp., ISBN 27081-0749-6, recensé par Paul Beaulieu. v. 42 , no 4,1987 , p. $881-883$

\section{EUDGE, $F$.}

ARTHURS, H.W. CARTER, D.D.

EUDGE, F. GLASBEEK, H.J.

Labour Law and Industrial Relations in Canada. 3rd ed., Toronto, Butterworths, 1988, 341 pp., ISBN 0-409-88891-5, ISBN 90-6544-389-4 (Kluwer), recensé par Pierre Verge.

v. 44 , no 4,1989, p. 959 


\section{$\mathbf{F}$}

FABI, Bruno

LAROCQUE, Alain BORDELEAU, Yvan

BOULARD, René FABI, Bruno

LAROUCHE, Viateur RONDEAU, Alain

Technologies nouvelles et aspects psychologiques. Sillery, Presses de l'Université du Québec, 1987, 171 pp., ISBN 27605-0450-6, recensé par Jean Mercier. v. 44, no 2,1989 , p. $469-470$

FELDMAN, Daniel C.

FELDMAN, Daniel C. ARNOLD, Hugh J. Managing Individual and Group Behavior in Organizations. Toronto, McCrowill-Hill Series in Management, 1983, 613 pp., ISBN 0-8359-3006-4, recensé par Laurent Bélanger.

v. 38 , no 3,1983 , p. $682-683$

\section{FERLAND, Gilles}

LAFLAMME, Gilles MURRAY, Gregor BÉLANGER, Jacques FERLAND, Gilles Flexibilité and Labour Markets in Canada and United States. (Research Series 94), Geneva, International Institute for Labour Studies, 1989, 317 p., ISBN 92-9014-4629 , recensé par Brad Pragnell. v. 45 , no 3,1990 , p. $643-647$

\section{FINCHAM, Robin}

FINCHAM, Robin RHODES, Peter S.

The Individual, Work and Organization. Behavioural Studies in Business and Management Students. London, Weidenfeld and Nicolson, 1988, 336 pp., ISBN 0-29779139-7, recensé par Alexander J. Matejko.

v. 44 , no 4,1989 , p. $961-962$

FINKELMAN, Jacob

FINKELMAN, Jacob

GOLDENBERG, Shirley B.

Collection Bargaining in the Public Service : the Federal Experience in Canada. Montréal, Institute for Research on Public Policy/L'Institut de recherches politiques, 1983, 825 pp., ISBN 0-920380-95-6 (vol. 1), ISBN 0-920380-97-2 (vol. 2), recensé par Maurice Lemelin.

v. 39 , no 3,1984, p. $633-634$
FOISY, C.H.

FOISY, C.H. LAVERY, D.E.

MARTINEAU, L.

Canada Labour Relations Board - Policies and Procedure. Toronto, Butterworths, 1986, 553 pp., ISBN 0-409-81975-1, recense par Pierre Verge.

v. 41 , no 4,1986 , p. $875-877$

\section{FOOTE WHITE, William}

FOOTE WHITE, William

KING WHITE, Kathleen

Making Mondragon : The Growth and Dynamics of the Worker Cooperative Complex. Ithaca, ILR Press, 1988, 317 pp., ISBN 0-87546-137-9, recensé par Hem C. Jain.

v. 44 , no 1,1989, p. $289-291$

FORD, B.

FORD, B. PLOWMAN, D.

Australian Unions : An Industrial Relations Perspectives. Melbourne, MacMillan Company of Australia, 1983, 576 pp., ISBN O333-33913-4, recensé par Gilles Dussault. v. 40 , no 1,1985 , p. $186-188$

FORSEY, Eugène FORSEY, Eugène

Trade Unions in Canada : 1812-1902. Toronto, University of Toronto Press, 1982, 600 pp., ISBN 0-8020-6388-8, recensé par James Thwaites.

v. 38, no 1,1983, p. $179-180$

FOSSUM, John A.

FOSSUM, John A.

Labor Relations : Development, Structure, Process (Revised Edition). Dallas, Business Publications, 1982, 521 pp., ISBN 0-256-02688-2, recensé par Kurt Wetzel. v. 37, no 4,1982 , p. $968-969$

FREEMAN, Richard $B$. FREEMAN, Richard B. MEDOFF, James $L$. What do Unions do? New York, Basic Books Inc., 1984, 293 pp., ISBN 0-46509133-4, recensé par Alexander J. Matejko. v. 40, no 1,1985 , p. $185-186$ 


\section{FRIEDBERG, Erhard} CROZIER, Michel FRIEDBERG, Erhard Actors and Systems. The Politics of Collective Action. Chicago, The University of Chicago Press, 1980, $333 \mathrm{pp}$., recense par Alexander J. Matejko.

v. 38, no 2,1983, p. $448-452$

\section{FRIEDMAN, Henry}

FRIEDMAN, Henry MEREDEEN, Sander The Dynamics of Industrial Conflict, Lessons from Ford. London, Croom Helm, 1980, 386 pp., recensé par Hervé Gauthier.

v. 37 , no 1,1982 , p. $246-248$

\section{FRIESEN, Peter H.,}

MILLER, Danny FRIESEN, Peter $\mathrm{H}_{\text {., }}$ Organizations : A Quantum View. Englewood Cliffs, Prentice-Hall, 1984, 320 pp., ISBN 0-13-641985-2, recensé par Michel Paquin.

v. 39 , no 3,1984 , p. $636-637$

\section{FUERBOECK, $\mathrm{K}$.}

TOMANDL, T. FUERBOECK, $K$.

Social Partnership - The Austrian System of Industrial Relations and Social Insurance. Ithaca, ILR Press, 1986, $165 \mathrm{pp}$., ISBN 0-87546-116-6, recensé par Pierre Verge.

v. 42 , no 3,1987 , p. $662-663$

\section{FULLERTON, David F.}

PORTIS, Bernard FULLERTON, David F. INGRAM, Paul $R$.

Quality Circles in Canada - A Review of Case Histories on Employee Involvement Programs. London, School of Business Administration, University of Western Ontario, 1986, 77 pp., recensé par Jean-Louis Bergeron.

v. 41 , no 4,1986 , p. $885-886$

\section{G}

\section{GAGNON, Chantal}

GAGNON, Chantal HÉBERT, Gérard Les grèves illégales dans les hôpitaux du Québec, 1977-1978. Montréal, École de relations industrielles, Université de Montreal, monographie no 14, 1982, 76 pp.,
ISBN 0708-9945, recensé par Gilles Dussault.

v. 39 , no 1,1984 , p. $209-210$

\section{GAGNON, Robert P.}

GAGNON, Robert P. LEBEL, Louis

VERGE, Pierre

Droit du travail. Québec, Presses de l'Université Laval, 1987, 933 pp., ISBN 2 7637-7123-8, recensé par Alain Barré.

v. 43, no 4,1988 , p. $974-980$

\section{GALLICK, Rosemary}

O'SULLIVAN, Judith

GALLICK, Rosemary

Workers and Allies : Female Participation in the American Trade Union Movement, 1824-1976. Washington, Smithsonian Institution Press, 1975, 96 pp., ISBN 0-87546138-1, recense par Micheline Dumont.

v. 43, no 1,1988, p. 205

\section{GATTIKER, Urs E.}

GATTIKER, Urs E. LARWOOD, Laurie

Managing Technological Development. Strategic and Human Resources Issues. Berlin, Walter de Gruyter, 1988, 232 pp., ISBN 3110110849, recensé par Alexander J. Matejko.

v. 44 , no 2,1989 , p. $470-472$

\section{GAUTHIER-MONTPLAISIR, Francine} GAUTHIER-MONTPLAISIR, Francine L'arbitrage des griefs et les infractions disciplinaires à caractère criminel. Cowansville, Les Éditions Yvon Blais Inc., 1983, 171 pp., ISBN 2-89073-170-7, recensé par André C. Cóté.

v. 39 , no 4,1984 , p. $795-797$

\section{GAZAILLE, Alain}

SIRARD, Ronald GAZAILLE, Alain

Comprendre et appliquer une convention collective. Montréal, Wilson et Lafleur, 1989, 165 p., ISBN 2-89127-151-3, recensé par Hélene Gascon.

v. 45, no 3,1990, p. $635-637$

\section{GELB, Alan}

BRADLEY, Keith GELB, Alan

Worker Capitalism. The New Industrial Relations. London, Heinemann, 1983, 186 pp., ISBN 0-435-82084-2, recensé par Alexander J. Matejko.

v. 39 , no 1,1984, p. 208-209 
GELB, Alan (suite)

BRADLEY, Keith GELB, Alan

Worker Capitalism. The New Industrial Relations. Cambridge, The MIT Press, 1983, 186 pp., ISBN 0-262-02191-0, recensé par Clive H.J. Gilson.

v. 40 , no 3,1985, p. $678-680$

\section{GEORGE, Kenneth}

GEORGE, Kenneth

Entre syndicats et patrons, fragile alliance. Montreal, Les Éditions Agence d'Arc, 1987, 137 pp., ISBN 2-89022-104-0, recensé par André Roy.

v. 43, no 1,1988 , p. 210

GERHART, Paul $\mathbf{F}$.

GERHART, Paul $F$.

Saving Plants and Jobs : Union Management Negotiations in the Context of Threatened Plant Closing. Kalamazoo, W.E. Upjohn Institute for Employment Research, 1987, 109 pp., ISBN 0-88099-047-3, recensé par Norman A. Solomon.

v. 44 , no 1,1989, p. $293-294$

\section{GERSUNY, Carl}

GERSUNY, Carl

Work Hazards and Industrial Conflict Hanover, University Press of New England, 1981, 162 pp., Lib of Congress Catalog Card no 80-51506, recensé par René Boulard.

v. 38 , no 3,1983 , p. 686

\section{GIANNINI, Mirella}

GIANNINI, Mirella

Giustizia e Mezzogiorno. Il caso dello statuto dei lavoratori. Venezia, Marsilio Editori, 1979, 162 pp., recensé par Danielle Picard.

v. 36, no 3,1981, p. $699-701$

\section{GILSON, C.H.J.}

GILSON, C.H.J.

Strikes in Nova Scotia, 1970-1985. Hantsport, Lancelot Press, 1986, 191 pp., ISBN 0-88999-314-9, recensé par David Frank.

v. 43 , no 1,1988, p. $200-203$

\section{GIUGNI, Gino}

GIUGNI, Gino MENGONI, Luigi

MORTILLARO, Felice WEISS, Dimitri

Possono migliorare te relazioni industriali in Italia? Torino, Gruppo Dirigenti Fiat,
1981, 140 pp., recensé par $P$. Joubert.

v. 38 , no 1,1983 , p. $190-192$

\section{GLADSTONE, Alan}

GLADSTONE, Alan

LANSBURY, Russel D.

STIEBER, Jack TREU, T. WEISS, M.

Current Issues in Labour Relations : An International Perspective. Berlin, Walter de Gruyter, 1989, 380 pp., ISBN 0-89925471-3, recensé par Jack Barbash.

v. 45 , no 1,1990 , p. $204-205$

WINDMULLER, John P. GLADSTONE, Alan

Employers' Associations and Industrial Relations : A Comparative Study. Oxford, Clarendon Press, 1986, 370 pp., ISBN 019-827260-X, recensé par James Thwaites. v. 44, no 1,1989, p. $295-297$

GLASBEEK, H.J. ARTHURS, H.W. CARTER, D.D.

EUDGE, F. GLASBEEK, H.J.

Labour Law and Industrial Relations in Canada. 3rd ed., Toronto, Butterworths, 1988, 341 pp., ISBN 0-409-88891-5, ISBN 90-6544-389-4 (Kluwer), recensé par Pierre Verge.

v. 44, no 4,1989 , p. 959

ARTHURS, H.W. CARTER, D.D.

GLASBEEK, H.J.

Labour Law and Industrial Relations in Canada. Deventer, Kluwer; Toronto; Butterworths, 1981, 291 pp., ISBN 90-3120139 (Kluwer), 0-409-81184-X (Butterworths), recense par Pierre Verge.

v. 37 , no 1,1982 , p. $241-243$

ARTHURS, H.W. CARTER, D.D.

GLASBEEK, H.J.

Labour Law and Industrial Relations in Canada. 2nd ed, Toronto, Butterworths, 1984, 316 pp., ISBN 0-409-81185-8, recensé par Pierre Verge.

v. 41 , no 1,1986 , p. 197-198

GLUECK, William F.

GLUECK, William $\mathrm{F}$.

Personnel : A Diganostic Approach, (3rd ed.). Business Publications Inc., 1982, 720 pp., ISBN 0-256-02429-4, recensé par Laurent Bélanger.

v. 37, no 2,1982 , p. $459-460$ 
GOLDENBERG, Shirley B.

FINKELMAN, Jacob GOLDENBERG, Shirley B.

Collection Bargaining in the Public Service : the Federal Experience in Canada. Montréal, Institute for Research on Public Policy/L'Institut de recherches politiques, 1983, 825 pp., ISBN 0-920380-95-6 (vol. 1), ISBN 0-920380-97-2 (vol. 2), recensé par Maurice Lemelin.

v. 39, no 3,1984, p. $633-634$

\section{GOODSELL, Charles T.}

GOODSELL, Charles T.

The Case for Bureaucracy : A Public Administration Polemic. Chatham, Chatham House Publishers, 1983, 179 pp., ISBN O934540-17-9, recensé par Alexander J. Matejko.

v. 40 , no 1,1985, p. $188-190$

\section{GORDON, David}

GORDON, David EDWARDS, Richard REICH, Michael

Segmented Work Divided Workers : The Historical Transformation of Labour in the United States. Cambridge, Cambridge University Press, 1982, 288 pp., recensé par Jerry $P$. White.

v. 40, no 2,1985, p. $410-411$

\section{GOULD, W.B.}

GOULD, W.B.

A Primer on American Labor Law. Cambridge, Mass., The MIT Press, 1982, 242 pp., ISBN 0-262-070-87-1, recensé par Pierre Verge.

v. 38 , no 1,1983, p. $180-181$

GOULD, W.B.

Japan's Reshaping of American Labor Law. Cambridge, Mass., The MIT Press, 1984, 193 pp., ISBN 0-262-07091-X, recensé par Pierre Verge.

v. 39, no 3,1984, p. $642-643$

GOULD, W.B.

Strikes, Disputes Procedures and Arbitration - Essays on Labor Law. Westport, Greenwood Press, 1985, 313 pp., ISBN 0313-24468-5, recensé par Pierre Verge. v. 41 , no 3,1986 , p. $650-651$

\section{GRAIS, Bernard}

GRAIS, Bernard

Les mises à pied et le travail à temps réduit dans quelques pays de l'OCDE. Paris, Institut national de la statistique et des études économiques et Conclusions du groupe de travail de l'OCDE sur les statistiques de l'emploi et du chómage, 1983, 154 pp., ISBN 92-64-22391-6, recensé par Lise Chrétien.

v. 40, no 4,1985, p. $904-905$

\section{GRAWITZ, Madeleine}

\section{GRAWITZ, Madeleine}

Lexique des sciences sociales. Paris, Dalloze, 1981, 376 pp., recensé par Dimitri Weiss.

v. 36 , no 4,1981 , p. $945-946$

\section{GROOTINGS, Peter}

GROOTINGS, Peter GUSTAVSEN, Bjorn HÉTHY, Lajos

New Forms of Work Organization and their Social and Economic Development. Budapest, Statistical Publishing House, 1986, 297 pp., ISBN 963-01-6862-6, recensé par Alexander J. Matejko.

v. 42 , no 3,1987 , p. $665-667$

GROSS, James A.

GROSS, James A.

The Reshaping of the National Labor Relations 3oard: National Labor Policy in Transition 1937-1947. Albany, State University of New York Press, 1981, 381 pp., ISBN 0-87395-270-7, recensé par Robert H. Babcock.

v. 38 , no 1,1983 , p. $188-189$

\section{GUILBAULT, Jacques}

BOIVIN, Jean GUILBAULT, Jacques

Les relations patronales-syndicales au Québec. Chicoutimi, Gaëtan Morin Editeur, 1982, 309 pp., ISBN 2-89105-087-8, recensé par Dimitri Weiss.

v. 37 , no 4,1982 , p. $969-970$

BOIVIN, Jean GUILBAULT, Jacques Les relations patronales-syndicales. Boucherville (Québec), 2ième éd., Gaétan Morin éditeur, 1989, 301 p. ISBN 2-89105322-2, recensé par Jacques-André Lequin. v. 45, no 3,1990, p. $627-629$ 
Relations Inoustrielles, vol. 45, no 4 (1990)

\section{GUILLEMARD, Anne-Marie}

GUILLEMARD, Anne-Marie

Le déclin du social, formation et crise des politiques de la vieillesse. Paris, Presses universitaires de France, 1986, 396 pp., ISBN 2-130-39565-1, recensé par Hélene David.

v. 42 , no 3,1987, p. $642-646$

GUIOT, Jean $M$.

GUIOT, Jean $M$.

Organisations sociales et comportements, Montréal, Les éditions Agence d'Arc Inc., Paris, Les editions Hommes et Techniques, 1980, 213 pp., recensé par Laurent Belanger.

v. 36 , no 4 , 1981 , p. $946-948$

\section{GUITTON, Jean}

GUITTON, Jean

Le travail intellectuel. Paris, Aubier-Montaigne, 1951 et 1986, 189 pp., ISBN 2707. 3409-2, recensé par Maurice Lebel.

v. 42 , no 1,1987, p. $225-227$

\section{GUNDERSON, Morley}

ANDERSON, John $\mathrm{C}$.

GUNDERSON, Morley PONAK, Allen

Union-Management Relations in Canada.

(2nd ed.), Don Mills, Ont., Addison-Wesley Publishers, 1989,498 pp., ISBN 0-201. 18621-7, recensé par Gene Swimmer.

v. 45 , no 2,1990 , p. $428-429$

\section{GUSTAVSEN, Bjorn}

GROOTINGS, Peter GUSTAVSEN, Bjorn HÉTHY, Lajos

New Forms of Work Organization and their Social and Economic Development. Budapest, Statistical Publishing House, 1986, 297 pp., ISBN 963-01-6862-6, recensé par Alexander J. Matejko.

v. 42 , no 3, 1987 , p. $665-667$

H

\section{HAGE, Jerald}

HAGE, Jerald

Theories of Organizations : Form, Process and Transformation. Newport, John Wiley \& Sons, 1980, 558 pp., ISBN 0471-338591, recensé par D. Briolat, $P$. Gagnon, $H$. Sidibe et Y. Gasse.

v. 39 , no 1,1984, p. $197-201$
HALLORAN, Jack

HALLORAN, Jack

Applied Human Relations, An Organizational Approach. (2nd ed), Englewood Cliffs, N.J., Prentice-Hall, 1983, 531 pp., ISBN O. 13-040808-5, recensé par Alain Rondeau. v. 38 , no 4,1983 , p. $920-922$

\section{HAMOWY, Ronald}

HAMOWY, Ronald

Canadian Medicine : A Study in Restricted Entry. Vancouver, The Fraser Institute, 1984, 394 pp., ISBN 0-88975-062-9, recensé par Gilles Dusseault.

v. 40, no 2,1985 , p. $412-414$

HANAMI, T.

HANAMI, T. BLANPAIN, R.

Industrial Conflict Resolution in Market Economies. Deventer, Kluwer, 1984 , 321 pp., ISBN 90-6544-1808, recensé par Pierre Verge.

v. 40, no 3,1985, p. 681.682

HARRIS, Rosemary

HARRIS, Rosemary

Power and Powerlessness in Industry: An Analysis of the Industrial Relations of Production. London, U.K., Tavistock Publ., 1987, pp. VIII + 245, ISBN 0-422-609-X, recensé par Alexander J. Matejko.

v. 45, no 3,1990, p. 634

\section{HÉBERT, Gérard}

GAGNON, Chantal HÉBERT, Gérard

Les grèves illégales dans les hópitaux du Québec, 1977-1978. Montréal, École de relations industrielles, Université de Montréal, monographie no 14, 1982, $76 \mathrm{pp}$., ISBN 0708-9945, recensé par Gilles DuSsault.

v. 39 , no 1,1984 , p. $209-210$

HÉBERT, Gérard TRUDEAU, Gilles

Les normes minimales du travail au Canada et au Québec : Étude juridique et institutionnelle. Montréal, Éditions Yvon Blais, 1987, 192 pp., ISBN 2-89073-611-3, recensé par Pierre Laporte.

v. 42 , no 4,1987 , p. $876-877$

HECKSCHER, Charles C.

HECKSCHER, Charles C.

The New Unionism : Employee Involvement in the Changing Corporation. New York, Basic Books, 1988, 320 pp., ISBN O465-05098-0, recensé par Michel Grant. v. 45 , no 1,1990 , p. $205-208$ 


\section{HEPPEL, Bob (Ed.)}

HEPPEL, Bob (Ed.)

The Making of Labour Law in Europe - A Comparative Study of Nine Countries up to 1945. London, Mansell Publishing Ltd, 1986, 401 pp., ISBN 0-7202-1697-X, recensé par Pierre Verge.

v. 42 , no 4,1987 , p. $884-886$

\section{HERMAN, E. Edward}

HERMAN, E. Edward KUHN, Alfred

SEEBER, Ronald

Collective Bargaining and Labor Relations. (2nd ed.), Englewood Cliffs, Prentice Hall, 1987, 621 pp., ISBN 0-13-140576-6, recensé par Norman A. Solomon.

v. 43 , no 1,1988 , p. $207-208$

\section{HERON, Craig}

HERON, Craig STOREY, Robert

On the Job: Confronting the Labour Process in Canada. Kingston and Montréal, McGill-Queen's University Press, 1986, 360 pp., ISBN 0.7735-0598-9, recensé par Robert $H$. Zieger.

v. 42 , no 4,1987 , p. $216-218$

\section{HÉTHY, Lajos}

GROOTINGS, Peter GUSTAVSEN, Bjorn HÉTHY, Lajos

New Forms of Work Organization and their Social and Economic Development. Budapest, Statistical Publishing House, 1986, 297 pp., ISBN 963-01-6862-6, recensé par Alexander J. Matejko.

v. 42 , no 3,1987 , p. $665-667$

\section{HIRSCHHORN, Larry}

HIRSCHHORN, Lary

Beyond Mechanization: Work and Technology in a Postindustrial Age. Cambridge, MIT Press, 1984, 187 pp., ISBN 0-26208132-3, recensé par Daniel Benedict. v. 40 , no 1,1985 , p. $192-194$

\section{HOLLEY, William $\mathrm{H}$.}

WOLTERS, Roger HOLLEY, William $\mathrm{H}$. Labour Relations : An Experiential and Case Approach. New York, the Dryden Press, 1988, 321 pp., ISBN 0-03-0693047, recensé par Mark Thompson. v. 44 , no 4,1989 , p. $957-958$
HOLT, James

\section{HOLT, James}

Compulsory Arbitration in New Zealand The First Forty Years. Auckland, Auckland University Press, 1986, 247 pp., ISBN 186940-0062, recensé par Donald D. Carter.

v. 44, no 2,1989, p. $468-469$

\section{HOUDE, Eugène}

HOUDE, Eugène

Émotivité et efficacité au travail. Montréal, Éditions de l'Homme, 1982, 112 pp., ISBN 2-7619-0213-0, recensé par Bernard Angers.

v. 37 , no 2,1982 , p. $458-459$

\section{HYMAN, Richard}

HYMAN, Richard STREECK, Wolfgang New Technology and Industrial Relations. Oxford, Basil Blackwell, 1988, 309 pp., ISBN 0-631-15982-7, recensé par Jacques Bélanger.

v. 45 , no 1,1990 , p. $208-209$

\section{I}

ILGEN, D.R.

NAYLOR, James C. PRITCHARD, R.D.

ILGEN, D.R.

A Theory of Behavior in Organizations. New York, Academic Press Inc., 1980, 299 pp., recensé par André Petit.

v. 36, no 3,1981, p. 703-704

INFANTE, Jaime T.

INFANTE, Jaime $T$.

The Political, Economic and Labor Climate in the Philippines. Industrial Research Unit, The Wharton School, University of Pennsylvania, 1980, 147 pp., recensé par Elias T. Ramos.

v. 37 , no 3,1982 , p. $720-722$

INGERMAN, Sidney $H$. INGERMAN, Sidney $\mathrm{H}$.

Economics for Canadian Trade Unionists. 2nd Ed., Ottawa, Labour College of Canada, 1985, 208 pp., ISBN 0-969-0345-1-1, recensé par Peter Larson.

v. 40 , no 4,1985 , p. 906 
INGRAM, Paul R.

PORTIS, Bernard FULLERTON, David F. INGRAM, Paul R.

Quality Circles in Canada - A Review of Case Histories on Employee Involvement Programs. London, School of Business Administration, University of Western Ontario, 1986, 77 pp., recensé par Jean-Louis Bergeron.

v. 41 , no 4,1986 , p. $885-886$

\section{J}

JACKSON, John $H$.

JACKSON, John H. MORGAN, Cyrill P. Organization Theory : A Macro Perspective for Management (2nd ed.). Englewood Cliffs, Prentice-Hall. Inc., 1982, 413 pp., ISBN 013-641415-X, recensé par Laurent Bélanger.

v. 37, no 2,1982 , p. $457-458$

JACKSON, John H. MORGAN, Cyrill P. PROLILLO, J.G.P.

Organization Theory: A Macro Perspective for Managing. Englewood Cliffs, PrenticeHall, 1986, 387 pp., ISBN 0-13-641572-501, recensé par James Thwaites.

v. 41 , no 4,1986 , p. $884-885$

JACKSON, Michael P.

JACKSON, Michael $P$.

Industrial Relations - A textbook. 3rd ed., London, Croom Helm, 1985, 294 pp., ISBN 0-7099-1474-1, recensé par Anthony Giles. v. 42 , no 3,1987 , p. $667-669$

\section{JACOBI, Otto}

ROBERTS, B.C. JACOBI, Otto

JESSOP, Bob KASTENDIEK, Hans

REGINI, Marino

Industrial Relations in Europe. The Imperatives of Change. Beckenham, England, 1986, 279 pp., ISBN 0-7099-4212-5, and Economic Crisis. Trade Unions and the State. Beckenham, England, 1986, 295 pp., ISBN 0-7099-1447-4, recensés par Roy J. Adams.

v. 42, no 2,1987 , p. $448-450$
JAIN, Hem C.

JAIN, Hem C.

Worker Participation : Success and Problems. New York, Praeger Publisher, 1980, 358 pp., recensé par Jacques Bélanger.

v. 36 , no 3,1981 , p. $690-692$

\section{JAVILLIER, Jean-Claude}

JAVILLIER, Jean-Claude

Le Droit du travail, 2ième éd., Paris, L.G.D.J., 1981, 700 pp., recensé par Dimitri Weiss.

v. 36 , no 2,1981 , p. $452-453$

\section{JESSOP, Bob}

ROBERTS, B.C. JACOBI, Otto

JESSOP, Bob KASTENDIEK, Hans

REGINI, Marino

Industrial Relations in Europe. The Imperatives of Change. Beckenham, England, 1986, 279 pp., ISBN 0-7099-4212-5, and Economic Crisis. Trade Unions and the State. Beckenham, England, 1986, 295 pp., ISBN 0-7099-1447-4, recensés par Roy J. Adams.

v. 42 , no 2,1987 , p. $448-450$

\section{JOBIN, Carol}

JOBIN, Carol

Les relations du travail dans l'industrie de la construction. Montréal, Wilson \& Lafleur, 1989, 568 pp., ISBN 2-89127-085-1, recensé par Jean Sexton.

v. 44 , no 2,1989 , p. 473

JURIS, $H$.

JURIS, H. THOMPSON, Mark

DANIELS, W.

Industrial Relations in a Decade of Economic Change. IRRA Series, Annual Research Volume, Madison, Wisconsin, IRRA, 1985, 407 pp., ISBN 0-913447-30-7, recense par Hem $C$. Jain.

v. 42 , no 2,1987 , p. $443-445$

\section{K}

KAHN, Joan

KAHN, Joan WESTLEY, William A.

The Working Conditions in Canadian Hospitals. Constraints and Opportunity. Ottawa, Minister of Supply and Services Canada, 1984, 112 pp., ISBN 0-662-52924-3, recensé par Alexander J. Matejko.

v. 39 , no 4,1984 , p. $815-816$ 
KAHN-FREUND, 0 .

KAHN-FREUND, $O$.

Labour Law and Politics in the Weimar Republic. Oxford, Basil Blackwell, 1981, ISBN 0-631-12825-5, recensé par Pierre Verge.

v. 37 , no 2 , 1982 , p. $454-455$

\section{KANTER, Rosabeth Moss}

KANTER, Rosabeth Moss

The Change Masters. Innovation and Entrepreneurship in the American Corporation. New York, Simon and Schuster, 1984, 432 pp., ISBN 0-671-42802-0, recensé par Alexander J. Matejko.

v. 40, no 2,1985 , p. $411-412$

\section{KASTENDIEK, Hans}

ROBERTS, B.C. JACOBI, Otto JESSOP, Bob KASTENDIEK, Hans REGINI, Marino

Industrial Relations in Europe. The Imperatives of Change. Beckenham, England, 1986, 279 pp., ISBN 0-7099-4212-5, and Economic Crisis. Trade Unions and the State. Beckenham, England, 1986, 295 pp., ISBN 0-7099-1447-4, recensés par Roy J. Adams.

v. 42, no 2,1987 , p. $448-450$

\section{KATZ, Harry C.}

KATZ, Harry C.

Shifting Gears : Changing Labor Relations in the U.S. Automobile Industry. London, MIT Press, 1985, 230 pp., ISBN 0-26211098-9, recensé par Michel Turner.

v. 40 , no 4,1985 , p. $900-901$

KATZ, Harry C.

Shifting Gears : Changing Labor Relations in the U.S. Automobile Industry. Cambridge, MIT Press, 1985, 230 pp., ISBN O262-11098-9, recensé par Solomon Barkin. v. 41, no 3,1986 , p. $654-658$

KOCHAN, Thomas C. KATZ, Harry C. Collective Bargaining and Industival Relations. Irwin Series in Management and the Behavioural Sciences, Homewood, 1988, 496 pp., ISBN 0-256-03025-1, recensé par Jean Boivin.

v. 45, no 1,1990 , p. 210
KAZANAS, H.C.

ROTHWELL, W.J. KAZANAS, H.C.

Strategic Human Resources Planning and Management. Englewood Cliffs, PrenticeHall, 1988, 512 pp., ISBN 0-13-851643-X, recensé par Michel Audet.

v. 45 , no 1,1990 , p. $211-212$

\section{KELLEY, Robert E.}

KELLEY, Robert E.

The Gold Collar Worker. Reading Massachusetts, Addison-Wesley Publishing Co., 1985, 196 pp., ISBN 0-201-11739-8, recensé par Paul Beaulieu.

v. 42, no 3,1987 , p. $664-665$

\section{KELLY, John E.}

KELLY, John E.

Scientific Management, Job Redesign and Work Performance. London, Academic Press, 1982, 257 pp., ISBN 012404020-9, recensé par Alain Vinet.

v. 38 , no 2,1983 , p. $453-455$

\section{KERR, Clark}

KERR, Clark STAUDOHAR, Paul D.

Industrial Relations in a New Age. San Francisco, Jossey-Bass Inc., 1986, 419 pp., ISBN 0-55542-013-3, recensé par Norman A. Solomon.

v. 42 , no 4,1987 , p. $883-884$

KESSLER, Francis

KESSLER, Francis

Le droit des conventions collectives de travail en République fédérale d'Allemagne. Francfort sur Mayenne, Peter Lang, 1988, 666 pp., ISBN 3-8204-1138-0, recensé par Pierre Verge.

v. 44 , no 3,1989 , p. $728-729$

KING WHITE, Kathleen

FOOTE WHITE, William

KING WHITE, Kathleen

Making Mondragon : The Growth and Dynamics of the Worker Cooperative Complex. Ithaca, LR Press, 1988, 317 pp., ISBN 0-87546-137-9, recensé par Hem C. Jain.

v. 44 , no 1,1989, p. $289-291$ 
Relations lndustrielles, vol. 45 , no 4 (1990)

\section{KLEINER, Morris $M$.}

KLEINER, Morris M. BLOCK, Richard N. ROOMKIN, Myron

SALSBURG, Sydney W.

Human Resources and the Performance of the Firm. Madison, Industrial Relations Research Association Series, 1987, 343 pp., ISBN 0-913447-382, recensé par Bernard Portis.

v. 44 , no 1,1989 , p. 297

\section{KOBRIN, Catherine}

SARFATI, Hedva KOBRIN, Catherine

La flexibilité du marché de l'empioi : un enjeu économique et social. Genève, Bureau international du travail, 1987, 384 pp., ISBN 92-2-205675-2, recensé par Lise Poulin-Simon.

v. 43, no 3,1988, p. $707-710$

KOCHAN, Thomas C.

KOCHAN, Thomas C. KATZ, Harry C. Collective Bargaining and Industrial Relations. Irwin Series in Management and the Behavioural Sciences, Homewood, 1988, 496 pp., ISBN 0-256-03025-1, recensé par Jean Boivin.

v. 45 , no 1,1990 , p. 210

\section{KOLB, Deborah M.}

KOLB, Deborah $M$.

The Mediators. Cambridge, Mass., The MIT Press, 1983, 230 pp., ISBN 0-26211088-1, recensé par Jean Sexton.

v. 39 , no 2,1984 , p. $391-392$

\section{KOZDROJ, Alicja}

KOZDROJ, Alicja

Grupa pracownicza jako przedmiot i podmiot motywowania. The Polish Academy of Sciences, Institute of Management, Wroclaw Ossolineum, 1988, 307 pp., ISBN 8304-02806-9, recensé par Alexander J. Matejko.

v. 45 , no 1,1990 , p. $213-214$

\section{KOZIARA, S}

KOZIARA, S MOSKOW, M.H.

TANNER, L.D.

Working Women : Past, Present, Future. Industrial Relations Research Association Series, Washington, The Bureau of National Affairs, 1987, 419 pp., ISBN 0-91344734-X, recensé par $P$. Andiappan.

v. 45, no 2,1990, p. $430-431$
KRAHN, Harvey J.

KRAHN, Harvey J. LOWE, Graham S.

Work, Industry and Canadian Society.

Scarborough, Nelson Canada, 1988, 310 pp., ISBN 0-17-603414-5, recensé par Alexander J. Matejko.

v. 43, no 3,1988, p. $718-719$

\section{KUECHLE, David}

PEACH, David A. KUECHLE, David

The Practise of Industrial Relations. 2nd Ed., Scarborouogh, McGraw Ryerson, 1985, 407 pp., ISBN 0-07-548909-0, recensé par Norman A. Solomon.

v. 42, rio 1,1987, p. $222-223$

\section{KUHN, Alfred}

HERMAN, E. Edward KUHN, Alfred

SEEBER, Ronald

Collective Bargaining and Labor Relations. (2nd ed.), Englewood Cliffs, Prentice Hall, 1987, 621 pp., IDBN 0.13-140576-6, recensé par Norman A. Solomon.

v. 43, no 1,1988, p. $207-208$

\section{KURTZ, David L.}

BOONE, Louis E. KURTZ, David L. L'entreprise d'aujourd'hui. (2ième éd), Montréal, Éditions études vivantes, 1989. 737 pp., ISBN 2-7607-0446-7, recensé par Laurent Belanger.

v. 45, no 2,1990, p. 432

\section{L}

\section{LAFLAMME, Gilles}

LAFLAMME, Gilles MURRAY, Gregor BÉLANGER, Jacques FERLAND, Gilles Flexibilité and Labour Markets in Canada and United States. (Research Series 94). Geneva, International Institute for Labour Studies, 1989, 317 p., ISBN 92-9014-4629 , recensé par Brad Pragnell. v. 45, no 3,1990 , p. $643-647$

\section{LAFLAMME, Marcel} MEUNIER, Pierre-Marc LAFLAMME, Marcei Dynamisme humain dans l'excellence organisationnelle. Montréal, Agence d'Arc, 1987, 431 pp., ISBN 2-89022-120-2, recensé par Roger Z. Vanié. v. 43, no 4,1988 , p. $972-973$ 


\section{LANDY, Frank J.}

LANDY, Frank J.

Psychology of Work Behavior. Homewood, The Dorsey Press, 1985, XN-622-R41-

11 pp., ISBN 0-256-03046-4, recensé par Alexander J. Matejko.

v. 40, no 4,1985, p. $896-898$

\section{LANSBURY, Russell D.}

BAMBER, Greg J. LANSBURY, Russell D. International and Comparative Industrial Relations. London, Allen \& Unwin, 1987, 289 pp., ISBN 0-04-331117-4, recensé par Anthony Giles.

v. 43, no 3,1988 , p. $703-707$

BAMBER, Greg J. LANSBURY, Russell D. New Technology International Perspectives on Human Resources and Industrial Relations. Winchester, Unwin Hyman, 1989, 267 pp., ISBN 0-04-445123-7, recensé par Martin S. Serediak.

v. 45, no 2,1990 , p. $435-438$

\section{GLADSTONE, Alan}

LANSBURY, RUSSEI D.

STIEBER, Jack TREU, T. WEISS, M.

Current Issues in Labour Relations : An International Perspective, Berlin, Walter de Gruyter, 1989, 380 pp., ISBN 0-89925-471.

3, recensé par Jack Barbash.

v. 45 , no 1, 1990 , p. 204-205

\section{LAPERRIERE, René}

LAPERRIERE, René

Bibliographie du droit du travail canadien et québecois. Montréal, Les Éditions Yvon Blais Inc., 1984, 70 pp., ISBN 2-89073515- $X$, recensé par Rodrigue Blouin.

v. 39 , no 4,1984, p. $812-813$

\section{LAPORTE, PIerre}

\section{LAPORTE, Pierre}

Code du travail du Québec (Légis/ation, Jurisprudence et Doctrine). Avec la collaboration d'Hélène Ouimet, Montréal, Wilson \& Lafleur, 1987, 475 pp., ISBN 289127-058-4, recensé par Rodrigue Blouin. v. 42 , no 2,1987 , p. $436-437$

\section{LAROCQUE, Alain}

LAROCQUE, Alain BORDELEAU, Yvan BOULARD, René FABI, Bruno LAROUCHE, Viateur RONDEAU, Alain Technologies nouvelles et aspects psy- chologiques. Sillery, Presses de l'Université du Québec, 1987, 171 pp., ISBN 27605-0450-6, recensé par Jean Mercier. v. 44 , no 2,1989 , p. $469-470$

\section{LAROUCHE, Viateur}

LAROCQUE, Alain BORDELEAU, Yvan BOULARD, René FABI, Bruno LAROUCHE, Viateur RONDEAU, Alain Technologies nouvelles et aspects psychologiques. Sillery, Presses de I'Université du Québec, 1987, 171 pp., ISBN 27605-0450-6, recensé par Jean Mercier. v. 44, no 2,1989 , p. $469-470$

\section{LARSON, Simeon}

LARSON, Simeon NISSEN, Bruce

Theories of the Labor Movement. Detroit, Wayne State University Press, 1987, 395 pp., ISBN 0-8143-1815-0, recensé par Michel Audet.

v. 43 , no 1,1988 , p. $199-200$

\section{LARWOOD, Laurie}

GATTIKER, Urs E. LARWOOD, Laurie Managing Technological Development. Strategic and Human Resources Issues. Berlin, Walter de Gruyter, 1988, 232 pp., ISBN 3110110849, recensé par Alexander J. Matejko.

v. 44, no 2,1989 , p. $470-472$

\section{LAVERY, D.E.}

FOISY, C.H. LAVERY, D.E.

MARTINEAU, L.

Canada Labour Relations Board - Policies and Procedure. Toronto, Butterworths, 1986, 553 pp., ISBN 0-409-81975-1, recensé par Pierre Verge.

v. 41, no 4,1986, p. $875-877$

\section{LAWRENCE, Peter}

LAWRENCE, Peter

Managers and Management in West Germany. London, Croom Helm, 1980, 202 pp., ISBN 0-85664-912-0, recensé par Michel Paquin.

v. 38, no 2,1983 , p. $455-456$

LEAB, Daniel J.

LEAB, Daniel J.

The Labor History Reader. Champaign, University of Illinois Press, 1985, 470 pp., ISBN 0-252-01197-X, recensé par Foster J.K. Griezic.

v. 41 , no 1,1986 , p. $202-203$ 
LEAB, Daniel J. (suite)

NEUFELD, Maurice F, LEAB, Daniel J. SWANSON, Dorothy

American Working Class History : A Representative Biography. New York - London, Bowker, 1983, 356 pp., ISBN 0-8352-17523 , recensé par James Thwaites.

v. 39 , no 2,1984 , p. 406

\section{LEARY, Virginia}

LEARY, Virginia

International Labour Conventions and $\mathrm{Na}$ tional Law. The Haque, Martinus Nijhoff Publishers, 1982, 191 pp., ISBN 90-2472551-8, recensé par Alain Barré.

v. 39 , no 1,1984 , p. $203-205$

\section{LEBEL, Louis}

GAGNON, Robert P. LEBEL, Louis

VERGE, Pierre

Droit du travail. Québec, Presses de I'Université Laval, 1987, 933 pp., ISBN 27637-7123-8, recensé par Alain Barré.

v. 43, no 4,1988, p. $974-980$

\section{LECLERC, Claudine}

LECLERC, Claudine SEXTON, Jean

La sécurité d'emploi dans l'industrie de la construction au Québec, un rêve impossible? Québec, Presses de l'Université Laval, 1983, 275 pp., ISBN 2-7637-7028-

2, recensé par Noël Mallette.

v. 39 , no 3,1984, p. $643-645$

\section{LECLERC, L.}

AOUST, Claude d' LECLERC, L.

TRUDEAU, Gilles

Les mesures disciplinaires : etude jurisprudentielle et doctrinale. Montréal, École de relations industrielles, Monographie no 13, 1982, 484 pp., ISBN 2-89067-012-0, ISSN 0708-9945, recensé par R. Blouin. v. 38 , no 2,1983 , p. $444-445$

\section{LEFEBVRE, Gérard}

LEFEBVRE, Gérard

Le coeur à l'ouvrage. Montréal, Les Éditions de l'Homme, 1982, 120 pp., ISBN 27619-0188-6, recensé par Dimitri Weiss.

v. 38 , no 1,1983 , p. $187-188$

\section{LEMELIN, Maurice}

LEMELIN, Maurice

Les négociations collectives dans les secteurs public et parapublic. Expérience québécoise et regard sur l'extérieur. Montreal, Éditions Agence d'Arc, 1984, 381 pp., ISBN 2-89022-067-2, recensé par Jacques Bélanger.

v. 40, no 3,1985, p. $675-676$

LEMELIN, Maurice MCNEIL, Jeannine Productivité et qualité de vie au travail. Montreal, Les éditions Agence d'Arc Inc., 1982, ISBN 2-89022-044-3, recensé par Laurent Bélanger.

v. 38 , no 2,1983, p. $452-453$

\section{LESTER, Richard A.}

LESTER, Richard A.

Labor Arbitration in State and Local Go vernment. Princeton, Princeton University, 1984, 210 pp., ISSN 0079-5305, recensé par André Roy.

v. 40 , no 1,1985, p. $181-183$

\section{LEVY-LEBOYER, C.}

LEVY-LEBOYER, C.

Le psychologue et l'entreprise. Montréal, Masson et Presses de l'Université de Montreal, 1980, 169 pp., recensé par Laurent Bélanger.

v. 36 , no 4,1981 , p. 948

\section{LEWCHUK, Wayne}

LEWCHUK, Wayne

American Technology and the British Vehicle Industry. Cambridge, Cambridge University Press, 1987, 304 pp., ISBN 0521 30269-2, recensé par Jacques Bélanger. v. 43, no 3,1988, p. $716-717$

\section{LEWICKI, Roy J.}

LEWICKI, Roy J. LITTERER, Joseph A. Negotiation. Homewood, Richard D. Inwin, 1985, 368 pp., ISBN 0-256-02633-5, recensé par Jean Sexton.

v. 40, no 4,1985, p. $894-895$

LEWICKI, Roy J. LITTERER, Joseph A. Negotiation : Readings, Exercices and Cases. Homewood, Richard D. Inwin, 1985, 633 pp., ISBN 0-256-02634-3, recensé par Gilles Ferland.

v. 40, no 4,1985, p. 895 


\section{LEWIS, Gary A.}

LEWIS, Gary A.

News from Somewhere : Connecting Health and Freedom at the Workplace. New York, Greenwood Press, 1986, 213 pp., ISBN 0-313-24869-9, recensé par Robert Sass.

v. 42 , no 3,1987, p. $653-655$

\section{LEWIS, R.}

LEWIS, R. CLARK, J.

WEDDERBURN, Lord of Chartton

Labour Law and Industrial Relations: Building on Kahn-Freund. Oxford, Clarendon Press, 1983, 250 pp., ISBN 0-19-8253931, recensé par Pierre Verge.

v. 38 , no 3,1983, p. $677-678$

\section{LINDENFELD, Frank}

LINDENFELD, Frank

ROTHSCHILD-WHITT, Joice

Workplace Democracy and Social Change.

Boston, Porter Sargent Publication, 447 pp., ISBN 0-87558-102-1, recensé par Alexander J. Matejko.

v. 38, no 3,1983, p. $687-688$

\section{LIPSET, Seymour Martin}

LIPSET, Seymour Martin

Unions in Transition. Entering the Second Century. San Francisco, ICS Press, 1986, 506 pp., ISBN 0-917616-74-X, recensé par Carla Lipsig.

v. 42 , no 2,1987 , p. $452-454$

\section{LIPSKI, David B.}

LIPSKI, David B. DONN, Clifford B.

Collective Bargaining in American Industry. Lexington, Lexington Books, 1987, 351 pp., ISBN 0-669-12595, recensé par Joseph $B$. Rose.

v. 43 , no 4,1988 , p. $980-981$

\section{LITTERER, Joseph A.}

LEWICKI, Roy J. LITTERER, Joseph A Negotiation. Homewood, Richard D. Irwin, 1985, 368 pp., ISBN 0-256-02633-5, recensé par Jean Sexton.

v. 40, no 4,1985 , p. $894-895$

LEWICKI, Roy J. LITTERER, Joseph A. Negotiation : Readings, Exercices and Cases. Homewood, Richard D. Irwin, 1985, 633 pp., ISBN 0-256-02634-3, recensé par Gilles Ferland.

v. 40, no 4,1985 , p. 895
LOJKINE, Jean

DURAND, Jean-Pierre

DURAND-SEBAG, Joyce LOJKINE, Jean MAHIEU, Christian

L'enjeu informatique : former pour changer l'entreprise. Paris, Merridiens Klincksieck, 1986, 192 pp., ISBN 2-86563-148-6, recensé par Guylaine Vallée.

v. 42 , no 3,1987 , p. $660-661$

LONG, Richard J.

NIGHTINGALE, Donald V.

LONG, Richard J.

Gain and Equity Sharing. Quality of Working Life. Case Study Series, Ottawa, Minister of Supply and Services, Canada, 1984, 51 pp., ISBN 0-662-13138-X, recensé par Alexander J. Matejko.

v. 39 , no 4,1984 , p. $813-814$

LOWE, Graham S.

KRAHN, Harvey J. LOWE, Graham S.

Work, Industry and Canadian Society. Scarborough, Nelson Canada, 1988, 310 pp., ISBN 0-17-603414-5, recensé par Alexander J. Matejko.

v. 43, no 3,1988, p. $718-719$

L.R.S.C.

L.R.S.C.

Labour Studies Research Bulletin. Labour Studies Resource Centre, Department of Political Science, Carleton University, 1981, 32 pp., recensé par James Thwaites.

v. 36, no 4,1981 , p. 958

\section{LUFT, Joseph}

LUFT, Joseph

Group Process. An Introduction to Group Dynamics. 3rd ed., Alto, Cal., Mayfield Publishing Company, 1984, 237 pp., ISBN 0-87484-542-4, recensé par Alexander J. Matejko.

v. 41, no 3,1986 , p. 667

\section{LYON-CAEN, Gérard}

LYON-CAEN, Gérard PÉLISSIER, Jean Les grands arrets de Droit du travail, 2ième éd., Paris, Editions Sirey, 1980, 406 pp., recensé par Dimitri Weiss. v. 36 , no 2,1981 , p. $454-455$ 


\section{M}

\section{MACKAY, Plerre}

BUREAU, Robert D. MACKAY, Pierre

Le droit dans tous ses états. Montréal, Wilson \& Lafleur, 1987, 620 p., ISBN 289127-07602, recensé par Fernand Morin. v. 45, no 3,1990, p. $640-643$

MAGUIRE, J.J.

ASHTON, D.N. MAGUIRE, J.J.

Young Adult in the Labour Market. Research Paper no 55, Leicester, University of Leicester, 1986, 163 pp., recensé par Lise Poulin Simon.

v. 42, no 4,1987, p. $887-889$

\section{MAHIEU, Christian}

DURAND, Jean-Pierre

DURAND-SEBAG, Joyce LOJKINE, Jean MAHIEU, Christian

L'enjeu informatique : former pour changer l'entreprise. Paris, Méridiens Klincksieck, 1986, 192 pp., ISBN 2-86563-148-6, recensé par Guylaine Vallée.

v. 42 , no 3,1987, p. $660-661$

\section{MAINWARING, John}

MAINWARING, John

The International Labour Organisation : A Canadian View. Ottawa, Canadian Government Publishing Centre, 1986, 206 pp., recensé par Garfield Clack.

v. 42 , no 4,1987 , p. $889-890$

\section{MALLETTE, Noël}

MALLETTE, Noël

La gestion des relations du travail au Québec. Le cadre juridique et institutionnel. Scarborough, Collection administration, McGraw-Hill, 1980, 642 pp., recensé par Rodrigue Blouin.

v. 36 , no 1,1981 , p. 275

\section{MANSELL, Jacquie}

MANSELL, Jacquie

Workplace Innovation in Canada. Reflections on the Past Prospects for the Future. A Study prepared for the Economic Council of Canada, Ottawa, Minister of Supply and Services Canada, 1987, 40 pp., ISBN 0-660-12554-4, recense par Alexander $J$. Matejko.

v. 43 , no 1,1988 , p. 211
MARIC, D.

MARIC, D.

La durée du travail dans les pays en voie de développement. Genève, Bureau international du Travail, 1981, 138 pp., ISBN 92-2-202732-9, recensé par Alain Larocque.

v. 37 , no 3,1982 , p. $718-719$

\section{MARS, Gerald}

MARS, Gerald

Cheats at Work. An Anthropology of Workplace Crime. London, Unwin Paperbacks, 1983, 242 pp., ISBN 0-04-301166-7, recensé par Alexander J. Matejko.

v. 41 , no 2,1986 , p. $431-432$

\section{MARSH, Arthur}

MARSH, Arthur

Trade Union Handbook, 2nd ed. Farnborough, England, Gower, 1980, 396 pp., recensé par Gerard Dion.

v. 36 , no 4,1981 , p. $948-949$

MARSH, Arthur RYAN, Victoria

Historical Directory of Trade Unions, vol. 1 : Non-Manual Unions. Farnborough, England, Gower, 1980, 256 pp., ISBN 0566-02160-9, recensé par Gérard Dion.

v. 37 , no 1,1982 , p. $245-246$

\section{MARTIN, Roger G.}

MARTIN, Roger $\mathbf{G}$.

Annuaire du travail 1986-1987. Montréal, Productions INFORT, 1986, recensé par Gérard Dion.

v. 41 , no 4,1986 , p. $886-887$

\section{MARTIN, Ross $M$.}

MARTIN, Ross $M$.

TUC : The Growth of a Pressure Group 1868-1976. Oxford, Oxford University Press, 1980, 394 pp., recensé par Jacques Bélanger.

v. 36 , no 4,1981 , p. $956-958$

MARTINEAU, L.

FOISY, C.H. LAVERY, D.E.

MARTINEAU, L.

Canada Labour Relations Board - Policies and Procedure. Toronto, Butterworths, 1986, 553 pp., ISBN 0-409-81975-1, recensé par Pierre Verge.

v. 41 , no 4,1986 , p. $875-877$ 


\section{MATTIOLI, Francesco}

PIRZIO AMMASSARI, Gloria

MATTIOLI, Francesco

I sindicalisti. Bar, De Donato, 1982, 158 pp., CL 07-0501-2, recensé par Dimitri Weiss.

v. 38 , no 4,1983 , p. $922-924$

\section{MAURICE, Marc}

MAURICE, Marc SELLIER, François

SILVESTRE, Jean-Jacques

Politique d'éducation et organisation industrielle en France et en Allemagne : essai d'analyse sociétale. Paris, PUF, collection 'Sociologies" dirigée par Raymond Boudon et François Bourricaud, 1982, 382 pp., ISBN 2-13-037472-7, recensé par Dimitri Weiss.

v. 38 , no 4,1983, p. $929-934$

MAURICE, Marc SElLIER, François

SILVESTRE, Jean-Jacques

The Social Foundations of Industrial Power : A Comparison of France and Germany. Traduit par Arthur Goldhammer, Cambridge, MIT Press, 1986, 292 pp., ISBN O262-13213-3, recensé par Anthony Giles. v. 43, no 3,1988 , p. $703-707$

\section{MCCARTHY, Maureen}

MCCARTHY, Maureen

ROSENBERG, Gail S.

Work Sharing: Case Studies. Kalamazoo, Michigan, W.E. Upjohn Institute for Employment Research, 1981, 277 pp., ISBN O911558-87-X, recensé par Alain Vinet.

v. 37, no 4,1982, p. 971

MCGAUGHEY, William Jr.

MCGAUGHEY, William Jr.

A Shorter Workweek in the 1980's. White Bear Lake, Minnesota, Thestterose Publications, 1981, 308 pp., ISBN 0-9605630$0-8$, recensé par Alain Larocque.

v. 37 , no 3,1982, p. $718-719$

MCKELVEY, Jean T.

MCKELVEY, Jean T.

Cleared for Takeoff : Airline Labour Relations since Deregulation. Ithaca, ILR Press, Corne/l University, 1988, 298 pp., ISBN 0-87546-110-7, recensé par Jean Boivin.

v. 44, no 3,1989, p. $733-734$
MCKERSIE, Robert B.

STIEBER, Jack MCKERSIE, Robert B.

QUINN MILLS, D.

U.S. Industrial Relations 1950-1980: A Critical Assessment. Madison, Industrial Relations Research Association, 1981, 361 pp., recensé par Mahmood A. Zaidi. v. 38 , no 1,1983 , p. $184-187$

\section{MCMILLAN, Charles J.}

MCMILLAN, Charles J.

The Japanese Industrial System. BerlinNew York, Walter de Gruyter, 1984, 356 pp., ISBN 3-11-008894-0, recensé par Alexander J. Matejko.

v. 39, no 4,1984 , p. $799-801$

MCNEIL, Jeannine

LEMELIN, Maurice MCNEIL, Jeannine Productivité et qualité de vie au travail. Montréal, Les éditions Agence d'Arc Inc., 1982, ISBN 2-89022-044-3, recensé par Laurent Bélanger.

v. 38, no 2,1983, p. $452-453$

\section{MEDOFF, James $L$.}

FREEMAN, Richard B.

MEDOFF, James L.

What do Unions do? New York, Basic Books /ric., 1984, 293 pp., ISBN 0-46509133-4, recensé par Alexander J. Matejko.

v. 40 , no 1,1985 , p. $185-186$

\section{MEREDEEN, Sander}

FRIEDMAN, Henry MEREDEEN, Sander The Dynamics of Industrial Conflict, Lessons from Ford London, Croom Helm, 1980, 386 pp., recensé par Hervé Gauthier.

v. 37, no 1,1982 , p. $246-248$

MEHLING, Jean

MEHLING, Jean

Analyse socio-économoique d'une grève. Montréal, HEC et Beauchemin, 1963, 218 pp., recensé par Jacques Gagnon.

v. 41 , no 3,1986 , p. $670-671$

MELTZ, Noah $M$.

MELTZ, Noah M. REID, Frank

SWATZ, Gérald S.

Sharing the Work: An Analysis of the /ssues in Worksharing and Jobsharing. Toronto, University of Toronto Press, 1981, 90 pp., recensé par Paul Blyton.

v. 37 , no 3,1982 , p. $717-718$ 
MELTZ, Noah M. (suite)

STONE, Thomas H. MELTZ, Noah M. Personnel Management in Canada. Toronto Holt, Rinehart \& Winston, 1983, 411 pp., ISBN 0-03-921591-1, recensé par Alexander J. Matejko.

v. 39 , no 1,1984, p. $205-206$

MEMFORD, Enid

COOPER, Cary L. MEMFORD, Enid

The Quality of Working Life in Western and Eastern Europe. Westport, Greenwood Press, 1979, 348 pp., recensé par Alexander J. Matejko.

v. 36, no 1,1981 , p. $285-288$

MENGONI, Luigi

GIUGNI, Gino MENGONI, Luigi

MORTILLARO, Felice WEISS, Dimitri

Possono migliorare le relazioni industriali in Italia? Torino, Gruppo Dirigenti Fiat, 1981, 140 pp., recensé par P. Joubert.

v. 38, no 1,1983, p. $190-192$

MERCHANT, Kenneth A.

MERCHANT, Kenneth A.

Control in Business Organization. Boston, Pitman, 1985, 161 pp., ISBN 0-273-019147 , recensé par Alexander J. Matejko.

v. 40 , no 3,1985 , p. $685-686$

\section{MEUNIER, Pierre-Marc}

MEUNIER, Pierre-Marc

LAFLAMME, Marcel

Dynamisme humain dans l'excellence organisationnelle. Montreal, Agence d'Arc, 1987, 431 pp., ISBN 2-89022-120-2, recensé par Roger Z. Vanié.

v. 43 , no 4,1988, p. $972-973$

MILKOVICH, George T.

MILKOVICH, George T.

NEWMAN, Jerry $M$.

Compensation. Plano, Texas, Business Publications Inc., 1984, 549 pp., ISBN O256-02204-6, recensé par Gilles Ferland. v. 42 , no 1,1987, p. $218-219$

MILLARD, $F$.

BLANPAIN, R. MILLARD, F.

Comparative Labour Law and Industrial Relations. Deventer, Kluwer, Washington, the Bureau of National Affairs, 1982, 411 pp., ISBN 0-87179-392-2, recensé par Pierre Verge.

v. 39 , no 1,1984 , p. 201-203
MILLER, Danny

MILLER, Danny FRIESEN, Peter $H_{\text {., }}$

Organizations : A Quantum View. Englewood Cliffs, Prentice-Hall, 1984, 320 pp., ISBN 0-13-641985-2, recensé par Michel Paquin.

v. 39 , no 3,1984, p. $636-637$

MILLWARD, Neil

DANIEL, W.W. MILLWARD, Neil

Workplace Industrial Relations in Britain. The DEIPSI/SSRC Survey, London, Heinemann Educational Books, 1983, 338 pp. ISBN 0-435-83191-7, recensé par Alexander J. Matejko.

v. 39, no 1,1984, p. $217-218$

MILLWARD, Neil STEVENS, Mark

British Workplace Industrial Relations 1980-1984 : The DE/ESRC/PSI/ACAS Surveys. Aldershot, Gower, 1986, $341 \mathrm{pp}$., ISBN 0-566-05396-9, recensé par Jacques Bélanger.

v. 42 , no 4,1987 , p. $891-893$

MINTZBERG, Henry

MINTZBERG, Henry

Le manager au quotidien. Les dix róles du cadre. Montréal, Les Editions Agence d'Arc, 1984, 220 pp., ISBN 2-7081-05582, recensé par Michel Paquin. v. 39 , no 4,1984 , p. $811-812$

MOLLOY, Edmond S.

CUMMINGS, Thomas $\mathrm{G}$

MOLLOY, Edmond S.

Improving Productivity and the Quality of Work Life. New-York, Prager Publications, 1977, 305 pp., ISBN 0-03-022601-5, recensé par Alexander J. Matejko.

v. 37 , no 3,1982, p. $715-717$

MONLÉON, Jacques de MONLÉON, Jacques de

Marx et Aristote. Perspectives sur l'homme. Paris, FAC éditions, 1984, ISBN 0317-0179, recensé par Maurice Lebel.

v. 41 , no 4,1986 , p. $883-884$

MORGAN, Cyrill P.

JACKSON, John $H$. MORGAN, Cyrill $P$.

Organization Theory: A Macro Perspective for Management (2nd ed.). Englewood Cliffs, Prentice-Hall. Inc., 1982, 413 pp., ISBN 013-641415-X, recensé par Laurent Belanger.

v. 37, no 2,1982, p. $457-458$ 
MORGAN, Cyrill P. (suite) JACKSON, John $H$. MORGAN, Cyrill P. PROLILLO, J.G.P

Organization Theory: A Macro Perspective for Managing. Englewood Cliffs, PrenticeHall, 1986, 387 pp., ISBN 0-13-641572-501 , recensé par James Thwaites.

v. 41 , no 4,1986 , p. $884-885$

\section{MORIN, Fernand}

MORIN, Fernand

Rapports collectifs du travail. Montreal, Editions Thémis, 1982, 619 pp., ISBN 2920376-21-7, recensé par André C. Cóté. v. 38 , no 2,1983 , p. $437-440$

MORIN, Fernand BLOUIN, Rodrigue Précis de l'arbitrage des griefs. Québec, Les Presses de I'Université Laval, 1980, 507 pp., recensé par Jean-Louis Dubé. v. 36 , no 2,1981 , p. $449-451$

MORIN, Fernand BLOUIN, Rodrigue Arbitrage des griefs. Montréal, Les Éditions Yvon Blais, 1986, 554 pp., ISBN 289083-569-9, recensé par Gilles Plante. v. 42 , no 2,1987 , p. $432-434$

MORRIS, Gillian S.

MORRIS, Gillian S.

Strikes in Essential Services. London and New York, Manselle Publishing Company, 1986, 221 pp., ISBN 0-7201-1791-7, recensé par Anthony Giles.

v. 43 , no 2,1988 , p. $462-466$

MORTILLARO, Felice

GIUGNI, Gino MENGONI, Luigi

MORTILLARO, Felice WEISS, Dimitri

Possono migliorare le relazioni industriali in Italia? Torino, Gruppo Dirigenti Fiat, 1981, 140 pp., recensé par $P$. Joubert.

v. 38 , no 1,1983 , p. $190-192$

\section{MORTON, Desmond}

MORTON, Desmond COOP, Terry

Working People, An Illustrated History of Canadian Labour. Ottawa, Deneau \& Greenberg, 1980, 349 pp., recensé par F.J.K. Griezic.

v. 37 , no 1,1982 , p. $243-245$

MOSKOW, M.H.

KOZIARA, S MOSKOW, M.H.

TANNER, L.D.

Working Women : Past, Present, Future. Industrial Relations Research Association
Series, Washington, The Bureau of National Affairs, 1987, 419 pp., ISBN 0-91344734X, recensé par $P$. Andiappan.

v. 45, no 2,1990, p. $430-431$

\section{MOURIAUX, René}

MOURIAUX, René

Les syndicats dans la sociéte française. Paris, Presses de la Fondation nationale des Sciences politiques, 1983, $272 \mathrm{pp}$., ISBN 2-7246-0480-6, recensé par Dimitri Weiss

v. 38 , no 4,1983 , p. $922-924$

\section{M.T.M.O.}

M.T.M.O.

Problèmes et perspectives d'emploi dans le secteur hôtelier du Québec. Québec, Centre de recherche et de statistiques sur le marché du travail, MTMO, 1980, 262 $p p$., recensé par André Roy.

v. 36 , no 2,1981 , p. $460-461$

\section{MURRAY, Gregor}

LAFLAMME, Gilles MURRAY, Gregor BÉLANGER, Jacques FERLAND, Gilles Flexibilité and Labour Markets in Canada and United States. (Research Series 94), Geneva, International Institute for Larour Studies, 1989, 317 p., ISBN 92-9014-462-

9, recensé par Brad Pragnell.

v. 45 , no 3,1990, p. $643-647$

\section{$\mathbf{N}$}

\section{NADEAU, Gaston}

NADEAU, Gaston

Le statut juridique du salarié-gréviste québécois. Québec, Les Presses de l'Université Laval, 1981, 186 pp., ISBN 2-76376938-1, recensé par Rodrigue Blouin.

v. 37 , no 4,1982 , p. $966-967$

NAJITA, Joyce $M$.

AARON, Benjamin NAJITA, Joyce $M$. STERN, James $L$.

Public Sector Bargaining. 2nd ed., Industrial Relations Research Association Series, Washington, Bureau of National Affairs, 1988, 334 pp., ISBN 0-87179-566-3, ISBN 0-913447-37-4, recensé par Jean Boivin. v. 44, no 3,1989, p. $730-732$ 
NAISBITT, John

NAISBITT, John ABURDENE, Patricia Reinventing the Corporation. Transforming your Job and your Company for the New Information Society. New York, Warner Books, 1985, 308 pp., ISBN 0-446-512842, recensé par Alexander J. Matejko.

v. 41 , no 4,1986 , p. $881-882$

NAYLOR, James C.

NAYLOR, James C. PRITCHARD, R.D. ILGEN, D.R.

A Theory of Behavior in Organizations. New York, Academic Press inc., 1980, 299 pp., recensé par André Petit.

v. 36 , no 3,1981 , p. $703-704$

\section{NEILSEN, Eric $\mathrm{H}$.}

NEILSEN, Eric $\mathrm{H}$.

Becoming an O.D. Practitioner. Englewood Cliffs, Prentice Hall, 1984, 280 pp., ISBN 0-13-071555-7, recensé par Laurent Bélanger.

v. 39, no 4,1984 , p. $809-811$

\section{NEUFELD, Maurice F.}

NEUFELD, Maurice F. LEAB, Daniel J. SWANSON, Dorothy

American Working Class History : A Representative Biography. New York - London, Bowker, 1983, 356 pp., ISBN 0-8352-1752-

3, recensé par James Thwaites.

v. 39, no 2,1984 , p. 406

NEWMAN, Jerry $M$.

MILKOVICH, George T.

NEWMAN, Jerry $M$.

Compensation. Plano, Texas, Business Publications Inc., 1984, 549 pp., ISBN 0256-02204-6, recensé par Gilles Ferland. v. 42 , no 1,1987 , p. $218-219$

\section{NICHLOSON, Nigel}

NICHLOSON, Nigel URSELL, Gill

BLYTON, Paul

The Dynamics of White Collar Unionism: A Study of Local Union Participation. London, Academic Press, 1981, 268 pp., ISBN 0-12-518020-9, recensé par Jacques Bélanger.

v. 39, no 1,1984 , p. $218-220$

NIGHTINGALE, Donald V.

NIGHTINGALE, Donald V.

Workplace Democracy : An Inquiry into
Employee Participation in Canadian Work Organizations. Toronto, University of Toronto Press, 1983, 313 pp., ISBN 0-80206471-1, recensé par Alexander J. Matejko. v. 38 , no 4,1983 , p. $927-929$

NIGHTINGALE, Donald V.

LONG, Richard J.

Gain and Equity Sharing. Quality of Working Life. Case Study Series, Ottawa, Minister of Supply and Services, Canada, 1984, 51 pp., ISBN 0-662-13138-X, recensé par Alexander J. Matejko.

v. 39 , no 4,1984, p. $813-814$

\section{NISSEN, Bruce}

LARSON, Simeon NISSEN, Bruce

Theories of the Labor Movement. Detroit, Wayne State University Press, 1987, 395 pp., ISBN 0-8143-1815-0, recensé par Michel Audet.

v. 43 , no 1,1988 , p. $199-200$

NORTHRUP, Herbert $R$.

NORTHRUP, Herbert R.

ROWAN, Richard $\mathrm{L}$.

Multinational Collective Bargaining Attempts. Multinational Industrial Relations Series no 6, Industrial Research Unit, Philadelphia, The Wharton School, University of Pennsylvaria, 1979, 580 pp., recensé par Jean Boivin.

v. 36 , no 1. 1981 , p. $280 \cdot 28 \hat{c}$

\section{0}

\section{OFFE, Claus}

OFFE, Claus

Contradictions of the Welfare Slate. Edited and introduced by John Keane, Cambridge, MIT Press, 1984, 310 pp., ISBN O262-65014-2, recensé par Anthony Giles. v. 41, no 3,1986 , p. $664-667$

O.I.T.

O.I.T.

International Labour Conventions and Recommandations. I.L.O., Geneva, 1982, 1167 pp., recensé par Alain Barré.

v. 38 , no 1,1983 , p. $189-190$ 


\section{ONDRACK, Dan}

ONDRACK, Dan STUART, Timperley

The Humanisation of Work. European

Perspective. London, Armstrong Publishing, 1982, 239 pp., ISBN 0-9506540-86, recensé par Alexander J. Matejko.

v. 39 , no 2,1984, p. $392-393$

\section{O'SULLIVAN, Judith}

O'SULLIVAN, Judith GALLICK, Rosemary Workers and Allies : Female Participation in the American Trade Union Movement, 1824-1976. Washington, Smithsonian Institution Press, 1975, 96 pp., ISBN 0-87546138-1, recensé par Micheline Dumont. v. 43, no 1,1988, p. 205

\section{$\mathbf{P}$}

\section{PACAULT, Emile}

\section{PACAULT, Emile}

La fin du chomage par le partage du travail. Édition établie avec introduction, notes et index par Maurice Lebel. Montreal, Éditions Paulines, 1985, 118 pp., ISBN 2-89039-046-2, recensé par Dimitri Weiss.

v. 41, no 1,1986, p. $193-196$

PALMER, Bryan D.

PALMER, Bryan D.

A Culture in Conflict: Skilled Workers and Industrial Capitalism in Hamilton. Ontario, 1860-1914, Montréal, McGill-Queen's University Press, 1979, 331 pp., recensé par Hugh Tuck.

v. 36 , no 1,1981 , p. $277-280$

PALMER, Bryon

PALMER, Bryon

Working-Class Experience: The Rise and Reconstitution of Canadian Labour, 18001980. Toronto, Butterworths, 1983, 320 pp., recensé par Jerry P. White.

v. 40, no 2,1985 , p. $408-409$

PALMER, E.E.

CARROTHERS, A.W.R. PALMER, E.E.

RAYNER, W.B.

Collective Bargaining Law in Canada. 2nd Ed., Toronto, Butterworths, 1986, 785 pp., ISBN 0-409-81879-8, recensé par Pierre Verge.

v. 42 , no 1,1987, p. $219-221$
PAQUET, GIIIES

PAQUET, Gilles

La pensée économique au Québec français. Témoignages et perspectives. Montreal, Association canadiennefrançaise pour l'avancement des sciences, 1989, 364 pp., ISBN 2-89245-987-7, recensé par Gérard Dion.

v. 45 , no 2,1990 , p. $441-442$

\section{PAQUIN, Michel}

PAQUIN, Michel

L'organisation du travail. Montréal, Agence d'Arc, 1986, 199 pp., ISBN 2-89022091-5, recensé par Alain Vinet.

v. 42, no 3,1987 , p. $663-664$

\section{PARENT, Réjean}

DELORME, François PARENT, Réjean

Les licenciements collectifs au Québec : un bilan partiel du dispositif public en vigueur. Monographie 12, Montréal, Ecole de relations industrielles, Université de Montréal, 1982, 106 pp., ISBN 2-89067011-2 et ISSN 0708-9945, recensé par Jean Sexton.

v. 38 , no 1,1983 , p. 178

\section{PARTRIDGE, $M$.}

BEAUMONT, P.B. PARTRIDGE, $M$

Job Satisfaction in Public Administration. Londres, Royal Institute of Public Administration, 1983, 41 pp., ISBN 0-900628-32-

4, recensé par Michel Paquin.

v. 41 , no 2,1986 , p. $426-427$

\section{PASMORE, William A.}

PASMORE, William A.

Designing Effective Organizations. The Sociotechnical Systems Perspective. Wiley Series on Organizational Assessment and Change, New York, John Wiley, 1988, 200 pp., ISBN 0-471-88785-4, recensé par Alexander J. Matejko.

v. 44 , no 4,1989 , p. $964-967$

\section{PEACH, David A.}

PEACH, David A. KUECHLE, David The Practise of Industrial Relations. 2nd Ed., Scarborouogh, McGraw Ryerson, 1985, 407 pp., ISBN 0-07-548909-0, recensé par Norman A. Solomon.

v. 42, no 1,1987, p. $222-223$ 


\section{PÉGANIOL, Claude} PÉGANIOL, Claude

Techniques et politiques d'amélioration des conditions de travail. Paris, Entreprise moderne d'édition, 162 pp., recensé par Laurent Bélanger.

v. 36 , no 4,1981 , p. $943-944$

\section{PÉLISSIER, Jean}

DESPAX, Michel PÉLISSIER, Jean

La gestion du personnel : aspects juridiques. Tome 1, 2ième éd., Paris, Éditions Cujas, 1984, 400 pp., ISBN 2-2548-40310 , recensé par Dimitri Weiss.

v. 41 , no 1,1986 , p. 190-191

LYON-CAEN, Gérard PÉLISSIER, Jean Les grands arrêts de Droit du travail, 2ième éd., Paris, Editions Sirey, 1980, 406 pp., recensé par Dimitri Weiss.

v. 36, no 2,1981, p. $454-455$

\section{PÉRUSSE, Lyne}

BOULARD, René PÉRUSSE, Lyne Les moyens organisationnels de prévention des maladies industrielles et des accidents du travail : essai de typologie et bibliographie annotée. Québec, Collection instruments de travail, Département des relations industrielles, Université Laval, 1981, 183 pp., recensé par Bernard Boucher.

v. 36 , no 3,1981 , p. $697-699$

\section{PETERS, T.J.}

PETERS, T.J. WATERMAN, R.H. Jr. in Search of Excellence : Lessons from America's Best-Run Companies. New York, Harper and Row, 1982, 350 pp., ISBN 0-06-015042-4, recensé par James Thwaites.

v. 39 , no 2,1984 , p. $403-406$

\section{PETERS, TOM}

PETERS, Tom

Thriving on Chaos. Handbook for a Managerial Revolution. New York, Alfred A. Knopf, 1987, 561 pp., ISBN 0-394-567846 , recensé par Alexander J. Matejko.

v. 44 , no 1,1989, p. 292-293

PETERS, Tom AUSTIN, Nancy

A Passion for Excellence. The Leadership Difference. New York, Random House, 1985, 437 pp., ISBN 0-394-54484, recensé par Alexander J. Matejko.

v. 41, no 4,1986, p. $881-882$

\section{PETIT, André}

BÉLANGEH, Laurent PETIT, André

BERGERON, Jean-Louis

Gestion des ressources humaines : une approche globale et intégrée. Chicoutimi, Gaëtan Morin éditeur, 1983, 451 pp., ISBN 2-89105-072-X, recensé par Dimitri Weiss.

v. 39 , no 2,1984 , p. $406-408$

\section{PHILLIPS, Erin}

PHILLIPS, Paul PHILLIPS, Erin

Women and Work : Inequality in the Labour Market. Toronto, James Lorimer \& Company, 1983, 200 pp., ISBN 0-8862608-8, recensé par Jennifer Stoddart.

v. 39, no 2,1984 , p. 400

\section{PHILLIPS, Paul}

PHILLIPS, Paul PHILLIPS, Erin

Women and Work : Inequality in the Labour Market. Toronto, James Lorimer \& Company, 1983, 200 pp., ISBN 0-8862608-8, recensé par Jennifer Stoddart.

v. 39 , no 2,1984 , p. 400

\section{PINDER, Craig C.}

PINDER, Craig $\mathrm{C}$.

Work Motivation. Theory, Issues and Applications. Glenview, Scott, Foresman and Corp., 1984, 365 pp., ISBN 0-673-157997, recensé par Alexander J. Matejko.

v. 40, no 3,1985 , p. 680

\section{PIRZIO AMMASSARI, Gloria}

PIRZIO AMMASSARI, Gloria

MATTIOLI, Francesco

I sindicalisti. Bar, De Donato, 1982, 158 pp., CL 07-0501-2, recensé par Dimitri Weiss.

v. 38 , no 4,1983 , p. $922-924$

\section{PIVETEAU, Jacques}

PIVETEAU, Jacques

L'entretien d'appréciation du personnel : pratiques en question. Toulouse, Éditions Erès, 1981, 207 pp., ISBN 2-86586-002-7, recensé par Roland Thériault. v. 39 , no 4,1984 , p. $806-809$ 


\section{PLANTE, Gilles}

PLANTE, Gilles

Le conflit du travail : stratégie et tactique. Collection Relations du travail, Québec, Presses de l'Université Laval, 1984, 166 pp., ISBN 2-7637-7041-X, recensé par Marcel Simard.

v. 40, no 4,1985, p. $891-892$

PLANTE, Gilles

Le conflit du travail : stratégie et tactique. Collection Relations du travail, Quebec, Presses de I'Université Laval, 1984, $166 \mathrm{pp}$, ISBN 2-7637-7041-X, recensé par Yvan Simonis.

v. 41 , no 1,1986, p. $200-202$

\section{PLASSARD, Jean-Michel}

PLASSARD, Jean-Michel

Discrimination sur le marché du travail et information imparfaite. Éditions du CNRS, France, 1987, 386 pp., ISBN 2-222-040779, recensé par Esther Déom

v. 45 , no 1,1990 , p. $203-204$

\section{PLASSE, Micheline}

\section{PLASSE, Micheline}

Santé et sécurité du travail. Montréal, Agence d'Arc inc., 1988, 155 p., ISBN 289022-138-5, recensé par Alain Vinet.

v. 45 , no 3,1990, p. $632-633$

PLOWMAN, D.

FORD, B. PLOWMAN, D.

Australian Unions : An Industrial Relations Perspectives. Melbourne, MacMillan Company of Australia, 1983, 576 pp., ISBN 0333-33913-4, recensé par Gilles Dussault. v. 40, no 1,1985, p. $186-188$

PONAK, Allen

ANDERSON, John C.

GUNDERSON, Morley PONAK, Allen Union-Management Relations in Canada. (2nd ed.), Don Mills, Ont., Addison-Wesley Publishers, 1989, 498 pp., ISBN 0-201. 18621-7, recensé par Gene Swimmer. v. 45 , no 2,1990 , p. $428-429$

\section{PONIATOWSKI, Michel} PONIATOWSKI, Michel

Les Technologies Nouvelles. Paris, Plon, 1986, 334 pp., ISBN 2-259-0143-9, recensé par Maurice Lebel.

v. 42, no 2,1987, p. $445-448$

\section{POOLE, Michael}

POOLE, Michael BROWN, William

RUBERY, Jill SISSON, Keith

TARLING, Roger WILKINSON, Frank

Industrial Relations in the Future. Trends

and Possibilities in Britain over the Next

Decade. Agincourt, Methuen Publications, 1984, 148 pp., ISBN 0.7102-0145-1, recensé par Roy J. Adams.

v. 42, no 2,1987, p. $437-439$

\section{PORTIS, Bernard}

PORTIS, Bernard FULLERTON, David F. INGRAM, Paul R.

Quality Circles in Canada - A Review of Case Histories on Employee Involvement Programs. London, School of Business Administration, University of Western Ontario, 1986, 77 pp., recensé par Jean-Louis Bergeron.

v. 41 , no $4,1986, p .885-886$

\section{POULIN-SIMON, Lise}

BELLEMARE, Diane POULIN-SIMON, Lise Le defi du plein emploi - un nouveau regard économique. Montréal, Éditions Saint-Martin, 1986, 530 pp., ISBN 2-89035131-9, recensé par Louis Ascah.

v. 42 , no 2,1987 , p. $450-451$

\section{PRATT, Michel}

PRATT, Michel

La grève à la United Aircraft. Montréal, Les Presses de I'Université du Québec, 1980, 115 pp., recensé par Jacques Gagnon.

v. 41 , no 3,1986 , p. $671-672$

\section{PRITCHARD, R.D.}

NAYLOR, James C. PRITCHARD, R.D. ILGEN, D.R.

A Theory of Behavior in Organizations. New York, Academic Press Inc., 1980, 299 pp., recensé par André Petit.

v. 36 , no 3,1981 , p. $703-704$

PROLILLO, J.G.P.

JACKSON, John $H$. MORGAN, Cyrill $P$. PROLILLO, J.G.P.

Organization Theory : A Macro Perspective for Managing. Englewood Cliffs, PrenticeHall, 1986, 387 pp., ISBN 0-13-641572-501 , recensé par James Thwaites.

v. 41 , no 4,1986 , p. $884-885$ 
QUINN MILLS, D.

STIEBER, Jack MCKERSIE, Robert B. QUINN MILLS, D.

U.S. Industrial Relations 1950-1980 : A

Critical Assessment. Madison, Industrial Relations Research Association, 1981, 361 pp., recensé par Mahmood A. Zaidi. v. 38 , no 1,1983 , p. 184-187

\section{$\mathbf{R}$}

\section{RACINE, Martin}

BRADET, Denis CLICHE, Bernard RACINE, Martin THIBAULT, France Droit de la santé et de la sécurité du travail - La loi et la jurisprudence commentée. Cowansville, Les Éditions Yvon Blais, 1986, 300 pp., ISBN 2-89073-567-2, recensé par Gilles Trudeau.

v. 42 , no 1,1987 , p. $215-216$

RAYNER, W.B.

CARROTHERS, A.W.R. PALMER, E.E. RAYNER, W.B.

Collective Bargaining Law in Canada. 2nd Ed., Toronto, Butterworths, 1986, 785 pp., ISBN 0-409-81879-8, recensé par Pierre Verge.

v. 42 , no 1,1987 , p. $219-221$

\section{REES, Albert}

REES, Albert

The Economics of Trade Unions. Chicago and London, The University of Chicago Press, 1977, 200 pp., ISBN 0-226-707024, recensé par Alexander J. Matejko. v. 39 , no 1,1984, p. $212-215$

REGAN, Thomas G.

CHEN, Mervin Y.T. REGAN, Thomas G. Work in the Changing Canadian Society. Toronto, Butterworths, 1985, 289 pp., ISBN 0-409-82335-X, recensé par Alexander $J$. Matejko.

v. 40, no 2,1985 , p. $405-407$

\section{REGINI, Marino}

ROBERTS, B.C. JACOBI, Otto

JESSOP, Bob KASTENDIEK, Hans

REGINI, Marino

Industrial Relations in Europe. The Imperatives of Change. Beckenham, England,
1986, 279 pp., ISBN 0-7099-4212-5, and Economic Crisis. Trade Unions and the State. Beckenham, England, 1986, 295 pp., ISBN 0-7099-1447-4, recensés par Roy J. Adams.

v. 42, no 2,1987, p. $448-450$

\section{REICH, Michael}

GORDON, David EDWARDS, Richard

REICH, Michael

Segmented Work Divided Workers : The Historical Transformation of Labour in the United States. Cambridge, Cambridge University Press, 1982, 288 pp., recensé par Jerry $P$. White.

v. 40, no 2,1985, p. $410-411$

\section{REID, Frank}

MELTZ, Noah M. REID, Frank

SWATZ, Gérald S.

Sharing the Work: An Analysis of the issues in Worksharing and Jobsharing. Toronto, University of Toronto Press, 1981 . 90 pp., recensé par Paul Blyton.

v. 37 , no 3,1982, p. $717-718$

\section{RENAUD, Marc}

RENAUD, Marc TRUDEAU, Gilles

SAINT-JACQUES, Chantal DUBÉ, Louise Le droit de refus: une révolution tranquille - étude de la mise en oeuvre d'un nouveau droit. École des relations industrielles, Université de Montréal, Monographie, no 21, 1989, 329 p., ISBN 2-89067-0201, ISSN 0708-9945, recensé par Rodrigue Blouin.

v. 45, no 3,1990, p. $637-640$

\section{REYNAUD, Jean-Daniel}

REYNAUD, Jean-Daniel

Sociologie des conflits du travail. Paris, Presses universitaires de France, 1982, 127 pp., recensé par Alexander J. Matejko.

v. 38 , no 3,1983, p. $681-682$

\section{RHODES, Peter S.}

FINCHAM, Robin RHODES, Peter S.

The Individual, Work and Organization. Behavioural Studies in Business and Management Students. London, Weidenfeld and Nicolson, 1988, 336 pp., ISBN 0-297. 79139-7, recensé par Alexander J. Matejko.

v. 44, no 4,1989 , p. $961-962$ 


\section{RICCI, Maurizio}

$\mathrm{RICCl}$, Maurizio

La struttura organizzativa del movimento sindicale, Dalle origini al 1949. Milano, Franco Angeli, 1986, 250 pp., recensé par Dimitri Weiss.

v. 42 , no 1,1987, p. 223-224

RICHARDSON, Reed C.

RICHARDSON, Reed C.

Collective Bargaining by Objectives : A Positive Approach. 2nd ed., New Jersey, Prentice-Hall, 1985, 325 pp., ISBN 0-13140476-8-01, recensé par Esther Déom.

v. 41 , no 1,1986, p. 191-192

\section{RIDDELL, W. Craig}

RIDDELL, W. Craig

Labour-Management Cooperation in Canada. Toronto, University of Toronto Press, 1986, 205 pp., ISBN 0-8020-7257-7, recensé par Alexander J. Matejko.

v. 43 , no 3,1988, p. $714-715$

RINEHART, James W.

RINEHART, James W.

The Tyranny of Work Alienation and the Labour Process. (2nd éd.), Toronto, Harcourt Brace Jovanovich, 1987, 226 pp., ISBN 0.7747-3067-6, recensé par Alexander J. Matejko.

v. 43, no 2,1988, p. $466-468$

RITA, Giuseppe De

RITA, Giuseppe De SILVESTRI, Umberto TEDESCHI, Michele WEISS, Dimitri ZUBOFF, Shoshana

"l ruolo del fattore "uomo" nello scenario economico-produttivo degli anni "80: i quadri. Roma, STET, 1982, 84 pp., recensé par $P$. Joubert.

v. 39, no 3,1984, p. $637-638$

RIVERO LAMAS, Juan

RIVERO LAMAS, Juan

Limitacion de los poderes empresariales y democracia industrial, Leccion inaugural del curso academico 1986-1987. Zaragoza, Universidad de Zaragoza, 1986, 144 pp., Deposito Legal : Z.I. 631-86, recensé par Pierre Verge.

v. 42 , no 3,1987 , p. $659-660$
ROBB, A.L.

DENTON, F.T. ROBB, A.L.

SPENCER, B.G.

Unemployment and Labour Force Behaviour of Young People : Evidence from Canada and Ontario. Ontario Economic Council Research Series no 20, Toronto, University of Toronto Press, 1980, 215 pp., ISSN 0708-3688, ISBN 0-8020-3379-2, recensé par Jacques Mercier.

v. 37 , no 1,1982 , p. $249-251$

\section{ROBBINS, Stephen P.}

ROBBINS, Stephen P.

Organizational Behavior. Concepts, Controversies and Applications. 3rd ed., Englewood Cliffs, Prentice-Hall, 1986, 554 pp., ISBN 0-13-641549-0-01, recensé par Hélène Bois.

v. 41 , no 4,1986 , p. $879-880$

\section{ROBERTS, B.C.}

ROBERTS, B.C.

Industrial Relations in Europe. The Imperatives of Change. Dover, New Hampshire, Crown Holm, 1985, 277 pp., recensé par Solomon Barkin.

v. 41 , no 2,1986, p. $417-422$

ROBERTS, B.C. JACOBI, Otto JESSOP, BOb KASTENDIEK, Hans REGINI, Marino

Industrial Relations in Europe. The Imperatives of Change. Beckenham, England, 1986, 279 pp., ISBN 0-7099-4212-5, and Economic Crisis. Trade Unions and the State. Beckenham, England, 1986, 295 pp., ISBN 0-7099-1447-4, recensés par Roy J. Adams.

v. 42, no 2,1987 , p. $448-450$

\section{ROBERTSON, David} ROBERTSON, David WAREHAM, Jeff Technological Change in the Auto Industry. Willowdale, CAW Technology Project, 56 pp., ISBN 0-969-2932-08, recensé par Bernard Portis.

v. 43 , no 1,1988 , p. $206-207$

ROMAGNOLI, Umberto

ROMAGNOLI, Umberto WEISS, Dimitri Pluralità : Italia ed Europa nei conflitti industriali. Roma, ASAP, 1983, 288 pp., recensé par $P$. Joubert. v. 38 , no 4,1983 , p. $916-920$ 


\section{RONDEAU, Alain}

LAROCQUE, Alain BORDELEAU, Yvan

BOULARD, René FABI, Bruno

LAROUCHE, Viateur RONDEAU, Alain

Technologies nouvelles et aspects psychologiques. Sillery, Presses de l'Université du Québec, 1987, 171 pp., ISBN 27605-0450-6, recensé par Jean Mercier.

v. 44, no 2,1989 , p. $469-470$

\section{ROOMKIN, Myron}

KLEINER, Morris M. BLOCK, Richard N.

ROOMKIN, Myron

SALSBURG, Sydney W.

Human Resources and the Performance of the Firm. Madison, Industrial Relations Research Association Series, 1987, 343 pp., ISBN 0-913447-382, recensé par Bernard Portis.

v. 44 , no 1, 1989, p. 297

ROSENBERG, Gail S.

MCCARTHY, Maureen

ROSENBERG, Gail S.

Work Sharing: Case Studies. Kalamazoo, Michigan, W.E. Upjohn Institute for Employment Research, 1981, 277 pp., ISBN O911558-87-X, recensé par Alain Vinet.

v. 37 , no 4,1982 , p. 971

ROTHSCHILD-WHITT, Joice

LINDENFELD, Frank

ROTHSCHILD-WHITT, Joice

Workplace Democracy and Social Change.

Boston, Porter Sargent Publication, 447 pp., ISBN 0-87558-102-1, recensé par Alexander J. Matejko.

v. 38 , no 3,1983 , p. $687-688$

\section{ROTHWELL, W.J.}

ROTHWELL, W.J. KAZANAS, H.C.

Strategic Human Resources Planning and Management. Englewood Cliffs, PrenticeHall, 1988, 512 pp., ISBN 0-13-851643-X, recensé par Michel Audet.

v. 45 , no 1,1990, p. 211-212

\section{ROWAN, Richard L.}

CAMPBELL, Duncan C.

ROWAN, Richard $L$.

Multinational Enterprises and the OECD Industrial Relations. Philadelphia, The Wharton School Industrial Research Unit, University of Pennsylvania, 1983, 280 pp., ISBN 0-89546-039-4 et ISSN 0149-0818, recensé par François Vandamme.

v. 39 , no 4,1984 , p. 797-799

NORTHRUP, Herbert $R$.

ROWAN, Richard L.

Multinational Collective Bargaining Attempts. Multinational Industrial Relations Series no 6, Industrial Research Unit, Philadelphia, The Wharton School, University of Pennsylvania, 1979, 580 pp., recensé par Jean Boivin.

v. 36, no 1,1981 , p. $280-282$

\section{ROY, Denis}

DOLAN, Shimon L. ROY, Denis

La sélection des cadres : principes et problèmes contemporains. Monographie no 13, Montréal, École de Relations Industrielles, Université de Montreal, 1982, ISBN 2-89067-010-4, ISSN 0708-9945, recensé par Normand Pettersen.

v. 38 , no 4,1983 , p. $910-912$

RUBERY, Jill

CRAIG, C. RUBERY, Jill

TARLING, Roger WILKINSON, Frank Abolition and After the Paper Box Wages Council. Research Paper no 12, London, Department of Employment, June 1980, $98 \mathrm{pp}$., recensé par David Bright.

v. 39 , no 2,1984 , p. $396-398$

POOLE, Michael BROWN, William

RUBERY, Jill SISSON, Keith

TARLING, Roger WILKINSON, Frank

Industrial Relations in the Future. Trends and Possibilities in Britain over the Next Decade. Agincourt, Methuen Publications, 1984, 148 pp., ISBN 0-7102-0145-1, recensé par Roy J. Adams.

v. 42, no 2,1987 , p. $437-439$

RUBINSTEIN, Sidney P.

RUBINSTEIN, Sidney $P$.

Participative Systems at Work : Creating Quality and Employment Security. New York, Human Sciences Press, 1987, 180 pp., ISBN 0-89885-338-9, recensé par Alexander J. Matejko.

v. 43 , no 2,1988 , p. $471-472$

\section{RYAN, Victoria}

MARSH, Arthur RYAN, Victoria

Historical Directory of Trade Unions, vol. 1 : Non-Manual Unions. Farnborough, England, Gower, 1980, 256 pp., ISBN 0-56602160-9, recensé par Gérard Dion.

v. 37, no 1,1982, p. $245-246$ 


\section{S}

SAGARDOY BENGOECHEA, J.A.

SAGARDOY BENGOECHEA, J.A.

BLANCO, David Leon

El poder sindical en Espana. Barcelona, Editorial Planeta/Instituto de Estudios Economicos, 1982, 210 pp., ISBN 84-3207318-0, recensé par $P$. Joubert.

v. 38 , no 1,1983 , p. $192-194$

SAHAl, Jugendra

SAHAl, Jugendra

Urban Complex of an Industrial City. Allahbad, India, Chugh Publishing Company, 1980, recensé par Hem C. Jain.

v. 38, no 1,1983, p. $194-195$

\section{SAINT-JACQUES, Chantal}

RENAUD, Marc TRUDEAU, Gilles

SAINT-JACQUES, Chantal DUBÉ, Louise Le droit de refus: une révolution tranquille - étude de la mise en oeurre d'un nouveau droit. École des relations industrieles, Université de Montréal, Monographie, no 21, 1989, 329 p., ISBN 2-89067-0201, ISSN 0708-9945, recensé par Rodrigue Blouin.

v. 45 , no 3,1990 , p. $637-640$

SALSBURG, Sydney W.

KLEINER, Morris M. BLOCK, Richard N. ROOMKIN, Myron

SALSBURG, Sydney W.

Human Resources and the Performance of the Firm. Madison, Industrial Relations Research Association Series, 1987, 343 pp., ISBN 0-913447-382, recensé par Bernard Portis.

v. 44 , no 1,1989, p. 297

SALUTIN, Rick

SALUTIN, Rick

Kent Rowley, The Organizer, A Canadian Union Life. Toronto, James Lorimer, 1980, 163 pp., recensé par F.J.K. Griezic.

v. 36 , no 3,1981 , p. $704-705$

\section{SARFATI, Hedva}

SARFATI, Hedva KOBRIN, Catherine La flexibilité du marché de l'emploi : un enjeu économique et social. Genève, Bureau international du travail, 1987, 384 pp., ISBN 92-2-205675-2, recensé par Lise Poulin-Simon.

v. 43 , no 3,1988 , p. $707-710$
SCHANZ, Günter

SCHANZ, Günter

Mitarbeiter beteiligung. Grundlagen-Befunde - Modelle. Munich, Verlag Franz Vahlen, 1985, 198 pp., ISBN 3-8006-11309, recensé par Alexander J. Matejko.

v. 42 , no 1,1987, p. $224-225$

SCHMIECHEN, James A.

SCHMIECHEN, James A.

Sweated Industries and Sweated Labor: The London Clothing Trades, 1860-1914. Urbana and Chicago, University of Illinois Press, 1984, 209 pp., ISBN 0-252-010248, recensé par James Thwaites.

v. 41 , no 4,1986 , p. $877-878$

SCHULER, Randall S.

DOLAN, Shimon L. SCHULER, Randall S. Personnel and Human Resource Management in Canada. St-Paul, Wiest Publishing, 1987, 620 pp., ISBN 0-314-32486-0, recensé par Michel Audet.

v. 42 , no 2,1987, p. $440-443$

DOLAN, Shimon L. SCHULER, Randall S. Canadian Readings in Personnel and Human Resource Management. St-Paul, West Publishing Company, 1987, 467 pp., ISBN 0-314-32487-9, recensé par Michel Audet.

v. 43 , no 1, 1988, p. 203-205

\section{sCHÜTT, Maria Teresa}

BUSANA, Osvaldo

SCHÜTT, Maria Teresa UNNIA, Mario L'impresa nel sistema socio-sindicale : dieci anni di contrattazione. Turin, ISVOR - FIAT, 1980, recensé par Dimitri Weiss. v. 36 , no 2,1981 , p. 459

\section{SCIARRA, Silvana}

SCIARRA, Silvana

Contratto collettivo e contrattazione in azienda. Milano, Franco Angeli, 1985, 226 pp., recensé par Dimitri Weiss.

v. 41 , no 1,1986, p. $198-200$

\section{SCULLION, Hugh}

EDWARDS, P.K. SCULLION, Hugh

The Social Organization of Industrial Conflict : Control and Resistance in the Workplace. Oxford, Basil Blackwell, 1982, 314 pp., ISBN 0-631-13127-2, recensé par Jacques Belanger.

v. 38 , no 1,1983 , p. 181-184 
SCULLION, Hugh (suite)

EDWARDS, P.K. SCULLION, Hugh

The Social Organization of Industrial Conflict. Control and Resistance in the Workplace. Oxford, Basil Blackwell, 1982, 314 pp., ISBN 0-631-13127-2, recensé par Alexander J. Matejko.

v. 38 , no 3,1983 , p. $685-686$

\section{SEEBER, Ronald}

HERMAN, E. Edward KUHN, Alfred

SEEBER, Ronald

Collective Bargaining and Labor Relations.

(2nd ed.), Englewood Cliffs, Prentice Hall, 1987, 621 pp., ISBN 0-13-140576-6, recensé par Norman A. Solomon.

v. 43 , no 1,1988 , p. $207-208$

\section{SEFTON MACDOWELL, Laurel} SEFTON MACDOWELL, Laurel

"Remember Kirkland Lake" : The History and Effects of the Kirkland Lake Gold Miners' Strike 1941-42. Toronto, University of Toronto Press, 1983, 292 pp., ISBN 08020-5585-0, recensé par Hugh Tuck.

v. 39, no 4,1984, p. $803-804$

\section{SELLIER, François}

MAURICE, Marc SELLIER, François

SILVESTRE, Jean-Jacques

Politique d'éducation et organisation industrielle en France et en Allemagne : essai d'analyse sociétale. Paris, PUF, collection "Sociologies" dirigée par Raymond Boudon et François Bourricaud, 1982, 382 pp., ISBN 2-13-037472-7, recensé par Dimitri Weiss.

v. 38 , no 4,1983 , p. $929-934$

MAURICE, Marc SELLIER, François

SILVESTRE, Jean-Jacques

The Social Foundations of Industrial Power : A Comparison of France and Germany. Traduit par Arthur Goldhammer, Cambridge, MIT Press, 1986, 292 pp., ISBN O262-13213-3, recensé par Anthony Giles. v. 43, no 3,1988, p. $703-707$

\section{SEN, Joya}

\section{SEN, Joya}

The Unemployment of Youth : The Importance of Education for their Adjustment in the Canadian Labour Market. Toronto,
Ontario Institute for Studies in Education, 1982, 125 pp., ISBN 0-7744-5053-3, recensé par Morley Gunderson.

v. 38 , no 4,1983 , p. $925-927$

\section{SERRUYS, Jacques $W$.}

SERRUYS, Jacques $W$.

Phychologie du commandement. Bruxelles, Vander Éditeur, 1984, 230 pp., ISBN 2-8008-0000-3, recensé par Hélène BoisBrochu.

v. 40, no 2,1985, p. $414-415$

\section{SEXTON, Jean}

LECLERC, Claudine SEXTON, Jean La sécurité d'emploi dans l'industrie de la construction au Québec, un rêve impossible? Québec, Presses de l'Université Laval, 1983, 275 pp., ISBN 2-7637-7028-

2, recensé par Noël Mallette.

v. 39 , no 3,1984 , p. $643-645$

\section{SHARMA, Baldev $R$.}

SHARMA, Baldev R.

Not by Bread Alone : A Stuoy of Organizational Climate and Employer-Employee Relations in India. New Delhi, Shri Ram Centre for Industrial Relations and Human Resources, 1987, 191 pp., resencé par Basu Sharma

v. 43 , no 1,1988 , p. $208-209$

\section{SHRAGGE, Eric}

\section{SHRAGGE, Eric}

Pensions Policy in Britain : A Socialist Analysis. London, Routledge \& Kegan Paul, 1984, 194 pp., ISBN 0-7100-9842-1, recensé par Anthony Giles.

v. 41 , no 2,1986 , p. 428.430

\section{SIEGEL, Irving $H$.}

SIEGEL, Irving $H$. WEINBERT, Edgar

Labor-Management Cooperation. The American Experience. Kalamazoo, Michigan, W.E. Upjohn Institute for Employment Research, 1982, 316 pp., ISBN 091155899-3, recensé par Jean Sexton

v. 37, no 4,1982 , p. 973 


\section{SILVESTRE, Jean-Jacques}

MAURICE, Marc SELLIER, François

SILVESTRE, Jean-Jacques

Politique d'éducation et organisation industrielle en France et en Allemagne : essai d'analyse sociétale. Paris, PUF, collection 'Sociologies' dirigée par Raymond Boudon et François Bourricaud, 1982, 382 pp., ISBN 2-13-037472-7, recense par Dimitri Weiss.

v. 38 , no 4,1983 , p. $929-934$

MAURICE, Marc SELLLER, François SILVESTRE, Jean-Jacques

The Social Foundations of Industrial Power : A Comparison of France and Germany. Traduit par Arthur Goldhammer, Cambridge, MIT Press, 1986, 292 pp., ISBN O262-13213-3, recense par Anthony Giles. v. 43 , no 3,1988 , p. $703-707$

\section{SILVESTRI, Umberto}

RITA, Giuseppe De SILVESTRI, Umberto TEDESCHI, Michele WEISS, Dimitri ZUBOFF, Shoshana

"I ruolo del fattore "uomo" nello scenario economico-produttivo degli anni "80 : i quadri. Roma, STET, 1982, 84 pp., recensé par $P$. Joubert.

v. 39 , no 3,1984, p. $637-638$

\section{SINGH, R.K.}

VENKATCHALAM, V. SINGH, R.K. The Political, Economic and Labor Climate in India. Philadelphia, The Wharton Schoof Industrial Research Unit, University of Pennsylvania, 1982, 147 pp., ISBN O89546-030-0, ISSN 149-0818, recensé par Hem C. Jain.

v. 39 , no 1,1984, p. $215-217$

\section{SIRARD, Ronald}

SIRARD, Ronald GAZAILLE, Alain Comprendre et appliquer une convention collective. Montréal, Wilson et Lafleur, 1989, 165 p., ISBN 2-89127-151-3, recensé par Hélène Gascon.

v. 45 , no 3,1990, p. $635-637$

\section{SISSON, Keith}

POOLE, Michael BROWN, William

RUBERY, Jill SISSON, Keith

TARLING, Roger WILKINSON, Frank Industrial Relations in the Future. Trends and Possibilities in Britain over the Next
Decade. Agincourt, Methuen Publications, 1984, 148 pp., ISBN 0-7102-0145-1, recensé par Roy J. Adams.

v. 42 , no 2,1987 , p. $437-439$

\section{SLOANE, Arthur A.}

SLOANE, Arthur $A$.

Personnel, Managing Human Resources. Englewood Cliffs, Prentice Hall, 1983, 619 pp., ISBN 0-13-658278-8, recensé par Paul Tessier.

v. 39 , no 4,1984, p. $804-805$

\section{SMUCKER, Joseph}

SMUCKER, Joseph

VAN DEN BERG, Axel

Capitalism vs Socialism? Canadian and Swedish Labour Market Policies Compared. Working Papers in Social Behaviour, no 88-8, Montreal, McGill University, 1988, 75 pp., recensé par Alexander J. Matejko. v. 44 , no 1,1989 , p. 294-295

\section{SNOW, Charles C.}

SNOW, Charles C.

Strategy, Organization Design, and Human Resource Management. Greenwich, JAI Press, 1989, 368 pp., ISBN 0-89232-807. $X$, recensé par Michel Audet. v. 44, no 3,1989 , p. $734-736$

\section{SOMERS, Gérald C.}

SOMERS, Gérald C.

Collective Bargaining : Contemporary American Experience. Madison, WI., Industrial Relations Research Association, 1980, 588 pp., recensé par Gérard Dion. v. 36 , no 4,1981 , p. 954

\section{SPENCER, B.G.}

DENTON, F.T. ROBB, A.L. SPENCER, B.G.

Unemployment and Labour Force Behaviour of Young People : Evidence from Canada and Ontario. Ontario Economic Council Research Series no 20, Toronto, University of Toronto Press, 1980, 215 pp., ISSN 0708-3688, ISBN 0-8020-3379-2, recensé par Jacques Mercier. v. 37 , no 1,1982 , p. $249-251$ 
Relations lmdustrielles, vol. 45, no 4 (1990)

\section{SPYROPOULOS, G.}

SPYROPOULOS, G.

Trade Unions Today and Tomorrow. 2 vol, Masestricht, Presses universitaires européennes, 1985 et 1987, 266 et 212 pp., ISBN 90-70776-17-0 et ISBN 9070776-18-9, recensé par Pierre Verge.

v. 44 , no 1,1989, p. $287-289$

\section{STAUDOHAR, Paul D.}

KERR, Clark STAUDOHAR, Paul D.

Industrial Relations in a New Age. San Francisco, Jossey-Bass Inc., 1986, 419 pp., ISBN 0-55542-013-3, recensé par Norman A. Solomon.

v. 42 , no 4,1987 , p. $883-884$

\section{STAUDOHAR, Paul D.}

The Sports Industry and Collective Bargaining. Ithaca, ILR Press, Cornell University, 1986, 195 pp., ISBN 0-87546-118-2, recensé par Jean Boivin.

v. 45 , no 1,1990, p. 212

\section{STEINBERG RATNER, Ronnie}

STEINBERG RATNER, Ronnie

Equal Employment Policy for Women : Strategies for Implementation in the United States, Canada and Western Europe. Philadelphia, Temple University Press, 1980, 520 pp., recensé par Carole Swan.

v. 36 , no 3,1981 , p. $692-696$

\section{STELLMAN, Jeanne M.}

STELLMAN, Jeanne M. DAUM, Susan M. Perdre sa vie à la gagner; manuel pour la santé des travailleurs. Montréal, Édition Parti pris, 1979, 493 pp., recensé par René Boulard et Michel Pérusse.

v. 36 , no 3,1981 , p. $702-703$

STERN, James L.

AARON, Benjamin NAJITA, Joyce M. STERN, James $L$.

Public Sector Bargaining. 2nd ed., Industrial Relations Research Association Series, Washington, Bureau of National Affairs, 1988, 334 pp., ISBN 0-87179-566-3, ISBN 0-913447-37-4, recensé par Jean Boivin.

v. 44, no 3,1989 , p. $730-732$

\section{STEVENS, Mark}

MILLWARD, Neil STEVENS, Mark

British Workplace Industrial Relations
1980-1984: The DE/ESRC/PSI/ACAS SUrveys. Aldershot, Gower, 1986, 341 pp., ISBN 0-566-05396-9, recensé par Jacques Bélanger.

v. 42 , no 4,1987, p. $891-893$

\section{STIEBER, Jack}

GLADSTONE, Alan

LANSBURY, Russel D. STIEBER, Jack

TREU, T. WEISS, M.

Current Issues in Labour Relations : An International Perspective. Berlin, Walter de Gruyter, 1989, 380 pp., ISBN 0-89925471-3, recensé par Jack Barbash.

v. 45 , no 1,1990 , p. $204-205$

STIEBER, Jack MCKERSIE, Robert $B$.

QUINN MILLS, D.

U.S. Industrial Relations 1950-1980 : A

Critical Assessment. Madison, Industrial Relations Research Association, 1981, 361 pp., recense par Mahmood A. Zaidi. v. 38 , no 1,1983 , p. $184-187$

\section{STODDART, Linda}

\section{STODDART, Linda}

Conditions of Work and Quality of Working Life, a Directory of Institutions. Geneva, International Labour Office, 1986, 306 pp., ISBN 92-2-105328-8, recensé par Gerard Dion.

v. 41 , no 4,1986 , p. $878-879$

\section{STONE, Thomas $H$.}

STONE, Thomas H. MELTZ, Noah $M$. Personnel Management in Canada. Toronto Holt, Rinehart \& Winston, 1983, 411 pp., ISBN 0-03-921591-1, recensé par Alexander J. Matejko.

v. 39 , no 1,1984, p. $205-206$

\section{STOREY, John}

\section{STOREY, John}

The Challenge to Management Control. Brookfield, Vermont, Renouf USA, 1981, 192 pp., ISBN 0-09-145941-9, recensé par Jacques Bélanger.

v. 37, no 2,1982, p. $455-457$

\section{STOREY, RObert}

HERON, Craig STOREY, Robert

On the Job : Confronting the Labour Process in Canada. Kingston and Montréal, McGill-Queen's University Press, 1986, 360 pp., ISBN 0-7735-0598-9, recensé par Robert $H$. Zieger.

v. 42, no 1,1987 , p. $216-218$ 


\section{STREECK, Wolfgang}

HYMAN, Richard STREECK, Wolfgang New Technology and Industrial Relations. Oxford, Basil Blackwell, 1988, 309 pp., ISBN 0-631-15982-7, recensé par Jacques Bélanger.

v. 45 , no 1,1990 , p. $208-209$

\section{STRUTHERS, James}

STRUTHERS, James

"No Fault of their Own". Unemployment and the Canadian Welfare State 19141940. The State and Economic Life Series, Mel Watkins and Leo Panitch eds, Toronto, University of Toronto Press, 1983, 238 pp., ISBN 0-8020-7, recensé par F.J.K. Griezic.

v. 39 , no 3,1984 , p. $638-641$

\section{STUART, TImperley}

ONDRACK, Dan STUART, Timperley

The Humanisation of Work. European Perspective. London, Armstrong Publishing, 1982, 239 pp., ISBN 0-9506540-86, recensé par Alexander J. Matejko.

v. 39 , no 2,1984 , p. $392-393$

\section{SWANSON, Dorothy}

NEUFELD, Maurice F. LEAB, Daniel J. SWANSON, Dorothy

American Working Class History : A Representative Biography. New York - London, Bowker, 1983, 356 pp., ISBN 0-8352-1752-

3 , recensé par James Thwaites.

v. 39 , no 2,1984, n. 406

\section{SWATZ, Gérald S.}

MELTZ, Noah M. REID, Frank

SWATZ, Gérald S.

Sharing the Work: An Analysis of the Issues in Worksharing and Jobsharing. Toronto, University of Toronto Press, 1981 . 90 pp., recensé par Paul Blyton.

v. 37 , no 3,1982 , p. $717-718$

\section{SWIMMER, Gene}

THOMPSON, Mark SWIMMER, Gene

Conflict or Compromise : The Future of Public Sector Industrial Relations. Montreal, Institut de recherches politiques, 1984, 476 pp., ISBN 0-88645-001-2, recensé par Jean Boivin.

v. 40 , no 3,1985, p. $676-678$

\section{T}

TAHAR, Gabrlel

TAHAR, Gabriel

La réduction de la durée du travail. Paris, Collection Repères, Éditions La découverte, 1985, 127 pp., ISBN 2-7071-1552-

5, recensé par Lise Chrétien.

v. 42 , no 3,1987 , p. $646-648$

TANNER, L.D.

KOZIARA, S MOSKOW, M.H.

TANNER, L.D.

Working Women : Past, Present, Future. Industrial Relations Research Association Series, Washington, The Bureau of National Affairs, 1987, 419 pp., ISBN 0-91344734-X, recensé par $P$. Andiappan.

v. 45 , no 2,1990 , p. $430-431$

\section{TARLING, Roger}

CRAIG, C. RUBERY, Jill

TARLING, Roger WILKINSON, Frank

Abolition and After the Paper Box Wages

Council. Research Paper no 12, London,

Department of Employment, June 1980, 98 pp., recensé par David Bright.

v. 39, no 2,1984, p. $396-398$

POOLE, Michael BROWN, William

RUBERY, Jill SISSON, Keith

TARLING, Roger WILKINSON, Frank Industral Relations in the Future. Trends and Possibilities in Britain over the Next Decade. Agincourt, Methuen Publications, 1984, 148 pp., ISBN 0-7102-0145-1, recensé par Roy J. Adams.

v. 42 , no 2,1987 , p. $437-439$

\section{TARNOPOLSKY, Walter Surma}

TARNOPOLSKY, Walter Surma

Discrimination and the Law in Canada. Toronto, Richard De Boo Ltd, 198\%, 595 pp., ISBN 0-88820-105-2, recensé par Rodrigue Blouin.

v. 38 , no 2,1983 , p. $441-442$

\section{TAYLOR, Robert}

TAYLOR, Robert

Workers and the New Depression. London, Macmillan Press, 1981, 212 pp., ISBN 0-333-33411-6, recensé par Alexander J. Matejko.

v. 39 , no 3,1984 , p. 636 


\section{TEDESCHI, Michele}

RITA, Giuseppe De SILVESTRI, Umberto TEDESCHI, Michele WEISS, Dimitri ZUBOFF, Shoshana

II ruolo del fattore "uomo" nello scenario economico-pronuttivo diegii anni "80:i quadri. Roma, STET, 1982, 84 pp., recensé par $P$. Joubert.

v. 39 , no 3,1984, p. $637-638$

\section{TÉGA, Vasile}

CÔTÉ, Marcel TÉGA, Vasile

La démocratie industrielle / The Industrial Democracy. Montréal, Les Editions Agence d'Arc, 1981, 518 pp., recensé par Jacques Bélanger.

v. 36, no 3,1981 , p. $689-690$

\section{THÉRIAULT, Roland}

THÉRIAULT, Roland

Gestion de la rémunération : Politiques et pratiques efficaces et équitables. Boucherville, Gaétan Morin Éditeur, 1983, 585 pp., ISBN 2-89105-109-2, recensé par Jacques Chouinard.

v. 38 , no 4,1983 , p. $913-915$

\section{THIBAULT, France}

BRADET, Denis CLICHE, Bernard

RACINE, Martin THIBAULT, France

Droit de la santé et de la sécurité du travail - La loi et la jurisprudence commentée. Cowansville, Les Éditions Yvon Blais, 1986, 300 pp., ISBN 2-89073-567-2, recensé par Gilles Trudeau.

v. 42 , no 1,1987 , p. $215-216$

\section{THOMPSON, Kenneth}

THOMPSON, Kenneth

Work, Employment and Unemployment. Perspectives on Work and Society. Milton Keynes, Open University Press, 1984, 280 pp., ISBN 03-3510-5947, recensé par Alexander J. Matejko.

v. 41, no 2,1986, p. $425-426$

\section{THOMPSON, Mark}

THOMPSON, Mark SWIMMER, Gene Conflict or Compromise : The Future of Public Sector Industrial Relations. Montreal, Institut de recherches politiques, 1984, 476 pp., ISBN 0-88645-001-2, recensé par Jean Boivin.

v. 40, no 3,1985 , p. $676-678$
JURIS, H. THOMPSON, Mark

DANIELS, $W$.

Industrial Relations in a Decade of Economic Change. IRRA Series, Annual Research Volume, Madison, Wisconsin, IRRA, 1985, 407 pp., ISBN 0-913447-30-7, recensé par Hem C. Jain.

v. 42 , no 2,1987 , p. $443-445$

\section{THOMPSON, Paul}

THOMPSON, Paul

The Nature of Work. An Introduction to Debates on the labour Process. London, The Macmillan Press, 1983, 305 pp., ISBN 0-333-33027-7, recensé par Alexander $J$. Matejko.

v. 39, no 3,1984, p. $641-642$

\section{THUROW, Lester C.}

THUROW, Lester C.

The Zero-Sum Society. Distribution and the Possibilities for Economic Change. New York, Basic Books Inc., 1980, 230 pp., recensé par Alexander J. Matejko. v. 36 , no 4,1981 , p. $949-951$

THUROW, Lester C.

The Management Challenge. Japanese Views. Cambridge, The MIT Press, 1985, 237 pp., ISBN 0-262-20053-8, recensé par Hélène Bois-Brochu.

v. 40, no 3,1985, p. $683-685$

\section{TIZIANO, Treu et al.}

TIZIANO, Treu et al.

Public Service Labour Relations : Recent Trends and Future Prospects. Geneve, Bureau international du travail, 1987, 287 pp., ISBN 92-2-106049-7, recensé par Maurice Lemelin.

v. 43 , no 4,1988 , p. 974

\section{TOMANDL, $T$.}

TOMANDL, T. FUERBOECK, $\mathrm{K}$.

Social Partnership - The Austrian System of Industrial Relations and Social Insurance. Ithaca, ILR Press, 1986, $165 \mathrm{pp}$., ISBN 0-87546-116-6, recensé par Pierre Verge.

v. 42 , no 3,1987, p. $662-663$ 
TRAVES, Tom

TRAVES, Tom

Essays in Canadian Business History. Toronto, McClelland and Steward, 1984, 212 pp., ISBN 0-7710-8570-2, recensé par James Thwaites.

v. 39, no 4,1984, p. $802-803$

\section{TREU, T.}

GLADSTONE, Alan LANSBURY, Russel D. STIEBER, Jack TREU, T. WEISS, M.

Current issues in Labour Relations: An International Perspective. Berlin, Walter de Gruyter, 1989, 380 pp., ISBN 0-89925-4713 , iecensé par Jack Barbash.

v. 45, no 1,1990 , p. 204-205

\section{TREVOR, Malcolm}

WHITE, Michael TREVOR, Malcolm Under Japanese Management. The Experience of British Workers. London, Heinemann, 1983, 162 pp., ISBN 0-435-839357 , recensé par Alexander J. Matejko.

v. 39, no 2,1984, p. $395-396$

\section{TRUDEAU, Gilles}

AOUST, Claude d' LECLERC, L. TRUDEAU, Gilles

Les mesures disciplinaires : étude jurisprudentielle et doctrinale. Montréal, École de relations industrielles, Monographie no 13, 1982, 484 pp., ISBN 2-89067-012-0, ISSN 0708-9945, recensé par $R$. Blouin.

v. 38 , no 2,1983 , p. $444-445$

HÉBERT, Gérard TRUDEAU, Gilles Les normes minimales du travail au Canada et au Québec : Étude juridique et institutionnelle. Montréal, Éditions Yvon Blais, 1987, 192 pp., ISBN 2-89073-611-3, recensé par Pierre Laporte.

v. 42, no 4,1987, p. $876-877$

RENAUD, Marc TRUDEAU, Gilles

SAINT-JACQUES, Chantal DUBÉ, Louise Le droit de refus: une révolution tranquille - étude de la mise en oeuvre d'un nouveau droit. École des relations industrielles, Université de Montréal, Monographie, no 21, 1989, 329 p., ISBN 2-89067-0201, ISSN 0708-9945, recensé par Rodrigue Blouin.

v. 45 , no 3,1990, p. $637-640$

\section{TRUDEAU, Pierre Elliott}

TRUDEAU, Pierre Elliott

La grève de l'amiante. Montréal, Éditions du Jour, 1970, 430 pp., recensé par Jacques Gagnon.

v. 41 , no 3,1986, p. $669-670$

\section{TURCOTTE, Pierre R.}

TURCOTTE, Pierre R.

Qualité de vie au travail : anti-stress et créativité. Montreal, Agences d'Arc, 1982, 184 pp., ISBN 2-89022-046- $X$, recensé par René Boulard.

v. 38 , no 3,1983 , p. $689-690$

\section{TURCOTTE, Pierre R.}

La QVT une voie vers l'excellence. Montreal, Les Éditions Agence d'Arc, 1988, 276 pp., ISBN 289022-134-2 et ISBN 27624-0066-X, recensé par Roger Zaoré. v. 44 , no 4,1989 , p. $962-963$

\section{U}

\section{UNNIA, Mario}

BUSANA, Osvaldo

SCHÜTT, Maria Teresa UNNIA, Mario L'impresa nel sistema socio-sindicale: dieci anni di contrattazione. Turin, ISVOR - FIAT, 1980, recensé par Dimitri Weiss. v. 36 , no 2,1981 , p. 459

\section{URSELL, Gill}

NICHLOSON, Nigel URSELL, Gill

BLYTON, Paul

The Dynamics of White Collar Unionism: A Study of Local Union Participation. London, Academic Press, 1981, 268 pp., ISBN 0-12-518020-9, recensé par Jacques Bélanger.

v. 39 , no 1,1984, p. $218-220$

\section{V}

VAISEY, Douglas G.

VAISEY, Douglas G.

The Labour Companion: A Bibliography of Canadian Labour History, Based on Materials Printed from 1950 to 1975. Halifax, Committee on Canadian Labour History, 1980, 128 pp., recensé par Mario Lajoie. v. 37, no 1,1982 , p. $251-252$ 
VAN DEN BERG, Axel

SMUCKER, Joseph

VAN DEN BERG, Axel

Capitalism vs Socialism? Canadian and Swedish Labour Market Policies Compared. Working Papers in Social Behaviour, no 88-8, Montréal, McGill University, 1988, $75 \mathrm{pp}$., recensé par Alexander J. Matejko. v. 44 , no 1,1989, p. $294-295$

\section{VECCHIO, Robert P.}

VECCHIO, Robert P.

Organizational Behavior. Chicago, Toronto, The Dryden Press, 1988, 576 pp., ISBN 0-000853-0, recensé par Bernard Portis.

v. 44 , no 4,1989 , p. $963-964$

\section{VENKATCHALAM, $V$.}

VENKATCHALAM, V. SINGH, R.K.

The Political, Economic and Labor Climate in India. Philadelphia, The Wharton School Industrial Research Unit, University of Pennsylvania, 1982, 147 pp., ISBN 089546-030-0, ISSN 149-0818, recensé par Hem C. Jain.

v. 39 , no 1,1984 , p. $215-217$

\section{VERDIER, Jean-Maurice}

VERDIER, Jean-Maurice

Syndicats et droit syndical. 2ième éd., v. II, Le droit syndical dans l'entreprise, tome 5 du Traite de droit du travail, publié sous la direction de G.H. Camerlynck, Paris, Daloz, 1984, 475 pp., ISBN 2-247-005160 , recensé par Pierre Verge.

v. 40 , no 1,1985, p. $178-180$

\section{VERDIER, Jean-Maurice}

Syndicats et droit syndical. 2ième éd., tome 5, vol. 1 du Traite de droit du travail publié sous la direction de G.H. Camerlynck, Paris, Dalloz, 1987, 671 pp., ISBN 2-247-00754-6, recensé par Pierre Verge.

v. 42, no 3,1987 , p. $657-658$

\section{VERGE, Pierre}

GAGNON, Robert P. LEBEL, Louis VERGE, Pierre

Droit du travail. Québec, Presses de l'Université Laval, 1987, 933 pp., ISBN 27637-7123-8, recensé par Alain Barré.

v. 43 , no 4,1988 , p. $974-980$
VERGE, Pierre

Le droit de grève, fondements et limites. Chicoutimi, Editions Yvon Blais, 1985, 229 pp., ISBN 2-89073-553-2, recensé par Fernand Morin.

v. 40 , no 4,1985 , p. $907-910$

\section{VÉZINA, Claude}

VÉZINA, Claude

Les clauses d'ancienneté et l'arbitrage des griers. Ottawa, Collection des travaux de la Faculté de droit de l'Université d'Ottawa, editions de l'Université d'Ottawa, 1979, 125 pp., recensé par Rodrigue Blouin.

v. 36 , no 1,1981 , p. $288-289$

\section{VÉZINA, Lucie}

VINET, Alain DUFRESNE, Francine VÉZINA, Lucie

Condition féminine en milieu ouvrier. Québec, Institut québécois de recherche sur la culture, 1982, 221 pp., ISBN 289224-018-2, recensé par Marie Françoise Marchis-Mouren.

v. 38 , no 3,1983 , p. $679-680$

\section{VIGEANT-GALLEY, Paulette} VIGEANT-GALLEY, Paulette

Les enseignants et le pouvoir : histoire de l'Alliance des professeurs de Montréal, les luttes syndicales et le développement social (1952-1958). Québec, CEQ-APM, décembre 1981, 128 pp., recensé par James Thwaites.

v. 37, no 3,1982 , p. $712-713$

\section{VINET, Alain}

VINET, Alain DUFRESNE, Francine

VÉZINA, Lucie

Condition féminine en milieu ouvrier. Québec, Institut québécois de recherche sur la culture, 1982, 221 pp., ISBN 289224-018-2, recensé par Marie Françoise Marchis-Mouren.

v. 38 , no 3,1983 , p. $679-680$

VON PRONDZYNSKI, F. VON PRONDZYNSKI, F.

Freedom of Association and Industrial Relations. London, Mansell Publishing, 1987, 248 pp., ISBN 0-7201-1775-5, recensé par Pierre Verge.

v. 42 , no 3,1987 , p. 651-652 


\section{w}

WALKER, Mlchael A.

WALKER, Michael A.

Freedom, Democracy and Economic Welfare. Vancouver, the Fraser institute, 1988, 369 pp., ISBN 0-88975-116-1, recensé par Morley Gunderson.

v. 44 , no 2,1989 , p. $465-466$

WALL, J.A. Jr.

WALL, J.A. Jr.

Negotiation: Theory and Practice. Glenview, Scott, Foresman and Company, 1985, 182 pp., ISBN 0-673-15865-9, recensé par Paul Beaulieu.

v. 42 , no 2,1987, p. 435

\section{WAREHAM, Jeff}

ROBERTSON, David WAREHAM, Jeff

Technological Change in the Auto Industry. Willowdale, CAW Technology Project, 56 pp., ISBN 0-969-2932-08, recensé par Bernard Portis.

v. 43 , no 1,1988 , p. $206-207$

WATERMAN, R.H. Jr.

PETERS, T.J. WATERMAN, R.H. Jr.

In Search of Excellence : Lessons from

America's Best-Run Companies. New York, Harper and Row, 1982, 350 pp., ISBN 0-06-015042-4, recensé par James Thwaites.

v. 39 , no 2,1984, p. $403-406$

WAY, Harold E.

WAY, Harold E. WEISS, Carla M.

Plant Closings : A Selected Bibliography of Materials Published Through 1985. Ithaca, NYSS/LR, Cornell University, 1988, 206 pp., recensé par Jean Sexton.

v. 43 , no 2,1988, p. 472

\section{WEDDERBURN, Lord of Charlton}

LEWIS, R. CLARK, J.

WEDDERBURN, Lord of Charlton

Labour Law and Industrial Relations : Building on Kahn-Freund. Oxford, Clarendon Press, 1983, 250 pp., ISBN 0-19-825393-

1 , recensé par Pierre Verge.

v. 38 , no 3,1983, p. $677-678$
WEINBERT, Edgar

SIEGEL, Irving $H$. WEINBERT, Edgar

Labor-Management Cooperation. The American Experience. Kalamazoo, Michigan, W.E. Upjohn Institute for Employment Research, 1982, 316 pp., ISBN 091155899-3, recensé par Jean Sexton.

v. 37, no 4,1982 , p. 973

\section{WEISS, Carla $M$.}

WAY, Harold E. WEISS, Carla M.

Plant Closings : A Selected Bibliography of Materials Published Through 1985. Ithaca, NYSSILR, Cornell University, 1988, 206 pp., recensé par Jean Sexton.

v. 43, no 2,1988 , p. 472

\section{WEISS, Dimitri}

GIUGNI, Gino MENGONI, Luigi

MORTILLARO, Felice WEISS, Dimitri

Possono migliorare le relazioni industriali in Italia? Torino, Gruppo Dirigenti Fiat, 1981, 140 pp., recensé par $P$. Joubert. v. 38 , no 1,1983 , p. $190-192$

RITA, Giuseppe De SILVESTRI, Umberto TEDESCHI, Michele WEISS, Dimitri ZUBOFF, Shoshana

II ruolo del fattore "uomo" nello scenario economico-produttivo degli anni "80:i quadri. Roma, STET, 1982, 84 pp., recensé par P. Joubert.

v. 39, no 3,1984 , p. $637-638$

ROMAGNOLI, Umberto WEISS, Dimitri Pluralità : Italia ed Europa nei conflitti industriali. Roma, ASAP, 1983, 288 pp., recensé par $P$. Joubert.

v. 38 , no 4,1983 , p. $916-920$

WEISS, Dimitri

Politica, partidos y sindicatos en la empresa. Madrid, Instituto de Estudios laborales y de la Seguridad social, 1982, 136 pp., ISBN 84-5007823-7, recensé par P. Joubert.

v. 38 , no 1,1983 , p. $192-194$

WEISS, Dimitri

Les relations du travail. Employeurs, personnel, syndicats, Etat. 5ième ed., Paris, Dunod, 1983, 448 pp., ISBN 2-04-0155457, ISSN 0335-3184, recensé par Gérard Dion.

v. 38 , no 4,1983 , p. $912-913$ 


\section{WEISS, DimitrI (suite)}

WEISS, Dimitri

"La participation". Enciclopedia de Dirreccion y Administracion de la Empresa, Fasciculo 82, Barcelona, Ediciones Orbis, 1985, ISBN (fascicule) 84-7530-585-7; (oeuvre complète) 84-7530-583-0; (volumeVI) 84-7634-230-6, recensé par $P$. Joubert.

v. 41 , no 2,1986 , p. $423-424$

\section{WEISS, Dimitri CHIROUZE, Yves}

Le consommerisme. Paris, Editions Sirey, collection Administration des entreprises, 1984, 387 pp., ISBN 2-248-00080-4, recensé par P. Joubert.

v. 39, no 3,1984, p. $634-635$

\section{WEISS, $M$.}

GLADSTONE, Alan LANSBURY, Russel D.

STIEBER, Jack TREU, T. WEISS, $M$.

Current Issues in Labour Relations : An International Perspective. Berlin, Walter de Gruyter, 1989, 380 pp., ISBN 0-89925-471-

3, recensé par Jack Barbash.

v. 45 , no 1,1990 , p. $204-205$

\section{WELLS, Don}

WELLS, Don

Soft Sell : "Quality of Working Life" Programs and the Productivity Race. Canadian Centre for Policy Alternatives, 150 pp., ISBN 088627-040-5, recensé par Robert Sass.

v. 41, no 2,1986 , p. $432-433$

\section{WESTLEY, William A.}

KAHN, Joan WESTLEY, William A.

The Working Conditions in Canadian Hospitals. Constraints and Opportunity. Ottawa, Minister of Supply and Services Canada, 1984, 112 pp., ISBN 0-662-52924-3, recensé par Alexander J. Matejko.

v. 39 , no 4,1984 , p. $815-816$

\section{WHEELER, Hoyt N.}

WHEELER, Hoyt $\mathrm{N}$.

Industrial Conflict. An Integrative Theory. Columbia, University of South Carolina Press, 1985, 293 pp., ISBN 0-87249-4594, recensé par Esther Déom. v. 41, no 3,1986 , p. $652-654$
WHITE, Julie

WHITE, Julie

Les femmes et le syndicalisme. Ottawa, Conseil consultatif canadien de la situation de la femme, Centre d'édition du gouvernement du Canada, 1980, 147 pp., recensé par Marie Lavigne.

v. 36 , no 2,1981 , p. $456-457$

\section{WHITE, Michael}

WHITE, Michael TREVOR, Malcolm

Under Japanese Management. The Experience of British Workers. London, Heinemann, 1983, 162 pp., ISBN 0-435-839357, recensé par Alexander J. Matejko.

v. 39 , no 2,1984 , p. $395-396$

\section{WILCZYNSKI, Jozef}

WILCZYNSKI, Jozef

Comparative Industrial Relations. Ideologies, Institutions, Practices and Problems under Social Systems with Special Reference to Socialist Planird Economies. London, The Macmillan Press, 256 pp., ISBN 0-333-33430-2, recense par Alexander J. Matejko.

v. 40, no 1,1985, p. $190-192$

WILKES, John

WILKES, John

The Future of Work. Sydney, G. Allen and Unwin, Australia Institute of Political Science, 1981, 164 pp., ISBN 0-86861-283-9, recensé par Alexander J. Matejko.

v. 38, no 3,1983 , p. 689

WILKINSON, Frank

CRAIG, C. RUBERY, Jill

TARLING, Roger WILKINSON, Frank

Abolition and After the Paper Box Wages Council. Research Paper no 12, London, Department of Employment, June 1980, $98 \mathrm{pp}$., recensé par David Bright. v. 39 , no 2,1984 , p. $396-398$

POOLE, Michael BROWN, William RUBERY, Jill SISSON, Keith TARLING, Roger WILKINSON, Frank Industrial Relations in the Future. Trends and Possibilities in Britain over the Next Decade. Agincourt, Methuen Publications, 1984, 148 pp., ISBN 0-7102-0145-1, recensé par Roy J. Adams.

v. 42, no 2,1987, p. $437-439$ 


\section{WILLMAN, Paul}

DAWSON, Sandra WILLMAN, Paul

BAMFORD, Martin CLNTON, Alain Safety at Work : The Limits of Self-Regulation. Cambridge, Cambridge University Press, 1988, 310 pp., ISBN 0-521-354978, recensé par Eric Tucker.

v. 44 , no 4,1989 , p. $959-961$

WINDMULLER, John P. WINDMULLER, John $\mathbf{P}$.

The International Trade Union Movement. Deventer, Kluwer, 1980, 174 pp., recensé par Gérard Dion.

v. 36 , no 2,1981 , p. $455-456$

WINDMULLER, John P.

Collective Bargaining in Industrialised Market Economies: A Reappraisal. Geneva, International Labour Office, 1987, 333 p., ISBN 92-2-105606-6, recensé par Anthony Giles.

v. 45, no 3,1990, p. 630

WINDMULLER, John P.

GLADSTONE, Alan

Employers' Associations and Industrial Relations : A Comparative Study. Oxford, Clarendon Press, 1986, 370 pp., ISBN 019-827260-X, recensé par James Thwaites. v. 44 , no 1,1989 , p. $295-297$

\section{WOLTERS, Roger}

WOLTERS, Roger HOLLEY, William $\mathrm{H}$.

Labour Relations : An Experiential and Case Approach. New York, the Dryden Press, 1988, 321 pp., ISBN 0-03-0693047 , recensé par Mark Thompson.

v. 44 , no 4,1989 , p. $957-958$

WONG, Jim

WONG, Jim

Employment Injuries and Occupational Illnesses 1972-1981. Ottawa Minister of Supply and Services Canada, 1984, 134 pp., ISBN 0-662-53002-0, recensé par Alexander J. Matejko.

v. 39, no 4,1984, p. $814-815$

\section{WOODS, H.D.}

WOODS, H.D.

Relations industrielles dans l'industrie de la construction du sud du Nouveau-Brunswick. Fredericton, N.B., Ministère du tra- vail et de la main-d'oeuvre, mars 1981, 617 pp., recensé par Jean Sexton.

v. 37 , no 1,1982 , p. $240-241$

\section{Z}

\section{ZUBOFF, Shoshana}

RITA, Giuseppe De SILVESTRI, Umberto TEDESCHI, Michele WEISS, Dimitri ZUBOFF, Shoshana

II ruolo del fattore "uomo" nello scenario economico-produttivo degli anni $80: i$ quadri. Roma, STET, 1982, 84 pp., recensé par $P$. Joubert.

v. 39, no 3,1984, p. $637-638$ 



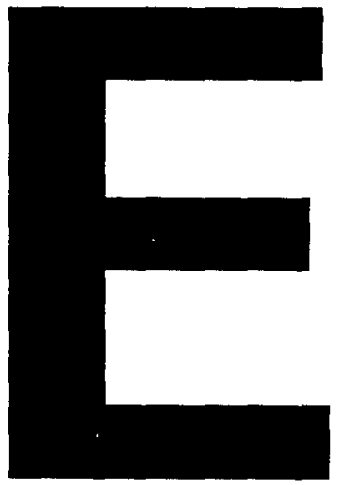

INDEX DES AUTEURS DES ARTICLES ET DES RECENSIONS

INDEX OF AUTHORS OF ARTICLES AND BOOK REVIEWS 
Liste alphabétique des auteurs des articles et des recensions.

\section{EXEMPLE:}

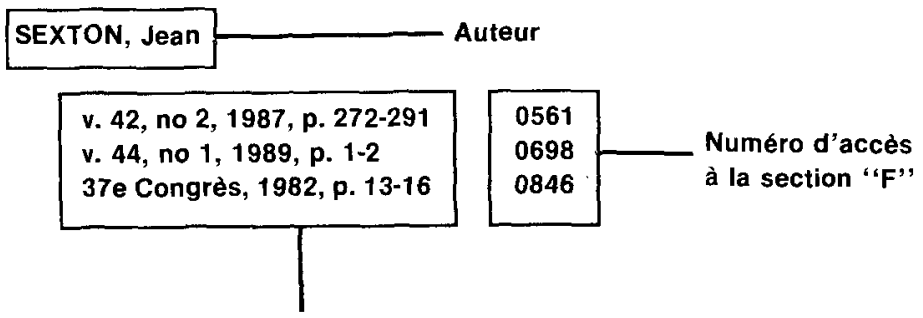

Références bibliographiques des articles

dans "Relations industrielles" ou dans

les "Congrès des relations industrielles"

Lorsqu'il s'agit de l'auteur d'une recension, son nom est immédiatement suivi par la lettre (R).

\section{EXEMPLE:}

SEXTON, Jean (R)

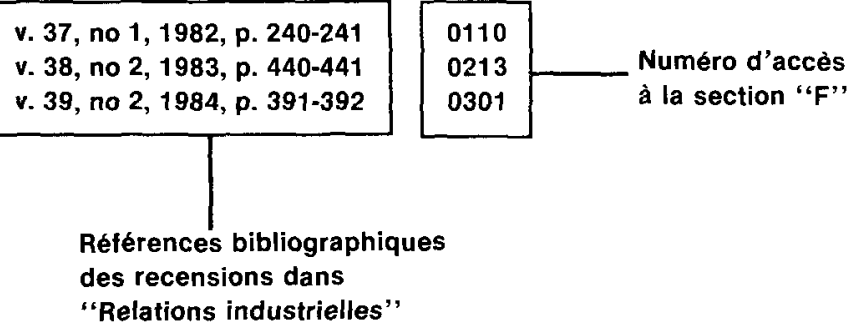

NOTE: Pour obtenir le titre de l'article ou de la recension il suffit pour l'utilisateur de référer à la section " $F$ " par le numéro d'accès. 
Alphabetical list of authors of articles and book reviews.

\section{EXAMPLE:}

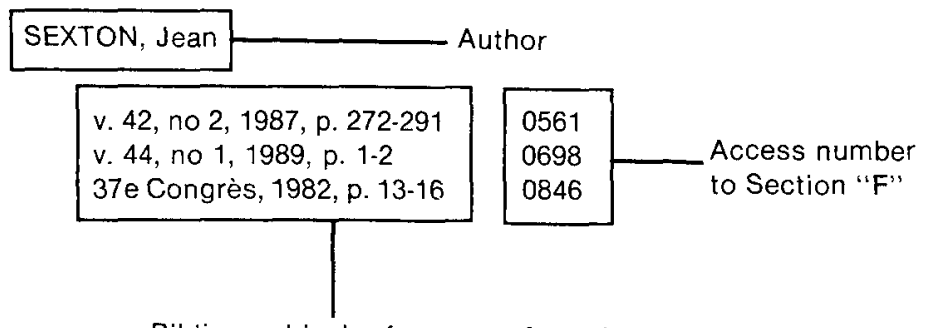

Bibliographical references of articles

in "Industrial Relations" or in "Congrès

des relations industrielles"

The name of the author of a book review is followed by the letter (R).

\section{EXAMPLE:}

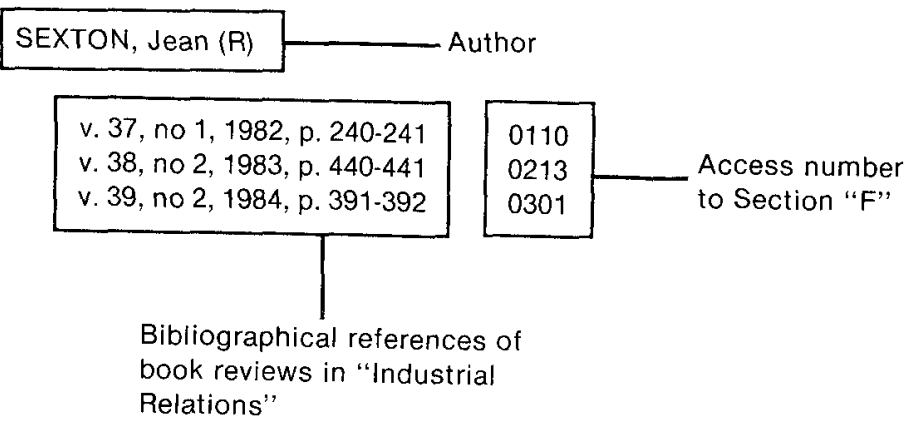

NOTE: To obtain the title of the article or of the book review, user must refer to section " $F$ " by access number. 



\section{A}

ABENHAIM, Lucien

v. 38, no 1,1983, p. $45-57$

ADAMS, Georges W.

v. 36 , no 1,1981 , p. $225-250$

v. 37 , no 3,1982 , p. $606-617$

v. 38 , no 3,1983 , p. 508-531

v. 39 , no 4,1984 , p. $655-667$

v. 42 , no 1,1987, p. $46-64$

ADAMS, Roy J.

v. 37, no 3,1982 , p. $606-617$

v. 38 , no 3,1983 , p. $508-531$

v. 39 , no 4,1984 , p. $655-667$

v. 42 , no 1,1987, p. $46-64$

ADAMS, Roy J. (R)

v. 42 , no 2,1987 , p. $437-439$

v. 42, no 2,1987, p. $448-450$

ADAMSON, Raymond

v. 41 , no 4,1986 , p. $851-859$

AGARWAL, Naresh C.

v. 37, no 4,1982, p. $780-804$

AGARWAL, Naresh C. (R)

v. 39, no 1,1984 , p. $206-208$

ALVI, S.A. (R)

v. 42, no 4,1987, p. $894-895$

AMBROISE, Gérald d'

v. 44, no 3,1989, p. $487-506$

AMERNIC, J.H.

v. 38, no 2,1983, p. $319-343$

\section{ANDIAPPAN, $P$.}

v. 37, no 4,1982 , p. $765-779$

v. 39, no 4,1984 , p. $680-694$

v. 41 , no 3,1986 , p. $491-504$

v. 41 , no 4,1986 , p. $758-777$

v. 44 , no 4,1989 , p. $827-849$

ANDIAPPAN, P. (R)

v. 42 , no 3,1987, p. $649-651$

v. 43, no 2,1988 , p. $469-471$

v. 45, no 2,1990 , p. $430-431$

ANGERS, Bernard (R)

v. 37 , no 2,1982 , p. $458-459$

ANGERS, Georges

44ième Congrès, 1989, p. 85-100 0960

0179

0012

0141

0224

0336

0540

0141

0224

0336

0540

0573

0577

0524

0159

0282

0625

0733

0206

0158

0338

0488

0517

0755

0592

0660

0804

0132
AOUST, Claude d'

v. 36 , no 1,1981 , p. $259-262 \quad 0014$

v. 36 , no 2,1981 , p. $418-424 \quad 0034$

v. 36 , no 4,1981 , p. $894-921 \quad 0078$

v. 38 , no 1, 1983, p. 155-158 0186

v. 38, no 2,1983, p. $415-417 \quad 0210$

v. 39 , no 2,1984 , p. $365-370 \quad 0299$

v. 41 , no 1,1986 , p. $157-180 \quad 0455$

v. 41 , no 2,1986 , p. $348-356 \quad 0473$

v. 43 , no 4,1988 , p. $909-9420690$

AOUST, Claude d' (R)

v. 44, no 3,1989 , p. $732-733 \quad 0748$

AOUST, Fernand d'

36ième Congrès, 1981, p. 219-246 0844

APPARICIO-VALDEZ, Luis

v. 44, no 1,1989, p. 124-148 0704

ARANYA, $\mathbf{N}$.

v. 38, no 2, 1983, p. 319-343 0206

ARSENAULT, André

v. 38, no 1,1983, p. $45-57 \quad 0179$

v. 39 , no 3,1984, p. $509-525 \quad 0317$

v. 42 , no 4,1987 , p. $774-789 \quad 0609$

42ième Congrès, 1987, p. 61-72 0928

ARTHURS, H.W.

v. 39 , no 4,1984, p. $753-761$

ASCAH, Louis (R)

v. 42, no 2,1987 , p. $450-451 \quad 0578$

AUCLAIR, Robert

v. 36, no 1,1981, p. $223-224 \quad 0011$

AUDET, Claude

v. 43, no 4,1988, p. $737-756 \quad 0682$

AUDET, Michel

v. 43 , no 1,1988, p. 3-31 0626

v. 44, no 1,1989 , p. 62-96 0702

41ième Congrès, 1986, p. 13-14 0905

AUDET, Michel (R)

v. 42, no 2,1987, p. $440-443 \quad 0574$

v. 43 , no 1,1988 , p. $199-200 \quad 0638$

v. 43 , no 1,1988 , p. $203-205 \quad 0640$

v. 43, no 2,1988, p. $461-462 \quad 0656$

v. 44 , no 3,1989 , p. $734-736 \quad 0750$

v. 45 , no 1,1990 , p. $211-212 \quad 0789$ 
AULD, D.A.L.

v. 42, no 1,1987, p. $132-149$

0544

\section{B}

BABA, Vishwanath V.

v. 37 , no 1,1982 , p. $126-140$

v. 37 , no 3,1982 , p. $618-633$

v. 42 , no 2,1987 , p. $325-344$

BABCOCK, Robert H. (R)

v. 38 , no 1,1983 , p. $188-189$

BACHAND, Gilles (R)

v. 38 , no 3,1983 , p. $676-677$

BADIN, François

v. 41 , no 1,1986, p. $69-90$

BAR-HAIM, Aviad

v. 39 , no 2,1984 , p. $301-312$

\section{BARBASH, Jack}

v. 42 , no 1,1987 , p. $168-179$

v. 43 , no 1,1988 , p. $32-42$

BARBASH, Jack (R)

v. 43 , no 4,1988 , p. $981-982$

v. 45 , no 1,1990 , p. $204-205$

\section{BARKIN, Solomon}

v. 36 , no 2,1981 , p. $361-370$

v. 38 , no 4,1983 , p. $713-729$

v. 41 , no 1,1986 , p. 3-27

v. 42 , no 1,1987, p. $12-44$

v. 43 , no 3,1988 , p. $491-508$

v. 44 , no 3,1989 , p. $691-702$

v. 44 , no 4,1989 , p. $905-914$

\section{BARKIN, Solomon (R)}

v. 41 , no 2,1986 , p. $417-422$

v. 41 , no 3,1986 , p. $654-658$

\section{BARRÉ, Alain}

v. 42 , no 4,1987, p. $831-851$

43ième Congrès, 1988, p. 131-156

0612

0949

\section{BARRÉ, Alain (R)}

v. 36, no 1, 1981, p. 291-294

v. 36, no 3,1981, p. $696-697$

v. 36, no 4, 1981, p. 951-954

v. 36 , no 4,1981 , p. 951-954

v. 38 , no 1,1983 , p. $189-190$

v. 39 , no 1,1984 , p. 203-205

v. 43, no 4,1988 , p. $974-980$

0100

0564

0194

0232

0450

0296

0546

0627

0697

0785

0028

0245

0447

0539

0664

0743

0759

0478

0503

0024

0064

0088

0089

0195

0280

0695
BARTH, Richard T.

v. 37 , no 1,1982 , p. $126-140 \quad 0100$

BEACH, Charles $M$.

v. 41, no 2,1986, p. 317-328 0471

v. 45 , no 1,1990 , p. $118-1350777$

BEATTY, Carol

v. 44 , no 3,1989 , p. $569-592 \quad 0737$

\section{BEAULIEU, LUC}

v. 38 , no 4,1983 , p. $880-903 \quad 0255$

v. 39 , no 1,1984 , p. 177-189 0277

v. 39, no 2,1984, p. $371-3850300$

v. 39 , no 3,1984 , p. 605-618 0323

v. 43 , no 2,1988 , p. $231-304 \quad 0647$

BEAULIEU, Paul (R)

v. 37, no 1,1982, p. 248

v. 40 , no 4,1985 , p. $892-893 \quad 0436$

v. 41 , no 3,1986 , p. 651-652 0501

v. 42 , no 2,1987, p. $435 \quad 0571$

v. 42, no 3, 1987, p. 664-665 0601

v. 42 , no 4,1987, p. $881-883$

0617

\section{BEAUMONT, P.B.}

v. 37, no 4,1982, p. $827-842 \quad 0161$

v. 38 , no 4,1983 , p. $744-766 \quad 0247$

v. 40 , no 2,1985 , p. $243-256 \quad 0384$

v. 40, no 4,1985 , p. $810-825 \quad 0429$

v. 43 , no 4,1988 , p. $819-846 \quad 0686$

\section{BEAUSOLEIL, Gilles}

36ième Congrès, 1981, p. 145-172 0841

39ième Congrès, 1984, p. 207-242 0885

\section{BÉGIN, Clermont}

v. 37 , no 4,1982 , p. $887-904 \quad 0164$

BÉGIN, Monique

37ième Congrès, 1982, p. 171-180 0854

BEIGBEDER, Yves

v. 37, no 2,1982 , p. 328-343 0122

BÉLAND, Claude

45ième Congrès, 1990, p. 7-14 0967

\section{BÉLANGER, Gérard}

37ième Congrès, 1982, p. 75-98 0850

BÉLANGER, Gérard (R)

v. 44 , no 1,1989 , p. 291-292 0713 v. 37, no 3, 1982, p. 704-709 0147 


\section{BÉLANGER, Jacques}

v. 41 , no 1,1986 , p. $28-52$

v. 41 , no 4,1986, p. $704-719$

38ième Congrès, 1983, p. 13-14

38ième Congrès, 1983 , p. $15-36$

40ième Congrès, 1985, p. 13-15

40ième Congrès, 1985, p. 245-259

BÉLANGER, Jacques (R)

v. 36 , no 3,1981 , p. $689-690$

v. 36 , no 3,1981 , p. $690-692$

v. 36 , no 4,1981 , p. $956-958$

v. 37, no 2,1982, p. $455-457$

v. 38 , no 1,1983, p. $181-184$

v. 39 , no 1,1984 , p. $218-220$

v. 40, no 3,1985 , p. $675-676$

v. 42 , no 4,1987 , p. $891-893$

v. 43 , no 3,1988 , p. $716-717$

v. 45 , no 1,1990 , p. $208-209$

\section{BÉLANGER, Laurent}

v. 44, no 1,1989, p. $62-96$

40ième Congrès, 1985, p. 171-196

41ième Congrès, 1986, p. 13-14

44ième Congrès, 1989, p. XV-XVI

BÉLANGER, Laurent (R)

v. 36 , no 4,1981 , p. $943-944$

v. 36 , no 4,1981 , p. $946-948$

v. 36, no 4,1981 , p. 948

v. 37, no 2,1982 , p. $457-458$

v. 37 , no 2,1982 , p. $459-460$

v. 38 , no 1,1983 , p. $195-196$

v. 38 , no 2,1983 , f $452-453$

v. 38 , no 3,1983 , p. $682-683$

v. 38, no 3,1983, p. $683-684$

v. 39 , no 4,1984 , p. $805-806$

v. 39, no 4,1984 , p. $809-811$

v. 45, no 2,1990, p. 432

\section{BÉLANGER, Marc}

44ième Congrès, 1989, p. 13-32

\section{BÉLANGER, Paul R.}

v. 42 , no 2,1987, p. $345-365$

\section{BELLEMARE, Diane}

45ième Congrès, 1990, p. 117-128 0978

\section{BEMMELS, Brian}

v. 41 , no 3,1986 , p. $596-621$

\section{BENEDICT, Daniel}

v. 40, no 1,1985, p. $27-47$
0958

0565

0496

0448

0514

0858

0859

0887

0903

0061

0062

0092

0130

0191

0290

0416

0623

0680

0787

0702

0898

0905

0956

0081

0084

0085

0131

0133

0200

0220

0236

0237

0353

0355

0805

0363

\section{BENEDICT, Daniel (R)}

v. 39 , no 3,1984 , p. $626-630 \quad 0324$

v. 40 , no 1,1985, p. $192-194 \quad 0380$

\section{BENOIT, Michel}

37ième Congrès, 1982, p. 35-44 0848

BERGERON, Jean-Louis

43ième Congrès, 1988, p. 51-62 0944

BERGERON, Jean-Louis (R)

v. 41 , no 4,1986 , p. $885-886$

0536

\section{BERNIER, Colette}

40ième Congrès, 1985, p. 137-160 0896

\section{BERNIER, Gilles}

42ième Congrès, 1987, p. 223-241 0937

BERNIER, Jean

v. 38 , no 3,1983, p. $532-544 \quad 0225$

BERNIER, Lionel

42ième Congrès, 1987, p. 177-211 0935

BETCHERMAN, Gordon

v. 41 , no 4,1986 , p. $802-816 \quad 0519$

BHÉRER, Harold

40ième Congrès, 1985, p. 205-222 0900

\section{BLACK, Errol}

v. $36,1,02,1981$, p. $414-417 \quad 0033$

v. 40, no 1,1985 , p. $140-161 \quad 0369$

v. 45, no 1, 1990, p. 146-165 0779

v. 45 , no 3,1990 , p. $612-615 \quad 0819$

BLAIS, Michel

44ième Congrès, 1989, p. 101-127 0961

BLANCHARD, Francis

v. 37, no 1,1982, p. 3-14 0094

v. 42 , no 1,1987 , p. 3-11 0538

BLANCHETTE, Sylvain

44ième Congrès, 1989, p. 129-154 0962

BLONDIN, Michel

45ième Congrès, 1990, p. 15-23 0968

BLOUIN, Rodrigue

v. 39 , no 3.1984 , p. $578-604 \quad 0322$

v. 42, no 2,1987 , p. $309-324 \quad 0563$

39ième Congrès, 1984, p. 15-16 0873

40ième Congrès, 1985, p. 13-15 0887

40ième Congrès, 1985, p. 17-32 0888

43ième Congrès, 1988, p. XV 0941 
Relations Industrielles, vol. 45, no 4 (1990)

\section{BLOUIN, Rodrigue (R)}

v. 36 , no 1,1981 , p. 275

v. 36 , no 1,1981, p. $288-289$

v. 36 , no 2,1981 , p. $451-452$

v. 36, no 2,1981, p. $453-454$

v. 37, no 4,1982 , p. $966-967$

v. 38 , no 2,1983 , p. $441-442$

v. 38 , no 2,1983 , p. $444-445$

v. 39, no 4,1984, p. $812-813$

v. 41, no 3,1986, p. $658-659$

v. 42 , no 2,1987 , p. $436-437$

v. 45 , no 3,1990 , p. $637-640$

BLYTON, Paul

v. 41 , no 4,1986, p. $851-859$

BLYTON, Paul (R)

v. 37, no 3,1982 , p. $717-718$

BOIS, Hélène (R)

v. 41 , no 4,1986 , p. $879-880$

BOIS-BROCHU, Hélène

40ième Congrès, 1985, p. 261-281 0904

BOIS-BROCHU, Hélène (R)

v. 40, no 2,1985 , p. $414-415$

v. 40, no 3,1985, p. $683-685$

BOISVERT, Maurice

v. 36 , no 4,1981, p. $775-802$

BOIVIN, Jean

v. 42 , no 1,1987, p. $179-196$

38ième Congrès, 1983, p. 13-14

41 ième Congrès, 1986, p. 13-14

41ième Congrès, 1986, p. 17-30

BOIVIN, Jean (R)

v. 36 , no 1,1981 , p. $275-276$

v. 36 , no 1,1981 , p. $280-282$

v. 40, no 3,1985, p. $676-678$

v. 44, no 3,1989, p. $730-732$

v. 44 , no 3,1989, p. $733-734$

v. 45 , no 1,1990 , p. 210

v. 45 , no 1,1990 , p. 212

BOIVIN, Suzanne $P$.

43ième Congrès, 1988, p. 97-113

0402

0422

0073

0547

0858

0905

0906

0017

0019

0417

0747

0749

0788

0790

0947

BOLDUC, Roch

v. 37, no 2,1982, p. $403-430$

0126

\section{BONENFANT, Claire}

37ième Congrès, 1982, p. 107-145 0852 38ième Congrès, 1983, p. 207-223 0871
BONIN, Bernard

38ième Congrès, 1983, p. 70-76

0861

BOUCHARD, Lucien

v. 37, no 2,1982 , p. $403-430 \quad 0126$

BOUCHARD, Robert

39ième Congrès, 1984, p. 113-135 0879

42ième Congrès, 1987, p. $72-86 \quad 0929$

BOUCHER, Bernard (R)

v. 36, no 3,1981, p. 697-699 0065

BOUCHER, Lysette

41ième Congrès, 1986, p. 77-84 0911

BOUDREAULT, Plerre

45ième Congrès, 1990, p. 103-116 0977

BOULARD, René

v. 36 , no 3,1981 , p. $648-662$

v. 38 , no 4,1983 , p. $847-862 \quad 0252$

36ième Congrès, 1981, p. 13-14 0832

39ième Congrès, 1984, p. 15-16 0873

39ième Congrès, 1984, p. 137-157 0880

BOULARD, René (R)

v. 36, no 3,1981 , p. 701-702 0067

v. 36, no 3,1981 , p. $702-703 \quad 0068$

v. 38, no 3,1983, p. $686 \quad 0239$

v. 38 , no 3,1983 , p. $689-690 \quad 0242$

BOURASSA, Raynald (R)

v. 37, no 3,1982, p. 720

0155

v. 39 , no 1,1984 , p. $220-221$

0291

BOURGEOIS, Robert-Paul

v. 42, no 3,1987, p. $528-543 \quad 0583$

v. 45, no 3,1990 , p. $512-530 \quad 0814$

BOUTEILLER, Dominique

v. 40, no 4,1985, p. $703-719 \quad 0424$

BRETON, Réjean (R)

v. 44, no 2,1989, p. $466-468 \quad 0728$

BRIGHT, David (R)

v. 39, no 2,1984, p. $396-398 \quad 0305$

BRIOLAT, Dominique (R)

v. 39 , no 1,1984 , p. $197-201 \quad 0278$

BRISSON, Chantal

v. 36 , no 1, 1981, p. 152-178 0007

42ième Congrès, 1987, p. 21-38 0925 


\section{BRODY, Bernard}

v. 40, no 3,1985 , p. $545-566$

0409

v. 45, no 1, 1990, p. 94-117

0776

42ième Congrès, 1987, p. 241-252

0938

\section{BROSSARD, Michel}

v. 36 , no 3,1981 , p. $552-575$

0053

v. 37, no 3,1982, p. $670-683$

v. 41 , no 2,1986 , p. $219-235$

v. 44, no 3,1989 , p. $552-568$

v. 45, no 1,1990, p. $3-21$

BROUILLET, Normand

45ième Congrès, 1990 , p. $70-81$

BRUCE, Christopher J.

v. 36, no 2,1981 , p. $386-402$

BRUCE, Peter G.

v. 45, no 3,1990 , p. $481-511$

BRUNET, Jacques

39ième Congrès, 1984, p. 195-206

BRUNET, LUC

v. 39, no 4,1984, p. $668-679$

0337

BRUNET, YveS $W$.

v. 43, no 2,1988, p. $231-304$

BRYCE, George $K$.

v. 40, no 2,1985, p. $257-283$

BURKE, Ronald J.

v. 39 , no 3.1984 , p. $570-577$

v. 40, no 1,1985 , p. $162-169$

BURT, Sandra

v. 41 , no 3,1986 , p. $541-551$

\section{C}

\section{CABELLY, Alan}

v. 36, no 1,1981 , p. $87-105$

\section{CAIRE, GuY}

v. 38 , no 1,1983 , p. $3-27$

0177

v. 39 , no 2,1984, p. $235-258$

0292

38 ième Congrès, 1983, p. 225-265 0872

\section{CAMERON, Samuel}

v. 38, no 4,1983, p. $767-784$

0248

v. 40, no 2,1985, p. $367-370$

0466

0736

0771

0385

0491
CAMIRÉ, André

v. 43, no 2,1988, p. $322-340 \quad 0649$

\section{CAMPBELL, Adrian}

v. 43, no 1,1988 , p. 115-132 0632

CANTIN, Isabelle

v. 43 , no 2,1988 , p. 231-304 0647

CARROTHERS, A.W.R.

V. 40 , no 2,1985, p. $351 \quad 0390$

v. 45 , no 3,1990 , p. $585-611 \quad 0818$

CARTER, Donald D.

v. 43 , no 2,1988 , p. $305-321 \quad 0648$

v. 44 , no 2,1989, p. $337-353 \quad 0720$

CARTER, Donald D. (R)

v. 44, no 2,1989 , p. $468-469 \quad 0729$

CASAVANT, Jean-Claude

41ième Congrès, 1986, p. 53-61 0908

CATTANEO, R. Julian

v. 39, no 4,1984 , p. $680-694 \quad 0338$

CHAISON, Gary N.

v. 36 , no 3,1981 , p. $530-551 \quad 0052$

v. 37, no 1,1982 , p. 198-206 0104

v. 37, no 4,1982 , p. $765-779 \quad 0158$

CHARLAND, Gaston

45ième Congrès, 1990, p. 128-148 0979

CHARLES, Lawrence $M$.

v. 40, no 3,1985, p. $495-511 \quad 0406$

CHAYKOWSKI, Richard $\mathbf{P}$.

v. 41 , no 4,1986 , p. $720-737 \quad 0515$

v. 45, no 2,1990, p. $326-356 \quad 0797$

CHERMESH, Ran

v. 36, no 3, 1981, p. 630-647 0057

CHIASSON, Denis-Émile 42ième Congrès, 1987, p. 9-19 0924

CHOUINARD, Jacques

v. 37, no 1, 1982, p. 207-214 0105

v. 37, no 1,1982, p. $220-224 \quad 0107$

CHOUINARD, Jacques (R)

v. 38 , no 4,1983 , p. $913-9150258$

CHRÉTIEN, Lise (R)

v. 40 , no 4,1985 , p. $902-903 \quad 0442$

v. 40, no 4,1985 , p. $904-905 \quad 0444$

v. 42 , no 3,1987 , p. $646-648 \quad 0591$ 
CHRISTENSEN, Sandra

v. 36 , no 3,1981 , p. $616-629$

CLACK, Garfield (A)

v. 42 , no 4,1987 , p. $889-890$

CLARKE, Graham

v. 44 , no 4,1989 , p. $933-946$

v. 45 , no 1,1990 , p. 187-197

v. 45 , no 3,1990, p. $616-624$

CLARKE, Oliver

v. 42 , no 1,1987, p. $196-202$

CLÉMENT, Pierre R.

39ième Congrès, 1984 , p. 113-135 0879

COOK, Alice $\mathrm{H}$.

v. 42, no 3,1987, p. $520-527$

COPP, Terry

v. 37, no 4,1982, p. $843-875$

CORMIER, Roger A.

v. 37 , no 4,1982 , p. $805-826$

CÔTÉ, André C.

v. 44 , no 4,1989 , p. $751-768$

CÔTÉ, André C. (R)

v. 38, no 2,1983 , p. $437-440$

v. 38, no 2,1983 , p. $446-448$

v. 38 , no 3,1983 , p. $690-691$

v. 39 , no 4,1984 , p. $795-797$

CÔTÉ, Marcel

40ième Congrès, 1985, p. 196-203 0899

COULOMBE, Gilles

v. 37 , no 4,1982 , p. $928-943$

0166

COUSINEAU, Jean-Michel

v. 36 , no 1,1982 , p. $214-220$

v. 38 , no 4,1983 , p. $730-743$

v. 39, no 1,1984 , p. 3-22

v. 41, no 3,1986 , p. $451-468$

v. 43 , no 1,1988 , p. $85-100$

v. 45, no 3,1990, p. $467-480$

CRAIG, Alton W.J. (R)

v. 39 , no 2,1984, p. $393-394$

v. 41, no 3,1986, p. $644-646$

CRAWFORD, Ronald L.

v. 36, no 2,1981, p. $325-335$

0782

0820

0548

0582

0162

0160

0751

0212

0218

0243

0346

0246

0268

0486

0630

0812

0303

0498

0026

\section{CRESTOHL, Mark}

v. 44 , no 4,1989 , p. $827-849$

0755

CROCKER, Olga

v. 43 , no 1, p. $167-182$

0635

CROWLEY, R.W.

v. 38 , no 3,1983 , p. $636-647 \quad 0230$

v. 43 , no 2,1988 , p. $394-411 \quad 0652$

v. 45 , no 2,1990 , p. $382-403 \quad 0799$

CUNNINGHAM, William B. (R)

v. 36 , no 2,1981, p. $461-462 \quad 0049$

DELORME, François

v. 36 , no 4,1981 , p. $894-921 \quad 0078$

DASTMALCHIAN, Ali

v. 41 , no 4,1986 , p. $851-8590524$

v. 45, no 2,1990 , p. $311-325 \quad 0796$

DAVID, Hélène (R)

v. 42, no 3,1987 , p. $642-646 \quad 0590$

DE KONINCK, Maria

39ième Congrès, 1984, p. 159-175 0881

DELAMOTTE, Yves

v. 42, no 1, 1987, p. $92-109 \quad 0542$

DELANEY, John T.

v. 44 , no 1,1989 , p. $97-123 \quad 0703$

v. 37 , no 3,1982 , p. $575-605 \quad 0140$

DEMERS, Marie

v. 38 , no 4,1983 , p. $785-814 \quad 0249$

\section{DENTON, Margaret}

v. 45, no 3,1990 , p. $531-547 \quad 0815$

\section{DÉOM, André}

36ième Congrès, 1981 , p. 219-246 0844

DÉOM, Esther

v. 37 , no 1, 1982 , p. $141-163$

v. 39, no 1,1984, p. $114-145 \quad 0274$

40ième Congrès, 1985, p. 161-170 0897

41ième Congrès, 1986, p. 13-14 0905 v. 42 , no 4,1987, p. $737-755 \quad 0607$ 
DÉOM, Esther (R)

v. 39 , no 4,1984 , p. $816-817$

v. 41 , no 1,1986 , p. 191-192

v. 41 , no 3,1986 , p. $652-654$

v. 45 , no 1,1990 , p. $203-204$

v. 45, no 2,1990 , p. $438-439$

DÉPATIE, Raymond

37ième Congrès, 1982, p. 98-105

DESCHÊNES, Jean-Paul

v. 39 , no 2,1982 , p. $313-327$

36ième Congrès, 1981, p. 13-14

36ième Congrès, 1981 , p. 136-144

37ième Congrès, 1982, p. 13-16

39ième Congrès, 1984, p. 15-16

DESMARAIS, Jacques

38ième Congrès, 1983, p. 101-116 0864

43ième Congrès, 1988, p. 157-195 0950

DEVORETZ, D.J.

v. 39 , no 3,1984 , p. $526-537$

0318

DICKINSON, John A.

v. 41 , no 2,1986 , p. $357-381$

0474

DILTS, David A.

v. 45, no 1,1990, p. $22-37$

0772

DIMMOCK, Stuart J.

v. 41, no 4,1986, p. $738-757$

DION, Gérard

v. 44 , no 1,1989, p. $1-2$

v. 44 , no 1,1989, p. $5-24$

38ième Congrès, 1983, p. 162-171

0516

0698

0699

0867

DION, Gérard (R)

v. 36 , no 2,1981 , p. $455-456$

0044

v. 36 , no 4,1981 , p. $948-949$

v. 36 , no 4,1981 , p. 954

v. 37 , no 1,1982 , p. $245-246$

v. 37, no 2,1982 , p. 460

v. 37, no 3,1982, p. $709-712$

v. 38, no 4,1983, p. $912-913$

v. 39, no 2,1984, p. $402-403$

v. 41 , no 4,1986 , p. $878-879$

v. 41 , no 4,1986 , p. $886-887$

v. 43, no 2,1988 , p. 473

v. 45 , no 2,1990, p. $429-430$

v. 45, no 2,1990, p. $441-442$

DION, Stéphane

v. 39 , no 3.1984 , p. $466-485$

0086

0090

0113

0134

0148

0257

0309

0529

0537

0663

0803

0811

0315
DIONNE, Denis

44ième Congrès, 1989, p. 155-173 0963

DIONNE, Pierre

v. 38, no 2,1983, p. $344-368 \quad 0207$

DOCQUIER, Gérard

41ième Congrès, 1986, p. 149-156 0917

DOLAN, Shimon L.

v. 38 , no 1, 1983, p. 45-57 0179

v. 39 , no 1,1984 , p. $167-176 \quad 0276$

v. 40 , no 1,1985 , p. $87-98 \quad 0366$

v. 42, no 4,1987, p. $774-789 \quad 0609$

DOOLEY, Martin D.

v. 42 , no 3,1987 , p. $594-611 \quad 0587$

DORIS DUFFY, Ann

v. 43, no 3,1988, p. $660-685 \quad 0672$

DORNSTEIN, Miriam

v. 41 , no 1,1986 , p. $91-110 \quad 0451$

DOUCET, René

40ième Congrès, 1985, p. 48-54 0890

DOW, Bradley

v. 41, no 2,1986, p. $236-255 \quad 0467$

DOWNIE, Bryan $M$.

v. 36, no 1,1981, p. $63-86 \quad 0003$

v. 45, no 1,1990, p. $169-186 \quad 0781$

DROUIN, Claude

39ième Congrès, 1984, p. 183-194 0883

DUBÉ, Jean-Louis (R)

v. 36 , no 2,1981 , p. $449-451 \quad 0037$

DUCHARME, Claude

40ième Congrès, 1985, p. 121-136 0895

43ième Congrès, 1988, p. 63-82 0945

DUCHESNE, André

42ième Congrès, 1987, p. 212-222 0936

DUFOUR, Ghislain

36ième Congrès, 1981, p. 45-59 0835

38ième Congrès, 1983, p. 207-223 0871

39ième Congrès, 1984, p. 243-276 0886

42ième Congrès, 1987, p. 253-276 0939 
Relations Industrielles, vol. 45, no 4 (1990)

DUGuaY, Plerre

42ième Congrès, 1987 , p. $72-86$

0929

DULUDE, Gilles

43ième Congrès, 1988, p. 195-208 0951

DULUDE, Yves

41ième Congrès, 1986, p. 111-1330914

\section{DUMAS, Anne-Chantal}

39ième Congrès, 1984 , p. 93-111 0878

DUMONT, Micheline (R)

v. 43, no 1,1988, p. 205

\section{DUPONT, Serge}

v. 43 , no 1,1988 , p. $153-166$

\section{DUSSAULT, Gilles}

v. 40 , no 2,1985 , p. $324-339$

43ième Congrès, 1988, p. 83-95

DUSSAULT, Gilles (R)

v. 39 , no 1,1984 , p. $209-210$

v. 39 , no 3,1984 , p. $630-631$

v. 40 , no 1,1985, p. $186-188$

v. 40, no 2,1985, p. $407-408$

v. 40, no 2,1985, p. $412-414$

\section{E}

\section{EASTMAN, Byron}

v. 38 , no 1,1983 , p. 58-71

v. 40, no 2,1985, p. $340-350$

EVANS, Martin G.

v. 41 , no 3,1986 , p. $572-577$

\section{$\mathbf{F}$}

FABI, Bruno

v. 39, no 2,1984, p. $313-334$

FAILLE, Jacques

37ième Congrès, 1982, p. 45-74

\section{FAJANA, Sola}

v. 44, no 3,1989 , p. $615-634$

\section{FALABELLA, Gonzalo}

v. 44 , no 1,1989 , p. $47-61$

0641

0634

0388

0946

0284

0325

0377

0397

0401

0180

0701
FARQUHAR, Alec

42ième Congrès, 1987, p. 115-130 0932

FASHOYIN, Tayo

v. 36 , no 1,1981, p. $207-2220010$

FAVA, Frank

39ième Congrès, 1984, p. 243-276 0886

\section{FERLAND, Gilles}

v. 36 , no 3,1981 , p. $475-498 \quad 0050$

36ième Congrès, 1981 , p. 87-121 0838

37ième Congrès, 1982, p. 13-16 0846

43ième Congrès, 1988, p. XV

0941

FERLAND, Gilles $(R)$

v. 40, no 4,1985, p. 895

0438

v. 42 , no 1,1987 , p. $218-2190552$

FINKELMAN, Jacob

V. 41 , no 4,1986 , p. $691-703 \quad 0513$

FISHER, E.G.

v. 37, no 2,1982, p. $284-312 \quad 0120$

v. 38 , no 2,1983 , p. $254-276 \quad 0203$

v. 39 , no 3,1984, p. $538-552 \quad 0319$

v. 40 , no 1,1985, p. $115-1390368$

v. 41, no 3,1986, p. $596-621 \quad 0496$

v. 41 , no 4,1986 , p. $778-801 \quad 0518$

FISHER, E.G. (R)

v. 38, no 4, 1983, p. 915-916 0259

FONTAINE, Claude

44ième Congrès, 1989, p. 33.84 0959

FORREST, Anne

v. 41 , no 4,1986 , p. $840-850 \quad 0523$

v. 44 , no 2,1989 , p. $393-408 \quad 0723$

FORTIN, Bernard

36ième Congrès, 1981, p. $75-86 \quad 0837$

FORTIN, Pierre

v. 39 , no 3,1984, p. $419-448 \quad 0313$

FRANCOEUR, Jean

41ième Congrès, 1986, p. 157-166 0918

FRANK, David (R)

v. 43 , no 1,1988, p. $200-203 \quad 0639$

FRANK, J.A. 


\section{FRAPPIER-DESROCHERS, Monique}

36ième Congrès, 1981, p. 193-218 0843

45ième Congrès, 1990, p. 25-39 0969

FRÉCHET, Guy (R)

v. 43, no 2,1988 , p. $468-4690659$

\section{FRÉCHETTE, Raynald}

38ième Congrès, 1983, p. 197-206 0870

FREEMAN, Richard $B$.

v. 44 , no 1,1989, p. $25-46$

0700

FRENETTE, Jean-Guy

44ième Congrès, 1989, p. 155-173 0963

FRICKE, John G.

v. 43, no 3,1988 , p. $633-658$

0671

FRIED, Yatzhak

v. 41 , no 2,1986 , p. $329-347$

0472

\section{G}

\section{GAGNON, Astrid}

44ième Congrès, 1989, p. 129-154 0962

\section{GAGNON, Denise}

44ième Congrès, 1989, p. 101-127 0961

\section{GAGNON, Jacques (R)}

v. 41 , no 3,1986, p. $669-670$

0510

v. 41 , no 3,1986 , p. $670-671$

0511

v. 41, no 3,1986 , p. $671-672$

0512

\section{GAGNON, Jean-Denis}

40ième Congrès, 1985, p. 33-47 0889

GAGNON, Mona-Josée

43ième Congrès, 1988, p. 222-236 0953

GAGNON, Normand

44ième Congrès, 1989, p. 85-100 0960

GAGnON, Pierre (R)

v. 39 , no 1,1984, p. $197-201$

0278

GAGNON, Robert P.

40ième Congrès, 1985, p. 116-119 0894

GAGNON, Yves-Chantal

v. 44 , no 2,1989, p. $421-447$

0725

\section{GALBRAITH, John Kenneth}

37ième Congrès, 1982, p. 193-213 0856

GALLAGHER, Daniel G.

v. 39 , no 3,1984, p. $486-508 \quad 0316$

v. 40 , no 4,1985, p. $793-809 \quad 0428$

GANDZ, Jeffrey

v. 44 , no 3,1989 , p. $569-592 \quad 0737$

\section{GARANT, Patrice}

43ième Congrès, 1988, p. 237-254 0954

GARNIER, Bernard

v. 38, no 2,1983 , p. 277-296 0204

GARON, Jacques

45ième Congrès, 1990, p. 103-116 0977

GASCON, Hélène (R)

v. 45 , no 3,1990, p. $635-637 \quad 0827$

GASSE, Yvon (R)

v. 39 , no 1,1984, p. 197-201 0278

GAUTHIER, Fernand

38ième Congrès, 1983, p. 183-195 0869

GAUTHIER, Hervé

36ième Congrès, 1981, p. 145-172 0841

GAUTHIER, Hervé (R)

v. 37 , no 1,1982 , p. $246-248 \quad 0114$

GAUTHIER, Michel

44ième Congrès, 1989, p. 101-1270961

GENDRON, Michel

42ième Congrès, 1987, p. 223-241 0937

GEORGE, Kenneth

v. 40 , no 3,1985, p. $512-528 \quad 0407$

v. 41 , no 3,1986, p. 583-595 0495

GERA, Surendra

v. 37 , no 1,1982, p. $65-92 \quad 0098$

GIASSON, Étienne

39ième Congrès, 1984, p. 113-1350879

GILES, Anthony

v. 37 , no 1,1982 , p. 93-125 0099

v. 40 , no 4,1985 , p. $747-774 \quad 0426$

v. 43 , no 4,1988 , p. $780-811 \quad 0684$ 
GILES, Anthony (R)

v. 41 , no 2,1986, p. $428-430$

v. 41 , no 3,1986, p. $664-667$

v. 42 , no 3,1987 , p. 667-669

v. 43 , no 2,1988 , p. $462-466$

v. 43, no 3,1988, p. $703-707$

v. 43, no 3,1988, p. $703-707$

v. 45, no 3,1990, p. 630

GILUS, L.P.

v. 42 , no 2,1987 , p. $256-271$

GILSON, Clive H.J.

v. 40, no 4,1985 , p. $856-864$

v. 41 , no 2,1986 , p. $390-396$

v. 42 , no 2,1987 , p. $256-271$

v. 42 , no 4,1987 , p. $756-773$

v. 44 , no 4,1989 , p. $785-804$

GILSON, Clive H.J. (R)

v. 40, no 3,1985 , p. $678-680$

GINGRAS, Claude

42ième Congrès, 1987, p. 212-222 0936

GOBEILLE, Kenneth R.

45ième Congrès, 1990, p. 48-58 0971

GODBOUT, Clément

40ième Congrès, 1985, p. 222-228 0901

44ième Congrès, 1989, p. 175-188 0964

GODIN, Jean

v. 41 , no 3,1986 , p. $505-518$

0489

GODIN, Michel

45ième Congrès, 1990 , p. 59-64

0972

GOLDENBERG, Shirley $B$.

v. 38, no 4,1983, p. $707-712$

GONTHIER, Jean-Marie

42ième Congrès, 1987, p. 72-86

0929

\section{GOSSELIN, Alain}

44ième Congrès, 1989, p. 155-173 0963

GOSSELIN, Louis (R)

v. 41 , no 1,1986 , p. $189-190$

0456

GRANT, Hugh $M$.

v. 45, no 1,1990 , p. $166-168$

0780

GRANT, Michel

v. 40, no 3,1985 , p. $473-494$
GRANT, Michel (R)

v. 45 , no 1,1990, p. $205-208 \quad 0786$

GRANVILLE, $\mathbf{S}$.

v. 44 , no 4,1989 , p. $785-804 \quad 0753$

GRAYSON, J. Paul

v. 44 , no 3,1989 , p. $635-653 \quad 0740$

GRIEZIC, F.J.K. (R)

v. 36 , no 3,1981 , p. 704-705 0070

v. 37 , no 1,1982, p. $243-245 \quad 0112$

v. 39 , no 3,1984 , p. $638-641 \quad 0332$

v. 41, no 1,1986 , p. 202-203 0464

GRUBEL, Herbert G.

v. 36 , no 4,1981 , p. $922-927 \quad 0079$

GUÉRARD, Yves

37ième Congrès, 1982, p. 107-145 0852

GUÉRIN, Gilles

v. 44 , no 2,1989, p. $354-375 \quad 0721$

v. 45, no 2,1990, p. $235-267 \quad 0792$

GUILLEMETTE, Michel

39ième Congrès, 1984, p. 113-135 0879

GUNDERSON, Morley

v. 40 , no 4,1985, p. $775-792 \quad 0427$

GUNDERSON, Morley (R)

v. 38, no 4,1983, p. $925-927 \quad 0264$

v. 44, no 2,1989 , p. $465-466 \quad 0727$

GURDON, Michael A.

v. 39 , no 2,1984 , p. $259-274$

v. 42, no 3,1987, p. $566-575 \quad 0585$

H

HACCOUN, Robert $R$.

v. 43 , no 1,1988 , p. 153-166 0634

HAMEED, Syed M.A.

v. 37, no 1,1982, p. $15-31$

v. 42, no 2,1987 , p. $243-255 \quad 0559$

HAMELIN, Jean-Marie

36ième Congrès, 1981, p. 87-121 0838

HARGUINDEGUY, Jean-Louis

42ième Congrès, 1987, p. 241-252 0938 
HARRIS, Philip

v. 37, no 4,1982, p. $905-926$

0165

HARRIS, R.I.D.

v. 43, no 4,1988 , p. $819-846$

0686

\section{HARVEY, Pierre}

36ième Congrès, 1981, p. 219-246 0844

\section{HASAN, Abrar}

v. 37 , no 1,1982, p. $65-92$

0098

\section{HÉBERT, Gérard}

v. 36, no 4,1981, p. $715-747$

0071

v. 37, no 2,1982, p. $403-430$

0126

v. 37 , no 3,1982 , p. $475-476$

v. 38 , no 4,1983 , p. $707-712$

v. 40, no 2,1985, p. $211-212$

v. 42 , no 3,1987 , p. $500-519$

v. 44 , no 1,1989 , p. 5-24

v. 44 , no 4,1989, p. $884-904$

v. 45, no 2,1990, p. $404-413$

HÉBERT, Gérard (R)

v. 37 , no 4,1982 , p. $962-963$

v. 41, no 3,1986 , p. $646-650$

0135

0244

0381

0581

0699

0758

0800

HÉBERT, Hervé

37ième Congrès, 1982, p. 147-169 0853

HÉBERT, Michel

v. 45, no 2,1990, p. $235-267$

0792

HEENAN, Roy

39ième Congrès, 1984, p. 159-175 0881

HÉTU, Jean-Paul

38ième Congrès, 1983, p. 207-223 0871

39ième Congrès, 1984 , p. 243-276 0886

41ième Congrès, 1986, p. 167-172 0919

HOLMES, R.H.

v. 39 , no 3,1984 , p. $553-569 \quad 0320$

v. 43 , no 1,1988 , p. $133-152 \quad 0633$

HUGUES, Karen D.

v. 44 , no 3,1989 , p. $654-679$

0741

HULL, Dennis

v. 43 , no 2,1988 , p. $394-411 \quad 0652$

HUMPHREYS, Edward $\mathrm{H}$.

v. 40 , no 3,1985, p. $495-511$

0406
HURTUBISE, Rolland (R)

v. 39 , no 4,1984, p. $801-802 \quad 0349$

v. 41, no 2,1986 , p. $433-435 \quad 0485$

HUTH, E.

v. 38 , no 3,1983 , p. $636-647$

0230

\section{I}

ICHNIOWSKI, Casey

v. 44 , no 1,1989, p. $97-123$

0703

INGERMAN, Sidney

38ième Congrès, 1983, p. 37-69 0860

IVANKOVICH, I.F.

v. 40 , no 1,1985, p. $115-139$

0368

\section{$\checkmark$}

JAIN, Hem C.

v. 36 , no 4,1981 , p. $748-774 \quad 0072$

v. 37, no 2,1982 , p. $344-366 \quad 0123$

v. 38, no 4,1983 , p. $869-879 \quad 0254$

v. 40, no 4,1985, p. $747-774 \quad 0426$

v. 41, no 4,1986 , p. $758-777 \quad 0517$

v. 42 , no 4,1987 , p. $790-805 \quad 0610$

v. 44, no 3,1989, p. $593-614 \quad 0738$

v. 44 , no 4,1989 , p. $850-8650756$

JAIN, Hem C. (R)

v. 38 , no 1, 1983, p. 194-195 0199

v. 39 , no 1,1984, p. $215-217 \quad 0288$

v. 42 , no 2,1987 , p. $443-445 \quad 0575$

v. 44 , no 1,1989 , p. $289-291 \quad 0712$

v. 45 , no 3,1990 , p. $631-6320823$

JAMAL, Muhammad

v. 36 , no 2,1981 , p. $325-3350026$

v. 37, no 3,1982 , p. $618-633 \quad 0142$

JECCHINIS, Chris (R)

v. 44 , no 3,1989 , p. $729-730 \quad 0746$

JÉRÔME-FORGET, Monique

42ième Congrès, 1987, p. 277-285 0940

JOHNSTON, Raymond

$43 i e ̀ m e$ Congrès, 1988 , p. 222-236 0953
JOLIVET, Thierry

v. 38, no 1, 1983, p. 142-154 0185 
JONES, J.C.H.

v. 39 , no 4,1984 , p. $695-709$

JOSEPH, P.A.

v. 38, no 2,1983, p. $380-414$

v. 38, no 4,1983 , p. $863-868$

JOUBERT, P. (R)

v. 38 , no 1,1983 , p. $190-192$

v. 38 , no 1,1983 , p. $192-194$

v. 38 , no 1,1983, p. $192-194$

v. 38 , no 4,1983 , p. $916-920$

v. 39, no 3,1984 , p. $634-635$

v. 39, no 3,1984, p. $637-638$

v. 41 , no 2,1986 , p. $423-424$

\section{K}

KALISKI, S.F.

v. 41 , no 2,1986 , p. $317-328$

KAMEL, Nawal

v. 43, no 2,1988, p. $412-430$

KAMINKA, Shlomit

v. 38, no 3,1983, p. $598-617$

KARIM, Ahmad

v. 45 , no 1,1990, p. $22-37$

KATASANEVAS, Theodoros

v. 40 , no 1,1985 , p. $99-114$

KELLER, Berndt

v. 43, no 2,1988, p. $431-446$

KELLY, Laurence

v. 42, no 3,1987, p. $475-499$

KELLY, Michael J.

v. 37 , no 2,1982 , p. $267-283$

KNIGHT, Thomas $R$.

v. 41 , no 2,1986, p. $281-298$

v. 42, no 4,1987, p. $716-736$

\section{KNOOP, Robert}

v. 42 , no 2,1987, p. $325-344$

v. 44 , no 2,1989 , p. $409-420$

KOCHAN, Thomas A.

v. 44, no 1, 1989, p. 224-248 0709

41ième Congrès, 1986, p. 181-189 0922

0471

0654

0580

0469

0606

0564

0724
KRAHN, Harvey

0339

v. 39 , no 1,1984 , p. $93-113$

0273

KRUGER, Arthur

v. 40 , no 1,1985 , p. $48-67$

0364

0253

KUMAR, Pradeep

v. 41 , no 2, 1986, p. 236-255 0467

0196

0197

0198

0260

0328

0331

0479

v. 42 , no 4,1987, p. $687-701$

v. 43, no 4,1988 , p. 757.779

KUSHNER, Stephen

v. 41 , no 4,1986, p. $778-801 \quad 0518$

0653

0228

0772

0367

LABELLE, Christiane

v. 44, no 2,1989, p. $354-375 \quad 0721$

v. 45 , no 3,1990, p. $512-530 \quad 0814$

LABERGE, Louis

39ième Congrès, 1984 , p. 243-276 0886 41ième Congrès, 1986, p. 173-175 0920 42ième Congrès, 1987, p. 253-276 0939 43ième Congrès, 1988, p. 255-272 0955

LACOSTE, Paul

v. 40 , no 2,1985 , p. $213-2180382$

LACROIX, Robert

v. 36, no 1, 1982, p. 214-220 0106

v. 38 , no 4,1983 , p. $730-743 \quad 0246$

v. 39 , no 1,1984 , p. 3-22 0268

v. 41 , no 1,1986, p. 111-127 0452

v. 43, no 4,1988, p. $812-828 \quad 0685$

LAFLAMME, Gilles

v. 42 , no 4,1987, p. $702-715 \quad 0605$

38ième Congrès, 1983 , p. 77-90 0862

42ième Congrès, 1987, p. 89-100 0930

43ième Congrès, 1988, p. XV 0941

LAFLAMME, Lucie

v. 39, no 3,1984, p. 509-525 0317

v. 43, no 3,1988 , p. $591-608 \quad 0669$

LAFONTAINE, Réal

\section{LAFOREST, Martial}

37ième Congrès, 1982, p. 107-145 0852

LAFRANCE, Victor

V. 37 , no 3,1982 , p. 575-605 0140 
LAJOIE, Mario

40ième Congrès, 1985, p. 261-281 0904

LAJOIE, Mario (R)

v. 37 , no 1,1982 , p. 251-252

0117

LALANDE, Serge

41ième Congrès, 1986, p. 137-146 0916

\section{LAMARCHE, André}

44ième Congrès, 1989, p. 101-127 0961

\section{LAMARCHE, Pierre}

41ième Congrès, 1986, p. 133-136 0915

\section{LAMONTAGNE, Maurice}

37ième Congrès, 1982, p. 213-218 0857

\section{LAMSON, Cynthla}

v. 41 , no 1,1986, p. $145-156$

\section{LAMY, Francine}

v. 40, no 2,1985 , p. $386-398$

v. 40, no 3,1985 , p. $659-662$

v. 40 , no 4,1985 , p. $880-885$

\section{LANDRY, Maurice}

V. 44 , no 2,1989, p. $421-447$

\section{LANGLOIS, Simon}

v. 45 , no 3,1990, p. 548-565

\section{LAPORTE, Pierre}

v. 42 , no 2,1987 , p. $398-414$

\section{LAPORTE, Pierre (R)}

$$
\text { v. } 42 \text {, no } 4,1987 \text {, p. } 876-877
$$

\section{LAROCQUE, Alain}

36ième Congrès, 1981, p. 13-14

36ième Congrès, 1981, p. 15-29

42ième Congrès, 1987 , p. 89-100

43ième Congrès, 1988, p. XV

0454

0394

0415

0434

0725

0816

0568

0614

0832

0833

0930

0941

\section{LAROCQUE, Alain (R)}

v. 37 , no 3,1982 , p. $718-719$

v. 37, no 3,1982, p. $718-719$

0153

0154

\section{LAROSE, Gérald}

39ième Congrès, 1984 , p. 243-276 0886 41ième Congrès, 1986, p. 177-179 0921 42ième Congrès, 1987 , p. 253-276 0939 43ième Congrès, 1988, p. 255-272 0955

\section{LAROUCHE, Vlateur}

v. 38, no 3,1983 , p. $568-597 \quad 0227$

v. 39 , no 1,1984 , p. $114-145 \quad 0274$

v. 43 , no 1,1988 , p. $3-31 \quad 0626$

v. 43 , no 3,1988 , p. $509-530 \quad 0665$

v. 45 , no 1,1990 , p. $38-62 \quad 0773$

41ième Congrès, 1986, p. 31-51 0907

LARRIVÉE, Llette

V. 44, no 3,1989, p. $487-506$

0733

LARSON, Peter E. (R)

v. 40, no 1,1985 , p. $180-181 \quad 0373$

v. 40, no 4,1985 , p. 906

0445

LATULIPPE, Gérard $P$.

v. 37 , no 3,1982, p. $634-655 \quad 0143$

LAUDADIO, L.

v. 39, no 4,1984 , p. $695-709 \quad 0339$

\section{LAVALLÉE, Diane}

45ième Congrès, 1990, p. 128-148 0979

\section{LAVERY, Daniel}

v. 36 , no 4,1981 , p. $928-936 \quad 0080$

v. 37 , no 1,1982 , p. $226-234 \quad 0109$

v. 37, no 2,1982 , p. $438-450 \cdot 0128$

v. 37, no 3,1982 , p. $684-696 \quad 0146$

v. 37 , no 4,1982 , p. $944-959 \quad 0167$

v. 38, no 1, 1983, p. 159-173 0187

v. 38 , no 2,1983 , p. $418-432 \quad 0211$

v. 38 , no 3,1983 , p. $658-666 \quad 0231$

LAVIGNE, Marie (R)

v. 36 , no 2,1981 , p. $456-457 \quad 0045$

v. 36 , no 2,1981 , p. $458 \quad 0046$

\section{LEBEAU, Ernest}

43ième Congrès, 1988, p. 222-236 0953

LEBEL, Hélène

38 ième Congrès, 1983 , p. 91-100 0863

\section{LEBEL, Maurice (R)}

v. 41 , no 4,1986 , p. $883-884 \quad 0533$

v. 42 , no 1,1987, p. $225-227 \quad 0558$

v. 42 , no 2,1987 , p. $445-448 \quad 0576$

LE BRASSEUR, Lola

44ième Congrès, 1989, p. 13-32 0958

LEBRASSEUR, Rolland

v. 44 , no 2,1989 , p. $376-392 \quad 0722$

v. 45, no 2,1990 , p. $268-282 \quad 0793$ 
Relatjons Industrielles, vol. 45, no 4 (1990)

LECLERC, Claudine

v. 36 , no 2,1981, p. $435-448$

LECLERCQ, Dominique

43ième Congrès, 1988 , p. 63-82

LEDUC, Jean-Guy

39ième Congrès, 1984 , p. $50-72$

LEE, Tanya

v. 44 , no 1,1989 , p. $195-223$

LEFÈBVRE, Marie-Claire

42ième Congrès, 1987, p. 155-176 0934

LE LOUARN, Jean-Yves

v. 37, no 2, 1982, p. 385-402 0125

v. 39 , no 1,1984, p. $36-50$

v. 41 , no 3,1986, p. $505-518$

v. 44 , no 2 , 1989 , p. $354-375$

45ième Congrès, 1990 , p. 93-103

LEMAIRE, Alain

41ième Congrès, 1986, p. 63-68

LEMELIN, Maurice

39ième Congrès, 1984 , p. 50-72

\section{LEMELIN, Maurice (R)}

v. 39, no 3,1984 , p. $633-634$

v. 43, no 4,1988 , p. 974

LÉONARD, Christine

v. 42 , no 4,1987 , p. $774-789$

0270

0489

0721

0976

0909

0876

0327

0694

0609

\section{LEPAGE, Brigitte}

45ième Congrès, 1990, p. 128-148 0979

LEQUIN, Jacques-André (R)

v. 45 , no 3 , 1990 , p. $627-629$

0821

LESAGE, Michel

42ième Congrès, 1987, p. 39-50

LESAGE, Pierre B.

v. 36 , no 4,1981 , p. $775-802$

44ième Congrès, 1989 , p. 33-84

LESPÉRANCE, André

v. 43, no 4,1988, p. $812-828$

LESSARD, Claude

v. 37 , no 4,1982 , p. $805-826$

LÉTOURNEAU, Yves

v. 45 , no 1,1990 , p. $94-117$

0926

0073

0959

0685

0776
LEVANONI, Eliahu

v. 44 , no 2,1989, p. $409-420$

0724

LEVASSEUR, Raymond

42ième Congrès, 1987, p. 155-176 0934

LÉVESQUE, Benoît

v. 42 , no 2,1987, p. $345-3650565$

LÉVESQUE, Christian

v. 40 , no 4,1985, p. $703-719 \quad 0424$

LEVINE, David

45ième Congrès, 1990 , p. 41-47 0970

\section{LEWIN, David}

v. 44 , no 1,1989 , p. $97-123 \quad 0703$

LIPSIG, Carla

44ième Congrès, 1989, p. XV-XVI 0956

LIPSIG, Carla (R)

V. 42 , no 2,1987 , p. $452-454 \quad 0579$

v. 45 , no 1,1990, p. $202-203 \quad 0783$

LIPSIG-MUMME, Carla

v. 38 , no 3,1983 , p. $545-567 \quad 0226$

v. 39, no 2, 1984, p. 275-284 0294

LISTON, Terrence

45ième Corıgrès, 1990, p. 65-69 0973

L'ITALIEN, Paul

44ième Congrès, 1989 , p. 13-32 0958

LIZOTTE, Jean-Paul

v. 38 , no 1,1983 , p. $45-57 \quad 0179$

LOCHHEAD, Clarence

v. 44 , no 3,1989, p. $507-531 \quad 0734$

LOCKWELL, LUC M.

36ième Congrès, 1981, p. 173-192 0842

LONG, Richard J.

v. 42 , no 1,1987, p. $65-91$

0541

v. 44 , no 4,1989 , p. $805-826 \quad 0754$

LORRAIN, Jean

v. 39, no 4,1984, p. $668-679 \quad 0337$

LORTIE, Pierre

36ième Congrès, 1981, p. 173-192 0842

LOUMÈDE, Catherine 
LOVELL, Malcolm R. Jr.

v. 44 , no 1. 1989 , p. $162-176$

LOWE, Graham $\mathbf{S}$.

v. 36 , no 4,1981, p. $865-893$

v. 39 , no 1,1984, p. $93-113$

LUCE, Sally $R$.

v. 40 , no 3,1985 , p. $529-544$

LUCENA, Hector

v. 44 , no 1,1989 , p. $249-282$

\section{M}

MACNAUGHTON, Bruce D.

v. 37 , no 2,1982, p. $267-283$

MAC NEIL, Michael

v. 43, no 4,1988, p. $847-868$

\section{MAGUN, Sunder}

v. 37 , no 3,1982 , p. $528-556$

v. 38 , no 1,1983, p. $72-94$

v. 40 , no 4,1985 , p. $720-746$

\section{MAKI, Dennis $R$.}

v. 36 , no 4,1981 , p. $922-927$

v. 37 , no 4,1982 , p. $876-886$

v. 38 , no 2,1983, p. $211-228$

v. 39 , no 1,1984, p. $77-92$

v. 41 , no 1,1986 , p. $54-68$

MAKI, Dennis R. (R)

v. 36 , no 1,1981 , p. $289-291$

0023

MALLETTE, Noël (R)

v. 39 , no 3,1984, p. $643-645$

0335

MANGA, Pran

v. 40 , no 2,1985 , p. $257-283$

0385

MARCHAND, Jean

40ième Congrès, 1985, p. 229-244 0902

MARCHAND, Michel

v. 38 , no 4,1983, p. $847-862 \quad 0252$

MARCHIS-MOUREN, Marie Françoise (R)

v. 38 , no 3,1983 , p. $679-680$

0234

\section{MARION, Gérald}

v. 37 , no 1,1982, p. 53-64

0181

0079

0163

0201

0272

0449

\section{MARKGRAF, Herb}

v. 38 , no 1,1983, p. $120-141 \quad 0184$

MARLEAU, Véronlque L.

v. 43, nว 4, 1988 , p. $953-962 \quad 0692$

v. 44 , no 2,1989 , p. $448-462 \quad 0726$

v. 44 , no 3,1989, p. $703-722 \quad 0744$

v. 45 , no 2,1990, p. $414-423 \quad 0801$

\section{MARTIN, Ross}

v. 36 , no 2,1981, p. $414-417 \quad 0033$

\section{MARTINEAU, LUC}

v. 36 , no 1,1981, p. $263-266 \quad 0015$

v. 36 , no 2,1981, p. $424-4290035$

v. 36 , no 3,1981, p. $589-615 \quad 0055$

v. 36 , no 3,1981, p. $671-680 \quad 0060$

MASSE, Gilles

36ième Congrès, 1981, p. 123-135 0839

MASSÉ, Henri

45ième Congrès, 1990, p. 128-148 0979

MATEJKO, Alexander J. (R)

v. 36 , no 1,1981, p. $285-288 \quad 0021$

v. 36 , no 4,1981, p. $949-951 \quad 0087$

v. 37 , no 3,1982, p. $715-717 \quad 0151$

v. 37, no 4,1982, p. $964-966$

0169

v. 38 , no 2,1983 , p. $448-452 \quad 0219$

v. 38 , no 3,1983, p. $681-682 \quad 0235$

v. 38 , no 3,1983, p. $685-686 \quad 0238$

v. 38 , no 3,1983 , p. $687-688 \quad 0240$

v. 38, no 3,1983, p. 689

0241

v. 38, no $4,1983$. p. $927-929 \quad 0265$

v. 39 , no $1,1984,0.205-206 \quad 0281$

v. 39, no 1, 1984, p. 208-209 0283

v. 39 , no 1,1984, p. $212-215 \quad 0286$

v. 39, no 1,1984, p. $212-215 \quad 0287$

v. 39 , no 1, 1984, p. $217-218 \quad 0289$

v. 39, no 2, 1984, p. 392-393 0302

v. 39 , no 2, 1984, p. 395-396 0304

v. 39 , no 2,1984, p. $398-399 \quad 0306$

v. 39 , no 3,1984, p. $636 \quad 0329$

v. 39 , no 3,1984, p. $641-642$

v. 39 , no 4,1984, p. $799-801 \quad 0348$

v. 39, no 4,1984, p. $813-814 \quad 0358$

v. 39 , no 4,1984, p. $814-8150359$

v. 39 , no 4,1984 , p. $815-816 \quad 0360$

v. 40 , no 1,1985, p. $185-186 \quad 0376$

v. 40 , no 1,1985, p. $188-190 \quad 0378$

v. 40, no 1,1985, p. $190-1920379$

v. 40 , no 2,1985, p. $405-407 \quad 0396$

v. 40 , no 2,1985, p. $411-412 \quad 0400$

v. 40, no 3,1985, p. 680

0419 
Relations Industrielles, vol. 45, no 4 (1990)

MATEJKO, Alexander J. (R) (suite)

v. 40 , no 3,1985 , p. $682-683$

v. 40, no 3,1985 , p. $685-686$

v. 40 , no 4,1985 , p. $896-898$

v. 41, no 2,1986 , p. $425-426$

v. 41 , no 2,1986 , p. $431-432$

v. 41 , no 3,1986, p. $662-664$

v. 41 , no 3,1986, p. 667

v. 41 , no 3, 1986, p. 667-668

v. 41 , no 4,1986 , p. $881-882$

v. 41 , no 4,1986 , p. $881-882$

v. 42 , no 1,1987, p. $224-225$

v. 42 , no 3,1987 , p. $665-667$

v. 43 , no 1,1988, p. 211

v. 43, no 2,1988 , p. $466-468$

v. 43 , no 2,1988 , p. $471-472$

v. 43 , no 3,1988 , p. $714-715$

v. 43 , no 3,1988 , p. $718-719$

v. 44 , no 1,1989 , p. $292-293$

v. 44, no 1,1989 , p. $294-295$

v. 44, no 2,1989 , p. $470-472$

v. 44 , no 4,1989 , p. $961-962$

v. 44, no 4,1989, p. $964-967$

v. 45 , no 1,1990 , p. $213-214$

v. 45 , no 2,1990 , p. 440

v. 45 , no 3,1990 , p. 634

\section{MATHIEU, René}

45ième Congrès, 1990 , p. $82-92$

MATON, BOb

v. 43 , no 4,1988 , p. $869-889$

MAUSER, Gary

v. 43, no 1,1988, p. $133-152$

MAXEY, Charles T.

v. 40, no 4,1985, p. $793-809$

MAYER, Udo (R)

v. 42 , no 4,1987 , p. $880-881$

MAZEROLLE, Maurice J. (R)

v. 45 , no 2,1990 , p. $432-434$

MCKERSIE, Robert B.

v. 44 , no 1,1989, p. $224-248$

MCPHILLIPS, David C.

v. 36 , no 4,1981 , p. $803-827$

v. 43 , no 1,1988 , p. $63-84$

MCSHANE, Steven L.

v. 38, no 3,1983 , p. $618-635$

v. 40, no 2,1985 , p. $284-302$

v. 45, no 2,1990, p. $357-381$
MCWATTERS, Catherine J.

v. 45 , no 1,1990 , p. $118-135$

0777

0423

0439

0480

0483

0506

0508

0509

0531

0532

0557

0602

0646

0658

0661

0679

0681

0714

0716

0731

0767

0770

0791

0810

0825

0975

MEALIA, David W.

v. 37, no 3,1982 , p. $656-669 \quad 0144$

MELTZ, Noah $M$.

v. 37, no 2,1982 , p. $431-437 \quad 0127$

v. 44, no 1,1989 , p. 149-161 0705

MÉNARD, Marius

44ième Congrès, 1989, p. 13-32 0958

MERCIER, Jacques

v. 40 , no 3,1985, p. $431-457 \quad 0403$

v. 41, no 1, 1986, p. 28-52 0448

v. 42 , no 4,1987 , p. $806-830$

41ième Congrès, 1986, p. 13-14 0905

45ième Congrès, 1990, p. 1-5 0966

MERCIER, Jacques (R)

v. 37 , no 1,1982 , p. $249-251 \quad 0116$

MERCIER, Jean

v. 41 , no 2,1986 , p. 299-316 0470

45ième Congrès, 1990, p. 128-148 0979

MERCIER, Jean (A)

v. 44, no 2,1989 , p. $469-470 \quad 0730$

0688

MERCILLE, Pierre

38ième Congrès, 1983, p. 117-134 0865

MEREDITH, Lindsay $\mathbf{N}$.

v. 41, no 1,1986, p. $54-68 \quad 0449$

0428

MICHAUD, Sharon

v. 37, no 1,1982 , p. $224-2250108$

0616

MILLER, Roger

v. 37, no 1, 1982, p. 164-176 0102

v. 40, no 1. 1985 , p. $68-86 \quad 0365$

0806

MILNE, William J.

v. 39 , no 4,1984 , p. $762-773 \quad 0343$

0709

0074

0629

0229

0386

0798

\section{MITCHELL, Lari}

v. 45 , no 2,1990 , p. $382-403 \quad 0799$

MITCHELL, Vance F.

v. 37, no 3,1982 , p. 618-633 0142

MONTMINY, Jean-Paul

36ième Congrès, 1981, p. 31-45 0834 
MOORE, Larry F.

v. 36 , no 1,1981 , p. $3-34$

v. 38 , no 1,1983, p. $120-141$

MOORE, William R.

v. 36, no 4,1981, p. $848-864$

\section{MORIN, Fernand}

v. 39 , no $4,1984, p .744-752$

v. 40 , no 2,1985, p. $371-378$

v. 40 , no 3,1985, p. $629-645$

v. 40 , no 3,1985 , p. $646-658$

v. 40 , no 4,1985, p. $847-855$

v. 41 , no 3,1986, p. $578-582$

v. 41 , no 4,1986 , p. $835-839$

v. 43 , no 4,1988, p. $943-952$

v. 44 , no 2,1989, p. $315-336$

v. 44 , no 4,1989, p. $921-932$

v. 45 , no 3,1990 , p. $566-584$

40ième Congrès, 1985, p. 13-15

40ième Congrès, 1985, p. 75-115

42ième Congrès, 1987 , p. 131-155

44ième Congrès, 1989, p. XV-XVI

44ième Congrès, 1989 , p. 1-12

MORIN, Fernand (R)

v. 37 , no 4,1982 , p. $967-968$

v. 39 , no 4,1984 , p. $793-795$

v. 40 , no 4,1985, p. $907-910$

v. 42 , no 3,1987, p. $655-656$

v. 42 , no 4,1987 , p. $893-894$

v. 45 , no 3,1990, p. $640-643$

Mosco, Vincent

v. 44 , no 3,1989 , p. 507-531

MOUNT, Joan

v. 38, no 4,1983 , p. $815-830$

\section{MROCZKOWSKI, Tomasz}

v. 38, no 2,1983, p. $297-318$

MUCKENBERGER, UIrich

v. 45 , no 1,1990, p. $136-145$

MURPHY, John

v. 39 , no 4,1984 , p. $680-694$

\section{MURRAY, Grégor}

v. 43, no 4,1988, p. $780-811$

\section{$\mathbf{N}$}

\section{NADEAU, Bertin}

38ième Congrès, 1983, p. 117-134
0001

0184

0076

0341

0392

0413

0414

0431

0494

0522

0691

0719

0761

0817

0887

0893

0933

0956

0957

0171

0345

0446

0595

0624

0829

0734

0250

0205

0778

0338

0684

\section{PAGÉ, Lorainne}

45ième Congrès, 1990, p. 103-116 0977

\section{PAQUETTE, Pierre}

45ième Congrès, 1990, p. 103-116 0977

0812

0910

0567

0752

0796

0308

0496

0143

0588

OLIVIER, Madeleine

38ième Congrès, 1983, p. 117-134 0865

ONDRACK, Daniel A.

v. 41 , no 3,1986 , p. $572-577 \quad 0493$

OSBERG, Lars

v. 40, no 4,1985 , p. $865-879 \quad 0433$

OUELLET, Florian

39ième Congrès, 1984, p. 73-92 0877

OUELLET, Gilles

v. 38, no 2,1983 , p. $344-368 \quad 0207$

OUELLET, Lionel

42ième Congrès, 1987, p. 101-113 0931

\section{P}

0865 
PAQUIN, Michel (R)

v. 38 , no 2,1983 , p. $455-456$

0222

v. 39 , no 3,1984 , p. $636-637$

v. 39, no 4,1984 , p. $811-812$

v. 41 , no 2,1986 , p. $426-427$

PARENT, Louise

40ième Congrès, 1985, p. 48-54 0890

PARIZEAU, Jacques

37ième Congrès, 1982, p. 181-192 0855

PARROT, Jean-Claude

43ième Congrès, 1988, p. 195-208 0951

PEACH, David A.

v. 37, no 1,1982 , p. 177-197 0103

PECCEI, Ricardo

v. 36 , no 1, 1981, p. 132-151 0006

PEITCHINIS, Stephen G.

v. 38, no 1,1983, p. 104-119 0183

PEPERMANS, Raymond

v. 43 , no 1,1988 , p. $183-187$

PÉPIN, Marcel

43ième Congrès, 1988, p. 209-221

0952

PERCY, M.B.

v. 38, no 2,1983, p. $254-276$

0203

\section{PERREAULT, Charles}

38ième Congrès, 1983, p. 173-182 0868

\section{PERRON, Jacques}

37ième Congrès, 1982, p. 147-169 0853

\section{PÉRUSSE, Michel}

39ième Congrès, 1984, p. 15-16

39ième Congrès, 1984, p. 17-31

42ième Congrès, 1987, p. 1-8

0873

0874

0923

44ième Congrès, 1989, p. XV-XVI 0956

PÉRUSSE, Michel (R)

v. 36, no 3,1981, p. $702-703$

0068

\section{PETERS, Frank}

v. 38 , no 1,1983, p. $95-103$

0182

PETERSON, Richard $B$.

v. 36 , no 1,1981 , p. 87-105

0004

\section{PETIT, André (R)}

v. 36, no 3, 1981, p. 703-704 0069

v. 36 , no 4,1981 , p. $944-9450082$

PETTERSEN, Normand (R)

v. 38, no 4,1983 , p. $910-9120256$

PHILLIPS, Paul (R)

v. 39, no 1, 1984, p. 210-211 0285

PICARD, Danielle (R)

v. 36, no 3, 1981, p. 699-701 0066

PIGANIOL, Claude

v. 39 , no 2,1984 , p. $285-300 \quad 0295$

PINFIELD, L.T.

v. 39 , no 3,1984 , p. $553-569 \quad 0320$

PIUZE, Guy

40ième Congrès, 1985, p. 69-74 0892

PLAMONDON, Denise

39ième Congrès, 1984, p. 183-194 0883

PLANTE, Gilles

v. 41 , no 4,1986, p. $817-834 \quad 0521$

PLANTE, Gilles (R)

v. 42, no 2,1987, p. $432-434 \quad 0570$

PLASSE, Micheline

v. $42, \mathrm{nc} 3,1987$, p. $544-565 \quad 0584$

PLISZKIEWICZ, Marek

v. 36 , no 1, 1981, p. 251-259 0013

POIRIER, André

v. 45, no 1,1990, p. $94-117$

0776

PONAK, Allen

v. 36, no 1, 1981, p. 3-34 0001

v. 39 , no 3,1984, p. $449-465 \quad 0314$

v. 41, no 3,1986 , p. $552-571 \quad 0492$

POOLE, Michael

v. 36, no 1,1981 , p. $35-620002$

PORTIS, Bernard (R)

v. 42, no 1, 1987, p. 221-222 0554

v. 43 , no 1,1988 , p. $206-207 \quad 0642$

v. 44, no 1,1989 , p. $297 \quad 0718$ v. 44 , no 4,1989, p. $963-964$

0769 


\section{POTHIER, Dianne}

v. 39 , no 4,1984 , p. $774-782$

0344

v. 40 , no 1,1985, p. $170-174$

0371

\section{POULIN-SIMON, LIse}

36 ième Congrès, 1981 , p. $75-86 \quad 0837$

37ième Congrès, 1982, p. 147-169 0853

45ième Congrès, 1990, p. 1-5 0966

POULIN-SIMON, Lise (R)

v. 42 , no 4,1987 , p. $887-889$

v. 43, no 3,1988, p. $707-710$

PRAGNELL, Brad (R)

v. 45 , no 3,1990 , p. $643-647$

PRÉVOST, Charles

42ième Congrès, 1987, p. 51-60

\section{PROULX, Pierre-Paul}

v. 37, no 3,1982 , p. $477-497$

v. 41 , no 2,1986 , p. $382-389$

36ième Congrès, 1981, p. 87-121

PUPO, Norene

v. 43, no 3,1988, p. $660-685$

0672

\section{$\mathbf{Q}$}

QUINTAL, Pierre

44ième Congrès, 1989, p. 129-154 0962

\section{$\mathbf{R}$}

\section{RABEAU, Yves}

v. 43 , no 1,1988 , p. $85-100$

\section{RAINVILLE, Jean-Marie}

v. 36 , no 2,1981 , p. $336-360$

v. 38 , no 4,1983, p. $831-846$

RAINVILLE, Jean-Marie (R)

v. 37 , no 4,1982 , p. $971-973$

RAMOS, Ellas T. (R)

v. 37 , no 3,1982 , p. $720-722$

\section{RaUSCH, Peter}

v. 37 , no 4,1982 , p. $905-926$

REDEKOP, Bruce

v. 45 , no 2,1990, p. $357-381$

0621

0676

0830

0927

0475

0838

0251

0175

0156

0165

0798
REED, Clyde G.

$$
\text { v. } 39 \text {, no } 3,1984, \text { p. } 526-537 \quad 0318
$$

REID, Frank

v. 43 , no 1,1988 , p. 101-114 0631

RENAUD, Robert

v. 44, no 2, 1989, p. 376-392 0722

\section{RESHEF, Yonatan}

v. 41 , no 2,1986 , p. $329-347 \quad 0472$

v. 42 , no 1,1987 , p. $150-167$

0545

v. 43 , no 1,1988 , p. $43-62 \quad 0628$

v. 44, no 3,1989 , p. $532-551 \quad 0735$

v. 45 , no 1,1990, p. $76-93$

0775

RICHARDS, John

v. 43 , no 1,1988 , p. $133-152 \quad 0633$

RICHER, Jean-Charles

v. 40 , no 2,1985 , p. $379-3850393$

RIOUX, Claude

44ième Congrès, 1989, p. 175-188 0964

ROBAKC, Léo

v. 40 , no 4,1985, p. $826-846 \quad 0430$

ROBB, Roberta Edgecome

v. 39 , no 1,1984 , p. $146-166 \quad 0275$

ROBICHAUD, Jean-Marc

v. 37 , no 1,1982, p. $220-224 \quad 0107$

ROCHER, GuY

43ième Congrès, 1988, p. 1-18 0942

RODRIGUE, Norbert

36ième Congrès, 1981, p. 45-59 0835

ROHAN, Paul

v. 40, no 3,1985 , p. $545-566 \quad 0409$

ROHLING, Thomas A.

v. 41 , no 4,1986 , p. $817-826 \quad 0520$

ROJOT, Jacques

43ième Congrès, 1988, p. 19-49 0943

ROMPRÉ, Louise

v. 40 , no 3,1985, p. $545-566 \quad 0409$

RONDEAU, Alain (R) 
RONDEAU, Claude

v. 41 , no 1,1986, p. $69-90$

36ième Congrès, 1981, p. 13-14

38ième Congrès, 1983, p. 13-14

38 ième Congrès, 1983, p. 15-36

43ième Congrès, 1988, p. XV

ROSE, Joseph B.

v. 36, no 3, 1981, p. 530-551

v. 41 , no 2,1986 , p. $256-280$

ROSE, Joseph B. (R)

v. 43 , no 4,1988 , p. $980-981$

ROSE, Ruth

v. 40 , no 3,1985, p. $473-494$

ROSEINSTEIN, Eliezer

v. 38, no 3,1983, p. $598-617$

ROSEN, Hjalmar

v. 41 , no 1,1986, p. $128-144$

ROSS, Claudette

v. 45 , no 1,1990, p. 3-21

Ross, Thomas $W$.

v. 39, no 4,1984, p. $762-773$

ROSSILION, Claude

v. 40 , no 1,1985, p. $03-26$

ROUSSEAU, Léontine

44ième Congrès, 1989, p. 189-252 0965

ROY, André (R)

v. 36, no 2,1981 , p. $460-461$

v. 40, no 1,1985, p. $181-183$

v. 43, no 1,1988 , p. 210

0048

0374

0645

ROY, Denis

v. 39 , no 1,1984 , p. 167-176

0276

ROY, Jean-Charles

v. 37, no 1,1982, p. 224-225 0108

ROY, Paul-Martel

v. 42 , no 3,1987, p. $577-593$

0586

v. 43, no 2,1988 , p. $412-430 \quad 0653$

v. 45 , no 2,1990 , p. $283-299 \quad 0794$

38ième Congrès, 1983, p. 183-195 0869

RYAN, Samuel G.

v. 37 , no 4,1982 , p. $905-926$

0165

\section{$\mathbf{S}$}

SACK, Jeffrey

v. 44 , no 1,1989 , p. $195-223 \quad 0708$

SAINT-GERMAIN, Catherine

v. 41 , no 2, 1986, p. $397-411 \quad 0477$

v. 41 , no 3,1986 , p. $622-637 \quad 0497$

v. 41 , no 4,1986, p. $860-868 \quad 0525$

v. 42 , no 1,1987, p. $203-209 \quad 0549$

v. 42 , no 2,1987, p. $415-427 \quad 0569$

v. 42 , no 3,1987, p. $628-6350589$

SAINT-JEAN, Sylvain

v. 41 , no 1,1986 , p. $157-180$

SAINT-LAURENT, Jacques

37ième Congrès, 1982, p. 13-16 0846

SALES, Carol $A$.

v. 44, no 2,1989, p. $409-420 \quad 0724$

SARTIN, Pierrette

v. 36, no 2,1981, p. $307-324 \quad 0025$

SASS, Robert

v. 40 , no 3,1985 , p. $591-6220411$

SASS, Robert (R)

v. 41 , no 2,1986, p. $432-433 \quad 0484$

v. 42 , no 3,1987, p. $653-655 \quad 0594$

SAUNDERS, George

v. 40 , no 2,1985 , p. $219-242$

SAUVÉ, Robert

39ième Congrès, 1984 , p. 243-276 0886

SAVOIE, Dominique

v. 43 , no 3,1988 , p. $509-530 \quad 0665$

v. 45 , no 1,1990 , p. $38-62 \quad 0773$

SCHELL, Bernadette

v. 44, no 2,1989, p. $376-392 \quad 0722$

SEFTON MACDOWELL, Laurel

v. 43 , no 3,1988, p. $609-632 \quad 0670$

SEN, Joya

V. 42 , no 2,1987 , p. 243-255 0559

SEREDIAK, Martin S. (R)

v. 45 , no 2,1990, p. $435-438 \quad 0808$

SÉRIEYX, Hervé

41ième Congrès, 1986, p. 85-97 0912 


\section{SETHI, Amarjt S.}

v. 41 , no 4,1986, p. $738-757$

\section{SEXTON, Jean}

v. 42 , no 2,1987 , p. $272-291$

v. 44 , no 1,1989 , p. $1-2$

37ième Congrès, 1982, p. 13-16 38ième Congrès, 1983, p. 13-14 40ième Congrès, 1985, p. 13-15

\section{SEXTON, Jean (R)}

v. 37 , no 1,1982 , p. $240-241$

v. 37, no 4,1982, p. 973

v. 38 , no 1,1983, p. 178

v. 38 , no 2,1983 , p. $440-441$

v. 39 , no 2,1984 , p. $391-392$

v. 40, no 4,1985 , p. $894-895$

v. 43, no 2,1988, p. 472

v. 44, no 2,1989, p. 473

\section{SHAND, Dan}

v. 41 , no 3,1986 , p. $491-504$

SHARMA, Basu (R)

v. 43 , no 1,1988 , p. $208-209$

SHERWOOD, L.M.

v. 39, no 3,1984, p. $538-552$

\section{SHIROM, Arie}

v. 40, no 2,1985, p. $303-323$

\section{SIDIBE, Hamadoun (R)}

v. 39 , no 1,1984 , p. 197-201

SIEGEL, Jacob P.

v. 38, no 4,1983, p. $815-830$

\section{SILVER, JIm}

v. 45 , no 1,1990, p. $146-165$

v. 45 , no 3,1990, p. $612-615$

\section{SIMARD, Jean}

44ième Congrès, 1989, p. 85-100

\section{SIMARD, Marcel}

v. 40, no 4,1985 , p. $703-719$

v. 41 , no 2,1986 , p. 219-235

39ième Congrès, 1984, p. 33-50

SIMARD, Marcel (R)

v. 40, no 4,1985, p. $891-892$
SIMARD, Monique

0516

39ième Congrès, 1984 , p. $93-1110878$

40ième Congrès, 1985, p. 121-136 0895

43ième Congrès, 1988, p. 114-130 0948
0561

0698

0846

0858

0887

0110

0176

0188

0213

0301

0437

0662

0732

0488

0644

0319

0387

0278

0250

0779

0819

0960

0424

0466

0875

0435
SIMONIS, Yvan (R)

v. 41 , no 1,1986 , p. $200-202 \quad 0463$

SIMPSON, Wayne

v. 38 , no 1,1983 , p. 95-103 0182

SINGH, Jang $B$.

v. 43 , no 1 , p. 167-182 0635

v. 44 , no 4,1989 , p. $827-849$

SLIGER, Raymond

38ième Congrès, 1983, p. 135-1620866

SLOTSVE, G.A.

v. 41 , no 4,1986 , p. $720-737 \quad 0515$

SMITH, Anthony $\mathrm{E}$.

v. 45 , no 1,1990, p. $63-75 \quad 0774$

SODERSTROM, Lee

v. 43 , no 2,1988 , p. $341-377 \quad 0650$

SOLASSE, Bernard

37ième Congrès, 1982, p. 17-34 0847

SOLOMON, Norman A.

v. 39, no 1,1984, p. $23-35 \quad 0269$

v. 40, no 3,1985, p. $458-472 \quad 0404$

v. 41 , no 3,1986 , p. $491-504 \quad 0488$

v. 43, no 2,1988, p. $378-393 \quad 0651$

SOLOMON, Norman A. (R)

v. 41 , no 4,1986 , p. $874-875 \quad 0526$

v. 42 , no 1,1987 , p. $222-223 \quad 0555$

v. 42 , no 4,1987 , p. $883-884 \quad 0618$

v. 43 , no 1,1988 , p. $207-208 \quad 0643$

v. 44, no 1,1989, p. $293-294 \quad 0715$

SPENCER, Ian S.

v. 42 , no 4,1987, p. $756-773 \quad 0608$

v. 44 , no 4,1989 , p. $785-804$

STAGER, David A.A.

v. 43, no 3,1988, p. $571-590 \quad 0668$

STODDART, Jennifer (R)

v. 39 , no 2,1984 , p. $400 \quad 0307$

v. 41 , no 1,1986 , p. 203-204 0465

STOREY, Robert H.

v. 42 , no 2,1987, p. $366-385 \quad 0566$ 
Relations lnoustrielles, vol. 45, no 4 (1990)

STRAND, Kenneth

v. 39 , no 1,1984 , p. $77-92$

0272

STRATTON, Kay

v. 45 , no 1,1990, p. $76-93$

0775

STURMTHAL, Adolf

v. 37, no 4,1982 , p. $739-764$

0157

SUBBARAO, A.V.

v. 40, no 3,1985, p. $567-590$

v. 43 , no 3,1988 , p. $547-570$

0410

0667

\section{SURET, Jean-Marc}

42ième Congrès, 1987, p. 223-241 0937

SWAN, Carole (R)

v. 36 , no 3,1981 , p. $692-696$

0063

SWIDINSKI, Robert

v. 36, no 2,1981 , p. $371-385$

0029

\section{SWIMMER, Gene}

v. 40, no 3,1985, p. $529-544$

v. 45, no 2,1990 , p. $300-310$

SWIMMER, Gene (R)

v. 45 , no 2,1990 , p. $428-429$

0802

\section{$T$}

\section{TAJGMAN, David}

v. 36 , no 3,1981 , p. $499-529$

0051

TANG, Roger Y.W.

v. 41, no 3,1986, p. $552-571$

0492

TELLIER, Marie

43ième Congrès, 1988, p. 114-130 0948

TEMPLER, Andrew

v. 43 , no 2,1988 , p. $378-393$

0651

TESSIER, Paul (R)

v. 39, no 4,1984 , p. $804-805$

0408

0795

v. 40, no 4,1985 , p. $898-899$

0352

0440

THACKER, James $W$.

v. 41 , no 1,1986 , p. 128-144

0453

THÉRIAULT, Gilles

v. 37, no 4,1982 , p. $887-904$

0164

THÉRIAULT, Roland

v. 36 , no 4,1981 , p. $775-802 \quad 0073$

v. 39, no 1, 1984, p. 36-50 0270

THÉRIAULT, Roland (R)

v. 39 , no 4,1984 , p. $806-809 \quad 0354$

THIBAULT, Laurent

40ième Congrès, 1985, p. 121-136 0895

THOMPSON, Mark

v. 39 , no 3,1984 , p. $449-465 \quad 0314$

THOMPSON, Mark (R)

v. 40 , no 4,1985, p. $903-904 \quad 0443$

v. 44 , no 4,1989, p. $957-958 \quad 0764$

THWAITES, James

v. 36 , no 2,1981 , p. $403-411 \quad 0031$

40ième Congrès, 1985, p. 261-281 0904

THWAITES, James (R)

v. 36, no 4, 1981, p. $958 \quad 0093$

v. 37 , no 1,1982 , p. $252-253 \quad 0118$

v. 37 , no 3,1982, p. $712-713 \quad 0149$

v. 38, no 1,1983, p. $179-180 \quad 0189$

v. 39 , no 2,1984, p. $403-406 \quad 0310$

v. 39 , no 2,1984, p. $406 \quad 0311$

v. 39 , no 4,1984, p. $802-803 \quad 0350$

v. 41 , no 4,1986 , p. $877-8780528$

v. 41 , no 4,1986 , p. $884-8850534$

v. 41 , no 4, 1986, p. $884-885$

v. 44 , no 1,1989, p. 295-297 0717

TOBIN, Edmund

42ième Congrès, 1987, p. 253-276 0939

TOULOUSE, Jean-Marie

v. 39, no 1,1984, p. $36-50 \quad 0270$

TOUPIN, Louis

v. 37 , no 4,1982 , p. $805-826 \quad 0160$

TOUPIN, Sylvain

v. 43 , no 3,1988 , p. $531-546 \quad 0666$

TOWNLEY, B.

V. 40 , no 4,1985, p. $810-825 \quad 0429$

TRACY, Lane

v. 36 , no 1,1981 , p. 87-105 0004

TREMBLAY, Johane

v. 42 , no 4,1987 , p. $852-861 \quad 0613$

v. 43 , no 1,1988 , p. $188-195 \quad 0637$

v. 43, no 2,1988, p. $447-452 \quad 0655$

v. 43 , no 3,1988 , p. $686-694 \quad 0673$ 
TRUDEAU, Gilles

v. 41 , no 1,1986, p. $157-180$

TRUDEAU, Gilles (R)

v. 42 , no 1,1987, p. $215-216$

v. 44 , no 4,1989, p. $953-957$

0550

0763

TRUDEAU, GuY J.

42ième Congrès, 1987, p. 101-113 0931

TRUDEL, Johanne

v. 38 , no 3,1983 , p. $568-597$

0227

TUCK, Hugh

v. 36 , no 1,1981 , p. $106-131$

0005

TUCK, Hugh (R)

v. 36 , no 1,1981, p. $277-280$

0018

v. 36 , no 4,1981 , p. $954-956$

v. 39 , no 4,1984 , p. $803-804$

TUCKER, Eric (R)

v. 44 , no 4,1989 , p. $959-961$

0766

\section{TURCOTTE, Fernand}

39ième Congrès, 1984, p. 177-183 0882

TURNER, Michel (R)

v. 40 , no 4,1985 , p. $900-901$

0441

TZINER, Aharon

v. 39 , no 1,1984, p. $167-176$

v. 40 , no 1,1985 , p. $87-98$

\section{V}

VALASKAKIS, Kimon

36ième Congrès, 1981, p. 61-74

\section{VALLÉE, Guylaine}

v. 42 , no 4,1987, p. $702-715$

v. 44 , no 3,1989, p. $680-690$

VALLÉE, Guylaine (R)

v. 42 , no 3,1987, p. $660-661$

VALOIS, Paul

v. 37 , no 4,1982 , p. $805-826$

VAN AMÉRINGEN, Marie-Reine

v. 42 , no 4,1987 , p. $774-789$

\section{VANDAMME, François}

v. 39 , no 1,1984, p. $51-76$

0160

0609
VANDAMME, François (R)

v. 39, no 4,1984 , p. $797-799 \quad 0347$

VANIE, Roger $Z$. (R)

v. 43, no 4,1988, p. $972-973 \quad 0693$

\section{VEILLEUX, Diane}

v. 38, no 2,1983 , p. 229-253 0202

\section{VERGE, Pierre}

v. 36, no 3,1981, p. $663-670 \quad 0059$

v. 38, no 3,1983 , p. $475-506 \quad 0223$

v. 39 , no 4,1984, p. $710-743 \quad 0340$

VERGE, Pierre (R)

v. 37, no 1, 1982, p. 241-243 0111

v. 37, no 2, 1982 , p. $454-455 \quad 0129$

v. 38 , no 1,1983, p. $180-181 \quad 0190$

v. 38 , no 2,1983 , p. $442-444 \quad 0215$

v. 38, no 2, 1983 , p. $445-446 \quad 0217$

v. 38 , no 3,1983 , p. $677-678 \quad 0233$

v. 39 , no 1,1984, p. $201-203 \quad 0279$

v. 39 , no 3,1984, p. $632-633 \quad 0326$

v. 39 , no 3,1984, p. $642-643 \quad 0334$

v. 40 , no 1,1985, p. $178-180 \quad 0372$

v. 40 , no 1,1985, p. $183-185 \quad 0375$

v. 40, no 2,1985, p. $403-405 \quad 0395$

v. 40 , no 3,1985, p. 681-682 0420

v. 41 , no 1,1986, p. 196-197 0460

v. 41, no 1,1986 , p. $197-198 \quad 0461$

v. 41 , no 3,1986, p. $650-651 \quad 0500$

v. 41 , no 3,1986 , p. 659-661 0505

v. 41 , no 4,1986, p. $875-877 \quad 0527$

v. 42 , no 1,1987, p. $219-221 \quad 0553$

v. 42 , no 3,1987 , p. $651-652 \quad 0593$

v. 42, no 3, 1987 , p. $657-658 \quad 0596$

v. 42, no 3,1987, p. $659-660 \quad 0597$

v. 42 , no 3,1987 , p. $662-663 \quad 0599$

v. 42, no 4,1987, p. $877-880 \quad 0615$

v. 42, no 4,1987, p. $884-886 \quad 0619$

v. 42 , no 4,1987 , p. $886-887 \quad 0620$

v. 43 , no 3,1988, p. $710-712 \quad 0677$

v. 43 , no 3,1988, p. $712-713 \quad 0678$

v. 44 , no 1,1989 , p. $287-289 \quad 0711$

v. 44 , no 3,1989, p. $728-729 \quad 0745$

v. 44 , no 4,1989 , p. $959 \quad 0765$

v. 45 , no 2,1990 , p. $434-435 \quad 0807$

VERMA, Anil

v. 42 , no 3,1987, p. $612-627 \quad 0588$

VÉZINA, Lucie (R)

v. 37, no 3,1982, p. $713-715 \quad 0150$ 
VÉZINA, Michel

42ième Congrès, 1987, p. 21-38 0925

VIEL, André

v. 37 , no 1,1982 , p. $207-214$

VINET, Alain

v. 37, no 4,1982 , p. $887-904$

v. 43, no 3,1988, p. $591-608$

v. 43 , no 4,1988 , p. $737-756$

39ième Congrès, 1984, p. 50-72

42ième Congrès, 1987, p. 21-38

VINET, Alain (R)

v. 37 , no 4,1982, p. 971

v. 38 , no 2,1983 , p. $453-455$

v. 42 , no 3,1987 , p. $663-664$

v. 45, no 3,1990, p. $632-633$

VINET, Bernard

v. 36 , no 4,1981 , p. $828-847$

\section{W}

WALSH, William D.

v. 37, no 2,1982, p. $367-384$

WALTERS, Vivienne

v. 45, no 3,1990, p. $531-547$

\section{WARNER, Malcolm}

v. 36 , no 1, 1981, p. 132-151

v. 38 , no 1,1983 , p. $27-44$

v. 42 , no 1,1987 , p. $65-91$

v. 43 , no 1,1988 , p. $115-132$

\section{WEIERMAIR, Klaus}

v. 37 , no 3,1982 , p. $557-574$

v. 41, no 3,1986 , p. $469-490$

WEISS, Dimitri

v. 36 , no 1,1981 , p. $192-206$

v. 36 , no 2,1981 , p. $412-413$

v. 38 , no 2,1983 , p. $369-379$

v. 44 , no 4,1989 , p. $914-920$

WEISS, Dimitri (R)

v. 36 , no 2 , 1981 , p. $452-453$

v. 36 , no 2,1981 , p. $454-455$

v. 36 , no 2,1981 , p. $454-455$

v. 36 , no 2,1981 , p. $454-455$

v. 36 , no 2,1981 , p. 459

v. 36 , no 4,1981 , p. $945-946$

v. 37 , no 4,1982 , p. $969-970$

0105

0164

0669

0682

0876

0925

0174

0221

0600

0824

0075

0124

0815

0006

0178

0541

0632

0139

0487

0009

0032

0208

0760

0039

0041

0042

0043

0047

0083

0173 v. 38 , no 1,1983 , p. $187-188$

0193

v. 38 , no 4,1983 , p. $922-924$

0262

v. 38 , no 4,1983 , p. $922-924$

v. 38, no 4,1983 , p. $929-934$

v. 38 , no 4,1983, p. $929-934$

0263

0266

v. 39 , no 2,1984 , p. $406-408$

0267

0312

v. 41 , no 1,1986 , p. $190-191$

v. 41 , no 1,1986 , p. $193-196$

v. 41 , no 1,1986 , p. $198-200$

v. 42 , no 1,1987, p. 223-224

v. 45, no 3,1990 , p. $647-649$

0457

0459

0462

0556

0831

\section{WEST, Leigh}

v. 43, no 4,1988 , p. $890-908$

0689

WESTMORELAND-TRAORE, Juanita

43ième Congrès, 1988, p. 63-82 0945

WETZEL, Kurt

v. 39 , no 3,1984 , p. $486-508 \quad 0316$

v. 40 , no 4,1985 , p. $793-809 \quad 0428$

v. 41 , no 2,1986 , p. $256-280 \quad 0468$

WETZEL, Kurt (R)

v. 37, no 4,1982 , p. $968-9690172$

WHITE, Jerry P. (R)

v. 40, no 2,1985, p. $408-4090398$

v. 40, no 2,1985 , p. $410-411 \quad 0399$

WHITE, Terrence $\mathrm{H}$.

v. 36 , no 1,1981 , p. 179-191 0008

\section{WILLIAMS, C. Glyn}

v. 36 , no 3,1981 , p. $576-588 \quad 0054$

v. 37, no 3,1982 , p. $498-527 \quad 0137$

v. 39, no 2,1984, p. $335-364 \quad 0298$

v. 41 , no 3,1986 , p. $519-540 \quad 0490$

v. 42 , no 1,1987, p. 110-131 0543

WILS, Thierry

v. 42, no 3,1987 , p. $528-543 \quad 0583$

v. 44, no 2,1989, p. $354-375 \quad 0721$

v. 45 , no 3,1990 , p. $512-530 \quad 0814$

41ième Congrès, 1986, p. 99-109 0913

WILTON, D.A.

v. 42 , no 1,1987 , p. $132-149 \quad 0544$

WRIGHT, David J.

v. 39 , no 2,1984, p. $259-274 \quad 0293$

WRIGHT, Michael D.

v. 44, no 4,1989, p. $866-883$

0757 


\section{$\mathbf{x}$}

XISHAN, Yang

v. 38 , no 1, 1983, p. 120-141 0184

\section{$\mathbf{Y}$}

YOUNG, Stanley

v. 37, no 1,1982, p. $32-52$

0096

$\mathbf{Z}$

ZABALA, Craig A. (R)

v. 36 , no 1,1981 , p. $282-285$

0020

ZAIDI, Mahmood A. (R)

v. 38, no 1, 1983, p. 184-187 0192

ZAORE, Roger (R)

v. 44 , no 4,1989 , p. $962-963 \quad 0768$

v. 45, no 3,1990 , p. $634-6350826$

ZAPATA, Francisco

v. 44 , no 1,1989 , p. $177-194$

0707

ZEYTINOGLU, Isik Urla

v. 42 , no 2,1987 , p. $292-3080562$

ZIEGER, Robert $H$. (R)

v. 42 , no 1,1987 , p. 216-218

0551

ZOREF, Uri

v. 41 , no 1,1986 , p. $91-110$

0451

\section{ZUREIK, Elia}

v. 44 , no 3,1989 , p. 507-531

0734 



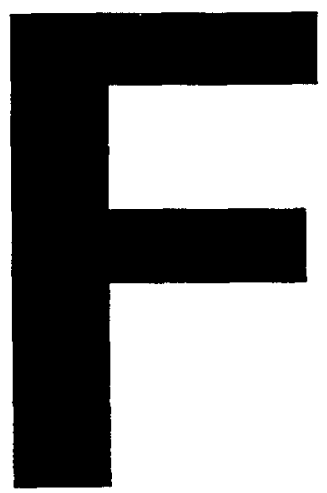

TABLE CHRONOLOGIQUE DES RÉFÉRENCES BIBLIOGRAPHIQUES

\author{
CHRONOLOGICAL TABLE OF \\ BIBLIOGRAPHICAL REFERENCES
}


Cette section fournit dans l'ordre chronologique les données bibliographiques (auteur(s), titre et référence bibliographique) de tous les articles et recensions parus dans les différents numéros de 'Relations industrielles"' (du v. 36, no 1, 1981 au v. 45, no 3,1990$)$ et de tous les articles publiés dans les différents "Congrès des relations industrielles"' (du 36e Congrès, 1981 au 45e Congrès, 1990). Elle fournit aussi un résumé de tous les articles.

\section{EXEMPLE:}

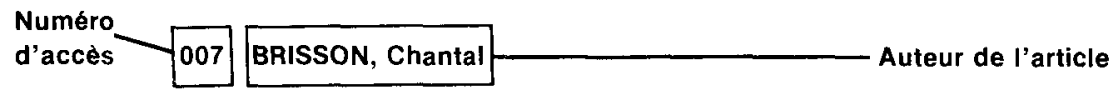

\begin{tabular}{l|l|l|}
$\begin{array}{l}\text { Titre et } \\
\text { référence }\end{array}$ & $\begin{array}{l}\text { La santé au travail et la convention collective } \\
\text { v. } 36, \text { no } 1,1981, \text { p. 152-178 }\end{array}$ \\
\hline
\end{tabular}

de l'article

\begin{tabular}{l} 
Résumé de $\begin{array}{l}\text { L'utilisation de la convention collective en tant } \\
\text { l'article } \\
\text { qu'outil d'intervention en matière de santé au } \\
\text { travail. Résultats d'une étude effectuée à partir de } \\
491 \text { conventions collectives négociées récemment } \\
\text { au Québec en matière de santé et sécurité au travail. } \\
\text { Les tendances évolutives - } 6 \text { tableaux. }\end{array}$ \\
\hline
\end{tabular}

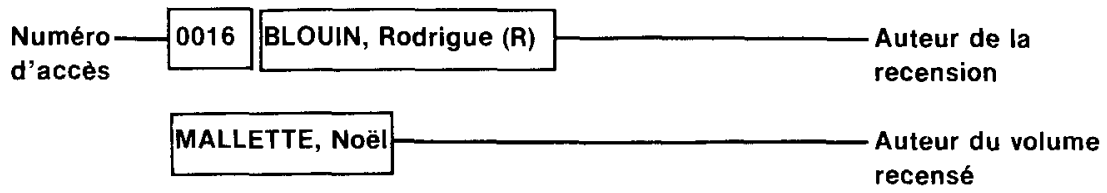

Titre et - La gestion des relations du travail au Québec.

référence Le cadre juridique et institutionnel. Scarborough,

du volume Collection administration, McGraw-Hill, 1980, 642 p.,

recensé recensé par Rodrigue Blouin.

v. 36 , no 1,1981 , p. 275

Référence de la recension 
This Section gives complete information (Author(s), title and bibliographical reference) for all articles and book reviews published in issues of "Industrial Relations" (From v. 36, no 1, 1981 to v. 45, no 3,1990 ) as well as for all articles published in the last ten (10) "Congrès des relations industrielles" (From no 36, 1981 to no 45, 1990). It also gives an abstract of all articles.

\section{EXAMPLE:}

Access
number 0631 REID, Frank $\begin{aligned} & \text { Author of the } \\ & \text { article }\end{aligned}$

Title and Economic Aspects of Mandatory Retirement reference The Canadian Experience.

of the v. 43 , no 1,1988, p. $101-114$

article

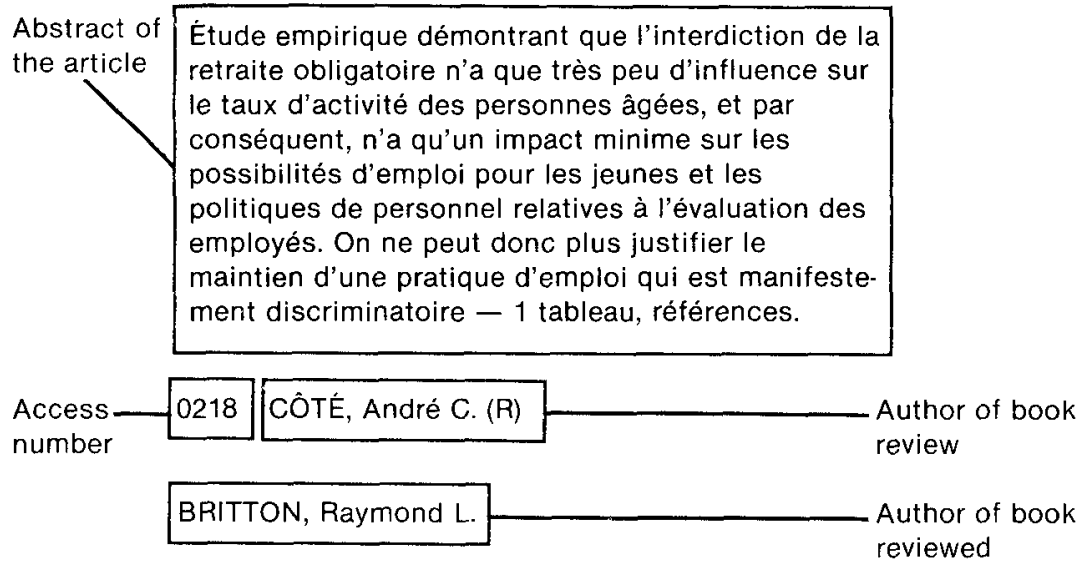

Title and The Arbitration Guide: A Case-Handing Manual reference Procedures and Practices in Dispute Resolutions. of the book Englewood Cliffs, New Jersey, Prentice Hall, 1982, reviewed 298 p., ISBN 0-13-043984-3, recensé par André C. Côté. reviewed

v. 38 , no 2,1983 , p. $446-448$

Reference of book review 

0001 PONAK, Allen

MOORE, Larry F.

Canadian Bank Unionism: Perspectives and Issues

v. 36 , no 1,1981 , p. $3-34$

La syndicalisation des employés de banque au Canada: coup d'oeil sur le milieu bancaire; la question de l'unité de négociation; l'action syndicale dans les banques; le contenu des conventions collectives; les conséquences de la syndicalisation - 1 figure, 5 tableaux.

0002 POOLE, Michael

Perceptions of Trade Union Members and the Social Action Perspective.

v. 36 , no 1,1981, p. 35-62

Présentation des résultats d'une enquête faite auprès des membres d'un syndicat britannique dans le secteur des transports. L'enquête visait à connaitre la perception qu'ont de l'action sociale les syndiqués tout en établissant un cadre théorique qui soit de nature à favoriser une accumulation systématique de connaissances sur le sujet - 6 tableaux.

0003 DOWNIE, Bryan M.

Some Thoughts on Public Policy and Industrial Peace.

v. 36 , no 1,1981, p. $63-86$

Inquietudes au sujet de l'état de santé du régime de négociations collectives et de relations professionnelles au Canada. Le vaste domaine des stratégies Je rechange dans les relations du travail peut se diviser en quatre groupes ou catégories: 1) La réforme politique; 2) la réforme des structures; 3) la réforme des mécanismes; 4) la réforme des comportements.

0004 PETERSON, Richard B. TRACY, Lane CABELLY, Alan

Problem Solving in Labor Negotiations: Retest of a Model

v. 36 , no 1, 1981, p. 87-105

La solution de problèmes en matière de négociations collectives: comparaison des résultats de deux enquêtes effectuées aux ÉtatsUnis à partir d'un même modèle de solution des problèmes en matière de négociations collectives - 1 figure, 7 tableaux, références.
0005 TUCK, Hugh

Canadian Railways and Unions in the Running Trades, 1865-1914

v. 36 , no 1,1981 , p. 106-131

Histoire des relations entre la direction des chemins de fer canadiens et les fraternités de cheminots depuis l'avènement du premier syndicat au Canada en 1865, jusqu'à la déclaration de la première guerre mondiale. $\mathrm{Ce}$ fut au cours de cette période que le modèle de syndicalisation du personnel itinérant s'implanta au Canada où il s'est maintenu presqu'inchangé jusqu'à nos jours.

\section{PECCEI, Ricardo}

WARNER, Malcolm

Industrial Relations, Strategic Importance, and Decision - Making.

v. 36 , no 1,1981 , p. $132-151$

Étude du processus de prise de décision dans les sociétés multinationales en ce qui concerne les relations du travail. L'étude se rapporte à une analyse des différences dans le processus de prise de décision selon les divisions de produits qu'on y fabrique en regard de recherches antérieures (Peccei et Warner, 1976) - 4 figures, 4 tableaux, références.

\section{BRISSON, Chantal}

La sante au travail et la convention collective v. 36 , no 1,1981 , p. $152-178$

L'utilisation de la convention collective en tant qu'outil d'intervention en matière de santé au travail. Résultats d'une étude effectuée à partir de 491 conventions collectives négociées récemment au Québec en matière de santé et sécurité au travail. Les tendances évolutives - 6 tableaux.

0008 WHITE, Terrence $\mathrm{H}$.

The Relative Importance of Work as a Factor in Life Satisfaction

v. 36, no 1,1981 , p. $179-191$

Étude mettant en parallèle les rapports entre la satisfaction au travail et la satisfaction dans la vie. Les résultats indiquent que la satisfaction au travail est un facteur explicatif relativement peu important de la satisfaction dans la vie. La vie familiale, les activités de loisirs et les relations d'amitié ont tendance à être des sources plus marquées de satisfaction dans la vie -4 tableaux, références. 
0009 WEISS, Dimitri

Politique, entreprise, syndicats

v. 36 , no 1,1981 , p. $192-206$

Quelques propos sur la polysémie du terme "politique" en relations industrielles: la "politique organisationnelle"; 'politique de l'entreprise' et "politique dans l'entreprise"; "politique syndicale" et "politique partisane"; les partis politiques dans l'entreprise; les "grèves politiques" - références.

0010 FASHOYIN, Tayo

State Regulation of Trade Disputes in Essential Services in Nigeria

v. 36 , no 1, 1981, p. 207-222

La réglementation concernant les conflits dans les services essentiels au Nigéria. Les mécanismes détaillés de règlement des conflits prévus dans a) le décret relatif aux conflits de travail (1976) et b) le décret concernant les services essentiels (1976).

\section{AUCLAIR, Robert}

Genève ignore Québec en matière de vocabulaire

v. 36 , no 1,1981 , p. $223-224$

Dans un article paru dans la "Revue internationale du travail" et portant sur les clauses de sécurité syndicale, le vocabulaire utilisé pour désigner les différentes clauses se compose en grande partie d'expressions anglaises, comme s'il n'existait aucune expression française correspondante. II y a belle lurette que les Québécois ont traduit ces termes en français.

0012 ADAMS, Georges W.

The Ontario Experience with Interest arbitration: Problems in Detecting Policy

v. 36 , no 1,1981 , p. $225-250$

L'expérience de l'Ontario en ce qui concerne l'arbitrage des conflits d'intérêt, particulièrement dans les secteurs d'activité représentant des zones critiques: policiers et pompiers, hôpitaux, fonction publique, enseignants.

\section{PLISZKIEWICZ, Marek}

Le licenciement en droit polonais.

v. 36 , no 1, 1981, p. 251-259

Résumé de la législation du travail en Pologne en matière de licenciement: a) La résiliation du travail avec préavis par l'établisse- ment; b) la résiliation du contrat de travail sans préavis par l'établissement.

0014 AOUST, Claude d'

Les réclamations à l'arbitrage de dommages résultant de la violation d'une clause de renonciation à la grève.

v. 36 , no 1,1981, p. $259-262$

La Cour suprême du Canada vient de rendre une décision qui affermit singulièrement l'autorité de l'arbitre de griefs québécois. Elle établit très clairement le pouvoir de l'arbitre d'octroyer des dommages lorsque le syndicat fait grève en dépit d'une clause de renonciation inscrite dans la convention collective.

\section{MARTINEAU, LUC}

Décisions rendues par le Conseil canadien des relations du travail.

v. 36 , no 1,1981 , p. $263-266$

Dans le différend opposant la Banque royale du Canada et les employés de six de ses succursales et trois syndicats affiliés à la Centrale des syndicats nationaux, le Conseil, par décision majoritaire, en est venu à la conclusion qu'il n'y avait pas lieu d'imposer une première convention collective.

\section{BLOUIN, Rodrigue (R)}

MALLETTE, Noël

La gestion des relations du travail au Québec. Le cadre juridique et institutionnel. Scarborough, Collection administration, McGraw-Hill, 1980, 642 pp., recensé par Rodrigue Blouin. v. 36 , no 1,1981 , p. 275

\section{BOIVIN, Jean (R)}

CHRISTENSEN, Sandra

Unions and the Public Interest: Collective Bargaining in the Government Sector. Vancouver, The Fraser Institute, 1980, 95 pp., recensé par Jean Boivin.

v. 36 , no 1,1981 , p. $275-276$

0018 TUCK, Hugh (R)

PALMER, Bryan D.

A Culture in Conflict : Skilled Workers and Industrial Capitalism in Hamilton. Ontario, 1860-1914, Montreal, McGill-Queen's University Press, 1979, 331 pp., recensé par Hugh Tuck. v. 36 , no 1,1981 , p. $277-280$ 
0019 BOIVIN, Jean (R)

NORTHRUP, Herbert R. ROWAN, Richard L. Multinational Collective Bargaining Attempts. Multinational Industrial Relations Series no 6 , Industrial Research Unit, Philadelphia, The Wharton School, University of Pennsylvania, 1979, 580 pp., recensé par Jean Boivin.

v. 36 , no 1,1981, p. $280-282$

0020 ZABALA, Craig A. (R)

BURAWOY, Michael

Manufacturing Consent: Changes in the Labor Process under Monopoly Capitalism. Chicago, III., The University of Chicago Press, 1979 , 267 pp., recensé par Craig A. Zabala.

v. 36 , no 1,1981 , p. $282-285$

0021 MATEJKO, Alexander J. (R)

COOPER, Gary L. MEMFORD, Enid

The Quality of Working Life in Western and Eastern Europe. Westport, Greenwood Press, 1979, 348 pp., recensé par Alexander J. Matejko.

v. 36 , no 1,1981 , p. $285-288$

\section{BLOUIN, Rodrigue $(\mathrm{R})$}

VÉZINA, Claude

Les clauses d'ancienneté et l'arbitrage des griefs. Ottawa, Collection des travaux de la Facuité de droit de l'Université d'Ottawa, éditions de l'Université d'Ottawa, 1979, 125 pp., recensé par Rodrigue Blouin.

v. 36 , no 1,1981, p. $288-289$

0023 MAKI, Dennis R. (R)

EHRENBERG, Ronald G. (Ed.)

Research in Labor Economics: An Annual Compilation of Research, Vol. 2, Greenwich, Connecticut, JAI Press Inc., 1978, recensé par Dennis R. Maki.

v. 36 , no 1, 1981, p. $289-291$

\section{BARRÉ, Alain (R)}

B.I.T.

Rapport de la Commission d'experts pour l'application des conventions et recommandations. Conference internationale du travail, 66ième session, Genève, Rapport III, 1980, Partie 4A, 251 pp., Partie 4B, Travailleurs migrants, 190 pp., recensé par Alain Barré. v. 36 , no 1,1981, p. 291-294

0025 SARTIN, Pierrette

Le travail à temps partiel

v. 36, no 2, 1981, p. 307-324

Définition de la notion de travail à temps par- tjel et présentation des différentes implications: Les personnes concernées; les secteurs concernés; les avantages et les inconvénients; les obstacles légaux, techniques et organisationnels, psychologiques; les salaires; les syndicats; perspectives d'avenir et ébauche de solutions.

\section{JAMAL, Muhammad} CRAWFORD, Ronald L.

Moonlighters: A Product of Deprivation or Aspiration?

v. 36 , no 2,1981, p. $325-335$

Les résultats d'une enquête sur le phénomène du double emploi et de ses effets sur: le besoin d'accomplissement au travail, la santé mentale, la performance au travail, l'absentéisme ou la possible mobilité d'emploi, l'activité sociale volontaire et les préférences concernant des prévisions de travail plus flexibles - 2 tableaux, références.

0027 RAINVILLE, Jean-Marie

Hiérarchie ethnique dans la grande entreprise industrielle montréalaise.

v. 36 , no 2,1981, p. $336-360$

Analyse empirique montrant comment les Canadiens-français occupent une position d'infériorité à l'intérieur de la grande entreprise industrielle montréalaise par rapport aux Canadiens-anglais. Pour ces derniers, les chances d'avancement sont nettement supérieures à celles des Canadiens-français. Présentation des mucièles de carrière propres à chaque groupe ethnique et des attitudes au travail qui en découlent - 8 tableaux, bibliographie.

\section{BARKIN, Solomon}

Productivity Measures in Collective Bargaining. v. 36, no 2, 1981, p. 361-370

La productivité physique n'est que l'une des dimensions auxquelles les parties doivent faire face à la table des négociations collectives. Les mesures de productivité n'apparaissent pas comme un enjeu véritable de la négociation de même que la productivité n'est pas ce qui détermine d'abord le niveau des gains.

0029 SWIDINSKI, Robert

The Effect of Bargaining Structure on Negotiated Wage Settlements.

v. 36, no 2,1981, p. $371-385$ 
Étude vérifiant l'hypothèse selon laquelle le type d'unités de négociation (unité de négociation par établissement, par compagnie, multipartite ou association d'employeurs) retenu dans la négociation collective a un effet sur les ententes salariales négociées dans le secteur privé au Canada. Les résultats obtenus se fondent sur l'étude de 2,338 conventions collectives régissant 200 salariés ou davantage qui ont été conclues entre 1966 et 1975 - 1 tableau, références.

\section{BRUCE, Christopher J.}

The Role of Information Concerning the Arbitrator's Preferences.

v. 36 , no 2, 1981, p. 386-402

Le degré de connaissance que possèdent les parties contractantes de la décision de l'arbitre s'il y avait impasse ou rupture est une caractéristique importante pour déterminer si les parties peuvent s'entendre de gré à gré. Évaluation du résultat de cette connaissance tant dans le système d'arbitrage traditionnel que sous le système d'arbitrage des propositions finales.

\section{THWAITES, James}

Business "Organizes": The Early Years in Quebec.

v. 36 , no 2,1981, p. $403-411$

L'organisation et la consolidation des groupements patronaux au Québec pendant la période de 1896 à 1915. Caractéristiques des associations d'employeurs ou groupements patronaux de cette époque.

\section{WEISS, Dimitri}

Qu'est-ce que les relations industrielles?

v. 36 , no 2,1981, p. $412-413$

Commentaires sur la confusion que crée parfois l'utilisation de l'expression relations industrielles".

\section{MARTIN, Ross BLACK, Errol}

Roy J. Adams' Proposal for a Training Levy Scheme.

v. 36 , no 2,1981 , p. $414-417$

Commentaires et critiques de l'articie de Roy J. Adams paru dans le volume 35, numéro 1, 1980 de la revue "Relations industrielles" et portant sur la formation professionnelle et sur les recommandations de la Commission d'enquête sur les congés éducatifs et la productivité.

\section{AOUST, Claude d'}

La juridiction de l'arbitre et de la Cour des petites créances devant la Cour suprême du Canada.

v. 36 , no 2 , 1981, p. $418-424$

Analyse de la décision de la Cour suprême du Canada sur la juridiction des tribunaux de droit commun en matière de réclamation de salaires. L'intérêt de cette décision tient à ce qu'elle est reliée plus généralement à l'étendue de la compétence de l'arbitre à régler complètement les litiges nés de l'application d'une convention collective.

\section{MARTINEAU, LuC}

Décisions rendues par le Conseil canadien des relations du travail.

v. 36, no 2, 1981, p. $424-429$

Ingérence dans la représentation des employés et congédiements illégaux: Analyse de la décision du Conseil qui a accueilli favorablement diverses plaintes de pratique déloyale logées par des employées de la Banque royale du Canada congédiées durant une grève légale.

\section{LECLERC, Claudine}

Thèses de maitrise en relations industrielles - Université Laval et Université de Montréal: 1940-1980

v. 36 , no 2,1981 , p. $435-448$

Liste des thèses de maitrise en relations industrielles déposées de 1940 à 1980 au Département des relations industrielles de l'Université Laval et à l'Ecole des relations industrielles de l'Université de Montréal.

0037 DUBÉ, Jean-Louis (R)

MORIN, Fernand BLOUIN, Rodrigue

Précis de l'arbitrage des griefs. Québec, Les Presses de l'Université Laval, 1980, 507 pp., recensé par Jean-Louis Dubé.

v. 36 , no 2,1981 , p. $449-451$

0038 BLOUIN, Rodrigue (R)

BÉRAUD, Jean-Marc

La suspension du contrat de travail. Paris, Editions Sirey, 1980, 294 pp., recense par Rodrigue Blouin.

v. 36 , no 2, 1981 , p. $451-452$ 
0039 WEISS, Dimitri (R)

JAVIUIER, Jean-Claude

Le Droit du travail, 2ième éd., Paris, L.G.D.J., 1981, 700 pp., recensé par Dimitri Weiss.

v. 36 , no 2,1981 , p. $452-453$

0040 BLOUIN, Rodrigue (R)

B.IT.

Conciliation and Arbitration Procedures in Labour Disputes. A Comparative Study. Genève, International Labour Office, 1980, 183 pp., recensé par Rodrigue Blouin.

v. 36 , no 2,1981 , p. $453-454$

0041 WEISS, Dimitri (R)

LYON-CAEN, Gérard PÉLISSIER, Jean

Les grands arrêts de Droit du travail, 2ième éd., Paris, Editions Sirey, 1980, 406 pp., recensé par Dimitri Weiss.

v. 36 , no 2,1981 , p. $454-455$

0042 WEISS, Dimitri (R)

BÉRAUD, Jean-Marc

La suspension du contrat de travail. Paris, Editions Sirey, 1980, 296 pp., recensé par Dimitri Weiss.

v. 36, no 2,1981 , p. $454-455$

0043 WEISS, Dimitri (R)

BAR-NIV, Zvi H.

International Labour Law Reports, vol. 2, 1979, 376 pp., recensé par Dimitri Weiss.

v. 36 , no 2,1981 , p. $454-455$

0044 DION, Gérard $(R)$

WINDMULLER, John P.

The International Trade Union Movement. Deventer, Kluwer, 1980, 174 pp., recensé par Gérard Dion.

v. 36 , no 2,1981 , p. $455-456$

0045 LAVIGNE, Marie (R)

WHITE, Julie

Les femmes et le syndicalisme. Ottawa, Conseil consultatif canadien de la situation de la femme, Centre d'édition du gouvernement du Canada, 1980, 147 pp., recensé par Marie Lavigne.

v. 36 , no 2,1981 , p. $456-457$

0046 LAVIGNE, Marie (R)

COOK, Alice $\mathrm{H}$.

The Working Mother, A Survey of Problems and Programs in Nine Countries. 2nd ed., Ithaca, New York State School of Industrial and Labor Relations, Cornell University, 1978, 71 pp., recensé par Marie Lavigne

v. 36, no 2,1981 , p. 458
0047 WEISS, Dimitri (R)

BUSANA, Osvaldo

SCHÜTT, Maria Teresa

UNNIA, Mario

L'impresa nel sistema socio-sindicale : dieci anni di contrattazione. Turin, ISVOR - FIAT, 1980, recensé par Dimitri Weiss.

v. 36 , no 2,1981 , p. 459

0048 ROY, André (R)

M.T.M.O.

Problèmes et perspectives d'emploi dans le secteur hótelier du Québec. Québec, Centre de recherche et de statistiques sur le marché du travail, MTMO, 1980, 262 pp., recensé par André Roy.

v. 36, no 2,1981 , p. $460-461$

0049 CUNNINGHAM, William B. (R)

CHRISTENSEN, Sandra

Unions and the Public Interest: Collective Bargaining in the Government Sector. Vancouver, The Fraser Institute, 1980, 95 pp., recensé par William Cunningham.

v. 36 , no 2,1981 , p. $461-462$

0050 FERLAND, Gilles

La politique de rémunération dans les secteurs public et parapublic au Québec.

v. 36 , no 3,1981 , p. $475-498$

Exploration d'une avenue différente de politique salariale pour le secteur public québécois qui consisterait à fixer le niveau des rémunérations des salariés de l'État à un niveau comparable à celui pratiqué par les industries du secteur privé en concurrence sur le marché international. Présentation des résultats qu'aurait permis d'obtenir l'application d'une telle politique dans le secteur public québécois - 8 tableaux.

\section{TAJGMAN, David}

Political Resolutions in the International Labor Organizations: The experience since 1964.

v. 36 , no 3,1981 , p. $499-529$

De plus en plus, depuis 1964, le thème des résolutions présentées ou adoptées à la Conférence internationale du travail a été détourné des questions traitant des politiques du travail vers des débats se rapportant aux problèmes de politique internationale. Étude des divers moyens de réduire le nombre de résolutions de caractère politique au sein de l'Organisation internationale du travail - 4 tableaux. 
0052

CHAISON, Gary N.

ROSE, Joseph B.

The Structure and Growth of the Canadian National Unions.

v. 36 , no 3,1981 , p. $530-551$

Le développement du syndicalisme national au Canada entre 1920 et 1972 . Survol de la croissance du syndicalisme et discussion des caractéristiques des syndicats nationaux. Mise au point d'un modèle de développement des syndicats qui puisse expliquer les changements dans les effectifs des syndicats nationaux canadiens - 1 figure, 5 tableaux.

0053 BROSSARD, Michel

Revendications, stratégie syndicale et organisation du travail dans l'entreprise.

v. 36 , no 3,1981 , p. $552-575$

Analyse de la stratégie élaborée par le syndicat pour obtenir une amélioration des conditions de travail des travailleurs. Cette analyse est concentrée sur les relations entre l'action revendicatrice d'un syndicat local et les caractéristiques de l'organisation du travail dans l'entreprise.

0054 WILLIAMS, C. Glyn Joan Robinson on Unions, Distribution and Inflation.

v. 36 , no 3,1981 , p. $576-588$

La pensée de Joan Robinson sur le rôle des syndicats dans l'économie mixte moderne, sur la répartition des biens et l'inflation. Selon elle, la survivance du capitalisme pivote autour de syndicats qui disposent de la juste mesure de pouvoir économique - Bibliographie.

0055 MARTINEAU, LUC

La "gestion" des conflits par le Conseil canadien des relations du travail.

v. 36 , no 3,1981 , p. $589-615$

Les modifications apportées au Code canadien du travail par le législateur en 1978 ont considérablement accru le pouvoir de manoeuvre du Conseil canadien des relations du travail. Analyse et bilan de ces trois années d'expérience - 1 tableau.

0056 CHRISTENSEN, Sandra

Collective Bargaining in Provincial Public Administration.

v. 36 , no 3,1981 , p. $616-629$
Jusqu'à quel point les taux de salaires dans le secteur public s'alignent sur ceux du secteur privé face aux changements cycliques de l'économie mesurés en tenant compte à la fois de l'inflation et des emplois disponibles sur le marché du travail. L'introduction de la négociation collective dans le secteur public a-t-elle modifié ce rapport de façon significative? - 1 tableau.

\section{CHERMESH, Ran}

Conflict in the Israeli Industrial Relations System: A Critical Description.

v. 36 , no 3,1981 , p. $630-647$

Analyse du système des relations profession. nelles en Israël au point de vue national et en tant qu'organisation. L'analyse s'appuie sur l'exposé d'Eldridge (1972) tiré du concept des relations professionnelles de Dunlop (1958) - 2 tableaux, bibliographie.

0058 BOULARD, René

Le professionnalisme au Québec: Une étude empirique.

v. 36 , no 3,1981 , p. $648-662$

Description de l'approche empirique utilisée dans une étude sur le professionnalisme des pharmaciens québécois. On montre de quelle façon it a été possible de mesurer le professionnalisme d'une population donnée, celle des pharmaciens - 2 tableaux, bibliographie.

0059 VERGE, Pierre

La mission-type d'un Conseil des relations du travail moderne.

v. 36 , no 3,1981 , p. $663-670$

Analyse présentant et examinant l'idée d'un nouveau type de conseil des relations du travail. La nature générale d'un tel conseil: son champ de compétence, sa composition et son mode de fonctionnement; la spécificité et la portée de ce conseil moderne.

DO60 MARTINEAU, LUC

Décisions rendues par le Conseil canadien des relations du travail.

v. 36 , no 3. 1981 , p. $671-680$

Analyse de deux décisions du Conseil portant sur le devoir de représentation juste. Dans les deux cas, le Conseil a rejeté des plaintes qui alléguaient que l'agent négociateur avait 
fait preuve de négligence lors du refus de poursuivre des griefs de congédiement à l'arbitrage.

0061 BÉLANGER, Jacques (R)

CÔTÉ, Marcel TÉGA, Vasile

La démocratie industrielle / The Industrial Democracy. Montréal, Les Editions Agence d'Arc, 1981, 518 pp., recensé par Jacques Bélanger.

v. 36 , no 3,1981 , p. $689-690$

0062 BÉLANGER, Jacques ( $R$ )

JAIN, Hem C.

Worker Participation : Success and Problems. New York, Praeger Publisher, 1980, 358 pp., recensé par Jacques Bélanger.

v. 36 , no 3,1981 , p. $690-692$

0063 SWAN, Carole (R)

STEINBERG RATNER, Ronnie

Equal Employment Policy for Women : Strategies for Implementation in the United States, Canada and Western Europe. Philadelphia, Temple University Press, 1980, 520 pp., recensé par Carole Swan.

v. 36 , no 3,1981 , p. $692-696$

0064 BARRÉ, Alain (R)

B.I.T.

Normes et déclarations de principes intéressant particulièrement les travailleuses. Genève, BIT, 1981, 138 pp., recensé par Alain Barré.

v. 36, no 3, 1981, p. 696-697

0065 BOUCHER, Bernard (R)

BOULARD, René PÉRUSSE, Lyne

Les moyens organisationnels de prévention des maladies industrielles et des accidents du travail : essai de typologie et bibliographie annotée. Québec, Collection instruments de travail, Département des relations industrielles, Université Laval, 1981, 183 pp., recensé par Bernard Boucher.

v. 36 , no 3,1981 , p. 697-699

0066 PICARD, Danielle (R)

GIANNINI, Mirella

Giustizia e Mezzogiorno. I/ caso dello statuto dei lavoratori. Venezia, Marsilio Editori, 1979, 162 pp., recensé par Danielle Picard.

v. 36 , no 3,1981 , p. $699-701$

0067 BOULARD, René (R)

DOLAN, Shimon L. ARSENEAULT, André

Stress, santé et rendement au travail, mono- graphie no 5. Montréal, École des relations industrielles de l'Université de Montreal, 1980, 186 pp., recensé par René Boulard.

v. 36 , no 3,1981 , p. $701-702$

0068 BOULARD, René (R)

PÉRUSSE, Michel (R)

STELLMAN, Jeanne M. DAUM, Susan M.

Perdre sa vie à la gagner; manuel pour la santé des travailleurs. Montréal, Édition Parti pris, 1979, 493 pp., recensé par René Boulard et Michel Pérusse.

v. 36 , no 3,1981, p. $702-703$

0069 PETIT, André (R)

NAYLOR, James C. PRITCHARD, R.D.

ILGEN, D.R.

A Theory of Behavior in Organizations. New York, Academic Press Inc., 1980, 299 pp., recensé par André Petit.

v. 36 , no 3,1981, p. $703-704$

0070 GRIEZIC, F.J.K. (R)

SALUTIN, Rick

Kent Rowley, The Organizer, A Canadian Union Life. Toronto, James Lorimer, 1980, 163 pp., recensé par F.J.K. Griezic.

v. 36 , no 3,1981 , p. $704-705$

0071 HÉBERT, Gérard

Les relations du travail au Québec: Bilan des années 1970

v. 36, no 4,1981 , p. $715-747$

Les années 1970 peuvent apparaître, sous un certain angle, comme un grand pas en avant; sous un autre, elles témoignent d'un recul irréparable; elles ont assuré la consolidation de la négociation collective, en même temps qu'elles en ont peut-être préparé la disparition éventuelle. Tel est le thème de la première partie de cette analyse. La seconde partie analyse le contenu des conventions collectives et leur évolution au cours de la décennie.

0072 JAIN, Hem C.

Disclosure of Corporate Information to Trade Unions in North America.

v. 36 , no 4,1981 , p. $748-774$

II n'y a que très peu de renseignements fournis aux syndicats par les entreprises en Amérique du Nord. Étude de la législation et des pratiques des entreprises relativement à la divulgation de l'information; les arguments favorables ou opposés à la divulgation; les problèmes que de telles mesures soulèvent; 
la nécessité pour les entreprises d'avoir une politique en cette matière; les initiatives possibles de la part des gouvernements.

\section{THÉRIAULT, Poland LESAGE, Pierre $B$. BOISVERT, Maurice}

L'absentéisme: importance, nature et remèdes. v. 36 , no 4,1981 , p. $775-802$

L'importance et l'étendue du phénomène d'absentéisme au sein des entreprises ainsi que des coûts impliqués. Identification des principaux modèles explicatifs ainsi que des limites inhérentes à ces modèles en vue d'en présenter un schéma intégrateur. Les diverses stratégies d'intervention à la portée de la direction des entreprises utilisées en pratique afin d'enrayer l'absentéisme et ses effets au sein des entreprises - 1 tableau, 1 schéma, bibliographie.

0074 MCPHILLIPS, David C.

Duty of Fair Representation: Recent Attitudes in British Columbia and Ontario.

v. 36 , no 4,1981 , p. $803-827$

L'exemple de la Colombie Britannique et de l'Ontario en ce qui a trait à l'obligation formelle pour le syndicat de représenter les salariés d'une façon équitable, c'est-à-dire sans mauvaise foi, sans discrimination et sans arbitraire. L'obligation formelle de représenter les salariés est envisagée sous deux aspects: les négociations et l'administration d'une convention collective.

0075 VINET, Bernard

Le problème de la retraite.

v. 36 , no 4,1981 , p. $828-847$

La définition de la retraite et le sens à donner au terme retraite. Aperçu historique, évolution et implications de la retraite. La nécessité de répartir différemment le travail pour une meilleure justice distributive. Arguments contre l'âge obligatoire de la retraite.

0076 MOORE, William R. Justice and the Grievance Procedure in the Federal Public Service.

v. 36 , no 4,1981 , p. $848-864$

La loi des relations de travail dans la fonction publique fédérale empêche les parties à une convention collective de porter certaines de leurs clauses à l'arbitrage. Par exemple, les griefs relatifs aux nominations, aux promotions, aux mises à pied, aux mutations, aux notations de même que plusieurs autres ne sont pas "arbitrables". Les restrictions imposées dans le cas de certains types de griefs créent un obstacle inutile à l'exercice de la justice -6 tableaux.

\section{LOWE, Graham S.}

Causes of Unionization in Canadian Banks.

v. 36, no 4,1981 , p. $865-893$

Le développement du syndicalisme dans les banques du Canada depuis 1976, secteur d'activité au niveau de syndicalisation très bas, la main-d'oeuvre étant en grande partie formée de femmes. Analyse du processus de syndicalisation sous quatre aspects différents - Bibliographie.

\section{AOUST, Claude d' DELORME, François}

The Origin of the Freedom of Association and of the Right to Strike in Canada: An Historical Perspective.

v. 36, no 4, 1981, p. 894-921

Quelques aspects historiques reliés à l'adoption, en 1872, de deux lois qui accordaient aux travailleurs le droit de se regrouper en syndicat et de faire la grève: La "Loi concernant les associations ouvrières" et la "Loi pour amender la loi criminelle relative à la violence, aux menaces et à la molestation". Quelques effets de ces lois sur l'évolution du statut légal des activités syndicales - Références.

\section{GRUBEL, Herbert $G$}

MAKI, Dennis R.

A Note on the Effects of Unemployment insurance, Minimum Wage Legislation and Trade Union Growth on Reported Unemployment Rates in Canada, 1950-75

v. 36 , no 4,1981 , p. $922-927$

Analyse à la fois théorique et empirique des effets de l'assurance-chômage, de la législation sur le salaire minimum et de la croissance du syndicalisme sur les taux de chômage rapportés au Canada de 1950 à 1975 - 1 tableau, bibliographie.

0080 LAVERY, Daniel

Décisions rendues par le Conseil canadien des relations du travail.

v. 36 , no 4,1981 , p. $928-936$ 
Résumé de cing décisions du Conseil: 1) Pratique déloyale: ingérence dans la représentation des employés; 2) Santé et sécurité au travail: définition de l'expression "danger imminent"; 3) Congédiement illégal et congédiement injuste; 4) "Employé" au sens du Code dans le secteur du débardage; 5) Vente d'entreprise au sens de l'article 144 du Code.

0081 BÉLANGER, Laurent (R)

PÉGANIOL, Claude

Techniques et politiques d'amélioration des conditions de travail. Paris, Entreprise moderne d'édition, 162 pp., recensé par Laurent Belanger.

v. 36 , no 4,1981, p. $943-944$

0082 PETIT, André (R)

BRIDDLE, Bruce J.

Role Theory: Expectations, Identities and Behaviors. New York, Academic Press, 1979, 416 pp., recensé par André Petit.

v. 36 , no 4,1981, p. $944-945$

0083 WEISS, Dimitri (R)

GRAWITZ, Madeleine

Lexique des sciences sociales. Paris, Dalloze, 1981, 376 p $\rho$., recensé par Dimitri Weiss.

v. 36 , no 4,1981 , p. $945-946$

0084 BÉLANGER, Laurent (R)

GUIOT, Jean $M$.

Organisations sociales et comportements. Montréal, Les éditions Agence d'Arc Inc., Paris, Les editions Hommes et Techniques, 1980, 213 pp., recensé pai Laurent Bélanger. v. 36 , no 4,1981, p. $946-948$

0085 BÉLANGER, Laurent (R)

LEW-LEBOYER, $\mathrm{C}$.

Le psychologue et l'entreprise. Montréal, Masson et Presses de l'Université de Montréal, 1980, 169 pp., recensé par Laurent Bélanger.

v. 36, no 4,1981, p. 948

0086 DION, Gérard (F)

MARSH, Arthur

Trade Union Handbook, 2nd ed. Farnborough, England, Gower, 1980, 396 pp., recensé par Gérard Dion.

v. 36 , no 4,1981, p. $948-949$

0087 MATEJKO, Alexander J. (R)

THUROW, Lester C.

The Zero-Sum Society. Distribution and the Possibilities for Economic Change. New York,
Basic Books inc., 1980, 230 pp., recensé par Alexander J. Matejko.

v. 36 , no 4 , 1981, p. $949-951$

0088 BARRÉ, Alain (R)

B.I.T.

Rapport de la Commission d'experts pour l'application des conventions et recommandations. Conférence internationale du travail, 67ième session, Genève, Rapport III (Partie 4A), BIT, 1981, 255 pp., recensé par Alain Barré.

v. 36 , no 4,1981 , p. $951-954$

0089

BARRÉ, Alain (R)

B.I.T.

Age minimum. Etude d'ensemble de la commission d'experts pour l'application des conventions et recommandations, Conférence internationale du travail, 67ième session, Genève, Rapport III (Partie 4B), BIT, 1981, 238 pp., recensé par Alain Barré.

v. 36 , no 4,1981 , p. $951-954$

0090 DION, Gérard (R)

SOMERS, Gérald C.

Collective Bargaining : Contemporary American Experience. Madison, WI., Industrial Relations Research Association, 1980, 588 pp., recensé par Gérard Dion.

v. 36 , no 4,1981 , p. 954

0091 TUCK, Hugh (R)

DANIEL, Cletus E.

The ACLU and the Wagner Act: An inquiry into the Depression. Era Crisis of American Liberalism. Cornell Studies in Ind. and Labor Rel. Number 20, N. Y. State School of Ind. and Labor Rel., Cornell Univ., Ithaca, N. Y., 1980, 142 pp., recensé par Hugh Tuck.

v. 36 , no 4,1981 , p. $954-956$

0092 BÉLANGER, Jacques (R)

MARTIN, Ross M.

TUC : The Growth of a Pressure Group 1868 1976. Oxford, Oxford University Press, 1980, 394 pp., recensé par Jacques Bélanger.

v. 36 , no 4,1981 , p. $956-958$

0093 THWAITES, James (R)

L.R.S.C.

Labour Studies Research Bulletin. Labour Studies Resource Centre, Department of Political Science, Carleton University, 1981, 32 pp., recensé par James Thwaites.

v. 36 , no 4,1981 , p. 958 


\section{BLANCHARD, Francis}

Les relations professionnelles et les defis des années 80 .

v. 37 , no 1,1982 , p. 3-14

Le directeur du Bureau international du travail évoque les différentes voies que devrait prendre la pratique des relations professionnelles pour s'adapter aux complexités de la prochaine décennie. Les défis à relever sont rangés sous quatre rubriques: La conjoncture économique et sociale, la participation, la fonction publique, les services. Les problèmes qui se posent aux pays du Tiers Monde.

0095 HAMEED, Syed M.A.

A Critique of Industrial Relations Theory.

v. 37 , no 1,1982, p. $15-31$

Examen critique des développements théoriques qui se sont produits dans le domaine des relations professionnelles, Les trois façons de concevoir théoriquement les relations professionnelles se réfèrent, dans une certaine mesure, au partage du pouvoir dans la société ou dans le régime des relations professionnelles. On fait de la notion de pouvoir, le thème central de la formulation d'une structure théorique des relations professionnelles.

0096 YOUNG, Stanley

Industrial Relations: A Paradigmatic Analysis. v. 37 , no 1,1982 , p. $32-52$

Deux exemples présentant l'ensemble des caractéristiques des paradigmes. Comparajson de la recherche normale et de la recherche paradigmatique en mettant en relief les traits particuliers de la recherche paradigmatique. Pour la discipline des relations professionnelles, cette forme de recherche est de toute première importance si on veut en arriver à une conception valide et réaliste des comportements en matière de relations du travail - 2 tableaux, bibliographie.

\section{MARION, Gérald}

La part des salaires dans le revenu national au Canada, 1910 à 1980.

v. 37 , no 1,1982, p. $53-64$

De 1910 à 1980, la part salariale dans le revenu national a augmenté de trente (30) points en pourcentage. Cette hausse s'accompagne d'une augmentation de la proportion de salariés dans la population ainsi que de changements de structure de l'économie. Les grandes tendances de la part salariale et quantification de l'importance des facteurs qui les sous-tendent.

\section{HASAN, Abrar}

GERA, Surendra

Reservation Wages in Canadian Labour Markets.

v. 37 , no 1,1982 , p. $65-92$

Les exigences salariales des chômeurs en quête de travail à partir de la théorie de la recherche d'emploi. Vérification empirique de l'hypothèse du salaire d'acception (salaire qu'un chômeur accepte plutôt que de continuer sa recherche d'emploi). L'influence du salaire d'acceptation sur la durée du chômage - 3 tableaux, 2 appendices, références.

\section{GILES, Anthony}

The Canadian Labour Congress and Tripartism.

v. 37 , no 1,1982 , p. $93-125$

Le Congrès du travail du Canada et le tripartisme: L'origine, le fonctionnement et le résultat des débats sur le tripartisme au cours de la période de l'application du programme de contrôle des prix et des salaires de 1976 à 1978. Les efforts en vue d'instituer une politique volontaire de restriction des revenus et évènements majeurs qui ont suivi la période des contrôles. La tendance au tripartisme est reliée à la politique du contrôle des revenus par le gouvernement - 1 tableau.

\section{BARTH, Richard T.}

BABA, Vishwanath V.

Alienation Among Professionnal Engineers: $A$ Canadian - American Comparison.

v. 37 , no 1,1982 , p. $126-140$

Recherche comparant l'intensité de l'aliénation parmi les ingénieurs canadiens et américains. Les variables spécifiques utilisées dans l'évaluation du degré d'aliénation consistaient dans le type de supervision, la liberté de s'adonner à la recherche, le climat professionnel et les encouragements de la part de l'employeur. L'étude révèle que les ingénieurs canadiens se sentent moins aliénés que les ingénieurs américains - 1 tableau, références.

0101 DÉOM, Esther

La négociation collective chez les fonctionnai- 
res et les enseignants québecois: 1975-1976. v. 37, no 1, 1982, p. 141-163

Présentation des résultats d'une étude sur la quatrième ronde de négociation, celle de 1975-1976, chez les fonctionnaires et les enseignants québécois: les éléments du cadre de références; les négociations des deux secteurs retenus: objectifs des parties, évènements importants des négociations, résultats obtenus, variables qui ont influencé les résultats de ces négociations; comparaison des deux négociations; commentaires sur le cadre de référence et sur le type de recherche réalisée.

0102 MILLER, Roger

Les formes d'organisation dans l'industrie de la construction au Québec.

v. 37 , no 1,1982, p. $164-176$

Les variables techniques et économiques qui influencent la structure et les stratégies des entreprises de construction au Québec. SeIon les dirigeants d'entreprises de construction, la stratégie porte non seulement sur le choix d'une mission économique spécialisée mais surtout sur la confection d'une structure organisationnelle qui permet le retranchement, l'expansion, la formalisation et la contraction en fonction du contexte économique - 3 tableaux.

0103 PEACH, David A.

The Canadair-I.A.M. Productivity: Improvement Plan.

v. 37 , no 1,1982 , p. 177-197

En 1968, Canadair Limited et I'International Association of Machinists ont négocié un plan d'amélioration de la productivité. Avec les années, ce plan a subi plusieurs changements. Histoire de ce plan: Comment il a débuté; comment il s'est développé; comment il est relié aux relations patronales-ouvrières à Canadair.

0104 CHAISON, Gary N.

A Note on the Critical Dimensions of the Union Merger Process.

v. 37, no 1, 1982, p. 198-206

L'activité de recherche récente signale le besoin d'établir au moins les fondements d'une thérie compréhensive et comparative du processus de fusion syndicale. Description des dimensions les plus importantes du pro- cessus à prendre en considération dans toute recherche pouvant mener à l'énoncé d'une telle théorie - Références.

\section{CHOUINARD, Jacques VIEL, André}

L'efficacité des clauses d'indexation des salaires

v. 37 , no 1,1982 , p. $207-214$

Commentaires critiques sur l'étude de JeanMichel Cousineau et de Robert Lacroix traitant de l'efficacité des clauses d'indexation des salaires et de sa mesure - 2 tableaux.

\section{COUSINEAU, Jean-Michel} LACROIX, Robert

L'efficacité des clauses d'indexation des salaires: Réplique.

v. 36 , no 1, 1982 , p. $214-220$

Réplique des auteurs à l'article de Jacques Chouinard et Pierre Lacroix, dans lequel ces derniers critiquaient l'étude qu'ils ont faites sur l'efficacité des clauses d'indexation des salaires.

\section{CHOUINARD, Jacques}

ROBICHAUD, Jean-Marc

L'efficacité des clauses d'indexation des salaires.

v. 37 , no 1,1982 , p. $220-224$

Commentaires critiques sur l'étude réalisée par Sharon Michaud et Jean-Charles Roy et traitant de l'efficacité des clauses d'indexation des salaires.

\section{MICHAUD, Sharon} ROY, Jean-Charles

L'efficacité des clauses d'indexation des salaires: Réplique.

v. 37 , no 1,1982 , p. $224-225$

Réplique des auteurs à l'article de Jacques Chouinard et de Jean-Marc Robichaud dans lequel ces derniers critiquaient l'étude qu'ils ont réalisées sur l'efficacité des clauses d'indexation des salaires.

0109 LAVERY, Daniel

Décisions rendues par le Conseil canadien des relations du travail.

v. 37, no 1, 1982, p. 226-234

Résumé de trois décisions rendues par le Conseil: 1) Le devoir de représentation juste 
Relations Industrielles, vol. 45, no 4 (1990)

et équitable pour un syndicat s'impose également dans le cadre de la négociation d'une convention collective. 2) Le Conseil décide qu'un syndicat n'est pas un "employeur" au sens du Code. 3) Décision sur le refus par un employé de s'acquitter des fonctions et des responsabilités d'un autre employé qui était en grève.

\section{SEXTON, Jean (R)}

WOODS, H.D.

Relations industrielles dans l'industrie de la construction du sud du Nouveau-Brunswick. Fredericton, N.B., Ministère du travail et de la main-d'oeuvre, mars 1981, 617 pp., recensé par Jean Sexton.

v. 37 , no 1,1982 , p. $240-241$

0111 VERGE, Pierre (R)

ARTHURS, H.W. CARTER, D.D.

GLASBEEK, H.J.

Labour Law and Industrial Relations in Canada. Deventer, Kluwer; Toronto; Butterworths, 1981, 291 pp., ISBN 90-312-0139 (Kluwer), 0409-81184-X (Butterworths), recensé par Pierre Verge.

v. 37 , no 1, 1982, p. 241-243

0112 GRIEZIC, F.J.K. (R) MORTON, Desmond COOP, Terry

Working People, An IIlustrated History of Canadian Labour. Ottawa, Deneau \& Greenberg, 1980, 349 pp., recensé par F.J.K. Griezic.

v. 37 , no 1, 1982, p. $243-245$

0113 DION, Gérard (R)

MARSH, Arthur RYAN, Victoria

Historical Directory of Trade Unions, vol. 1 : Non-Manual Unions. Farnborough, England, Gower, 1980, 256 pp., ISBN 0-566-02160-9, recensé par Gerard Dion.

v. 37, no 1,1982 , p. $245-246$

0114 GAUTHIER, Hervé (R)

FRIEDMAN, Henry MEREDEEN, Sander

The Dynamics of Industrial Conflict, Lessons from Ford. London, Croom Helm, 1980, 386 pp., recensé par Hervé Gauthier.

v. 37, no 1,1982 , p. $246-248$

0115 BEAULIEU, Paul (R)

DELPLANQUE, Bernard

Le partage de l'emploi, vers une societé sans chómage. Paris, Editions Entente, 1980, 141 pp., recensé par Paul Beaulieu.

v. 37 , no 1,1982, p. 248
0116 MERCIER, Jacques ( $R$ )

DENTON, F.T. ROBB, A.L.

SPENCER, B.G.

Unemployment and Labour Force Behaviour of Young People : Evidence from Canada and Ontario. Ontario Economic Council Research Series no 20, Toronto, University of Toronto Press, 1980, 215 pp., ISSN 0708-3688, ISBN 0-8020-3379-2, recensé par Jacques Mercier. v. 37 , no 1, 1982, p. 249-251

\section{LAJOIE, Mario (R)}

VAISEY, Douglas $\mathrm{G}$.

The Labour Companion : A Bibliography of Canadian Labour History, Based on Materials Printed from 1950 to 1975. Halifax, Committee on Canadian Labour History, 1980, 128 pp., recensé par Mario Lajoie.

v. 37 , no 1,1982 , p. $251-252$

0118 THWAITES, James (R)

CRAVEN, Paul

"An Impartial Umpire" : Industrial Relations and the Canadian State 1900-1911. Toronto, University of Toronto Press, 1980,386 pp., ISBN 0-8020-5505-2, recensé par James Thwaites.

v. 37 , no 1,1982, p. $252-253$

0119 FRANK, J.A. KELLY, Michael J. MACNAUGHTON, Bruce D.

Legislative Change and Strike Activity in Canada, 1926-1974

v. 37 , no 2,1982, p. $267-283$

Explication des variations à long terme des grèves au Canada analysant les effets de diverses variables d'ordre économique et organisationnel sur les types de conflits du travail. Comment les modifications apportées à la législation fédérale du travail ont influencé au cours des années les types de grèves survenues au Canada - 5 tableaux, références.

0120 FISHER, E.G.

Strike Activity and Wildcat Strikes in British Columbia: 1945-1975.

v. 37 , no 2,1982 , p. $284-312$

Présentation et analyse du modèle des grèves en Colombie britannique de 1945 à 1975. Classement des grèves en trois groupes distincts. Les grèves déclenchées 1) à l'occasion d'une première convention collective; 2 ) lors du renouvellement de la convention; 3) en cours de convention. L'analyse traite en particulier de ces dernières qu'on appelle 
aussi grèves sauvages, illégales, inautorisées ou de protestation - 8 tableaux, références.

\section{DESCHÊNES, Jean-Paul}

L'évaluation des emplois et la convention collective.

v. 39 , no 2,1982 , p. $313-327$

Analyse du rôle de chacune des parties dans 1) l'élaboration et l'implantation d'une méthode d'évaluation, 2) l'évaluation proprement dite du groupe d'emploi qu'elles ont déterminé, 3) la réévaluation des emplois modifiés, 4) la procédure à suivre en cas de grief et d'arbitrage en matière d'évaluation.

\section{BEIGBEDER, Yves}

Individual Grievance Procedures in United Nations Secretariats.

v. 37, no 2,1982 , p. $328-343$

Les procédures de recours individuel dans les organisations des Nations Unies, procédures destinées à examiner et régler les conflits internes entre employés et employeurs. Le processus de règlement des griefs pour les 46,000 employés des Nations Unies - 2 tableaux.

0123 JAIN, Harish C.

Race ans Sex Discrimination in Employment in Canada: Theories, Evidence and Policies.

v. 37, no 2,1982 , p. $344-366$

La discrimination dans l'emploi fondée sur la race et le sexe au Canada est analysée selon trois approches différentes: 1) L'approche fondée sur le marché du travail interne d'une entreprise; 2) L'approche se rapportant au marché du travail divisé en deux secteurs: primaire et secondaire; 3) L'approche dite de capital humain. Analyse de la législation contre la discrimination dans l'emploi adoptée par le gouvernement fédéral et l'ensemble des provinces - 5 tableaux, références.

0124 WALSH, William D.

A Time Series Analysis of Female Labour Force Participation Rates Disaggregated by Marital Status.

v. 37 , no 2,1982, p. $367-384$

Étude qui a pour objet d'apporter une double contribution à l'analyse des taux d'activité de la main-d'oeuvre féminine: 1) Par l'utilisation de statistiques canadiennes encore inexploi- tées, de façon à ajouter la dimension du statut matrimonial aux groupes d'âge ordinaire; 2) Par l'application d'un modèle empirique qui mesure séparément les effets du travailleur additionnel et du travailleur découragé - 2 tableaux, références.

\section{LE LOUARN, Jean-Yves}

Les professeurs d'université et leur travail. v. 37 , no 2,1982 , p. $385-402$

Analyse des résultats d'une étude faite auprès d'un échantillon de professeurs de l'Université de Montréal au sujet de leur satisfaction au travail. Les résultats révèlent des différences marquantes suivant l'aspect du travail considéré et les caractéristiques du professeur interrogé - 3 tableaux, références.

0126 BOLDUC, Roch BOUCHARD, Lucien HÉBERT, Gérard

Le régime québécois de négociation des secteurs public et parapublic

v. 37 , no 2,1982 , p. $403-430$

Trois textes décrivant le régime de négociation collective dans les secteurs public et parapublic québécois depuis les années 1940 jusqu'à ce jour: Bilan de la situation avant 1964; changement majeur en 1964 et conséquences; fonctionnement et évolution du régime; les réussites et les échecs du régime; les équivoques du régime; perspectives pour les années 1980.

0127 MELTZ, Noah M.

Labour Market Infcrmation in Canada: The Current Situation and a Proposal.

v. 37, no 2,1982 , p. $431-437$

En ce qui a trait à l'information sur le marché du travail, on constate qu'il y a eu diminution dans les types de statistiques publiées disponibles, nécessaires pour contrôler les développements du marché du travail au Canada. Vue d'ensemble sur le sujet et esquisse d'une proposition pour améliorer la situation en cette matière - Références.

0128 LAVERY, Daniel

Décisions rendues par le Conseil canadien des relations du travail.

v. 37 , no 2,1982 , p. $438-450$

1) Le Conseil accueille une plainte formulée à l'encontre d'un syndicat ayant failli à son 
devoir de représentation juste et équitable à l'endroit d'un employé congédié alors qu'il était en période de probation. 2) Le gel statuaire de l'article 148b du Code entrant en vigueur suite à l'envoi d'un avis de négocier par l'une des parties. 3) Le Conseil juge qu'il a juridiction pour entendre une plainte de congédiement illégal par un employé ayant rédigé et remis une lettre de démission suite à des pressions de l'employeur.

0129 VERGE, Pierre (R)

KAHN-FREUND, 0 .

Labour Law and Politics in the Weimar Republic. Oxford, Basil Blackwell, 1981, ISBN O631-12825-5, recensé par Pierre Verge.

v. 37 , no 2,1982 , p. $454-455$

0130 BÉLANGER, Jacques (R)

STOREY, John

The Challenge to Management Control. Brookfield, Vermont, Renouf USA, 1981, 192 pp., ISBN 0-09-145941-9, recensé par Jacques Belanger.

v. 37 , no 2,1982, p. $455-457$

0131 BÉLANGER, Laurent (R) JACKSON, John H. MORGAN, Cyrill $P$.

Organization Theory : A Macro Perspective for Management (2nd ed.). Englewood Cliffs, Prentice-Hall. Inc., 1982, 413 pp., ISBN 013$641415-X$, recensé par Laurent Bélanger.

v. 37 , no 2,1982 , p. $457-458$

0132 ANGERS, Bernard ( $R$ )

HOUDE, Eugène

Émotivité et efficacité au travail. Montréal, Éditions de l'Homme, 1982, 112 pp., ISBN 27619-0213-0, recensé par Bernard Angers. v. 37 , no 2,1982 , p. $458-459$

0133 BÉLANGER, Laurent (R)

GLUECK, William F.

Personnel : A Diganostic Approach, (3rd ed.). Business Publications inc., 1982, 720 pp., ISBN 0-256-02429-4, recensé par Laurent Bélanger.

v. 37 , no 2,1982 , p. $459-460$

0134 DION, Gérard (R)

CEREQ

L'évolution des systèmes de travail dans l'économie moderne. Paris, Editions du CNRS, 1981, 287 pp., ISBN 2-222-02935-X, recensé par Gerard Dion.

v. 37 , no 2,1982 , p. 460
0135 HÉBERT, Gérard

Distinction 1982 de l'Association canadienne des relations industrielles: Gerard Dion.

v. 37 , no 3,1982 , p. $475-476$

Éloges à l'endroit de l'abbé Gérard Dion, récipiendaire de la Distinction de l'Association canadienne des relations industrielles. Professeur au Département des relations industrielles de l'Université Laval depuis 1943, il a à son crédit de nombreuses réalisations, entre autres: La fondation de la revue "Relations industrielles' en 1945; l'inauguration des Congrès annuels des relations industrielles en 1945; le 'Dictionnaire canadien des relations du travail".

\section{PROULX, Pierre-Paul}

Rémunération dans les secteurs public et parapublic au Québec: Éléments d'une nouvelle politique.

v. 37 , no 3,1982 , p. $477-497$

Examen de la situation actuelle en regard des politiques de rémunération de la main-d'oeuvre dans les secteurs publics fédéral et québécois. Présentation d'une nouvelle démarche en expliquant le modèle d'estimation des écarts de rémunération entre les secteurs public et privé.

0137 WILLIAMS, C. Glyn

The Role of Unions in Inflation: A Survey Article

v. 37 , no 3,1982 , p. $498-527$

Description du large éventail de points de vue exprimés par de nombreux auteurs sur le rôle des syndicats en tant que responsable de l'inflation et sur leurs réactions devant celleci. Utilisation d'un système de classification pour diviser les auteurs par catégorie. Les moyens par lesquels les auteurs ont mesuré le pouvoir des syndicats. Évaluation des études sur le militantisme des syndicats en tant qu'aspect distinct de leur rôle - 5 figures, 1 tableau.

0138 MAGUN, Sunder

The Rise of Service Employment in the Canadian Economy.

v. 37 , no 3,1982 , p. $528-556$

L'emploi dans le secteur des services au Canada a augmenté rapidement au cours des trois dernières décennies. Les raisons de 
l'augmentation de l'emploi dans les entreprises de services et hypothèse pouvant l'expliquer. Cet accroissement s'explique soit par une demande plus forte de services que de produits manufacturés, soit par une augmentation plus lente de la productivité par employé dans les services - 10 tableaux, 5 graphiques, références.

\section{WEIERMAIR, Klaus}

Apprenticeship Training in European Countries: The Lessons for Canada.

v. 37 , no 3,1982, p. 557-574

Les caractéristiques et les éléments fondamentaux des programmes de formation professionnelle existant dans les pays européens. Définition de l'apprentissage comme un processus général de formation qui permet d'acquérir des connaissances. Les conditions qui, en Europe, ont contribué au maintien de cette forme d'apprentissage de type industriel: Législation et financement des programmes par les gouvernements. Problèmes et déficiences en matière d'apprentissage au Canada.

\section{DELORME, François LAFRANCE, Victor}

La concurrence dans un contexte de pluralisme syndical: Quelques données sur le Québec.
v. 37 , no 3,1982 , p. $575-605$

Description de l'activité sur le plan de la concurrence entre les diverses organisations syndicales oeuvrant au Québec, au cours de la période comprise entre le 15 juin 1978 et le 16 juillet 1979. Les coordonnées juridiques relatives à ce phénomène de concurrence. Identification des sources de données dans lesquelles ont été puisés les renseignements et analyse des résultats obtenus - 8 tableaux, bibliographie.

\section{ADAMS, Roy J.}

The Federal Government and Tripartism. v. 37 , no 3 , 1982, p. 606-617

Depuis la deuxième guerre mondiale, la plupart des pays occidentaux ont établi un système de prise de décision tripartite qui regroupe les syndicats, les associations d'employeurs et l'État en vue de rechercher un consensus en matière de politique économique et sociale. Les pays d'Europe qui ont instauré des systèmes tripartites ont mieux réussi durant la dernière décennie que les États-Unis et le Canada où le tripartisme est sous-développé. Les difficultés d'implanter le tripartisme au Canada.

\section{JAMAL, Muhammad \\ BABA, Vishwanath $V$. MITCHELL, Vance F.}

The Naturea of Need Structure in Nonwork. v. 37 , no 3,1982 , p. $618-633$

Résultats d'une enquête portant sur la nature des besoins chez les gens en dehors des heures de travail. L'étude regroupait un échantillon de 403 salariés appartenant à des entreprises du Vancouver métropolitain dont le nombre d'employés varie entre 100 et 300 . Les données furent recueillies au moyen d'un questionnaire conçu pour la méthode d'enquête sur le terrain. Description de la méthodologie utilisée pour mesurer la satisfaction des besoins en dehors du travail - 3 tableaux, références.

\section{LATULIPPE, Gérard P.} O'FARRELL, Kevin

Le comite paritaire: Anachronisme ou formule d'avenir.

v. 37, no 3,1982, p. $634-655$

Les dimensions théoriques et pratiques du paritarisme et comment ce concept traditionnel peut répondre aux besoins nouveaux. Les origines historiques et l'évolution du paritarisme au Québec. L'alternative paritaire et son application dans divers domaines, particulièrement dans le domaine de la santé et de la sécurité ainsi que dans celui de la formation des travailleurs.

\section{MEALIA, David W.}

A Macro/Micro Decision Model for the Training and Development Specialist.

v. 37 , no 3,1982 , p. $656-669$

Réponse à une carence apparente dans les études relatives à la formation et au développement, l'article a pour objet de mettre au point un modèle de décision destiné à guider les spécialistes en formation et développement. Le modèle de prise de décision se situe à deux niveaux, global et fragmentaire. Enfin, on insiste sur les facteurs fondamentaux à considérer dans l'application du modèle, soit les mécanismes d'appréciation et de rétroaction - Références. 
Relations Inoustrielles, vol. 45, no 4 (1990)

0145 BROSSARD, Michel

La stratégie syndicale face aux groupes semiautonomes de production: Hypothèse pour l'analyse du cas Rushton.

v. 37, no 3,1982, p. $670-683$

Par l'analyse de l'expérience à la mine Rushton, on vérifie la validité de l'hypothèse que l'action syndicale est articulée autour d'une stratégie comportant trois dimensions complémentaires, la survie, la vigilance et le contrôle qui caractérisent, selon des agencements différents, toutes les phases de la stratégie.

0146 LAVERY, Daniel

Décisions rendues par le Conseil canadien des relations du travail.

v. 37 , no 3,1982 , p. $684-696$

Résumé de trois décisions du Conseil: 1) Saisi d'une requête en accréditation, le Conseil décide d'élargir l'unité d'accréditation proposée. 2) L'élargissement de l'unité d'accréditation découlant de l'ajout d'un groupe d'employés à une unité existante et les droits des nouveaux employés. 3) Le Conseil accueille une plainte de pratique déloyale portant sur l'obligation de négocier de bonne foi.

0147 BÉLANGER, Gérard (R) CONSEIL ÉCONOMIQUE DU CANADA

Pénuries et carences. Travailleurs qualifiés et emplois durant les années 80 . Ottawa, ministère des Approvisionnements et Services Canada, 1982, 141 pp., ISBN 0-660-90874-3, recensé par Gérard Bélanger.

v. 37 , no 3,1982 , p. $704-709$

0148 DION, Gérard (R)

COUSINEAU, Jacques

L'Église d'ici et le social 1940-1960, 1. La Commission sacerdotale d'Études sociales, Montréal, Les Éditions Bellarmin, 1982, 287 pp., ISBN 2-89007-462-5, recensé par Gérard Dion.

v. 37 , no 3,1982 , p. $709-712$

0149 THWAITES, James (R)

VIGEANT-GALLEY, Paulette

Les enseignants et le pouvoir : histoire de l'Alliance des professeurs de Montréal, les luttes syndicales et le développement social (1952-1958). Québec, CEQ-APM, décembre 1981, 128 pp., recensé par James Thwaites.

v. 37 , no 3,1982 , p. $712-713$
0150 VÉZINA, Lucie (R)

BOISVERT, Maurice

La qualité de la vie au travail. Regard sur l'expérience québécoise. Le Groupe d'étude de travail des HEC, Montréal, Éditions Agence d'Arc Inc., 1981, 461 pp., recensé par Lucie Vézina.

v. 37 , no 3,1982 , p. $713-715$

0151 MATEJKO, Alexander J. (R)

CUMMINGS, Thomas $\mathrm{G}$.

MOLLOY, Edmond S.

Improving Productivity and the Quality of Work Life. New-York, Prager Publications, 1977, 305 pp., ISBN 0-03-022601-5, recensé par Alexander J. Matejko.

v. 37 , no 3,1982 , p. $715-717$

0152 BLYTON, Paul (R)

MELTZ, Noah M. REID, Frank

SWATZ, Gérald S.

Sharing the Work: An Analysis of the Issues in Worksharing and Jobsharing. Toronto, University of Toronto Press, 1981, 90 pp., recense par Paul Bhyton.

v. 37 , no 3,1982 , p. $717-718$

0153 LAROCQUE, Alain (R)

MARIC, D.

La durée du travail dans les pays en voie de développement. Genève, Bureau international du Travail, 1981, 138 pp., ISBN 92-2-2027329 , recensé par Alain Larocque.

v. 37 , no 3,1982 , p. $718-719$

0154 LAROCQUE, Alain (R)

MCGAUGHEY, William Jr.

A Shorter Workweek in the 1980's. White Bear Lake, Minnesota, Thestterose Publications, 1981, 308 pp., ISBN 0-9605630-0-8, recensé par Alain Larocque.

v. 37 , no 3,1982 , p. $718-719$

0155 BOURASSA, Raynald (R)

CONNAGHAN, Charles J.

L'expérience japonaise. Relations industrielles au Japon moderne. Ottawa, Travail Canada, 1982, 143 pp., ISBN 0-662-91608-5, recensé par Raynald Bourassa.

v. 37, no 3,1982, p. 720

0156 RAMOS, Elias T. (R)

INFANTE, Jaime T.

The Political, Economic and Labor Climate in the Philippines. Industrial Research Unit, The Wharton School, University of Pennsylvania, 1980, 147 pp., recensé par Elias T. Ramos. v. 37, no 3,1982 , p. $720-722$ 
0157 STURMTHAL, Adolf Unemployment, Inflation and "Guest Workers": Comparative Study of Three European Countries.

v. 37 , no 4,1982 , p. $739-764$

Trois pays occidentaux ont trouvé une solution partielle et possiblement temporaire au problème de parvenir à un degré élevé d'emploi tout en gardant leur taux d'inflation à un niveau relativement bas. Ce sont la Suède, l'Allemagne de l'ouest et l'Autriche. Le taux de croissance du produit national brut de même que l'existence dans ces pays de ce qu'on est convenu d'appeler "un contrat social" sont deux éléments pouvant expliquer le succès de ces trois pays - 4 tableaux.

0158 CHAISON, Gary N. ANDIAPPAN, P.

Characteristics of Female Union Officer in Canada.

v. 37 , no 4,1982 , p. $765-779$

Résultats d'une enquête effectuée auprès des femmes qui faisaient partie de comités exécutifs syndicaux en 1980. Caractéristiques de ces femmes occupant des postes de commande dans les syndicats au Canada et obstacles qui s'opposent à leur participation à la direction de ces institutions - 3 tableaux, références.

0159 AGARWAL, Naresh C.

Male-Female Pay Inequity and Public Policy in Canada and the U.S.

v. 37 , no 4,1982 , p. $780-804$

Revue détaillée des études empiriques qui ont traité, au cours des deux dernières décennies, des différences de salaires entre les hommes et femmes aux États-Unis et au Canada. Examen de la législation existante dans ces deux pays en matière d'égalité de traitement pour les hommes et les femmes qui occupent des tâches semblables - Références.

0160 TOUPIN, Louis LESSARD, Claude CORMIER, Roger A. VALOIS, Paul La satisfaction au travail chez les enseignantes et enseignants au Québec.

v. 37 , no 4,1982 , p. $805-826$

Résultats d'une enquête effectuée en 1978 auprès de 4,000 enseignants des écoles pri- maires et secondaires des secteurs public et privé, francophone et anglophone du Québec, portant sur la satisfaction au travail. L'article examine si l'enseignant est satisfait de son travail, s'il veut le quitter et quels sont les motifs les plus probables qui peuvent l'amener à le vouloir - 2 tableaux, références.

0161 BEAUMONT, P.B.

Third Party Conciliation and Trade Union Recognition: Some British Evidence.

v. 37 , no 4,1982 , p. $827-842$

L'utilisation des mécanismes de la conciliation volontaire pour résoudre les différends entre les syndicats et les employeurs a une longue histoire en Grande-Bretagne. On étudie ici le résultat de la totalité des requêtes en reconnaissance syndicale entendues par conciliation volontaire en Ecosse entre 1976 et 1980 par le Bureau régional du service consultatif de conciliation et d'arbitrage - 2 tableaux.

\section{COPP, Terry}

The Rise of Industrial Unions in Montreal 1935-1945

v. 37, no 4,1982, p. $843-875$

Étude de cas portant sur le développement des syndicats industriels dans une ville nordaméricaine (Montréal) au cours d'une décennie décisive dans la montée du syndicalisme. Description de la législation du travail exceptionnelle qui était alors en vigueur dans la province de Québec et des initiatives subséquentes du premier gouvernement Duplessis. Revue des quatorze principales industries de Montréal en fonction de l'activité syndicale dans les années 1930 - 2 tableaux.

\section{MAKI, Dennis R.}

Political Parties and Trade Union Growth in Canada.

v. 37 , no 4,1982 , p. $876-886$

Étude démontrant qu'il y a une relation de cause à effet entre le parti politique au pouvoir et le taux de croissance du syndicalisme. Étant donné que la législation du travail au Canada est du ressort des provinces, la vérification de cette hypothèse s'est effectuée à partir des statistiques par province relatives au développement des syndicats. Ces statistiques portaient sur la période de 1962 à 1978 inclusivement - 1 tableau. 
Relations lndustrielles, vol. 45, no 4 (1990)

0164 VINET, Alain BÉGIN, Clermont THÉRIAULT, Gilles

La santé des employés de bureau: Le cas de la fonction publique québécoise.

v. 37 , no 4,1982 , p. $887-904$

Résumé des résultats d'une enquête de santé effectuée auprès d'un échantillon de 1,300 employés du gouvernement du Québec. Les données furent recueillies à l'aide d'examens médicaux et de questionnaires concernant les habitudes de vie et de travail des participants. Les troubles psychiques et la consommation excessive de somnifères et de tranquillisants sont des problèmes réels que l'employeur et les syndicats doivent considérer de plus près - 5 tableaux.

\section{HARRIS, Philip RAUSCH, Peter} RYAN, Samuel G.

Ineffectiveness in Teacher Bargaining: The Anatomy of a First Strike.

v. 37 , no 4,1982 , p. $905-926$

Dans l'étude et la pratique de la négociation collective, on se heurte toujours à la possibilité d'une grève qui peut être néfaste. Lorsque, de plus, les parties sont des novices dans l'art de négocier et qu'elles font face à un état de grève pour la première fois, il est probable qu'elles auront un comportement qui laisse à désirer. Identification des faits qui caractérisent une situation de première grève et analyse des évènements qui ont entouré la première grève d'un syndicat d'enseignants - 1 tableau, références.

\section{COULOMBE, Gilles}

Le système de gestion des cas du Conseil canadien des relations du travail (CCRT).

v. 37 , no 4,1982 , p. $928-943$

Les pratiques et les procédures établies pour assurer le fonctionnement efficace du C.C.R.T. ainsi que le système utilisé pour mesurer le rendement des opérations de cet organisme.

0167 LAVERY, Daniel

Décisions rendues par le Conseil canadien des relations du travail.

v. 37 , no 4,1982 , p. $944-959$

Après l'étude de la notion d'employé" que l'on retrouve à l'article 107 du Code canadien du travail, le Conseil conclut que toute personne visée par une demande d'accréditation, économiquement subordonnée à un employeur et qui n'est pas un entrepreneur, est un "employé" au sens du Code. Le Conseil a également énoncé sa politique quant aux délais de présentation et la recevabilité de requêtes en accréditation et celle quant aux requêtes en révision.

\section{HÉBERT, Gérard $(R)$}

DEMERS, François

Chroniques impertinentes du 3ième (sic) Front commun Syndical, 1979-1980. Montreal, Nouvelle optique, 1982, 170 pp., ISBN 2-89017-035-7, recensé par Gerard Hébert. v. 37 , no 4,1982 , p. $962-963$

0169 MATEJKO, Alexander J. (R)

BAUM, Gregory

The Priority of Labour. A Commentary on Laborem Exercens, Encyclical Letter of Pope John Paul II. New York/Ramsey, Paulist Press, 1982, 152 pp., ISBN 0-8091-2479-3, recensé par Alexander J. Matejko.

v. 37 , no 4,1982 , p. $964-966$

0170 BLOUIN, Rodrigue (R) NADEAU, Gaston

Le statut juridique du salarié-gréviste québécois. Québec, Les Presses de l'Université Laval, 1981, 186 pp., ISBN 2-7637-6938-1, recensé par Rodrigue Blouin.

v. 37 , no 4,1982 , p. $966-967$

\section{MORIN, Fernand $(\mathrm{R})$}

COPLAND LTÉE.

Index et résumés de sentences arbitrales de griefs, vol. 1, 1970-1980. Montreal, Copland Ltée, 1982, ISBN 2-920391-002, recensé par Fernand Morin.

v. 37 , no 4,1982 , p. $967-968$

0172 WETZEL, Kurt (R)

FOSSUM, John A.

Labor Relations : Development, Structure, Process (Revised Edition). Dallas, Business Publications, 1982, 521 pp., ISBN 0-256-02688-2, recensé par Kurt Wetzel.

v. 37 , no 4,1982 , p. $968-969$

0173 WEISS, Dimitri (R)

BOIVIN, Jean GUILBAULT, Jacques

Les relations patronales-syndicales au Qué-

bec. Chicoutimi, Gaëtan Morin Éditeur, 1982, 309 pp., ISBN 2-89105-087-8, recensé par Dimitri Weiss.

v. 37, no 4,1982 , p. $969-970$ 
0174 VINET, Alain (R) MCCARTHY, Maureen ROSENBERG, Gail S. Work Sharing : Case Studies. Kalamazoo, Michigan, W.E. Upjohn Institute for Employment Research, 1981, 277 pp., ISBN 0-911558-87-X, recensé par Alain Vinet.

v. 37, no 4,1982 , p. 971

0175 RAINVILLE, Jean-Marie (R)

BOULET, Jac-André

La langue et le revenu du travail à Montréal. Ottawa, Ministère des Approvisionnements et Services Canada, Etude préparée par le Conseil économique du Canada, 1980, 135 pp., ISBN 0-660-90515-9, recensé par Jean-Marie Rainville.

v. 37, no 4,1982 , p. $971-973$

0176 SEXTON, Jean ( $R)$

SIEGEL, Irving $H$. WEINBERT, Edgar

Labor-Management Cooperation. The American Experience. Kalamazoo, Michigan, W.E. Upjonn Institute for Employment Research, 1982, 316 pp., ISBN 0911558-99-3, recensé par Jean Sexton.

v. 37 , no 4,1982 , p. 973

0177 CAIRE, Guy

Procédures de règlement pacifique des conflits collectifs en France.

v. 38, no 1,1983, p. 3-27

Description et interprétation du particularisme de la procédure d'arbitrage des conflits collectifs du travail en France qui offre l'image contradictoire de l'échec des modalités officielles de règlement et de la vertu des procédures informelles - 5 tableaux.

0178 WARNER, Malcolm

Corporatism, Participation and Society

v. 38 , no 1,1983, p. $27-44$

Les changements qui peuvent se produire en Angleterre dans le domaine de la participation des travailleurs à la vie des entreprises parallèlement à la croissance des tendances corporatistes qui se répandent en Europe continentale. Alors que l'expérience britannique a conduit à plusieurs réalisations favorables à la démocratie industrielle, il n'y a guère eu émergence d'institutions proprement dites qui se soient implantées - 2 tableaux, références.
0179 DOLAN, Shimon L.

ARSENAULT, André

LIZOTTE, Jean-Paul

ABENHAIM, Lucien

L'absentéisme hospitalier au Québec: Aspects culturels et socio-démographiques.

v. 38, no 1, 1983, p. 45-57

Résultats d'une étude portant sur certains aspects de l'absentéisme en milieu hospitalier au Québec. Identification des variables sociodémographiques et analyse de la complexité des relations entre ces variables: âge, statut civil, responsabilités familiales, niveau occupationnel, contenu de la tâche et son contexte, ancienneté, éducation, salaire etc. On démontre particulièrement que les modèles basés sur la corrélation linéaire ne sont plus adéquats pour comprendre le phénomène de l'absentéisme - 8 tableaux.

\section{EASTMAN, Byron}

Canadian Union Growth

v. 38 , no 1,1983 , p. $58-71$

La seule analyse antérieure de la croissance des syndicats canadiens en est une de type d'équation simple. Or, plusieurs forces exercent une infiuence sur le développement des syndicats ainsi que sur l'augmentation des salaires et des prix. Les trois variables dépendantes s'influencent les unes les autres. On tente ici de combler le manque d'équations simultanées pour apprécier la croissance des syndicats canadiens en présentant une analyse empirique des variations dans le temps des effectifs des syndicats de 1947 à 1970 - 1 tableau, références.

\section{MAGUN, Sunder}

The Placement Activity of the Canadian Employment Agency.

v. 38 , no 1,1983 , p. $72-94$

Le service de placement de l'Agence canadienne d'emplois fournit des services rapides de références et de placement d'emplois. En réduisant le temps de recherche de travail, le service public de placement améliore le fonctionnement du marché du travail en diminuant le chômage frictionnel. Cependant, les centres d'emplois du Canada n'alimentent encore qu'une faible partie du marché du travail - 5 tableaux, 3 graphiques, bibliographie. 


\section{SIMPSON, Wayne} PETERS, Frank

The Economics of Mileage Restrictions for Railway Workers in Western Canada. v. 38, no 1, 1983, p. 95-103

La convention collective conclue entre le Canadien Pacifique et le Syndicat des employés des transports dans l'Ouest du Canada contient un système de rémunération fondé sur un taux de salaire au parcours comportant un kilométrage maximal et minimal. On étudie ici les données économiques de ce système de rémunération au parcours de manière à identifier et à faire comprendre les problèmes de négociation collective qu'il engendre - 2 figures, références.

0183 PEITCHINIS, Stephen G.

The Attitude of Trade Unions Towards Technological Changes.

v. 38 , no 1,1983 , p. $104-119$

Résultats d'une enquête sur le comportement des syndicats à l'égard des changements technologiques. En général, les syndicats adopteront des attitudes positives dans les industries où il y a augmentation de l'emploi et négatives dans les industries où il $\mathrm{y}$ a déclin dans l'emploi. Enfin, on note une attitude d'indifférence chez les syndicats dont les membres n'ont pas été touchés par des changements technologiques - 2 tableaux.

\section{XISHAN, Yang MOORE, Larry $F$.} MARKGRAF, Herb

Behavioural Science Application in Vancouver Based Firms.

v. 38 , no 1,1983 , p. $120-141$

Les controverses qui entourent l'utilisation des découvertes de la science des comportements (BEHAVIORISME) dans les entreprises nord-américaines. Identification des applications de cette science et de ses réalisations dans l'Ouest canadien et des vues disparates de divers groupes à son endroit. Résumé des constatations d'une enquête auprès de 39 entreprises de Vancouver consistant en plus de 50 entrevues personnelles avec différents cadres de ces entreprises - Références.

0185 JOLIVET, Thierry

La réduction de la durée du travail est-elle créatrice d"emploi?

v. 38 , no 1,1983 , p. $142-154$
La réduction de la durée du travail est considérée comme un moyen possible de réponse au chômage. Pour vérifier cette hypothèse, on aborde dans une première partie l'aspect macro-économique et théorique du problème, avant de centrer l'analyse, dans une deuxième partie, sur la firme à la fois micro-économique et plus concrète - 1 tableau.

\section{AOUST, Claude d'}

Les ententes individuelles et la convention collective.

v. 38 , no 1,1983 , p. $155-158$

Les deux écoles de pensée concernant la relation entre le contrat individuel de travail et la convention collective: 1) La convention collective prend toute la place; 2) Les conditions de travail négociées sont minimales et peuvent être améliorées par contrat individuel de travail. Analyse d'une décision basée sur le Code scolaire albertain qui permet expressément la détermination partielle des conditions de travail par entente individuelle.

0187 LAVERY, Daniel

Décisions rendues par le Conseil canadien des relations du travail.

v. 38 , no 1,1983 , p. $159-173$

Résumé de deux décisions rendues par le Conseil: 1) Le Conseil est saisi d'un conflit de distribution du travail entre syndicats rivaux engendré par l'absence de quelque mention relative à la fonction disputée dans leurs certificats d'accréditation ou conventions collectives. 2) Le Conseil énonce pour la première fois sa politique relative aux droits de succession entre syndicats.

0188 SEXTON, Jean ( $R$ )

DELORME, François PARENT, Réjean

Les licenciements collectifs au Québec : un bilan partiel du dispositif public en vigueur. Monographie 12, Montréal, Ecole de relations industrielles, Université de Montréal, 1982, 106 pp., ISBN 2-89067-011-2 et ISSN 0708-9945, recensé par Jean Sexton.

v. 38 , no 1,1983, p. 178

0189 THWAITES, James (R)

FORSEY, Eugène

Trade Unions in Canada : 1812-1902. Toronto, University of Toronto Press, 1982, $600 \mathrm{pp}$., ISBN 0-8020-6388-8, recensé par James Thwaites.

v. 38 , no 1,1983 , p. $179-180$ 
0190 VERGE, Pierre (R)

GOULD, W.B.

A Primer on American Labor Law. Cambridge, Mass., The MIT Press, 1982, 242 pp., ISBN 0262-070-87-1, recensé par Pierre Verge.

v. 38 , no 1,1983 , p. $180-181$

0191 BÉLANGER, Jacques (R)

EDWARDS, P.K. SCULLION, Hugh

The Social Organization of Industrial Conflict : Control and Resistance in the Workplace. Oxford, Basil Blackwell, 1982, 314 pp., ISBN 0-631-13127-2, recensé par Jacques Bélanger. v. 38 , no 1,1983, p. $181-184$

0192 ZAIDI, Mahmood A. (R) STIEBER, Jack MCKERSIE, Robert $B$. QUINN MILLS, D.

U.S. Industrial Relations 1950-1980 : A Critical Assessment. Madison, Industrial Relations Research Association, 1981, 361 pp., recensé par Mahmood A. Zaidi.

v. 38, no 1,1983, p. 184-187

0193 WEISS, Dimitri ( $R$ )

LEFEBVRE, Gérard

Le coeur à l'ouvrage. Montréal, Les Éditions de l'Homme, 1982, 120 pp., ISBN 2-76190188-6, recensé par Dimitri Weiss.

v. 38 , no 1,1983 , p. $187-188$

0194 BABCOCK, Robert H. (R)

GROSS, James A.

The Reshaping of the National Labor Relations Board : National Labor Policy in Transition 1937-1947. Albany, State University of New York Press, 1981, 381 pp., ISBN 0-87395-2707 , recensé par Robert H. Babcock.

v. 38 , no 1,1983 , p. $188-189$

0195 BARRÉ, Alain (R)

O.I.T.

International Labour Conventions and Recommandations. I.L.O., Geneva, 1982, 1167 pp., recensé par Alain Barré.

v. 38 , no 1,1983 , p. $189-190$

0196 JOUBERT, P. (R)

GIUGNI, Gino MENGONI, Luigi

MORTILLARO, Felice WEISS, Dimitri

Possono migliorare le relazioni industriali in Italia? Torino, Gruppo Dirigenti Fiat, 1981, 140 pp., recensé par $P$. Joubert.

v. 38, no 1,1983 , p. 190-192
0197 JOUBERT, P. (R)

WEISS, Dimitri

Politica, partidos y sindicatos en la empresa.

Madrid, Instituto de Estudios laborales y de la Seguridad social, 1982, 136 pp., ISBN 845007823-7, recensé par $P$. Joubert.

v. 38, no 1, 1983, p. 192-194

0198 JOUBERT, P. (R)

SAGARDOY BENGOECHEA, J.A.

BLANCO, David Leon

El poder sindical en Espana. Barcelona, Editorial Planeta/Instituto de Estudios Economicos, 1982, 210 pp., ISBN 84-320-7318-0, recensé par $P$. Joubert.

v. 38 , no 1,1983 , p. $192-194$

0199 JAIN, Hem C. (R)

SAHAL, Jugendra

Urban Complex of an Industrial City. Allahbad, India, Chugh Publishing Company, 1980, recensé par Hem C. Jain.

v. 38 , no 1,1983 , p. 194-195

0200 BÉLANGER, Laurent (R)

COLLERETTE, Pierre DELISLE, Gilles

Le changement planifié. Montréal, Agence d'Arc, 1982, 213 pp., ISBN 0-89022-045-1, recensé par Laurent Bélanger.

v. 38 , no 1,1983 , p. 195-196

0201 MAKI, Dennis R.

Trade Unions and Productivity: Conventional Estimates.

v. 38, no 2,1983 , p. $211-228$

Les études portant sur le rôle des syndicats sur la productivité sont plutôt rares et prennent le plus souvent la forme d'études de cas. En critiquant certains travaux récents sur le sujet et en discutant les questions et les concepts en jeu, on ouvre ici de nouvelles voies vers une recherche future. Au Canada, les statistiques dont on dispose pour porter un jugement manquent généralement. Certains résultats empiriques sur l'industrie manufacturière canadienne -3 tableaux, références.

0202 VEILLEUX, Diane

Les clauses de promotion et de transfert chez les infirmières et les infirmiers.

v. 38 , no 2,1983 , p. $229-253$

Les mécanismes de promotion et de transfert chez les infirmiers et infirmières du Québec. Comment la promotion et le transfert (muta- 
tion) chez les infirmiers et infirmieres sont encadrés par les conventions collectives et par les spécificités de la profession exercée - 1 tableau, références.

0203 FISHER, E.G. PERCY, M.B.

The Impact of Unanticipated Output and Consumer Prices on Wildcat Strikes. v. 38 , no 2,1983 , p. 254-276

Les conséquences des changements imprévus du rendement et de l'indice des prix à la consommation sur les grèves sauvages ou en cours de convention qui ont eu lieu dans l'industrie du cuivre en Colombie Britannique pendant la période 1967 à 1979. Exposé d'une théorie des grèves sauvages basée sur le rendement et l'indice des prix à la consommation -2 tableaux, références.

\section{GARNIER, Bernard}

La gestion des conflits interpersonne/s en milieu universitaire.

v. 38 , no 2,1983 , p. $277-296$

L'utilisation et l'efficacité des méthodes de gestion de conflit interpersonnel utilisées par les doyens d'université. La façon dont les doyens gèrent les conflits avec leurs directeurs de département et leurs professeurs. Les implications théoriques de l'étude pour la gestion des conflits ainsi que les implications pratiques pour l'administrateur - 2 graphiques, 3 tableaux, bibliographie.

0205 MROCZKOWSKI, Tomasz

Theory Z: Myths, Realities and Alternatives. v. 38 , no 2,1983 , p. $297-318$

La théorie $Z$ a beaucoup attiré l'attention comme modèle abstrait de coopération entre employeurs et salariés à base de paternalisme, laquelle serait susceptible d'aider les entreprises américaines en y implantant certains éléments empruntés au régime de travail mis en place au Japon. Les régimes de relations professionnelles du Japon, non plus que ceux de l'Europe, ne paraissent pas être des régimes immédiatement adaptables aux ÉtatsUnis - 4 tableaux, 2 figures, références.

0206 AMERNIC, J.H. ARANYA, N.

Organizational Commitment: Testing Two Theories.

v. 38 , no 2,1983 , p. $319-343$
Résultats d'une recherche portant sur l'étude de l'appartenance à 'entreprise chez un groupe représentatif de Canadiens membres d'une profession libérale, soit les comptables agréés. La recherche examine la valeur potentielle de deux théries de l'appartenance à l'entreprise, celle dite d'échange et celle dite d'investissement. Description de la méthode de recherche utilisée et résultats obtenus - 5 tableaux, références.

\section{DIONNE, Pierre \\ OUELLET, Gilles}

Repenser l'évaluation.

v. 38 , no 2,1983 , p. $344-368$

Réflexion fondamentale sur l'état de la recherche en évaluation du potentiel humain. A partir des synthèses des études et recherches faites sur le sujet, on peut conclure qu'il est possible d'aborder la recherche sur l'évaluation du potentiel humain sous de nouveaux aspects plus prometteurs - 1 illustration.

\section{WEISS, Dimitri}

Du concept de "professionnalité" dans les relations industrielles italiennes.

v. 38 , no 2,1983 , p. $369-379$

Le concept, typiquement italien, de "professionnalité', engendre des prises de positions multiples des partenaires sociaux et un discours envahissant, original mais non privé de rhétorique, des observateurs du système des relations industrielles. Les différentes significations le plus souvent attribuées à ce concept qui ne se confond pas toujours avec le "professionnalisme".

\section{JOSEPH, P.A.}

Perfecting the Administrative Solution to Labour Disputes: The British Columbia Experiment.

v. 38 , no 2,1983 , p. $380-414$

Le nouveau Code du travail de la Colombie Britannique a innové en écartant la compétence des cours en matière de conflits du travail. La nouvelle loi recherche une solution administrative plutôt que judiciaire aux conflits du travail. Les moyens par lesquels le Code du travail de cette province cherche à mettre au point la solution administrative aux différends du travail - Références. 
0210 AOUST, Claude d'

Les dispositifs d'une sentence arbitrale: quelques pièges à éviter.

v. 38 , no 2,1983 , p. $415-417$

Une sentence arbitrale peut s'analyser en un syllogisme, dont les faits constituent la mineure et la règle de droit applicable la majeure; le dispositif en est la conclusion. Le dispositif met un terme au litige né du grief et ferme la boucle du processus arbitral. La juridiction de l'arbitre s'éteint habituellement avec la rédaction du dispositif et les formalités qui s'ensuivent. La prudence est donc de mise à ce stade de la décision.

\section{LAVERY, Daniel}

Décisions rendues par le Conseil canadien des relations du travail.

v. 38 , no 2,1983 , p. $418-432$

Résumé d'une décision rendue par le Conseil et dans laquelle celui-ci a étudié la légalité de certaines clauses des protocoles de retour au travail, le statut des substituts engagés et promus durant la grève ainsi que la doctrine de la conversion d'une grève économique en une grève dite de pratiques déloyales. $\mathrm{Au}$ terme de cette décision, il a déclaré l'employeur coupable.

0212 CÓTÉ, André C. (R)

MORIN, Fernand

Rapports collectifs du travail. Montréal, Éditions Thémis, 1982, 619 pp., ISBN 2-92037621-7, recensé par André C. Cóté.

v. 38 , no 2,1983 , p. $437-440$

0213 SEXTON, Jean (R)

Code du travail F.M., lois connexes et règlements. Les Éditions FM à feuilles mobiles Enr., Farnham, 1981, pagination variée, recensé par Jean Sexton.

v. 38 , no 2,1983 , p. $440-441$

0214 BLOUIN, Rodrigue (R)

TARNOPOLSKY, Walter Surma

Discrimination and the Law in Canada. Toronto, Richard De Boo Ltd, 1982, 595 pp., ISBN 0-88820-105-2, recensé par Rodrigue Blouin. v. 38 , no 2,1983 , p. $441-442$

0215 VERGE, Pierre (R)

AUBERT, Gabriel

L'obligation de paix du travail - étude du droit suisse et comparé. Genève, Librairie de I'Uni- versité, Georg et Cie S.A., 1981, 306 pp., ISBN 2-8257-0078-9, recensé par Pierre Verge.

v. 38, no 2,1983, p. $442-444$

0216 BLOUIN, Rodrigue (R)

AOUST, Claude d' LECLERC, L.

TRUDEAU, Gilles

Les mesures disciplinaires : étude jurisprudentielle et doctrinale. Montréal, École de relations industrielles, Monographie no 13, 1982, 484 pp., ISBN 2-89067-012-0, ISSN 0708-9945, recensé par $R$. Blouin.

v. 38 , no 2,1983 , p. $444-445$

0217 VERGE, Pierre $(\mathrm{R})$

A l'enseigne du droit social belge. 3ième ed., dans Revue de l'Université de Bruxelles, 1978, Bruxelles, Éditions de l'Université de Bruxelles, 1982, 392 pp., recensé par Pierre Verge.

v. 38 , no 2,1983 , p. $445-446$

0218 CÔTÉ, André C. (R)

BAITTON, Raymond $\mathrm{L}$.

The Arbitration Guide : A Case-Handing Manual Procedures and Practices in Dispute Resolutions. Englewood Cliffs, New Jersey, Prentice Hall, 1982, 298 pp., ISBN 0-13043984-3, recensé par André C. Cóté.

v. 38 , no 2,1983 , p. $446-448$

0219 MATEJKO, Alexander J. (R)

CROZIER, Michel FRIEDBERG, Erhard

Actors and Systems. The Politics of Collective Action. Chicago, The University of Chicago Press, 1980, 333 pp., recense par Alexander J. Matejko.

v. 38 , no 2,1983 , p. $448-452$

0220 BÉLANGER, Laurent (R)

LEMELIN, Maurice MCNEIL, Jeannine

Productivité et qualité de vie au travail. Montréal, Les éditions Agence d'Arc Inc., 1982, ISBN 2-89022-044-3, recensé par Laurent Belanger.

v. 38 , no 2,1983 , p. $452-453$

0221 VINET, Alain (R)

KELLY, John E.

Scientific Management, Job Redesign and Work Performance. London, Academic Press, 1982, 257 pp., ISBN 012404020-9, recensé par Alain Vinet.

v. 38 , no 2,1983 , p. $453-455$

0222 PAQUIN, Michel (R)

LAWRENCE, Peter

Managers and Management in West Germany. 
Relations lmoustrielles, vol. 45, no 4 (1990)

London, Croom Helm, 1980, 202 pp., ISBN O85664-912-0, recensé par Michel Paquin.

v. 38, no 2,1983 , p. $455-456$

\section{VERGE, Pierre}

Syndicalisation de la grève.

v. 38 , no 3,1983 , p. $475-506$

Initialement affirmé en fonction des individus qui se coalisent, le droit de grève envisagé par le Code du travail du Québec s'acquiert par l'association accréditée. Interrogations sur les conséquences juridiques d'une telle dissociation du droit de grève des individus qui participent au mouvement collectif: limites de la liberté des salariés; responsabilité de l'instance syndicale et des grévistes eux-mêmes à l'occasion des grèves illégales; la situation particulière des salariés qui ont assumé des charges au sein de l'association accréditée.

0224 ADAMS, Roy J.

Competing Paradigms in Industrial Relations. v. 38 , no 3,1983 , p. $508-531$

Les travaux conceptuels récents ont dégagé un ensemble de principes qui devraient permettre le développement d'une tradition cohérente de recherche en matière de relations professionnelles. Considéré dans la perspective systémique, le rôle des relations professionnelles est d'identifier et de décrire la structure et le processus des relations entre le travail (sous ses aspects individuel et collectif), les employeurs et l'État dans des entreprises, des industries et des pays différents - Références.

\section{BERNIER, Jean}

L'extension juridique des conventions collectives au Québec: Une approche comparative. v. 38 , no 3,1983 , p. $532-544$

Après un rappel des nombreuses études que le gouvernement du Québec a fait réaliser depuis une vingtaine d'années sur les régimes européens d'extension juridique des conventions collectives, on recourt à l'approche comparative pour tenter de dégager un certain nombre de questions susceptibles de favoriser un réexamen du système québécois d'extension juridique.

0226 LIPSIG-MUMME, Carla The Renaissance of Homeworking in Develop- ped Economies.

v. 38 , no 3,1983, p. 545-567

Le travail salarié à domicile est de nouveau en train de se développer pour la première fois depuis plus d'un siècle dans les pays capitalistes développés. Analyse des causes et des implications de la renaissance du travail à domicile en Europe de l'ouest, en Amérique du Nord et en Australie depuis les années 1960 .

\section{LAROUCHE, Viateur} TRUDEL, Johanne

La qualité de vie au travail et l'horaire variable. v. 38 , no 3,1983, p. $568-597$

L'étude a pour objet de vérifier si la transformation des horaires de travail peut affecter directement la qualité de vie au travail. Après définition du nouveau concept de qualité de vie au travail, on fait le tour des différents types d'horaires alternatifs et on analyse d'une façon particulière l'horaire variable à la lumière de quelques cas pratiques - 1 tableau, bibliographie.

\section{KAMINKA, Shlomit ROSEINSTEIN, Eliezer}

Shop Stewards, Workers, and Managers in Israëli Industry.

v. 38 , no 3,1983 , p. $598-617$

Description et analyse de la fonction du Comité des délégués du personnel dans les relations professionnelles au niveau de l'établissement, en accordant une attention particulière aux relations entre les membres du comité et les travailleurs. A partir d'un échantillon de 70 établissements en israël, analyse des relations entre les trois groupes suivants: les comités de délégués du personnel, les travailleurs et les employeurs ou la direction - 1 tableau, 4 figures.

\section{MCSHANE, Steven L.}

Reasonable Notice Criteria in Common Law Wrongful Dismissal Cases.

v. 38 , no 3,1983, p. $618-635$

Pour les travailleurs canadiens non régis par une convention collective ni protégés par la législation du travail, la protection contre un renvoi injustifié n'est possible que par le recours a la doctrine du common law en matière de respect des conditions du travail. Analyse de 199 jugements rapportés de 1960 
à 1982 dans des affaires de renvoi injustifié. Identification des critères selon lesquels les tribunaux ont fixé la longueur des délaiscongé suite à un renvoi injustifié - 2 tableaux, références.

\section{CROWLEY, R.W. HUTH, E.}

An International Comparison of Work Sharing Programs.

v. 38 , no 3,1983 , p. $636-647$

Étude comparative des programmes de travail partagé en Amérique du nord et en Europe de l'ouest. Caractéristiques de ces programmes de main-d'oeuvre qui ont pour but de maintenir le niveau d'emploi dans les entreprises qui se voient dans l'obligation de réduire temporairement leur production et leurs effectifs. Les critères d'éligibilité des employés et des entreprises à ces programmes - 2 tableaux.

\section{LAVERY, Daniel}

Décisions rendues par le Conseil canadien des relations du travail.

v. 38 , no 3, 1983 , p. $658-666$

Le panel du Conseil décide majoritairement que l'octroi d'un certificat d'accréditation ne confère pas au syndicat un droit absolu au maintien des fonctions qui y sont énumérées et l'employeur peut, en raison de ses droits de gérance, altérer la nature de ces fonctions au point même de les soustraire au pouvoir de représentation du syndicat.

\section{BACHAND, Gilles (R)}

\section{CUTLER, Philip}

Code du travail du Québec. Montréal, Les Livres Toundra, 1983, 539 pp., ISBN 88776135-6, recensé par Gilles Bachand.

v. 38 , no 3,1983, p. $676-677$

0233 VERGE, Pierre (R)

LEWIS, R. CLARK, J.

WEDDERBURN, Lord of Charlton

Labour Law and Industrial Relations : Building on Kahn-Freund. Oxford, Clarendon Press, 1983, 250 pp., ISBN 0-19-825393-1, recensé par Pierre Verge.

v. 38 , no 3,1983 , p. $677-678$
0234 MARCHIS-MOUREN, Marie Françoise $(R)$

VINET, Alain DUFRESNE, Francine

VÉZINA, Lucie

Condition féminine en milieu ouvrier. Québec, Institut québécois de recherche sur la culture, 1982, 221 pp., ISBN 2-89224-018-2, recensé par Marie Françoise Marchis-Mouren.

v. 38 , no 3, 1983 , p. $679-680$

0235 MATEJKO, Alexander J. (R)

REYNAUD, Jean-Daniel

Sociologie des conflits du travail. Paris, Presses universitaires de France, 1982, 127 pp., recensé par Alexander J. Matejko.

v. 38 , no 3,1983 , p. $681-682$

0236 BÉLANGER, Laurent (R)

FELDMAN, Daniel C. ARNOLD, Hugh J.

Managing Individual and Group Behavior in Organizations. Toronto, McCrowill-Hill Series in Management, 1983, 613 pp., ISBN 0-83593006-4, recensé par Laurent Bélanger.

v. 38, no 3,1983 , p. $682-683$

0237 BÉLANGER, Laurent ( $\mathrm{R})$

CASCIO, Wayne E. AWARD, Elias M.

Human Resources Management. An Information System Approach. Virginia, Reston Publishing Co., 1981, 594 pp., ISBN 0-07-0203865 , recensé par Laurent Bélanger.

v. 38 , no 3,1983, p. $683-684$

0238 MATEJKO, Alexander J. (R)

EDWARDS, P.K. SCULLION, Hugh

The Social Organization of Industrial Conflict. Control and Resistance in the Workplace. Oxford, Basil Blackwell, 1982, 314 pp., ISBN 0-631-13127-2, recensé par Alexander J. Matejko.

v. 38 , no 3,1983 , p. $685-686$

0239 BOULARD, René (R)

GERSUNY, Carl

Work Hazards and Industrial Conflict. Hanover, University Press of New England, 1981, 162 pp., Lib of Congress Catalog Card no 8051506, recensé par René Boulard.

v. 38, no 3,1983 , p. 686

0240 MATEJKO, Alexander J. (R)

LINDENFELD, Frank

ROTHSCHILD-WHITT, Joice

Workplace Democracy and Social Change. Boston, Porter Sargent Publication, 447 pp., ISBN 0-87558-102-1, recensé par Alexander J. Matejko.

v. 38 , no 3,1983 , p. $687-688$ 
0241 MATEJKO, Alexander J. (R)

WILKES, John

The Future of Work Sydney, G. Allen and Unwin, Australia Institute of Political Science, 1981, 164 pp., ISBN 0-86861-283-9, recensé par Alexander J. Matejko.

v. 38 , no 3, 1983, p. 689

0242 BOULARD, René (R)

TURCOTTE, Pierre R.

Qualité de vie au travail : anti-stress et créativité. Montreal, Agences d'Arc, 1982, 184 pp., ISBN 2-89022-046-X, recensé par René Boulard.

v. 38, no 3,1983 , p. $689-690$

0243 CÔTÉ, André C. (R)

BAR-NIV, Zvi H.

International Labour Law Reports, vol. 3. Alphen aan den Rijn, Hollande, Sijthoff \& Noordhoff, 1980, 399 pp., ISBN 90-286-0060-4, recensé par André C. Cóté.

v. 38 , no 3,1983 , p. $690-691$

0244 GOLDENBERG, Shirley B. HÉBERT, Gérard

Harry Douglas Woods, 1907-1983

v. 38 , no 4,1983 , p. 707-712

La vie et la carrière de H.D. Woods, personnalité bien connue dans le monde des relations industrielles, décédé le 7 août 1983. Professeur à l'Université MCGILL, on lui doit le Rapport Woods publié en 1968, la fondation du Centre de relations industrielles en 1948 et de nombreuses publications.

\section{BARKIN, Solomon}

Troubled Worker Militancy: Challenges Confronting Western Industrial Relations Systems. v. 38 , no 4,1983 , p. $713-729$

Histoire du militantisme syndical et des systèmes de relations industrielles dans les pays occidentaux au cours des cinquante dernières années. Les nouveaux défis auxquels sont confrontées les organisations syndicales. Évolution de l'action syndicale vers de nouveaux domaines de revendication.

\section{COUSINEAU, Jean-Michel LACROIX, Robert}

Le climat des relations du travail à Montreal et à Toronto

v. 38 , no 4,1983 , p. $730-743$

Étude comparative de l'activité de grève à
Montréal et à Toronto et du "potentiel" de grève des deux villes. Les divers indicateurs du climat des relations du travail et de l'activité de grève dans les deux villes. Résultats et principales conclusions de l'étude -6 tableaux.

0247 BEAUMONT, P.B.

Statutory Recognition Provisions in Britain, 1976-80

v. 38 , no 4,1983 , p. $744-766$

En Grande-Bretagne, les syndicats s'en sont remis traditionnellement à la reconnaissance volontaire des employeurs. Certains changements importants dans le système de relations professionnelles ont favorisé la mise en place d'un régime de reconnaissance légale des syndicats. On évalue les gains syndicaux obtenus pendant la période où fut appliqué ce mécanisme législatif de reconnaissance syndicale, soit de février 1976 à août 1980 , et on analyse les stipulations relatives à la reconnaissance des syndicats -4 tableaux.

\section{CAMERON, Samuel}

An International Comparison of the Volatility of Strike Behavior

v. 38 , no 4,1983 , p. $767-784$

Les statistiques en matière de grève diffèrent tellement d'un pays à l'autre qu'il est impossible d'établir des comparaisons valables sur le sujet. Le modèle utilisé ici permet d'en arriver à certaines prévisions parmi les indicateurs de grève. Le taux de variabilité se fonde à la fois sur le nombre de jours de travail perdus, le nombre de travailleurs touchés et le nombre de conflits. L'analyse étudie les statistiques publiées dans 62 pays sur une période de 10 ans. -1 figure, 2 tableaux.

\section{DEMERS, Marie}

Chômage chez les jeunes: Conséquences psychologiques et sociales.

v. 38 , no 4 , 1983, p. $785-814$

A partir d'une revue de la littérature en provenance des États-Unis, de la France, de la Grande-Bretagne et de l'Australie et portant sur le chômage chez les jeunes, on dégage les changements qui se sont opérés ainsi que les problèmes sociaux qu'entraine ce phénomène - Bibliographie. 


\section{MOUNT, Joan}

SIEGEL, Jacob P.

Resources and Results in Union Rivalny: $A$ Case Study

v. 38 , no 4, 1983, p. $815-830$

L'Association des employés de soutien de l'Université Laurentienne s'est méritée en 1973 le titre de premier syndicat indépendant d'un groupe d'employés de soutien à être accrédité sous la Loi des relations du travail dans une université d'Ontario. Analyse rétrospective et approfondie de la lutte que lui a livrée le Syndicat canadien de la fonction publique. Les moyens utilisés par les deux syndicats concurrents pour prôner leurs avantages respectifs - 1 figure, références.

\section{RAINVILLE, Jean-Marie}

La responsabilité fonctionnelle chez les cadres subalternes: Facteurs d'evolution des systèmes d'autorité.

v. 38 , no 4,1983 , p. $831-846$

Examen d'un cas particulier qui pose le problème de l'évolution des systèmes d'autorité aux échelons inférieurs des organisations administratives. Comment fonctionnent les rapports d'autorité entre les cadres subalternes et leurs supérieurs dans la section psychiatrique d'un hôpital montréalais. Comment l'évolution du système d'autorité a été compromise suite à une décision juridique refusant l'accréditation syndicale des cadres concernés.

\section{BOULARD, René MARCHAND, Michel}

La loi sur la santé et la sécurité du travail et les conventions collectives.

v. 38 , no 4,1983, p. $847-862$

On examine la situation dans les secteurs prioritaires de la Commission de la santé et de la sécurité au travail pour évaluer si la "Loi sur la santé et la sécurité du travail" a eu un impact sur les clauses de conventions collectives - 5 tableaux.

0253 JOSEPH, P.A.

Perfecting the Administrative Solution to Labour Disputes.

v. 38 , no 4,1983 , p. $863-868$

La Cour suprême du Canada avait à décider si le Tribunal des professions du Québec avait la compétence pour exercer les vastes pouvoirs qui lui sont attribués par le code des professions du Québec ou si l'exercice de ces pouvoirs violait la section 96 de l'Acte de l'Amérique du Nord Britannique. Pour la première fois, la Cour suprême a déclaré sans équivoque qu'un tribunal légalement constitué sous une loi provinciale ne peut pas, constitutionnellement, être immunisé contre la révision des décisions sur les questions de juridiction.

\section{JAIN, Harish C.}

Micro-electronics Technology and Industrial Relations.

v. 38 , no 4,1983 , p. $869-879$

Dans son rapport de novembre 1982 au Ministre du travail du Canada, la 'Commission d'enquête sur la microélectronique et l'emploi" examinait les implications qu'ont, pour les relations professionnelles, l'émergence et l'adoption sur une grande échelle de la technologie microélectronique. Définition du mandat de la Commission d'enquête, hypothèses importantes et recommandations faites par celle-ci - 1 tableau.

\section{BEAULIEU, LUC}

Décisions rendues par le Conseil canadien des relations du travail.

v. 38 , no 4,1983 , p. $880-903$

Résumé de différentes décisions rendues par le Conseil et portant sur l'interprétation de la notion de "vente d'entreprise", contenue à l'article 144 du Code canadien du travail.

\section{PETTERSEN, Normand (R)}

DOLAN, Shimon L. ROY, Denis

La sélection des cadres: principes et prob/èmes contemporains. Monographie no 13, Montréal, École de Relations Industrielles, Université de Montréal, 1982, ISBN 2-89067010-4, ISSN 0708-9945, recensé par Normand Pettersen.

v. 38 , no 4,1983 , p. $910-912$

0257 DION, Gérard $(R)$

WEISS, Dimitri

Les relations du travail. Employeurs, personnel, syndicats, Etat. 5ième éd., Paris, Dunod, 1983, 448 pp., ISBN 2-04-015545-7, ISSN 0335-3184, recensé par Gerard Dion.

v. 38 , no 4,1983 , p. $912-913$ 
Relations Industrielles, vol. 45, no 4 (1990)

0258 CHOUINARD, Jacques $(R)$

THÉRIAULT, Roland

Gestion de la rémunération : Politiques et pratiques efficaces et équitables. Boucherville, Gaétan Morin Éditeur, 1983, 585 pp., ISBN 2-89105-109-2, recensé par Jacques Chouinard.

v. 38 , no 4,1983 , p. $913-915$

0259 FISHER, E.G. (R)

The Bargaining Process and Mediation. Toronto, Education Relations Commission, 1983, recensé par E.G. Fisher.

v. 38 , no 4,1983 , p. $915-916$

0260 JOUBERT, $\mathrm{P}$. (R)

ROMAGNOLI, Umberto WEISS, Dimitri

Pluralità : Italia ed Europa nei conflitti industriali. Roma, ASAP, 1983, 288 pp., recensé par $P$. Joubert.

v. 38 , no 4,1983 , p. $916-920$

0261 RONDEAU, Alain (R)

HALLORAN, Jack

Applied Human Relations, An Organizational Approach. (2nd ed), Englewood Cliffs, N.J., Prentice-Hall, 1983, 531 pp., ISBN 0-13040808-5, recensé par Alain Rondeau.

v. 38 , no 4,1983 , p. $920-922$

0262 WEISS, Dimitri $(R)$

MOURIAUX, René

Les syndicats dans la societé française. Paris, Presses de la Fondation nationale des Sciences politiques, 1983, $272 \mathrm{pp}$., ISBN 27246-0480-6, recensé par Dimitri Weiss.

v. 38 , no 4,1983 , p. $922-924$

0263 WEISS, Dimitri (R)

PIRZIO AMMASSARI, Gloria

MATTIOLI, Francesco

I sindicalisti. Bar, De Donato, 1982, 158 pp., CL 07-0501-2, recensé par Dimitri Weiss.

v. 38 , no 4,1983 , p. $922-924$

0264 GUNDERSON, Morley (R)

SEN, Joya

The Unemployment of Youth : The Importance of Education for their Adjustment in the Canadian Labour Market. Toronto, Ontario Institute for Studies in Education, 1982, 125 pp., ISBN 0-7744-5053-3, recensé par Morley Gunderson.

v. 38 , no 4,1983 , p. $925-927$
0265 MATEJKO, Alexander J. (R) NIGHTINGALE, Donald V.

Workplace Democracy : An Inquiry into Employee Participation in Canadian Work Organizations. Toronto, University of Toronto Press, 1983, 313 pp., ISBN 0-8020-6471-1, recensé par Alexander J. Matejko.

v. 38, no 4,1983 , p. $927-929$

0266 WEISS, Dimitri $(\mathrm{R})$

BOLTANSKI, LUC

Les cadres : la formation d'un groupe social.

Paris, Éditions Minuit, Collection "Le sens commun" dirigée par Pierre Bourdieu, 1982, 523 pp., ISBN 2-7078-0617-7, recensé par Dimitri Weiss.

v. 38 , no 4,1983 , p. $929-934$

0267 WEISS, Dimitri ( $R$ )

MAURICE, Marc SELLIER, François

SILVESTRE, Jean-Jacques

Politique d'éducation et organisation industrielle en France et en Allemagne : essai d'analyse sociétale. Paris, PUF, collection "Sociologies" dirigee par Raymond Boudon et François Bourricaud, 1982, 382 pp., ISBN 213-037472-7, recensé par Dimitri Weiss.

v. 38 , no 4,1983 , p. $929-934$

0268 COUSINEAU, Jean-Michel LACROIX, Robert

La détermination des avantages sociaux au Canada

v. 39 , no 1, 1984, p. $3-22$

Après une revue des modèles économiques usuels de détermination des avantages sociaux qui, pour la plupart, ont été développés et testés aux États-Unis, on présente des résultats inédits pour le Canada et on en tire un certain nombre d'implications pour les finances publiques, la politique économique et la compréhension du fonctionnement des marchés du travail - 1 tableau, bibliographie.

0269 SOLOMON, Norman A.

The Negociation of First Agreement in Ontario. v. 39, no 1,1984 , p. $23-35$

En Ontario, la Commission des relations du travail n'a pas autorité pour imposer une première convention collective. On analyse les unités de négociation où l'on a réussi à conclure une première convention collective et celles où on n'y est pas parvenu, soit en fonction de faits résultant de l'expérience de la Commission en matière d'accréditation, soit 
en fonction de faits se rapportant aux caractéristiques fondamentales de l'unité de négociation - 5 tableaux.

\section{LE LOUARN, Jean-Yves THÉRIAULT, Roland TOULOUSE, Jean-Marie}

Le travail des deux conjoints: Effets sur la progression de carrière du cadre.

v. 39 , no 1,1984 , p. $36-50$

Hypothèses, méthode et résultats d'une recherche ayant pou but d'étudier l'effet du mariage à une personne qui travaille à l'extérieur sur la progression de carrière de l'individu dans l'entreprise. Le concept de progression de carrière est mesuré par trois variables: 1) le niveau de salaire, 2) le niveau hiérarchique et 3) le nombre de promotions obtenues par l'individu - 3 tableaux, références.

\section{VANDAMME, François}

L'information des travailleurs dans les grandes firmes: L'approche spécifique de la CEE.

v. 39 , no 1, 1984, p. 51-76

La contribution de la Communauté économique européenne au débat sur l'information et la consultation des travailleurs des grandes firmes à sièges multiples au sujet des projets du groupe. La Communauté doit exercer ses responsabilités politiques par l'adoption d'une réglementation allant plus loin que les codes de conduite volontaires tout en s'inscrivant dans leur prolongement.

\section{MAKI, Dennis R. STRAND, Kenneth}

The Determinants of Strike Activity: An Interindustry Analysis

v. 39 , no 1,1984 , p. $77-92$

Vérification et adaptation du modèle Chamberlain-Kuhn en matière de coûts entre un accord et un désaccord en vue d'expliquer l'incidence et la durée des grèves en tant que fonctions des seuls facteurs économiques. Les résultats indiquent que le modèle a failli à expliquer l'incidence des grèves, mais qu'il a réussi davantage à en expliquer la durée, lorsqu'il y avait eu grève - 1 figure, 1 tableau, références.

\section{KRAHN, Harvey}

LOWE, Graham S.

Community Influences on Attitudes Towards
Unions.

v. 39 , no 1,1984, p. $93-113$

Etude explorant l'influence de la collectivité locale sur l'attitude de la population envers les syndicats. Une étude approfondie des relations professionnelles confirme l'opinion selon laquelle la collectivité locale constitue une des variables communautaires importante pouvant influencer les relations professionnelles au niveau local. A partir de données recueillies à Edmonton et à Winnipeg, on vérifie et on teste cette proposition générale - 3 tableaux.

\section{LAROUCHE, Viateur}

DÉOM, Esther

L'approche systémique en relations industrielles

v. 39 , no 1,1984 , p. $114-145$

Dans une première partie, on présente et discute les différents éléments propres à la notion de système dans le but de retenir certains de ces éléments jugés pertinents pour l'utilisation de l'approche systémique en relations industrielles. Dans une deuxième partie, on applique ces caractéristiques à la notion de relations industrielles et on essaie de dégager une conception plus claire de ce champ d'étude - 3 graphiques, 1 tableau, bibliographie.

\section{ROBB, Roberta Edgecome}

Occupational Segregation and Equal Pay for Work of Equal Value.

v. 39 , no 1,1984 , p. $146-166$

La Charte canadienne des droits et libertés, entrée en vigueur en 1978, déclare qu'il y a pratique discriminatoire lorsqu'un employeur établit et maintient des écarts de salaires entre les hommes et les femmes dans un établissement où les uns et les autres effectuent un travail de valeur égale. On essaie d'évaluer l'effet de l'application de cette loi sur le marché du travail - 1 figure, 2 graphiques, bibliographie.

0276 DOLAN, Shimon L. TZINER, Aharon ROY, Denis

A Real Estate Agency's Level Analysis of the Climate-Performance Relationship.

v. 39 , no 1,1984, p. 167-176

On tente d'établir la variance explicative du climat organisationnel sur le rendement au 
Relations Imoustrielles, vol. 45, no 4 (1990)

travail en tenant compte des variables socio-démographiques pertinentes au niveau de l'unité d'opération. L'échantillon était composé de 29 agences de courtage immobilier. L'analyse des résultats révèle que le climat organisationnel dans les agences contribue fortement à expliquer le rendement au travail des agents - 2 tableaux.

0277 BEAULIEU, LUC

Décisions rendues par le Conseil canadien des relations du travail.

v. 39 , no 1,1984, p. $177-189$

Résumé de trois décisions du Conseil: 1) Le Conseil a rejeté une requête en révision d'une décision qu'il avait rendue et a profité de l'occasion pour exprimer un nouveau concept qui sera appliqué dans sa politique de révision. 2) Le Conseil interprète les dispositions du Code canadien du travail relatives aux grèves illégales. 3) Le Conseil décide que le retrait partiel des prestations de travail constitue une grève illégale.

0278 BRIOLAT, Dominique (R)

GAGNON, Pierre (R)

SIDIBE, Hamadoun (R)

GASSE, Yvon (R)

HAGE, Jerald

Theories of Organizations : Form, Process and Transformation. Newport, John Wiley \& Sons, 1980, 558 pp., ISBN 0471-33859-1, recensé par D. Briolat, P. Gagnon, H. Sidibe et Y. Gasse.

v. 39 , no 1,1984, p. $197-201$

0279 VERGE, Pierre (R)

BLANPAIN, R. MILLARD, F.

Comparative Labour Law and Industrial Relations. Deventer, Kluwer, Washington, the $B u-$ reau of National Affairs, 1982, 411 pp., ISBN O87179-392-2, recensé par Pierre Verge.

v. 39 , no 1, 1984, p. 201-203

0280 BARRÉ, Alain (R)

LEARY, Virginia

International Labour Conventions and National Law. The Haque, Martinus Nijhoff Publishers, 1982, 191 pp., ISBN 90-247-2551-8, recensé par Alain Barré.

v. 39, no 1, 1984, p. 203-205

0281 MATEJKO, Alexander J. (R)

STONE, Thomas $H$. MELTZ, Noah $M$.

Personnel Management in Canada. Toronto Holt, Rinehart \& Winston, 1983, 411 pp., ISBN 0-03-921591-1, recensé par Alexander J. Matejko.

v. 39 , no 1, 1984, p. 205-206
0282 AGARWAL, Naresh C. (R) ARGYRIS, Chris

Reasoning, Learning and Action. San Francisco, Jossey-Bass, 1982, 499 pp., ISBN O. 87589-524-7, recensé par Naresh C. Aganwal. v. 39, no 1, 1984, p. 206-208

0283 MATEJKO, Alexander J. (R)

BRADLEY, Keith GELB, Alan

Worker Capitalism. The New Industrial Relations. London, Heinemann, 1983, 186 pp., ISBN 0-435-82084-2, recensé par Alexander J. Matejko.

v. 39 , no 1,1984, p. $208-209$

0284 DUSSAULT, Gilles (R)

GAGNON, Chantal HÉBERT, Gérard

Les grèves illégales dans les hópitaux du Québec, 1977-1978. Montréal, École de relations industrielles, Université de Montréal, monographie no 14, 1982, 76 pp., ISBN 07089945, recensé par Gilles Dussault.

v. 39 , no 1, 1984 , p. $209-210$

0285 PHILLIPS, Paul (R)

CLARK, Paul F.

The Mines Fight for Democracy. Arnold Miller and Reform of the United Mine Workers. New York State School of Industrial and Labour Relations, Cornell University, 1981, 190 pp., recensé par Paul Phillips.

v. 39 , no 1,1984, p. $210-211$

0286 MATEJKO, Alexander J. (R)

CROUCH, Colin

Trade Unions : The Logic of Collective Action. Glasgow, Fontana Paperbacks, 1982, 251 pp., ISBN 0-00-635873X, recensé par Alexander $J$. Matejko.

v. 39 , no 1,1984, p. $212-215$

0287 MATEJKO, Alexander J. (R)

REES, Albert

The Economics of Trade Unions. Chicago and London, The University of Chicago Press, 1977, 200 pp., ISBN 0-226-70702-4, recensé par Alexander J. Matejko.

v. 39 , no 1,1984, p. $212-215$

0288 JAIN, Hem C. (R)

VENKATCHALAM, V. SINGH, R.K.

The Political, Economic and Labor Climate in India. Philadelphia, The Wharton School Industrial Research Unit, University of Pennsylvania, 1982, 147 pp., ISBN 0-89546-030-0, ISSN 149-0818, recensé par Hem C. Jain.

v. 39 , no 1,1984, p. $215-217$ 
0289 MATEJKO, Alexander J. (R)

DANIEL, W.W. MILLWARD, Neil

Workplace Industrial Relations in Britain. The DE/PSI/SSRC Survey, London, Heinemann Educational Books, 1983, 338 pp., ISBN 0435-83191-7, recensé par Alexander J. Matejko.

v. 39 , no 1,1984 , p. $217-218$

0290 BÉLANGER, Jacques ( $R$ )

NICHLOSON, Nigel URSELL, Gill

BLYTON, Paul

The Dynamics of White Collar Unionism : A Study of Local Union Participation. London, Academic Press, 1981, 268 pp., ISBN 0-12$518020-9$, recensé par Jacques Bélanger. v. 39 , no 1,1984 , p. $218-220$

\section{BOURASSA, Raynald (R)}

La médiation pré-arbitrale en matière de conflits de droits (griefs). Journée d'étude à l'École de relations industrielles, Université de Montréal, 1981, 91 pp., recensé par Rodrigue Blouin.

v. 39 , no 1,1984, p. $220-221$

\section{CAIRE, GuY}

\section{Les lois Auroux}

v. 39 , no 2,1984, p. 235-258

Les grandes lignes des changements profonds apportés au Code du travail français par les lois Auroux en 1982. Les motivations qui éclairent leur présentation ainsi que l'accueil qui leur a été fait.

\section{GURDON, Michael A. WRIGHT, David J.}

Collective Bargaining in the Maritime Fishing Industry: Recent Developments

v. 39 , no 2,1984 , p. $259-274$

Au cours des dernières années, des changements importants se sont produits dans l'industrie de la pêche au Canada, spécialement dans la région des Maritimes avec la promulgation par le Nouveau-Brunswick de la "Loi sur la négociation collective dans l'industrie de la pêche". Après avoir décrit ces changements majeurs et donné quelques détails sur la législation du Nouveau-Brunswick et sur la formation d'un syndicat représentant les pêcheurs, on présente les résultats d'une enquête portant sur la dépendance des pêcheurs.
0294 LIPSIG-MUMME, Carla

La crise du syndicalisme nord-américain: elements d'interprétation.

v. 39 , no 2,1984 , p. $275-284$

A travers toute l'Amérique du Nord, la dernière décennie fut loin d'être fructueuse pour les mouvements syndicaux. On explore deux avenues de ce qu'il faut déjà entrevoir comme la crise contemporaine du syndicalisme nord-américain: 1) Le contexte politico-économique, 2) Les deux causes importantes de l'incapacité des syndicats à articuler et à promouvoir une vision alternative à leur rôle légitime.

0295 PIGANIOL, Claude

Relations professionnelles et gestion des ressources humaines.

v. 39 , no 2,1984 , p. $285-300$

L'existence de deux approches du facteur humain dans l'entreprise, celle de l'analyse des relations du travail et celle des techniques de gestion du personnel, paraît relever au premier abord de démarches incompatibles. Une connaissance approfondie de leurs apports respectifs est nécessaire pour comprendre qu'en réalité la synthèse se fait sur le terrain en raison de la nature indissociable des aspects de la gestion du personnel que chacune éclaire. Au niveau de la recherche et de l'enseignement une fusion des deux approches devrait se faire.

\section{BAR-HAIM, Aviad}

The Desire for Workers' Participation: Conflicting Forces in the Workplace.

v. 39, no 2,1984, p. $301-312$

Résultats d'une enquête effectuée auprès de 248 travailleurs non qualifiés et spécialisés et portant sur le désir de participation des travailleurs aux prises de décision dans l'entreprise. L'enquête révèle deux résultats importants: 1) On remarque un affaiblissement du désir de participation lorsqu'il y a satisfaction au travail; 2) On trouve un désir bien ancré de participation qui va s'accroissant lorsqu'il s'accompagne d'une véritable adhésion de l'esprit - 3 tableaux, références.

\section{$0297 \mathrm{FABI}, \mathrm{Bruno}$}

Privé ou public: choix et transfert du secteur organisationnel.

v. 39, no 2,1984, p. $313-334$ 
Les principaux résultats d'une étude longitudinale explorant quelques variables psychologiques relatives au choix et au transfert de secteur organisationnel chez des diplômés possédant une maîtrise en administration des affaires (M.B.A.) d'une université francophone québécoise. Le groupe de répondants était constitué de sujets oeuvrant respectivement aux secteurs privé (50\%), parapublic (28\%) et public (22\%) - 4 tableaux, références.

\section{WILLIAMS, C. Brian}

The Impact of Labor Migration: The International Molders and Allied Workers Union in Canada, 1860-1885.

v. 39, no 2,1984, p. $335-364$

Étude de l'émergence de l'un des premiers syndicats à structure internationale au Canada, soit l'International Molders and Allied Workers Union. En utilisant la fondation et le développement de ce syndicat comme étude de cas, on peut affirmer que l'implantation de cette structure fut le résultat des circonstances prévalant sur les marchés des biens, des capitaux et du travail qui se trouvaient inter-reliés - 2 tableaux.

\section{AOUST, Claude d'}

Effets d'annulation d'un avis de congédiement par un arbitre

v. 39 , no 2,1984, p. $365-370$

L'annulation du congédiement pour raison de vice de procédure entraîne-t-elle l'incapacité de l'employeur de procéder à un nouveau congédiement fondé sur les mêmes faits? Selon une jurisprudence relativement récente, il semble bien que ce soit le cas.

0300 BEAULIEU, LUC

Décisions rendues par le Conseil canadien des relations du travail

v. 39, no 2,1984, p. $371-385$

Résumé de deux décisions rendues par le Conseil: 1) Le Conseil établit une distinction entre les activités syndicales protégées par le Code et celles qui ne le sont pas. II affirme aussi qu'un plaignant alléguant violation par son syndicat du devoir de représentation juste doit coopérer avec son syndicat. 2) Dans une autre affaire, le Conseil fait l'étude de ses pouvoirs de redressement et de sa politique en matière du devoir de négocier de bonne foi.

\section{SEXTON, Jean (R)}

KOLB, Deborah M.

The Mediators. Cambridge, Mass., The MIT Press, 1983, 230 pp., ISBN 0-262-11088-1, recense par Jean Sexton.

v. 39, no 2,1984, p. $391-392$

0302 MATEJKO, Alexander J. (R)

ONDRACK, Dan

STUART, Timperley

The Humanisation of Work. European Perspective. London, Armstrong Publishing, 1982, 239 pp., ISBN 0-9506540-86, recensé par Alexander J. Matejko.

v. 39 , no 2,1984, p. $392-393$

0303 CRAIG, Alton W.J. (R)

ANTONIDES, Harry

Renewal in the Workplace : A Critical Look at Collective Bargaining. London, Ontario, Christian Labour Association of Canada, 1982, 82 pp., ISBN 0-919663-00-1, recensé par Alton W.J. Craig.

v. 39 , no 2,1984, p. $393-394$

0304 MATEJKO, Alexander J. (R)

WHITE, Michael TREVOR, Malcolm

Under Japanese Management. The Experience of British Workers. London, Heinemann, 1983, 162 pp., ISBN 0-435-83935-7, recensé par Alexander J. Matejko.

v. 39 , no 2,1984, p. $395-396$

0305 BRIGHT, David (R)

CRAIG, C. RUBERY, Jill

TARLING, Roger WILKINSON, Frank

Abolition and After the Paper Box Wages

Council. Research Paper no 12, London, Department of Employment, June 1980, 98 pp., recensé par David Bright.

v. 39 , no 2,1984 , p. $396-398$

0306 MATEJKO, Alexander J. (R)

CATALYST, The Staff of

Upward Mobility. New-York, Holt, Rinehart and Winston, 1982, 292 pp., ISBN 0-03-056163-9, recensé par Alexander J. Matejko.

v. 39 , no 2, 1984, p. 398-399

0307 STODDART, Jennifer (R)

PHILLIPS, Paul PHILLIPS, Erin

Women and Work : Inequality in the Labour Market. Toronto, James Lorimer \& Company, 1983, 200 pp., ISBN 0-8862-608-8, recensé par Jennifer Stoddart.

v. 39 , no 2,1984, p. 400 
0308 NOLLET, Jean (R)

Collection "Technologie et travail". Montréal, Institut national de productivité, 1983, 8 textes de 43 pp. à 202 pp., recensé par Jean Nollet. v. 39 , no 2,1984 , p. $400-402$

\section{DION, Gérard (R)}

DELAMOTTE, Yves

Le droit du travail en pratique. Paris, Les Éditions d'organisation, 1983, 319 pp., ISBN 27081-0541-8, recensé par Gérard Dion.

v. 39 , no 2 , 1984 , p. $402-403$

\section{THWAITES, James $(R)$}

PETERS, T.J. WATERMAN, R.H. Jr.

In Search of Excellence : Lessons from America's Best-Run Companies. New York, Harper and Row, 1982, 350 pp., ISBN 0-06-0150424, recensé par James Thwaites.

v. 39 , no 2,1984 , p. $403-406$

0311 THWAITES, James (R)

NEUFELD, Maurice F. LEAB, Daniel J.

SWANSON, Dorothy

American Working Class History : A Representative Biography. New York - London, Bowker, 1983, 356 pp., ISBN 0-8352-1752-3, recensé par James Thwaites.

v. 39, no 2,1984 , p. 406

0312 WEISS, Dimitri (R)

BÉLANGER, Laurent PETIT, André

BERGERON, Jean-Louis

Gestion des ressources humaines : une approche globale et intégrée. Chicoutimi, Gaëtan Morin éditeur, 1983, 451 pp., ISBN 289105-072-X, recensé par Dimitri Weiss.

v. 39 , no 2,1984 , p. $406-408$

\section{FORTIN, Pierre}

Le chômage des jeunes au Québec: Aggravation et concentration, 1966-1982

v. 39 , no 3,1984 , p. $419-448$

L'aggravation du chômage des jeunes Québécois depuis 1966 est principalement due à la raréfaction progressive de l'emploi depuis 1974 (et surtout depuis 1979) ainsi qu'à la vulnérabilité particulière des jeunes à la conjoncture. Ce chômage est aussi fort concentré chez les jeunes décrocheurs en état chronique de privation d'emploi. Sept propositions pour une politique qui s'attaque à la pénurie générale d'emploi et à sa concentration parmi les jeunes de régions ou de milieux défavorisés - 7 tableaux.

\section{PONAK, Allen THOMPSON, Mark}

Faculty Collective Bargaining: The Voice of Experience

v. 39 , no 3,1984 , p. $449-465$

La négociation collective est maintenant un fait accompli pour la plupart des professeurs des universités canadiennes. Résultats d'une enquête effectuée dans six universités canadiennes et portant sur l'opinion des professeurs travaillant sous les régimes de conventions collectives - 2 tableaux, références.

\section{DION, Stéphane}

Syndicats et politique au niveau municipal en France.

v. 39 , no 3. 1984 , p. $466-485$

Sur la base d'une enquête menée en 1981 dans cinq municipalités d'union de la gauche situées dans la banlieue "rouge" de Paris, on examine le comportement d'une section cégétiste d'employés municipaux face à un maire communiste et les réactions de la CGT et de la CFDT.

\section{GALLAGHER, Daniel G WETZEL, Kurt}

Local Employer and Union Perceptions of Two-Tier Bargaining

v. 39 , no 3,1984 , p. $486-508$

En Saskatıhewan, les négociations collectives entre les enseignants et les commissions scolaires qui auparavant s'effectuaient au niveau local, ont fait place à une structure à deux paliers consistant en une négociation multipartite pour toute la province et aussi à des pourparlers au plan local. Analyse de la structure de négociation à double palier en Saskatchewan et évaluation empirique des avantages et des limitations de la négociation multipartite - 4 tableaux.

\section{LAFLAMME, Lucie} ARSENAULT, André

Rémunération, postes de travail et accidents: Une relation interactive.

v. 39 , no 3,1984 , p. $509-525$

Comparaison des fréquences d'accidents entre des travailleurs rémunérés au temps et des travailleurs rémunérés au rendement dans différentes catégories d'occupations du secteur du meuble et des articles d'ameuble- 
Relations Inoustrielles, vol. 45, no 4 (1990)

ment. L'étude révèle qu'il existe un lien significatif entre le mode de rémunération et le risque d'accidents qui est intéractif avec le poste de travail - 7 tableaux, bibliographie.

\section{DEVORETZ, D.J.}

REED, Clyde G.

Evidence from the Skilled-Unskilled Canadian Wage Index.

v. 39 , no 3 , 1984 , p. $526-537$

Les études contemporaines démontrent que, au cours des décennies récentes, il y aurait eu un mouvement significatif dans le sens de l'égalisation des salaires entre les travailleurs canadiens qualifiés et non qualifiés. On révise cette opinion en corrigeant les erreurs de mesure les plus manifestes: on oppose les salaires réels aux salaires nominaux; on utilise les "ratios" de salaires au lieu des différences pour mesurer les changements dans les taux de salaires; on remplace les comparaisons annuelles par l'analyse de régression - 2 figures, 2 tableaux, références.

\section{FISHER, E.G.}

SHERWOOD, L.M.

Fairness and Managerial Rights in Canadian Arbitral Jurisprudence

v. 39, no 3,1984, p. $538-552$

Après une étude des décisions arbitrales et judiciaires récentes on peut conclure ceci: Le devoir d'équité, qui a été transposé du droit administratif dans la jurisprudence arbitrale, peut prendre place à l'intérieur des paramètres de la convention collective. Mais là où la clause des droits de la direction confère clairement à la direction le droit exclusif de décider certaines questions, un tribunal d'arbitrage est sans compétence pour réviser soit la justesse de la décision, soit le processus par lequel on y est arrivé.

0320 HOLMES, R.H. PINFIELD, L.T.

Logit Analysis of Employee Turnover in a Remote Community

v. 39 , no 3,1984, p. $553-569$

Vérification expérimentale d'un modèle de roulement de la main-d'oeuvre dans une localité isolée au Canada. L'analyse "logit" des données recueillies chez les travailleurs d'un grand établissement industriel d'une région isolée de la Colombie Britannique, permet de déterminer l'effet sur la probabilité de départ qu'entraînent chez les travailleurs leur perception de l'emploi qu'ils occupaient, celle de la vie dans une région isolée, ainsi que leurs caractéristiques personnelles - 1 figure, références.

\section{BURKE, Ronald J.}

Trainee Experiences in Industrial Retraining Programs: A Case Study

v. 39 , no 3.1984 , p. $570-577$

Suite à la fermeture de l'usine de "Canadian Admiral" à Cambridge, Ontario, en novembre 1981, on fait le compte rendu de l'expérience de 58 anciens employés de cette usine qui se sont inscrits à des programmes de formation académique ou professionnelle financés par le gouvernement fédéral. L'analyse met l'accent sur leur rôle d'étudiant et sur leurs aspirations pour l'avenir, alors que tous étaient encore en période de reformation - 1 tableau, références.

\section{BLOUIN, Rodrigue}

La réforme du Code du travail du Québec: une démarche fragmentaire.

v. 39 , no 3.1984 , p. 578-604

A la suite de la création d'une commission consultative et d'une conférence socio-économique sur le travail devant aboutir à la réforme du "Code du travail" au Québec, on met en doute la validité de l'approche fragmentaire envisagée.

\section{BEAULIEU, LUC}

Décisions rendues par le Conseil canadien des relations du travail.

v. 39 , no 3,1984, p. $605-618$

Résumé de deux décisions rendues par le Conseil: 1) Le Conseil décide qu'il possède la juridiction nécessaire pour condamner aux frais judiciaires. 2) A la suite d'une cession de travail à l'extérieur (sous-traitance) faite par un employeur alléguant des motifs de rentabilisation de son entreprise, le Conseil décide qu'une telle cession, si elle est effectuée sans motivation anti-syndicale, ne constitue pas une violation du Code canadien du travail.

\section{BENEDICT, Daniel (R)}

BARKIN, Sol

Worker Militancy and its Consequences, 2nd Ed. The Changing Climate of Western Indus- 
trial Relations, New York, Praeger Publisher, 1983, 400 pp., ISBN 0-03-061793-6, recensé par Daniel Benedict.

v. 39 , no 3,1984, p. $626-630$

\section{DUSSAULT, Gilles (R)}

DONN, C.D.

The Australian Council of Trade Unions. History and Economic Policy. New York, University Press of America, 1983, 366 pp., ISBN a8191-2728-9, recensé par Gilles Dussault. v. 39, no 3,1984, p. $630-631$

\section{VERGE, Pierre (R)}

BARBAGELATA, H.H.

Introduction aux institutions du travail en Amérique Latine. Louvain, Presses universitaires de Louvain, 1980, 303 pp., ISBN 90-6186-1012 , recense par Pierre Verge.

v. 39 , no 3,1984 , p. $632-633$

0327 LEMELIN, Maurice (R)

FINKELMAN, Jacob

GOLDENBERG, Shirley B.

Collection Bargaining in the Public Service: the Federal Experience in Canada. Montreal, Institute for Research on Public Policy/L'Institut de recherches politiques, 1983, 825 pp., ISBN 0-920380-95-6 (vol. 1), ISBN 0-920380-97-2 (vol. 2), recensé par Maurice Lemelin.

v. 39 , no 3,1984, p. 633-634

\section{JOUBERT, P. (R)}

WEISS, Dimitri CHIROUZE, Yves

Le consommérisme. Paris, Editions Sirey, collection Administration des entreprises, 1984, 387 pp., ISBN 2-248-00080-4, recensé par $P$. Joubert.

v. 39 , no 3,1984, p. $634-635$

0329 MATEJKO, Alexander J. (A)

TAYLOP, Robert

Workers and the New Depression. London, Macmillan Press, 1981, 212 pp., ISBN 0-33333411-6, recensé par Alexander J. Matejko. v. 39, no 3,1984, p. 636

\section{PAQUIN, Michel (R)}

MILLER, Danny FRIESEN, Peter $H$, Organizations : A Quantum View. Englewood Cliffs, Prentice-Hall, 1984, 320 pp., ISBN 0-13641985-2, recensé par Michel Paquin.

v. 39, no 3, 1984, p. $636-637$
0331 JOUBERT, P. (R)

RITA, Giuseppe De SILVESTRI, Umberto TEDESCHI, Michele WEISS, Dimitri ZUBOFF, Shoshana

II ruolo del fattore "uomo" nello scenario economico-produttivo degli anni "80: i quadri. Roma, STET, 1982, 84 pp., recensé par $P$. Joubert.

v. 39 , no 3,1984 , p. $637-638$

0332 GRIEZIC, F.J.K. (R)

STRUTHERS, James

"No Fault of their Own". Unemployment and the Canadian Welfare State 1914-1940. The State and Economic Life Series, Mel Watkins and Leo Panitch eds, Toronto, University of Toronto Press, 1983, 238 pp., ISBN 0-8020-7, recensé par F.J.K. Griezic.

v. 39 , no 3,1984, p. $638-641$

0333 MATEJKO, Alexander J. (R)

THOMPSON, Paul

The Nature of Work. An Introduction to Debates on the labour Process. London, The Macmillan Press, 1983, 305 pp., ISBN 0-33333027-7, recensé par Alexander J. Matejko.

v. 39 , no 3,1984 , p. $641-642$

0334 VEÀGE, Pierre $(A)$

GOULD, W.B.

Japan's Reshaping of American Labor Law. Cambridge, Mass., The MIT Press, 1984, 193 pp., ISBN 0-262-07091- $X$, recensé par Pierre Verge.

v. 39 , no 3,1984, p. $642-643$

0335 MALLETTE, Noël (R)

LECLEPC, Claudine SEXTON, Jean

La sécurité d'emploi dans l'industrie de la construction au Québec, un réve impossible? Québec, Presses de I'Université Laval, 1983, 275 pp., ISBN 2-7637-7028-2, recensé par Noël Mallette.

v. 39 , no 3,1984, p. $643-645$

0336 ADAMS, ROy J.

The Extent of Collective Bargaining in Canada v. 39 , no 4,1984, p. $655-667$

Après une analyse des problèmes techniques se rapportant aux enquêtes annuelles de Travail Canada sur "Les conditions de travail dans l'industrie canadienne" et l'enquête sur l'effectif syndical de la "Loi sur les déclarations des corporations et des syndicats ouvriers", on a procédé à une évaluation de l'effectif syndical par industrie pour l'année 
1977. De ce calcul, on peut retenir que $44 \%$ des travailleurs canadiens vivaient sous le régime de conventions collectives en 1977 - 7 tableaux, références.

\section{LORRAIN, Jean BRUNET, Luc}

Climat organisationnel, satisfaction au travail et perception du syndicalisme

v. 39 , no 4,1984, p. $668-679$

Analyse des résultats d'une étude empirique qui tentait de vérifier la relation entre le climat organisationnel et la perception de l'instrumentalité des syndicats tout en considérant l'effet de la satisfaction au travail sur la relation entre ces deux variables. L'échantillon étudié se composait de 625 cadres inférieurs travaillant pour une entreprise spécialisée dans les télécommunications - 3 tableaux, références.

0338 ANDIAPPAN, P. CATTANEO, R. Julian MURPHY, John

Interest Arbitration in Ontario Hospitals: Result of an Attitude Survey of Union and Manage. ment Officials.

v. 39 , no 4,1984 , p. $680-694$

Résultats d'une étude portant sur les attitudes que les dirigeants patronaux et syndicaux adoptent à propos de l'arbitrage des conflits d'intérêt dans les hôpitaux ontariens. Les procédures d'arbitrage des conflits d'intérêt sont mises en place pour faciliter le règlement des différends lorsque le droit de grève n'existe pas - 6 tableaux, références.

\section{JONES, J.C.H.}

\section{LAUDADIO, L.}

Organized Labour, Regional Political Bias and the Canadian Tariff Structure

v. 39 , no 4 , 1984, p. 695-709

L'objectif de l'étude est de tenter d'évaluer l'influence des syndicats, en tant que groupes de pression, sur la politique tarifaire canadienne. Une des conclusions fondamentales de l'étude, c'est que le degré de syndicalisation ne semble pas avoir une valeur significative dans la détermination des mesures tarifaires - 2 tableaux, références.

\section{VERGE, Pierre}

Evolution de la protection juridictionnelle de l'accès au syndicat.

v. 39 , no 4,1984, p. $710-743$
Le syndicat, d'organisme purement privé qu'il était en une période initiale au Canada et au Québec, est devenu au cours des années 1940 un organisme de représentation officiel sur le plan de la détermination et de la protection des conditions de travail. On examine si l'intervention juridictionnnelle relative à la protection de l'accès au syndicat a fait montre d'une évolution correspondante à celle de la nature juridique du syndicat.

\section{MORIN, Fernand}

Nouvelle présence de l'État dans les relations du travail.

v. 39 , no 4,1984, p. $744-752$

Analyse de certains changements apportés au Code du travail du Québec et des effets de ces changements sur la gestion des entreprises. Les modifications de 1978 et 1982 apportées au Code du travail peuvent être rangées en trois sous-groupes: 1) Le nouveau rôle de l'État pour la tenue de rapports collectifs, 2) l'intervention de l'État dans le rapport syndicat/salarié, 3) la modification du statut et des pouvoirs de l'employeur.

0342 ARTHURS, H.W.

Understanding "Understanding": Industrial Relations Research and Policy in Canada from 1969 to $1984 \ldots$ and Beyond.

v. 39 , no 4,1984, p. $753-761$

Le Rapport de la Commission Woods portant sur les relations du travail au Canada a été publié en décembre 1968. A cette époque, les membres de la Commission souhaitaient deux choses: Que le Rapport apporterait une plus grande compréhension des problèmes dans le domaine des relations industrielles au Canada et qu'il stimulerait davantage la recherche dans ce domaine. Qu'en est-il exactement quinze ans après la publication du Rapport.

\section{MILNE, William J. \\ ROSS, Thomas W}

The Cyclical Variation of Wage Premiums in the Canadian Manufacturing Industries v. 39 , no 4,1984, p. $762-773$

Le changement dans les taux de salaires payés dans une économie sera influencé, d'une façon largement prévisible, par le taux d'inflation et le taux actuel et récent de chômage. Après avoir présenté un nouveau 
modèle macro-économique de variation cyclique de la structure salariale, interindustrielle, on teste empiriquement ce modèle en utilisant les données annuelles du secteur manufacturier canadien pour les années 1958-1981 - 1 figure, 2 tableaux, références.

\section{POTHIER, Dianne}

Décisions rendues par le Conseil canadien des relations du travail.

\section{v. 39 , no 4,1984 , p. $774-782$}

Une compagnie transfère toutes ses affaires à une de ses filiales à part entière afin de changer de convention collective et de se soustraire à la compétence fédérale. Dans cette cause, le Conseil 1) a rejeté la requête portant sur la question d'employeur unique (article 133); 2) a admis qu'il y avait eu vente d'entreprise (article 144); 3) a rejeté la requête portant sur la confusion (article 144-3a)

\section{MORIN, Fernand (R)}

BARBASH, Jack

The Elements of Industrial Relations. Madison, Wisc., The University of Wisconsin Press, 1984, 153 pp., ISBN 0-299-09610-6, recensé par Fernand Morin.

v. 39 , no 4,1984 , p. $793-795$

0346 CÔTÉ, André C. (R)

GAUTHIER-MONTPLAISIR, Francine

L'arbitrage des griefs et les infractions disciplinaires à caractère criminel. Cowansville, Les Éditions Yvon Blais Inc., 1983, 171 pp., ISBN 2-89073-170-7, recensé par André C. Cóté.

v. 39 , no 4,1984 , p. $795-797$

0347 VANDAMME, François (R)

CAMPBELL, Duncan C. ROWAN, Richard L. Multinational Enterprises and the OECD Industrial Relations. Philadelphia, The Wharton School Industrial Research Unit, University of Pennsylvania, 1983, 280 pp., ISBN 0-89546039-4 et ISSN 0149-0818, recensé par François Vandamme.

v. 39 , no 4,1984, p. $797-799$

0348 MATEJKO, Alexander J. (R)

MCMILLAN, Charles J.

The Japanese Industrial System. Berlin-New York, Walter de Gruyter, 1984, 356 pp., ISBN 3-11-008894-0, recensé par Alexander J. Matejko.

v. 39 , no 4,1984 , p. $799-801$
0349 HURTUBISE, Rolland (R)

BARANSON, Jack

Robots in Manufacturing : Key to International

Competitiveness. Mt Airy, Maryland, Lomond Publications, 1983, 152 pp., ISBN 0-91233839-3, recensé par Rolland Hurtubise.

v. 39 , no 4,1984 , p. $801-802$

0350 THWAITES, James (R)

TRAVES, TOm

Essays in Canadian Business History. Toronto, McClelland and Steward, 1984, 212 pp., ISBN 0-7710-8570-2, recensé par James Thwaites.

v. 39, no 4,1984 , p. $802-803$

0351 TUCK, Hugh (R)

SEFTON MACDOWELL, Laurel

"Remember Kirkland Lake" : The History and Effects of the Kirkland Lake Gold Miners' Strike 1941-42. Toronto, University of Toronto Press, 1983, 292 pp., ISBN 0-8020-5585-0, recensé par Hugh Tuck.

v. 39 , no 4,1984 , p. $803-804$

0352 TESSIER, Paul (R)

SLOANE, Arthur A.

Personnel, Managing Human Resources. Englewood Cliffs, Prentice Hall, 1983, 619 pp., ISBN 0-13-658278-8, recensé par Paul Tessier. v. 39, no 4, 1984, p. 804-805

0353 BÉLANGER, Laurent $(\mathrm{R})$

DUBRIN, Andrew J.

Foundations of Organizational Behavior : An Applied Perspective. Englewood Cliffs, Prentice-Hall, 1984, 500 pp., ISBN 0-13-329367-X, recensé par Laurent Bélanger.

v. 39 , no 4,1984 , p. $805-806$

0354 THÉRIAULT, Roland (R)

PIVETEAU, Jacques

L'entretien d'appréciation du personnel : pratiques en question. Toulouse, Éditions Erès, 1981, 207 pp., ISBN 2-86586-002-7, recensé par Roland Thériault.

v. 39 , no 4,1984 , p. $806-809$

0355 BÉLANGER, Laurent (R)

NEILSEN, Eric $\mathrm{H}$.

Becoming an O.D. Practitioner. Englewood Cliffs, Prentice Hall, 1984, 280 pp., ISBN 013-071555-7, recensé par Laurent Bélanger.

v. 39 , no 4,1984 , p. $809-811$ 
0356 PAQUIN, Michel (R)

MINTZBERG, Henry

Le manager au quotidien. Les dix róles du cadre. Montreal, Les Éditions Agence d'Arc, 1984, 220 pp., ISBN 2-7081-0558-2, recensé par Michel Paquin.

v. 39 , no 4,1984, p. $811-812$

0357 BLOUIN, Rodrigue (R)

LAPERRIERE, René

Bibliographie du droit ou travail canadien et québécois. Montreal, Les Éditions Yvon Blais Inc., 1984, 70 pp., ISBN 2-89073-515-X, recensé par Rodrigue Blouin.

v. 39 , no 4,1984, p. $812-813$

0358 MATEJKO, Alexander J. (R)

NIGHTINGALE, Donald V. LONG, Richard J. Gain and Equity Sharing. Quality of Working Life. Case Study Series, Ottawa, Minister of Supply and Services, Canada, 1984, 51 pp., ISBN 0-662-13138-X, recense par Alexander J. Matejko.

v. 39 , no 4,1984, p. $813-814$

0359 MATEJKO, Alexander J. (R)

WONG, Jim

Employment Injuries and Occupational IIInesses 1972-1981. Ottawa Minister of Supply and Services Canada, 1984, 134 pp., ISBN 0-66253002-0, recensé par Alexander J. Matejko. v. 39, no 4, 1984, p. 814-815

0360 MATEJKO, Alexander J. (R) KAHN, Joan WESTLEY, William A.

The Working Conditions in Canadian Hospitals. Constraints and Opportunity. Ottawa, Minister of Supply and Services Canada, 1984, 112 pp., ISBN 0-662-52924-3, recensé par Alexander J. Matejko.

v. 39 , no 4,1984 , p. $815-816$

0361 DÉOM, Esther $(\mathrm{R})$

ARMSTRONG, Pat ARMSTRONG, Hugh

Une majorité laborieuse. Les femmes qui gagnent leur vie, mais a quel prix. Ottawa, Conseil consultatif canadien de la situation de la fermme, 1983, 329 pp., ISBN 0-660-910268, recensé par Esther Déom.

v. 39 , no 4,1984 , p. $816-817$

0362 ROSSILION, Claude

L'OIT et l'élimination de la discrimination dans l'emploi

v. 40 , no 1,1985, p. $03-26$

Les différents aspects des conventions et recommandations adoptées par l'Organisation internationale du travail en ce qui concerne l'élimination de la discrimination et la promotion de l'égalité des chances en matière d'emploi et de profession. Les précisions qui ont été apportées à la définition de la discrimination et à la détermination des mesures à prendre pour son élimination.

\section{BENEDICT, Daniel}

The 1984 GM Agreement in Canada: Significance and Consequences.

v. 40 , no 1,1985, p. $27-47$

Les négociations et la grève de $1984 \mathrm{chez}$ General Motors au Canada sont un point tournant dans les relations entre les syndicats américains et canadiens. On explique les différents éléments de la crise et ses conséquences possibles sur l'avenir du mouvernent ouvrier canadien.

\section{KRUGER, Arthur}

Collective Bargaining in Ontario Public Hospitals

v. 40 , no 1,1985 , p. $48-67$

L'évolution de la négociation collective dans les hôpitaux publics en Ontario. La croissance et la répartition de l'emploi dans les hôpitaux. La législation en vigueur dans le secteur. Comment les employeurs et les syndicats s'arrangent pour négocier compte tenu de la législation et d'autres forces qui sont à l'oeuvre. Le champ de la négociation et son développement chronologique. Les attitudes des parties par rapport au système actuel - 4 tableaux, bibliographie.

0365 MILLER, Roger

La stratégie d'entreprise et la gestion des ressources humaines.

v. 40 , no 1. 1985 , p. $68-86$

$L$ 'harmonisation de la gestion des ressources humaines à la stratégie d'entreprise est souvent conçue dans une perspective statique qui a le résultat imprévu, au fil du temps, de rendre difficile l'adaptation de l'entreprise aux exigences concurrentielles et techniques nouvelles. On esquisse, de manière dynamique, la relation entre la gestion des ressources humaines et la stratégie afin d'assurer le progrès de l'entreprise - 2 tableaux, 2 graphiques, bibliographie. 


\section{TZINER, Aharon}

DOLAN, Shimon L.

Identifying Female Officer Potential: An Exploration in Predictors' Payoff

v. 40, no 1,1985 , p. $87-98$

Examen de l'efficacité d'un système de prédiction à multiples prédicteurs et comparaison de ce système avec d'autres systèmes n'utilisant qu'un seul prédicteur dans le but de mettre au point un système efficace d'identification du potentiel des officiers. Deux groupes de cadets de l'armée composés exclusivement de femmes ont participé à l'étude de la validité des prédicteurs pendant leur période de service militaire obligatoire - Références.

0367 KATASANEVAS, Theodoros

Trade Unions in Greece

v. 40 , no 1,1985 , p. $99-114$

Histoire, développement et structure du syndicalisme en Grèce. Les principaux facteurs économiques qui ont influencé d'une façon décisive la croissance du syndicalisme et le développement d'une structure organisationnelle très complexe du syndicalisme grec - 2 tableaux.

0368 FISHER, E.G. IVANKOVICH, I.F.

Alberta's Occupational Health and Safety Amendment Act, 1983.

v. 40 , no 1,1985 , p. $115-139$

Les modifications apportées à la Loi sur la santé et la sécurité au travail en Alberta en 1983, dont l'objet est la réorientation des mesures réglementaires, sont à la fois progressives sur certains points et conservatrices sur d'autres. Description des principaux éléments du nouveau texte législatif.

\section{BLACK, Errol}

In Search of "Industrial Harmony": The Process of Labour Law Reform in Manitoba, 1984.

v. 40 , no 1,1985 , p. $140-161$

En 1981, le Nouveau parti démocratique fut élu au Manitoba suite à des promesses de réformes législatives favorables aux syndicats. a) Les sources de la controverse sur les relations du travail à l'intérieur du NPD manitobain. b) Le cheminement suivi par le gouvernement élu pour remplir ses engagements envers les syndicats. c) Les conditions qui ont permis aux hommes d'affaires du Manitoba d'obliger le gouvernement à battre en retraite.

0370 BURKE, Ronald J.

Consequences of not Working Sixteen Months After a Plant Closing

v. 40 , no 1,1985, p. $162-169$

En novembre 1981, l'usine de Canadian Admiral à Cambridge, Ontario, fermait soudainement ses portes. Résultats d'une étude analysant les conséquences d'être toujours en chômage seize mois après la fermeture de l'usine - 3 tableaux, références.

\section{POTHIER, Dianne}

Décisions rendues par le Conseil canadien des relations du travail.

v. 40 , no 1, 1985, p. $170-174$

Le Conseil décide qu'un employeur, même s'il n'agit pas dans un esprit antisyndical, se rend coupable de pratique déloyale lorsque, à moins de raisons d'affaires graves, il interdit la solicitation d'adhésions syndicales dans ses locaux pendant les heures libres ou le port de l'insigne du syndicat pendant les heures de travail.

\section{VERGE, Pierre (R)}

VERDIER, Jean-Maurice

Syndicats et droit syndical. 2ième éd., v. II, Le droit syndical dans l'entreprise, tome $5 d u$ Traité de droit du travail, publié sous la direction de G.H. Camerlynck, Paris, Daloz, 1984, 475 pp., ISBN 2-247-00516-0, recensé par Pierre Verge.

v. 40, no 1,1985, p. $178-180$

0373 LARSON, Peter E. (R) ADDISON, John T. BURTON, John

Trade Unions and Society. Vancouver, The Fraser Institute, 1984, 189 pp., ISBN 0-88975056-4, recensé par Peter E. Larson.

v. 40, no 1,1985, p. $180-181$

0374 ROY, André (R)

LESTER, Richard A.

Labor Arbitration in State and Local Government. Princeton, Princeton University, 1984, 
210 pp., ISSN 0079-5305, recensé par André Roy.

v. 40 , no 1,1985, p. $181-183$

0375 VERGE, Pierre (R)

DORSEY, $J$.

Canada Labour Relations Board - Federal Law and Practice. Toronto, the Carswell Company 1983, 323 pp., ISBN 0-459-35850-2, recensé par Pierre Verge.

v. 40, no 1,1985, p. $183-185$

0376 MATEJKO, Alexander J. (R) FREEMAN, Richard B. MEDOFF, James $L$. What do Unions do? New York, Basic Books Inc., 1984, 293 pp., ISBN 0-465-09133-4, recense par Alexander J. Matejko.

v. 40 , no 1,1985 , p. $185-186$

0377 DUSSAULT, Gilles (R)

FORD, B. PLOWMAN, D.

Australian Unions : An Industrial Relations Perspectives. Melbourne, MacMillan Company of Australia, 1983, 576 pp., ISBN 0-333-339134, recensé par Gilles Dussault.

v. 40 , no 1,1985 , p. $186-188$

0378 MATEJKO, Alexander J. (R)

GOODSELL, Charles T.

The Case for Bureaucracy : A Public Administration Polemic. Chatham, Chatham House Publishers, 1983, 179 pp., ISBN 0-934540-17-

9, recensé par Alexander J. Matejko.

v. 40 , no 1,1985, p. $188-190$

0379 MATEJKO, Alexander J. (R)

WILCZYNSKI, Jozef

Comparative Industrial Relations. Ideologies, Institutions, Practices and Problems under Social Systems with Special Reference to Socialist Planned Economies. London, The Macmillan Press, 256 pp., ISBN 0-333-33430-2, recensé par Alexander J. Matejko.

v. 40 , no 1, 1985, p. $190-192$

0380 BENEDICT, Daniel (R)

HIRSCHHORN, Larry

Beyond Mechanization : Work and Technology in a Postindustrial Age. Cambridge, MIT Press, 1984, 187 pp., ISBN 0-262-08132-3, recensé par Daniel Benedict.

v. 40 , no 1, 1985 , p. $192-194$

0381 HÉBERT, Gérard In memoriam: Émile Bouvier, S.J. 1906-1985

v. 40 , no 2,1985 , p. $211-212$
A l'occasion de son décès, rétrospective de la carrière du Père Émile Bouvier, Jésuite, fondateur de l'École des relations industrielles de l'Université de Montréal en 1945.

0382 LACOSTE, Paul

L'École des relations industrielles de l'Université de Montréal: Quarantième anniversaire.

v. 40 , no 2,1985 , p. $213-218$

A l'occasion du quarantième anniversaire de l'École des relations industrielles, le recteur de l'Université de Montréal brosse une esquisse historique de cette institution et décerne la Médaille de l'Université à son fondateur, le R.P. Emile Bouvier.

\section{SAUNDERS, George}

Employment and the Productivity Slowdown: 1958-1980

v. 40 , no 2,1985 , p. $219-242$

Le ralentissement de la productivité qui se généralise dans les pays industriels d'Occident demeure un mystère. En s'inspirant des mesures de la productivité totale par facteur récemment mises au point et dont dispose le Conseil économique du Canada, on examine les rapports entre les facteurs de production, principalement celui du travail, et la productivité -5 tableaux

0384 BEAUMONT, P.B.

The Diffusion of Human Resource Management Innovations.

v. 40 , no 2,1985 , p. $243-256$

Très peu de chercheurs se sont intéressés à la question de la vulgarisation des innovations en matière de gestion des ressources humaines. La présente étude se fonde sur un échantillon de quelque 300 établissements en Grande-Bretagne et examine la portée et la nature de la vulgarisation d'innovations d'une entreprise à l'autre. L'innovation considérée a trait à un programme conjoint syndicat-employeur de traitement de l'alcoolisme sur les lieux du travail - 2 tableaux.

\section{BRYCE, George $K$.} MANGA, Pran

The Effectiveness of Health and Safety Committees.

v. 40 , no 2,1985 , p. $257-283$

La présente étude traite des principaux fac- 
teurs d'évaluation de l'efficacité des comités paritaires de santé et de sécurité au travail. En examinant les quelques travaux sur le sujet, on peut énumérer une série de mesures de la valeur de ces comités. Les données accessibles en Alberta et en Saskatchewan sont évaluées à la lumière de ces mesures de façon à savoir si les comités paritaires de santé et de sécurité professionnelles sont efficaces -6 tableaux.

\section{MCSHANE, Steven L.}

Sources of Attitudinal Union Militancy

v. 40, no 2,1985 , p. $284-302$

Le militantisme syndical revêt deux dimensions: le militantisme en milieu de travail et le militantisme du syndicat lui-même. Résultats d'une étude ayant pour objectif de déceler l'importance des facteurs démographiques, familiaux, sociaux, professionnels et d'activisme en milieu de travail, pour expliquer les attitudes militantes syndicales. Les données sont tirées d'une enquête effectuée auprès de 297 membres d'un syndicat d'employés municipaux du sud de l'Ontario - 3 tableaux, références.

\section{SHIROM, Arie}

The Labor Relations System: A Proposed Conceptual Framework

v. 40 , no 2,1985 , p. $303-323$

Présentation d'un cadre conceptuel d'un système de relations du travail (SRT). Après avoir approfondi les premiers essais d'application de la théorie des systèmes, on définit et explique les composantes d'un SRT à partir des concepts et de la terminologie de l'approche systémique en fonction de leur application aux relations du travail en milieu de travail - 1 figure, 1 tableau, références.

\section{DUSSAULT, Gilles}

The Future of Professional Monopolies.

v. 40, no 2,1985, p. $324-339$

Analyse sociologique de la situation des professions dont les membres ont le monopole de la production de certains biens et services, comme les médecins, les dentistes, les avocats, les comptables et les ingénieurs. Après une revue de la littérature récente sur le professionnalisme, on peut affirmer que les changements récents à l'intérieur des professions et dans le contexte dans lequel celles-ci opèrent, réduiront la capacité des professions de conserver leurs privilèges monopolistiques - Bibliographie.

\section{EASTMAN, Byron}

A Catastrophe Theory on Union Behaviour.

v. 40, no 2,1985 , p. $340-350$

Application de la théorie de la catastrophe au comportement d'un syndicat en période de négociation d'une convention collective. L'idée fondamentale en est qu'un syndicat peut devenir très docile ou, tout à coup très agressif, selon les risques ou les provocations qu'il a a affronter. Ces changements draconiens de comportement peuvent expliquer certains arrêts de travail inattendus et certains cas de rupture des négociations - 4 figures, références.

\section{CARROTHERS, A.W.R.}

A Way of Thinking about Collective Bargaining: Circumstance, Policy, Law and Actuality.

v. 40 , no 2,1985 , p. 351

Présentation d'un système de classification pouvant aider à l'étude multidimensionnelle d'un système de négociation collective. Étude des systèmes britannique, américain, canadien, suédois et ouest-allemand selon quatre grands thèmes: circonstances, politique, législatiun et actualité.

\section{CAMERON, Samuel}

Historical Variations in the Impact of Union Density on Strike Frequency: Some U.K. Evidence

v. 40 , no 2,1985 , p. $367-370$

II existe un important débat à propos de l'influence du membership syndical sur la fréquence des grèves. Cette étude empirique effectuée à partir des données sur le Royaume-Uni à différentes périodes, démontre qu'il y a une relation positive entra la fréquence des grèves et les effectifs syndicaux - 1 tableau, références.

\section{MORIN, Fernand}

Les tenants et les aboutissants de la convention collective.

v. 40 , no 2,1985 , p. $371-378$

La durée de la convention collective constitue un point d'ancrage important des rapports collectifs du travail selon l'aménagement im- 
Relations Industrielles, vol. 45, no 4 (1990)

posé au Code du travail du Québec. Le tribunał du travail a eu récemment l'occasion de prendre position à l'égard de plusieurs sous-questions rattáchées à la durée de la convention collective. Rappel de cette affaire et appréciation des éléments pertinents à la décision du tribunal.

\section{RICHER, Jean-Charles}

La Corporation professionnelle des conseillers en relations industrielles du Québec devant la Commission Beaudry.

v. 40 , no 2,1985, p. $379-385$

Le 13 décembre 1984, la Corporation professionnelle des conseillers en relation industrielle du Québec présentait son mémoire à la Commission consultative sur le travail présidée par le juge René Beaudry. Extraits de ce mémoire donnant les 20 "propositions d'actions" et les quelques commentaires sous-jacents présentés à la Commission Beaudry.

0394 LAMY, Francine

Décisions rendues par le Conseil canadien des relations du travail.

v. 40 , no 2,1985 , p. $386-398$

Le Conseil décide que la sollicitation syndicale au lieu d'emploi est une activité protégée par la Charte canadienne des droits et libertés car elle constitue l'exercice des libertés d'association et d'expression. II reconnaît l'employeur coupable de pratiques déloyales suite à ses agissements.

0395 VERGE, Pierre (R)

BLANPAN, R.

Principes de droit du travail. Bruges-Bruxelles, La Charte, 1984, 313 pp., ISBN 90-6400029-0, recensé par Pierre Verge.

v. 40 , no 2,1985 , p. $403-405$

0396 MATEJKO, Alexander J. (R)

CHEN, Mervin Y.T. REGAN, Thomas G.

Work in the Changing Canadian Society. Toronto, Butterworths, 1985, 289 pp., ISBN 0409-82335-X, recensé par Alexander J. Matejko.

v. 40, no 2,1985, p. $405-407$

0397 DUSSAULT, Gilles (P)

Le travail : quête de sens, quête d'emploi. Cahiers de recherche éthique, no 10, Montreal, Fides, 1984, 238 pp., ISBN 2-7621-1222-

2, recensé par Gilles Dussault.

v. 40 , no 2,1985 , p. $407-408$
0398 WHITE, Jerry P. (A)

PALMER, Bryon

Working-Class Experience : The Rise and Reconstitution of Canadian Labour, 1800-1980. Toronto, Butterworths, 1983,320 pp., recensé par Jerry $P$. White.

v. 40, no 2,1985, p. $408-409$

0399 WHITE, Jerry P. (R)

GORDON, David EDWARDS, Richard

$\mathrm{REICH}$, Michael

Segmented Work Divided Workers : The Historical Transformation of Labour in the United States. Cambridge, Cambridge University Press, 1982, 288 pp., recensé par Jerry $P$. White.

v. 40 , no 2,1985, p. $410-411$

0400 MATEJKO, Alexander J. (R)

KANTER, Rosabeth Moss

The Change Masters. Innovation and Entrepreneurship in the American Corporation. New York, Simon and Schuster, 1984, 432 pp., ISBN 0-671-42802-0, recensé par Alexander J. Matejko.

v. 40 , no 2,1985, p. $411-412$

0401 DUSSAULT, Gilles (R)

HAMOWY, Ronald

Canadian Medicine : A Study in Restricted Entry. Vancouver, The Fraser Institute, 1984, 394 pp., ISBN 0-88975-062-9, recensé par Gilles Dusseault.

v. 40, no 2,1985, p. $412-414$

0402 BOIS-BROCHU, Hélène (R)

SERRUYS, Jacques $W$.

Phychologie du commandement. Bruxelles, Vander Editeur, 1984, 230 pp., ISBN 2-80080000-3, recensé par Hélène Bois-Brochu.

v. 40 , no 2,1985 , p. $414-415$

0403 MERCIER, Jacques

Les effets du salaire minimum sur l'emploi des jeunes au Québec.

v. 40 , no 3,1985 , p. $431-457$

Estimation des effets du salaire minimum sur les taux d'emploi des jeunes pour la période de 1966 à 1981 faite à l'aide de l'analyse de régression. Les diverses mesures utilisées pour les variables explicatives et les spécifications des équations de régression. Présentation et discussion des résultats statistiques - 3 tableaux, bibliographie. 
0404 SOLOMON, Norman A.

The Negociation of First Agreements under the Canada Labour Code.

v. 40, no 3,1985 , p. $458-472$

Analyse des unités de négociation qui sont arrivées à négocier une première convention collective et de celles qui n'ont pas réussi à y parvenir, à partir des données reliées a) à l'expérience des accréditations accordées par le Conseil canadien des relations du travail, b) aux caractéristiques de l'unité de négociation de base, $c$ ) au processus de négociation -4 tableaux

\section{GRANT, Michel} ROSE, Ruth

Encadrement du travail à domicile dans lindustrie du vêtement au Québec.

v. 40 , no 3,1985, p. $473-494$

Analyse du contexte économique de l'industrie du vêtement et du rôle qu'y joue le travail à domicile. Bilan des tentatives de contrôle du travail à domicile dans le cadre de notre régime actuel de relations du travail et perspectives pour l'avenir. Résultats d'une enquête tant auprès des intervenants patronaux, syndicaux et gouvernementaux qu'auprès d'une cinquantaine de travailleuses à domicile pour la plupart clandestines - 3 tableaux

0406 CHARLES, Lawrence M. HUMPHREYS, Edward $\mathrm{H}$. Bargaining to Achieve Teacher Control in Ontario

v. 40 , no 3,1985, p. $495-511$

Les écrits traitant du professionnalisme suggèrent qu'obtenir le contrôle sur l'emploi ou obtenir le droit de participer aux prises de décision avant l'application d'une politique constituent des aspects majeurs si l'on souhaite atteindre le professionnalisme. On examine jusqu'à quel point les enseignants ontariens se sont servis des négociations collectives pour améliorer leur statut en participant aux prises de décision et en exerçant une autorité en millieu de travail - 4 tableaux, bibliographie

0407 GEORGE, Kenneth

Les comités de santé et de sécurité du travail: Tables de concertation ou de négociation?

v. 40, no 3,1985, p. $512-528$
Évaluation de l'expérience des comités de santé et de sécurité du travail par rapport à certains des objectifs visés à l'origine par la loi québécoise sur la santé et la sécurité du travail. On essaie de voir si, via ces comités d'entreprise, on réussit à sortir les enjeux de la santé et de la sécurité du travail du champ de la négociation pour en faire des objets de concertation. La situation dans les entreprises syndiquées et non-syndiquées.

\section{SWIMMER, Gene} LUCE, Sally $R$.

Asbestos Exposure and Attitudes Toward Occupational Health.

v. 40 , no 3,1985 , p. $529-544$

En 1981, le gouvernement de l'Ontario institua une commission royale d'enquête sur les questions de santé et de sécurité se rapportant à l'amiante. Une des études de recherche portait sur un relevé des attitudes des travailleurs exposés à l'amiante à l'endroit de la santé industrielle. Présentation des résultats d'une nouvelle analyse des données de cette étude - 4 tableaux.

\section{BRODY, Bernard ROHAN, Paul} ROMPRÉ, Louise

Les accidents industriels au Canada: Le portrait d'une décennie

v. 40 , no 3,1985 , p. $545-566$

Dans la décennie 1971-1980, les accidents industriels au Canada ont connu une très forte croissance en dépit des nombreuses interventions gouvernementales dans le domaine de la santé et de la sécurité du travail. Pareillement, les coûts totaux ont quadruplé en dollars courants. Analyse de ce phénomène du point de vue de l'incidence quantitative et de divers aspects économiques - 2 tableaux, 5 graphiques, bibliographie.

\section{SUBBARAO, A.V.}

Impasse Choice in the Canadian Federal Service: An Innovation and an Intrigue

v. 40 , no 3,1985 , p. $567-590$

Dans un jugement prononcé en 1982, la Cour suprême du Canada a décidé que la Commission des relations du travail dans la fonction publique, organisme administratif, n'est autorisée à fixer ni les modalités des services essentiels, ni le nombre de salariés pour les 
Relations Industrielles, vol. 45, no 4 (1990)

assurer. Les effets de ce jugement sur le règlement des conflits d'intérêt relatifs aux services essentiels dans la Fonction publique fédérale.

0411 SASS, Robert The Saskatchewan Trade Union Act. 1983: The Public Battie.

v. 40, no 3,1985 , p. $591-622$

En 1983, le gouvernement du parti progressiste conservateur de la Saskatchewan adoptait un projet de loi qui modifiait la loi sur les syndicats. La Fédération du travail de Saskatchewan et ses syndicats affiliés critiquèrent ces modifications qu'ils taxaient d'anti-syndicalisme. Les réactions et la lutte des milieux syndicaux contre ces nouvelles modifications à la loi.

0412 CÔTÉ, André C.

L'Acte des manufactures de Québec, 1885: Un centenaire

v. 40, no 3,1985, p. $623-628$

II y a cent ans était sanctionnée la première grande loi québécoise du travail, l'Acte pour protéger la vie et la santé des personnes employées dans les manufactures, autrement désignée sous son titre abrégé comme l'Acte des manufactures de Québec, 1885. Résumé du contenu de cette loi.

0413 MORIN, Fernand

Rapports collectifs du travail dans les secteurs publics québécois ou le nouvel équilibre selon la loi du 19 juin 1985.

v. 40, no 3,1985, p. $629-645$

L'adoption de la "Loi sur le régime de négociation des conventions collectives dans les secteurs public et parapublic" en 1985 concrétisait la réforme du régime annoncée depuis plus d'un an par le gouvernement du Québec. Analyse des principales modifications apportées au régime au sujet de la déconcentration proposée, des institutions et agents mis à contribution et de l'exercice du droit de grève.

0414 MORIN, Fernand

Le "raisonnable" déraisonnable! ou la rationalité du raisonnable

v. 40 , no 3,1985 , p. $646-658$

Dans tous les recueils de jurisprudence, on retrouve d'innombrables variations sur le thème du raisonnable et du déraisonnable. Le 22 novembre 1984 la Cour suprême du Canada rendait deux jugements tendant à réduire l'usage de ce double qualificatif marquant trop de subjectivité et conduisant parfois à l'incohérence. Rappel des questions soulevées lors de ces deux affaires. Commentaires sur la portée de ces deux jugements au sujet de l'usage du critère de la 'raisonnabilité" pour fins de contrôle judiciaire.

\section{LAMY, Francine}

Décision rendue par le Conseil canadien des relations du travail.

v. 40 , no 3,1985 , p. $659-662$

Le Conseil décide qu'un employeur contrevient aux dispositions du Code canadien du travail lorsqu'il impose des mesures disciplinaires à ses employés pour avoir refusé de fournir leurs prestations de travail conformément à la pratique antérieure dans le cadre d'une grève légale. A cette occasion, le Conseil a défini ce qui constitue une grève au sens du Code.

0416 BÉLANGER, Jacques ( $R$ )

LEMELIN, Maurice

Les negociations collectives dans les secteurs public et parapublic. Expérience québecoise et regard sur l'extérieur. Montreal, Éditions Agence d'Arc, 1984, 381 pp., ISBN 2-89022067-2, recensé par Jacques Bélanger.

v. 40, no 3,1985 , p. $675-676$

0417 BOIVIN, Jean (R)

THOMPSON, Mark SWIMMER, Gene

Conflict or Compromise : The Future of Public

Sector Industrial Relations. Montreal, Institut de recherches politiques, 1984, 476 pp., ISBN 0-88645-001-2, recensé par Jean Boivin.

v. 40, no 3,1985 , p. $676-678$

0418 GILSON, Clive H.J. (R)

BRADLEY, Keith GELB, Alan

Worker Capitalism. The New Industrial Relations. Cambridge, The MIT Press, 1983, 186 pp., ISBN 0-262-02191-0, recensé par Clive H.J. Gilson.

v. 40, no 3,1985 , p. $678-680$

0419 MATEJKO, Alexander J. (R) PINDER, Craig C.

Work Motivation. Theory, Issues and Applications. Glenview, Scott, Foresman and Corp., 
1984, 365 pp., ISBN 0-673-15799-7, recensé par Alexander J. Matejko.

v. 40, no 3,1985, p. 680

0420 VERGE, Pierre (R)

HANAMI, T. BLANPAIN, R.

Industrial Conflict Resolution in Market Econo-

mies. Deventer, Kluwer, 1984, 321 pp., ISBN

90-6544-1808, recensé par Pierre Verge.

v. 40, no 3,1985, p. $681-682$

0421 MATEJKO, Alexander J. (R)

CROCKER, Olga CHIU, Johnny Sik Leung

CHARNEY, Cyril

Quality Circles. A Guide to Participation and Productivity. Toronto, Methuen, 294 pp., ISBN 0-458-97360-2, recensé par Alexander J. Matejko.

v. 40 , no 3,1985 , p. $682-683$

0422 BOIS-BROCHU, Hélène (R)

THUROW, Lester C.

The Management Challenge. Japanese Views. Cambridge, The MIT Press, 1985, 237 pp., ISBN 0-262-20053-8, recensé par Hélène BoisBrochu.

v. 40, no 3,1985, p. $683-685$

0423 MATEJKO, Alexander J. (R)

MERCHANT, Kenneth A.

Control in Business Organization. Boston, Pitman, 1985, 161 pp., ISBN 0-273-01914-7, recensé par Alexander J. Matejko.

v. 40, no 3,1985 , p. $685-686$

0424 SIMARD, Marcel

BOUTEILLER, Dominique

LÉVESQUE, Christian

Prévention des accidents du travail: Contexte législatif québécois et efficacité organisationnelle.

v. 40 , no 4,1985 , p. $703-719$

L'ensemble des moyens pertinents pour la prévention des accidents du travail prévus dans la législation québécoise et leur capacité de réduire efficacement les accidents du travail. Résultats d'une recherche exploratoire réalisée auprès de dix établissements de l'industrie des textiles primaires portant sur les facteurs d'efficacité organisationnelle en matière de prévention - 4 tableaux

0425 MAGUN, Sunder

The Effects of Technological Changes on the Labour Market in Canada

v. 40, no 4,1985 , p. $720-746$
Analyse des effets des changements technologiques sur la situation de l'emploi par industrie et par occupation en utilisant le modèle canadien intrant-extrant. Dans quelles industries l'emploi diminue ou augmente. Dans quelles professions ou métiers l'emploi diminue ou augmente - 4 tableaux, références.

\section{JAIN, Hem C.} GILES, Anthony

Workers' Participation in Western Europe: Implications for North America.

v. 40, no 4,1985 , p. $747-774$

Étude des progrès récents de la participation ouvrière en Amérique du nord et en Europe de l'ouest en vue d'analyser les facteurs qui sont de nature à les promouvoir ou à les retarder. Contrairement à l'expérience européenne, aux États-Unis, on a réalisé peu de progrès en matière de représentation ouvrière au niveau de la direction - 1 tableau

\section{GUNDERSON, Morley}

Spline Functions Estimates of the Impact of Equal Pay Législation: The Ontario Experience v. 40, no 4,1985 , p. $775-792$

Recherche mezurant à l'aide de l'analyse de régression l'impact de la législation en matière d'égalité salariale sur la réduction de l'écart de salaires entre sexes en Ontario. L'intérêt de l'approche réside dans la spécification fonctionnelle de la variable dépendante, le différentiel de salaires entre sexes, par rapport à la législation et au temps écoulé - 2 tableaux, références.

0428 WETZEL, Kurt GALLAGHER, Daniel G. MAXEY, Charles T.

Impact of Nursing Unionism in the Hospital Industry: The Saskatchewan Experience.

v. 40 , no 4,1985 , p. $793-809$

Les perceptions que direction et syndicat se font de l'influence d'une association professionnelle d'infirmiers et d'infirmières au plan local dans les hôpitaux de la Saskatchewan où il existe un mécanisme de négociation collective centralisé en matière de soins hospitaliers. Tant du côté de la direction que de celui des syndicats, on a une perception positive de l'influence du syndicalisme au sein des institutions - 2 tableaux. 


\section{BEAUMONT, P.B.} TOWNLEY, $B$.

Non-Union American Plants in Britain: Their Employment Practices.

\section{v. 40 , no 4,1985, p. $810-825$}

Caractéristiques des pratiques en matière d'emploi communes aux entreprises non syndiquées américaines en Grande-Bretagne, particulièrement en Angleterre et en Écosse. Ces pratiques sont efficaces pour limiter le désir des employés de s'associer et peuvent être qualifiées de tactiques antisyndicales américaines exportées en Grande-Bretagne -5 tableaux.

\section{ROBAKC, Léo}

Les travailleurs du Québec au XXième siècle. v. 40 , no 4,1985, p. $826-846$

Résumé des grandes étapes du mouvement ouvrier au Québec depuis le début du siècle en les situant dans le contexte économique, social et politique de chaque période, et en soulignant quelques-uns de ses thèmes préférés.

\section{MORIN, Fernand}

La survie de droits subjectifs à la convention collective.

v. 40 , no 4,1985 , p. $847-855$

Résumé et critique d'un jugement de la cour d'appel du Québec qui a décidé qu'advenant le retour de deux contreman̂res à titre de salarié, ceux-ci disposaient encore du même droit d'ancienneté prévalant au moment de leur nomination et que les dispositions de la convention collective en vigueur au moment de leur nomination devaient encore s'appliquer, nonobstant les dispositions de l'actuelle convention collective.

\section{GILSON, Clive H.J.}

Changes in the Nature of Grievance Issues Over the Last Ten Years: Labor Management Relations and the "Frontier of Control".

v. 40 , no 4,1985 , p. $856-864$

Le contrôle total signifierait qu'un employeur peut faire ce qu'il veut et comme if le veut. La "frontière du contrôle" est vue comme une ligne changeante dans une masse de réglementations sur des sujets que la question de contrôle peut ne jamais avoir soulevée. A la lumière des changements survenus au cours des dix dernières années dans les questions matières à grief, on peut dire que la négociation du contrôle est une tendance répandue qui remet en cause les définitions des "relations industrielles" - Références.

\section{OSBERG, Lars}

A Note on the Incomes of Lawyers

v. 40 , no 4,1985 , p. $865-879$

II y a de bonnes raisons de croire que la structure du marché des services légaux n'est pas la même aux États-Unis et au Canada. En utilisant des données canadiennes, on présente les estimations des revenus moyens des avocats dans les provinces de l'Atlantique et l'impact de deux scénarios sur l'offre future des services légaux - 2 tableaux, bibliographie.

0434 LAMY, Francine

Décision rendue par le Conseil canadien des relations du travail

v. 40 , no 4,1985 , p. $880-885$

A l'occasion d'une requête en accréditation, le Conseil refuse la preuve des adhésions syndicales et de la date de versement des droits d'entrée présentée par le syndicat requérant.

\section{SIMARD, Marcel (R)}

PLANTE, Gilles

Le conflit du travail : stratégie et tactique. Collection Relations du travail, Québec, Presses de l'Université Laval, 1984, 166 pp., ISBN 2-7637-7041-X, recensé par Marcel Simard. v. 40 , no 4,1985 , p. $891-892$

\section{BEAULIEU, Paul $(R)$}

DUPONT, Christophe

La négociation. Conduite, théorie, application.

Paris, Dalloz, 1982, 276 pp., ISBN 2-247$00315-X$ recensé par Paul Beaulieu.

v. 40 , no 4,1985, p. $892-893$

0437 SEXTON, Jean (R)

LEWICKI, Roy J. LJTTERER, Joseph A.

Negotiation. Homewood, Richard D. Irwin, 1985, 368 pp., ISBN 0-256-02633-5, recensé par Jean Sexton.

v. 40, no 4,1985, p. $894-895$

0438 FERLAND, Gilles ( $R$ )

LEWICKI, Roy J. LITTERER, Joseph A.

Negotiation : Readings, Exercices and Cases. 
Homewood, Richard D. Inwin, 1985, 633 pp., ISBN 0-256-02634-3, recensé par Gilles Ferland.

v. 40, no 4,1985 , p. 895

0439 MATEJKO, Alexander J. (R)

LANDY, Frank J.

Psychology of Work Behavior. Homewood, The Dorsey Press, 1985, XIV-622-R41-11 pp., ISBN 0-256-03046-4, recensé par Alexander J. Matejko.

v. 40 , no 4,1985 , p. $896-898$

0440 TESSIER, Paul (R)

BEAUDOIN, Pierre

La gestion par projet: aspects stratégiques. Montréal, Agence d'Arc, 1984, 251 pp., ISBN 2-89-22-065-6, recensé par Paul Tessier.

v. 40 , no 4,1985 , p. $898-899$

0441 TURNER, Michel (R)

KATZ, Harry C.

Shifting Gears : Changing Labor Relations in the U.S. Automobile Industry. London, MIT Press, 1985, 230 pp., ISBN 0-262-11098-9, recensé par Michel Turner.

v. 40 , no 4,1985 , p. $900-901$

0442 CHRÉTIEN, Lise (R)

Sécurité sociale, chômage et retraite anticipée. Association internationale de la securité sociale, Etudes et recherches no 22, Genève, 1985, 191 pp., ISBN 92-843-2021-6, recensé par Lise Chrétien.

v. 40 , no 4,1985, p. $902-903$

0443 THOMPSON, Mark (R)

BEAN, $R$.

Comparative Industrial Relations : An Introduction to Cross National Perspectives. London, Crom Helm, 1985, 261 pp., ISBN 0-7099-32519 recense par Mark Thompson.

v. 40, no 4,1985 , p. $903-904$

0444 CHRÉTIEN, Lise ( $\mathrm{R}$ )

GRAIS, Bernard

Les mises à pied et le travail à temps réduit dans quelques pays de l'OCDE. Paris, Institut national de la statistique et des études économiques et Conclusions du groupe de travail de l'OCDE sur les statistiques de l'emploi et du chomage, 1983, 154 pp., ISBN 92-6422391-6, recensé par Lise Chrétien.

v. 40 , no 4,1985 , p. $904-905$
0445 LARSON, Peter E. (R)

INGERMAN, Sidney $\mathrm{H}$.

Economics for Canadian Trade Unionists. 2nd Ed., Ottawa, Labour College of Canada, 1985, 208 pp., ISBN 0-969-0345-1-1, recensé par Peter Larson.

v. 40, no 4,1985 , p. 906

0446 MORIN, Fernand (R)

VERGE, Pierre

Le droit de grève, fondements et limites. Chicoutimi, Editions Yvon Blais, 1985, 229 pp., ISBN 2-89073-553-2, recensé par fernand Morin.

v. 40 , no 4,1985 , p. $907-910$

0447 BARKIN, Solomon

The Current Unilateralist Counterattack on Unionism and Collective Bargaining

v. 41, no 1, 1986, p. 3-27

La tendance au conservatisme en politique a encouragé les groupements antisyndicaux à affaiblir ou à détruire les mouvements syndicaux et les partis ouvriers dans plusieurs pays occidentaux en rétablissant l'autorité unilatérale des employeurs par le processus de contrôle des prises de décision. Analyse des développements et des contenus des nouvelles politiques en matière de relations du travail.

0448 BÉLANGER, Jacques MERCIER, Jacques

Le plafonnement de la densité syndicale au Québec et au Canada.

v. 41 , no 1,1986 , p. $28-52$

Le choix des données retenues pour mesurer la densité syndicale. Les effets des modifications structurelles dans l'emploi et la main-d'oeuvre et des facteurs d'ordre institutionnel sur la densité syndicale. Rapport entre certaines caractéristiques du régime des relations du travail et le phénomène de saturation de la syndicalisation. Les implications pour l'avenir de la syndicalisation - 5 tableaux, références.

0449 MAKI, Dennis R.

MEREDITH, Lindsay N.

The Effects of Unions on Profitability: Canadian Evidence

v. 41 , no 1,1986 , p. $54-68$

En utilisant les statistiques provenant de vingt grandes industries manufacturières compilées 
pendant la période 1971-1981, on analyse l'influence des syndicats sur le niveau des profits et le taux de rendement sur le capital, les ventes et les actifs. Les constatations tendent vers un effet négatif de la syndicalisation sur les profits - 3 tableaux, références.

\section{RONDEAU, Claude}

BADIN, François

Le contenu non salarial des conventions collectives dans les industries manufacturières au Québec.

v. 41 , no 1,1986 , p. $69-90$

Les études sur le contenu non salarial des conventions collectives sont peu nombreuses. Pour le Québec, nous disposons de travaux sur les clauses de conventions collectives dans certains domaines spécifiques comme la protection du revenu, le changement technologique et les clauses de sous-traitance. L'objet de l'étude est d'étendre l'analyse à l'ensemble des clauses non salariales. Les données proviennent de 2192 conventions collectives signées au Québec - 3 tableaux, références.

\section{DORNSTEIN, Miriam ZOREF, Uri}

Motivation for Changing Jobs, Personal Background Characteristics and Perceived Opportunity

v. 41 , no 1,1986, p. $91-110$

A partir d'un échantillon de 159 travailleurs engagés dans un processus de mutation d'emploi d'une entreprise à une autre ou d'une occupation à une autre, on étudie deux questions fondamentales: a) La relation entre les caractéristiques personnelles antérieures et le roulement de la main-d'oeuvre; b) le rôle des occasions d'emploi en perspective dans le roulement lui-même - 2 tableaux, références.

\section{LACROIX, Robert}

A Microeconometric Analysis of the Effects of Strikes on Wages

v. 41 , no 1,1986 , p. 111-127

On remet en cause l'hypothèse d'une relation positive entre les grèves et les ententes salariales. En partant de trois modèles largement reconnus, on tente d'inférer quelle serait la conséquence d'une grève sur les salaires. On découvre que, dépendant du modèle rete- nu, on peut aussi bien inférer un effet positif ou négatif que nul de la grève sur l'entente salariale - 4 figures, 2 tableaux, références.

0453 THACKER, James $W$. ROSEN, Hjalmar

Dynamics of Employee Reactance to Company and Union Dual Allegiance Revisited and Expanded.

v. 41 , no 1,1986, p. $128-144$

L'avènement des efforts de coopération entre les syndicats et les employeurs a ranimé l'intérêt pour la notion de "double allégeance". La présente recherche décrit les types d'engagement envers le syndicat et l'employeur dans un cadre à quatre volets: 1) Double engagement, 2) double rejet, 3) engagement unilatéral envers l'entreprise, 4) engagement unilatéral envers le syndicat. Les résultats n'indiquent aucune réciprocité significative entre la loyauté à l'employeur et au syndicat - 2 tableaux, 2 figures, références.

\section{LAMSON, Cynthia}

On the Line: Women and Fish Plant Jobs in Atlantic Canada.

v. 41 , no 1,1986 , p. $145-156$

Quand on parle des difficultés financières des grandes usines de poissons ou de fruits de mer des provinces Maritimes, on fait référence au nombre d'emplois perdus mais rarement à ceux qui perdront leur emploi. Un grand nombre de femmes travaillent sur les lignes de production de ces usines mais on ne connait pas les caractéristiques de ces femmes. Analyse des résultats d'une étude pilote effectuée en 1983 et venant combler le manque d'information sur ce sujet - Références.

\section{AOUST, Claude d' SAINT-JEAN, Sylvain TRUDEAU, Gilles \\ L'obligation de civilité du salarié \\ v. 41 , no 1,1986, p. $157-180$}

L'étude a pour but de mettre en évidence les relations interpersonnelles qui naissent à l'occasion de l'exécution du travail et de montrer à quelles normes les parties au contrat de travail doivent se conformer dans ces relations. La violation de ces normes peut entraîner des sanctions de la part du groupe social lui-même (rejet de l'individu par ses compagnons de travail) ou de la part de l'em- 
ployeur. Examen de certaines décisions judiciaires ou sentences arbitrales relatives à ce type de problème.

0456 GOSSELIN, Louis (R)

Entreprises d'intérét collectif et création d'emplois. Initiatives locales de création d'emplois, OCDE, Paris, 1984, 77 pp., ISBN 92-64-225692 , recensé par Louis Gosselin.

v. 41 , no 1,1986, p. $189-190$

0457 WEISS, Dimitri (R)

DESPAX, Michel PÉLISSIER, Jean

La gestion du personnel : aspects juridiques. Tome 1, 2ième éd., Paris, Éoitions Cujas, 1984, 400 pp., ISBN 2-2548-4031-0, recensé par Dimitri Weiss.

v. 41 , no 1,1986, p. $190-191$

0458 DÉOM, Esther (R)

RICHARDSON, Reed C.

Collective Bargaining by Objectives : A Positive Approach. 2nd ed., New Jersey, PrenticeHall, 1985, 325 pp., ISBN 0-13-140476-8-01, recensé par Esther Déom.

v. 41 , no 1, 1986, p. 191-192

0459 WEISS, Dimitri (R)

PACAULT, Emile

La fin du chómage par le partage du travail. Edition établie avec introduction, notes et index par Maurice Lebel. Montréal, Éditions Paulines, 1985, 118 pp., ISBN 2-89039-046-2, recensé par Dimitri Weiss.

v. 41 , no 1, 1986, p. 193-196

0460 VERGE, Pierre (R)

ZISKIND, David

Concerning Human Aspirations - Essays in comparative Labor Law. Los Angeles, The Litlaw Foundation, 1985, 172 pp., ISBN 0-9615761-1-1, recensé par Pierre Verge.

v. 41 , no 1, 1986, p. 196-197

0461 VERGE, Pierre (R)

ARTHURS, H.W. CARTER, D.D.

GLASBEEK, H.J.

Labour Law and Industrial Relations in Canada. 2nd ed, Toronto, Butterworths, 1984, 316 pp., ISBN 0-409-81185-8, reçensé par Pierre Verge.

v. 41 , no 1,1986 , p. $197-198$

0462 WEISS, Dimitri (R)

SCIARRA, Silvana

Contratto collettivo e contrattazione in azienda. Milano, Franco Angeli, 1985, 226 pp., recensé par Dimitri Weiss.

v. 41 , no 1,1986 , p. $198-200$

0463 SIMONIS, Yvan (R)

PLANTE, Gilles

Le conflit du travail : stratégie et tactique. Collection Relations du travail, Québec, Presses de l'Université Laval, 1984, 166 pp., ISBN 2-7637-7041-X, recensé par Yvan Simonis.

v. 41, no 1,1986 , p. $200-202$

0464 GRIEZIC, F.J.K. (R)

LEAB, Daniel J.

The Labor History Reader. Champaign, University of Illinois Press, 1985, 470 pp., ISBN O252-01197-X, recensé par Foster J.K. Griezic. v. 41 , no 1,1986 , p. $202-203$

0465 STODDART, Jennifer (R)

BRETON, Albert

Le mariage, la population et le taux d'activité des femmes. Conseil économique du Canada, Ottawa, ministère des Approvisionnements et Services Canada, 1983, 36 pp., ISBN O660-91184-1, recensé par Jennifer Stoddart. v. 41 , no 1,1986, p. $203-204$

\section{BROSSARD, Michel SIMARD, Marcel}

Problématique de la différenciation de la main-d'oeuvre et changement organisationnel.

v. 41 , no $2,1986, p, 219-235$

Pour rendre compte des différences observées dans les positions adoptées par les travailleurs vivant un changement organisationnel consistant à aménager le travail en groupe semi-autonome, il faut tenir compte du jeu complexe de facteurs caractérisant le contexte organisationnel, l'organisation du travail et aussi de facteurs d'ordre personnel. Résultats d'une recherche portant sur la réorganisation du travail d'un centre de distribution de produits alimentaires selon la formule des groupes semi-autonomes de travail - 5 tableaux.

\section{KUMAR, Pradeep}

DOW, Bradley

Econometric Analysis of Union Membership Growth in Canada, 1935-1981

v. 41, no 2,1986, p. 236-255

On étudie les déterminants empiriques principaux du développement global des effectifs syndicaux au Canada. On propose un modèle de la progression des syndicats fondé 
Relations Imdustrielles, vol. 45, no 4 (1990)

sur le dynamisme de deux modèles bien connus et on incorpore à ce modèle plusieurs hypothèses rattachant la croissance syndicale aux indicateurs économiques, à l'état de la législation et aux changements de la composition de la main-d'oeuvre - 1 tableau, références.

0468 ROSE, Joseph B. WETZEL, Kurt

Outcomes of Bargaining Structures in the Ontario and Saskatchewan Construction Industries.

v. 41 , no 2,1986 , p. $256-280$

Les effets des structures de négociation dans l'industrie de la construction en Ontario et en Saskatchewan sur l'activité de grève et sur les ententes salariales et non salariales négociées. Comparaison des expériences antérieures et postérieures à l'établissement de structure de négociations provinciales obligatoires. Comment la redéfinition des structures de négociation a influencé les organisations patronales et syndicales - 5 tableaux

0469 KNIGHT, Thomas R.

Correlates of Informal Grievance Resolution Among Fist-Line Supervisors

v. 41 , no 2,1986 , p. $281-298$

L'expérience et la formation des agents de maîtrise (contremaîre) influencent d'une façon importante le pourcentage de règlements informels des griefs et donc, l'efficacité du processus des réclamations. De même, le type de relations entre les contremaitres et les représentants syndicaux et patronaux peuvent influencer ce pourcentage - 4 tableaux, références.

0470 MERCIER, Jean

L'informatique, ses "filtres" et ses effets.

v. 41 , no 2,1986 , p. $299-316$

Même si on peut soutenir que la technologie informatique incitera, à long terme, les organisations productrices de biens et services a adopter des formules de gestion plus souples et plus décentralisées, certains facteurs individuels, organisationnels et culturels (qu'on appelle "filtres") peuvent retarder ou inhiber l'apparition de ces nouvelles formules de gestion.

\section{BEACH, Charles $M$. \\ KALISKI, S.F.}

The Impact of Recession on the Distribution of Annual Unemployment.

v. 41 , no 2,1986 , p. $317-328$

La longueur moyenne des périodes de chômage fut plus courte en 1980 alors que le taux de chômage était de $7.5 \%$, et plus prononcée en 1982 alors que le taux de chômage était de $11 \%$. Afin de mieux interpréter l'impact de la récession de 1981-82 au Canada sur la concentration ou le poids relatif $d u$ chômage, on présente un ensemble de comparaisons cycliques fondé sur la distribution totale des périodes de chômage - 1 figure, 5 tableaux, références.

\section{RESHEF, Yonatan} FRIED, Yatzhak

Strikes in Israell: The Histadrut in a Sectorial Perspective, 1965-1982

v. 41 , no 2,1986 , p. $329-347$

Israël est probablement la seule démocratie industrialisée dont l'économie soit fondée sur trois grands secteurs: le secteur public, le secteur privé et le troisième secteur qui appartient à l'Histadrut (La Fédération générale du travail d'Israël). Le recours à la grève parmi les travailleurs des entreprises de I'Histadrut est compare aux conflits de travail des autres secteurs pour vérifier s'il y a quelque différence de principe concernant les moyens de grève que chaque secteur choisit - 3 figures, 3 tableaux, références.

\section{AOUST, Claude d'}

Le controle judiciaire des decisions arbitrales: Reflexions sur les moyens d'y echapper.

v. 41 , no 2 , 1986, p. 348-356

Le contrôle judiciaire des décisions des tribunaux inférieurs, notamment des tribunaux d'arbitrage, a fait couler beaucoup d'encre au cours des dernières années. On cherche ici a répondre à la question posée par le professeur Fernand Morin dans le volume 40 , no 3 de cette revue, à savoir: Comment le caractère raisonnable d'une décision peut-il servir de critère déterminant pour l'exercice du contrôle judiciaire?

0474 DICKINSON, John A.

La législation et les travailleurs québécois, 1894-1914

v. 41, no 2,1986, p. $357-381$ 
C'est entre 1894 et 1914 que les législateurs canadiens et québécois commencent enfin à prendre conscience des problèmes engendrés par l'industrialisation et à établir un système législatif capable d'encadrer la croissance industrielle. Analyse des lois fédérales et provinciales, et des principaux règlements municipaux des villes de Québec et de Montréal, visant à protéger les travailleurs québécois et canadiens - 4 tableaux.

0475 PROULX, Pierre-Paul

Maîtriser la technologie: pourquoi, quelles technologies, comment?

v. 41 , no 2,1986, p. $382-389$

Les industries électroniques ont généré en 1984 environ 82,000 emplois dans le secteur des ordinateurs, 4,000 dans le secteur des logiciels et 22,000 emplois indirects au Canada. "Domestiquer" les nouvelles technologies signifie qu'il faut les apporter et les développer au pays. II ne faut pas simplement développer au pays. II ne faut pas simplement s'adapter de façon passive à ces technologies envahissantes mais plutôt développer des buts, des processus et des politiques afin de les maîtriser.

0476 GILSON, Clive H.J.

Bargaining Exercises: Beyond Simulation, a Touch of Reality.

v. 41, no 2, 1986 , p. 390-396

Les exercices de simulation de négociation collective constituent pour les étudiants universitaires une expérience de formation qui fait le lien entre la théorie et la pratique, entre l'apprentissage académique et le monde du travail. Quelques suggestions sur les méthodes à utiliser pour que ces exercices de simulation se rapprochent davantage de la réalité - Références.

0477 SAINT-GERMAIN, Catherine Décisions rendues par le Conseil canadien des relations du travail.

v. 41 , no 2,1986, p. $397-411$

Résumé de deux décisions du Conseil: 1) Le Conseil rend une décision sans précédent en accordant une accréditation visant tous les employées de toutes les succursales de la Banque Nationale de Rimouski. 2) Le Conseil établit un autre précédent dans le secteur bancaire en réglant les modalités de la pre- mière convention collective entre la Banque de commerce canadienne impériale et le Syndicat des employés de banque.

\section{BARKIN, Solomon (R)}

ROBERTS, B.C.

Industrial Relations in Europe. The Imperatives of Change. Dover, New Hampshire, Crown Holm, 1985, 277 pp., recensé par Solomon Barkin.

v. 41 , no 2,1986, p. $417-422$

0479 JOUBERT, P. (R)

WEISS, Dimitri

"La participation". Enciclopedia de Dirreccion y Administracion de la Empresa, Fasciculo 82, Barcelona, Ediciones Orbis, 1985, ISBN (fascicule) 84-7530-585-7; (oeuvre complète) 847530-583-0; (volume VI) 84-7634-230-6, recensé par $P$. Joubert.

v. 41 , no 2,1986 , p. $423-424$

0480 MATEJKO, Alexander J. (R)

THOMPSON, Kenneth

Work, Employment and Unemployment. Perspectives on Work and Society. Milton Keynes, Open University Press, 1984, 280 pp., ISBN 03-3510-5947, recensé par Alexander J. Matejko.

v. 41 , no 2,1986 , p. $425-426$

0481 PAQUIN, Michel (R)

BEAUMONT, P.B. PARTRIDGE, M.

Job Satisfaction in Public Administration. Londres, Royal Institute of Public Administration, 1983, 41 pp., ISBN 0-900628-32-4, recensé par Michel Paquin.

v. 41 , no 2,1986 , p. $426-427$

\section{GILES, Anthony (R)}

SHRAGGE, Eric

Pensions Policy in Britain : A Socialist Analysis. London, Routledge \& Kegan Paul, 1984, 194 pp., ISBN 0-7100-9842-1, recensé par Anthony Giles.

v. 41 , no 2,1986 , p. $428-430$

0483 MATEJKO, Alexander J. (R)

MARS, Gerald

Cheats at Work. An Anthropology of Workplace Crime. London, Unwin Paperbacks, 1983, 242 pp., ISBN 0-04-301166-7, recensé par Alexander J. Matejko.

v. 41 , no 2,1986 , p. $431-432$ 
0484 SASS, Robert (R)

WELLS, Don

Soft Sell : "Quality of Working Life" Programs and the Productivity Race. Canadian Centre for Policy Alternatives, 150 pp., ISBN 088627040-5, recensé par Robert Sass.

v. 41 , no 2,1986 , p. $432-433$

0485 HURTUBISE, Rolland (R)

B.I.T.

Technological Change : The Tripartite Response, 1982-85. Genève, Bureau international du Travail, 1985, 355 pp., ISBN 92-2-1051625 , recensé par Rolland Hurtubise.

v. 41 , no 2,1986 , p. $433-435$

0486 COUSINEAU, Jean-Michel

Objectifs et modalités de l'assurance-chômage au Canada: 1940-1986

v. 41 , no 3,1986 , p. $451-468$

Historique du programme d'assurancechômage canadien né en 1940. Les caractéristiques du programme tel que nous pouvons l'observer dans sa forme actuelle et l'évolution des objectifs prioritaires du programme à partir de l'analyse de la dynamique de ses modalités à travers le temps - 5 tableaux, bibliographie.

\section{WEIERMAIR, Klaus}

Secular Changes in Youth Labour Markets and Youth Unemployment in Canada.

v. 41 , no 3,1986 , p. $469-490$

L'évolution du chômage chez les jeunes Canadiens et modèles de comportement s'y rapportant sur les marchés du travail de ce groupe de salariés lorsqu'on les considère dans une perspective de longue durée. Les nombreuses hypothèses concourantes du chômage chez les jeunes. Les principales causes du chômage chez les jeunes. Analyse empirique des changements de longue durée dans les taux de chômage des jeunes- 3 graphiques, 2 tableaux, bibliographie.

\section{SOLOMON, Norman A.}

ANDIAPPAN, P. SHAND, Dan

Canadian National Union Presidents: An Empirical Study.

v. 41 , no 3,1986 , p. 491-504

On analyse dans quelle mesure divers facteurs influencent le temps nécessaire à un membre d'un bureau syndical pour accéder au poste de président d'un syndicat national au Canada. Au moyen d'une analyse de régression multiple, on vérifie un modèle fondé tant sur les caractéristiques personnelles des dirigeants syndicaux que celles des syndicats qu'ils administrent - 3 tableaux.

0489 GODIN, Jean

$$
\text { LE LOUARN, Jean-Yves }
$$

Les mentors ont-ils un effet sur la progression de carrière?

v. 41 , no 3,1986, p. $505-518$

Le mentor est une personne cadre située à un niveau hiérarchique élevé qui prend un intérêt personnel dans le développement de la carrière d'une personne ayant un rang inférieur dans la même organisation ou dans une autre. A partir des réponses à un questionnaire, on vérifie la relation entre le "mentorship" et la progression de carrière, plus précisément le nombre de promotions de l'individu en carrière - 1 tableau, références.

0490 WILLIAMS, C. Brian

International Trade Unionism: The United Mine Workers in Eastern Canada, 1900-1920

v. 41 , no 3,1986 , p. $519-540$

Le caractère international du mouvement syndical canadien est non seulement une particularité de son histoire et de son évolution, mais c'est aussi un cas unique à l'intérieur de la grande collectivité du syndicalisme. Historique des activités des Mineurs unis d'Amérique dans l'est du Canada au cours de la période 1900 à 1920.

\section{BURT, Sandra}

Voluntary Affirmative Action. Does it Work? v. 41, no 3,1986 , p. 541-551

Résultats d'une enquête effectuée auprès d'entreprises des villes jumelées Kitchener-Waterloo en Ontario et portant sur la promotion sociale du personnel féminin . Dans la plupart des entreprises, on décèle la présence de ségrégation sexuelle: concentration de femmes dans le travail de bureau et d'hommes dans les emplois de production et de direction. Peu d'employeurs comprennent la notion de promotion sociale ce qui remet en question l'approche volontaire en ce domaine- 6 tableaux. 
0492 TANG, Roger Y.W. PONAK, Allen

Employer Assessment of Strike Costs. v. 41 , no 3,1986 , p. $552-571$

Résultats d'une enquête effectuée auprès d'entreprises canadiennes sur l'évaluation, par les employeurs, des coûts pécuniaires et non pécuniaires reliés à une grève ou à un loc$k$-out. Les données furent recueillies en 1980 et l'échantillon comprenait des entreprises manufacturières et minières ayant subi une grève ou un lock-out impliquant plus de 100 travailleurs dans les deux années précédentes - 4 tableaux, références.

0493 EVANS, Martin G. ONDRACK, Daniel A.

The Effect of Unionization on Wages: Some Canadian Evidence

v. 41 , no 3,1986, p. $572-577$

Résultats d'une étude qui confirme les découvertes des autres chercheurs à savoir que, dans la plupart des cas, l'effet de la syndicalisation est d'accroître les niveaux de salaires et de réduire les différences salariales. Cependant, les effets observés dans l'industrie pétrochimique sont quelque peu différents: les variances des deux paramètres se rétrécissent; les employés non-syndiqués ont des salaires supérieurs - Références

0494 MORIN, Fernand

Le rationnel et le raisonnable: Deux nécessités distinctes et conjugées en droit.

v. 41 , no 3,1986, p. $578-582$

En 1985, la Cour suprême du Canada retenait le critère du raisonnable pour justifier et, du même coup, limiter le contrôle judiciaire des sentences arbitrales. On cherche à justifier l'utilisation de ce critère et à mieux connaître sa portée exacte et si possible, savoir comment on reconnait ou distingue une décision raisonnable d'une décision déraisonnable.

\section{GEORGE, Kenneth}

L'accès à la syndicalisation

v. 41, no 3,1986 , p. $583-595$

Revue de la littérature scientifique québécoise sur le problème de l'accès à la syndicalisation. Les universitaires ont attiré l'attention sur quatre types de variables qui pourraient avoir un impact sur l'accès à la syndicalisation: 1) la législation et les mécanismes institutionnels, 2) les caractéristiques et les attitudes des travailleurs, 3) le contexte économique et 4) les caractéristiques de l'entreprise -2 tableaux.

\section{BEMMELS, Brian FISHER, E.G. NYLAND, Barbara}

Canadian-American Jurisprudence on "Good Faith' Bargaining.

v. 41 , no 3,1986 , p. $596-621$

Analyse comparative de la jurisprudence canadienne et américaine établie sur la négociation de bonne foi. L'analyse porte d'abord sur les infractions à l'obligation légale de négocier de bonne foi, mais traite aussi d'autres questions concernant la négociation collective, comme les droits des grévistes, par exemple - Bibliographie.

\section{SAINT-GERMAIN, Catherine}

Décisions rendues par le Conseil canadien des relations du travail

v. 41, no 3,1986, p. $622-637$

Résumé de deux décisions du Conseil: 1) Le Conseil exempte un travailleur de l'obligation d'appartenance à un syndicat et du versement de la cotisation syndicale en raison de convictions religieuses. 2) Le Conseil condamne un employeur pour refus de négocier de bonne foi et pour pratiques déloyales en période de renouvellement de la convention collective.

0498 CRAIG, Alton W.J. (R)

DION, Gérard

Dictionnaire canadien des relations du travail. 2ième éd., Québec, Les Presses de l'Université Laval, 1986, 993 pp., ISBN 2-7637-69756 , recensé par Alton W.J. Craig.

v. 41 , no 3,1986 , p. $644-646$

0499 HÉBERT, Gérard ( $\mathrm{A}$ )

DION, Gérard

Dictionnaire canadien des relations du travail. 2ième éd., Québec, Les Presses de I'Université Laval, 1986, 993 pp., ISBN 2-7637-69756 , recensé par Gérard Hébert. v. 41 , no 3,1986 , p. $646-650$

0500 VERGE, Pierre (R)

GOULD, W.B.

Strikes, Disputes Procedures and Arbitration Essays on Labor Law. Westport, Greenwood 
Press, 1985, 313 pp., ISBN 0-313-24468-5, recensé par Pierre Verge.

v. 41 , no 3,1986, p. $650-651$

0501 BEAULIEU, Paul (R)

B.I.T.

Collective Bargaining : A Response to the Recession in Industrialised Market Economy Countries. Genève, Bureau international du Travail, 1984, 275 pp., ISBN 92-20103628-6, recensé par Paul Beaulieu.

v. 41, no 3, 1986, p. 651-652

0502 DÉOM, Esther (R)

WHEELER, Hoyt N.

Industrial Conflict. An Integrative Theory. Columbia, University of South Carolina Press, 1985, 293 pp., ISBN 0-87249-459-4, recensé par Esther Déom.

v. 41 , no 3,1986, p. $652-654$

0503 BARKIN, Solomon (R)

KATZ, Harry C.

Shifting Gears : Changing Labor Relations in the U.S. Automobile Industry. Cambridge, MIT Press, 1985, 230 pp., ISBN 0-262-11098-9, recensé par Solomon Barkin.

v. 41 , no 3,1986 , p. $654-658$

0504 BLOUIN, Rodrigue (R)

Index et résumés de sentences arbitrales de griefs. Tome II, Montréal, Coplanam, 1985, ISBN 2-920391-03-8, recensé par Rodrigue Blouin.

V. 41 , no 3,1986, p. $658-659$

0505 VERGE, Pierre (R)

ADAMS, G.W.

Canadian Labour Law - A Comprehensive Text. Aurora, Canada Law Book Inc., 1985, 983 pp., ISBN 0-888-04-030-X, recensé par Pierre Verge.

v. 41 , no 3,1986 , p. $659-661$

0506 MATEJKO, Alexander J. (R)

ALSTOM, Jon P.

The American Samurai. Blending American and Japanese Managerial Practices. BerlinNew York, Walter de Gruyter, 1986, 369 pp., ISBN 0-89925-063-7, recensé par Alexander J. Matejko.

v. 41 , no 3,1986, p. $662-664$

0507 GILES, Anthony (R)

OFFE, Claus

Contradictions of the Welfare State. Edited and introduced by John Keane, Cambridge, MIT Press, 1984, 310 pp., ISBN 0-262-650142 , recense par Anthony Giles.

v. 41 , no 3, 1986, p. 664-667
0508 MATEJKO, Alexander J. (R)

LUFT, Joseph

Group Process. An Introduction to Group Dynamics. 3rd ed., Alto, Cal., Mayfield Publishing Company, 1984, 237 pp., ISBN 0-87484542-4, recensé par Alexander J. Matejko.

v. 41 , no 3,1986 , p. 667

0509 MATEJKO, Alexander J. (R)

BERNSTEIN, Paul

Workplace Democratization. Its Internal Dyna mics. New Brunswick, Transaction Books, 1983, 133 pp., ISBN 0-87855-711-3, recensé par Alexander J. Matejko.

v. 41 , no 3,1986 , p. $667-668$

0510 GAGNON, Jacques (R) TRUDEAU, Pierre Elliott

La grève de l'amiante. Montréal, Editions du Jour, 1970, 430 pp., recensé par Jacques Gagnon.

v. 41, no 3,1986, p. $669-670$

0511 GAGNON, Jacques (R)

MEHLING, Jean

Analyse socio-économoique d'une grève. Montréal, HEC et Beauchemin, 1963, 218 pp., recensé par Jacques Gagnon.

v. 41 , no 3,1986 , p. $670-671$

0512 GAGNON, Jacques (R)

PRATT, Michel

La grève à la United Aircraft. Montréal, Les Presses de I'Université du Québec, 1980, 115 pp., recensé par Jacques Gagnon.

v. 41 , no 3,1986, p. $671-672$

0513 FINKELMAN, Jacob

Public Sector Collective Bargaining

v. 41 , no 4, 1986, p. 691-703

Origines et développement de la négociation dans le secteur public au Canada. Les aspects particuliers du secteur public en ce qui concerne la détermination des unités de négociation, la définition de ce qui est négociable, les principaux problèmes rencontrés et les façons de les résoudre, la détermination des services essentiels. A la lumière de quarante années d'expérience, on peut conclure que ce fut une erreur d'accorder le droit de grève dans le secteur public.

0514 BÉLANGER, Jacques

Le contróle ouvrier sur l'organisation du travail: Étude de cas en Grande-Bretagne.

v. 41 , no 4,1986 , p. $704-719$ 
Sur la base d'une recherche dans deux entreprises de l'industrie mécanique, on montre le degré de contrôle exercé par les ouvriers de la production sur l'organisation et l'exécution de leur travail, malgré la réforme des relations du travail. On analyse principalement l'intéraction des facteurs techniques et sociaux pouvant expliquer ce type de régulation sociale - Références.

\section{CHAYKOWSKI, Richard P.}

\section{SLOTSVE, G.A.}

Union Seniority Rules as a Determinant of Intra-Firm Job-Changes.

v. 41 , no 4,1986, p. $720-737$

Observation du processus des changements d'occupation dans une entreprise déterminée, en utilisant une grille d'analyse qui inclut tous les changements d'occupation qui peuvent se produire. Principalement, on examine les effets de l'ancienneté dans les caractérisations des changements d'occupation selon les modèles strictement définis: changements d'occupation avec augmentation du salaire, sans modification du salaire et avec diminution du salaire - 2 tableaux, références.

\section{DIMMOCK, Stuart J.}

SETHI, Amarjit $S$.

The Role of Ideology and Power in Systems Theory: Some Fundamental Shortcomings.

v. 41 , no 4,1986, p. $738-757$

Les agissements des employeurs et des gouvernements contre les syndicats depuis le début de la décennie 1980 aux États-Unis (mais aussi au Canada et en Grande-Bretagne) ont mis en doute la valeur de la théorie des systèmes de John Dunlop. La principale faiblesse de la théorie des systèmes est qu'elle conçoit mal l'idéologie et le pouvoir, ce qui restreint fondamentalement sa capacité d'expliquer les évènements récents dans le domaine des relations du travail. Comparaison avec d'autres théories dites traditionnelles - Bibliographie.

\section{JAIN, Harish C.} ANDIAPPAN, $P$.

Sexual Harassment in Employment in Canada: Issues and Policies.

v. 41 , no 4,1986, p. $758-777$

Toutes les législatures provinciales, de même que le Parlement canadien, ont adopté des lois sur les droits des personnes, lois qui interdisent la discrimination sexuelle et autres formes de discrimination en matière d'emploi, incluant le harcèlement sexuel. Analyse de toutes les affaires judiciaires qui ont eu lieu au Canada entre 1980 et 1984 concernant les attitudes discriminatoires qui furent considérées comme du harcèlement sexuel. Nécessité d'une politique préventive efficace en cefte matière - 4 tableaux.

\section{FISHER, E.G.} KUSHNER, Stephen

Alberta's Construction Labour Relations During the Recent Downturn

v. 41 , no 4,1986, p. $778-801$

Description et analyse, en ce qui a trait aux relations professionnelles, de la transformation rapide de lindustrie de la construction en Alberta pendant la dernière récession. De 1975 à 1982, 70 à 80 pour cent des chantiers étaient syndiqués et régis par des conventions collectives, alors qu'au début de 1984, il n'y en avait plus que 5 à 10 pour cent. Les facteurs qui ont concouru à ce phénomène dont la rapidité est sans précédent - 4 tableaux.

0519 BETCHERMAN, Gordon

Labour Market Imbalances in Canada, 1966-1983

v. 41 , no 4,1986, p. $802-816$

Les statistiques des vacances d'emploi furent colligées au Canada de 1971 à 1978 et sont utilisées ici comme la base des séries de données sur le nombre des postes à pourvoir associées aux demandes d'assistance. Au moyen de cette mesure et des statistiques usuelles du chômage, on étudie les inadaptations du marché du travail au Canada de 1966 à 1983, les tendances au déséquilibre qui ont caractérisé le marché du travail canadien depuis 1966 et les facteurs pouvant expliquer ces tendances - 1 figure, 4 tableaux.

0520 ROHLING, Thomas A.

Screening and Human Capital Theory: An Empirical Test

v. 41 , no 4,1986, p. $817-826$

Le débat sur le rôle de la formation supérieure a donné naissance à deux théories opposées: la théorie des ressources humai- 
Relatyons Inoustrielles, vol. 45, no 4 (1990)

nes et la théorie du tamisage. Application du test mis au point par Wiles en 1974 à une enquête sur les diplômés des universités et collèges canadiens. Les résultats de l'enquête tendent à confirmer la théorie des ressources humaines et à affaiblir la théorie du tamisage sur le rôle de la formation supérieure - 1 tableau, références.

\section{PLANTE, Gilles}

La permission d'appel au tribunal du travail v. 41 , no 4,1986, p. $817-834$

II existe une dérnarcation entre la compétence du Tribunal du travail du Québec siégeant en appel et celle du juge siégeant en permission d'appel. Pour obtenir la permission d'appel, le plaideur doit faire naître dans l'esprit du juge, un doute sérieux sur la valeur juridique de la décision du commissaire au travail. Mais alors, que faut-il plaider pour faire naîte un tel doute?

\section{MORIN, Fernand}

La double personnalité d'un concierge!

v. 41 , no 4,1986 , p. $835-839$

Le Tribunal du travail vient de qualifier d'entrepreneur un travailleur préposé à l'entretien ménager puisqu'il était lié au donneur d'ouvrage par le truchement de sa "corporation". Le concierge était à la fois président de sa "corporation' et principal salarié d'exécution. Rappel des principaux faits de l'affaire et des motifs de la décision du tribunal.

\section{FORREST, Anne} Bargaining Units and Bargaining Power v. 41 , no 4,1986, p. $840-850$

L'étude examine comment le récent amendement à la Loi sur les relations du travail en Ontario qui établit l'arbitrage obligatoire dans le cas d'une première convention collective s'harmonise avec le cadre légal existant - Références.

\section{DASTMALCHIAN, Ali}

ADAMSON, Raymond BLYTON, Paul Developing a Measure of Industrial Relations Climate

v. 41 , no 4,1986 , p. $851-859$

L'étude vise à mettre au point un instrument de mesure du climat des relations du travail dans les organisations en combinant un en- semble d'échelles qui tient compte des nombreux éléments à considérer lorsqu'on veut mesurer un tel climat - 4 tableaux, références.

\section{SAINT-GERMAIN, Catherine}

Décisions rendues par le Conseil canadien des relations du travail.

v. 41 , no 4,1986 , p. $860-868$

Résumé de deux décisions du Conseil: 1) Le Conseil se déclare sans juridiction pour entendre la requête en accréditation présentée par une association d'employés de la Gendarmerie royale canadienne. 2) Le Conseil conclut qu'il a juridiction pour aborder et décider la question du statut des entrepreneurs postaux et celle de la constitutionnalité de la disposition de la 'Loi sur la Société canadienne des postes" qui exclut ces entrepreneurs de la définition "d'employé" du Code canadien du travail.

\section{SOLOMON, Norman A. (R)}

CRAIG, Alton W.J.

The System of Industrial Relations in Canada. (2nd ed.), Englewood Cliffs, Prentice-Hall, 1986, 510 pp., ISBN 0-13-881194-6, recensé par Norman A. Solomon.

v. 41 , no 4,1986 , p. $874-875$

0527 VERGE, Pierre (R)

FOISY, C.H. LAVERY, D.E.

MARTINEAU, L.

Canada Labour Relations Board - Policies and Procedure. Toronto, Butterworths, 1986, 553 pp., ISBN 0-409-81975-1, recensé par Pierre Verge.

v. 41 , no 4,1986, p. $875-877$

0528 THWAITES, James (A)

SCHMIECHEN, James A.

Sweated Industries and Sweated Labor: The London Clothing Trades, 1860-1914. Urbana and Chicago, University of Illinois Press, 1984, 209 pp., ISBN 0-252-01024-8, recensé par James Thwaites.

v. 41 , no 4,1986 , p. $877-878$

0529 DION, Gérard (R)

STODDART, Linda

Conditions of Work and Quality of Working Life, a Directory of Institutions. Geneva, International Labour Office, 1986, 306 pp., ISBN 92-2-105328-8, recensé par Gérard Dion.

v. 41 , no 4,1986, p. $878-879$ 
0530 BOIS, Hélène $(R)$

ROBBINS, Stephen $P$.

Organizational Behavior. Concepts, Controversies and Applications. 3rd ed., Englewood Cliffs, Prentice-Hall, 1986, 554 pp., ISBN 0-13641549-0-01, recensé par Hêlène Bois.

v. 41, no 4,1986 , p. $879-880$

0531 MATEJKO, Alexander J. (R)

PETERS, Tom AUSTIN, Nancy

A Passion for Excellence. The Leadership Difference. New York, Random House, 1985, 437 pp., ISBN 0-394-54484, recensé par Alexander J. Matejko.

v. 41 , no 4,1986 , p. $881-882$

0532 MATEJKO, Alexander J. (R)

NAISBITT, John ABURDENE, Patricia

Reinventing the Corporation. Transforming your Job and your Company for the New Information Society. New York, Warner Books, 1985, 308 pp., ISBN 0-446-51284-2, recensé par Alexander J. Matejko.

v. 41 , no 4,1986 , p. $881-882$

0533 LEBEL, Maurice ( $\mathrm{R})$

MONLÉON, Jacques de

Marx et Aristote. Perspectives sur l'homme. Paris, FAC éditions, 1984, ISBN 0317-0179, recensé par Maurice Lebel.

v. 41 , no 4,1986 , p. $883-884$

0534 THWAITES, James (R)

JACKSON, John H. MORGAN, Cyrill P.

PROLILLO, J.G.P.

Organization Theory : A Macro Perspective for Managing. Englewood Cliffs, Prentice-Hall, 1986, 387 pp., ISBN 0-13-641572-5-01, recensé par James Thwaites.

v. 41 , no 4,1986 , p. $884-885$

0535 THWAITES, James (R)

DESSLER, Gary

Organization Theory: Integrating Structure and Behavior. Englewood Cliffs, Prentice-Hall, 1986, 477 pp., ISBN 0-13-641-903-8-01, recensé par James Thwaites.

v. 41 , no 4,1986 , p. $884-885$

0536 BERGERON, Jean-Louis $(\mathrm{R})$

PORTIS, Bernard FULLERTON, David F.

INGRAM, Paul R.

Quality Circles in Canada - A Review of Case Histories on Employee Involvement Programs. London, School of Business Administration, University of Western Ontario, 1986, 77 pp., recensé par Jean-Louis Bergeron.

v. 41 , no 4,1986 , p. $885-886$

\section{DION, Gérard (R)}

MARTIN, Roger $G$.

Annuaire du travail 1986-1987. Montreal, Productions INFORT, 1986, recensé par Gérard Dion.

v. 41 , no 4,1986 , p. $886-887$

0538 BLANCHARD, Francis

L'OIT face au problème des relations professionnelles

v. 42 , no 1,1987 , p. $3-11$

Le nouveau contexte dans lequel s'inscrit le fonctionnement des relations professionnelles présente aux partenaires et aux pouvoirs publics une série de défis qu'ils doivent affronter. On évoque diverses questions à partir de l'expérience de l'Organisation internationale du travail et on donne quelques indications sur les perspectives que l'Organisation s'efforce de tracer et de proposer.

0539 BARKIN, Solomon

The Flexibility Debate in Western Europe: The Current Drive to Restore Managements' Rights Over Personnel and Wages.

v. 42 , no 1,1987, p. $12-44$

En Europe de l'Ouest, les employeurs revendiquent leurs droits de diriger leur personnel selon leurs propres critères économiques et réclament l'abolition des mécanismes qui restreignent leur contrôle. L'OCDE a donné son appui à cette cause en proposant une politique de fiexibilité de la main-d'oeuvre et des salaires. Résumé du débat que cette politique a soulevé entre les partisans d'une politique active de main-d'oeuvre et les tenants de la flexibilité de la main-d'oeuvre et des salaires.

0540 ADAMS, Roy J.

Employment Standards in Ontario: An Industrial Relations Systems Analysis.

v. 42 , no 1,1987 , p. $46-64$

Dans cette étude, on s'est appliqué à analyser le fonctionnement des normes du travail en se référant aux cadres des systèmes de relations professionnelles. On imagine le senice des normes du travail comme s'il s'agissait d'un sous-système de relations professionnelles. Pour l'illustrer, on y considère les normes du travail dans leurs principaux aspects en les comparant aux divers régimes de négociation collective qu'on retrouve en Ontario - 3 tableaux, références. 
0541 LONG, Richard J.

WARNER, Malcolm

Organizations, Participation and Recession: An Analysis of Recent Evidence.

v. 42 , no 1, 1987, p. 65-91

Étude des modes de participation ouvrière dans l'entreprise au cours des évènements récents de la récession économique dans les pays occidentaux. L'impact principal de la récession en regard de la participation ouvrière a eu pour conséquence de rendre les dirigeants d'entreprises de même que les travailleurs de plus en plus conscients de l'imperfection des structures organisationnelles traditionnelles ainsi que des relations entre employeurs et travailleurs - Références.

0542 DELAMOTTE, Yves

La loi et la négociation collective en France: Réflexion sur l'expérience 1981-1985.

v. 42 , no 1,1987 , p. $92-109$

Quatre ans après la publication des lois Auroux touchant la négociation collective, on essaie d'en apprécier les effets pratiques et on s'interroge sur les pouvoirs et les limites de la loi lorsqu'elle cherche à agir sur les relations collectives du travail.

0543 WILLIAMS, C. Brian

International Unionism: The Papermakers in Eastern Canada, 1930-1945

v. 42 , no 1,1987, p. $110-131$

L'étude traite du cas de l'United Papermakers and Paperworkers et de ses conflits avec la Fédération nationale des travailleurs de la pulpe et du papier dans la province de Québec. L'influence qu'ont pu avoir le contrôle et la propriété de l'industrie par les américains, les migrations ouvrières et les échanges commerciaux à l'intérieur de marchés limitrophes ainsi que le rôle de l'Église catholique romaine sur l'activité professionnelle de l'une et de l'autre des organisations.

\section{AULD, D.A.L.}

WILTON, D.A.

The Impact of Public Sector Wage Controls in Ontario

v. 42 , no 1,1987, p. $132-149$

Le programme de contrôle des salaires dans le secteur public en Ontario en 1982-1983 a exercé un effet négatif plutôt modeste sur les ententes salariales dans les sous-secteurs des conseils scolaires, des municipalités et de la fonction publique provinciale. II a augmenté le volume des règlements salariaux dans le sous-secteur des institutions de la santé. Par contre, le programme n'a eu qu'un effet minimal sur les majorations salariales dans le secteur privé qui n'était soumis à aucun contrôle - 4 tableaux.

0545 RESHEF, Yonatan

A Typology of Shop Stewards: A Confirmatory Factor Analysis

v. 42 , no 1,1987 , p. $150-167$

II n'existe que peu de recherches sur les comportements et les attitudes des délégués du personnel aux États-Unis. L'étude, dans un effort destiné à augmenter nos connaissances, conçoit et apprécie cinq modalités différentes de leur comportement: radical, changeant, coopérateur, passif et malhabile. La typologie théorique est soumise au test empirique de l'analyse confirmative des facteurs - 1 figure, 1 tableau, références.

\section{BARBASH, Jack}

Like Nature, Industrial Relations Abhors a Vaccum: The Case of the Union-Free Strategy.

v. 42 , no 1,1987, p. $168-179$

Depuis les années 80 , le contexte économique et politique ayant complètement changé, l'idée de relations industrielles sans syndicat a été rendue philosophiquement et pratiquement possible. C'est le nouveau système de relations industrielles. Dans la mesure où il n'existe pas de contrepouvoir syndical pour favoriser l'application du principe d'équité, la "loi de l'équilibre" en relations industrielles signifie qu'un autre mécanisme se développera pour combler ce vide.

0547 BOIVIN, Jean

Les relations industrielles: Une pratique et une discipline

v. 42 , no 1,1987 , p. $179-196$

Les relations industrielles peuvent être considérées de façon empirique ou analytique. On discute des principaux concepts utilisés en relations industrielles et on examine si la façon de présenter les connaissances en ce domaine est basée sur la réalité concrète - 3 graphiques. 
0548 CLARKE, Oliver

Industrial Relations Theory and Practice: $A$ Note

v. 42 , no 1,1987 , p. $196-202$

Commentaires sur le fossé qui sépare les théoriciens et les praticiens en relations industrielles. On propose aux théoriciens en relations industrielles une liste indicative de huit grands sujets à étudier et à examiner pour rendre la théorie en relations industrielles utiles aux praticiens.

0549 SAINT-GERMAIN, Catherine Décision rendue par le Conseil canadien des relations du travail.

v. 42 , no 1,1987, p. $203-209$

Le Conseil décide qu'une vente en justice effectuée suite à la faillite d'une entreprise peut donner lieu à la transmission de droits et obligations résultant du Code qui s'y rattachent si cette entreprise se continue entre les mains de l'acheteur.

0550 TRUDEAU, Gilles (R)

BRADET, Denis CLICHE, Bernard

RACINE, Martin THIBAULT, France

Droit de la santé et de la sécurité du travail .

La loi et la jurisprudence commentée. Cowansville, Les Éditions Yvon Blais, 1986, 300 pp., ISBN 2-89073-567-2, recensé par Gilles Trudeau.

v. 42 , no 1,1987 , p. $215-216$

0551 ZIEGER, Robert $H$. (R)

HERON, Craig STOREY, Robert

On the Job: Confronting the Labour Process in Canada. Kingston and Montréal, McGillQueen's University Press, 1986, 360 pp., ISBN 0-7735-0598-9, recense par Robert $H$. Zieger. v. 42 , no 1,1987 , p. $216-218$

0552 FERLAND, Gilles (R)

MILKOVICH, George T. NEWMAN, Jerry M. Compensation. Plano, Texas, Business Publications Inc., 1984, 549 pp., ISBN 0-256-022046 , recensé par Gilles Ferland.

v. 42 , no 1,1987, p. $218-219$

0553 VERGE, Pierre (R)

CARROTHERS, A.W.R. PALMER, E.E.

RAYNER, W.B.

Collective Bargaining Law in Canada. 2nd Ed., Toronto, Butterworths, 1986, 785 pp., ISBN 0-409-81879-8, recensé par Pierre Verge. v. 42 , no 1,1987 , p. $219-221$
0554 PORTIS, Bernard (R)

Industrial Democracy and Employee Participation. Digest of Case Studies. vol. 1, Department of Employment and Industrial Relations, Sydney, Australia, 1985, 207 pp., ISBN 08149739, recensé par Bernard Portis.

v. 42 , no 1, 1987, p. 221-222

0555 SOLOMON, Norman A. (R)

PEACH, David A. KUECHLE, David

The Practise of Industrial Relations. 2nd Ed., Scarborowogh, McGraw Ryerson, 1985, 407 pp., ISBN 0-07-548909-0, recensé par Norman A. Solomon.

v. 42 , no 1,1987 , p. $222-223$

0556 WEISS, Dimitri $(R)$

RICCl, Maurizio

La struttura organizzativa del movimento sindicale, Dalle origini al 1949. Milano, Franco Angeli, 1986, 250 pp., recensé par Dimitri Weiss.

v. 42 , no 1,1987 , p. $223-224$

0557 MATEJKO, Alexander J. (R)

SCHANZ, Günter

Mitarbeiter beteiligung. Grundlagen-Befunde - Modelle. Munich, Verlag Franz Vahlen, 1985, 198 pp., ISBN 3-8006-1130-9, recensé par Alexander J. Matejko.

v. 42 , no 1,1987, p. $224-225$

0558 LEBEL, Maurice (R)

GUITTON, Jean

Le travail intellectuel. Paris, Aubier-Montaigne, 1951 et 1986, 189 pp., ISBN 2707-3409-2, recensé par Maurice Lebel.

v. 42, no 1, 1987, p. 225-227

0559 HAMEED, Syed M.A.

SEN, Joya

A Power Theory of Third Party Intervention in Labour Management Relations.

v. 42 , no 2,1987 , p. $243-255$

Dans la littérature en matière de relations du travail, on ne trouve aucune théorie du pouvoir se rapportant à la tierce partie, celle qui n'est pas mêlée au conflit mais en facilite le règlement. On présente les sources du pouvoir ainsi que certains concepts théoriques dans la mesure où ils peuvent s'appliquer à la motivation, au conflit lui-même et à la négociation. L'origine et l'efficacité du pouvoir de l'intervenant, qu'il soit conciliateur, arbitre ou médiateur - Références. 
0560 GILSON, Clive H.J.

GILLIS, L.P.

Grievance Arbitration in Nova Scotia.

v. 42 , no 2,1987, p. $256-271$

De 1978 à 1985, les 730 griefs soumis à l'arbitrage en Nouvelle-Écosse peuvent être divisés en quatre grandes catégories: questions d'ordre pécuniaire, de discipline, d'unités de négociation et de sécurité d'emploi. On caractérise l'arbitrage des griefs en y incluant plusieurs autres variables: secteur public et secteur privé, griefs individuels et griefs collectifs, arbitres uniques ou tribunal d'arbitrage, interprétation et étendue des conventions collectives, gains des employeurs ou des syndicats - 3 tableaux, références.

\section{SEXTON, Jean}

L'arbitrage de première convention collective au Québec: 1978-1984

v. 42 , no 2,1987 , p. $272-291$

Le mécanisme d'arbitrage de première convention collective utilisé afin de solutionner les conflits de reconnaissance syndicale, existe au Québec depuis 1978. Rappel du contenu de la législation québécoise à cet égard et présentation de l'expérience de relations du travail après qu'une décision arbitrale eut imposé une première convention collective. Analyse des résultats recueillis auprès des parties elles-mêmes suite aux 88 décisions arbitrales rendues au Québec de 1978 à 1984 - 7 tableaux.

0562 ZEYTINOGLU, Isik Urla

The LO Standards and Canadian Labour Légi"slation.

v. 42 , no 2,1987, p. $292-308$

La convention de l'Organisation internationale du travail relative à la liberté d'association a influencé les lois canadiennes du travail avant et après sa ratification. Avant, parce qu'il a fallu que le gouvernement tédéral consulte les provinces de façon à ajuster les lois du travail aux dispositions de la convention. Après, puisque la ratification requiert que les lois nationales soient conformes aux normes ratifiées - 3 tableaux, références.

0563 BLOUIN, Rodrigue

Le titre réservé de CRI et la déontologie de la profession

v. 42 , no 2,1987 , p. $309-324$
L'adoption du Code des professions en 1974 a consacré l'existence de la Corporation des conseillers en relations industrielles du Québec (CRI) comme la seule corporation habilitée au plan légal à surveiller la déontologie de ses membres. Les moyens de mise en oeuvre de la déontologie et la difficulté d'ap. plication du régime déontologique -2 tableaux.

\section{BABA, Vishwanath V. KNOOP, Robert}

Organizational Commitment and Independence Among Canadian Managers

v. 42 , no 2,1987, p. $325-344$

Les résultats de cette enquête effectuée auprès d'un échantillon de cadres canadiens démontrent que la nature des liens qui unissent les individus aux organisations dépend des perceptions qu'ils ont de leur dépendance ou de leur indépendance à l'égard de celle-ci. On ne peut donc que partiellement supporter le modèle prédictif d'appartenance à l'organisation tiré des recherches antérieures puisque ce modèle ne tient pas compte de cette variable primordiale qu'est l'indépendance - 3 figures, 2 tableaux, références.

\section{BÉLANGER, Paul R.}

LÉVESQUE, Benoît

Le mode de vie determine-t-il l'éthique du travail? Etude de cas.

v. 42 , no 2,1987, p. $345-365$

Présentation de la méthode et des résultats d'une recherche visant à vérifier l'hypothèse de la prédominance du mode de vie sur la crise du travail. La conclusion de la recherche effectuée dans un Centre local de services communautaires fut que le mode de vie n'exerçait pas une influence prédominante sur le retrait au travail constaté chez les professionnels et semi-professionnels - Bibliographie

\section{STOREY, Robert $\mathrm{H}$}

The Struggle to Organize Steico and Dofasco v. 42 , no 2,1987 , p. $366-385$

On tente de répondre à la question suivante: pourquoi la lutte pour le syndicalisme industriel a-t-il réussi à Stelco et non pas à Dofasco? Pour répondre à cette question, on re late l'évolution des évènements durant les années 1930 et 1940, car c'est durant cette période que les politiques et les programmes 
adoptés par la direction et les travailleurs furent contestés et consolidés dans les deux entreprises.

0567 NG, Ignace

Determinants of Wildcat Strikes in Canada Manufacturing Industries.

v. 42 , no 2,1987 , p. $386-397$

De 1970 à 1979, 23.2 pour cent de toutes les grèves et lock-outs survinrent en cours de convention, ce qui indique clairement que les grèves sauvages sont parties intégrantes du système canadien des relations industrielles. L'étude tente d'identifier et de tester les effets de plusieurs facteurs sur l'occurrence des grèves sauvages au Canada - 2 tableaux, bibliographie.

\section{LAPORTE, Pierre}

Le caractère d'ordre public des dispositions de la Loi sur les normes du travail.

v. 42 , no 2,1987 , p. $398-414$

Après avoir situé la Loi sur les normes du travail dans son contexte historique et dégagé les règles d'interprétation applicables à une telle législation, on analyse le caractère d'ordre public des normes du travail et la portée des dérogations autorisées par le législateur.

0569 SAINT-GERMAIN, Catherine Décisions rendues par le Conseil canadien des relations du travail

v. 42 , no 2,1987 , p. $415-427$

Résumé de trois décisions du Conseil: 1) Le Conseil décide que le devoir de juste représentation d'un syndicat s'applique aux négociations intervenant au cours d'une convention collective. 2) Le Conseil fait part de son désir d'élargir ses conceptions antérieures du devoir de juste représentation d'un syndicat. 3) Le Conseil refuse de résilier le certificat d'accréditation attaché à une succursale bancaire fermée depuis 4 ans.

0570 PLANTE, Gilles (R) MORIN, Fernand BLOUIN, Rodrigue Arbitrage des griefs. Montréal, Les Éditions Yvon Blais, 1986, 554 pp., ISBN 2-89083-5699 , recensé par Gilles Plante. v. 42, no 2,1987, p. $432-434$
0571 BEAULIEU, Paul (R)

WALL, J.A. Jr.

Negotiation : Theory and Practice. Glenview, Scott, Foresman and Company, 1985, 182 pp., ISBN 0-673-15865-9, recensé par Paul Beaulieu.

v. 42, no 2,1987, p. 435

0572 BLOUIN, Rodrigue (R)

LAPORTE, Pierre

Code du travail du Québec (Législation, Jurisprudence et Doctrine). Avec la collaboration d'Hélène Ouimet, Montreal, Wilson \& Lafleur, 1987, 475 pp., ISBN 2-89127-058-4, recenss par Rodrigue Blouin.

v. 42 , no 2,1987 , p. $436-437$

0573 ADAMS, Roy J. (R)

POOLE, Michael BROWN, William

RUBERY, Jill SISSON, Keith

TARLING, Roger WILKINSON, Frank

Industrial Relations in the Future. Trends and Possibilities in Britain over the Next Decade. Agincourt, Methuen Publications, 1984, 148 pp., ISBN 0-7102-0145-1, recensé par Roy J. Adams.

v. 42 , no 2,1987 , p. $437-439$

0574 AUDET, Michel (R)

DOLAN, Shimon L. SCHULER, Randall S.

Personnel and Human Resource Management in Canada. St-Paul, West Publishing, 1987, 620 pp., ISBN 0-314-32486-0, recensé par Michel Audet.

v. 42, no 2,1987 , p. $440-443$

0575 JAIN, Hem C. (R)

JURIS, $H$. THOMPSON, Mark

DANIELS, W.

Industrial Relations in a Decade of Economic Change. IRRA Series, Annual Research Volume, Madison, Wisconsin, IRRA, 1985, 407 pp., ISBN 0-913447-30-7, recensé par Hem C. Jain.

v. 42 , no 2,1987 , p. $443-445$

0576 LEBEL, Maurice (R)

PONIATOWSKI, Michel

Les Technologies Nouvelles. Paris, Plon, 1986, 334 pp., ISBN 2-259-0143-9, recensé par Maurice Lebel.

v. 42, no 2,1987, p. $445-448$ 
0577 ADAMS, Roy J. (R)

ROBERTS, B.C. JACOBI, Otto JESSOP, Bob KASTENDIEK, Hans

REGINI, Marino

Industrial Relations in Europe. The Imperatives of Change. Beckenham, England, 1986, 279 pp., ISBN 0-7099-4212-5, and Economic Crisis. Trade Unions and the State. Beckenham, England, 1986, 295 pp., ISBN 0-70991447-4, recensés par Roy J. Adams.

v. 42 , no 2,1987, p. $448-450$

0578 ASCAH, Louis (R)

BELLEMARE, Diane POULIN-SIMON, Lise

Le défi du plein emploi - un nouveau regard économique. Montréal, Editions Saint-Martin, 1986, 530 pp., ISBN 2-89035-131-9, recensé par Louis Ascah.

v. 42 , no 2,1987 , p. $450-451$

0579 LIPSIG, Carla (R)

LIPSET, Seymour Martin

Unions in Transition. Entering the Second Century. San Francisco, ICS Press, 1986, 506 pp., ISBN 0-917616-74-X, recensé par Carla Lipsig.

v. 42 , no 2,1987, p. $452-454$

0580 KELLY, Laurence

Industrial Relations at Queen's: The First Fifty Years.

v. 42 , no 3,1987, p. $475-499$

En 1987, l'Université Queen fête le cinquantième anniversaire de fondation du Centre des relations industrielles qui fut la première institution de ce genre au Canada. Historique de la fondation du Centre et des personnes qui y furent associées et bilan de ces cinquante années d'existence.

\section{HÉBERT, Gérard}

L'évolution du syndicalisme au Canada: Comment un mouvement devient institution.

v. 42 , no 3,1987 , p. $500-519$

Les principaux changements qu'ont connus les syndicats ouvriers au cours de leur cent ans d'histoire, comment leurs objectifs, leurs structures, leurs membres et leurs méthodes d'action ont évolué au gré des périodes et de l'environnement social. Interrogations sur les caractéristiques actuelles du mouvement, sur le bien fondé aujourd'hui des privilèges qui leur ont été accordés et sur leur statut présent - 2 tableaux.
0582 COOK, Alice $\mathrm{H}$.

Family and Work: Challenger to Labor, Management and Government v. 42 , no 3,1987 , p. $520-527$

Au cours de ce siècle, les deux institutions que sont la famille et le travail ont connu de profonds changements. Présentement, ces deux institutions sont sources de demandes conflictuelles. II faut trouver des compromis pour à la fois réaliser l'égalité à la maison et restructurer le travail de façon à permettre aux deux parents d'avoir du temps pour la famille.

\section{BOURGEOIS, Robert-Paul WILS, Thierry}

Career Concepts, Personality and Values of Some Canadian Workers: An Exploratory Study v. 42 , no 3,1987, p. $528-543$

Résultats d'une recherche consistant à explorer les dynamiques de personnalité, de valeurs et de motivation au travail susceptibles de caractériser des travailleurs s'identifiant à un des quatre cheminements de carrière possible: le linéaire, l'homéostatique, le transitoire et le spiral. Les résultats confirment que le type de cheminement de carrière d'un individu est fonction de ses valeurs et de ses éléments profonds de personnalité -4 tableaux, bibliographie.

\section{PLASSE, Micheline}

Santé et sécurité du travail au Québec: Le défi de la concertation patronale - syndicale.

v. 42, no 3,1987, p. $544-565$

La réponse du milieu de travail à la mise en oeuvre de la Loi sur la santé et la sécurité du travail: a) Résumé des principales composantes de cette loi; b) éléments d'analyse nécessaires à la compréhension de la loi prise au sens de politique; c) les deux principaux types de politique que contient la loi: réglementaire et constitutionneile; d) réponse que les parties patronale et syndicale ont donnée à ces deux types de politiques - 3 tableaux.

\section{GURDON, Michael A.}

Divergent Paths: Civil Service Employment Relations in Australia and Canada.

v. 42 , no 3,1987, p. $566-575$

Les gouvernements fédéraux des deux pays 
ont cherché à améliorer l'efficacité du fonctionnement de leur fonction publique respective. Cependant, les politiques d'emploi dans ce secteur se sont développées dans des directions différentes au cours de la dernière décennie. Après une description et une analyse du rôle accru du syndicalisme dans les prises de décision dans la fonction publique australienne, on se demande s'il y a des leçons à en tirer pour le Canada.

0586 ROY, Paul-Martel

Licenciements collectifs, licenciements individuels et emploi au Québec: 1979-1984

v. 42 , no 3,1987 , p. $577-593$

On analyse d'abord la réalité économique des licenciements collectifs relativement aux licenciements individuels et à l'emploi au Québec de 1979 à 1984, pour proposer ensuite un critère de décision qui est en même temps un critère d'équité face à la question du dédommagement des travailleurs touchés par un licenciement collectif - 8 tableaux, 1 schéma, bibliographie.

0587 . DOOLEY, Martin D.

Within-Cohort Earnings Inequality Among Canadian Men: 1971-1982

v. 42, no 3, 1987 , p. 594-611

L'étude expose les résultats des derniers changements survenus dans la disparité des salaires a l'intérieur des groupes d'hommes au Canada par rapport aux niveaux d'éducation et d'âge. Les données de l'étude sont tirées de sept enquêtes sur les dépenses de consommation au cours de la période 1971-1982 - 4 tableaux, références.

0588 OHTSU, Makoto VERMA, Anil

Intra-Organizational Bargaining: Wage Differentials Among Saskatchewan Schoolteachers. v. 42 , no 3,1987, p. $612-627$

L'étude est basée sur les relevés statistiques (1949-1967) des différences de salaires entre deux catégories d'enseignants des écoles publiques de la Saskatchewan (ceux qui ont un baccalauréat et ceux qui n'en n'ont pas). L'étude confirme l'hypothèse que les différences de salaires à l'intérieur d'une unité de négociation sont influencées par le pouvoir relatif des différents groupes professionnels à l'intérieur du syndicat - 2 tableaux, références.
0589 SAINT-GERMAIN, Catherine Décision rendue par le Conseil canadien des relations du travail.

v. 42 , no 3,1987 , p. $628-635$

Saisi d'une requête en accréditation visant les employés d'une entreprise de téléphone exploitée par une société d'état provinciale (AIberta Government Telephone), le Conseil affirme sa compétence et sa juridiction puisqu'il s'agit d'une entreprise reliant une province à d'autres provinces.

0590 DAVID, Hélène (R) GUILLEMARD, Anne-Marie Le déclin du social, formation et crise des politiques de la vieillesse. Paris, Presses universitaires de France, 1986, 396 pp., ISBN 2130-39565-1, recensé par Hélène David.

v. 42 , no 3,1987 , p. $642-646$

0591 CHRÉTIEN, Lise (R)

TAHAR, Gabriel

La réduction de la durée du travail. Paris, Collection Reperres, Éditions La découverte, 1985, 127 pp., ISBN 2-7071-1552-5, recensé par Lise Chrétien.

v. 42 , no 3,1987 , p. $646-648$

0592 ANDIAPPAN, P. (R)

CHAISON, Gary N.

When Union Merge. Lexington, Lexington Books, 1986, 186 pp., ISBN 0-669-11081-7, recensé par $P$. Andiappan.

v. 42 , no 3,1987 , p. $649-651$

0593 VERGE, Pierre (R)

VON PRONDZYNSKI, $F$.

Freedom of Association and Industrial Rela tions. London, Mansell Publishing, 1987, 248 pp., ISBN 0-7201-1775-5, recensé par Pierre Verge.

v. 42 , no 3,1987 , p. $651-652$

0594 SASS, Robert (R)

LEWIS, Gary A.

News from Somewhere : Connecting Health and Freedom at the Workplace. New York, Greenwood Press, 1986, 213 pp., ISBN 0-31324869-9, recensé par Robert Sass.

v. 42 , no 3, 1987, p. 653-655

0595 MORIN, Fernand (R)

DUBÉ, Jean-Louis DI LORIO, Nicola

Les normes du travail. Sherbrooke, Les Éditions Revue de Droit, Université de Sherbrooke, 1987, 442 pp., ISBN 2-920003-08-9, recensé par Fernand Morin.

v. 42 , no 3,1987 , p. $655-656$ 
Relations lnoustrielles, vol. 45, no 4 (1990)

0596 VERGE, Pierre (R)

VERDIER, Jean-Maurice

Syndicats et droit syndical. 2ième ed., tome 5, vol. 1 du Traité de droit du travail publié sous la direction de G.H. Camerlynck, Paris, Dalloz, 1987, 671 pp., ISBN 2-247-00754-6, recensé par Pierre Verge.

v. 42 , no 3,1987, p. $657-658$

0597 VERGE, Pierre (R)

RIVERO LAMAS, Juan

Limitacion de los poderes empresariales $y$ democracia industrial, Leccion inaugural del curso academico 1986-1987. Zaragoza, Universidad de Zaragoza, 1986, 144 pp., Deposito Legal : Z.I. 631-86, recensé par Pierre Verge. v. 42 , no 3,1987 , p. $659-660$

0598 VALLÉE, Guylaine (R)

DURAND, Jean-Pierre

DURAND-SEBAG, Joyce LOJKINE, Jean

MAHIEU, Christian

L'enjeu informatique : former pour changer l'entreprise. Paris, Méridiens Klincksieck, 1986, 192 pp., ISBN 2-86563-148-6, recensé par Guylaine Vallée.

v. 42 , no 3,1987 , p. $660-661$

0599 VERGE, Pierre (R)

TOMANDL, T, FUERBOECK, $\mathrm{K}$.

Social Partnership - The Austrian System of Industrial Relations and Social Insurance. Ithaca, ILR Press, 1986, 165 pp., ISBN 087546-116-6, recensé par Pierre Verge.

v. 42 , no 3,1987, p. $662-663$

0600 VINET, Alain (R)

PAQUIN, Michel

L'organisation du travail. Montreal, Agence d'Arc, 1986, 199 pp., ISBN 2-89022-091-5, recensé par Alain Vinet.

v. 42 , no 3,1987, p. $663-664$

0601 BEAULIEU, Paul (R)

KELLEY, Robert E.

The Gold Collar Worker. Reading Massachusetts, Addison-Wesley Publishing Co., 1985, 196 pp., ISBN 0-201-11739-8, recensé par Paul Beaulieu.

v. 42, no 3,1987, p. $664-665$

0602 MATEJKO, Alexander J. (R) GROOTINGS, Peter GUSTAVSEN, Bjorn HÉTHY, Lajos

New Forms of Work Organization and their Social and Economic Development. Budapest, Statistical Publishing House, 1986, 297 pp., ISBN 963-01-6862-6, recensé par Alexander J.
Matejko.

v. 42 , no 3,1987 , p. $665-667$

0603 GILES, Anthony (R)

JACKSON, Michael $P$.

Industrial Relations - A textbook. 3rd ed., London, Croom Helm, 1985, 294 pp., ISBN O7099-1474-1, recensé par Anthony Giles.

v. 42 , no 3,1987 , p. $667-669$

0604 KUMAR, Pradeep

Recent Wage Deceleration in Canada: Short-run Response or Structural Change?

v. 42 , no 4,1987 , p. 687-701

Les mouvements récents des salaires au $\mathrm{Ca}$ nada indiquent que les taux moyens d'augmentation des salaires sont inférieurs au taux d'inflation. Comment expliquer ce phénomène? Les équations salariales indiquent que le frainage des salaires au cours des quatre dernières années est pour beaucoup attribuable au déclin de l'inflation des prix et aux taux élevés de chômage qui persistent depuis 1982 et que ce phénomène est une réaction temporaire de courte durée -3 tableaux, références.

\section{LAFLAMME, Gilles}

VALLÉE, Guylaine

Changements technologiques et modes régu"lateurs des relations du travail.

v. 42 , no 4,1987 , p. $702-715$

L'étude envisage le changement technologique comme enjeu des rapports du travail plutôt que comme source d'impacts et adapte l'approche stratégique à l'étude des relations du travail et du contrôle syndical du changement. a) Les traits principaux de l'approche stratégique. b) La notion de contrôle syndical. c) Les pratiques de contrôle syndical observées dans quelques entreprises. d) Les modes régulateurs des relations du travail qui ressortent de l'étude.

0606 KNIGHT, Thomas R.

The Role of The Duty of Fair Representation in Union Grievance Decisions.

v. 47 , no 4,1987 , p. $716-736$

Résultats d'une enquête auprès de représentants syndicaux au sujet de l'effet du devoir de représentation juste en matière de procédure de règlement des griefs: a) Les plaintes concernant la juste représentation reflètent l'existence de factions au sein des syndicats. 
b) Le recours a cette doctrine de la part des plaignants a incité les syndicats à présenter des griefs sans valeur - 7 tableaux, références.

\section{DÉOM, Esther}

La syndicalisation des journalistes dans quelques quotidiens québécois.

v. 42 , no 4,1987 , p. 737-755

L'origine de la fondation des syndicats de journalistes dans cinq quotidiens d'information québécois, francophones et anglophones. L'organisation de la profession avant l'apparition du syndicalisme. Les motifs de la syndicalisation des journalistes et le choix de leur affiliation syndicale - 1 tableau.

0608 GILSON, Clive H.J. SPENCER, lan S.

Trade Union Growth: A Marketing Model. v. 42 , no 4,1987, p. $756-773$

Le développement du syndicalisme vu selon le modèle du 'marketing'. a) Revue de la littérature sur la croissance du syndicalisme. b) Utilisation des concepts de base du marketing comme moyen de catégorier l'orientation du développement du syndicalisme et d'expliquer les changements dans les structures syndicales. c) Résultats d'une recherche exploratoire sur les méthodes de recrutement des membres - 1 tableau, 3 figures, bibliographie.

0609 LÉONARD, Christine VAN AMÉRINGEN, Marie-Reine DOLAN, Shimon $L$. ARSENAULT, André

Absentéisme et assiduité au travail: Deux moyens d'adaptation au stress.

v. 42 , no 4 , 1987 , p. $774-789$

L'étude teste de nouveau les relations entre d'une part le stress intrinsèque et extrinsèque et d'autre part l'absence et l'assiduité au travail et détermine la nature des relations entre le stress au travail et différentes mesures de fréquence des absences et de temps perdu - 2 tableaux, 1 graphique, bibliographie.

0610 JAIN, Harish C.

Recruitment of Racial Minorities in Canadian Police Forces.

v. 42 , no 4,1987 , p. $790-805$
L'étude effectue une revue des politiques de recrutement et de sélection des policiers à travers le Canada, identifie les barrières auxquelles les minorités se heurtent pour entrer dans la police et recommande des moyens afin de permettre l'augmentation du nombre de personnes appartenant à des minorités visibles parmi les policiers canadiens $-3 \mathrm{ta}$ bleaux, références.

\section{MERCIER, Jacques}

Effets du salaire minimum sur l'emploi: Résultats des études économétriques canadiennes et québécoises.

v. 42 , no 4,1987 , p. $806-830$

Synthèse des résultats des études économétriques canadiennes et québécoises des effets du salaire minimum sur l'emploi. Quelques caractéristiques générales des études économétriques et principales conclusions qui se dégagent des études canadiennes et québécoises, avec référence à la littérature américaine sur le sujet - 1 tableau, bibliographie.

\section{BARRÉ, Alain}

Le cadre juridique de la négociation collective dans le secteur public au Québec: une perspective internationale.

v. 42 , no 4,1987 , p. $831-851$

Le comité de la liberté syndicale du Conseil d'administration de l'Organisation internationale du travail a rendu sa décision concernant des plaintes présentées en 1985 et 1986 relatives à la "Loi sur le régime de négociation des conventions collectives dans les secteurs public et parapublic". Commentaires sur divers aspects de la décision: le niveau de la négociation collective, les restrictions apportées à l'exercice du droit de grève et la détermination des services essentiels.

0613 TREMBLAY, Johane

Décisions rendues par le Conseil canadien des relations du travail.

v. 42 , no 4,1987 , p. $852-861$

Résumé de deux décisions importantes dans le secteur des postes canadiennes, dans lesquelles le Conseil précise les notions d'entrepreneur indépendant et de vente d'entreprise: 1) Le Conseil reconnaît aux facteurs ruraux le statut d'employé au sens du Code canadien du travail. 2) Le Conseil décide qu'il y a eu vente d'entreprise par suite d'un contrat de franchise conclu entre la Société 
canadienne des postes et Sheldon Manly Drugs Ltd.

0614 LAPORTE, Pierre (R)

HÉBERT, Gérard TRUDEAU, Gilles

Les normes minimales du travail au Canada et au Québec : Étude juridique et institutionnelle. Montreal, Éditions Yvon Blais, 1987, 192 pp., ISBN 2-89073-611-3, recensé par Pierre Laporte.

v. 42 , no 4,1987 , p. $876-877$

0615 VERGE, Pierre (R)

BEATTY, D.M.

Putting the Charter to Work Designing a Constitutional Labour Code. Kingston et Montréal, McGill-Queen's University Press, 1987, 251 pp., ISBN 0-7735-0601-2, recensé par Pierre Verge.

v. 42 , no 4,1987 , p. $877-880$

0616 MAYER, Udo (R)

ENGLAND, Geoffrey

Part-time, Casual and Other Atypical Workers

: A Legal View. Research and Current Issues Series No 48, Kingston, Industrial Relations Centre, Queen's University, 1987, 81 pp., ISBN 0-88886-154-0, recensé par Udo Mayer.

v. 42 , no 4,1987 , p. $880-881$

0617 BEAULIEU, Paul (R)

BOYER, LUC EQUILBEY, Noël

Le projet d'entreprise. Paris, Les Éditions d'Organisation, 1986, 135 pp., ISBN 2-70810749-6, recensé par Paul Beaulieu.

v. 42 , no 4,1987 , p. $881-883$

0618 SOLOMON, Norman A. (R)

KERR, Clark STAUDOHAR, Paul D.

Industrial Relations in a New Age. San Francisco, Jossey-Bass Inc., 1986, 419 pp., ISBN 0-55542-013-3, recensé par Norman A. Solomon.

v. 42 , no 4,1987 , p. $883-884$

0619 VERGE, Pierre (R)

HEPPEL, Bob (Ed.)

The Making of Labour Law in Europe - A Comparative Study of Nine Countries up to 1945. London, Mansell Publishing Ltd, 1986, 401 pp., ISBN 0-7202-1697-X, recensé par Pierre Verge.

v. 42 , no 4,1987 , p. $884-886$

0620 VERGE, Pierre (R)

Compulsory Arbitration - The Australian Way? Industrial Relations Society of Australia, Papers presented at the 1986 National Convention, Adelaide, September 1986, 192 pp., recensé par Pierre Verge.

v. 42 , no 4,1987 , p. $886-887$

0621 POULIN-SIMON, Lise $(R)$

ASHTON, D.N. MAGUIRE, J.J.

Young Adult in the Labour Market. Research Paper no 55, Leicester, University of Leicester, 1986, 163 pp., recensé par Lise Poulin Simon. v. 42 , no 4 , 1987 , p. $887-889$

0622 CLACK, Garfield (R)

MAINWARING, John

The International Labour Organisation : A Canadian View. Ottawa, Canadian Government Publishing Centre, 1986, 206 pp., recensé par Garfield Clack.

v. 42 , no 4,1987 , p. $889-890$

0623 BÉLANGER, Jacques (R)

MILLWARD, Neil STEVENS, Mark

British Workplace Industrial Relations 19801984 : The DE/ESRC/PSI/ACAS Surveys. Aldershot, Gower, 1986, 341 pp., ISBN 0-56605396-9, recensé par Jacques Bélanger.

v. 42 , no 4,1987 , p. $891-893$

0624 MORIN, Fernand $(\mathrm{R})$

Labour Law. Cases, Material and Commentary. (4th ed.), compiled by The Labour Law Casebook Group, Industrial Relations Centre, Kingston, Queen's University, 1986, 593 pp., ISBN 0-88886-139-7, recensé par Fernand Morin.

v. 42 , no 4,1987, p. $893-894$

0625 ALVI, S.A. (R)

EASTMAN, Byron D.

Labour Market Theory and the Canadian Experience. Toronto, Harcourt Brace Jovanovich, 1987, 350 pp., ISBN 077-4730-757, recensé par S.A. Alvi.

v. 42, no 4,1987, p. $894-895$

0626 AUDET, Michel

LAROUCHE, Viateur

Paradigmes, écoles de pensée et théories en relations industrielles.

v. 43 , no 1,1988 , p. $3-31$

Essai de classification des principales théories de relations industrielles en fonction des paradigmes largement popularisés en sciences sociales. Cette classification repose sur des caractéristiques traditionnellement reconnues dans le domaine scientifique, ce qui la distingue de la littérature existante -3 graphiques. 
0627 BARBASH, Jack

The New Industrial Relations in the US: Phase II.

v. 43 , no 1,1988, p. $32-42$

Les nouvelles relations industrielles (NRI) aux États-Unis consistent en une stratégie offensive du patronat face aux syndicats et se résument a la doctrine d'absence de syndicats. Le patronat américain reconnaît la légitimité d'une équité dans l'emploi et croit qu'il peut assumer lui-même cette fonction d'équité à meilleur coût et sans l'intrusion de syndicats.

0628 RESHEF, Yonatan

Changing Environments and Management IR Practices: Implications for U.S. Trade Unions. v. 43, no 1,1988 , p. $43-62$

Alors que le milieu et les méthodes des employeurs se modifient en matière de relations professionnelles, les syndicats industriels américains doivent affronter de nombreux problèmes. L'orientation économique de la stratégie syndicale devient de plus en plus difficile à appliquer dans le domaine des relations du travail à mesure que l'environnement change et que la philosophie patronale délaisse les attitudes d'opposition traditionnelles - Références.

0629 MCPHILLIPS, David C.

The Appropriate Bargaining Unit: The Need for Policy Consistency by Canadian Labour Boards.

v. 43 , no 1,1988, p. 63-84

il existe au Canada un grand manque d'uniformité à l'intérieur de Commissions des relations du travail de même qu'entre elles lorsqu'elles ont à disposer des questions touchant la structure de l'unité de négociation appropriée, les regroupements, les fragmentations, les fusions, les consolidations et les maraudages partiels. Les Commissions doivent rendre leurs décisions selon un ordre des priorités uniforme pour tous les types de requêtes.

0630 COUSINEAU, Jean-Michel

RABEAU, Yves

Une méthodologie de comparaison des salaj-res pour les emplois spécifiques du secteur public.

v. 43, no 1,1988, p. $85-100$
Un grand nombre d'emplois du secteur public n'ont pas de point de comparaison dans le marché. Pour résoudre ce problème, on propose une méthode de comparaison non pas avec des emplois, mais avec des professions comparables. Cette méthode s'appuie essentiellement sur la théorie du capital humain. Application de la méthode proposée au cas des policiers de la Communauté urbaine de Montréal - 1 tableau, 1 graphique, bibliographie.

\section{REID, Frank}

Economic Aspects of Mandatory Retirement: The Canadian Experience.

v. 43 , no 1,1988, p. 101-114

Étude empirique démontrant que l'interdiction de la retraite obligatoire n'a que très peu d'influence sur le taux d'activité des personnes âgées, et par conséquent, n'a qu'un impact minime sur les possibilités d'emploi pour les jeunes et les politiques de personnel relatives à l'évaluation des employés. On ne peut donc plus justifier le maintien d'une pratique d'emploi qui est manifestement discriminatoire - 1 tableau, références.

\section{CAMPBELL, Adrian WAFNEA, Malcolm}

Workplace Relations, Skills-Training and Technological Change at Plant-Level.

v. 43 , no $1,1988, p .115-132$

On étudie les relations en milieu de travail, la formation professionnelle et les changements technologique en Grande-Bretagne en se demandant s'ils sont nécessairement reliés les uns aux autres d'une manière rigoureuse, spécialement lorsqu'il s'agit de microélectronique. De nouveaux métiers apparaissent là où la technologie et des marchés différenciés agissent les uns sur les autres, ce qui a d'importantes conséquences sur les stratégies de formation professionnelle -2 tableaux, 1 figure, références.

\section{RICHARDS, John}

MAUSER, Gary HOLMES, Richard

What Do Workers Want? Attitudes Towards Collective Bargaining and Participation in Management.

v. 43 , no 1,1988, p. $133-152$

Résultats d'une étude exploratoire, effectuée à Vancouver, sur le comportement du public 
Relaijons Industrielles, vol. 45, no 4 (1990)

en général en matière de démocratie indus trielle. On peut retenir, de ce sondage exploratoire, que la frustration existe à l'endroit du statuquo et de la volonté d'une participation plus forte à la gestion. Les revendications en matière de participation à la gestion ne sont pas un substitut aux attitudes pro-syndicales ou anti-syndicales - 6 tableaux, 1 figure, références.

\section{HACCOUN, Robert R.} DUPONT, Serge

Une analyse des comportements de travailleurs masculins et féminins selon deux formes d'absence au travail.

v. 43 , no 1,1988, p. $153-166$

On utilise des données provenant d'entrevues afin de décrire les activités hors-travail d'un échantillon d'employés d'hôpital lors de journées passées à l'extérieur du travail. Cette étude est la première à tenter d'analyser les comportements manifestés lors d'absence et se distingue de la recherche traditionnelle sur l'absentéisme - 1 tableau, 1 graphique, références.

\section{SINGH, Jang $B$. CROCKER, Olga}

Operative and Espoused Personnel Selection Criteria of Managers.

v. 43 , no 1 , p. $167-182$

Les dirigeants d'entreprises recourent à deux catégories de critères de sélection du personnel: les critères opérationnels et les critères favorisés. Les résultats de l'étude démontrent que les critères opérationnels les plus utilisés étaient le comportement, l'enthousiasme, l'honnêteté, le dévouement et la compétence. Du côté des critères favorisés, les plus communément retenus étaient la loyauté, l'honnêteté, la formation, le savoir et l'expérience - 5 tableaux, 1 graphique, références.

0636 PEPERMANS, Raymond

Line, staff et functional

v. 43 , no 1,1988, p. 183-187

La désignation des structures de l'organisation utilise en anglais trois termes principaux: "line", "functional" et "staff and line". Après avoir défini les caractéristiques de chacune de ces structures, on propose leur désignation en français par: hiérarchique (line), de consultation (staff), fonctionnelle (functional), mixte (staff and line).
0637 TREMBLAY, Johane

Décisions rendues par le Conseil canadien des relations du travail.

v. 43 , no $t, 1988$, p. $188-195$

Résumé de deux décisions du Conseil: 1) Le Conseil donne une interprétation généreuse de la notion "représentation des employés par un syndicat" de façon à inclure la représentation auprès du public et décide que tout représentant élu peut exercer ce droit de représentation pour le syndicat. 2) Le Conseil décide que les représentants syndicaux, à l'occasion d'une grève légale, jouissent d'une latitude plus grande en terme de liberté d'expression.

0638 AUDET, Michel (R)

LARSON, Simeon NISSEN, Bruce

Theories of the Labor Movement. Detroit, Wayne State University Press, 1987, 395 pp., ISBN 0-8143-1815-0, recensé par Michel Audet.

v. 43 , no 1,1988, p. $199-200$

0639 FRANK, David (R)

GILSON, C.H.J.

Strikes in Nova Scotia, 1970-1985. Hantsport, Lancelot Press, 1986, 191 pp., ISBN 0-88999314-9, recensé par David Frank.

v. 43 , no 1,1988, p. $200-203$

0640 AUDET, Michel (R)

DOLAN, Shimon L. SCHULER, Randall S.

Canadian Readings in Personnel and Human Resource Management. St-Paul, West Publishing Company, 1987, 467 pp., ISBN 0-31432487-9, recense par Michel Audet.

v. 43 , no 1,1988, p. $203-205$

0641 DUMONT, Micheline (R)

O'SULLIVAN, Judith GALLICK, Rosemary

Workers and Allies: Female Participation in the American Trade Union Movement, 18241976. Washington, Smithsonian Institution Press, 1975, 96 pp., ISBN 0-87546-138-1, recensé par Micheline Dumont.

v. 43 , no 1, 1988, p. 205

0642 PORTIS, Bernard (R)

ROBERTSON, David WAREHAM, Jeff

Technological Change in the Auto Industry. Willowdale, CAW Technology Project, $56 \mathrm{pp}$., ISBN 0-969-2932-08, recensé par Bernard Portis.

v. 43 , no 1,1988 , p. $206-207$ 
0643 SOLOMON, Norman A. (R) HERMAN, E. Edward KUHN, Alfred SEEBER, Ronald

Collective Bargaining and Labor Relations. (2nd ed.), Englewood Cliffs, Prentice Hall, 1987, 621 pp., ISBN 0-13-140576-6, recensé par Norman A. Solomon.

v. 43 , no 1,1988 , p. $207-208$

0644 SHARMA, Basu (R)

SHARMA, Baldev R.

Not by Bread Alone : A Study of Organizational Climate and Employer-Employee Relations in India. New Delhi, Shri Ram Centre for Industrial Relations and Human Resources, 1987, 191 pp., resencé par Basu Sharma.

v. 43 , no 1,1988, p. $208-209$

\section{ROY, André (R)}

GEORGE, Kenneth

Entre syndicats et patrons, fragile alliance. Montréal, Les Éditions Agence d'Arc, 1987, 137 pp., ISBN 2-89022-104-0, recensé par André Roy.

v. 43 , no 1,1988 , p. 210

0646 MATEJKO, Alexander J. (R)

MANSELL, Jacquie

Workplace Innovation in Canada. Reflections on the Past Prospects for the Future. A Study prepared for the Economic Council of Canada, Ottawa, Minister of Supply and Services Canada, 1987, 40 pp., ISBN 0-660-12554-4, recensé par Alexander J. Matejko.

v. 43, no 1, 1988, p. 211

\section{BRUNET, Yves W. BEAULIEU, LUC} CANTIN, Isabelle

La nouvelle Commission québécoise des relations du travail (1988)

v. 43 , no 2,1988 , p. 231-304

Les motifs allégués par le Ministre du travail du Québec au soutien du projet de loi 30 et les objectifs qu'il y visait. Analyse des changements institutionnels apportés par la "Loi constituant la Commission des relations du travail et modifiant diverses dispositions législatives" adoptée par l'Assemblée nationale le 18 décembre 1987. Précisions sur le rôle, le mandat, le fonctionnement et les pouvoirs de la Commission - 5 tableaux.

0648 CARTER, Donald D.

Canadian Labour Relations Under the Charter: Exploring the Implications.

v. 43 , no 2,1988, p. $305-321$
La Charte des droits et libertés est devenue la principale source d'influence légale sur le régime des relations professionnelles au $\mathrm{Ca}$ nada. La Charte a amplifié les pouvoirs des tribunaux canadiens et a créé la possibilité que notre régime de relations du travail puisse être transformé par les décisions judiciaires. Lors de l'application de la Charte par les tribunaux, ceux-ci mettront-ils l'accent sur la protection des droits individuels ou des droits collectifs?

\section{CAMIRÉ, André}

La productivité des intelligences.

v. 43 , no 2,1988 , p. $322-340$

La gestion des ressources humaines est l'art de mobiliser des individus à la réalisation d'un objectif commun. Pourtant, la grande majorité des activités reliées à la gestion des ressources humaines ne tiennent pas toujours compte de la nature et des caractéristiques des individus. On propose ici une typologie des styles de gestion qui remet en cause la façon traditionnelle d'aborder la gestion des personnes et la productivité organisationnelle - 2 graphiques.

\section{SODERSTROM, Lee}

Some Effects of Unemployment on the Health of Unemployed Quebec Workers.

v. 43 , no 2,1988 , p. $341-377$

Résultats d'une recherche visant à estimer les conséquences du chômage sur la santé des travailleurs sans emploi au Québec. Le chômage engendre certaines conséquences négatives considérables sur l'état de santé de certains sans-travail tout comme il peut avoir quelques effets positifs ou bénéfiques chez d'autres individus - 6 tableaux, référence.

\section{TEMPLER, Andrew SOLOMON, Norman A.}

Unions and Technology: A Survey of Union Use of Information Technology.

v. 43 , no 2,1988 , p. $378-393$

Présentation des résultats d'une enquête portant sur l'utilisation de l'informatique par les syndicats canadiens dans leur administration et sur la perception syndicale des attitudes face aux nouvelles technologies - 3 tableaux, références. 
0652 CUNNINGHAM, Barton HULL, Dennis

A Union Member's Perspective on Technological Change.

v. 43 , no 2,1988 , p. $394-411$

Illustration des conséquences humaines des changements technologiques par l'analyse des transformations récentes dans l'industrie des télécommunications. On observe plus particulièrement la situation à la Compagnie de téléphone de la Colombie-Britannique où on assiste à un mouvement massif vers l'informatisation de tous les services. Le rôle du syndicat face aux changements technologiques.

\section{KAMEL, Nawal}

ROY, Paul-Martel

Temps supplémentaire et création d'emplois: Le cas du Canada, du Québec et de l'Ontario. v. 43 , no 2,1988 , p. $412-430$

Analyse de la problématique du temps supplémentaire et compte rendu des études réalisées sur le sujet au Canada et au Québec. Etude du phénomène du temps supplémentaire dans l'ensemble des branches d'activité au Canada, au Québec et en Ontario pour la période de 1975 à 1984, globalement puis selon le statut d'emploi, le sexe et la profession. Les perspectives de transformation du temps supplémentaire en emplois à plein temps - 7 tableaux, bibliographie.

0654 KELLER, Berndt

Mediation as a Conflict-Solving Device in Collective Industrial Disputes.

v. 43 , no 2,1988 , p. $431-446$

Enquête sur les résultats récents d'études théoriques et de recherches empiriques sur la médiation. Par médiation, il faut entendre le processus de règlement des différends industriels collectifs en matière de conflits d'intérêts, généralement par l'intervention d'une tierce partie neutre - Références.

0655 TREMBLAY, Johane

Décisions rendues par le Conseil canadien des relations du travail.

v. 43 , no 2,1988 , p. $447-452$

Le 18 décembre 1985, le Conseil accréditait le Syndicat des employés des Banques Natio- nales de Rimouski pour représenter tous les employés des cinq succursales de la Banque à Rimouski. Près de deux ans plus tard, le Conseil révoquait le certificat d'accréditation et expliquait les mécanismes du Code en pareilles circonstances.

\section{AUDET, Michel (R)}

La flexibilité des marchés du travail : essai bibliographique/Labour Market Flexibility : A Bibliography Essay. Série bibliographique no 12, Genève, Institut international d'études sociales, 1987, 101 pp., ISBN 92-9014-418-1, recensé par Michel Audet.

v. 43 , no 2,1988 , p. $461-462$

0657 GILES, Anthony (R)

MORRIS, Gillian S,

Strikes in Essential Services. London and New York, Manselle Publishing Company, 1986, 221 pp., ISBN 0-7201-1791.7, recensé par Anthony Giles.

v. 43, no 2,1988 , p. $462-466$

0658 MATEJKO, Alexander J. (R)

RINEHART, James $W$.

The Tyranny of Work Alienation and the Labour Process. (2nd ed.), Toronto, Harcourt Brace Jovanovich, 1987, 226 pp., ISBN 0-7747-30676 , recensé par Alexander J. Matejko.

v. 43 , no 2,1988 , p. $466-468$

0659 FRÉCHET, Guy (R)

DAVIES, Annette

Industrial Relations and New Technology. UWIST /Croom Helm, London, Sydney, Dover, 1986, ISBN 0-7099-0882-2, recensé par Guy Fréchet.

v. 43, no 2,1988, p. $468-469$

0660 ANDIAPPAN, P. (R)

AGGARWAL, Arjun P.

Sexual Harassment in the Workplace. Toronto, Butterworths, 1987, 230 pp., ISBN 0-40980535-1, recensé par P. Andiappan.

v. 43, no 2,1988, p. $469-471$

0661 MATEJKO, Alexander J. (R)

RUBINSTEIN, Sidney $P$.

Participative Systems at Work: Creating Quality and Employment Security. New York, Human Sciences Press, 1987, 180 pp., ISBN O89885-338-9, recensé par Alexander J. Matejko.

v. 43, no 2,1988, p. $471-472$ 
0662 SEXTON, Jean (R)

WAY, Harold E. WEISS, Carla M.

Plant Closings : A Selected Bibliography of Materials Published Through 1985. Ithaca, NYSSILR, Cornell University, 1988, 206 pp., recensé par Jean Sexton.

v. 43 , no 2,1988 , p. 472

0663 DION, Gérard (R)

DELAMOTTE, Yves

Le droit du travail en pratique. (3ième éd.), Paris, Les Editions de l'organisation, 1987, 328 pp., ISBN 1-7081-0845-X, recensé par Gérard Dion.

v. 43, no 2,1988 , p. 473

\section{BARKIN, Solomon}

Institutional Economics and the American

Trade Union Movement.

v. 43 , no 3,1988 , p. $491-508$

Le fondement idéologique du syndicalisme américain, c'est le syndicalisme d'affaires soutenu par les économistes institutionnalistes. Suite aux changements majeurs survenus dans la décennie des années 1980 , les syndicats ont été obligés de réévaluer leurs structures et leurs stratégies. Pour affronter ce nouveau défi, les institutionnalistes peuvent être d'un grand secours aux dirigeants syndicaux car ils partagent avec eux de nombreux principes fondamentaux.

\section{SAVOIE, Dominique} LAROUCHE, Viateur

Le harcelement sexuel au travail: Définition et mesure du phénomène.

v. 43 , no 3,1988 , p. $509-530$

Après avoir défini ce qu'il faut entendre par harcèlement sexuel au travail, on propose une grille d'identification permettant de classifier selon la forme et le degré les différents comportements qui caractérisent le harcèlement sexuel au travail - 2 tableaux, bibliographie.

\section{TOUPIN, Sylvain}

Le harcelement sexuel en milieu de travail. v. 43 , no 3,1988 , p. $531-546$

Définition de la notion de harcèlement sexuel. Analyse de quelques décisions rendues par les tribunaux canadiens sur ie sujet. A la lumière de la décision rendue par la Cour suprême du Canada dans l'affaire Robichaud, il incombe à l'employeur d'adopter un programme concernant ce problème.
0667 SUBBARAO, A.V.

Criteria in Arbitration of Wage Disputes: Theory and Practice in the Canadian Federal Public Service.

v. 43 , no 3,1988 , p. $547-570$

Revue des recherches théoriques et pratiques sur l'arbitrage des différends sur les salaires dans la fonction publique fédérale du Canada. A partir des sentences rendues au cours d'une période de cinq ans (1969-1974), l'étude identifie les critères dont tiennent compte les arbitres lorsqu'ils rendent leurs décisions dans les différends en matière de salaires - 5 tableaux, références.

\section{STAGER, David A.A.}

Lawyers' Earnings in the Canadian Private and Public Sectors.

v. 43 , no 3,1988 , p. $571-590$

A partir des données extraites des recensements de 1971 et 1981, on analyse les différences de revenus entre les avocats de pratique privée et ceux des services gouvernementaux ainsi que les changements relatifs qui se sont produits depuis 1970 pour les deux groupes - 4 tableaux, références.

\section{LAFLAMME, Lucie} VINET, Alain

Accidents du travail et modernisation du processus de production: le cas de l'industrie forestière québécoise.

v. 43 , no 3,1988 , p. $591-608$

L'étude tente de savoir si la modernisation du processus de production est un facteur d'amélioration des conditions de sécurité du travail. Réalisée dans l'industrie forestière, l'étude traite des effets de la mécanisation de l'abattage et de l'ébranchage sur la fréquence et la gravité des accidents du travail ainsi que sur les conditions dans lesquelles ceux-ci se produisent -3 tableaux, bibliographie.

\section{SEFTON MACDOWELL, Laurel}

The Career of a Canadian Trade Union Leader: C.H. Millard 1937-1946

v. 43 , no 3,1988, p. $609-632$

Résumé de la carrière de $C . H$. Millard entre 1937 et 1946. Pendant cette période, sa carrière syndicale contribua à la transformation de la société canadienne et à l'émergence du mouvement des syndicats industriels modernes. 
Relations Industrielles, vol. 45, no 4 (1990)

0671 FRICKE, John G.

Worker Participation in Canada: Some Lessons from the Past.

v. 43, no 3,1988, p. $633-658$

Le concept de la participation ouvrière est réévalué en établissant une distinction entre "la participation au règlement des problèmes" et "la participation à la prise de décisions fondée sur la gestion des ressources". Application de cette distinction à la façon dont les entreprises canadiennes orientent cette participation et au rôle que l'État a joué historiquement dans sa promotion de la participation ouvrière - Références.

\section{PUPO, Norene} DORIS DUFFY, Ann

The Ontario Labour Relations Board and the Part Time Workers.

v. 43, no 3,1988, p. $660-685$

L'analyse des décisions de la Commission des relations du travail de l'Ontario relatives aux travailleurs à temps partiel entre 1976 et 1986 démontre, même si on peut noter certaines victoires syndicales, que la Commission se comporte de manière à maintenir le statuquo pour les temps partiels - 1 tableau, bibliographie.

0673 TREMBLAY, Johane

Décisions rendues par le Conseil canadien des relations du travail.

v. 43 , no 3,1988 , p. $686-694$

$\mathrm{Au}$ terme d'une analyse détaillée de onze plaintes de pratique déloyale, le Conseil juge que le paragraphe du Code qui impose à l'employeur le fardeau de la preuve est conforme aux dispositions de la Charte canadienne des droits et libertés.

0674 GILES, Anthony (R)

MAURICE, Marc SELLIER, François

SILVESTRE, Jean-Jacques

The Social Foundations of Industrial Power : A Comparison of France and Germany. Traduit par Arthur Goldhammer, Cambridge, MIT Press, 1986, 292 pp., ISBN 0-262-13213-3, recensé par Anthony Giles.

v. 43 , no 3,1988 , p. $703-707$
0675 GILES, Anthony (R)

BAMBER, Greg J. LANSBURY, Russell D. International and Comparative Industrial Relations. London, Allen \& Unwin, 1987, 289 pp., ISBN 0-04-331117-4, recensé par Anthony Giles.

v. 43, no 3,1988, p. $703-707$

0676 POULIN-SIMON, Lise (R)

SARFATI, Hedva KOBRIN, Catherine

La flexibilité du marché de l'emploi : un enjeu économique et social. Genève, Bureau international du travail, 1987, 384 pp., ISBN 92-2205675-2, recensé par Lise Poulin-Simon.

v. 43, no 3,1988 , p. $707-710$

0677 VERGE, Pierre (R)

BEN-ISRAEL, Ruth

International Labour Standards : The Case of Freedom to Strike. Deventer, Kluwer, 1988, 133 pp., ISBN 90-6544-317-7, recensé par Pierre Verge.

v. 43, no 3,1988, p. $710-712$

0678 VERGE, Pierre (R)

B.I.T.

La liberté syndicale - Manuel d'éducation ouvrière. (2ième éd.), Genève, Bureau international du travail, 1988, 153 pp., ISBN 92-2205782-1, recensé par Pierre Verge.

v. 43 , no 3,1988 , p. $712-713$

0679 MATEJKO, Alexander J. (R)

RIDDELL, W. Craig

Labour-Management Cooperation in Canada. Toronto, University of Toronto Press, 1986, 205 pp., ISBN 0-8020-7257-7, recensé par Alexander J. Matejko.

v. 43 , no 3,1988, p. $714-715$

0680 BÉLANGER, Jacques ( $\mathrm{R})$

LEWCHUK, Wayne

American Technology and the British Vehicle Industry. Cambridge, Cambridge University Press, 1987, 304 pp., ISBN 0521-30269-2, recensé par Jacques Bélanger.

v. 43 , no 3,1988 , p. $716-717$

0681 MATEJKO, Alexander J. (R) KRAHN, Harvey J. LOWE, Graham S.

Work, Industry and Canadian Society. Scarborough, Nelson Canada, 1988, 310 pp., ISBN 017-603414-5, recensé par Alexander J. Matejko.

v. 43, no 3,1988, p. $718-719$ 


\section{AUDET, Claude \\ VINET, Alain}

Bureautique et organisation du travail de secrétariat dans la fonction publique québécoise. v. 43, no 4,1988 , p. $737-756$

L'étude s'intéresse aux modifications survenues dans l'organisation du travail de secrétariat suite à l'implantation dans la fonction publique québécoise de la technologie bureautique du traitement de texte. On essaje de déterminer si l'introduction du traitement de texte a favorisé une accentuation de la division et de la spécialisation du travail de secrétariat - 8 travaux, références

\section{KUMAR, Pradeep}

Estimates of Unionism and Collective Bargaining in Canada.

v. 43 , no 4,1988 , p. $757-779$

Description du nouveau mode de calculs tiré d'une étude complémentaire récente de l'Enquête sur la population active du Canada, soit une enquête sur l'adhésion syndicale (EAS) datant de décembre 1984. En comparant ces statistiques tirées de la nouvelle enquête de Statistique Canada avec celles utilisées habituellement pour calculer les effectifs syndicaux et la densité syndicale, on constate une variation importante dans les différentes évaluations - 8 tableaux, références.

\section{GILES, Anthony} MURRAY, Grégor

Towards an Historical Und'crstanding of Industrial Relations Theory in Canada.

v. 43 , no 4,1988 , p. $780-811$

La compréhension des approches théoriques peut être enrichie par une approche historique laquelle cherche à relier les développements théoriques particuliers des différentes périodes au développement historique des relations industrielles canadiennes. Identification des quatre périodes de développement théorique. L'approche systémique développée dans la période d'après-guerre trace maintenant la voie à l'émergence de nouvelles approches - Références.

\section{LACROIX, Robert LESPÉRANCE, André}

Les nouvelles lois du travail et l'activité de grève.

v. 43, no 4,1988, p. $812-828$
On évalue l'effet de deux types de lois du travail sur l'activité de grève, à savoir celles qui visent à réduire telle activité et celles qui peuvent l'affecter indirectement par leur incidence sur le rapport de force syndical-patronal. On montre que le meilleur modèle pour anticiper l'effet de diverses lois du travail sur l'activité de grève est le "modèle d'accident" de la grève - 1 tableau, bibliographie.

\section{BEAUMONT, P.B.} HARRIS, R.I.D.

High Technology Industries and Non-Union Establishments in Britain.

v. 43 , no 4,1988 , p. $819-846$

L'étude s'efforce de remédier aux déficiences actuelles de la recherche en Grande-Bretagne où les débats concernant le syndicalisme dans les industries de haute technologie se sont toujours limités à des études courtes et partielles tirées de l'industrie de l'électronique. En se fondant sur la définition officielle des industries de haute technologie, on vérifie si réellement ce secteur regroupe une forte proportion d'entreprises non syndiquées - 7 tableaux.

\section{MAC NEIL, Michael}

Unions, Politics and Law in Canada.

v. 43, no 4,1988 , p. $847-868$

Le mouvement syndical est engagé dans un processus de réévaluation de son rôle au sein de la société canadienne. On passe en revue quelques unes des options que les syndicats ont choisies dans le passé et on examine certains types d'activité politique vers lesquels ils peuvent aujourd'hui s'orienter. L'accent est mis sur les contraintes juridiques pouvant entraver l'action politique des syndicats - 2 tableaux.

\section{MATON, Bob}

Socio-Technical Systems: Conceptual and Implementation Problems

v. 43 , no 4 , 1988 , p. $869-889$

Comment peut-on expliquer la résistance venant à la fois des travailleurs et de la direction quant à l'implantation des systèmes socio-techniques (SST). Pour plusieurs, les SST sont considérés comme une alternative capable de répondre aux exigences du taylorisme. Les SST proposent de remplacer les rapports humains rigides des entreprises taylorisées 
Relations Industrielles, vol. 45, no 4 (1990)

par des relations plus souples et suggèrent un nouvel équilibre entre les aspirations des travailleurs et les exigences technologiques - Bibliographie.

\section{WEST, Leigh}

The Exclusive Remedy Provision in Canadian Worker Compensation Law: The Need for Legislative Reform.

v. 43 , no 4,1988 , p. $890-908$

Les diverses lois sur les accidents du travail au Canada enlèvent aux travailleurs la possibilité d'intenter une action en dommages contre les employeurs. La Charte des droits et libertés a donné lieu à diverses offensives pour faire déclarer inconstitutionnelle la clause d'exclusion des employeurs en matière de responsabilité civile. On propose quelques corrections à apporter au système de compensation des accidents du travail tout en conservant intacte l'intégrité du système.

\section{AOUST, Claude d'}

L'amnistie des fautes disciplinaires.

v. 43 , no 4,1988 , p. $909-942$

L'amnistie des fautes du salarie en efface les conséquences, en ce qu'elles sont restées impunies, ou que la sanction, partiellement ou entièrement purgée, n'est plus retenue contre lui. On étudie l'effet atténuant de l'écoulement du temps qui, à la limite, rend inopposable aujourd'hui une faute qu'on aurait dû reprocher hier au salarié ou, en certain cas, qui lui a même mérité une sanction.

0691 MORIN, Fernand

Un préavis de licenciements ou son équivalent.

v. 43 , no 4,1988 , p. $943-952$

L'article 82 de la Loi sur les normes du travail confère au salarié un droit à un préavis de licenciement ou de mise à pied en fonction de la durée de son service. Analyse de la jurisprudence et de certaines interprétations judiciaires données en marge de l'article 82 de la Loi sur les normes du travail.

0692 MARLEAU, Véronique $L$. Décisions rendues par le Conseil canadien des relations du travail.

v. 43 , no 4,1988 , p. $953-962$

Résumé de deux décisions du Conseil dans lesquelles celui-ci précise les conditions d'exercice du droit de refus par un employé de travailler en cas de danger associé, dans le premier cas, à la violence qui peut se manifester sur une ligne de piquetage, et dans le second cas, à la possibilité d'une attaque terroriste.

0693 VANIÉ, Roger Z. (R)

MEUNIER, Pierre-Marc LAFLAMME, Marcel

Dynamisme humain dans l'excellence organisationnelle. Montréal, Agence d'Arc, 1987, 431 pp., ISBN 2-89022-120-2, recensé par Roger Z. Vanié.

v. 43, no 4,1988, p. $972-973$

\section{LEMELIN, Maurice (R)}

TIZIANO, Treu et al.

Public Service Labour Relations : Recent Trends and Future Prospects. Genève, Bureau international du travail, 1987, $287 \mathrm{pp}$., ISBN 92-2-106049-7, recensé par Maurice Lemelin.

v. 43 , no 4,1988 , p. 974

0695 BARRÉ, Alain (R)

GAGNON, Robert P. LEBEL, Louis

VERGE, Pierre

Droit du travail. Québec, Presses de l'Université Laval, 1987, 933 pp., ISBN 2-7637-7123-

8, recensé par Alain Barré.

v. 43, no 4,1988 , p. $974-980$

0696 ROSE, Joseph B. (R)

LIPSKI, David B. DONN, Clifford B.

Collective Bargaining in American Industry. Lexington, Lexington Books, 1987, 351 pp., ISBN 0-669-12595, recensé par Joseph B. Rose.

v. 43 , no 4,1988 , p. $980-981$

\section{BARBASH, Jack (A)}

New Departures in Industrial Relations : Developments in the U.S., the U.K. and Canada. Occasional Paper published by British - North American Committee, Toronto, C.D. Howe Institute, April 1988, 74 pp., ISBN 0-89068092-2, recensé par Jack Barbash.

v. 43 , no 4,1988 , p. $981-982$

0698 SEXTON, Jean

DION, Gérarơ

L'avenir des relations industrielles dans les Ameriques: Introduction.

v. 44 , no 1,1989 , p. $1-2$ 
Ce numéro spécial comprend les douze principaux exposés présentés lors du premier Congrès des relations industrielles des Amériques qui s'est tenu à Québec du 24 au 26 août 1988 et qui était organisé par l'Association canadienne des relations industrielles (ACRI) en collaboration avec l'Association internationale des relations professionnelles (AIRP).

\section{DION, Gérard HÉBERT, Gérard \\ L'avenir du syndicalisme au Canada. \\ v. 44 , no 1,1989, p. $5-24$}

Le syndicalisme au Canada jouit d'une situation assez avantageuse par rapport à celle de la plupart des pays industrialisés, mais ies transformations rapides dans l'économie et dans la composition du marché du travail de même que les interprétations que les tribunaux donneront à la Charte canadienne des droits et libertés l'affecteront sûrement. L'avenir des syndicats dépend de la stratégie qu'ils adopteront face aux problèmes qui se dessinent pour les années 1990 - 2 tableaux.

0700 FREEMAN, Richard $B$.

What Does the Future Hold for U.S. Unionism? v. 44 , no 1, 1989, p. $25-46$

Au cours des deux dernières décennies, le taux de syndicalisation dans le secteur privé aux États-Unis s'est effondré très rapidement. On revoit les causes du déclin du syndicalisme américain et on décrit quatre scénarios relatifs aux changements possibles du syndicalisme dans l'avenir - 3 tableaux, bibliographie.

\section{FALABELLA, Gonzalo}

El sindicato en el ano 2000 temas, y desafios. v. 44 , no 1,1989, p. $47-61$

La modernisation aura des implications positives sur le développement du mouvement syndical. Celui-ci se renforcera suite à la tendance au déplacement des processus industriels vers l'Amérique Latine. Dans ce contexte, on identifie six défis que le syndicalisme aura à affronter - Bibliographie.

\section{AUDET, Michel} BÉLANGER, Laurent

Nouveaux modes de gestion et relations in dustrielles au Canada.

v. 44 , no 1,1989, p. $62-96$
Suite à l'évolution des modes de gestion des organisations, on tente d'analyser ces nouveaux paramètres organisationnels en se servant de l'approche de l'adaptation des organisations aux défis que pose l'environnement canadien des années 1990. Pour ce faire, on présente certaines dimensions de l'environnement (l'économie, la technologie et les valeurs) et les paramètres qui s'en dégagent pour en évaluer l'impact sur le système canadien des relations industrielles - 1 tableau.

\section{ICHNIOWSKI, Casey}

DELANEY, John T. LEWIN, David

The New Resource Management in U.S. Workplaces: Is it Really New and is it Only Nonunion?

v. 44 , no 1,1989, p. 97-123

Résultats de la première étude exhaustive portant sur les politiques et pratiques de gestion des ressources humaines dans les entreprises, autant syndiquées que non syndiquées, aux États-Unis, Contrairement aux prétentions de certains observateurs, les résultats suggèrent qu'il est pour le moins prématuré de parler de l'existence d'un "nouveau modèle" de gestion des ressources humaines dans les entreprises non syndiquées - 4 figures, 2 tableaux, références.

\section{APPARICIO-VALDEZ, Luis}

La gestion empresarial en latinoamérica y su impacto en las relaciones laborales

v. 44 , no 1,1989, p. $124-148$

Les relations du travail en Amérique Latine se caractérisent par la présence traditionnelle d'un autoritarisme étatique ainsi que par un conflit permanent. De nouvelles tendances, encore embryonnaires, commencent cependant à faire leur apparition. La gestion patronale a joué un rôle important dans cette évolution. On présente ces nouvelles tendances et on identifie les facteurs internes et externes qui les ont générées - Références.

\section{MELTZ, Noah M.}

Job Security in Canada

v. 44, no 1, 1989, p. 149-161

Environ le tiers des salariés couverts par les grandes conventions collectives au Canada jouissent d'une certaine forme de sécurité d'emploi par la présence, dans les conventions, de clauses réglementant le recours à la 
sous-traitance, favorisant l'application du critère d'ancienneté en cas de licenciement etc. Les clauses de sécurité d'emploi prennent davantage d'importance de sorte qu'on peut s'attendre à une certaine continuité en cette matière - 3 tableaux.

0706 LOVELL, Malcolm R. Jr. Employment Security

v. 44 , no 1. 1989, p. $162-176$

Analyse d'un certain nombre de programmes sociaux qui touchent la sécurité d'emploi aux États-Unis. Les programmes publics et les efforts du secteur privé visant à aider les désavantagés à mieux se défendre sur le marché du travail ont connu un succès marginal. C'est à ce domaine que la société américaine doit donner une priorité particulière si l'on veut que la sécurité d'emploi devienne une réalité pour tous - 4 tableaux.

0707 ZAPATA, Francisco

La proteccion del empleo en las américas.

v. 44 , no 1,1989, p. $177-194$

La protection de l'emploi en Amérique Latine: a) Les principales caractéristiques de l'évolution de la main-d'oeuvre en Amérique Latine de 1950 à 1980 . b) L'impact sur l'emploi de la crise économique que traversent les pays latino-américains et la mise en place de programmes d'urgence pour faire face au chômage généré par cette crise. c) Les implications de la politique de rationalisation productive (ou reconversion industrielle) sur le marché du travail - 9 tableaux.

\section{SACK, Jeffrey}

$$
\text { LEE, Tanya }
$$

The Role of the State in Canadian Labour Relations.

v. 44 , no 1,1989 , p. $195-223$

Historique du rôle de l'État dans les relations du travail au Canada. De 1944 à la fin des années 1970, la législation canadienne a protégé la négociation collective en reconnaissant le droit d'association, le droit de grève et l'obligation pour l'employeur de négocier. Dans les années 1980, l'État s'est immiscé dans le processus général de négociation, incluant même l'utilisation de la loi de retour au travail spécialement dans le secteur public. Suggestions pour améliorer le système de base de négociation collective - 1 tableau.

\section{KOCHAN, Thomas A.} MCKERSIE, Robert $B$.

Future Directions for American Labor and Human Resources Policy.

v. 44 , no 1, 1989 , p. $224-248$

Revue des quatre approches faisant actuellement l'objet de discussions quant à l'aveñir de la politique américaine dans les domaines du travail et des ressources humaines: a) La poursuite des politiques de déréglementation et de laisser-faire des années 1980. b) Le retour à une politique du travail plus étendue et plus intensive. c) Un réengagement envers les principes du "New Deal". d) Une approche intégrée reliant les stratégies macroéconomiques à une nouvelle politique en matière de relations du travail et de ressources humaines.

0710 LUCENA, Hector

Papel del Estado en las relaciones industriales en América Latina

v. 44 , no 1,1989 , p. $249-282$

Le cadre dans lequel évoluent les relations industrielles en Amérique Latine est caractérisé par l'interventionnisme étatique. Les pays analysés ici sont l'Argentine, le Brésil, la Colombie, le Mexique, le Pérou, l'Uruguay et le Vénézuéla. Dans tous ces pays, le rôle de l'État évolue en vue de promouvoir un développement qui puisse assurer le succès de l'économie, dans un contexte qui n'est pas trop perturbé par des conflits sociaux ou de travail - 4 tableaux, bibliographie.

0711 VERGE, Pierre (R)

SPYROPOULOS, $\mathrm{G}$

Trade Unions Today and Tomorrow. 2 vol, Masestricht, Presses universitaires européennes, 1985 et 1987, 266 et 212 pp., ISBN 9070776-17-0 et ISBN 90-70776-18-9, recensé par Pierre Verge.

v. 44 , no 1,1989, p. $287-289$

0712 JAIN, Hem C. (R)

FOOTE WHITE, William

KING WHITE, Kathleen

Making Mondragon : The Growth and Dynamics of the Worker Cooperative Complex. Ithaca, ILR Press, 1988, 317 pp., ISBN 0 87546-137-9, recensé par Hem C. Jain. v. 44 , no 1,1989, p. $289-291$ 
0713 BÉLANGER, Gérard ( $R$ )

BROWN, Malcolm C.

Caring for Profit : Economic Dimensions of Canada's Health industry. Vancouver, The Fraser Institute, 1987, 182 pp., ISBN 0-88975106-04, recensé par Gérard Bélanger.

v. 44 , no 1,1989 , p. $291-292$

0714 MATEJKO, Alexander J. (R)

PETERS, TOM

Thriving on Chaos. Handbook for a Managerial Revolution. New York, Alfred A. Knopf, 1987, 561 pp., ISBN 0-394-56784-6, recensé par Alexander J. Matejko.

v. 44 , no 1,1989, p. 292-293

0715 SOLOMON, Norman A. (R)

GERHART, Paul F.

Saving Plants and Jobs : Union Management Negotiations in the Context of Threatened Plant Closing. Kalamazoo, W.E. Upjohn Institute for Employment Research, 1987, 109 pp., ISBN 0-88099-047-3, recensé par Norman A. Solomon.

v. 44 , no 1, 1989, p. 293-294

0716 MATEJKO, Alexander J. (R)

SMUCKER, Joseph VAN DEN BERG, Axel

Capitalism vs Socialism? Canadian and Swedish Labour Market Policies Compared. Working Papers in Social Behaviour, no 88-8, Montréal, McGill University, 1988, 75 pp., recensé par Alexander J. Matejko.

v. 44 , no 1,1989 , p. 294-295

0717 THWAITES, James (R)

WINDMULLER, John P. GLADSTONE, Alan Employers' Associations and Industrial Relations : A Comparative Study. Oxford, Clarendon Press, 1986, 370 pp., ISBN 0-19-827260$X$, recensé par James Thwaites.

v. 44, no 1,1989, p. $295-297$

0718 PORTIS, Bernard $(\mathrm{R})$

KLEINER, Morris M. BLOCK, Richard N. ROOMKIN, Myron SALSBURG, Sydney W. Human Resources and the Performance of the Firm. Madison, Industrial Relations Research Association Series, 1987, 343 pp., ISBN a 913447-382, recensé par Bernard Portis.

v. 44 , no 1,1989, p. 297

0719 MORIN, Fernand

D'un entrepreneur à un autre: l'accréditation ne suit pas

v. 44 , no 2,1989, p. $315-336$
Un donneur d'ouvrage confie le même service à un autre entrepreneur spécialisé, ce dernier ne peut être lié par l'accréditation et la convention collective du prédécesseur-concurrent. Telle serait la portée pratique d'un récent jugement de la Cour suprême du Canada commenté ici. L'approche retenue par la Cour pour justifier cette révision, celle de la question juridictionnelle, y est fortement critiquée parce qu'elle inciterait les tribunaux judiciaires à servir d'instances d'appel là où le législateur voulut écarter cette voie.

\section{CARTER, Donald D.}

Grievance Arbitration and the Charter: The Emergency Issues.

v. 44 , no 2,1989 , p. $337-353$

La Charte canadienne des droits et libertés a eu comme effet de jeter une ombre sur nos institutions établies en relations du travail. On tente d'établir dans quelle mesure la Charte peut s'appliquer au mécanisme d'arbitrage des griefs et d'expliquer les difficultés pour les arbitres et les juges d'obtenir un consensus clair sur le sujet.

0721 WILS, Thierry LABELLE, Christiane GUÉRIN, Gilles

LE LOUARN, Jean-Yves

La gestion stratégique des ressources humaines: Un reniement du róle social de l'entreprise?

v. 44, no 2,1989, p. $354-375$

Jusqu'à présent, l'objet de la gestion stratégique des ressources humaines (GSRH) en tant que domaine de recherche a été principalement d'étudier l'alignement des pratiques de gestion des ressources humaines (GRH) sur la stratégie d'entreprise. Or, les études empiriques indiquent que la stratégie d'entreprise n'explique pas toute la variation des pratiques de GRH. Afin de réduire cet inexpliqué, on propose une reformulation du cadre théorique de la GSRH - 7 graphiques, bibliographie.

\section{SCHELL, Bernadette LEBRASSEUR, Rolland RENAUD, Robert}

Predictors of Acceptance of Early Retirement Offers for Workers.

v. 44, no 2,1989 , p. $376-392$

Résultats d'une enquête par questionnaire ayant pour but de mettre au point une équation qui pourrait réussir à distinguer les tra- 
vailleurs qui acceptaient la préretraite de ceux qui refusaient l'offre des entreprises. L'étude se fonde sur huit facteurs qui peuvent contribuer à la formation des comportements et au choix de la décision de prendre ou non sa retraite - 2 tableaux, références.

\section{FORREST, Anne}

The Rise and Fall of National Bargaining in the Canadian Meat-Packing Industry.

v. 44, no 2, 1989, p. 393-408

Depuis près de quarante ans, les négociations collectives multipatronales avaient soustrait les salaires à la concurrence dans l'industrie des abattoirs au Canada, mais ce régime s'est effondré en 1984 sous la pression des employeurs. Ces derniers exigèrent les négociations unité par unité pour vaincre la forte opposition du syndicat. Les facteurs qui, en quatre ans, ont amené l'effondrement d'une structure qui semblait à toute épreuve - Références.

0724 SALES, Carol A. LEVANONI, Eliahu KNOOP, Robert

Employee Penformance as a Function of Job Orientation and Job Design.

v. 44 , no 2,1989, p. $409-420$

L'étude examine l'orientation des tâches en tant que substitut à la force du désir de progrès (FDP) comme modérateur entre les exigences de l'emploi, le rendement, la satisfaction au travail et le degré de motivation de l'employé. L'échantillonnage de l'enquête comprenait 333 salariés appartenant à 47 groupes de travail et leurs contremaitres dans 18 organisations diversifiées - 3 tableaux, références.

\section{GAGNON, Yves-Chantal LANDRY, Maurice}

Les changements technologiques: Une stratégie d'étude exploratoire

v. 44 , no 2,1989 , p. $421-447$

Après avoir identifié deux catégories différentes d'études exploratoires, on expose et discute une stratégie d'investigation reliée à l'implantation de changements technologiques dans les milieux syndiqués. La stratégie d'étude exploratoire présentée et illustrée ici, fait appel à des concepts qui sont au coeur de la théorie des organisations élaborée par Crozier et Friedberg (1977) - 1 graphique, références.
0726 MARLEAU, Véronique $L$.

Décisions rendues par le Conseil canadien des relations du travail.

v. 44, no 2, 1989 , p. $448-462$

Saisi de cinq requêtes complexes impliquant un groupe important de professionnels, le Conseil effectue pour la première fois une étude exhaustive des dispositions du Code canadien du travail qui traitent du statut des professionnels dans la détermination des unités de négociation et adopte une interprétation restrictive de la notion de "professionnel" au sens du Code.

0727 GUNDERSON, Morley (R)

WALKER, Michael A.

Freedom, Democracy and Economic Welfare. Vancouver, the Fraser Institute, 1988, 369 pp., ISBN 0-88975-116-1, recensé par Morley Gunderson.

v. 44, no 2, 1989, p. $465-466$

0728 BRETON, Réjean (R)

AUST, A.E.

Le contrat d'emploi. Cowansville, Les Editions Yvon Blais, 1988, 235 pp., ISBN 2-89073-675-

$X$, recensé par Réjean Breton.

v. 44 , no 2,1989, p. $466-468$

0729 CARTER, Donald D. (R)

HOLT, James

Compulsory Arbitration in New Zealand - The First Forty Years. Auckland, Auckland University Press, 1986, 247 pp., ISBN 1-86940-0062, recensé par Donald D. Carter.

v. 44 , no 2,1989 , p. $468-469$

0730 MERCIER, Jean $(R)$

LAROCQUE, Alain BORDELEAU, Yvan

BOULARD, René FABI, Bruno

LAROUCHE, Viateur RONDEAU, Alain

Technologies nouvelles et aspects psychologiques. Sillery, Presses de l'Université du Québec, 1987, 171 pp., ISBN 2-7605-0450-6, recensé par Jean Mercier.

v. 44 , no 2,1989, p. $469-470$

0731 MATEJKO, Alexander J. (R) GATTIKER, Urs E. LARWOOD, Laurie Managing Technological Development. Strategic and Human Resources issues. Berlin, Walter de Gruyter, 1988, 232 pp., ISBN 3110110849, recensé par Alexander J. Matejko.

v. 44 , no 2,1989, p. $470-472$ 
0732 SEXTON, Jean (R)

JOBIN, Carol

Les relations du travail dans lindustrie de la construction. Montréal, Wilson \& Lafleur, 1989, 568 pp., ISBN 2-89127-085-1, recensé par Jean Sexton.

v. 44, no 2,1989 , p. 473

\section{LARRIVÉE, Liette}

\section{AMBROISE, Gérald d'}

Difficultés de recrutement dans les PME québécoises: Quelques causes et solutions possibles.

v. 44 , no 3,1989 , p. $487-506$

Résultats d'une étude visant à mieux comprendre le problème de recrutement de main-d'oeuvre de production qualifiée dans les PME québécoises: a) Comment se manifeste une difficulté de recrutement de main-d'oeuvre de production dans le sous-secteur de la fabrication de machinerie. b) Vérification de l'existence d'une difficulté de recrutement dans ce secteur et mesure de son incidence et de son intensité. c) Prévision, selon les propriétaires-dirigeants, de la probabilité que celle-ci soit encore présente dans cinq ans - 1 schéma, 2 tableaux, bibliographie.

\section{ZUREIK, Elia MOSCO, Vincent} LOCHHEAD, Clarence

Telephone Workers' Reaction to the New Technology

v. 44 , no 3,1989 , p. $507-531$

Résultats d'une enquête ayant pour but précis d'examiner les données touchant les réactions de trois catégories différentes d'employés des services de téléphonie à la suite de la mise en place de technologies qui reposent sur l'utilisation des ordinateurs: soit les communications (opératrices et services qui leurs sont rattachés), les métiers (techniciens préposés aux installations et aux réparations), les cols blancs (employés de bureau et préposés aux ordinateurs) - 2 tableaux, références.

\section{RESHEF, Yonatan}

Negotiating Wage Settlements: A Structural Approach.

v. 44, no 3,1989 , p. $532-551$

Une double analyse, à la fois structurelle et économique, a été retenue pour tenter d'expliquer les résultats des négociations salaria- les et d'identifier les facteurs, autres que les facteurs économiques, qui peuvent influencer les accords salariaux. Une analyse de 405 conventions collectives déposées au Ministère du travail de l'Alberta en 1987, montre que des variables telles la durée de la convention, la structure de négociation et la taille de l'unité de négociation influencent les ententes salariales - 3 tableaux, références.

\section{BROSSARD, Michel}

Les limites du modèle-type du fonctionnement des cercles de qualité

v. 44 , no 3,1989 , p. $552-568$

Le modèle-type utilisé pour guider le fonctionnement des cercles de qualité n'a pas le caractère général que cherche à lui attribuer la documentation sur cette forme de gestion participative dans l'entreprise. On montre, à raide d'une étude de cas, que ce modèle-type correspond à une étape spécifique ('limplantation et les premiers mois) du développement de la formule et on oriente le fonctionnement des cercles de qualité, une fois franchie la période immédiate consécutive à leur création.

\section{BEATTY, Carol GANDZ, Jeffrey}

After the Strike: Changing the Teacher-Board Relationship.

v. 44 , no 3,1989 , p. $569-592$

Les grèves chez les enseignants, tout en ayant des conséquences nocives, peuvent être curatives et permettre l'établissement de meilleures relations du travail. La présente étude traite des suites de quatre grèves où des tentatives eurent lieu en vue d'améliorer les relations professionnelles entre enseignants et conseils scolaires. Deux d'entre elles ont été un succès et les deux autres, un échec - 4 figures, références.

0738 JAIN, Harish C.

Racial Minorities and Affirmative Action/Employment Equity Légis/ation in Canada.

v. 44 , no 3,1989 , p. $593-614$

Au Canada, les minorités visibles ne sont pas assez présentes dans les secteurs public et privé, compte tenu du pourcentage de la population et de la main-d'oeuvre qu'elles représentent. Analyse de la législation fédérale et provinciale en matière d'action positive 
et d'équité dans l'emploi. Suggestions pour augmenter l'efficacité des mesures fédérales pour assurer l'équité dans l'emploi - 2 tableaux, références.

\section{FAJANA, Sola}

The Systems Approach as Theory for Multinational Industrial Reiations in Developing Countries.

v. 44 , no 3,1989 , p. $615-634$

On soulève différentes questions de nature à montrer que l'influence des multinationales dans les systèmes de relations professionnelles des pays en voie de développement est beaucoup trop forte. A moins qu'elle ne soit modifiée, la théorie des systèmes peut fort bien n'être pas en mesure d'expliquer, de prévoir et de formuler les politiques en matière de relations du travail dans ces pays en particulier ainsi que dans les autres nations en général - Bibliographie.

\section{GRAYSON, J. Paul}

The Political Consequences of Unemployment: An Application of the "Power Model" of Blue- Collar Radicalism

\section{v. 44 , no 3,1989, p. 635-653}

Pour être bien comprises, les attitudes des chômeurs, relatives à leur engagement politique, doivent être vues conncurremment avec la présence ou l'absence d'organismes bien structurés capables, selon eux, de faciliter leur action politique, de traduire et canaliser leur mécontentement, et d'améliorer leur sort. A partir de l'analyse de deux fermetures d'usines au Canada, on montre que cette approche, compatible avec le "modèle de pouvoir" du radicalisme des cols bleus, est apte à comprendre les conséquences politiques du chômage - 4 graphiques.

\section{HUGUES, Karen D.}

Office Automation: A Review of the Litterature v. 44 , no 3,1989 , p. $654-679$

Au cours des dernières années, les bureaux sont devenus le lieu principal des changements technologiques, ce qui a soulevé d'importantes interrogations sur les perspectives d'avenir des employés de bureau au Canada. Même s'il y eu beaucoup de recherches sur le sujet, on n'en a guère fait l'analyse critique. Cette étude s'efforce de combler cette lacune en se préoccupant surtout de trois points: l'emploi, la qualification et la qualité de vie au travail - Bibliographie.

\section{VALLÉE, Guylaine}

Les accords "atypiques" et le droit des rapports collectifs du travail.

v. 44 , no 3,1989 , p. $680-690$

Les accords atypiques échappent au régime juridique de la convention collective en raison des libertés prises au regard des règles de forme. Ces accords prennent la forme d'ententes écrites mais non déposées, d'ententes non ratifiées par un vole des membres du syndicat ou d'ententes verbales convenues entre un syndicat accrédité et un employeur. Esquisse des principaux problèmes d'ordre juridique soulevés par l'existence de ces accords.

\section{BARKIN, Solomon}

Human Resources Management Examines itself and its Limitations

v. 44 , no 3,1989 , p. $691-702$

Critique de deux articles publiés dans la revue 'Relations industrielles" (Québec, v. 44, no 1, 1989) sur la gestion des ressources humaines dans les entreprises américaines. Dans les deux articles, les auteurs admettent I'hypothèse de la suprématie des employeurs, de leur pouvoir de décision unilatéral et de leur autorité absolue dans les entreprises.

0744 MARLEAU, Véronique L.

Décisions rendues par le Conseil canadien des relations du travail.

v. 44, no 3,1989, p. 703-722

Résumé de deux décisions du Conseil dans le contexte de la privatisation du secteur des postes. A deux reprises, mais pour des motifs différents, le Conseil conclut que la concession de l'exploitation d'un bureau de poste avec marge brute à l'intérieur d'une pharmacie ne constituait pas une vente d'entreprise au sens du Code canadien du travail parce qu'aucun transfert au sens des dispositions relatives aux droits de successeur n'avait été établi.

0745 VERGE, Pierre ( $\mathrm{A})$

KESSLER, Francis

Le droit des conventions collectives de travail en République fédérale d'Allemagne. Francfort sur Mayenne, Peter Lang, 1988, 666 pp., 
ISBN 3-8204-1138-0, recensé par Pierre Verge. v. 44 , no 3,1989 , p. $728-729$

0746 JECCHINIS, Chris (R)

ADAM, Jan

Employment Policies in the Soviet Union and Eastern Europe. 2nd ed., London, The MacMillan Press, 1987, 224 pp., ISBN 0-33341775-5, recensé par Chris Jecchinis.

v. 44 , no 3,1989 , p. $729-730$

0747 BOIVIN, Jean (R)

AARON, Benjamin NAJITA, Joyce M.

STERN, James $L$.

Public Sector Bargaining. 2nd ed., Industrial Relations Research Association Series, Washington, Bureau of National Affairs, 1988, 334 pp., ISBN 0-87179-566-3, ISBN 0-913447-374 , recensé par Jean Boivin.

v. 44, no 3,1989, p. $730-732$

0748 AOUST, Claude d' (R)

AUDET, Georges BONHOMME, Robert

Le congédiement en droit québécois en matière de contrat individuel de travail. 2ième éd., Cowansville, Les Éditions Yvon Blais, 1988, 487 pp., ISBN 2-89073-678-4, recensé par Claude D'Aoust.

v. 44 , no 3,1989, p. $732-733$

0749 BOIVIN, Jean $(R)$

MCKELVEY, Jean T.

Cleared for Takeoff : Airline Labour Relations since Deregulation. Ithaca, ILR Press, Cornell University, 1988, 298 pp., ISBN 0-87546-110-

7 , recensé par Jean Boivin.

v. 44, no 3,1989, p. $733-734$

0750 AUDET, Michel (R)

SNOW, Charles C.

Strategy, Organization Design, and Human Resource Management. Greenwich, JAI Press, 1989, 368 pp., ISBN 0-89232-807-X, recensé par Michel Audet.

v. 44 , no 3,1989, p. $734-736$

0751 CÔTÉ, André C.

Nouvelles technologies et droit du travail au Canada.

v. 44 , no 4,1989 , p. $751-768$

Les nouvelles technologies ont une profonde influence sur l'organisation du travail et provoquent la disparition et la dislocation parfois radicale des staturs ou conditions de travail. Le Canada s'interroge sur l'adéquation de ses catégories juridiques face aux impératifs des nouvelles conditions d'exercice du travail subordonné. L'étude examine le cadre légal de même que les dispositions conventionnelles visant à encadrer et à régir la planification et l'implantation des changements technologiques.

0752 NG, Ignace

Determinants of Union Commitment Among University Faculty.

v. 44 , no 4 , 1989 , p. $769-784$

Etude des rapports entre le degré d'engagement syndical des professeurs d'université et divers facteurs comme la satisfaction au travail, la confiance en la valeur de l'action syndicale, le comportement des professeurs à l'égard de l'institution d'enseignement elle-même, certains aspects du travail et les caractéristiques démographiques. L'étude a été effectuée auprès des professeurs de l'Université de la Saskatchewan - 1 tableau, références.

\section{GILSON, Clive H.J. SPENCER, Ian S. GRANVILLE, $S$.}

The Impact of a Strike on the Attitudes and Behavior of a Rural Community.

v. 44, no 4,1989 , p. $785-804$

A partir de lanalyse d'une grève touchant onze employée: d'une succursale bancaire à Antigonish en Nouvelle-Ecosse, l'étude vise à mesurer l'impact d'une grève sur le comportement d'une communauté rurale. Jusqu'à quel point la population de la ville a-t-elle donné son appui aux grévistes? L'influence de la grève sur l'attitude des gens postérieurement à l'arrêt de travail - 4 tableaux, bibliographie.

0754 LONG, Richard J.

Patterns of Workplace Innovation in Canada. v. 44, no 4,1989 , p. $805-826$

Résultats d'une vaste enquête portant sur neuf modèles d'innovation en milieu de travail au Canada: participation aux bénéfices, partage des gains de productivité, rémunération du savoir, partage des tâches, enrichissement des tâches, groupes de travail semi-autonomes, cercles de qualité, comités patronaux-ouvriers et autres groupes de règlement des difficultés. Pour chaque type d'innovation, on évalue les modèles d'implantation, leur durée de survie et les tendances dans leur mise en oeuvre - 4 tableaux, références. 
0755 ANDIAPPAN, P. CRESTOHL, Mark SINGH, Jang $B$.

Racial Discrimination in Employment in Canada.

v. 44 , no 4,1989 , p. $827-849$

Examen des développements récents concernant la nature des solutions juridiques en matière de discrimination raciale au Canada. A partir de l'analyse des cas de discrimination raciale publiés dans le "Canadian Human Rights Reporter" entre 1980 et 1987, on étudie trois sujets importants: le harcèlement racial, la responsabilité des employeurs pour les actes discriminatoires posés par leurs salariés, l'option entre le recours à l'arbitrage des griefs ou aux lois sur les droits de la personne comme solution à la discrimination raciale au travail - 7 tableaux.

0756 JAIN, Hem C.

AIDS: Need for Policy in the Workplace.

v. 44 , no 4,1989 , p. $850-865$

Conséquences résultant de la présence de sidéens en milieu de travail et le rôle des directeurs de personnel sur la question du SIDA et sur la nécessité d'établir des programmes d'éducation et des normes écrites en cette matière - Réiérences.

\section{WRIGHT, Michael D.}

The Legislation of Labour Relations at Canada Post.

v. 44 , no 4,1989, p. $866-883$

L'accroissement du recours aux lois spéciales de retour au travail exige de revoir la théorie traditionnelles des relations professionnelles. On aborde cette question à la lumière de la "Loi sur le maintien des services postaux" de 1987 qui marque une orientation nouvelle et majeure dans l'utilisation de telles lois.

\section{HÉBERT, Gérard}

L'état de la discipline en relations industrielles au Canada: Un brin d'histoire et une postface./The State of the Art in Industrial Relations: A Brief History and a Postcript.

v. 44, no 4,1989 , p. $884-904$

L'Association canadienne des relations industrielles (ACRI) a publié, en français et en anglais, un ouvrage composé d'études sur 'L'état de la discipline en relations industrielles au Canada:. Les péripéties qui ont marqué la préparation de l'ouvrage; les principaux problèmes soulevés par sa réalisation; les prochaines étapes à envisager dans la poursuite d'une théorie des relations industrielles.

\section{BARKIN, Solomon}

The State of the Art in Industrial Relations: A U.S. Commentary.

v. 44 , no 4, 1989, p. 905-914

Commentaires sur l'ouvrage publié par l'Association canadienne des relations industrielles (ACRI) et intitulé "Létat de la discipline en relations industrielles au Canada". L'ACRI a produit un volume remarquable, habilement rédigé, utile, intéressant et d'une grande valeur pour les étudiants en relations industrielles de partout.

\section{WEISS, Dimitri}

L'état de la discipline en relations industrielles au Canada: Un commentaire européen.

v. 44 , no 4 , 1989 , p. $914-920$

Commentaires sur l'ouvrage publié par l'Association canadienne des relations industrielles (ACRI) et intitulé 'L'état de la discipline en relations industrielles au Canada". Cet ouvrage trouvera sa place sur la même étagère que le monumental "Dictionnaire canadien des relations de travail" de Gérard Dion, au titre des contributions majeures du Canada à l'intelligence de notre domaine commun. Car sa portée, à l'instar de l'oeuvre de Dion, dépasse les frontières du pays.

\section{MORIN, Fernand}

Liberté d'expression et droit au travail: l'arbitrage de la Cour supréme du Canada.

v. 44 , no 4,1989 , p. $921-932$

Le 4 mai 1989, la Cour suprême du Canada précisait le pouvoir de réparation de l'arbitre de griefs suite à un congédiement imposé par un employeur: Cet arrêt comporte un double intérêt: la reconnaissance que la réparation par équivalence ou en espèces ne peut toujours suffire et la nécessité de contenir l'exercice des libertés garanties à la Charte canadienne des droits et libertés. Résumé de cet arrêt et commentaires.

\section{CLARKE, Graham}

Décisions rendues par le Conseil canadien des relations du travail.

v. 44, no 4,1989 , p. $933-946$ 
Résumé de deux décisions du Conseil: 1) Le Conseil juge valide le droit de refus invoqué par trois chauffeurs de la Société canadienne des postes qui ont refusé de franchir une ligne de piquetage avec leurs camions. 2) Le Conseil reconnaît le droit d'un agent négociateur de communiquer avec les employés sur les lieux du travail en utilisant les services internes de distribution du courrier et des tableaux d'affichage appartenant à l'employeur.

\section{TRUDEAU, Gilles ( $\mathrm{A}$ )}

l.abour Law under the Charter. Kingston, Ontario, Queen's Law Journal and Industrial Relations Center. 1988, 332 pp., recensé par Gilles Trudeau.

v. 44, no 4,1989, p. $953-957$

0764 THOMPSON, Mark (R)

WOLTERS, Roger HOLLEY, William $H$.

Labour Relations : An Experiential and Case Approach. New York, the Dryden Press, 1988, 321 pp., ISBN 0-03-069304-7, recensé par Mark Thompson.

v. 44 , no 4,1989 , p. $957-958$

0765 VERGE, Pierre (R)

ARTHURS, H.W. CARTER, D.D.

EUDGE, F. GLASBEEK, H.J.

Labour Law and Industrial Relations in Canada. 3rd ed., Toronto, Butterworths, 1988, 341 pp., ISBN 0-409-88891-5, ISBN 90-6544389-4 (Kluwer), recensé par Pierre Verge.

v. 44 , no 4,1989 , p. 959

0766 TUCKER, Eric (R)

DAWSON, Sandra WILLMAN, Paul

BAMFORD, Martin CLINTON, Alain

Safety at Work: The Limits of Self-Regulation. Cambridge, Cambridge University Press, 1988, 310 pp., ISBN 0-521-35497-8, recensé par Eric Tucker.

v. 44 , no 4,1989 , p. $959-961$

0767 MATEJKO, Alexander J. (R)

FINCHAM, Robin RHODES, Peter S.

The Individual, Work and Organization. Behavioural Studies in Business and Management Students. London, Weidenfeld and Nicolson, 1988, 336 pp., ISBN 0-297-79139-7, recensé par Alexander J. Matejko.

v. 44, no 4,1989, p. $961-962$
0768 ZAORÉ, Roger (R)

TURCOTTE, Pierre R.

La QVT une voie vers l'excellence. Montréal, Les Éditions Agence d'Arc, 1988, 276 pp., ISBN 289022-134-2 et ISBN 2-7624-0066-X, recensé par Roger Zaoré.

v. 44 , no 4,1989 , p. $962-963$

0769 PORTIS, Bernard (R)

VECCHIO, Robert P.

Organizational Behavior. Chicago, Toronto, The Dryden Press, 1988, 576 pp., ISBN 0000853-0, recensé par Bernard Portis.

v. 44 , no 4,1989 , p. $963-964$

0770 MATEJKO, Alexander J. (R)

PASMORE, William A.

Designing Effective Organizations. The Sociotechnical Systems Perspective. Wiley Series on Organizational Assessment and Change, New York, John Wiley, 1988, 200 pp., ISBN 0471-88785-4, recensé par Alexander J. Matejko.

v. 44, no 4,1989 , p. $964-967$

\section{ROSS, Claudette}

BROSSARD, Michel

La conciliation volontaire est-elle plus efficace que la conciliation obligatoire? Le cas du Québec

v. 45 , no 1,1990 , p. $3-21$

Les grandes lignes du débat sur l'efficacité de la conciliation volontaire et de la conciliation obligatoire au Québec. Étude de l'efficacité des deux régimes en utilisant plus de 3 500 dossiers de conciliation déposés au ministère du travail avant et après 1978. L'impact réel du régime légal sur l'efficacité de la conciliation - 1 graphique, 2 tableaux, bibliographie.

0772 DILTS, David A. KARIM, Ahmad

The Effect of Mediators' Qualities and Strategies on Mediation Outcomes

v. 45, no 1,1990, p. $22-37$

L'étude se fonde sur une analyse discriminante pour déterminer si ies qualités personnelles des médiateurs et les stratégies qu'ils utilisent permettent de prédire le succès de la médiation dans le règlement des impasses dans le secteur public en lowa. Les données sont tirées de questionnaires distribués aux négociateurs patronaux et syndicaux dans 214 situations d'impasse qui se sont produites en lowa en 1986 - 4 tableaux, références. 
Relations Inoustrielles, vol. 45, no 4 (1990)

0773 SAVOIE, Dominique

LAROUCHE, Viateur

Le harcèlement sexuel au travail: Résultats de deux études québécoises.

v. 45 , no 1,1990, p. $38-62$

Résultats de deux études québécoises menées en 1983 et 1986 sur le harcèlement sexuel au travail présentés sous sept rubriques distinctes: types de comportements harcelants; caractéristiques de la personne harcelée; caractéristiques du harceleur; lien d'autorité existant entre la personne harcelée et le harceleur; réactions des personnes harcelées, des tiers et des harceleurs; conséquences vécues par les personnes harcelées et les harceleurs; caractéristiques des milieux du travail des personnes harcelées - 6 tableaux.

0774 SMITH, Anthony E.

Innovation by Negotiation: Case Studies Among British White-Collar Unions.

v. 45 , no 1,1990, p. $63-75$

En Grande-Bretagne, l'influence des syndicats d'employés de bureau (cols blancs) sur l'implantation des changements technologiques dépend de la nature des rapports existant entre les employeurs et les syndicats. Là où les relations harmonieuses prévalaient, l'employeur était souvent disposé à faire des concessions en vue d'un consensus, ce qui n'était pas le cas là où conflits et antagonisme caractérisaient les relations du travail - Références.

\section{STRATTON, Kay RESHEF, Yonatan}

Private Sector Unions and Strategic Planning: A Research Agenda

v. 45 , no 1,1990, p. $76-93$

Pour enrayer l'érosion de la position du syndicalisme dans les années qui viennent, les syndicats devront s'engager dans une planification stratégique comparable à celle établie dans les entreprises. La décision de développer des plans stratégiques dépend des qualités des dirigeants du syndicat et des ressources de l'organisation. Cinq hypothèses de recherche ayant pour objet la nécessité de ressources organisationnelles - Références.
0776 BRODY, Bernard LÉTOURNEAU, Yves POIRIER, André

Le coût des accidents du travail: État des connaissances.

v. 45 , no 1,1990, p. $94-117$

Évolution du concept des coûts indirects des accidents du travail depuis la première étude d'Heinrich en 1931 jusqu'à ce jour. Constatation d'une très grande hétérogénéité dans les résultats obtenus par les études répertoriées. Ces écarts s'expliquent par des dissemblances au niveau des définitions, des méthodologies de recherche utilisées et des populations visées - 2 graphiques, 1 tableau, bibliographie.

\section{MCWATTERS, Catherine J.}

$\mathrm{BEACH}$, Charles $\mathrm{M}$.

Factors Behind the Changes in Canada's Family Income Distribution and the Share of the Middle Class.

v. 45 , no 1,1990, p. $118-135$

L'étude vise à identifier si des changements évidents sont survenus dans les caractéristiques principales de la répartition des revenus familiaux au Canada au cours des deux dernières décennies, et plus particulièrement dans la part des revenus de la classe moyenne. Revue des explications possibles et des déterminants de ces changements selon les différentes hypothèses qui ressortent de la littérature sur ce sujet et vérification de plusieurs de ces hypothèses - 3 tableaux, 1 figure, références.

0778 MUCKENBERGER, Ulrich

The Regulation of Strike Law in Times of New Technologies and Deregulation: The Case of West Germany.

v. 45 , no 1,1990, p. $136-145$

Le système des relations du travail de l'Allemagne de l'ouest se caractérise par une législation abondante. Bien que la loi de la grève soit demeurée relativement inchangée dans la dernière décennie, de nombreux amendements législatifs spécifiques (notamment en ce qui concerne le paiement des prestations d'assurance-chômage pendant un conflit de travail, la négociation collective et la représentation des employés dans l'entreprise) pourraient bien influencer l'activité de grève. On examine les effets cumulatifs de ces changements qui se produisent dans un 
contexte de changements technologiques et de déréglementation - 1 figure, 2 tableaux, références.

\section{BLACK, Errol} SILVER, Jim

Contradictions and Limitations of Final Offer Selection: The Manibota Experience.

v. 45 , no 1,1990, p. $146-165$

On analyse l'expérience manitobaine en ma tière d'arbitrage des propositions finales (APF) afin de clarifier les causes du conflit qu'il a généré. Les résultats du fonctionnement de l'APF pendant sa première année d'activité démontrent que l'APF est une pièce législative défectueuse qu'il faut abroger -5 tableaux.

0780 GRANT, Hugh M.

Contradictions and Limitations of Final Offer Selection: The Manitoba Experience. A Comment.

v. 45 , no 1,1990 , p. $166-168$

Commentaire sur l'article précédent dans lequel les auteurs Errol Black et Jim Silver font le bilan de l'expérience manitobaine en matière d'arbitrage des propositions finales.

0781 DOWNIE, Bryan M.

The Role of the State in Industrial Relations: A Comment.

v. 45 , no 1,1990 , p. $169-186$

Commentaire sur l'article de Jeffrey Sach et Tany Lee paru dans le volume 44, no 1 (1989) de "Relations industrielles" et portant sur le rôle de l'État dans les relations du travail au Canada. Le commentaire souligne que lapproche de la Colombie-Britannique et de l'Ontario en matière de règlement des conflits ne représente pas comme l'affirment Sach et Lee, un retranchement dans la politique canadienne des relations du travail - 3 tableaux, références.

0782 CLARKE, Graham

Décisions rendues par le Conseil canadien des relations du travail.

v. 45 , no 1,1990 , p. $187-197$

Résumé de deux décisions du Conseil: 1) Le Conseil décide que l'employeur a le droit d'engager des briseurs de grève plutôt que des employés non syndiqués mais membres d'une unité de négociation en grève. 2) Le Conseil donne raison à un employeur qui a envoyé une lettre directement à ses employés expliquant son offre finale puisque la lettre n'ajoutait rien à ce qui avait déjà été présenté au syndicat. De plus, le Conseil juge que le lock-out sélectif ne constitue pas une pratique déloyale.

0783 LIPSIG, Carla (R)

DAVID, Hélène

Femmes et emploi : le défi de l'égalité. Montréal, Les Presses de l'Université du Québec, 1986, 477 pp., ISBN 2-7605-0393-3, recensé par Carla Lipsig.

v. 45 , no 1,1990, p. $202-203$

0784 DÉOM, Esther $(R)$

PLASSARD, Jean-Michel

Discrimination sur le marché du travail et information imparfaite. Éditions du CNRS, France, 1987, 386 pp., ISBN 2-222-04077-9, recensé par Esther Déom.

v. 45 , no 1,1990, p. $203-204$

0785 BARBASH, Jack (R)

GLADSTONE, Alan LANSBURY, Russel D.

STIEBER, Jack TREU, T. WEISS, M.

Current issues in Labour Relations : An International Perspective. Berlin, Walter de Gruyter, 1989, 380 pp., ISBN 0-89925-471-3, recensé par Jack Barbash.

v. 45 , 701,1990, p. $204-205$

0786 GRANT, Michel (R) HECKSCHER, Charles $C$.

The New Unionism : Employee Involvement in the Changing Corporation. New York, Basic Books, 1988, 320 pp., ISBN 0-465-05098-0, recensé par Michel Grant.

v. 45 , no 1,1990 , p. $205-208$

0787 BÉLANGER, Jacques (R) HYMAN, Richard STREECK, Wolfgang New Technology and Industrial Relations. Oxford, Basil Blackwell, 1988, 309 pp., ISBN 0-631-15982-7, recensé par Jacques Bélanger. v. 45 , no 1, 1990, p. $208-209$

0788 BOIVIN, Jean (R) KOCHAN, Thomas C. KATZ, Harry C. Collective Bargaining and Industrial Relations. Irwin Series in Management and the Behaviou$\mathrm{ral}$ Sciences, Homewood, 1988, 496 pp., ISBN 0-256-03025-1, recensé par Jean Boivin.

v. 45, no 1,1990, p. 210 
0789 AUDET, Michel (R)

ROTHWELL, W.J. KAZANAS, H.C.

Strategic Human Resources Planning and Management. Englewood Cliffs, Prentice-Hall, 1988, 512 pp., ISBN 0-13-851643-X, recensé par Michel Audet.

v. 45 , no 1,1990 , p. $211-212$

0790 BOIVIN, Jean $(R)$

STAUDOHAR, Paul D.

The Sports Industry and Collective Bargaining. Ithaca, ILR Press, Cornell University, 1986, 195 pp., ISBN 0-87546-118-2, recensé par Jean Boivin.

v. 45 , no 1,1990, p. 212

0791 MATEJKO, Alexander J. (R)

KOZDROJ, Alicja

Grupa pracownicza jako przedmiot i podmiot motywowania. The Polish Academy of Sciences, Institute of Management, Wroclaw Ossolineum, 1988, 307 pp., ISBN 83-04-02806-9, recensé par Alexander J. Matejko.

v. 45 , no 1,1990, p. $213-214$

0792 GUÉRIN, Gilles

HÉBERT, Michel

Les obstacles rencontrés par des personnes de 45 aे 64 ans à la recherche d'un emploi. v. 45 , no 2,1990, p. $235-267$

La littérature suggère que les personnes âgées de 45 à 64 ans rencontrent trois groupes de difficultés dans la recherche d'un emploi: les problèmes découlant de l'accessibjlité aux programmes gouvernementaux, les pratiques discriminatoires des employeurs et les caractéristiques et faiblesses des personnes concernées. L'étude vérifie l'importance relative de ces obstacles auprès de 207 répondants. - 2 graphiques, 9 tableaux, bibliographie.

0793 LEBRASSEUR, Rolland Retirement and Skill Issues in Northern Ontario Industries.

v. 45 , no 2,1990, p. $268-282$

Résultats d'une étude consistant à définir des profils de professions et métiers pour les travailleurs de 45 à 64 ans dans l'industrie de la transformation et de la fabrication du nord de l'Ontario. Ces profils étaient composés des variables suivantes: démographique, santé, revenu présent et anticipé, et l'âge prévu et idéal de retraite. Les données utilisées ont été recueillies par Schell et al. (Rela- tions industrielles, v. 44, 1989) auprès d'employés âgés de 45 ans et plus. - 3 tableaux, références.

\section{ROY, Paul-Martel}

Aspects dynamiques du marché du travail au Québec.

v. 45, no 2,1990 , p. $283-299$

L'accord du libre-échange entre le Canada et les États-Unis entraînera vraisemblablement le déplacement d'un nombre important de travailleurs québécois. A partir des données d'une enquête spéciale de Statistique Canada, on examine d'abord la dynamique du chômage au Québec comparativement à celle de l'Ontario et de l'ensemble du Canada. On étudie ensuite la situation spécifique au Québec en se concentrant sur les travailleurs déplacés et recyclés. - 6 tableaux, bibliographie.

\section{SWIMMER, Gene}

Gender Based Differences in Promotions of Clerical Workers.

v. 45, no 2,1990 , p. $300-310$

L'objet de l'étude est de déterminer de façon empirique si les employés de bureau de sexe téminin sont moins susceptibles que les homimes occupant des posies de nature identique d'obtenir des promotions lorsque la scolarité et les autres qualifications mesurables sont comparables. Les résultats empiriques indiquent que les femmes ont beaucoup moins de chances de passer des emplois de bureau aux postes administratifs. - 3 tableaux, références.

\section{DASTMALCHIAN, Ali NG, Ignace}

Industrial Relations Climate and Grievance Outcomes.

v. 45 , no 2,1990, p. $311-325$

Résultats d'une étude examinant l'interaction entre le climat des relations du travail et l'issue finale des griefs. Les données, tirées de six organismes du gouvernement fédéral de l'Ouest du Canada, indiquent que là où les relations du travail sont bonnes, il y a davantage de probabilité que les griefs soient accueillis. - 2 tableaux, 1 figure, références. 
0797 CHAYKOWSKI, Richard P.

Union and Firm Preferences for Bargaining Outcomes in the Private Sector.

v. 45 , no 2,1990 , p. $326-356$

Afin de donner un certain aperçu de l'importance que syndicats et employeurs accordent aux différents enjeux en négociation, l'étude examine un ensemble de données uniques, tirées d'une enquête portant sur les objectifs poursuivis dans la négociation par les parties dans le secteur privé au Canada. Suit une analyse empirique exploratoire d'un ensemble d'équations caractérisant le classement que font les parties de plusieurs enjeux de négociation. - 1 figure, 4 tableaux, bibliographie.

\section{MCSHANE, Steven L. REDEKOP, Bruce}

Compensation Management and Canadian Wrongful Dismissal: Lessons from Litigation. v. 45 , no 2,1990 , p. $357-381$

La forme la plus courante de congédiements déguisés se produit lorsque l'employeur modifie de façon substantielle et unilatérale les conditions de travail d'un employé en diminuant, retirant ou retenant toute partie appréciable de sa rémunération. A partir de 110 jugements récents touchant des congédiements injustes reliés a un changement des pratiques de rémunération, l'étude identifie les principes qui se dégagent de la jurisprudence en "common law" canadienne. - 4 tableaux, réferences.

\section{CUNNINGHAM, Barton MITCHELL, Lari}

Privatization in British Columbia: What the Experts Say Will Happen.

v. 45, no 2,1990, p. $382-403$

Enquête auprès de spécialistes en relations du travail ayant pour objet d'examiner les effets de la privatisation des services gouver. nementaux sur les relations du travail en Colombie-Britannique. Les conclusions de l'enquête sont que la privatisation affectera la stabilité de la main-d'oeuvre et des salaires, qu'elle amènera la fragmentation de la partie patronale à la table des négociations et qu'elle rendra l'activité syndicale financièrement plus coûteuse. - 4 tableaux.

\section{HÉBERT, Gérard}

Le renouvellement du régime des décrets de convention collective.

v. 45 , no 2,1990 , p. $404-413$

Le régime de l'extension juridique des conventions collectives s'est toujours maintenu malgré une hostilité quasi constante. Avant de se demander s'il faut renouveler cette institution, il importe de savoir si elle a une chance de survie, si les principes de base sur lesquels elle repose sont encore valables, enfin si le renouvellement dont elle pourrait avoir besoin vise des aspects substantiels et constitutifs, ou accessoires et accidentels.

0801 MARLEAU, Véronique $L$.

Décision rendue par le Conseil canadien des relations du travail.

v. 45 , no 2,1990, p. $414-423$

Saisi de la question de savoir si une personne opérant un comptoir postal est un employé au sens du Code canadien du travail, le Conseil redéfinit le test applicable en matière de détermination du statut d'entrepreneur indépendant. Le Conseil déclare donc qu'une telle personne est un entrepreneur indépendant et qu'en cette qualité, elle n'est pas un employé de la Société canadienne des poste.

0802 SWIMMER, Gene (R)

ANDERSON, John C. GUNDERSON, Morley PONAK, Allen

Union-Management Relations in Canada. (2nd ed.), Don Mills, Ont., Addison-Wesley Publishers, 1989, 498 pp., ISBN 0-201-18621-7, recensé par Gene Swimmer.

v. 45 , no 2,1990 , p. $428-429$

0803 DION, Gérard (R)

Trade Unions of the World 1989-1990. (2ieme ed.), Longman Group UK Limited, The High, Harlow, 1989, 480 pp., ISBN 0-582-03908-8, Distributed exclusively in United States and Canada by St James Press, ISBN 0-55862014-1, recensé par Gérard Dion.

v. 45 , no 2,1990 , p. $429-430$

0804 ANDIAPPAN, P. (R) KOZIARA, S MOSKOW, M.H.

TANNER, L.D.

Working Women : Past, Present, Future. Industrial Relations Research Association Series, Washington, The Bureau of National Affairs, 
Relations Industrielles, vol. 45, no 4 (1990)

1987, 419 pp., ISBN 0-913447-34-X, recensé par $P$. Andiappan.

v. 45, no 2,1990 , p. $430-431$

0805 BÉLANGER, Laurent (R)

BOONE, Louis E. KURTZ, David L.

L'entreprise d'aujourd'hui. (2ième ed.), Montréal, Editions études vivantes, 1989, 737 pp., ISBN 2-7607-0446-7, recensé par Laurent Bélanger.

v. 45, no 2,1990, p. 432

0806 MAZEROLLE, Maurice J. (R) COOK, Robert F.

Worker Dislocation : Case Studies of Causes and Cures. Kalamazoo, Michigan, W.E. Upjohn Institute for Employment Research, 1987, 219 pp., ISBN 0-88099-052-X, recensé par Maurice J. Mazerolle.

v. 45 , no 2, 1990 , p. $432-434$

0807 VERGE, Pierre (R)

DESPAX, Michel

Négociations, conventions et accords collectifs. (2ième éd.), tome sept de Droit du travail, publié sous la direction de G.H. Camerlynck, Paris, Dalloz, 1989, 586 pp., ISBN 2247-00890-2, recensé par Pierre Verge.

v. 45 , no 2,1990, p. $434-435$

0808 SEREDIAK, Martin S. (R)

BAMBER, Greg J.

LANSBURY, Russell D.

New Technology International Perspectives on Human Resources and Industrial Relations. Winchester, Unwin Hyman, 1989, 267 pp., ISBN 0-04-445123-7, recensé par Martin $S$. Serediak.

v. 45 , no 2,1990, p. $435-438$

0809 DÉOM, Esther (R)

Les principes de l'équité salariale et les approches dans le secteur public québécois. Montréal, Institut de recherche et d'information sur la rémunération, IRI-050, octobre 1989, 99 pp., ISBN 2-551-12193-O, recensé par Esther Déom

v. 45, no 2,1990, p. $438-439$

0810 MATEJKO, Alexander J. (R)

ANDERSEN, Svein $S$.

British and Norwegian Offshore Industrial Relations : Pluralism and Neo-Corporatism as Contexts of Strategic Adaptation. Aldershot, U.K., and Brookfield, Vermont, USA, Averbury/Gover, 1988, 213 pp., ISBN 0-566-055317, recensé par Alexander J. Matejko.

v. 45 , no 2,1990, p. 440
0811 DION, Gérard (R)

PAQUET, Gilles

La pensée économique au Québec français. Témoignages et perspectives. Montréal, Association canadienne-française pour l'avancement des sciences, 1989, 364 pp., ISBN 289245-987-7, recensé par Gérard Dion.

v. 45, no 2,1990 , p. $441-442$

0812 COUSINEAU, Jean-Michel

NAJEM, Elmustapha

L'effet du développement de la petite entreprise sur l'évolution du syndicalisme au Canada.

v. 45, no 3,1990, p. $467-480$

Au Canada, l'apparente stabilité du mouvement syndical dissimule une tendance de fond au déclin du syndicalisme dans le secteur privé. En complément des hypothèses traditionnellement avancées pour expliquer le déclin dans les taux de syndicalisation, on vérifie l'incidence du développement de la petite entreprise sur l'évolution des effectifs syndicaux dans quatre grands sous-secteurs du secteur privé de l'économie canadienne. - 2 tableaux, bibliographie, 1 annexe.

0813 BRUCE, Peter G.

The Processing of Unfair Labor Practice Cases in the United States and Ontario.

v. 45 , no 3,1990 , p. $481-511$

Comparaison des méthodes utilisées par le National Labor Relations Board (États-Unis) et par l'Ontario Labour Relations Board dans le traitement des affaires de pratique déloyale de travail (PDT). Comparaison de la facilité avec laquelle les plaintes des travailleurs et des syndicats en matière de PDT peuvent donner lieu à des auditions formelles devant l'une et l'autre commissions et de la rapidité avec laquelle les deux organismes procèdent à l'audition des plaintes. - 5 tableaux, bibliographie.

\section{WILS, Thierry}

BOURGEOIS, Robert-Paul

LABELLE, Christiane

Planification des ressources humaines dans la fonction publique fédérale.

v. 45, no 3,1990 , p. $512-530$

La planification des ressources humaines en tant que nouveau style de gestion des ressources humaines est en train d'émerger 
dans le secteur privé. A partir d'une enquête par entrevue et par questionnaire, la présente recherche indique que la planification des ressources humaines à fait son apparition dans certaines parties de la fonction publique fédérale. - 6 tableaux, références.

\section{WALTERS, Vivienne} DENTON, Margaret

Workers' Knowledge of their Legal Rights and Resistance to Hazardous Work.

v. 45 , no 3, 1990 , p. 531.547

Résultats d'une étude portant sur les connaissances, les perceptions et les actions des travailleurs en matière de santé et de sécurité au travail. l'étude visaít à découvrir s'il existe une corrélation entre leur connaissance de la loi et leurs actions face à des risques. Pour ce faire, on a interviewé 492 travailleurs provenant de huit établissements du sud de I'Ontario. - 3 tableaux, bibliographie.

0816 LANGLOIS, Simon

Le travail à temps partiel: Vers une polarisation de plus en plus nette.

v. 45, no 3,1990, p. $548-565$

Analyse de l'évolution du travail à temps partiel depuis 1975 au Québec. La polarisation de l'emploi à temps partiel résulte de la rencontre entre deux types de transformations sociales: celles qui affectent les entreprises et le marché du travail et celles qui marquent les modes de vie et les préférences des individus. - 4 tableaux, références.

\section{MORIN, Fernand}

Modification unilatérale des conditions de travail au terme d'une négociation collective! v. 45 , no 3,1990 , p. $566-584$

À l'expiration d'une convention collective et au terme d'une négociation infructueuse, les salariés maintiennent néanmoins leur prestation de travail et l'employeur modifie seul leurs conditions de travail. Commentaires sur la décision de la Cour suprême du Canada dans l'affaire CAIMAW contre Paccar of Canada Ltd. 1) La difficile identification d'une décision manifestement déraisonnables. 2) Les limites aux initiatives faites de bonne foi. 3) L'effet juridique de la convention collective auprès des salariés.
0818 CARROTHERS, A.W.R.

Labour Law Through the Prism of Paccar v. 45 , no 3, 1990, p. 585-611

Commentaires sur la décision majoritaire de la Cour suprême du Canada dans l'affaire CAIMAW contre Paccar of Canada Ltd. Dans cette affaire, les parties voulaient savoir si l'employeur pouvait modifier unilatéralement les conditions de travail alors que la convention collective était expirée, mais avant que l'employeur ait recouvré le droit au lock out.

\section{BLACK, Errol} SILVER, Jim

FOS in Manitoba. A Rejoinder. v. 45 , no 3,1990 , p. $612-615$

Dans un premier article paru dans le volume 45, no 1 de "Relations industrielles", les auteurs analysaient l'expérience manitobaine en matière d'arbitrage des propositions finales. Cette analyse faisait ensuite l'objet d'une critique rédigée par Hugh $M$. Grant, critique que les auteurs n'ont pas appréciée. Dans le présent article, les auteurs répliquent à cette critique de M. Grant.

\section{CLARKE, Graham}

Décision rendue par le Conseil canadien des relations du travail.

v. 45 , no ?, 1990, p. $616-624$

Quatre employeurs fusionnent pour ne former qu'une seule entreprise et demandent au Conseil à être déclarés employeur unique. Analyse de la décision du Conseil qui a examiné si le Code canadien du travail lui permettait de former une unité de négociation globale composée d'unités accréditées et d'unités volontairement reconnues.

0821 LEQUIN, Jacques-André (R)

BOIVIN, Jean GUILBAULT, Jacques

Les relations patronales-syndicales. Boucherville (Québec), 2ième éd., Gaétan Morin éditeur, 1989, 301 p. ISBN 2-89105-322-2, recensé par Jacques-André Lequin.

v. 45 , no 3,1990 , p. $627-629$

\section{GILES, Anthony (R)}

WINDMULLER, John P.

Collective Bargaining in Industrialised Market Economies: A Reappraisal. Geneva, international Labour Office, 1987, 333 p., ISBN 92-2105606-6, recensé par Anthony Giles.

v. 45 , no 3,1990 , p. 630 
Relations Inoustrielles, vol. 45, no 4 (1990)

0823 JAIN, Hem C. (R)

CHALMERS, Norma $\mathrm{J}$.

Industrial Relations in Japan. The Peripheral Workforce. New York, Routledge, Chapman \& Hall, 1989, 283 p., ISBN 0-415-00008-4, recensé par Hem C. Jain.

v. 45 , no 3,1990 , p. $631-632$

0824 VINET, Alain (R)

PLASSE, Micheline

Santé et sécurité du travail. Montréal, Agence d'Arc inc., 1988, 155 p., ISBN 2-89022-138-5, recensé par Alain Vinet.

v. 45, no 3,1990, p. $632-633$

0825 MATEJKO, Alexander J. (R)

HARRIS, Rosemary

Power and Powerlessness in Industry: An Analysis of the Industrial Relations of Production. London, U.K., Tavistock Publ., 1987, pp. VIII + 245, ISBN 0-422-609-X, recensé par Alexander J. Matejko.

v. 45 , no 3,1990 , p. 634

\section{ZAORÉ, Roger (R)}

BEATTY, Carol

The Implementation of Technological Change. Kingston, Industrial Relations Centre, Queen's University, 1987, 137 p., ISBN 0-88886-172-9, recensé par Roger Zaoré.

v. 45, no 3,1990, p. $634-635$

\section{GASCON, Hélène (R)}

SIRARD, Ronald GAZAILLE, Alain

Comprendre et appliquer une convention collective. Montreal, Wilson et Lafleur, 1989, 165 p., ISBN 2-89127-151-3, recensé par Hélène Gascon.

v. 45, no 3,1990 , p. $635-637$

0828 BLOUIN, Rodrigue (R)

RENAUD, Marc TRUDEAU, Gilles

SAINT-JACQUES, Chantal DUBÉ, Louise

Le droit de refus: une revolution tranquille étude de la mise en oeuvre d'un nouveau droit. École des relations industrielles, Université de Montréal, Monographie, no 21, 1989, 329 p., ISBN 2-89067-020-1, ISSN 0708-9945, recensé par Rodrigue Blouin.

v. 45 , no 3,1990 , p. $637-640$

0829 MORIN, Fernand (R)

BUREAU, Robert D. MACKAY, Pierre

Le droit dans tous ses états. Montréal, Wilson \& Lafleur, 1987, 620 p., ISBN 2-8912707602, recensé par Fernand Morin.

v. 45 , no 3,1990 , p. $640-643$
0830 PRAGNELL, Brad (R)

LAFLAMME, Gilles MURRAY, Gregor

BÉLANGER, Jacques FERLAND, Gilles

Flexibilité and Labour Markets in Canada and

United States. (Research Series 94), Geneva, International Institute for Labour Studies, 1989, 317 p., ISBN 92-9014-462-9, recensé par Brad Pragnell.

v. 45 , no 3,1990 , p. $643-647$

0831 WEISS, Dimitri (R)

CROZIER, Michel

L'entreprise à l'écoute. Paris, Inter Éditions, 1990, 218 p., recensé par Dimitri Weiss.

v. 45, no 3,1990, p. $647-649$

0832 BOULARD, René

DESCHÊNES, Jean-Paul

LAROCQUE, Alain RONDEAU, Claude

Introduction - La réduction de la durée du travail.

36ième Congrès, 1981, p. 13-14

Présentation du thème du 36ième Congrès des relations industrielles qui aborde la question de la réduction de la durée du travail sous ses multiples facettes.

\section{LAROCQUE, ALAIN}

La réduction du temps de travail: un droit ou un privilege?

36ième Congrès, 1981, p. 15-29

La détermination du temps passé au travail vue à travers l'histoire. Les formes multiples de la réduction du temps de travail depuis le début du siècle: La réduction de la journée, de la semaine, de l'année et de la vie de travail. - 1 tableau, bibliographie.

\section{MONTMINY, Jean-Paul}

La signification du travail en 1980: émergence de valeurs nouvelles?

36ième Congrès, 1981, p. 31-45

Interrogations sur la signification du travail aujourd'hui et sur l'émergence de valeurs nouvelles du travail. L'État, les Syndicats, l'Église et le Patronat, constituent les principaux définisseurs des valeurs du travail. Chacun d'eux, en accord avec leur position sociale et leur idéologie particulière, propose des valeurs différentes du travail. - Bibliographie. 


\section{DUFOUR, Ghislain}

RODRIGUE, Norbert

Commentaires - La signification du travail en 1980: émergence de valeurs nouvelles?

36ième Congrès, 1981, p. 45-59

Un représentant du monde patronal et un représentant du monde syndical commentent l'exposé de Jean-Paul Montminy portant sur le sens véritable du travail à notre époque et l'émergence de valeurs nouvelles en ce domaine.

\section{VALASKAKIS, Kimon}

Le temps libére: à quels coûts?

36ième Congrès, 1981, p. 61-74

Les augmentations énormes de production générées par l'automation amènent nécessairement une réduction de la demande de travail et une augmentation du chômage structurel. La meilleure réponse à cette situation est le scénario du temps libéré qui permettrait à tout le monde de jouir des bienfaits du progrès technologique par une meilleure répartition du temps entre le travail et le loisir. On pourra ainsi créer la société des loisirs à un coût minime et réaliser "Athènes sans les esclaves'. - 3 tableaux.

\section{POULIN-SIMON, Lise}

FORTIN, Bernard

Commentaires - Le temps liberé: à quels coûts?

36ième Congrès, 1981, p. 75-86

Commentaires sur l'exposé de $M$. Valaskakis qui propose le scénario du temps libéré pour compenser les effets de la hausse de la productivité. Celui-ci est alarmiste lorsqu'il dit que l'évolution du marché du travail se traduira par une accélération du chômage structurel et sa vision est utopique en disant que l'automation affranchira l'homme du travail et lui permettra de s'épanouir dans une société de temps libéré.

\section{FERLAND, Gilles \\ HAMELIN, Jean-Marie \\ PROULX, Pierre-Paul}

Table ronde - Sommes-nous tous égaux face à la diminution du temps passé au travail? 36ième Congrès, 1981, p. 87-121

L'objectif des travailleurs de voir réduire la durée de leur travail a-t-il été atteint par tous de la même manière? Voit-on actuellement se dessiner une tendance à l'égalité pour tous dans la durée du travail? Sinon, quelles sont les principales inégalités que l'on peut constater sur les marchés du travail? Telles sont les questions auxquelles tentent de répondre les participants à cette table ronde. - 5 tableaux.

\section{MASSE, Gilles}

Le temps passé au travail: un élément encore négociable?

36ième Congrès, 1981, p. 123-135

Les horaires de travail compressibles sont en train de devenir un des éléments les plus importants des clauses marginales des conventions collectives et la possibilité de les négocier ne fait que débuter. Résumé de la situation des horaires de travail des policiers de la communauté urbaine de Montréal qui bénéficient de la semaine de quatre jours. - 3 annexes.

\section{DESCHÊNES, Jean-Paul}

Commentaires - Le temps passé au travail: un élément encore négociable?

36ième Congrès, 1981, p. 136-144

Oui, le temps passé au travail est un élément encore négociable mais pas à n'importe quel prix. La réduction des heures de travail est réalisable si elle s'accompagne d'une augmentation de la productivité. Plusieurs techniques peuvent servir à augmenter la productivité: la rationalisation du processus de productivité: la rationalisation du processus de production (étude des temps et mouvements), l'amélioration des conditions physiques de travail etc. Ces techniques ne doivent cependant pas ignorer le facteur humain, l'homme, le travailleur.

\section{BEAUSOLEIL, Gilles} GAUTHIER, Hervé

La législation: consolidation ou innovation? 36ième Congrès, 1981, p. 145-172

On tente d'évaluer le rôle de l'action gouvernementale dans la détermination de la durée du travail. On entend par action gouvernementale toute intervention, soit directe (établissement des normes légales sur la durée du travail), soit indirecte (fiscalité, programmes gouvernementaux divers). Revue des politiques pouvant influencer la durée hebdomadaire du travail et le temps consacré au 
Relations Industrielles, vol. 45, no 4 (1990)

travail au cours de la vie. Quelques trait de l'évolution future.

0842 LOCKWELL, LUC M. LORTIE, Pierre

Commentaire - La législation: consolidation ou innovation?

36ième Congrès, 1981, p. 173-192

Commentaires sur l'exposé de MM. Gilles Beausoleil et Hervé Gauthier portant sur l'effet des mesures gouvernementales sur le temps passé au travail.

0843 FRAPPIER-DESROCHERS, Monique La technologie: un substitut à la durée du travail?

36ième Congrès, 1981, p. 193-218

Que ce soit d'une façon directe ou indirecte, les développements technologiques affectent la durée du travail. L'apparition des nouvelles technologies pose la problématique suivante: d'une part, elle offre des possibilités énormes au niveau de la réduction de la durée du travail en augmentant la productivité; d'autre part, ces gains de productivité conduisent à des pertes d'emplois. - 1 graphique.

\section{AOUST, Fernand d'}

$$
\text { DÉOM, André HARVEY, Pierre }
$$

Table ronde - La réduction du temps passé au travail: un moyen de lutte contre le chómage. 36ième Congrès, 1981, p. 219-246

Quelle est la portée de la réduction de la durée du travail comme moyen de lutter contre le chômage? Les trois participants à cette table ronde expriment différents points de vue sur ce sujet. - 5 annexes.

\section{CROZIER, Michel}

La diminution du temps de travail: un phénomène inéluctable?

36ième Congrès, 1981, p. 247-263

Exposé présentant certaines contraintes qui affectent la réduction de la durée du temps au travail et situant les perspectives humaines et stratégiques qui doivent guider la recherche et l'innovation en matière de temps passé au travail.
0846 DESCHÊNES, Jean-Paul FERLAND, Gilles

SAINT-LAURENT, Jacques SEXTON, Jean

Introduction - Les régimes de retraite. 37ième Congrès, 1982, p. 13-16

Compte rendu des différents exposés présentés lors du 37ième Congrès des relations industrielles dont le thème était "Les régimes de retraites".

\section{SOLASSE, Bernard}

La retraite: votre probleme.

37ième Congrès, 1982, p. 17-34

Dans un premier temps, on essaie de préciser la notion de retraite et les principaux enjeux économiques et sociaux qu'elle recouvre. Dans un seccnd temps, on examine les principales caractéristiques de l'expérience québécoise en cette matière. Dans un troisième temps, on tente de dresser un inventaire des questions et des solutions mises de l'avant pour y remédier, en insistant sur les conditions requises pour les mettre en application.

\section{BENOIT, Michel}

Des solutions aux problèmes de la retraite.

37ième Congrès, 1982, p. 35-44

Présentation descriptive de différents rapports publiés depuis quelques années et portant sur le problème de la retraite. En s'appuyant sur ces rapports, on aborde d'une façon comparative l'acquisition du droit à la rente, le remboursement des contributions avec intérêt, la transférabilité et la protection de la rente différée contre l'inflation.

0849 FAlLLE, Jacques

Les principaux régimes de retraite et leur contenu.

37ième Congrès, 1982, p. 45-74

Présentation descriptive de trois paliers du système québécois de revenu de retraite: 1) Le régime de Sécurité de la vieillesse et du Supplément de revenu garanti; 2) Le régime de rentes du Québec; 3) Les régimes complémentaires de rentes mis sur pied en contexte employeur - employés. Les avantages et les inconvénients des différents régimes.

0850 BÉLANGER, Gérard

Les aspects économiques de la réforme des pensions.

37ième Congrès, 1982 , p. $75-98$ 
Survol de la question de la garantie financière des personnes ágées. Analyse des différents programmes du secteur public et étude des faiblesses et des lacunes des régimes privés de retraite. La réserve du régime des rentes du Québec (R.R.Q.) deviendra nulle vers l'an 2002, ce qui implique une augmentation du taux de cotisation ou une diminution des prestations. Dans les deux cas, il n'y a aucun bénéfice supplémentaire offerts aux citoyens. - 3 tableaux.

\section{DÉPATIE, Raymond}

Commentaire - Les aspects économiques de la reforme des pensions.

37ième Congrès, 1982, p. 98-105

On amène l'idée d'accroître substantiellement le rôle du R.R.Q. pour solutionner le problème du revenu des retraités. Les faiblesses et les lacunes du système actuel militent en faveur d'une intervention gouvernementale, essentielle et souhaitable. Le risque trop important pour les entreprises, la transférabilité des régimes et ses conséquences sur la mobilité de la main-d'oeuvre justifient une intervention accrue de l'État.

0852 BONENFANT, Claire GUÉRARD, Yves LAFONTAINE, Réal

LAFOREST, Martial

Table ronde - La protection du revenu à la retraite: une responsabilité partagée?

37ième Congrès, 1982, p. 107-145

Mme Bonenfant souhaite l'intervention de l'État pour éliminer les disparités qui existent entre hommes et femmes dans les régimes de retraite. M. Guérard favorise les régimes privés de retraite suite à la mauvaise performance de l'État comme gestionnaire des différents régimes. $M$. Lafontaine se fait le défenseur du R.R.Q. et invite les travailleurs à une plus grande participation à l'administration de leur régime de retraite. Pour M. Laforest, la solution se trouve dans un régime public mieux structuré. - 1 tableau, 1 graphique.

0853 HÉBERT, Hervé PERRON, Jacques POULIN-SIMON, Lise

Table ronde - Qui doit administrer les régimes de retraite: l'employeur ou le syndicat?

37ième Congrès, 1982, p. 147-169
M. Hébert suggère de confier l'administration des régimes de retraite aux plus compétents: l'employeur ou le syndicat. Selon M. Perron les employés doivent administrer majoritairement leurs régimes. Mme Poulin-Simon préfère, pour sa part, l'administration partagée, recommande la non-intervention de l'État et favorise l'amélioration du régime des rentes du Québec.

\section{BÉGIN, Monique}

Les politiques gouvernementales en matière de retraite.

37ième Congrès, 1982, p. 171-180

La politique du gouvernement fédéral en matière de retraite prévoit principalement une série de réajustements des cotisations pour éviter de vider le fond de réserve et pour établir certains aménagements en ce qui concerne ia retraite des femmes. Quant aux régimes privés, ils devront permettre la transférabilité, la couverture obligatoire pour les travailleurs et l'indexation. L'uniformité d'une province à une autre sera essentielle à une politique cohérente.

\section{PARIZEAU, Jacques}

Les politiques gouvernementales en matière de retraite.

37ième Congrès, 1982, p. 181-192

Le Ministre des finances du Québec aborde la situation urgente des régimes de retraite au Québec et au Canada. Un changement global des régimes privés apparaît nécessaire pour favoriser la mobilité de la main-d'oeuvre, spécialement au niveau de la transférabilité des régimes. Une bonification du régime des rentes est souhaitable, donnant ainsi lieu à des cotisations qui impliquent un taux de capitalisation acceptable dès le départ.

\section{GALBRAITH, John Kenneth}

Réflexions sur les problèmes de la retraite dans les années 80.

37ième Congrès, 1982, p. 193-213

À travers une vision macro-économique de la société, le professeur Galbraith se livre à une critique du "consensus" dans les pays industrialisés, consensus marqué par les interventions des grandes corporations qui inventent nos goûts, notre perception du monde et qui ont instauré avec les régimes de retraite, l'assistance sociale, et toutes les autres dis- 
positions similaires, un ordre artificiel dans le but d'amoindrir les effets tranchants du capitalisme.

\section{LAMONTAGNE, Maurice}

Commentaire - Reflexions sur les problèmes de la retraite dans les années 80.

37ième Congrès, 1982, p. 213-218

Deux questions générales et fondamentales se posent au-delà des problèmes plus spécifiques que soulèvent les régimes privés et publics de retraite. 1) Notre société pourra-t-elle procurer des revenus réels suffisants à un nombre croissant de retraités? 2) Notre société pourra-t-elle rendre les retraités plus heureux, en supposant que l'argent ne fait pas nécessairement le bonheur?

\section{RONDEAU, Claude}

\section{BÉLANGER, Jacques}

BOIVIN, Jean SEXTON, Jean

Introduction - La syndicalisation dans le secteur privé au Québec.

38ième Congrès, 1983, p. 13-14

Compte rendu des différents exposés présentés lors du 38ième congrès des relations industrielles dont le thème était "L'accès à la syndicalisation dans le secteur privé au Québec."

\section{RONDEAU, Claude}

\section{BÉLANGER, Jacques}

Le syndicalisme dans l'entreprise: tendances récentes et analyse.

38ième Congrès, 1983, p. 15-36

Si les tendances de la syndicalisation observées au Québec depuis 20 ans se maintiennent dans l'avenir, il pourrait en résulter une stagnation des effectifs syndicaux, et une diminution du taux de syndicalisation. La motivation des salariés à former un syndicat est inhérente à un régime de salariat comme celui de l'entreprise privée. L'effet de la syndicalisation dans l'entreprise peut aussi bien être positif que négatif. - 1 tableau.

0860 INGERMAN, Sidney

La syndicalisation dans le contexte économique québécois.

38ième Congrès, 1983, p. 37-69

Le développement du syndicalisme au Québec est étroitement relié à l'évolution de l'éco- nomie. L'analyse porte sur les questions suivantes: a) L'État de l'économie québécoise durant la crise actuelle et au cours de la période qui l'a précédée; b) La relation entre la croissance du syndicalisme et la croissance économique et les changements dans la structure de l'économie; c) La place de la concertation et d'un contrat social dans la politique économique gouvernementale. -14 tableaux.

\section{BONIN, Bernard}

Commentaires - La syndicalisation dans le contexte économique québécois.

38ième Congrès, 1983, p. 70-76

Évaluation de l'état actuel de l'économie québécoise et des changements structurels actuellement en cours. Énumération de cuelques problèmes à résoudre: a) Trouver les moyens de faire repartir la consommation; b) Rétablir la situation de l'emploi alors que le taux de chômage aura atteint près de $16 \%$ au cours de la récession; c) Trouver la solution aux problèmes de la structure industrielle du Québec.

\section{LAFLAMME, Gilles}

L'impact du régime de relations du travail sur la syndicalisation.

38ième Congrès, 1983 , p. 77-90

Le système des relations du travail peut-ij avoir un impact sur l'accès au syndicalisme au Québec? En se situant dans une perspective historique, on montre que les traits essentiels de notre régime de relations du travail sont le résultat de rapports sociaux et aussi le reflet des valeurs véhiculées dans la société, on discute des effets d'un tel régime sur la syndicalisation et enfin, on formule quelques commentaires sur le syndicalisme.

\section{LEBEL, Hélène}

Commentaires - L'impact du régime de relations du travail sur la syndicalisation.

38 ième Congrès, 1983, p. 91-100

Notre législation du travail freine souvent les progrès de la syndicalisation dans le secteur privé. Parmi les obstacles à la syndicalisation que l'on retrouve dans nos lois et dans le fonctionnement de nos institutions, il faut noter: a) les problèmes administratifs et b) la double juridiction sur les pratiques déloyales. 
0864 DESMARAIS, Jacques

Les idées de réforme sur la syndicalisation au Québec depuis 1964.

38ième Congrès, 1983, p. 101-116

Inventaire des multiples idées de réforme des dispositions légales relatives à la syndicalisation, mises de l'avant au Québec dans les vingt dernières années. On regroupe ces idées de réforme sous trois titres: comment améliorer le régime d'accréditation syndicale 1) quant aux personnes qui y ont accès; 2) quant à son fonctionnement; 3) quant à son extension.

0865 MERCILLE, Pierre NADEAU, Bertin OLIVIER, Madeleine

Table ronde - Organisation syndicale: difficultés et motifs de résistance.

38ième Congrès, 1983, p. 117-134

Selon M. Mercille, le Code du travail du Québec actuel ne permet pas un véritable accès à la syndicalisation car il contient de nombreux obstacles de plus en plus difficiles à surmonter. Pour M. Nadeau, la syndicalisation accrue n'est pas la solution à l'amélioration de la justice sociale. Quant à Mme Olivier, elle situe à trois niveaux les difficultés et les motifs de résistance à l'organisation syndicale dans le secteur privé.

\section{SIMARD, Monique}

SLIGER, Raymond

Pourquoi et comment accroître la syndicalisation dans le secteur privé?

38ième Congrès, 1983, p. 135-162

Les éléments qui militent en faveur de l'accroissement de la syndicalisation: La réduction de l'écart de rémunération entre syndiqués et non-syndiqués; l'uniformisation des relations du travail; l'accès de tous les travailleurs à l'information sur leurs droits; l'égalité des femmes sur le marché du travail. Dans une optique futuriste, on définit ce que devraient être les nouvelles responsabilités sociales d'un syndicalisme plus dynamique et plus représentatif. - 5 tableaux.

\section{DION, Gérard}

Commentaires - Pourquoi et comment accrốtre la syndicalisation dans le secteur privé? 38ième Congrès, 1983, p. 162-171

Pourquoi accroitre la syndicalisation? C'est pour le bien des travailleurs eux-mêmes en leur assurant une meilleure représentation vis-à-vis de l'État, du patronat et du public. Cependant, la syndicalisation totale est non seulement une chimère, mais elle serait préjudiciable. Comment accroitre la syndicalisation? Quand on aura compris qu'il existe aussi des intérêts communs entre travailleurs et employeurs, un grand pas sera franchi pour accroitre la syndicalisation.

0868 PERREAULT, Charles

L'entreprise peut-elle se passer d'un syndicat? 38ième Congrès, 1983, p. 173-182

Le syndicalisme canadien est en crise. Le militantisme syndical est à la source de ces problèmes car il consiste essentiellement à convaincre les travailleurs que l'employeur est un exploiteur et leur ennemi. Les travailleurs non-syndiqués se méfient du langage des chefs syndicaux. Pour ces derniers, la syndicalisation obligatoire apparaît comme la solution miracle à l'érosion de leur membership. II n'y a aucun avantage à tirer de la syndicalisation obligatoire.

\section{GAUTHIER, Fernand ROY, Paul-Martel}

Commentaires - L'entreprise peut-elle se passer d'un syndicat?

38ième Congrès, 1983, p. 183-195

Critique sévère de l'exposé de $M$. Charles Perreault qui adopte dans son texte une attitude antisyndicale sans nuance. Au niveau des faits, de la logique et de l'approche générale, la thèse développée par $M$. Perreault apparaît comme éminemment critiquable.

\section{FRÉCHETTE, Raynald}

La politique gouvernementale en matière de syndicalisation.

38ième Congrès, 1983, p. 197-206

À la lumière d'une expérience de près de vingt ans, il ressort que le Code du travail du Québec doit être continuellement adapté à la conjoncture. Les amendements qui seront apportés bientôt au Code du travail visent à améliorer le climat, l'environnement et le fonctionnement des relations du travail et assureront un meilleur accès à la syndicalisation. L'auteur est le Ministre du travail du Québec. 


\section{1}

BONENFANT, Claire

DUFOUR, Ghislain HÉTU, Jean-Paul

Table ronde - Les réactions du milieu.

38ième Congrès, 1983, p. 207-223

Mme Bonenfant brosse un portrait des travailleuses non syndiquées au Québec et suggère d'amender le Code du travail actuel pour lever certains obstacles à la syndicalisation. M. Dufour rejette les arguments avancés par le Ministre du travail pour justifier les amendements au Code du travail. Pour M. Hétu, les propositions d'amendements au Code ne sont pas des moyens qui visent à assurer le développement de la syndicalisation. - 2 tableaux.

0872 CAIRE, GuY

Syndicalisme, nouvelles technologies et incertitudes économiques.

38ième Congrès, 1983, p. 225-265

En premier lieu, l'exposé dégage les implications sociales essentielles des nouvelles technologies. En second lieu, il précise les forces conjoncturelles et structurelles qui sont constitutives des incertitudes économiques majeures de notre temps. Enfin, il esquisse les lignes que le syndicalisme ouvrier est susceptible de prendre dans cette réponse adaptive au défi majeur auquel il se trouve confronté.

\section{BLOUIN, Rodrigue BOULARD, René DESCHÊNES, Jean-Paul PÉRUSSE, Michel}

Introduction - Régimes de santé et sécurité et relations du travail.

39ième Congrès, 1984, p. 15-16

Introduction au rapport du 39ième Congrès des relations industrielles qui procède à un bilan critique des principaux points qui ont fait et font encore l'objet de discussions chaque fois qu'il est question de la Loi sur la santé et la sécurité du travail, adoptée le 21 décembre 1979 par l'Assemblée nationale du Québec.

\section{PÉRUSSE, Michel}

Régimes de santé et sécurité et relations du travail.

39ième Congrès, 1984, p. 17-31

Conférence d'introduction situant chacune des interventions dans le thème du Congrès et les unes par rapport aux autres. Les principaux mécanismes prévus par la Loi sur la santé et la sécurité du travail sont abordés. II est question des coûts, de la place des professionnels en santé et sécurité du travail et surtout de l'impact, des réalisation passées et potentielles de la loi. - 3 graphiques, références.

\section{SIMARD, Marce!}

Priorités en santé et sécurité du travail: secteur public et secteur privé. 39ième Congrès, 1984, p. 33-50

Questionnement sur la pertinence de la Loi sur la santé et la sécurité du travail pour les secteurs public et parapublic québécois. Le secteur public présente-t-il des problèmes de santé et de sécurité du travail justifiant qu'on l'ait inclus dans le champ d'application de la loi? Les mécanismes de cette loi ont-ils des chances réelles de fonctionner dans le secteur public et parapublic?

0876 LEDUC, Jean-Guy LEMELIN, Maurice VINET, Alain

Commentaires - Priorités en santé et sécurité du travail: secteur public et secteur privé. 39ième Congrès, 1984, p. 50-72

Commentaires sur l'exposé de M. Marcel Simard traitant des priorités en santé et sécurité du travail et questionnant la pertinence de la Loi sur la santé et la sécurité du travail pour les secteurs public et parapublic.

\section{OUELLET, Florian}

Concertation et participation: mythe ou réalité?

39ième Congrès, 1984, p. 73-92

Le thème de la concertation en santé et sécurité du travail demeure au centre du débat sur les relations entre l'État, le patronat et les syndicats. L'exposé se veut une analyse de la concertation telle qu'elle se vit dans le domaine de la santé et sécurité du travail et s'intéresse d'abord à la dynamique même de la concertation, à titre d'expérience de relations du travail. - Bibliographie.

\section{DUMAS, Anne-Chantal} SIMARD, Monique

Commentaires - Concertation et participation: mythe ou réalité.

39ième Congrès, 1984, p. 93-111

Commentaires sur l'exposé de $\mathrm{M}$. Florian 
Ouellet qui analyse la dynamique même de la concertation en santé et sécurité du travail, à titre d'expérience de relations du travail.

\section{BOUCHARD, Robert}

CLÉMENT, Pierre R.

GIASSON, Étienne

GUILLEMETTE, Michel

Table ronde - La santé et la sécurité dans l'entreprise.

39ième Congrès, 1984, p. 113-135

Table ronde regroupant des intervenants patronaux et syndicaux pour débattre de la santé et de la sécurité dans l'entreprise. Les thèmes de discussion portent sur l'élimination des risques et l'amélioration de la qualité du milieu du travail, sur les solutions proposées par la loi et les difficultés que présente leur implantation. - 1 tableau.

0880 BOULARD, René

L'impact de la Loi sur les conventions collectives.

39ième Congrès, 1984, p. 137-157

Avec l'adoption de la Loi sur la santé et la sécurité du travail en 1979, une partie du domaine de la santé et de la sécurité a été soustraite du champ du négociable. À partir de données concrètes, on se demande comment, de fait, tout cela s'est concrétisé dans les conventions collectives. Se retrouve-t-il dans les conventions plus, moins ou autant de clauses qu'avant l'adoption de la Loi? 12 tableaux, références.

\section{DE KONINCK, Maria HEENAN, Roy}

Droit de refus et retrait préventif: succès ou échec?

39ième Congrès, 1984, p. 159-175

Les divergences et les controverses entre les parties sur l'application de deux mécanismes ou droits prévus dans la Loi sur la santé et la sécurité du travail: 1) Le droit au retrait préventif de la femme enceinte ou qui allaite. 2) Le droit pour un travailleur de refuser un travail s'il a des motifs raisonnables de croire que l'exécution de ce travail l'expose à un danger.

0882 TURCOTTE, Fernand La prévention: une utopie?

39ième Congrès, 1984, p. 177-183
On démontre pourquoi et à quelles conditions la prévention en milieu de travail ne saurait être tenue pour une utopie. Par prévention on entend l'ensemble des mesures préventives qu'il convient de mettre en oeuvre contre certains risques de maladies ou de problèmes de santé d'origine professionnelle. La prévention est possible et réalisable pour toutes les maladies professionnelles.

0883 DROUIN, Claude

PLAMONDON, Denise

Commentaires - La prévention: une utopie?

39ième Congrès, 1984, p. 183-194

Commentaires sur l'exposé de M. Fernand Turcotte qui démontrait que la prévention des maladies professionnelles et des problèmes de santé en milieu de travail est prossible et réalisable, et ne saurait être tenue pour une utopie.

\section{BRUNET, Jacques}

Santé et sécurité du travail: une affaire de professionnels?

39ième Congrès, 1984, p. 195-206

Analyse de l'impact majeur de deux facteurs sur l'état de santé des individus: le niveau de vie et l'environnement de travail. Examen du rôle des travailleurs, des employeurs et des professionnels en santé et sécurité en vue d'atteindre les objectifs de la Loi sur la santé et la sécurité du travail. L'amélioration de la santé et sécurité au travail doit s'effectuer dans le milieu de travail lui-même et non par voie réglementaire.

\section{BEAUSOLEIL, Gilles}

Les coûts et les bénéfices du régime.

39ième Congrès, 1984, p. 207-242

L'objet de l'exposé est d'apporter un certain éclairage à la question de bénéfices et des coûts de la santé et de la sécurité au travail dans le contexte de l'expérience québécoise contemporaine. On traite plus précisément des implications économiques et des coûts de la prévention des accidents et des maladies du travail et de la réparation de ceux-ci. - 7 tableaux, bibliographie. 
0886 DUFOUR, Ghislain FAVA, Frank

HÉTU, Jean-Paul LABERGE, Louis

LAROSE, Gérald SAUVÉ, Robert

Table ronde - Déceptions et espoirs.

39ième Congrès, 1984, p. 243-276

À titre de conclusion et de synthèse sur les problèmes de santé et de sécurité au travail abordés au cours du Congrès, six intervenants de marque font le point et se prononcent sur les forces, les faiblesses et l'impact du régime québécois de santé et sécurité au travail. Ils estiment également les résultats qu'il faut escompter du régime dans les 5 années à venir. - 1 tableau.

0887 BÉLANGER, Jacques

BLOUIN, Rodrigue MORIN, Fernand SEXTON, Jean

Introduction - Le statut de salarié en milieu de travail.

40ième Congrès, 1985, p. 13-15

Texte d'introduction au 40ième Congrès des relations industrielles qui avait pour thème le statut de salarié en milieu de travail au Québec.

\section{BLOUIN, Rodrigue}

Le statut de salarié en milieu de travail: la problématique.

40ième Congrès, 1985, p. 17-32

On met en relief un certain nombre d'indicateurs de la distorsion entre le statut de salarié en droit et dans les faits dans le contexte québécois. Pour mieux cerner cette distorsion, on aborde les trois principaux volets de la question, à savoir les problèmes liés 1) à la technique de qualification de la relation employeur-salarié; 2) aux voies privilégiées pour assurer une présence du salarié dans l'entreprise; 3) aux moyens retenus pour mieux arrêter les conditions de travail en raison du statut de salarié.

\section{GAGNON, Jean-Denis}

Les notions de salarié en droit du travail.

40ième Congrès, 1985, p. 33-47

Lorsqu'on analyse les lois québécoises et canadiennes portant sur les rapports de travail, on constate la multiplicité des expressions utilisées pour désigner une personne qui accomplit un travail sous l'autorité d'une autre: "salarié", "employé", "travailleur", "entrepreneur indépendant". Pour qu'un véritable droit du travail prenne forme au Québec, un effort important de systématisation et de conceptualisation doit être entrepris.

\section{DOUCET, René \\ PARENT, Louise}

Commentaires - Les notions de salarié en droit du travail.

40ième Congrès, 1985, p. 48-54

Après un bref historique de la relation de travail et du statut de salarié du point de vue juridique, $M$. Doucet fait la distinction entre "salarié", "entrepreneur" et "cadre". Pour Mme Parent, le salarié est d'abord une personne humaine qui devient un facteur de production, un coût de production, lorsqu'il se retrouve en milieu de travail. Pour que le salarié se réalise dans son travail, la démocratisation de l'entreprise est essentielle.

0891 CÓTÉ, André C.

Évolution des conditions de travail des saiariés établies d'autorité.

40ième Congrès, 1985, p. 55-69

Les caractéristiques de l'évolution des interventions directes de l'État dans la fixation des conditions de travail. La récente prolifération de ces interventions législatives, située en perspective, permet de constater non seulement un accroissement du nombre d'objets d'intervention et leur élargissement, mais, sous certains aspects, un changement de principe et de modalités d'intervention.

\section{PIUZE, GuY}

Commentaires - Évolution des conditions de travail des salariés établies d'autorité.

40ième Congrès, 1985, p. 69-74

Depuis une vingtaine d'années, le législateur a multiplié les interventions visant à déterminer à l'avance les conditions de travail. $\mathrm{Ce}$ faisant, l'État favorisait une approche qui consiste à imposer à l'employeur le devoir de respecter certaines normes minimales quant au contenu du contrat de travail et à rééquilibrer ainsi les rapports de force.

\section{MORIN, Fernand}

L'institutionnalisation des rapports collectifs du travail: Réalité d'aujourd'hui et de demain.

40ième Congrès, 1985, p. 75-115 
L'évolution de notre régime juridique des rapports collectifs du travail depuis quarante ans démontre la publicisation progressive des relations du travail. L'omniprésence de l'État fait que les relations du travail ne sont plus du domaine privé mais relèvent davantage de l'organisation générale de la société. L'État établit maintenant un seuil de conditions de travail, impose sa présence et peut même se substituer aux parties.

\section{GAGNON, Robert P.}

Commentaires - L'institutionnalisation des rapports collectifs du travail: Réalité d'aujourd'hui et de demain.

40ième Congrès, 1985, p. 116-119

Pour faire face à l'omniprésence de l'État et à la publicisation progressive des relations du travail au cours des dernières années, il est absolument nécessaire de développer une éthique de l'État. II apparaît de première importance que l'État, tount employeur qu'il soit lui aussi, demeure crédible comme législateur, administrateur des lois et pacificateur.

\section{DUCHARME, Claude}

SIMARD, Monique THIBAULT, Laurent Table ronde - Le régime actuel de travail des salariés: où en sommes-nous?

40ième Congrès, 1985, p. 121-136

À l'aide d'exemples dans l'industrie de l'automobile, $M$. Ducharme démontre que l'énergie des employeurs doit servir à développer les P.M.E. et non pas à combattre les syndicats. Pour Mme Simard, le statut de salarié dans une entreprise ne peut plus désormais être confiné au seul rôle de pourvoyeur de force de travail. M. Thibault décrit la démarche qui a amené les gestionnaires de l'Association des manufacturiers canadiens à revoir et à changer leur façon de penser.

\section{BERNIER, Colette}

Évolution du statut du salarié en raison de nouvelles formes d'emploi: L'exemple du travail à temps partiel au Québec.

40ième Congrès, 1985, p. 137-160

Depuis une dizaine d'années, on a remarqué une croissance de diverses formes particulières d'emplois comme le travail à domicile, le travail temporaire, à la pige, en sous-traitance, à temps partiel. Définition et importance de ces formes nouvelles d'emplois. Comment et en quoi, le travail a temps partiel, tel qu'il s'est développé au Québec, constitue une forme d'emploi précaire et a des conséquences très nettes sur le statut de la personne salarié.

\section{DÉOM, Esther}

Commentaires - Evolution du statut du salarié en raison des nouvelles formes d'emplois: L'exemple du travail à temps partiel au Québec.

40ième Congrès, 1985, p. 161-170

Après avoir dégagé les principaux points de l'exposé de Mme Bernier, on reprend ces points en les regroupant 1) quant à la stratégie à la base du développement du travail à temps partiel et aux critères qui permettent de le qualifier d'emploi précaire; 2) quant aux besoins qui seraient non satisfaits dans un emploi précaire.

\section{BÉLANGER, Laurent}

Nouvelles formes d'organisation du travail, nouveaux modes de gestion et leur incidence sur le statut du salarié.

40ième Congrès, 1985, p. 171-196

Description des facteurs qui sont à l'origine d'une préoccupation renouvelée à l'endroit du statut du salarié dans l'entreprise. La notion de statut de salarié replacée dans le temps et envisagée sur un continuum. Rappel des nouvelles formes d'organisation du travail et des nouveaux modes de gestion. Les possibilités d'élargissement du statut du salarié que recèlent ces nouvelles formes et ces nouveaux modes. - 1 graphique.

\section{CÓTÉ, Marcel}

Commentaires - Nouvelles formes d'organisation du travail, nouveaux modes de gestion et leur incidence sur le statut du salarié. 40ième Congrès, 1985, p. 196-203

Les nouvelles formes d'organisation du travail et les nouveaux modes de gestion permettent l'élargissement du statut du salarié tant sur le plan de l'autonomie que sur celui du contenu de son travail. Mais s'agit-il là d'une façon de démocratiser davantage les lieux du travail ou plutôt d'une façon de récupérer les salariés afin de diminuer leur engagement? On tente de répondre à cette question en adoptant une démarche méthodologique. 
0900 BHÉRER, Harold

Le salarié et la gestion générale de l'entreprise.

40ième Congrès, 1985, p. 205-222

Après avoir défini le concept de stratégie d'entreprise et précisé ce qu'on entend par décision stratégique et par entreprise participative, on se demande: a) Quel peut être ou que devrait être le statut de salarié par rapport à la gestion générale de l'entreprise? b) Existe-t-il une volonté latente ou exprimée des travailleurs québécois d'exercer une influence et un contrôle sur les décisions stratégiques de l'entreprise?

0901 GODBOUT, Clément

Commentaires - Le salarié et la gestion générale de l'entreprise.

40ième Congrès, 1985, p. 222-228

L'employeur aurait intérêt à encourager la participation des travailleurs syndiqués à la gestion de l'entreprise car l'expérience des travailleurs constitue la plus belle richesse pour une entreprise. Les travailleurs veulent participer à l'organisation du travail, au choix des méthodes de production et de la machinerie industrielle. Pour eux, c'est une question de sécurité d'emploi.

0902 MARCHAND, Jean

Les rapports collectifs du travail: Rétrospective et perspective.

40ième Congrès, 1985, p. 229-244

Historique de l'évolution du syndicalisme ouvrier, de la législation du travail et des rapports collectifs du travail au Québec depuis le début des années 1940. Les évènements qui ont conduit le système québécois des relations du travail aux distorsions que nous constatons aujourd'hui. Comment on pourrait en arriver à un système plus rationnel et plus efficace.

0903 BÉLANGER, Jacques

Annexe - La participation des travailleurs aux decisions dans l'entreprise.

40ième Congrès, 1985, p. 245-259

Ce document de travail cherche d'abord à situer la question de la participation en référence à ce thème plus large du statut du salarié en milieu de travail. II traite ensuite de la participation dans notre régime de relations du travail. Finalement, il présente une distinction entre les différentes formes de participation aux décisions, en faisant référence à notre contexte et aux publications québécoises et canadiennes.

\section{THWAITES, James LAJOIE, Mario} BOIS-BROCHU, Hélène

Supplément - Quarante ans au service des relations industrielles

40ième Congrès, 1985, p. 261-281

Cette année, le Congrès des relations industrielles fête son quarantième anniversaire. Depuis 1946, il est devenu graduellement une organisation annuelle d'envergure dans le monde des relations du travail au Québec. On évoque ici l'origine du Département des relations industrielies de Laval et de son Congrès et on définit certaines des caractéristiques de ce dernier. - 6 graphiques.

0905 AUDET, Michel BÉLANGER, Laurent BOVIN, Jean DÉOM, Esther MERCIER, Jacques

Introduction - La mobilisation des ressources humaines: tendances et impact.

41ième Congrès, 1986, p. 13-14

Texte d'introduction au rapport du 41ième Congrès des relations industrielles qui a retenu comme thème "La gestion des ressources humaines: mariage ou divorce?"

0906 BOIVIN, Jean

Émergence d'une réalité nouvelle en relations industrielles.

41ième Congrès, 1986, p. 17-30

Définition des concepts "relations industrielles', "relations du travail". Comment la définition de ces concepts s'est élargie au fur et à mesure que la réalité du travail s'est modifiée. Analyse des changements survenus au sein de la fonction "personnel". Appréciation des rapports entre "gestion des ressources humaines" et "relations du travail". - 2 graphiques.

\section{LAROUCHE, Viateur}

La mobilisation des ressources humaines - orientations récentes.

41ième Congrès, 1986, p. 31-51

Résumé des huit principes de gestion des entreprises de troisième type. Les principales contraintes qui se sont exercées sur les en- 
treprises au cours de la dernière décennie. Les orientations récentes en matière de gestion des ressources humaines qui occupera, dans l'ère qui commence, une place importante dans les organisations.

\section{CASAVANT, Jean-Claude}

La stratégie de gestion des ressources humaines chez Abitibi Price Inc.

41 ième Congrès, 1986, p. 53-61

Sur quoi repose la stratégie de la gestion des ressources humaines (GRH) mise en place chez Abitibi Price; quels en sont les principes directeurs; le rôle joué par les professionnels en ressources humaines; comment les syndicats ont accueilli la stratégie de GRH; l'influence de cette stratégie sur les relations du travail.

\section{LEMAIRE, Alain}

La communication directe chez Cascades Inc. 41ième Congrès, 1986, p. 63-68

Identification de quelques éléments qui caractérisent la gestion des ressources humaines telle que pratiquée par les dirigeants de Cascades Inc.: Disponibilité et accessibilité des dirigeants, participation des employés aux décisions, structure hiérarchique simplifiée et non formalisée, climat de confiance, activités sociales, promotion interne, partage des profits etc.

\section{NÉRON, Roger}

Le projet d'entreprise de Culinar Inc.

41ième Congrès, 1986, p. 69-76

Description du projet d'entreprise de Culinar Inc. qui tout en voulant faire de l'entreprise l'une des plus rentables dans le secteur alimentaire canadien, vise à favoriser la croissance et le développement de tous ses employés sur le plan professionnel et personnel.

\section{BOUCHER, Lysette}

Les limites des nouvelles approches en gestion des ressources humaines.

41ième Congrès, 1986, p. 77-84

Ce commentaire tente de répondre à la question suivante: Pourquoi des entreprises comme Cascades, Abitibi Price et Culinar ont-elles dû, pour réussir, tenir compte des valeurs humaines et sociales? On amorce une réflexion vers un schéma d'explication qui pourrait articuler les deux champs d'études que sont la gestion des ressources humaines et les relations du travail, en regardant de plus près la réalité des organisations industrielles.

\section{SÉRIEYX, Hervé}

L'entreprise du troisième type.

41ième Congrès, 1986, p. 85-97

En tenant compte des nouvelles contraintes qui les touchent et des nouvelles attitudes de femmes et d'hommes face à leur vie, à leur travail en particulier, des entreprises s'organisent différemment, ce qui amène des syndicats à prendre d'autres points d'insertion dans la vie de l'entreprise, ce qui amène également des dirigeants à adopter de nouvelles conduites. Tout cela, c'est ce que l'on appelle l'entreprise du troisième type.

0913 WILS, Thierry

Les travailleurs seront-ils du troisième type? 41ième Congrès, 1986, p. 99-109

Depuis la dernière récession, les dirigeants semblent être particulièrement hantés par la baisse de la productivité. Dans l'exposé précédent, $M$. Hervé Sérieyx propose une solution qui peut paraitre attrayante pour les employeurs. Cette solution va-t-elle plaire également aux travailleurs? Les travailleurs sont-ils prêts à se défoncer? Est-il possible de mettre en oeuvre une stratégie de mobilisation des travailleurs?

\section{DULUDE, Yves}

Le conflit: la gestion au banc des accusés. 41 ième Congrès, 1986, p. 111-133

Le Service de médiation préventive du Ministère du travail est offert sur une base volontaire. L'intervention de médiation préventive se fait pendant la durée de la convention collective et vise à améliorer les rapports dans l'entreprise. L'exposé analyse les problèmes soulevés par les représentants syndicaux et les contremaitres dans les entreprises où le Senvice de médiation préventive est intervenu et fait part des conclusions qui s'en dégagent. - 1 graphique.

\section{LAMARCHE, Pierre}

Commentaires - Le conflit: la gestion au banc des accusés.

41ième Congrès, 1986, p. 133-136 
Les interventions de médiation préventive portent sur un ensemble de facteurs de relations humaines comme par exemple les rapports entre les contremaitres et leurs subordonnés. Les syndicats doivent pouvoir résoudre ces problèmes. De leur côté, les contremaîtres se doivent d'être des personnes représentatives de l'employeur et aptes à prendre des décisions au nom de l'employeur.

\section{LALANDE, Serge}

L'expérience de la compagnie Gaz Métropolitain.

41ième Congrès, 1986, p. 137-146

Suite à une intervention du Service de médiation préventive chez la compagnie Gaz Métropolitain en 1983, l'entreprise a connu une amélioration majeure et radicale de ses relations du travail. Après avoir brossé un tableau "avant et après" la médiation préventive, on apprécie l'influence majeure que peuvent avoir sur une entreprise les conclusions d'une telle médiation.

\section{DOCQUIER, Gérard}

Ressources humaines et defis du syndicalisme.

41 ième Congrès, 1986, p. 149-156

Le mouvement syndical ne doit pas ignorer que la grande entreprise est en train d'utiliser les ressources humaines dans le but de réaliser une croissance et des profits considérables et dans certains cas, pour éviter le syndicalisme. Si l'entreprise veut transformer le milieu des relations du travail par le biais de la stratégie de gestion des ressources humaines, il va falloir passer par la négociation collective, moyen efficace pour acquérir la démocratie industrielle.

0918 FRANCOEUR, Jean

Le syndicalisme et le nouveau travail.

41ième Congrès, 1986, p. 157-166

L'observateur a parfois l'impression de vivre simultanément les quatre âges des relations du travail: la loi d'airain, la reconnaissance du syndicalisme, l'áge d'or du syndicalisme et le nouveau travail. A l'ère du nouveau travail, l'avenir de l'entreprise repose sur la productivité et sur de nouvelles formes d'organisation du travail. Pour faire face à un défi tout à fait inédit, le syndicalisme doit se remettre en question et s'adapter à un nouvel environnement économique.
0919 HÉTU, Jean-Paul

La nouvelle gestion des ressources humaines - mythe ou réalite?

41 ième Cor.grès, 1986, p. 167-172

Description des grandes lignes du projet syndical de "gestion démocratique sur les lieux du travail" mis au point et expérimenté depuis plus de cinq ans par la Centrale des syndicats démocratiques. Pour jouer ce nouveau rôle dans la gestion démocratique ou participative, les syndicats se doivent de modifier certains comportements dans l'entreprise et adopter l'approche de la concertation en milieu de travail.

\section{LABERGE, Louis}

Les préalables à une réorientation des relations du travail au Québec.

41ième Congrès, 1986, p. 173-175

Lorsque l'on voit le nombre effarant de requê"tes en accréditation syndicale qui sont contestées devant les tribunaux par les employeurs, force est de constater que la situation n'a pas beaucoup changé dans le domaine des relations du travail au Québec. La reconnaissance de l'existence du mouvement syndical s'avère un élément important pour améliorer la situation.

\section{LAROSE, Gérald}

L'adaptation du syndicalisme - un phénomène de continuité.

41ième Congrès, 1986, p. 177-179

Devant l'émergence de phénomènes profondément nouveaux qui entraînent le fractionnement des lieux du travail et des modifications profondes de l'organisation du travail, le syndicalisme québécois saura s'adapter aux changements comme il a toujours su le faire par le passé.

0922 KOCHAN, Thomas A. L'avenir de la négociation collective. 41 ième Congrès, 1986 , p. 181-189

Pour bien comprendre les facteurs susceptibles d'influencer la négociation collective, on doit tenir compte de deux autres niveaux d'opérations: un palier inférieur d'opérations, celui des lieux mêmes du travail; un palier supérieur, là où se prennent les décisions d'ordre stratégique de l'entreprise. À l'aide de ce cadre d'analyse, on porte un jugement 
sur l'évolution des relations industrielles au cours des dernières années et sur les nouveaux défis posés par les changements economiques et sociaux que l'on observe actuellement.

\section{PÉRUSSE, Michel}

Les lésions professionnelles - une problématique.

42ième Congrès, 1987, p. 1-8

Présentation des principaux éléments de la problématique du régime québécois d'indemnisation des lésions professionnelles instauré en 1985 par la "Loi sur les accidents du travail et les maladies professionnelles". Synthèse des différents exposés présentés sur ce thème lors du 42ième Congrès des relations industrielles.

\section{CHIASSON, Denis-Émile}

Synopsis sur le nouveau régime.

42ième Congrès, 1987, p. 9-19

Rappel des objectifs du législateur lors de l'adoption de la "Loi des accidents du travail" en 1931. Les raisons qui ont motivé l'adoption de la "Loi sur les accidents du travail et les maladies professionnelles" en 1985. Description du fonctionnement du régime instauré par cette loi qui introduisait des changements majeures par rapport à l'ancienne loi.

\section{VINET, Alain VÉZINA, Michel BRISSON, Chantal}

Des lésions professionnelles méconnues: le cas des opératrices de l'industrie du vêtement.

42ième Congrès, 1987, p. 21-38

Rappel du modèle prévalant dans la définition des lésions professionnelles. Présentation d'un modèle complémentaire qui, en guidant les travaux sur le terrain et les enquêtes, a permis une meilleure compréhension de la réalité. Analyse des résultats concrets de recherche, provenant de l'industrie du vêtement, qui viennent étayer ce modèle et appuyer l'existence de lésions professionnelles méconnues. - 7 graphiques.

0926 LESAGE, Michel

Les lésions professionnelles: Point de vue d'un médecin.

42ième Congrès, 1987, p. 39-50
La "Loi sur les accidents du travail et les maladies professionnelles" veut qu'il appartienne uniquement à l'employeur de payer l'ensemble des coûts de la réparation des lésions professionnelles. Même si les revenus de cotisation à la CSST dépassent le milliard de dollars, celle-ci prévoyait un déficit de 300 millions de dollars pour l'année 1987. Le fardeau les lésions professionnelles devrait être supporté par tous les intervenants, ceci pour le plus grand bien de l'individu et de la société.

\section{PRÉVOST, Charles}

La sous-estimation des atteintes à la santé causées par les mauvaises conditions de travail.

42ième Congrès, 1987 , p. 51-60

Chaque année, la CSST reçoit environ 350,000 réclamations dont seulement 4,000 pour maladies. Ces chiffres révèlent une sous-déclaration des atteintes à la santé subies par les travailleuses et les travailleurs à cause des mauvaises conditions de travail et par conséquent, une sous-estimation des coûts reliés à ces atteintes. Certaines politiques de la CSST visent à réduire les droits des victimes de lésions professionnelles dans le but de diminuer davantage les coûts.

\section{ARSENAULT, André}

La reconnaissance d'une maladie professionnelle est-elle négociable?

42ième Congrès, 1987 , p. $61-72$

La reconnaissance d'une maladie professionnelle fait l'objet d'une négociation par les parties en cause, c'est-à-dire les groupements patronaux, les centrales syndicales, la CSST, la communauté scientifique de même que plusieurs ministères. II convient aux parties de décider si elles veulent en expliciter les enjeux, clarifier leurs rôles et aborder la question directement.

\section{DUGUAY, Pierre BOUCHARD, Robert GONTHIER, Jean-Marie}

Commentaires - La reconnaissance d'une maladie professionnelle est-elle négociable? 42ième Congrès, 1987 , p. 72-86

Par quel mécanisme en vient-on à la reconnaissance d'une maladie professionnelle? Est-ce le résultat d'un processus de négociation et d'entente entre les parties en cause. 
Sur ce point, les employeurs, les travailleurs et leurs associations respectives semblent, à première vue, avoir un apport plutôt restreint dans le processus décisionnel.

\section{LAFLAMME, Gilles} LAROCQUE, Alain

Lésions et maladies professionnelles: objet de négociation?

42ième Congrès, 1987, p. 89-100

Les interventions législatives dans le domaine de la santé et de la sécurité du travail avaient pour objectif avoué de soustraire ces questions au processus de négociation. Est-ce bien ce qui est arrivé? D'une façon plus spécifique, les lésions professionnelles sont-elles encore matière à litige en milieu de travail? Constituent-elles un thème de revendications des salariés? - 3 tableaux.

\section{TRUDEAU, GuY J. OUELLET, Lionel}

La comparaison en matière de systèmes de santé et de sécurité du travail.

42ième Congrès, 1987, p. 101-113

Résultats d'une étude comparative des différents systèmes de santé et de sécurité du travail (SST) au Canada et aux États-Unis: Le système québécois ainsi que les autres systèmes SST au Canada présentent des avantages marqués sur les systèmes américains. Le système américain, du reste, nous indique qu'il y aurait, au Canada, d'énormes possibilités d'amélioration de la gestion des système SST, notamment en ce qui concerne les demandes de prestations. - 1 tableau, 1 graphique, bibliographie.

\section{FARQUHAR, Alec}

Le régime des accidents du travail: Le cas de l'Ontario.

42ième Congrès, 1987, p. 115-130

La problématique du régime des accidents du travail s'est transformée de façon dramatique au cours des quinze dernières années en Ontario comme partout ailleurs au Canada. Le présent exposé trace les grandes lignes du régime en vigueur en Ontario et des principaux champs d'application susceptibles d'être modifiés.

\section{MORIN, Fernand}

Le régime actuel d'indemnisation pour lésions professionnelles: Accessibilité et efficacité. 42ième Congrès, 1987, p. 131-155

Les moyens retenus en 1985 par le législateur et mis en application par les organes ainsi constitués en vue d'établir l'indemnité due a l'accidenté du travail respectent-ils la finalité historique et pratique du régime. $\mathrm{Ca}$ ractéristiques des quatre organes responsables de l'indemnisation des accidentés du travail; le champ respectif de compétence et les moyens d'action dont disposent la CSST, le médecin-arbitre, les bureaux de révision et la Commission d'appel.

\section{LEFÈBVRE, Marie-Claire LEVASSEUR, Raymond}

Commentaires - Le régime actuel d'indemnisation pour lésions professionnelles: Accessibilité et efficacite.

42ième Congrès, 1987, p. 155-176

Mme Lefèbvre donne le point de vue des victimes d'accidents du travail et de maladies professionnelles et dénonce le régime d'indemnisation qui semble avoir oublié son objectif principal qui était d'offrir une juste réparation à tout accidenté du travail. $M$. Levasseur explique comment la Commission d'appel en matière de lésions professionnelles a su répondre à l'objectif du régime d'indemnisation des lésions professionnelles avec célérité, efficacité et cohérence.

\section{BERNIER, Lionel}

Équité, indemnisation des victimes de lésions professionnelles et coûts à l'entreprise.

42ième Congrès, 1987, p. 177-211

L'équité du régime actuel de réparation des lésions professionnelles consiste pour les deux parties (employeurs et accidentés du travail) à pouvoir être entendues, en temps utile, dans un délai raisonnable, par une autorité indépendante et impartiale, sur toute question litigieuse découlant de l'administration de la loi. On examine les aspects du régime actuel qui paraissent les plus équitables et ceux qui semblent manquer d'équité pour l'une ou l'autre des parties.

\section{GINGRAS, Claude}

$$
\text { DUCHESNE, André }
$$

Commentaires - Équité, indemnisation des 
victimes de lésions professionnelles et coûts à l'entreprise.

42ième Congrès, 1987, p. 212-222

Un représentant syndical et un représentant patronal donnent leur avis sur l'équité du régime actuel de réparation des lésions professionnelles. Pour le premier, il reste beaucoup à faire pour assurer l'équité pour les travailleurs en matière de santé et sécurité du travail. Pour le second, l'équité ne peut être atteinte sous le régime actuel parce que les lois elles-mêmes visent à donner le plus possible à l'un aux dépens de l'autre.

\section{SURET, Jean-Marc}

GENDRON, Michel BERNIER, Gilles

Le processus de gestion des risques, les lésions professionnelles et la CSST.

42ième Congrès, 1987, p. 223-241

À la CSST, les coûts de la prévention pour l'année 1985 ne représentaient que $5.2 \%$ des coûts directs de réparation des lésions professionnelles. Après avoir présenté le cadre d'analyse proposé par la discipline de la gestion des risques, on utilise ce cadre pour l'analyse coûts/avantages de la prévention et de la réparation en matière de lésions professionnelles. - 3 tableaux, 3 graphiques, bibliographie.

\section{HARGUINDEGUY, Jean-Louis BRODY, Bernard}

Commentaires - Le processus de gestion des risques, les lésions professionnelles et la CSST.

42ième Congrès, 1987, p. 241-252

En matière de lésions professionnelles, la réparation est-elle moins coûteuse que la prévention? Selon les deux intervenants, la réponse est non. Des programmes de prévention doivent être mis en place pour créer un milieu de travail exempt de risques. La baisse des dépenses en santé et sécurité du travail passe par la prévention et non seulement par un réaménagement de la CSST. - Bibliographie.

\section{TOBIN, Edmund LAROSE, Gérald}

DUFOUR, Ghislain LABERGE, Louis

Table ronde - Financement de la santé et paritarisme.

42ième Congrès, 1987, p. 253-276
Les cotisations des employeurs représentaient en 1985 plus de $75 \%$ des revenus de la CSST. Étant donné qu'on a voulu introduire le paritarisme dans l'ensemble des questions de santé et sécurité du travail, on devrait l'appliquer aussi au financement du régime; donc les travailleurs devraient également supporter une partie du fardeau financier. $\mathrm{Ce}$ raisonnement est-il défendable? Les participants à cette table ronde émettent des opinions divergentes sur le sujet.

\section{JÉRÓME-FORGET, Monique}

La politique québécoise en matière de lésions professionnelles à un point tournant.

42ième Congrès, 1987, p. 277-285

La présidente et directrice générale de la CSST fait part du défi que représente la gestion du régime québécois de santé et de sécurité du travail, régime administré par la CSST. Le plan d'action de la CSST pour réduire les lésions professionnelles et en contrôler les coûts repose sur des mesures de gestion. Description des grande orientations de la CSST en matière de prévention.

0941 BLOUIN, Rodrigue FERLAND, Gilles LAFLAMME, Gilles LAROCQUE, Alain RONDEAU, Claude

Introduction - Les Chartes des droits et les relations industritiles.

43ième Congrès, 1988, p. XV

Texte d'introduction au rapport du 43ième Congrès des relations industrielles qui se demande si les Chartes des droits et libertés qui ont vu le jour au Canada et au Québec au cours des dernières années ne remettent pas en cause les acquis de notre régime de relations industrielles.

\section{ROCHER, Guy}

Les fondements de la societé libérale, les relations industrielles et les Chartes.

43ième Congrès, 1988, p. 1-18

Les Chartes des droits et libertés représentent un très important virage juridique, politique et idéologique et, peut-être, le début d'une ère nouvelle au Canada. En adoptant une démarche historique, on montre que les Chartes canadienne et québécoise sont le produit d'une évolution complexe des fondements du libéralisme. 
0943 ROJOT, Jacques

Droits collectifs et droits individuels: Les situations française, americaine et anglaise. 43ième Congrès, 1988, p. 19-49

L'accent mis sur les droits individuels ou les droits collectifs n'est pas sans conséquences: a l'intérieur des cadres nationaux, il va orienter les solutions du droit positif. Pour mettre en évidence ce double effet, on définit d'abord un certain nombre de concepts au moyen d'une comparaison du traitement de la négociation collective dans les cadres opposés de la France et des États-Unis avant 1980. On passe ensuite en revue l'évolution récente en France et Grande-Bretagne.

\section{BERGERON, Jean-Louis}

La gestion de l'embauche, de la promotion et du licenciement revue et corrigée par les Chartes.

43ième Congrès, 1988 , p. 51-62

L'impact des Chartes des droits et libertés sur la gestion des ressources humaines, plus particulièrement sur l'embauche (recrutement, sélection, accueil, placement) et la mobilité du personnel (promotion, mutation, rétrogradation, congédiement). Comment devrait-on procéder pour remplir ces fonctions de la meilleure façon possible? Les Chartes sontelles un support ou un obstacle à une bonne gestion des ressources humaines?

\section{DUCHARME, Claude LECLERCQ, Dominique \\ WESTMORELAND-TRAORE, Juanita}

Commentaires - La gestion de l'embauche, de la promotion et du licenciement revue et corrigée par les Chartes.

43ième Congrès, 1988, p. 63-82

Dans l'ensemble, les prescriptions des Chartes des droits et libertés contribuent à améliorer les choses dans le domaine de l'embauche et de la promotion. L'application des Chartes permettra à tous les groupes faisant l'objet de discrimination (femmes, groupes ethniques) de faire valoir leurs droits.

0946 DUSSAULT, Ginette

A travail equivalent, salaire égal: Un droit difficile à appliquer?

43ième Congrès, 1988 , p. 83-95

L'application du concept d'équité salariale réserve un bon nombre de difficultés. Après avoir présenté un aperçu du contenu des Chartes des droits et libertés québécoise et canadienne en ce domaine, on développe succinctement la méthodologie pertinente à la mesure de l'équivalence des emplois et on souligne certains problèmes concrets d'application.

\section{BOIVIN, Suzanne P.}

Le Canadien National: Un cas riche d'enseignements.

43ième Congrès, 1988, p. 97-113

Compte rendu de la cause opposant Action travail des femmes (ATF) et le Canadien National. Dans cette affaire, l'ATF revendiquait l'accès pour les femmes à des postes de col bleu. À l'aide d'une preuve statistique l'ATF a su démontrer devant les tribunaux que le $\mathrm{CN}$ avait adopté des pratiques de recrutement discriminatoires qui avaient pour effet d'exclure les femmes des emplois visés.

\section{SIMARD, Monique TELLIER, Marie}

Commentaires - Le Canadien National: Un cas riche d'enseignements.

43ième Congrès, 1988, p. 114-130

Mme Simard introduit la problématique de l'équité salariale, aborde les moyens pour l'atteindre et les effets que cela produit sur le marché du travail, et identifie les défis qui se posent aux syndicats, aux employeurs et au gouvernement en cette matière. Mme Tellier, du Canadien National ( $\mathrm{CN}$ ), décrit la façon dont le CN s'est plié au jugement de la Cour Suprême qui a reconnu le caractère discriminatoire des pratiques de recrutement du $\mathrm{CN}$.

\section{BARRÉ, Alain}

Le régime des rapports collectifs et les Chartes.

43ième Congrès, 1988, p. 131-156

On rappelle, dans le cadre d'observations préliminaires, le statut respectif de la "Charte des droits et libertés de la personne' du Québec et de la "Charte canadienne des droits et libertés', on explique leur fonctionnement interne et on discute de manière spécifique de l'impact des deux Chartes sur certains aspects du régime des rapports collectifs du travail. 
0950 DESMARAIS, Jacques

Les moyens de pression: Les chartes en redéfinissent-elles les limites?

43ième Congrès, 1988, p. 157-195

La Charte canadienne des droits et libertés et la Charte des droits et libertés de la personne du Québec ont-elles fait reculer les limites des moyens de pression comme la grève et le piquetage? Les limites imposées au cours des ans à la grève et au piquetage sont-elles maintenant contestables et menacées d'invalidité parce qu'elles sont incompatibles avec les Chartes? Telles sont les questions auxquelles on tente de répondre.

\section{DULUDE, Gilles LOUMĖDE, Catherine PARROT, Jean-Claude}

Commentaires - Les moyens de pression: Les Chartes en redéfinissent-elles les limites?

43ième Congrès, 1988, p. 195-208

Les Chartes canadienne et québécoise des droits et libertés ont des incidences sur le régime des relations du travail au Canada et au Québec. Les Chartes doivent nous guider dans la recherche d'un équilibre social mais ne doivent nullement démolir les règles du jeu et les droits si durement acquis au cours des années par les travailleurs.

0952 PÉPIN, Marcel

L'actualisation du mouvement syndical.

43ième Congrès, 1988, p. 209-221

Le 9 avril 1987, les dirigeants syndicaux du Canada ont été estomaqués en apprenant de la Cour Suprême que le droit à la négociation collective et le droit de grève ne sont pas inclus dans la liberté d'association. Bon nombre de juristes croient que la Charte canadienne des droits et libertés cachent d'autres surprises qui pourraient bouleverser tout le régime des relations du travail. On tente d'identifier ce qui pourrait être remis en cause et quelles en seraient les conséquences pour les syndicats et leurs membres.

\section{GAGNON, Mona-Josée LEBEAU, Ernest JOHNSTON, Raymond}

Commentaires - l'actualisation du mouvement syndical.

43ième Congrès, 1988, p. 222-236

Certains jugements des tribunaux basés sur la Charte canadienne des droits et libertés semblent remettre en question la reconnaissance du droit à la négociation collective et du droit de grève. Face à cette situation qui crée un nouveau champ de lutte pour les organisations syndicales, le mouvement syndical doit être fort et présent pour combattre les dérives engendrées par la Charte. De plus, le législateur doit reconnaître officiellement les associations de cadres.

\section{GARANT, Patrice}

Statut et pouvoirs des organismes du travail en regard des Chartes.

43ième Congrès, 1988, p. 237-254

Les organismes du travail sont des autorités statutaires chargées d'appliquer la législation du travail. Ceux-ci comprennent des organismes investis de fonctions administratives et des organismes quasi-judiciaires qualifiés de "tribunaux". De quelle façon les Chartes canadienne et québécoise sur les droits et libertés s'appliquent-elles aux différents organismes du travail fédéraux et provinciaux.

0955 DUFOUR, Ghislain LABERGE, Louis LAROSE, Gérald

Table ronde - Les Chartes impliquent-elles un réalignement des politiques syndicales et patronales?

43ième Congrès, 1988, p. 255-272

Le représentant patronal explique en quoi les entreprises ont dû établir de nouvelles politiques afin de se conformer aux Chartes canadienne et québécoise sur les droits et libertés. Pour les représentants syndicaux, les Chartes sont essentielles mais l'abus qu'on peut en faire risque de mettre en danger les droits collectifs. Ceux-ci doivent être protégés car ils sont la garantie de la protection des droits individuels.

\section{BÉLANGER, Laurent LIPSIG, Carla} MORIN, Fernand PÉRUSSE, Michel Introduction - Acquisition ou fusion d'entreprises et emplois.

44ième Congrès, 1989, p. XV-XVI

Introduction au rapport du 44ième Congrès des relations industrielles qui analyse les effets des fusions ou des acquisitions d'entreprises sur le personnel des entreprises absorbées ou acquises. 
Relations Inoustrielles, vol. 45, no 4 (1990)

0957 MORIN, Fernand

Acquisition ou fusion d'entreprises et emplois: La problématique sous-jacente.

44ième Congrès, 1989, p. 1-12

L'intensité et la multiplicité incessantes du phénomène des acquisitions ou des fusions d'entreprises imposent que nous nous arrêtions à l'étude de ses répercussions sur l'emploi, sur les conditions de travail et sur les personnes impliquées dans le processus. Identification des causes et des conséquences du phénomène d'acquisition ou de fusion d'entreprises. - 3 tableaux.

\section{BÉLANGER, Marc}

LE BRASSEUR, Lola L'ITALIEN, Paul MÉNARD, Marius

Rappel de quelques expériences vécues.

44ième Congrès, 1989, p. 13-32

Diverses expériences d'acquisition ou de fusion d'entreprises: 1) Le cas de la fusion de deux hôpitaux de la Beauce. 2) L'acquisition de Téléglobe Canada par Mémotec Data Inc. 3) Le cas de SECUR Inc., société née de l'achat de Bririk's Québec par Sécurité Desjardins. 4) L'intégration de la fromagerie d'Agrinove à la Durantaye à l'usine de Beauceville. 5) La fusion des entrepôts de Métro-Richelieu et Épiciers Unis de Québec. 6) La fusion des mines d'amiante dans la région de Thetford-Mines. 7) La fusion des Caisses d'entraide économique et des Caisses d'établissement du Québec.

0959 CÔTÉ, André C. FONTAINE, Claude LESAGE, Paul

Aspects et implications juridiques des restructurations.

44ième Congrès, 1989, p. 33-84

Les incidences légales des acquisitions et des fusions d'entreprises sur la sécurité d'emploi et les autres normes du travail. La notion d'entreprise dans la législation québécoise et fédérale. Les modes d'acquisition ou de restructuration d'entreprises. Les droits et obligations de l'acquéreur de l'entreprise en matière de maintien de l'accréditation et de la convention collective, de mises à pied ou licenciements.

\section{ANGERS, Georges}

GAGNON, Normand SIMARD, Jean

Le droit et le devoir à l'information.

44ième Congrès, 1989, p. 85-100
Dans le cas d'une fusion ou d'une acquisition d'entreprises, le seul devoir légal d'information auquel sont contraintes les entreprises vise à sauvegarder l'intégrité du marché financier, avant l'entente, et à protéger les consommateurs, après l'entente. II n'existe en effet aucune obligation légale d'information à l'égard des employés. Cependant, pour assurer le succès de la réorganisation de l'entreprise après l'entente, il faut informer en priorité les employés concernés de ce que représente pour eux cette entente.

\section{LAMARCHE, André}

GAUTHIER, Michel BLAIS, Michel GAGNON, Denise

Les conditions de travail au lendemain d'une fusion ou d'une réorganisation.

44ième Congrès, 1989, p. 101-127

L'annonce de l'acquisition ou de la fusion d'une entreprise crée inévitablement un climat d'insécurité, de confusion et de méfiance dans l'organisation nouvellement acquise. L'acquéreur a donc intérêt à s'empresser de faire valoir, aux gestionnaires, au syndicat et aux employés, les perspectives d'un avenir promerteur pour l'ensemble de l'organisation.

\section{GAGNON, Astrid}

\section{BLANCHETTE, Sylvain}

QUINTAL, Pierre

Fusion d'entreprises publiques.

44ième Congrès, 1989, p. 129-154

a) Les effets de la privatisation et du désengagement de l'État sur l'emploi et les conditions de l'emploi dans les services de santé et services sociaux, secteur où l'on assiste à la création d'une classe de "cheap labor". b) Le cas d'une fusion de commissions scolaires où les emplois et les conditions de travail ont été protégés par des mesures contractuelles ou législatives. c) Le cas de la fusion qui a donné naissance à la ville de Jonquière en 1974 en regard des relations du travail et de l'évolution du niveau des emplois.

\section{GOSSELIN, Alain}

FRENETTE, Jean-Guy

DIONNE, Denis

Les acteurs d'une fusion ou d'une prise de pouvoir.

44ième Congrès, 1989 , p. 155-173

Les fusions et les acquisitions d'entreprises impliquent une transformation fondamentale de l'entreprise. a) Le rôle et les responsabili- 
tés des professionnels en ressources humaines dans le cas d'une fusion ou d'une acquisition. b) L'attitude des syndicats face au phénomène croissant des fusions et des acquisitions d'entreprises. c) La politique du Fonds de solidarité des travailleurs du Québec (FTQ) qui appuie les entreprises québécoises qui fusionnent ou qui acquièrent d'autres entreprises.

\section{GODBOUT, Clément} RIOUX, Claude

Le libre-échange canado-américain et le marché du travail.

44ième Congrès, 1989, p. 175-188

Le traité de libre-échange aura des répercussions sur le marché du travail canadien. a) Le traité nécessitera une réorientation des programmes d'adaptation, de formation et de recyclage de la main-d'oeuvre. b) L'une des stratégies qui sera retenue par certaines entreprises pour s'ajuster au contexte du libre-échange sera leur réorganisation dans une perspective de concentration de leur production.

0965 ROUSSEAU, Léontine Annexe - Acquisitions, fusions, offres publiques d'achat: Notions de base et facteurs considérés.

44ième Congrès, 1989, p. 189-252

Exposé sommaire des notions de base et des facteurs généralement considérés par les initiateurs d'une fusion ou d'une acquisition d'entreprises. L'acquisition en tant que moyen d'assurer la croissance de l'entreprise et les limites possibles de cette croissance. Les comportements prévisibles dans les années 1990 en matière d'acquisition et de fusion d'entreprises. -3 tableaux, 7 figures, bibliographie.

\section{MERCIER, Jacques} POULIN-SIMON, Lise

Le défi de la gestion des emplois: La problématique.

45ième Congrès, 1990 , p. 1-5

Présentation du thème du 45ième Congrès des relations industrielles de l'Université Laval, lequel mettait en parallèle l'efficacité de gestion des emplois dans l'entreprise et la sécurité économique des individus.
0967 BÉLAND, Claude

Gestion des emplois et sécurité économique des employés.

45ième Congrès, 1990, p. 7-14

Dans un premier temps, on se demande qui a la responsabilité de la permanence de la sécurité économique des employés. Dans un deuxième temps, on tente de répondre à la question: Comment gérer efficacement les emplois dans les entreprises sans nuire au maintien de la sécurité économique des employés?

\section{BLONDIN, Michel}

Le défi de la gestion de l'emploi: Pourquoi le relever?

45ième Congrès, 1990, p. 15-23

La sécurité d'emploi est un luxe réservé à une proportion plutôt faible de la main-d'oeuvre. Les entreprises du secteur privé ne savent pas relever le défi de la gestion des emplois. Comment en arriver à un pacte social sur la sécurité d'eniploi? Que peut faire le mouvement syndical pour ce qui est de la sécurité d'emploi?

0969 FRAPPIER-DESROCHERS, Monique La pratique de gestion des ressources humaines dans les entreprises.

45ième Congrès, 1990 , p. 25-39

Les eftorts du ministère de la Main-d'oeuvre, de la Sécurité du revenu et de la Formation professionnelle doivent porter sur l'amélioration de la gestion des ressources humaines dans les entreprises. 1) Examen de certaines tendances qui ont entraîné un accroissement de la valeur accordée aux ressources humaines comme facteur de production. 2) La situation des ressources humaines dans l'économie québécoise. 3) La gestion de ces ressources dans la PME aujourd'hui.

0970 LEVINE, David

Négocier la flexibilite: Reconversion des heu res de la liste de rappel en postes à temps complet.

45ième Congrès, 1990, p. 41-47

Présentation en détail du projet de reconversion des heures de la liste de rappel en postes à temps complet, projet mis en place au Centre hospitalier de Verdun et pouvant servir de modèle aux autres établissements de 
santé qui voudraient considérer la possibilité de créer de nouveaux emplois à temps complet dans leur établissement.

0971 GOBEILLE, Kenneth R.

Négocier la flexibilite: Le travail à temps partiel.

45ième Congrès, 1990 , p. $48-58$

Plus de la moitié des 17,000 employés des marchands affiliés à Provigo Distribution inc. sont des salariés à temps partiel. L'expérience de Provigo Distribution démontre qu'une gestion équitable de l'emploi à temps partiel peut éventuellement présenter des perspectives plus encourageantes. Par exemple, on peut maintenant envisager une gamme complète d'avantages sociaux pour les salariés à temps partiel du secteur de l'alimentation.

\section{GODIN, Michel}

Négocier la flexibilité: La sous-traitance. 45ième Congrès, 1990, p. 59-64

Les municipalités doivent maintenant puiser le maximum de rendement dans leurs ressources. Présentation de deux ententes conclues entre la Ville de Montréal et le Syndicat canadien de la fonction publique (section locale 301) pour transférer en régie des travaux qui étaient normalement exécutés en sous-traitance.

0973 LISTON, Terrence

Négocier la flexibilité: La polyvalence des emplois.

45ième Congrès, 1990, p. 65-69

La MIL Davie Inc. a pu conclure avec le Syndicat des travailleurs du chantier naval de Lauzon, une convention collective donnant plus de flexibilité à ses travailleurs. Présentation des principaux détails des changements qui ont été négociés pour atteindre une plus grande flexibilité de la main-d'oeuvre.

\section{BROUILIET, Normand}

Négocier la flexibilité: Réaction syndicale aux stratégies patronales.

45ième Congrès, 1990 , p. $70-81$

Plus que jamais en 1990, les fermetures, les licenciements et les mises à pied préoccupent les syndicats au plus haut point. La flexibilité dans l'emploi de la main-d'oeuvre, recherchée par une multitude d'entreprises, se traduit pourtant par le recours intensif aux employés à temps partiel ou au travail précaire. Les stratégies patronales consistent plutôt à éloigner le personnel de toute forme d'adhésion aux objectifs et aux stratégies de l'entreprise.

\section{MATHIEU, René}

Négocier la flexibilité: La recherche d'équité par la négociation.

45ième Congrès, 1990, p. 82-92

Le contexte économique actuel arriène les entreprises à rechercher de nouveaux moyens pour faire face à la concurrence. Le syndicat, de son côté, s'efforce de protéger la qualité de vie et la sécurité d'emploi des travailleurs. La flexibilité des conditions de travail et la flexibilité des relations de travail représentent une bonne approche qui permettrait aux deux parties de trouver des solutions satisfaisantes par la négociation.

0976 LE LOUARN, Jean-Yves

Les emplois atypiques et l'efficacité de la gestion des ressources humaines.

45ième Congrès, 1990, p. 93-103

Quatre points sont abordés dans cet exposé: 1) Les raisons pour lesquelles les entreprises, publiques ou privées, recherchent de plus en plus la flexibilité: 2) Les différentes formes de la flexibilité du travail et de la main-d'oeuvre; 3) Les gains et les coûts d'une stratégie de flexibilité des ressources humaines; 4) La stabilité d'emploi, une chance à saisir pour l'entreprise? - Références.

\section{BOUDREAULT, Pierre \\ GARON, Jacques PAGÉ, Lorainne PAQUETTE, Pierre}

Les emplois atypiques et l'efficacité de la gestion des ressources humaines - Commentaires.

45ième Congrès, 1990, p. 103-116

Commentaires sur l'exposé de M. Le Louarn, portant sur: 1) La recherche de la flexibilité par les entreprises; 2) Les formes de flexibilité du travail et de la main-d'oeuvre; 3) Les gains et les coûts d'une stratégie de flexibilité des ressources humaines; 4) La stabilité d'emploi. 
0978 BELLEMARE, Diane

Les emplois de l'avenir et les défis de la société.

45ième Congrès, 1990, p. 117-128

Le Conseil économique du Canada a entamé il y a déjà plus de deux ans une recherche devant essentiellement porter sur l'emploi et le secteur des services. Cet exposé présente les principaux résultats et recommandations de l'étude du Conseil économique du Canada, "L'emploi au futur", Ottawa, 1990.

0979 LAVALLÉE, Diane CHARLAND, Gaston LEPAGE, Brigitte MASSÉ, Henri MERCIER, Jean

Les emplois de l'avenir et les défis de la société - Commentaires.

45ième Congrès, 1990, p. 128-148

Commentaires sur l'exposé de Mme Diane Bellemarre, exposé présentant les principaux résultats et recommandations de l'étude du Conseil économique du Canada, 'L'emploi au futur", Ottawa, 1990. 


\title{
DICTIONNAIRE CANADIEN DES RELATIONS DU TRAVAIL
}

\author{
Deuxième édition
}

par Gérard DION

La deuxième édition du Dictionnaire canadien des relations du travail forme un ouvrage de 1024 pages.

Les nombreux usagers de la première édition y trouveront beaucoup plus qu'une simple mise à jour tenant compte des multiples changements survenus depuis dix ans dans le domaine des relations du travail.

En voici les principales caractéristiques:

- Un total de 19,422 entrées comprenant 2,552 nouveaux termes en français;

- Un choix élargi de termes et expressions pour répondre aux besoins des traducteurs;

- De nombreuses définitions révisées et améliorées afin de les rendre plus opérationnelles;

- Un index anglais-français de 12,812 entrées, complètement restructuré pour faciliter la consultation et donner la possibilité de trouver à la suite tous les mots, qu'ils soient seuls ou qu'ils soient éléments d'un syntagme;

- Une nouvelle partie présentant plus de 600 locutions ou maximes latines avec leur traduction en français et en anglais;

- Une mise à jour des annexes déjà existantes: sigles des organisations, statistiques syndicales, chronologie de la législation du travail et des événements importants;

- Quatre nouvelles annexes: liste de toutes les conventions et recom. mandations adoptées par l'Organisation internationale du travail depuis sa fondation; relevé de toutes les lois d'exception édictées par le gouvernement du Canada et ceux des provinces depuis 1955 pour faire face à des situations spéciales en relations du travail; textes de la Charte canadienne des droits et libertés et de la Charte des droits et libertés de la personne (Québec).

Tous ces éléments font de la deuxième édition du Dictionnaire canadien des relations du travail - déjà considéré comme un classique dans le domaine des relations professionnelles au Canada - l'ouvrage le plus complet du genre paru jusqu'à maintenant en langue française.

ISBN 2-7637-6975-6

1 volume relié-1024 pages: $\$ 65.00$

LES PRESSES DE L'UNIVERSITE LAVAL, C.P. 2447, QUÉBEC G1K 7R4 


\section{RÈGLES DE PUBLICATION}

ARTICLES: Ils peuvent être rédigés en français ou en anglais. Le manuscrit ne doit pas excéder 25 pages dactylographiées de $21,5 \mathrm{~cm}$ par $28 \mathrm{~cm}(81 / 2$ " x 11 ") à double espace sur un seul côté de feuille d'égale dimension avec une marge de $4 \mathrm{~cm}$ (11/2"). Quatre exemplaires du manuscrit sont requis. La soumission d'un article atteste du fait qu'il est présenté en exclusivité et que les droits de reproduction du texte ne sont pas déjà réservés. Chaque article est évalué anonymement par au moins deux personnes spécialisées dans le sujet traité. Leur évaluation guide la direction de la Revue, qui est responsable de la décision finale.

RÉFÉRENCES BIBLIOGRAPHIQUES: Elles seront identifiées dans le texte par le nom et l'année et, s'il y a lieu, la page. Exemple: (Dion 1986:219). Les ouvrages cités seront placés à la fin du texte (après les notes) par ordre alphabétique et pour chacun des auteurs par ordre de date de publication. Ils seront dactylographiés à double interligne en respectant le protocole bibliographique suivant:

Dion, Gérard. 1986. Dictionnaire canadien des relations du travail. $2^{\mathrm{e}}$ éd. Québec: Presses de l'Université Laval.

GuÉRin, Gilles et Michel Hébert. 1990. «Les obstacles rencontrés par des personnes de 45 à 64 ans à la recherche d'un emploi». Relations industrielles, vol. $45, \mathrm{n}^{\circ} 2$, 235-267.

NOTES: Compte tenu de la méthode de références utilisée, leur usage doit être limité. Elles seront numérotées de façon continue pour tout l'article et présentées ensemble à la fin du texte, à double interligne.

TABLEAUX ET GRAPHIQUES: Leur nombre doit être limité. Ils doivent être présentés clairement et correctement, sous forme de prêt à photographier, sur des feuilles séparées, numérotées consécutivement en chiffres arabes (v.g. Tableau 1, Graphique 1) et porter un titre. L'endroit précis du texte où ils doivent être placés doit être indiqué.

ÉQUATIONS MATHÉMATIQUES OU ÉCONOMÉTRIQUES: Leur usage doit être réduit au minimum et seuls leurs résultats doivent être discutés dans le texte. Des annexes techniques doivent aussi être disponibles sur demande.

PRÉCIS: Chaque manuscrit doit être accompagné d'un bref précis (enviton 100 mots).

RÉSUMÉ: Un résumé proportionné à la longueur du manuscrit doit être soumis (entre 1/12 et 3 pages). Celui-ci devrait être en anglais, si l'original est en français; et en français si l'original est en anglais.

NOTES BIOGRAPHIQUES: Chaque signataire d'un manuscrit doit faire parvenir ses notes biographiques: nom, titre, fonctions actuelles, adresse, numéros de téléphone et de télécopieur.

Pour renseignements additionnels, s'adresser à:

La direction, revue Relations industrielles, Université Laval, Québec, G1K 7P4, Canada, téléphone (418) 656-2468, télécopieur (418) 656-3175 


\section{NOTES FOR CONTRIBUTORS}

ARTICLES: They may be written in English or French, and should not exceed 25 pages in length $(81 / 2 \times 11$ "). Four copies of the manuscript should be submitted in typescript, double-spaced with $1_{1 / 2}$ inch margins, typed on only one side of the page. Submission of a paper implies that it is not already protected by copyright and is not currently under consideration by another journal. All manuscripts are reviewed by at least two anonymous referees. The final decision on publication is made by the Editor.

REFERENCES: They should be identified in the text by last name of author, year of publication, and pagination where appropriate. Example: (Thompson 1990:217). References mentioned in the text must appear alphabetically in a list at the end of the article, typed in double spacing in the following format:

Anderson, John C., Morley Gunderson, and Allen Ponak. 1989. UnionManagement Relations in Canada. 2nd ed. Don Mills, Ont.: Addison-Wesley Publishers.

Swimmer, Gene. 1990. "Gender Based Differences in Promotions of Clerical Workers." Relations industrielles/Industrial Relations, Vol. 45, No. 2, 300-309.

FOOTNOTES: With the author-date system, they should be restricted and numbered consecutively throughout the manuscript, typed double-spaced on a separate sheet and placed at the end of the article.

TABLES AND CHARTS: Their number should be limited, and they must be carefully and clearly presented. They should also be numbered consecutively in arabic numerals throughout the manuscript, and each must have a title. Their exact location in the text, moreover, should be indicated. Charts should be presented in a camera-ready form.

MATHEMATICAL AND ECONOMETRIC EQUATIONS: They should be kept to a minimum and restricted to their results. Technical appendices should be available to readers on request.

ABSTRACT: Authors must furmish a brief abstract (around 100 words).

SUMMARY: A summary covering the main points of the argument presented, and proportional to the article's length (11/2-3 typed pages), should be enclosed. This summary should be in French if the original text is in English and vice versa.

AUTOBIOGRAPHICAL NOTE: Authors should furnish details concerning their name, titles, present position, address, telephone and fax numbers.

For further information contact:

The Editor, Relations industrielles/Industrial Relations, Laval University, Québec, Canada, G1K 7P4, telephone (418) 656-2468, fax (418) 656-3175 